\title{
Raphaëlle Soulignac
}

Les Scories de forge

du Pays dogon (Mali)

Entre ethnoarchéologie, archéologie expérimentale et archéométrie

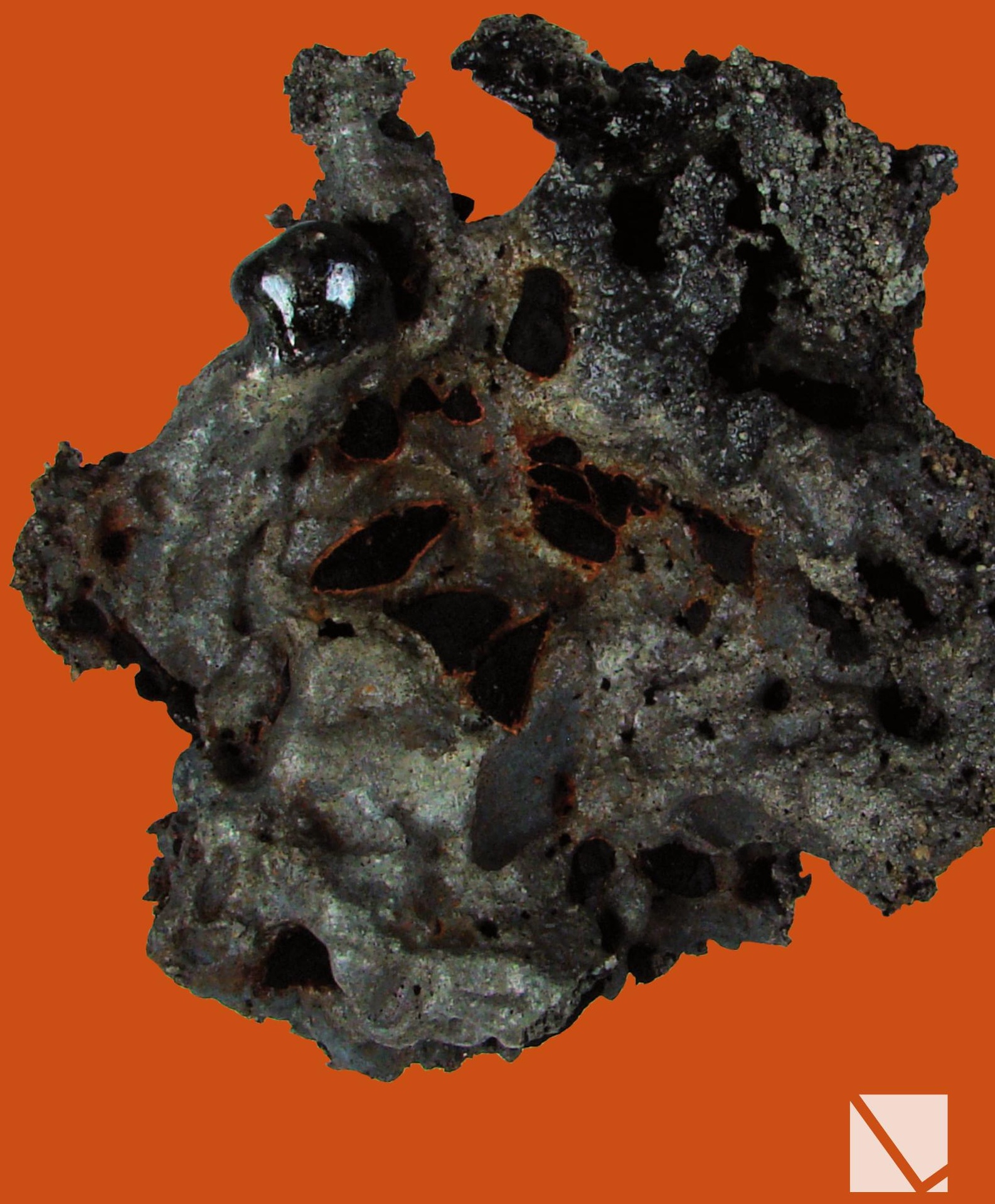




\section{Les Scories de forge du Pays dogon (Mali)}

Entre ethnoarchéologie, archéologie expérimentale et archéométrie 
(C) 2017, Raphaëlle Soulignac and LIBRUM Publishers \& Editors LLC, Hochwald/Basel (Switzerland)

All rights reserved.

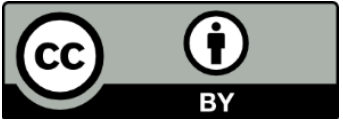

ISBN 978-3-9524542-9-9

Première de couverture :

Scorie de forge de Kobo (S022).

Dernière de couverture :

Haut : village de Kobo.

Bas, gauche : feu du foyer de forge de Kobo.

Bas, centre : forgeage, soudure à Kobo.

Bas, droite : forgeage d'une houe à Doundé.

Photographies : R. Soulignac

www.librum-publishers.com

www.LIBRUMopen.com 
Département des Géosciences

Université de Fribourg (Suisse)

\title{
Les Scories de forge du Pays dogon (Mali)
}

\section{Entre ethnoarchéologie, archéologie expérimentale et archéométrie}

\author{
THESE DE DOCTORAT \\ $\mathrm{n}^{\circ} 1836$ \\ Présentée à la Faculté des Sciences de l'Université de Fribourg (Suisse) \\ pour l'obtention du grade de Doctor rerum naturalium \\ par
}

Raphaëlle Soulignac

de

Bernède (France) et Foumban (Cameroun) 


\section{Note au lecteur :}

A ce manuscrit, s'ajoute un catalogue complet des Scories de forge, qui recense toutes les caractéristiques de ces matériaux. Ce catalogue, comme annexe à la thèse, est disponible librement :

1- Sur le portail OpenAccess de Librum Publishers \& Editors :

http://librum-publishers.com/librum-open/

2- Sur un serveur de l'Université de Fribourg, en deux parties :

http://www.unifr.ch/geoscience/geology/assets/files/Site\%20Science\%20de\%20la\%20terre/PhD\%20thesis/Listetheses-ST-19.09.2016.pdf 
Acceptée par la Faculté des Sciences de l’Université de Fribourg (Suisse) sur la proposition de

Prof. Dr. Vincent Serneels Dr. Ing. Philippe Dillmann Prof. Dr. Eric Huysecom Prof. Dr. David Killick Dr. Marc Leroy Prof. Dr. Reynald Delaloye
Département des Géosciences, Fribourg (Suisse) Laboratoire Métallurgie et Cultures, Saclay (France) Département de Génétique et Evolution, Genève (Suisse) Département d'Anthropologie, Tucson (USA) Laboratoire d'Archéologie des Métaux, Nancy (France) Département des Géosciences, Fribourg (Suisse)
Directeur de thèse Expert

Expert

Expert

Expert

Président du Jury

Fribourg, le 16 avril 2014

Le Directeur de thèse

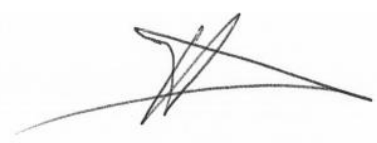

Prof. Dr. Vincent Serneels
Le Doyen

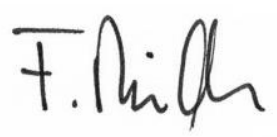

Prof. Dr. Fritz Müller 


\section{Table des matières}

Résumé......................................10

Abstract...........................................11

Remerciements...............................13

PARTiE 1 . PrÉAMbule 15

1.1. Pourquoi et comment mener cette étude ? 17

1.1.1. Constats et questionnements ..................... 17

1.1.2. Choix de la démarche du travail................. 22

1.1.3. Déroulement du travail .............................. 23

1.1.4. Cadre scientifique de la recherche............. 24

1.1.5. Structure du manuscrit ............................. 25

1.2. Aspects techniques de la forge..................... 27

1.2.1. Chaîne opératoire de la sidérurgie ancienne 27

1.2.2. Atelier de forge ....................................... 28

1.2.3. Travail du fer à la forge ............................. 29

1.2.4. Déchets de forgeage ................................. 30

1.3. Pays dogon et métallurgie du fer ............... 35

1.3.1. Carte d'identité.......................................... 35

1.3.2. Métallurgie du fer...................................... 36

1.3.3. Diversité des cultures dogon ...................... 37

1.3.4. Forgerons et société.................................... 38

PARTIE 2 . LES ATELIERS de FORGE ................ 39

2.1. Introduction aux ateliers .............................. 41

2.1.1. La forge, espace de travail et de croyances 41

2.1.2. Présentation des ateliers ............................. 41

2.1.3. Diversité des installations de forge ........... 42

2.1.4. Outils fabriqués ......................................... 43

2.2. Forge de Fiko .................................................. 44

2.2.1. Forgerons ….............................................. 44

2.2.2. Atelier ..................................................... 44

2.3. Forge de Kakoli .......................................... 48

2.3.1. Forgerons ...................................................... 48

2.3.2. Atelier ....................................................... 48

2.4. Forge de Kobo ............................................... 51

2.4.1. Forgerons ……….................................... 51

2.4.2. Atelier .................................................... 51

2.5. Forge de Doundé ........................................... 54
2.5.1. Forgerons …............................................. 54

2.5.2. Atelier .................................................. 54

2.6. Comparaison entre les ateliers de forge .... 57

PARTIE 3 . LES OPÉRATIONS DE FORGEAGE ... 61

3.1. Présentation des matériaux de la forge ..... 63

3.1.1. Matière première ferreuse ......................... 63

3.1.2. Le combustible .......................................... 66

3.1.3. Les matériaux siliceux............................... 67

3.2. Chaîne opératoire d'une lame de houe...... 68

3.2.1. Etape $1:$ le préformage .............................. 69

3.2.2. Etape $2:$ le formage ................................. 71

3.2.3. Etape 3 : les finitions................................ 73

3.2.4. Incidents au cours du travail ...................... 73

3.2.5. Comparaison des étapes de travail ............ 75

3.3. Durée du travail ........................................... 77

3.3.1. Durée totale du travail ............................... 77

3.3.2. Rapport chauffe/martelage (C/M) ............. 79

3.3.3. Type de barre ........................................... 81

3.3.4. Teneur en carbone .................................... 83

3.3.5. Synthèse des différents paramètres

influençant la durée du travail.............................. 85

3.4. Le produit : la $d a b a$...................................... 87

3.4.1. Présentation de la daba ............................. 87

3.4.2. Variabilités morphométriques ................... 88

3.4.3. Les défauts dans le métal ........................... 89

3.4.4. Métallographie ......................................... 91

3.5. Forgeage et perte en fer ................................. 96

3.5.1. Mesure de la perte en fer ............................ 96

3.5.2. Forgeage d'une barre de fer ...................... 97

3.5.3. Forgeage de deux barres de fer ............... 101

3.5.4. Forgeage avec incidents ......................... 101

3.5.5. Paramètres influençant la perte en fer ...... 103

3.6. Comparaison des opérations de forgeage 104

PARTIE 4 . LES DÉCHETS DE FORGEAGE ........ 105

4.1. Techniques de laboratoire et Méthodes de calculs .................................................................. 107

4.1.1. Préparation des échantillons .................... 107 
4.1.3. Méthode de calcul de surface...

4.2.1. Minéralogie des silicates 115

4.2.2. Minéralogie des oxydes 118

4.2.3. Chimie 119

4.3. Matériaux et Structure dans les scories .. 122

4.3.1. Description générale du corpus de scories 122

4.3.2. Morphométrie 124

4.3.3. Matériaux et minéralogie

4.3.4. Conditions de forgeage 136

4.3.5. Stratification 137

4.3.6. Comparaison des groupes de scories 140

4.4. Différentes contributions à la formation des scories ..................................................................... 143

4.4.1. Matière première métallique ................... 143

4.4.2. Combustible ........................................... 151

4.4.3. Inclusions des barres de fer ...................... 153

4.4.4. Ajouts siliceux........................................ 155

4.4.5. Apport chimique par atelier .................... 156

4.4.6. Récapitulatif des apports dans les scories 162

4.5. Relations entre forgeages et scories 164

PARTIE 5 . CONCLUSION................................ 167

PARTIE 6 . ANNEXES .................................. 175

PARTIE 7 . BIBLIOGRAPHIE 199 


\section{Résumé}

De très nombreux sites archéologiques livrent des scories, déchets résultant du travail du fer à la forge. De nombreuses études leur sont consacrées, mettant en évidence une importante variabilité. Ces différences reflètent la variabilité des conditions de travail à la forge mais restent très délicates à interpréter. Notre travail s'appuie sur une approche ethnoarchéologique sur le terrain et une étude archéométrique au laboratoire et contribue à une meilleure compréhension des facteurs qui influencent la formation des scories de forge.

La première phase du travail s'est déroulée dans quatre villages différents (Fiko, Kakoli, Kobo, Doundé) du Pays dogon (Mali), appartenant à deux traditions techniques distinctes (Mombo, Tomo). Une centaine d'opérations de forgeage de lames de houe organisées dans des conditions contrôlées ont été enregistrées selon un protocole défini. Les matières premières, les déchets (scories et battitures) et les produits ont été échantillonnés.

$\mathrm{Au}$ cours de la seconde phase du travail, ces matériaux ont été étudiés au Département des Géosciences de l'Université de Fribourg. Les méthodes utilisées se basent sur une étude morphométrique, ainsi que l'utilisation de plusieurs méthodes de caractérisation physico-chimiques, en particulier les analyses chimiques par fluorescence des rayons $\mathrm{X}$, la diffraction des rayons $\mathrm{X}$, la microscopie optique et la microscopie électronique à balayage.

Dans la région Mombo, l'atelier de forge est un hangar regroupant plusieurs unités de travail dont les composantes sont mobiles. Dans la région Tomo, l'atelier est un abri qui regroupe une seule unité de travail dont les composantes sont fixes.

L'observation du travail des forgerons a permis de comprendre la chaîne opératoire de fabrication des lames de houe, depuis la matière première jusqu'au produit final. Le préformage est une étape très variable qui dépend de la matière première utilisée, en particulier la taille et le nombre de barres (1 ou 2). Cette étape est suivie du formage et des finitions qui sont très peu variables. Au préformage, les températures sont élevées et la chauffe est très importante. Il existe plusieurs manières de souder deux barres de fer. L'emploi d'ajouts siliceux est réservé aux ateliers de la région Tomo. Les trempes favorisent l'apparition de défauts dans le métal. Au formage, les températures sont moyennes à élevées et la chauffe reste importante. Aux finitions, les températures sont moyennes à basses et le martelage est important.

Les scories se forment dans le foyer de forge par l'accumulation de différents matériaux: la matière première (fer), le combustible (charbon), le foyer (paroi, tuyère, sol) et des ajouts volontaires siliceux. La perte en fer est la quantité de fer de matière première métallique perdue dans le foyer de forge au cours du travail, sous la forme de croûtes d'oxydes de fer, battitures et particules de fer métallique. La perte en fer est un processus complexe variable (8-53\%) qui dépend des nombreuses conditions de travail (température, durée du travail, forme de la barre, nombre de soudures etc).

Les travaux de forge rapides, à partir d'une seule barre de fer, génèrent des scories argilosableuses SAS et informes SI. Les SAS comportent 80-100\% de matériaux siliceux, roches et verre, et elles sont pauvres en fer : elles contiennent moins de $2 \%$ de la masse du fer initial.

Les scories SI, de Doundé, sont des petites pièces $(<4 \mathrm{~cm})$ de différentes natures qui contiennent beaucoup de minéraux non ferreux comme augite, leucite, akermanite ou hedenbergite. Elles sont le reflet de conditions de formation difficiles dans le foyer.

Les travaux de forge à partir de deux barres de fer sont plus longs et ils génèrent des scories grises denses (SGD) et ferreuses rouillées (SFR). Environ 5 à $15 \%$ de la masse du fer initial est perdu dans la scorie. Les SGD comportent entre 50 et $100 \%$ de fayalite (ou kirschstéinite, équivalent calcique) et en moyenne $10 \%$ de particules ferreuses en coupe. A Kakoli, les scories comportent de nombreux oxydes de fer et un apport important de calcium à cause d'un ciment utilisé pour enduire le foyer. Les scories SFR proviennent surtout de forgeages ratés ou avec des trempes. Elles comportent jusqu'à $90 \%$ de fer en coupe et de nombreux oxydes de fer. Quelques cas de fonte sont observés. 


\begin{abstract}
\end{abstract}
Numerous archeological sites have produced slags originating from iron smithing workshops. These slags have been shown to exhibit great variability which is generally interpreted as reflecting different iron smithing techniques, but this interpretation remains tentative. This work is based on an ethno-archaeological approach in the field and archaeometrical laboratory studies and contributes to a better understanding of the factors influencing the formation of smithing slags.

The first step of the work took place at four different villages (Fiko, Kakoli, Kobo, Doundé) within Dogon Country, Mali, that reflect two different technical traditions (i.e., Mombo, Tomo). About a hundred smithing works of hoe blades, were recorded according to a defined protocol. Raw materials, waste (e.g., slags and hammerscale) and final products were sampled as well.

During the second step of the work, these specimens were studied at the Department of the Geosciences of the University of Fribourg. The methods include a morphometric study and characterization of the sample using a selection of physical and chemical methods, in particular, chemical analyses by X-ray fluorescence, X-ray diffraction, optical microscopy, and scanning electron microscopy.

In the Mombo area, the workshop is a large shed containing several working areas with mobile structures. In the Tomo area, the workshop is a shelter containing a single working area with fixed structures.

The observation of the work of the smiths helped us to understand the chaîne opératoire of hoe blades, from the raw material to the production of the finished blade. Preforming is a highly variable step in the production of hoes as it depends on the raw materials being utilized, in particular the size and number of bars (one or two) being used. This step is followed by the forming and the finishing steps, which show little variability. In the preforming step, temperatures are high and heating is important. There are several ways to weld two iron bars. The use of siliceous additions is restricted to the Tomo workshops. Quenching increases the formation of defects in the metal. During the forming step, temperatures are intermediate to high and heating therefore remains important. During the finishing step, temperatures are low to average and hammering is important.

Slag is formed at the bottom of the hearth by the accumulation of several materials : raw material (iron), fuel (coal), hearth (lining, tuyere, soil), and siliceous additions. Iron is lost from the raw material into the hearth forge during smithing work in the form of iron oxides crusts, hammerscale, and particles of metallic iron. Loss of iron is a complex and variable process $(8-53 \%)$ that depends on various work conditions (temperature, duration of the work, shape of the bar, number of welds, etc).

Fast smithing of a single bar of iron generates sandy-clayey slags (SAS) and shapeless slags (SI). SAS slags contain 80 to $100 \%$ of siliceous materials, such as rocks and glass, and they are poor in iron. They contain less than $2 \%$ of the mass of the initial iron bar.

SI slags from Doundé are small $(<4 \mathrm{~cm})$ and contain a lot of non-ferrous minerals, such as as augite, leucite, åkermanite, or hedenbergite. They reflect the difficult conditions of formation within the hearth.

Smithing works based on of two iron bars are longer and generate dense grey slags (SGD) and ferrous rusty slags (SFR). In this case, approximately $5-15 \%$ of the mass of the initial iron is lost in the slag.

SGD slags contains between 50 and $100 \%$ fayalite (or kirschsteinite, the $\mathrm{Ca}$ rich equivalent) and an average of $10 \%$ ferrous particles. Slags from Kakoli contain iron oxides and a large amount of calcium originating from cement used to coat the hearth.

SFR slags results especially from failed smithing works or from quenchings. They contain up to $90 \%$ iron and iron oxides. In some cases, cast iron is observed.

Traduction Walter Joyce 


\section{Remerciements}

C'est par un premier heureux hasard que, devant faire un choix de spécialité pour mon Master, je tombe sur le Centre de Recherches en Physique Appliquée à l'Archéologie (Université de Bordeaux, France) : ça y est, enfin une application concrète et très intéressante des sciences dures ! Cela m'évitera, sans regret, de jouer avec un laser, un plasma ou une onde électromagnétique dans une cave sombre pour la prochaine décennie ...

Par un second heureux hasard, je tombe sur une annonce de recrutement d'un(e) assistant(e) universitaire: métal, fer, scorie, terrain, Afrique, Suisse, laboratoire. Et me voilà embarquée pour une longue aventure !

Mes remerciements se dirigent en premier lieu vers Vincent Serneels, mon Directeur de thèse, qui m'a accueillie au sein du Département des Géosciences, dans son groupe d'Archéométrie, en me confiant la délicate mission d'explorer de plus près ces énigmatiques scories. De nature indépendante, il m'a laissée mener mon travail à ma convenance tout en gardant un œil bienveillant et recadrant son élève de temps en temps ! Il est de ces personnes très occupées, et malgré cela toujours présentes quand il le faut. Merci pour ces discussions toujours enrichissantes, merci pour tous les services personnels et professionnels rendus tout au long de ces années. Je n'oublierai pas !

J'adresse mes plus vifs remerciements aux membres du projet «Peuplement humain et paléoenvironnement en Afrique de l'Ouest » avec qui j'ai passé d'inoubliables moments au Pays dogon, dans un décor de paysages grandioses vertigineux. Merci à ceux qui m'ont aidée, en particulier Ancé mon infaillible interprète, Sékou de Fiko et Michel Mauvilly. A Caroline qui m'a prise sous son aile...elle, qui est la plus dure négociatrice de prix de tout l'Afrique de l'Ouest !

Je tiens également à remercier tous les membres du Jury qui ont relevé le challenge de relire et d'évaluer ce travail en un temps très court !

J'adresse toute ma gratitude au personnel logistique et technique du Département: Nicole, Patrick, Paulo, Alexandre. Je tiens aussi à remercier l'ensemble du corps professoral auprès de qui j'ai pu apprendre un peu de Géologie en excursions, en TP ou en discussions ! J'adresse des remerciements très spéciaux à Bernard et Ildiko mes irremplaçables voisins de bureau.

Mes remerciements vont aussi à tous les doctorant(e)s et post-docs du labo, passés et présents, qui ont rendu mes journées de travail agréables, ils se reconnaîtront. Je tiens à remercier Romain qui a trié et pesé mes battitures, Seb si passionnant, Akram et Silvia avec qui j'ai entretenu des discussions toujours très intéressantes, spécialement au moment des repas !

Ma gratitude et mon amitié vont à mon ex-co-bureau, certes parfois envahissant: le Yack (Martin). J'ai pu observer l'animal et apprendre un peu plus de son domaine obscur, la Tectonique. Finalement il se fait vite apprivoiser avec un café et nous voilà à refaire le monde des heures durant...

Fraîchement débarquée dans le groupe d'Archéométrie, je me suis très rapidement nouée d'amitié avec deux compatriotes Mama (Maëlle) et Clairette (Claire). Une longue histoire commencée à l'Uni et terminée dans une zone finalement très restreinte, entre le Court-Chemin et la Neuveville : carnaval des Bolzes, paniers de légumes à partager, livraisons de yaourts frais, auberge des Boulangers, café du Paon, grillades à la Sarine, soirées viande, la Farandole dans le bus, cat-sitting, virées Ikéa ou Emmaüs, ramassage de courrier ou autre arrosage de plantes. Merci de votre soutien et de votre amitié indéfectible, les filles !

A ceux qui m'ont accompagnée de près ou de loin, tout au long de mon cursus universitaire : Yo le binôme, Lolo le trinôme, Ghis, Fifi l'ami des toutpetits et Jean-Phi. Et à M. Lucbert de Langon qui m'a donné l'envie d'explorer la Physique !

Merci à mes parents qui m'ont toujours laissée une entière liberté, pour leur soutien moral et financier pendant mes études, et pour leur relecture de ce travail. Merci à mes sœurs Marie et Sara qui ont toujours une bonne oreille et de bons conseils !

Enfin, où en serais-je sans Sébastien... 
Préambule 


\subsection{Pourquoi et COMMENT MENER CETTE Étude ?}

Cette thèse de doctorat a été effectuée au Département des Géosciences à l'Université de Fribourg, sous la direction scientifique du Professeur Vincent Serneels, entre 2007 et 2014.

Le point central de cette recherche concerne une classe de mobilier archéologique que sont les scories de forge : ce sont les déchets qui se forment au fond du foyer, au cours du forgeage traditionnel d'objets en fer. Du point de vue de leur fonction, il s'agit de déchets et ils sont donc rejetés après le travail de la forge, dans des dépotoirs ou des fosses à proximité du lieu de forgeage.

C'est ainsi que, des siècles plus tard, les scories sont retrouvées lors des fouilles archéologiques d'anciens ateliers de forge, en Europe ou en Afrique. Ils sont d'ailleurs souvent, avec le sol de forge sombre et magnétique, les seuls éléments matériels qui subsistent à ces emplacements, témoignant d'une activité ancienne du travail du fer. Les scories de forge sont ainsi les derniers témoins de ce travail ancien et elles renferment potentiellement de riches informations.

Leur étude est donc d'une importance considérable pour l'interprétation des vestiges archéologiques, la compréhension de leurs modes de formation dans le foyer, ainsi que la compréhension des techniques de travail des sociétés anciennes.

\subsubsection{Constats et questionnements}

Les premières études concernant les scories de forge ont commencé il y a environ trois décennies, lorsque l'hypothèse a été émise que ces matériaux pouvaient avoir un mode de formation complètement différent d'autres types de déchets métallurgiques déjà étudiés par ailleurs, en particulier les scories de réduction. L'hypothèse a été émise qu'il s'agissait d'une gamme de matériaux totalement distincts, par leur mode de formation mais aussi par leurs propriétés morphométriques et physico-chimiques (McDonnell 1984, 1986, 1991).

Depuis ces temps, les scories de forge font l'objet d'études systématiques que cela soit en Allemagne, en Grande-Bretagne, en Suisse (liste non exhaustive: Anderson et al. 2003; Anderson \& Castella 2007; Anderson et al. 2000; Berti Rossi \& May Castella 2005; Eschenlohr et al. 2007; Hofmann
Rognon 2005; König 2011; Mauvilly et al. 1998; Nuoffer \& Menna 2001; Perret 2009; Reymond et al. 2009; Schucany 2006; Serneels 1993, 2001, 2006, 2011; Serneels \& Doswald 2005; Serneels et al. 2005; Serneels \& Perret 2007) ou bien en France (par exemple : Bauvais 2007b; Bauvais \& Fluzin 2005; Bauvais et al. 2007; Berranger \& Fluzin 2007; Chardron-Picault \& Pernot 1999; Dunikowski et al. 1996; Faivre et al. 1998; Gruat et al. 2007; Le Carlier et al. 2007; Leroy \& Merluzzo 2007; Mangin et al. 2000a; Mangin et al. 2000b; Menez et al. 2007; Milcent 2007; Orengo et al. 2000; Pagès et al. 2005; Serneels et al. 1999).

Toutes ces études montrent que les scories présentent une grande variabilité macroscopique, chimique et minéralogique qui témoigne en réalité de l'immense diversité des travaux de forge. Les recherches actuelles visent donc naturellement à établir un lien entre les critères intrinsèques des scories que l'on peut mesurer facilement, et les activités passées de la forge que l'on cherche à caractériser.

\section{Formation et stratification des scories}

Avant de commencer un travail à la forge, le forgeron vérifie le bon état de la soufflerie, du foyer et de la tuyère ; il rassemble ses outils et le charbon près de lui et vidange son foyer.

Au cours du forgeage, différents matériaux sont introduits dans le foyer volontairement ou non (Figure 1). Sous l'effet de la chaleur, ces matériaux subissent une fusion plus ou moins importante et s'accumulent à l'aplomb de la tuyère. A la fin du travail, lors du refroidissement du foyer, ils se solidifient pour former une scorie (McDonnell 1991).

La scorie ne se forme que si une quantité suffisante de matériaux est introduite dans le foyer avec un apport important de chaleur : au moins une température de plus de $700{ }^{\circ} \mathrm{C}$, maintenue suffisamment longtemps (Figure 9).

Quels sont ces matériaux et d'où viennent-ils ? 


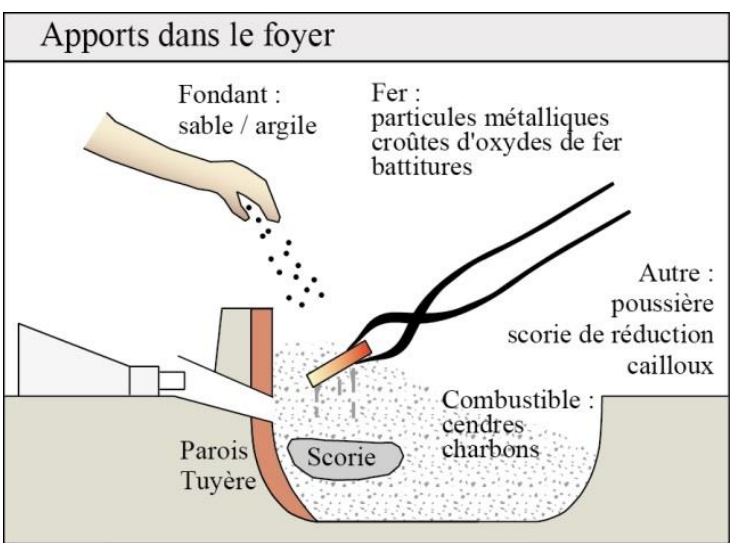

Figure 1 - Apports dans le foyer qui contribuent à la formation de la scorie. D'après Serneels \& Perret 2003 : figure 2

1 - Les analyses chimiques de scories montrent que la barre de fer travaillée est le matériau qui contribue le plus à la formation de la scorie. Cette contribution est complexe: des croûtes d'oxydes $\left(\mathrm{Fe}_{\mathrm{x}} \mathrm{O}_{\mathrm{y}}\right)$, des particules de métal $\left(\mathrm{Fe}_{\mathrm{M}}\right)$ qui seront plus ou moins oxydées au cours du travail, et des battitures provenant de l'enclume $\left(\mathrm{Fe}_{\mathrm{x}} \mathrm{O}_{\mathrm{y}}\right.$, contribution minime). S'il s'agit d'un fer ancien, l'apport des inclusions de scorie de réduction est à prendre en compte.

2 - Le second apport majoritaire dans les scories provient du combustible utilisé : il s'agit des pièces de charbon de bois et de ses cendres.

3 - Des apports optionnels et involontaires concernent : les éléments en argile du foyer (parois et tuyère) dans le cas où la température élevée du foyer puisse provoquer soit une fusion partielle de ces matériaux soit un détachement; une pollution vis-à-vis des autres métaux travaillés dans ce même foyer ; des fragments de roche et cailloux ajoutés en même temps que les charbons.

4 - Des apports optionnels et volontaires : des ajouts siliceux ajoutés par le forgeron pour nettoyer la surface du fer lors d'une soudure et pour protéger le fer d'une oxydation excessive.

Ainsi, les scories sont des matériaux stratifiés, la stratification faisant référence à une superposition des différents matériaux qui la composent, au cours du forgeage. Il est fréquent d'observer des scories qui comportent des particules de métal en partie basse des scories et des matériaux argilo-sableux en partie haute.
Ce travail cherchera à mieux comprendre les modalités de formation des scories; cette stratification représente-t-elle :

- une évolution du travail du forgeron dans le temps (la première étape du travail formant la base de la scorie, la dernière étape formant le haut de la scorie)?

- une séparation des matériaux par leur densité (matériaux lourds en bas et légers en haut)?

\section{Morphologie des scories}

Les scories de forge les plus typiques sont globalement hémisphériques (Figure 2). On parle de «scorie en forme de calotte», de «culot», de «culot plano-convexe » ou de «culots de forge » (Leroy $1997: 189$; Serneels et al. 2004 : 101). La notion de polarité est importante pour les scories car elles comportent une surface supérieure et une surface inférieure. La face supérieure est concave, plane ou convexe ; parfois il existe une dépression due au flux d'air de la tuyère, ou bien un bourrelet argilo-sableux. La surface inférieure est généralement plate ou convexe si les scories se forment en contact direct sur la sole du foyer, parfois irrégulière.

Cependant, une autre morphologie récurrente est observée. Il s'agit de scories de petite taille présentant le plus souvent une absence de polarité. Ces scories sont généralement nommées «informes » (Leroy 1997 : 193 ; Perret 2002 : 36-39 ; Ploquin et al. 1999 : 177). Ce terme n'est cependant pas tout-àfait adéquat car certaines pièces ont bien une forme : en rognon, en nodule, en bille, en coulure ou bien en «crispies». Ces scories informes sont décrites comme étant le résultat d'une scorie formée dans des conditions qui ne sont pas habituelles. Lesquelles ?

Différentes mesures de la scorie sont à prendre en compte pour décrire la pièce : grande dimension (longueur), petite dimension (largeur) et hauteur maximale (Figure 2), la hauteur, le poids. En déterminant la position de la soufflerie et/ou du bord du foyer, l'ellipticité peut être calculée : l'élongation est parallèle à la longueur ou bien à la largeur de la pièce. Il en est de même pour le facteur d'aplatissement. D'autre part, plus le flux d'air est fort et plus la scorie aura tendance à se former loin de la paroi du foyer.

Face à ces critères macroscopiques, nous chercherons à déterminer :

- si la morphologie des scories est liée à la forme du foyer, 


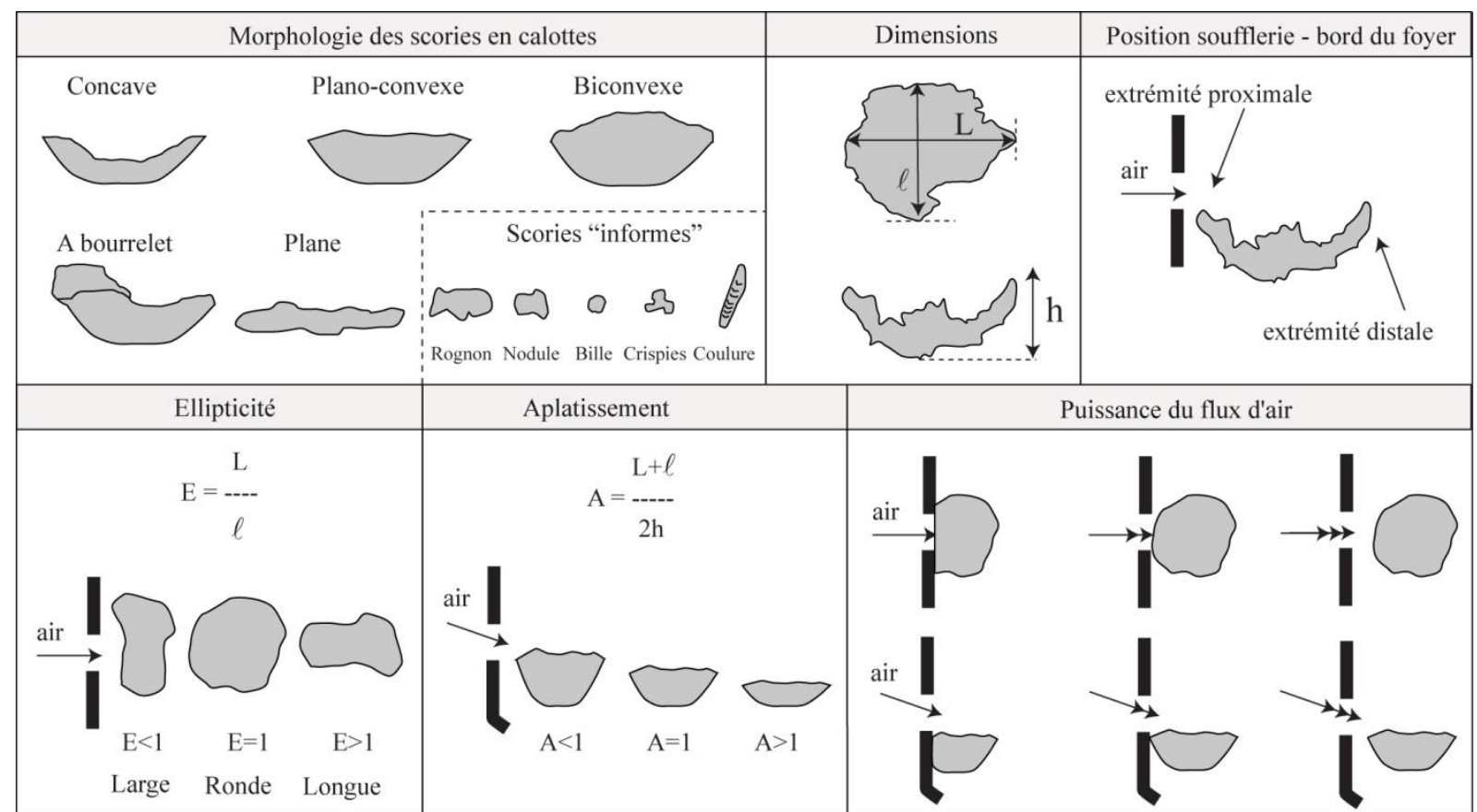

Figure 2 - Critères morphologiques généralement pris en compte lors de l'étude de scories de forge : morphologie, dimensions, position de la soufflerie par rapport au bord du foyer, ellipticité, aplatissement des pièces et puissance du flux d'air D'après Serneels \& Perret $2003: 474$.

- si le volume des pièces est dépendant ou non de la quantité de travail fourni (temps, intensité, nombre de forgeages successifs),

- sous quelles conditions de travail (foyer, température etc) se forment les scories en calotte,

- dans quels cas se forment les scories « informes ».

\section{Formation du bourrelet argilo-sableux}

Les scories à bourrelet, fréquemment décrites, représentent également un type de stratification (Figure 2). Le bourrelet est généralement vitreux et comporte des pièces de roche (cailloux, sable) incomplètement fondues. Il est toujours situé audessus de la pièce mais jamais en dessous. Une interprétation est proposée concernant la formation des scories surmontées d'un bourrelet sableux : elles seraient le résultat d'une session de forgeage à chaud suivie d'une phase de finitions plus courte pendant laquelle le forgeron jette du sable dans son foyer afin de protéger son outil d'une oxydation excessive.

Le mode de formation du bourrelet argilosableux reste à ce jour obscur et cette recherche vise à éclaircir cet aspect important des scories. Nous chercherons à identifier :

- durant quelle séquence du travail se forme le bourrelet,

- quelles conditions du travail (température du foyer etc) sont requises pour sa formation.

\section{Culots multiples}

Les dépotoirs de forge délivrent souvent des pièces formées de plusieurs paquets de scories empilés les uns sur les autres. Ces culots multiples résultent de plusieurs forgeages successifs, et se forment si le foyer n'est pas vidangé avant de débuter le travail. La scorie la plus récente recouvre alors la scorie plus ancienne (Mauvilly et al. 1998: 151; Serneels \& Perret 2007 : 140).

Cette stratification est plus ou moins marquée et plusieurs indices de délimitation entre les paquets de scorie peuvent être observés: des fines lignes millimétriques de bulles ou des cavités allongées (Dunikowski et al. 1996: 105), deux séquences différentes de cristallisation associées d'un changement de minéralogie (Bauvais 2007b : fig. 65) ou bien la présence d'un niveau argilo-sableux et/ou de charbons (Bauvais \& Fluzin 2005 : 122).

Comprendre les modes de formation des culots multiples peut permettre de mieux caractériser le travail du forgeron. Ce travail a pour but de comprendre la formation des culots multiples :

- Dans quelles conditions de travail (forgeage chaud/froid, plusieurs forgeages par jour/ un forgeage par jour, foyer vidangé ou non vidangé etc) ?

- Peut-on toujours affirmer que plusieurs forgeages successifs entrânent systématiquement la formation de ces culots multiples? 


\section{Nature des matériaux}

Les études archéométriques des scories de forge montrent qu'il s'agit de matériaux fortement hétérogènes. On remarque qu'entre les matériaux majoritaires qui composent les scories, les nuances varient fortement, montrant une incroyable diversité des scories de forge. La classification proposée à la figure 3 a été utilisée sur plusieurs sites en Suisse (Anderson et al. 2003, Perret 2009, Mauvilly et al. 1998, Tauber 1998).

Si l'apport prédominant provient de matériaux riches en silice (parois, tuyères, ajouts), alors se forment des scories argilo-sableuses (SAS). Ces matériaux sont complètement fondus, soit partiellement fondus soit non fondus. Ces matériaux sont soit complètement fondus (couleur noirâtre, bleuâtre ou verdâtre, aspect réfléchissant vitreux), soit partiellement fondus (couleur claire, aspect granuleux) soit non fondus (grains de quartz, cailloux centimétriques). Les scories SAS sont décrites comme résultant de travaux au cours desquels le forgeron a utilisé du sable de façon importante, pour limiter l'oxydation du métal travaillé lors de phases de soudure.

Si l'apport majoritaire vient des oxydes de fer qui se détachent du métal lors de sa chauffe et des matériaux argilo-sableux, alors se forment des scories grises et denses (SGD) à base de fayalite $\mathrm{Fe}_{2} \mathrm{SiO}_{4}$. La matrice de la scorie résulte de la fusion d'un liquide riche en fer et en silice, entre $1200^{\circ} \mathrm{C}$ et $1400{ }^{\circ} \mathrm{C}$, puis de sa cristallisation. Des oxydes de fer également à l'état liquide, peuvent cristalliser en même temps que la fayalite: comme la wüstite (majoritaire) et plus rarement la magnétite et l'hématite. Les nuances de couleur que l'on peut généralement observer dans les cristaux de fayalite (jaunâtre-gris-gris métallique) pourraient provenir : de l'inégalité entre les différents apports de fer et de silice; des différentes vitesses de refroidissement conduisant à divers habitus de la fayalite. Les scories SGD sont décrites comme résultant de travaux de forge à haute température sur des cycles plus ou moins longs.

Si l'apport majoritaire vient de la barre de fer travaillée (sous forme métallique et oxydes), alors se forment des scories ferreuses et rouillées (SFR). Dans un milieu humide comme l'est le sol, le métal se transforme en hydroxydes de fer et la scorie revêt alors une couleur rouille. Les scories SFR sont décrites comme résultant de travaux de forge complexes ou avec une matière première insuffisamment compactée.

La classification des scories, à partir de la nature des matériaux qui les composent, semble également un excellent point de départ pour caractériser les activités anciennes. La méthode de calcul de cette classification est détaillée à la figure 68. La classification proposée à figure 3 a été utilisée sur plusieurs sites de forge en Suisse (Anderson et al. 2003, Perret 2009, Mauvilly et al. 1998, Tauber 1998). On remarque qu'entre les trois matériaux majoritaires qui composent les scories, les nuances varient fortement, montrant une incroyable diversité des scories de forge.

Cependant les interprétations restent toujours très peu fiables. Ainsi, ce travail vise à donner des éléments de réponse importants :

- comment se forment les scories SAS, SGD et SFR ?

- les SAS proviennent-elles de travaux de soudure avec des ajouts siliceux ?

- quelles conditions de travail (température, savoir-faire etc) permettent la formation de scories SGD et SFR ?

- au cours de quelles étapes de la chaîne opératoire les scories SAS, SGD et SFR se formentelles?

- quelle est l'influence qualitative et quantitative de ces différents apports de matériaux dans les scories de forge?

\section{Quantité de fer travaillée}

Un enjeu important de l'archéométallurgie est d'évaluer la consommation de matière première (fer) au cours de la période de fonctionnement d'un atelier de forge. Pour procéder à cette évaluation, il faudrait connaître le poids individuel et la quantité des outils fabriqués, ces données étant généralement manquantes. Cependant il est possible de retrouver ces informations avec le nombre de scories comptabilisées sur le site et leur teneur en fer.

Au cours d'un forgeage à chaud dans un milieu riche en oxygène, comme c'est le cas d'un foyer de forge, la surface de la matière première s'oxyde, entraînant indéniablement une perte du métal en train d'être travaillé. Plusieurs auteurs ont déjà fait des expérimentations et mesuré cette perte de matière entre la matière première initiale et l'outil final, cependant soit de manière très ponctuelle (Serneels \& Huysecom A paraître), soit pour des séries de forgeages très différents sans pouvoir 


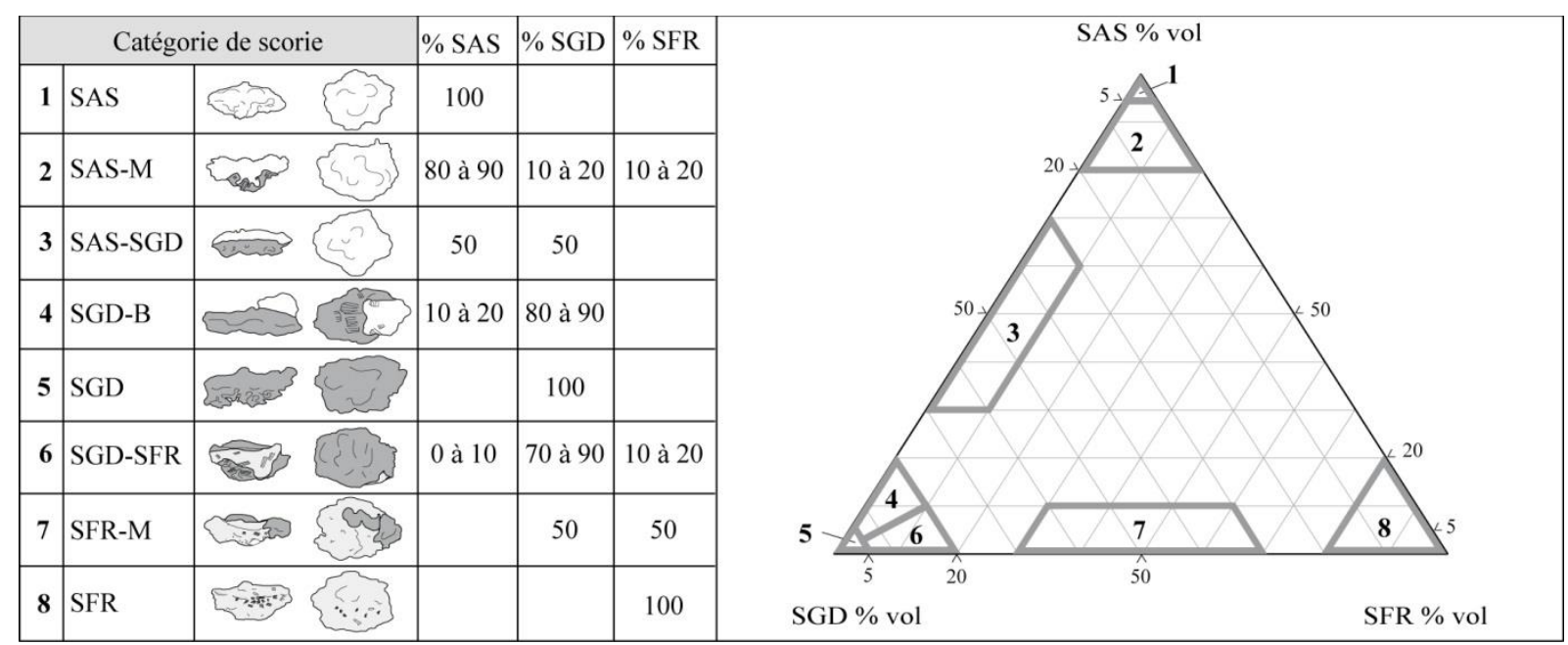

Figure 3 - Classifications des scories de forge d'après Serneels \& Perret $2003: 475$.

comparer réellement ces données entre elles (Sim 1998). Ils montrent que les forgeages de travaux simples génèrent environ $10 \%$ de pertes et jusqu'à $30 \%$ pour des travaux complexes.

Le fer perdu au cours du forgeage se retrouve à deux endroits : le foyer (dans la scorie) et l'enclume (les battitures). Cependant aucune étude n'a pu estimer la proportion et la quantité de fer perdu qui intègre les scories et les battitures.

Avec ce travail de recherche, nous souhaitons donner une évaluation de la quantité de fer travaillée à partir de la masse de fer contenue dans la scorie :

- en évaluant de façon plus systématique la part et quantité de fer initial perdu, au cours de différentes conditions de travail (nombre de barres à forger, température du foyer, durée de chauffe, quantité de déformation, expérience de l'artisan etc),

- en évaluant la part et la quantité du fer perdu qui intègre la scorie, en fonction de différentes conditions de forgeage.

\section{Métallographie}

Les scories de forge peuvent contenir du fer à l'état métallique, sous la forme de petites particules, de grosses particules, en petite quantité ou en grande quantité. Dans la littérature sur les scories de forge, on admet que la teneur en carbone maximale observée reflète celle qui a été travaillée, cependant aucune étude systématique d'expérimentation n'a pu prouver cette hypothèse.

De plus, les forgerons réalisent fréquemment des trempes sur certains outils dont ils veulent durcir une zone. Ces trempes laissent des facies microscopiques caractéristiques dans l'outil qui devraient également être observés dans les scories.
Concernant l'étude du métal dans les scories, notre étude vise à comprendre :

- le lien entre la nature du fer travaillé par le forgeron et la nature du fer contenu dans les scories,

- s'il existe un lien entre les conditions des forgeages (température du foyer, durée du travail etc) et la taille/quantité des particules de métal contenues dans les scories de forge,

- si l'on retrouve les faciès métallographiques de trempes des outils dans les scories.

\section{Ateliers de forge}

L'atelier de forge, dans lequel le forgeron procède au travail du fer, est une structure organisée qui possède de nombreuses composantes : un foyer, une soufflerie, des outils de frappe ou des outils percuteurs. Les installations de forge et les ateliers sont ainsi très différents d'une région à une autre, d'une tradition métallurgique à une autre, alors que tous les forgeages mènent à des déchets comme les scories.

Avec ce travail, nous souhaitons comparer plusieurs ateliers de forge et comprendre si les installations qu'elles contiennent (foyer, soufflerie, outils) peuvent avoir une influence sur les scories qui se forment au cours du forgeage.

\section{Opérations de forgeage}

Le travail $\mathrm{du}$ forgeron consiste à mettre en forme des objets de fer à partir d'une matière première ferreuse qui peut être très variée en termes de poids, dimensions ou composition. Ce travail de mise en forme passe par la réalisation d'une chaîne opératoire, c'est-à-dire une succession de gestes et d'étapes à suivre. 
Notre recherche vise à décrire de façon précise une chaîne opératoire et à en déterminer les principales parties, afin de déterminer quelles étapes de la chaîne opératoire sont essentielles à la formation des scories.

Enfin, nous avons souhaité savoir quels paramètres de la matière première, entre leur forme (trapue/allongée), leur nombre (une/plusieurs), leur origine (moderne/ancien) et leur composition (inclusions, teneur en carbone) laissent une signature visible sur les scories de forge produites.

Notre travail s'axe donc comme une recherche élargie, sur le thème des scories de forge, à propos de questionnements concernant les techniques, les matériaux et les traditions métallurgiques, dans l'optique de contribuer à une meilleure compréhension des processus de formation de la scorie, issue des travaux de forge.

\subsubsection{Choix de la démarche du travail}

Cette étude tente de donner des éléments de réponse pour mieux comprendre et mieux décrire les activités de forgeage grâce à l'étude de ses déchets : pour cela, nous avons souhaité comparer l'enregistrement complet des activités de forgeage avec les traces matérielles produites.

Ainsi, nous avons développé une démarche originale et novatrice à la confluence de l'ethnoarchéologie, l'archéologie expérimentale et l'archéométrie, qui combine l'étude de scories de forge (au laboratoire), en lien avec l'étude des opérations de forgeage (sur le terrain) qui les ont produites.

Le travail de laboratoire, réalisé au Département des Géosciences de l'Université de Fribourg, propose une approche analytique, en utilisant les techniques modernes d'analyses couramment appliquées dans le cas d'études archéométriques de déchets de forge. Celles-ci proposent une caractérisation macroscopique, chimique et minéralogique des matériaux anciens (Dillmann \& Fluzin 2003; Fluzin et al. 2004; Fluzin et al. 2000). Toutefois notre recherche ne se situe pas exactement dans une démarche archéométrique: nous n'étudions pas au laboratoire des matériaux provenant de sites archéologiques anciens, mais bien des scories modernes issues de forgeages réalisés dans des conditions de travail contrôlées traditionnelles.

Le travail sur le terrain, quant à lui, permet d'observer et d'enregistrer des traditions métallurgiques anciennes, en allant à la rencontre d'artisans contemporains qui utilisent des techniques de forge traditionnelles (Serneels \& Huysecom A paraître). Nous leur avons demandé de fabriquer des lames de houe à partir de plusieurs choix de barres de fer initiales (poids, nombre, taille, teneur en carbone etc), afin de mesurer l'influence de ces paramètres sur les caractéristiques des scories.

Toutes les données de terrain ont été récoltées lors de trois séjours d'un mois menés au Pays dogon entre 2008 et 2010 (Figure 5). Pourquoi le Pays dogon ?

- Le contact avec les forgerons est déjà établi à travers un projet de recherche établi sur place depuis une vingtaine années (voir ci-dessous).

- Dans cette région du Mali, il existe encore de nombreux villages comportant des forgerons.

- Le Pays dogon présente des régions où les traditions métallurgiques sont fortement contrastées, ce qui permet de visiter et de confronter des ateliers de traditions différentes.

- Dans ces ateliers dont l'accès à l'électricité et aux techniques modernes de forge est restreint, les forgerons ne connaissent pas d'autre manière de travailler le fer que celle enseignée par leurs parents, à l'ancienne.

- Les forgerons travaillent toujours de nos jours régulièrement dans leur atelier: c'est une tâche habituelle pour eux de fabriquer des outils.

Le travail de terrain se situe, à mi-chemin entre ethnoarchéologie et archéologie expérimentale, dans le cadre d'une expérimentation dirigée, durant laquelle différents paramètres sont contrôlés. Nous avons souhaité observer, auprès de deux groupes de forgerons dogons, la chaîne opératoire de fabrication d'un outil agricole commun. Il a été fourni au maître de forge, pour chaque forgeage, une sorte de matière première parmi 23 sortes différentes (Figure 28). Il a été demandé de fabriquer à chaque fois une lame de houe, outil forgé couramment utilisé dans cette région rurale, qui ne présente pas de difficulté particulière de fabrication (Figure 44). A l'issue des opérations de forgeage, au nombre de 126, chaque déchet constitue alors un élément pour la constitution d'un référentiel original de scories de forge. 
Cette recherche ne se profile pas dans une perspective ethnoarchéologique, car le sens premier de notre travail de terrain n'est pas de faire des observations actualistes répétées d'opérations de forgeage pour définir des régularités et établir des scénarios et des mécanismes visant à caractériser les techniques anciennes de forgeage. Cette recherche ne se situe pas non plus dans un créneau d'archéologie expérimentale: l'objectif de notre étude n'est en effet pas d'effectuer soi-même des expérimentations pour restituer les méthodes de fabrication et les fonctions des objets, en comparant les objets originaux avec les objets nouvellement fabriqués.

Il s'agit bien d'un travail interventionniste au cours duquel nous avons en toute conscience interféré dans le travail des forgerons: en leur demandant d'utiliser des matières premières spécifiques, de nettoyer le foyer avant d'y travailler, de fabriquer un type d'outil bien particulier, d'utiliser (parfois) lors des soudures, des matières siliceuses que nous leur avons fournies, de ramasser le plus possible les battitures éjectées lors du martelage, de stopper (parfois) le travail pour faire des photographies, de dégager et conserver précieusement la scorie, et en les rémunérant pour chaque journée de travail.

Ces interventions auprès du forgeron obligent à s'interroger sur la pertinence des données observées et enregistrées, sur lesquelles nous basons l'ensemble de notre travail. Ont-elles pu entraver ou réduire les observations scientifiques?

- la rémunération pourrait conduire à ce que le forgeron essaie de mieux travailler, pour livrer un outil de bonne qualité à son client ;

- sachant dès le départ que les outils fabriqués ne seraient pas utilisés dans des champs en Suisse, le forgeron pourrait sciemment négliger les finitions du travail, essentielles à la qualité d'un outil agricole ;

- l'intrusion de personnes étrangères dans son atelier pourrait inciter le forgeron à utiliser des outils de forge rarement utilisés afin de les montrer avec fierté :

- l'intrusion d'une femme blanche sur le lieu de forge pourrait conduire à ce que certains outils de forge spéciaux aient été volontairement gardés à l'abri des yeux indiscrets ;

- comme femme étrangère $\mathrm{j}$ 'ai eu la permission de circuler partout dans l'atelier et de m'installer dans la forge pour observer le travail, alors qu'habituellement les femmes dogons sont tolérées à proximité du lieu de travail mais pas pour autant admises dans l'enceinte de la forge.

Toutefois, cette démarche de travail, au croisement de plusieurs disciplines scientifiques, ne remet pas en cause le fait de pouvoir mettre en évidence des savoir-faire locaux traditionnels de la métallurgie du Pays dogon qui sont actuellement en voie de disparition.

\subsubsection{Déroulement du travail}

L'observateur s'installe à une place située hors de la zone d'action des forgerons afin de ne pas gêner leurs mouvements, mais d'où il peut voir l'intérieur du foyer et tous les gestes techniques des forgerons (Figure 4).

A notre demande le foyer est nettoyé avant de procéder aux forgeages, mais c'est aussi le cas dans un contexte de travail habituel. S'ils existent, les fragments de parois, de tuyère et de déchets sont évacués et les charbons sont renouvelés. L'enclume et ses alentours sont débarrassés des anciennes battitures par un rapide balayage du sol.

L'intégralité du déroulement de la chaîne opératoire est retranscrite sur une fiche d'enregistrement où sont rapportées les informations suivantes (Figure 6) :

- durée des plages de chauffe et de martelage : à l'aide d'un chronomètre ;

- nature des matériaux ajoutés dans le foyer ou utilisés pour faciliter les soudures ;

- couleur du fer avant et après martelage : observation visuelle donnant une idée approximative de la température du fer en cours de forgeage, à environ $100{ }^{\circ} \mathrm{C}$ près ;

- nom et fonction des forgerons en train de travailler : maître de forge, frappeurs et souffleur ;

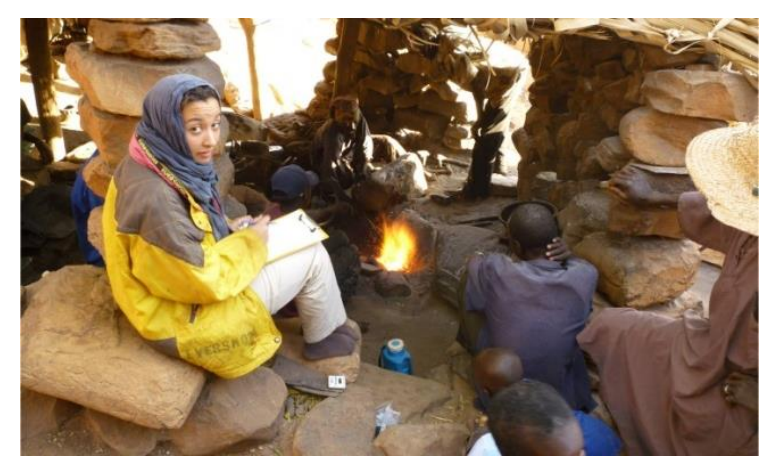

Figure 4 - L'observateur à sa place d'observation à Kakoli. 


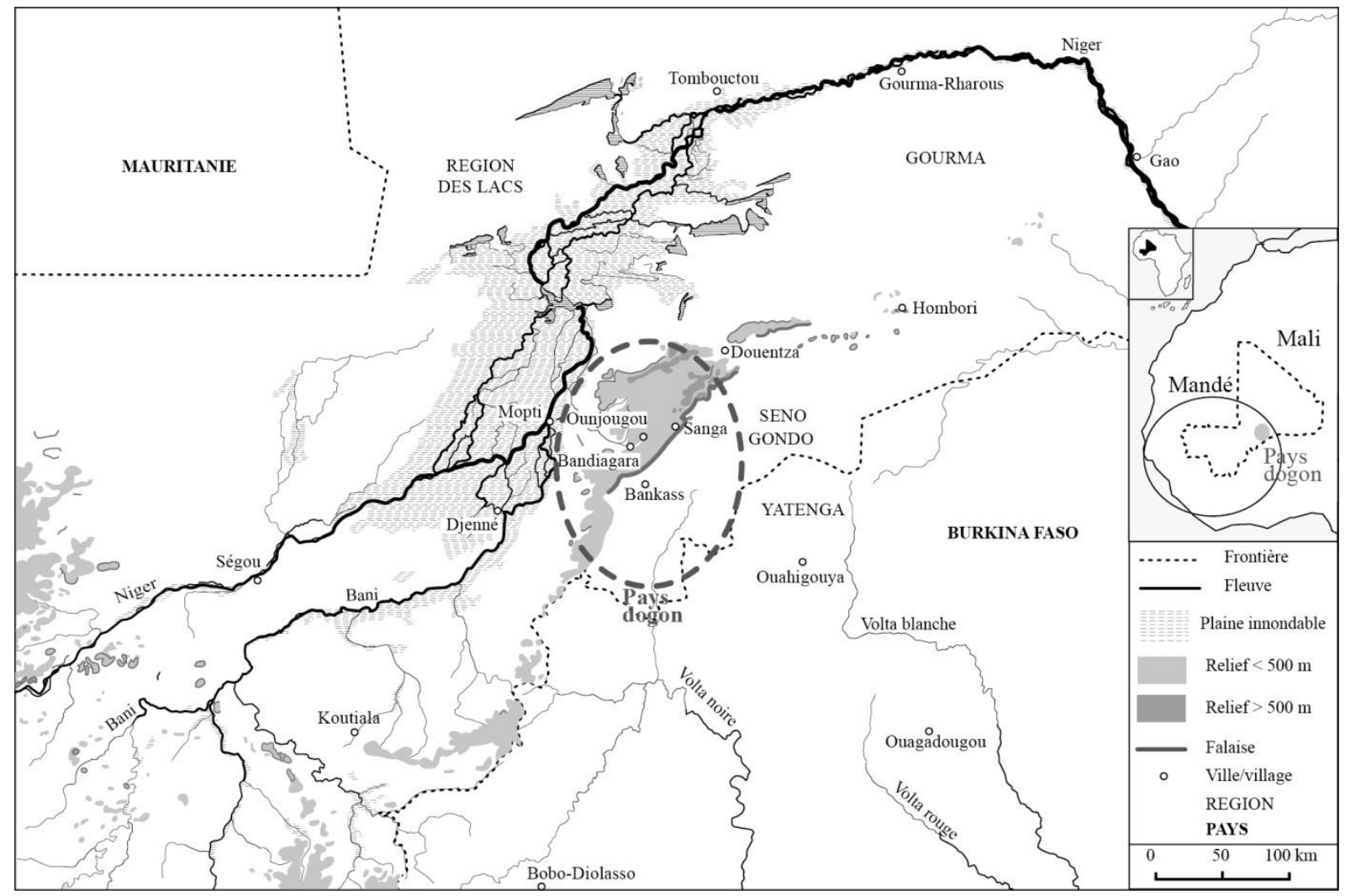

Figure 5 - Carte de la boucle du Niger et du Pays dogon, d'après Mayor 2011 : 7.

- nature des marteaux (à l'aide d'un code rapide à écrire : MM1, PM2 etc) et des enclumes utilisés ;

- description succincte des procédés techniques effectués, appuyée par des photographies.

Après chaque phase de martelage, les battitures sont récupérées sur et à proximité immédiate de l'enclume à l'aide d'une balayette, d'une pelle et d'un aimant. Lors de certaines phases de martelage où le fer est porté au blanc, des battitures peuvent être expulsées jusqu'à $5 \mathrm{~m}$ autour de l'enclume. La collecte des battitures sur et autour de l'enclume a été réalisée de manière à donner une image qualitative plus que quantitative des types de matériaux formés.

La scorie a été récoltée dans le foyer à la fin d'une opération de forgeage ou bien à la fin d'une journée de travail, soit deux à trois forgeages. Si la scorie est faite d'un seul bloc compact, alors la totalité du matériel a été récoltée. Si la scorie est fracturée, alors nous avons contrôlé que la totalité des fragments avaient été extraits du foyer. En revanche, si la scorie se compose de plusieurs pièces de petite taille, alors il n'est pas exclu que des morceaux soient restés dans le foyer au milieu des charbons.
La lame produite a été pesée, ses dimensions et les défauts qu'elle comporte ont été relevés. Certaines lames ont été données aux paysans du village, d'autres ont été ramenées à Fribourg pour une investigation plus approfondie. Enfin, le foyer et la soufflerie sont décrits ; Michel Mauvilly (Service Archéologique de Fribourg) a procédé à un relevé des ateliers. Battitures, scorie et lame ont été analysées sous différents aspects au laboratoire à Fribourg.

Par ce travail, toutes les variabilités de la chaîne opératoire sont consignées. La prise en compte de tous ces paramètres est primordiale pour comprendre quelles différences de travail entre les ateliers mènent aux variabilités observées des scories.

\subsubsection{Cadre scientifique de la recherche}

Cette recherche a été largement portée par le projet «Peuplement humain et paléo-environnement en Afrique de l'Ouest » (Huysecom 2002) initié par le Professeur Eric Huysecom en 1997, suite à la découverte en 1988 du site archéologique d'Ounjougou sur le plateau de Bandiagara (Pays dogon) qui évoquait une occupation antérieure à l'âge des métaux et aux périodes d'occupation 
connues (Huysecom et al. 2004). Ce vaste projet vise à mettre en relation les variations culturelles, climatiques mais aussi environnementales et historiques du Pays dogon en relation avec l'Afrique de l'Ouest, sur une période chronologique longue : du Pléistocène à nos jours. Pour cela, les recherches sont menées par une équipe internationale de chercheurs confirmés, débutants, d'étudiants, de personnels techniques œuvrant dans les domaines de la géomorphologie, la géographie, l'archéologie, l'ethnoarchéologie, l'ethnohistoire, la botanique et la linguistique.

Malheureusement, les évènements récents liés à la fois à la prise du pouvoir par un coup d'Etat en mars 2012 par la junte militaire menée par le capitaine Amadou Haya Sanogo et à la percée de groupes terroristes ont contraint ce projet de recherches, après plus de vingt années d'activités au Mali, à se recentrer sur une autre région, au Sénégal.

Pour plus d'informations, tous les rapports annuels du projet «Peuplement humain et paléoenvironnement en Afrique de l'Ouest», sont disponibles à l'adresse internet ci-contre : http://www.slsa.ch/Projekte/OunjougouF.htm.

Depuis 1995, de nombreuses études reliées à ce vaste projet de recherche au Pays dogon se sont intéressées à l'étude de la métallurgie du fer :

- Un film documentaire intitulé «Inagina, l'ultime maison du fer » - La métallurgie du fer chez les Dogons a fait l'objet d'une étude ethnoarchéologique particulière qui a permis d'observer et de filmer la réduction du minerai de fer dans un bas fourneau ainsi que le forgeage du fer obtenu. Cette étude a conduit à de nombreuses recherches concernant les forgerons dogons, à travers le biais de l'archéologie, de l'histoire, de la linguistique ou de l'archéométrie (Huysecom \& Agustoni 1996; Serneels et al. 1997).

- Une thèse défendue à Genève en 2008 au Département d'Anthropologie et d'Ecologie par Caroline Robion-Brunner - Etude mêlant archéologie et ethnohistoire, qui retrace l'histoire des forgerons et de la production du fer sur le plateau de Bandiagara depuis le $13^{\text {ème }}$ siècle jusqu'au milieu du $20^{\text {ème }}$ siècle (Robion-Brunner 2010).

- Une thèse en cours à l'Université de Fribourg par Sébastien Perret - Etude consacrée à la caractérisation des technologies et des volumes de production associés à quelques sites majeurs de la production du fer du Pays dogon (Perret \& Serneels 2009).

- Entre 2001 et 2011 par Vincent Serneels (Université de Fribourg, archéométrie), Caroline Robion-Brunner (Université de Genève, archéologie), Barbara Eichhorn (Université de Francfort, botanique) et Sébastien Perret (Université de Fribourg, archéométrie) - Etude du développement de la production locale et régionale du fer (les acteurs, les technologies, les volumes de production) sur le long terme avec le souci de comprendre les implications de ces activités sur le paysage (traces d'exploitation des matières premières) et la société (commerce, développement des états) (Serneels et al. 2006).

\subsubsection{Structure du manuscrit}

Après une partie introductive des aspects techniques de la forge et une présentation du Pays dogon (Partie 1), seront décrits la structure et l'organisation des ateliers de travail de forge dans lesquels ont été réalisés les travaux demandés (Partie 2).

La Partie 3 analyse le détail du déroulement des opérations de forgeage (au nombre de 126). Un résumé est donné à l'Annexe 1 .

Le travail de laboratoire permettra de mettre en évidence des groupes de scories et de proposer une réflexion sur leurs modalités de formation, en lien avec les opérations de forgeage (Partie 4).

La conclusion générale de cette recherche et les perspectives de travail sont indiquées à la Partie 5 .

La Partie 6 recense l'ensemble des données de chimie et de minéralogie des différents matériaux rencontrés et analysés: Annexe 2 - Combustible ; Annexe 3 - Matériaux siliceux; Annexe 4 - Scories. Un résumé des données analytiques par type de scorie et par atelier est donné à l'Annexe 5.

L'ensemble des références bibliographiques utilisées est consigné à la Partie 7 de ce manuscrit. Enfin, un catalogue additionnel en ligne recense toutes les données morphologiques, chimiques et minéralogiques concernant l'ensemble des scories de forge. 


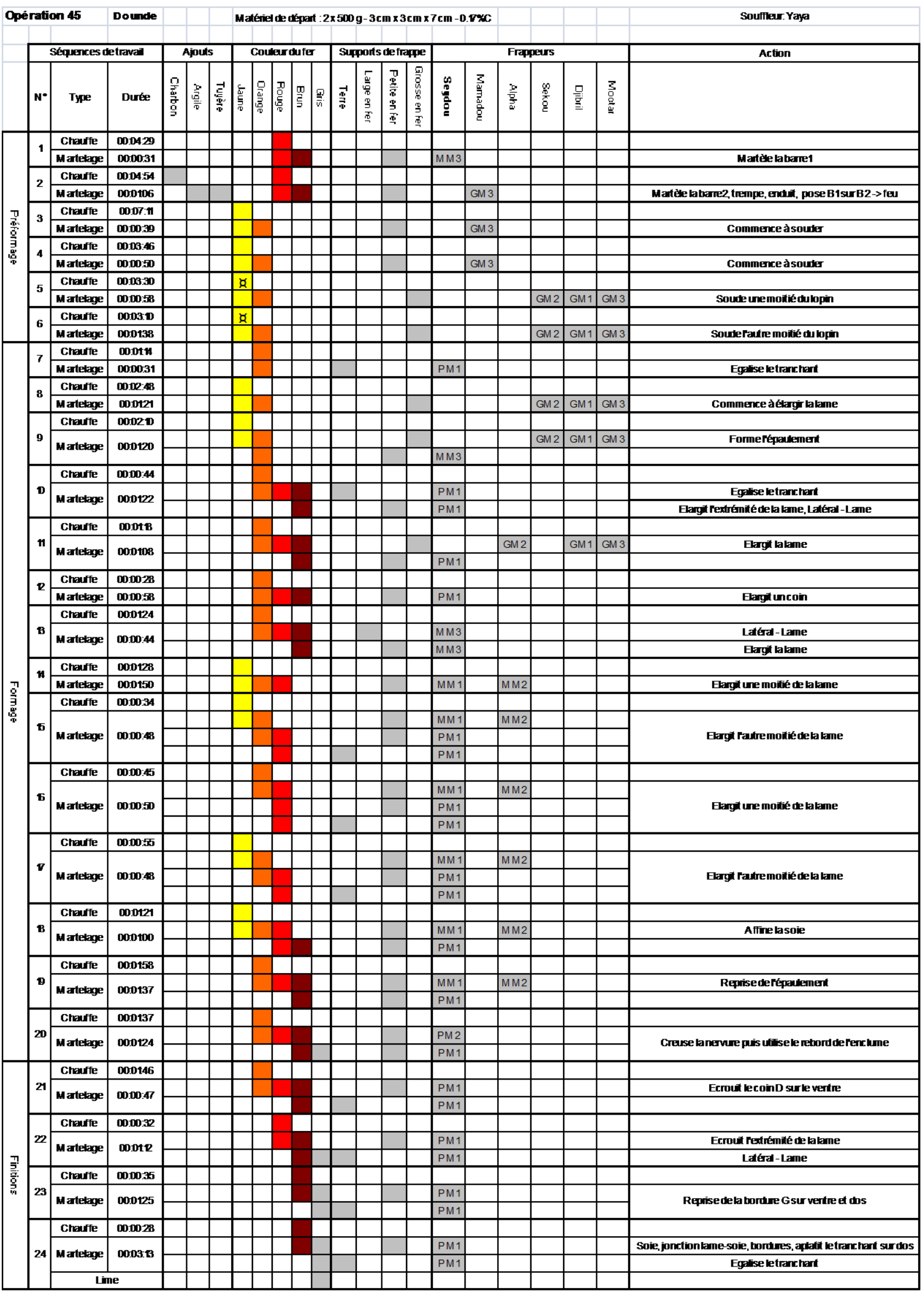

Figure 6 - Exemple de fiche d'enregistrement type, ici pour le forgeage F046 dans le village de Doundé. Ce forgeage a été réalisé à partir de deux barres de fer à $0.17 \% \mathrm{C}$ de $3 \mathrm{~cm}$ de section et $7 \mathrm{~cm}$ de longueur chacune et pesant $500 \mathrm{~g}$ chacune. Le maître Seydou a été aidé par Yaya à la soufflerie et cinq frappeurs : Mamadou, Alpha, Sekou, Djibril et Moctar. Légende : $\alpha=$ blanc crépitant. 


\subsection{ASPECTS TECHNIQUES DE LA FORGE}

\subsubsection{Chaîne opératoire de la sidérurgie ancienne}

La méthode directe de production du fer vise la transformation des oxydes de fer du minerai en une masse brute de fer ou d'acier, à l'état solide dans un bas-fourneau. La chaîne opératoire technique regroupe l'ensemble des procédés techniques depuis l'extraction du minerai jusqu'à l'élaboration des objets en fer. (Figure 7).

Extraction - L'homme creuse dans le sous-sol des puits et des galeries ou bien afin d'y extraire le minerai de fer, ou bien récolte le minerai en surface (cuirasses latéritiques etc).

Les principaux minéraux utilisés sont la magnétite $\mathrm{Fe}_{3} \mathrm{O}_{4} \quad(72.4 \% \mathrm{Fe})$, l'hématite $\mathrm{Fe}_{2} \mathrm{O}_{3}$ $(69.9 \% \mathrm{Fe})$, la goethite $\mathrm{FeO}(\mathrm{OH})(62.9 \% \mathrm{Fe})$ et la limonite (Serneels $2004: 27$ ). Cette dernière est un ensemble d'oxydes et d'hydroxydes de fer qui composent, entre autres, les chapeaux de fer.

Différentes techniques d'abattage permettent de détacher le minerai de la paroi rocheuse : au feu, à la poudre ou à l'aide de pics (Forbes 1963 : 201-202).

Concentration - Le minerai abattu subit ensuite divers traitements : tri, lavage, concassage ou calcination. Ce dernier facilite le concassage des gros blocs (par la création de fissurations) pour les amener à des tailles plus petites. Tous ces traitements visent à concentrer les particules ferreuses pour obtenir une matière première susceptible de fournir une plus grande quantité de fer métallique.

Réduction - C'est l'étape de fabrication du fer métallique. Le minerai de fer est introduit dans un bas-fourneau en alternance avec du charbon: les oxydes de fer qui sont dans le minerai sont réduits à l'état métallique par l'interaction à haute température avec les gaz de combustion (Figure 8). La combustion est assurée par une ventilation naturelle ou forcée, à l'aide de soufflets. La réaction se déroule dans des températures relativement basses, entre 1000 et $1250{ }^{\circ} \mathrm{C}$ (Figure 11) et le fer est toujours à l'état solide ou pâteux. Le produit est une éponge de fer, les déchets les plus caractéristiques étant les scories de réduction.
Epuration - L'éponge de fer n'est pas forgeable directement : elle comporte de nombreux matériaux non métalliques (charbons et scorie) qui doivent d'abord être évacués par un martelage à froid et à chaud à l'aide d'un marteau, de pics ou de burins. Ensuite les nombreuses cavités sont comblées par compactage. L'épuration est réalisée dans un atelier de forge ou bien sur place, directement après sa sortie du bas-fourneau afin de profiter de la chaleur résiduelle du métal. Ainsi, le fer est nettoyé et compacté (Serneels et al. 2004 : 82). Une fois épuré, le fer peut être conditionné sous forme de demiproduit à commercialiser, de poids et dimensions variables (Fluzin et al. 2004 : 166). Les déchets engendrés par l'épuration sont des battitures, des fragments hétérogènes de l'éponge et également une scorie dans le foyer.

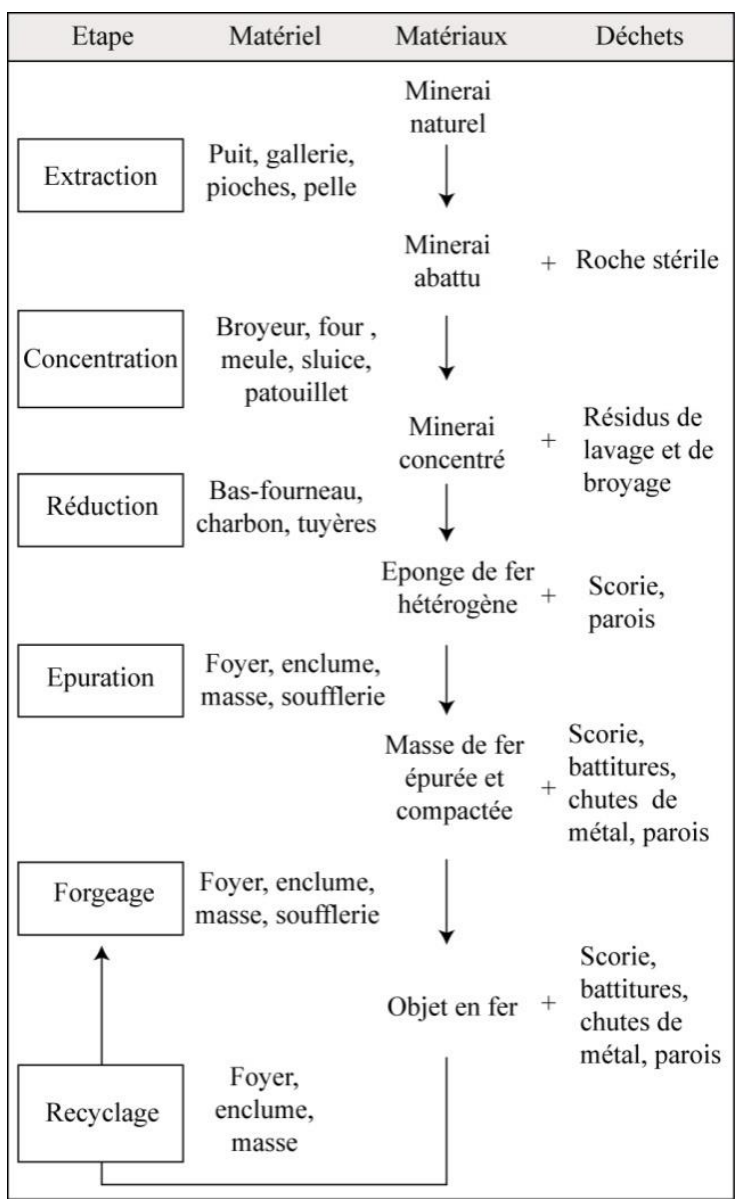

Figure 7 - Chaîne opératoire de la sidérurgie directe. D'après Mangin $2004: 8$. 


$$
\begin{array}{r}
\mathrm{O}_{2 \text { air }}+\mathrm{C}_{\text {charbon }} \rightarrow \mathrm{CO}_{2} \\
\mathrm{CO}_{2}+\mathrm{C}_{\text {charbon }} \rightarrow 2 \mathrm{CO} \\
\mathrm{CO}+3 \mathrm{Fe}_{2} \mathrm{O}_{3 \text { minerai }} \rightarrow \mathrm{CO}_{2}+2 \mathrm{Fe}_{3} \mathrm{O}_{4} \\
\mathrm{Fe}_{3} \mathrm{O}_{4}+\mathrm{CO} \rightarrow \mathrm{CO}_{2}+3 \mathrm{FeO} \\
\mathrm{FeO}+\mathrm{CO} \rightarrow \mathrm{Fe}+\mathrm{CO}_{2}
\end{array}
$$

Figure 8 - Réaction chimique de la réduction des oxydes de fer. D'après Leroy \& Merluzzo 2004 : 49.

Forgeage - Il s'agit de mettre en forme le métal épuré en un objet fonctionnel et de lui donner les propriétés physiques désirées, par déformation plastique à chaud et par des traitements thermiques (trempe, revenu, recuit) ou thermochimiques (carburation, nitruration) dans un atelier de forge (Serneels et al. 2004: 84-87). Notre travail s'intéresse uniquement à cette partie de la chaîne opératoire de la sidérurgie ancienne (1.2.3).

Recyclage - En fin de vie, les objets de fer peuvent être reforgés. Au cours de l'utilisation, ils sont aiguisés plusieurs fois, certaines parties remplacées. Plusieurs petits objets usés peuvent être réassemblés à la forge en un objet de plus gros volume par soudure (Serneels et al. 2004 : 85).

\subsubsection{Atelier de forge}

L'atelier de forge est le lieu où le forgeron travaille le fer. Cet espace contient un certain nombre d'installations dédiées à la mise en forme des outils de fer (Doswald et al. 1997 : 42).

\section{Unité / complexe spatial de travail}

Dans l'atelier, l'équipe des forgerons est installée au sein d'une unité spatiale de travail. Il s'agit d'un espace organisé qui comprend tous les éléments permettant la mise en forme d'un morceau de fer en un outil : foyer, soufflerie, enclume, marteaux, sièges pour le souffleur, pour le frappeur et pour le maître de forge, bac à eau.

Parfois, l'atelier ne compte qu'une seule unité de travail, parfois elle en compte plusieurs. Nous parlerons alors d'un complexe spatial. Au sein d'un complexe spatial, plusieurs activités peuvent coexister : travail du fer, du bois ou d'autres métaux.

\section{Foyer et soufflerie}

Le foyer est l'endroit dans lequel le fer est chauffé. Plusieurs modèles sont possibles : soit une simple fosse, soit un foyer aménagé au niveau du sol ou bien surélevé. Les parois peuvent être nues, consolidées par des pierres, revêtues d'argile etc. La taille et la forme du foyer diffèrent selon : la quantité de métal travaillé, les dimensions de l'outil fabriqué et surtout de la culture technique des artisans.

La soufflerie est le dispositif destiné à produire le vent nécessaire à la combustion du charbon de bois dans le foyer, afin d'atteindre les températures adéquates pour le travail du métal (Figure 9). Plusieurs modèles existent: soufflerie manuelle, hydraulique, électrique, avec/sans soufflets. Anciennement, la soufflerie comprend des soufflets qui peuvent être actionnés à une main, à deux mains ou au pied par une seule personne.

\section{Outils}

L'enclume est l'outil de frappe fixe qui présente une surface plane appelée table sur laquelle le fer est battu. En bois, en pierre ou en acier, l'enclume est de taille et poids variable. Elle est directement posée sur le sol ou enchâssée dans un support qui sert à la stabiliser lors du martelage.

Le marteau est un outil percuteur qui sert à donner une forme précise au fer chaud qui est posé sur l'enclume. Ses dimensions et son poids dépendent de sa fonction. Le forgeron le manipule à une ou deux mains. Le marteau est très souvent constitué d'un manche et d'une tête métallique (table et pane).

Le forgeron a besoin d'un instrument pour saisir et manipuler le fer lors de sa mise en forme. Cet outil indispensable est formé de deux bras en acier articulés munis de mâchoires. Celui qui sert à maintenir fortement le fer pour la frappe sur l'enclume est nommé tenaille. L'outil qui sert à manipuler le fer dans le foyer est une pince. Il en existe une grande variété (Guillaumet 1998).

Le forgeron tient à sa disposition un bac à eau qui sert soit à refroidir ses pinces, soit à effectuer la trempe d'outils, soit à refroidir les abords du foyer. Par ailleurs, pour certains travaux spécifiques, le forgeron tient à sa disposition d'autres outils : lime, 
burin, poinçon, tisonnier, pierre à aiguiser, goupillon etc.

\subsubsection{Travail du fer à la forge}

Le travail du forgeron est centré sur la mise en forme des objets en fer. Le terme de forgeage recouvre l'ensemble des traitements mécaniques de mise en forme des objets en fer et des traitements thermiques exécutés à la forge (Mangin et al. 2004 : 223 ). Ainsi, «Le forgeage a pour but de donner au métal, à l'aide d'un outillage approprié, une forme parfaitement déterminée tout en améliorant ses qualités » (Lagardelle 1926 : 7).

Le forgeron fabrique un outil en suivant une chaîne opératoire, c'est-à-dire une succession d'étapes au cours desquelles la matière première est progressivement amenée en un outil fonctionnel (3.2). Comme le choix des actes techniques mais aussi la succession des gestes sont propres à chaque forgeron, il peut arriver que d'un artisan à un autre, la chaîne opératoire varie légèrement.

\section{Déformation plastique à chaud}

Le travail du fer à la forge est une succession de cycles thermomécaniques au cours desquels le fer est chauffé («chaude») dans le foyer puis martelé sur l'enclume («passe ») à l'aide de marteaux jusqu'à l'obtention simultanée de la forme et des propriétés mécaniques désirées. Pour cela, le forgeron est guidé dans son travail par la couleur mais aussi la réponse du métal face aux coups qui lui sont portés.

Pour être forgé, le fer a besoin d'être chauffé dans une plage thermique précise (Figure 9). Plus sa température augmente et plus il est malléable. D'un point de vue microscopique, les phases de chauffe permettent la croissance des grains et leur recristallisation.
Le martelage est réalisé jusqu'à une certaine limite : si le fer est trop martelé, des défauts peuvent apparaître (3.4.3). Le martelage sur l'enclume déforme les grains de fer, diminue leur taille et réduit leur plasticité.

Par ailleurs, au cours du forgeage, le fer s'oxyde au contact de l'air (Figure 76) : les oxydes formés se détachent et tombent dans le foyer et autour de l'enclume. Cette perte de matière métallique est un inconvénient pour l'artisan qui souhaite préserver au maximum la masse de métal disponible.

Ainsi, la confection d'un outil n'est pas un acte simple mais il est le fruit du travail de forgerons expérimentés: la déformation plastique à chaud entraîne de profondes modifications sur la microstructure du métal (taille, forme des grains) et ses propriétés physico-chimiques (dureté, composition chimique) (Philibert et al. 1997 : 787).

\section{Procédés techniques de la forge}

Pour l'accomplissement d'un forgeage, le forgeron dispose d'une vaste gamme de gestes et de procédés techniques (Doswald et al. 1997 : 40-41 ; Leblanc 2002 : 71-72). Le choix final de l'un ou l'autre des procédés est imposé par :

- la matière première disponible : nature et volume,

- les connaissances techniques de l'artisan : maîtrise des gestes,

- les installations et les (bons) outils disponibles,

- le type d'objets à réaliser: complexité, qualité, volume.

Une liste des procédés techniques rencontrés au cours de notre étude est donnée à l'Encart 1 Procédés techniques.

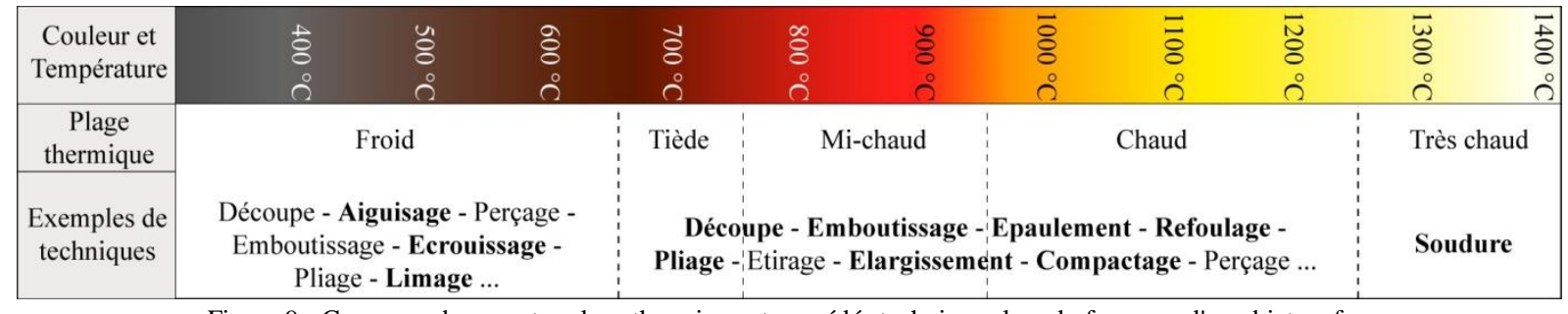

Figure 9 - Correspondance entre plage thermique et procédés techniques lors du forgeage d'un objet en fer. En gras sont indiqués les procédés techniques rencontrés dans le cadre de cette étude. D'après Leblanc $2002: 22$. 


\section{Encart 1 - Procédés techniques}

L'aiguisage consiste à rendre plus tranchant le côté affilé d'un outil coupant.

Le limage consiste à entailler une pièce avec une lime pour le dégrossir, réduire ses aspérités etc.

La découpe consiste à diviser une pièce métallique en plusieurs morceaux à l'aide d'un instrument tranchant, à chaud ou à froid.

L'emboutissage est la mise en forme d'une plaque de métal par serrage sur une forme provenant d'un support.

Le pliage consiste à rabattre une partie d'une pièce de métal avec un certain angle.

L'élargissement consiste à étendre la surface d'une pièce tout en l'affinant. Contraire de compactage.

L'écrouissage consiste en un martelage de l'outil à basse température, ce qui provoque une diminution de la taille des grains de fer, de leur élasticité et donc une augmentation générale de la dureté de l'outil.

L'épaulement consiste en un changement brusque de la section d'une pièce.

Le refoulage est une opération qui consiste à

\subsubsection{Déchets de forgeage}

Pendant de nombreuses années, les recherches archéométallurgiques ont tout d'abord cherché à établir des critères pour différencier quels déchets sont produits lors de la réduction du minerai de fer et quels déchets le sont lors du travail du fer à la forge (Eschenlohr et al. 1991 ,McDonnell 1986)

Concernant le travail de la forge, les techniques mises en œuvre sont très diversifiées (Figure 9), engendrant des déchets variés et de différentes natures (McDonnell 1991, Serneels 1993 ; Serneels et al. 2004; Serneels \& Perret 2003). comprimer une pièce dans le sens de la longueur.

Le compactage consiste à comprimer au maximum une pièce de métal.

La soudure est une technique de forge qui consiste à assembler deux pièces par une recristallisation des grains à l'interface des surfaces à réunir pour créer une liaison métallique entre ces deux pièces. Elle ne peut être effectuée qu'à très haute température $(>$ $1250{ }^{\circ} \mathrm{C}$, blanc soudant).

Pour que la jonction soit correctement réalisée, il faut que la surface des pièces à réunir soit exempte d'impuretés et notamment d'oxydes de fer. Ainsi, le forgeron tente de ralentir leur développement et d'expulser ceux qui sont déjà présents en enduisant les surfaces du fer d'un ajout siliceux dit «fondant». La silice se combine aux oxydes de fer pour former une pellicule de silicates de fer très fluide que le martelage chasse plus facilement (Lagardelle 1926 : 135-136).

La trempe est un traitement thermique visant à chauffer un métal au-dessus d'un point de transformation puis à la refroidir plus ou moins rapidement dans un fluide de refroidissement comme l'eau. Le but est de fabriquer des constituants hors équilibre riches en carbone pour augmenter la dureté de l'outil

\section{Scories}

Ce sont les déchets les plus caractéristiques du travail de la forge et les plus fréquemment retrouvés lors des fouilles archéologiques. Ces matériaux sont traités en intégralité dans la Partie 4 . : Les déchets de forgeage.

\section{Battitures}

Autour de l'enclume, le sol de la forge est sombre et très magnétique, à cause de la présence de croûtes d'oxyde de fer ( $\mu \mathrm{m}$ à $\mathrm{mm}$ ) appelées battitures. Les battitures sont des croûtes d'oxydes de fer qui se forment sur la surface des barres lorsqu'elles sont portées à haute température dans un milieu riche en oxygène comme dans le foyer de forge. 


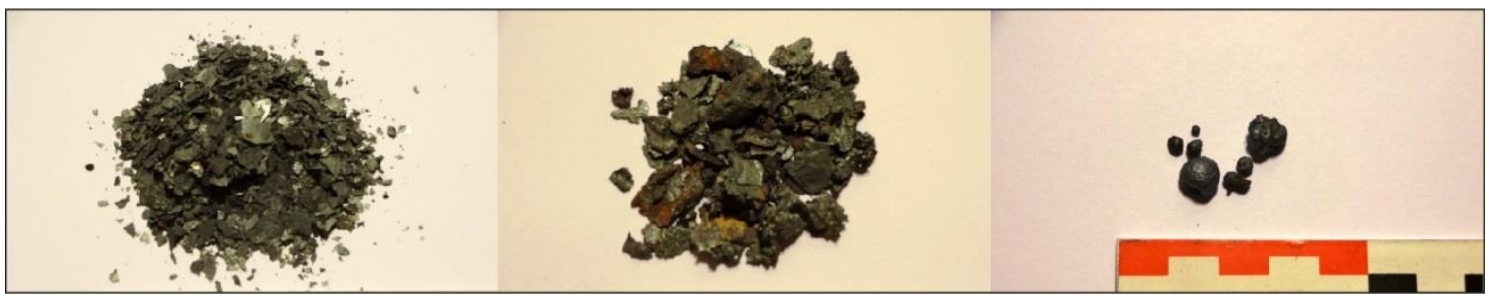

Figure 10 - Différentes morphologies des battitures : lamellaire, granulaire et globulaire.

Sous les coups de marteaux du forgeron, elles se détachent de la barre de fer, et tombent sur et à proximité de l'enclume (Dunikowski et al. 1996; Mills \& McDonnell 1992; Starley 1995).

Du point de vue chimique, elles sont composées majoritairement de wüstite, magnétite, hématite, dans un rapport 90: 10:3 avec éventuellement des silicates de fer (Figure 76). L'épaisseur des écailles est proportionnelle au temps et à la température d'oxydation (Birosca et al. 2004; Iordanova et al. 2000).

Plusieurs auteurs ont proposé des classifications morphologiques et microstructurales de battitures de sites archéologiques (Allen 1986; McDonnell 1984; Unglik 1991). Pour comprendre quelles sont les conditions du travail nécessaires à la formation de telle ou telle morphologie (température, nature de la matière première), divers travaux expérimentaux de forge ont été menés jusqu'à récemment (Crew 1991; Dungworth \& Wilkes 2007; Sim 1998). En particulier, J.-C. Leblanc a établi un corpus de références de battitures en lien avec différentes étapes de la chaine opératoire de forgeage (Leblanc 2002).

Il existe deux morphologies principales de battitures, qui dépendent du contexte du forgeage effectué : la plus commune est une écaille lamellaire, la seconde est globulaire. Parfois, la morphologie granulaire peut encore être différenciée (Figure 10).

\section{Les débris métalliques}

Dans l'espace occupé par les artisans, il n'est pas rare de trouver des débris de métal comme des ébauches, des ratés ou des chutes de découpe (Anderson et al. 2003: 104-133; Eschenlohr et al. 2007 : 57-60). Une étude a montré que les angles formés sur les débris peuvent renseigner sur les outils utilisés et les gestes des forgerons (Anderson et al. 2003: 133-147; Duvauchelle \& Agustoni 2002). La nature du métal retrouvé renseigne également sur la nature du métal travaillé (fer pur ou alliage) et sur les traitements thermiques, déformations plastiques et donc sur l'histoire de l'élaboration de ces objets : \% C, dureté etc.

Les travaux de forge que nous avons menés n'ont produit des débris métalliques que dans un seul cas : une surchauffe lors d'une soudure complexe, ayant entraîné une faiblesse du métal puis un détachement de matière pendant le martelage (F028).

\section{Les éléments du foyer}

Les éléments du foyer comme des fragments de parois ou de tuyère sont réalisés en argile contenant éventuellement du sable, des fragments de roche et des fibres végétales. Ces matériaux sont reconnaissables car en général ils comportent une surface altérée par la chaleur (dure et plus ou moins vitrifiée) tandis que l'autre face est cuite, plus fragile, d'aspect granuleux (Eschenlohr et al. $2007: 69)$.

\section{Encart 2 - Caractéristiques physico-chimiques du fer et ses alliages}

La métallographie contribue de manière importante à l'identification des phases métalliques et aux observations portant sur l'aspect des matériaux ferreux ou non ferreux (inclusions) dans la matrice étudiée. L'étude du métal est proposée à tous les stades de la chaîne opératoire : scories afin de déterminer à quelle phase de la chaîne opératoire elles appartiennent (Fluzin 2002), éponges de fer afin d'évaluer la qualité du métal fabriqué (Serneels et al. 1997) ou objets en fer afin de comprendre les techniques et les savoir-faire anciens (Fluzin 2000).

\section{Le fer métallique}

Le fer est un métal de transition du tableau périodique des éléments de symbole $\mathrm{Fe}$ et de numéro atomique $\mathrm{Z}=26$. Il est présent en quantité abondante dans la croûte terrestre $(\approx 7 \%)$ mais très rarement 
sous forme métallique non allié (seulement dans certaines météorites).

Pur, il s'agit d'un métal mou $(\approx 80 \mathrm{Hv}$ - Tableau 1$)$ de couleur bleuâtre. Poli, il réfléchit fortement la lumière. Au contact d'oxygène et d'eau, il rouille facilement dans toute son épaisseur. Le fer est également connu pour ses propriétés magnétiques (magnétisme en dessous de $769{ }^{\circ} \mathrm{C}$ ), électriques, thermiques (matériau conducteur d'électricité et de chaleur), et mécaniques (ductilité, malléabilité). Le fer est constitué de grains dont la taille et la forme varient selon la durée et température de chauffe, de martelage et des traitements thermiques appliqués (Higgins 1993: 255). Il possède trois variétés allotropiques (Tableau 2).

Jusqu'à $912^{\circ} \mathrm{C}$, le fer en présence est du fer $\alpha$ (ferrite) qui cristallise dans un réseau cubique centré (CC). Le fer $\gamma$ (austénite) apparaît jusqu'à $1394{ }^{\circ} \mathrm{C}$ dans un assemblage cubique à faces centrées (CFC), induisant une dilatation de la maille initiale. A des températures plus élevées et jusqu'à $1536{ }^{\circ} \mathrm{C}\left(\mathrm{T}_{\mathrm{f}}\right.$ fer pur), c'est le fer $\delta$ qui est présent, cristallisant dans une maille cubique centrée de taille similaire à celle de la ferrite (Figure 75).

\begin{tabular}{|c|c|c|c|}
\hline Nom & \multicolumn{2}{|c|}{$\% \mathrm{C} \min -\max$} & Dureté (Hv) \\
\hline Ferrite & \multicolumn{2}{|c|}{$0.00-0.02$} & 80-90 \\
\hline Acier hypoeutectoïde & \multicolumn{2}{|c|}{$0.02-0.77$} & \\
\hline Acier extra doux & \multicolumn{2}{|c|}{$0.02-0.15$} & \\
\hline Acier doux & \multicolumn{2}{|c|}{$0.10-0.30$} & \\
\hline Acier mi-doux & \multicolumn{2}{|c|}{$0.30-0.40$} & \\
\hline Acier mi-dur & \multicolumn{2}{|c|}{$0.40-0.60$} & \\
\hline Acier dur & \multicolumn{2}{|c|}{$0.60-0.77$} & \\
\hline Acier eutectoïde & \multicolumn{2}{|c|}{0.77} & 230 \\
\hline Acier hypereutectoïde & \multicolumn{2}{|c|}{$0.77-2.11$} & \\
\hline Fonte hypoeutectique & \multicolumn{2}{|c|}{$2.11-4.30$} & \\
\hline Fonte eutectique & \multicolumn{2}{|c|}{4.30} & \\
\hline Fonte hypereutectique & \multicolumn{2}{|c|}{$4.30-6.67$} & 800 \\
\hline \multicolumn{4}{|c|}{$\begin{array}{l}\text { Tableau } 1 \text { - Dénominations-teneurs en C- dureté des alliages } \\
\text { Fe-C. D'après Higgins } 1993: 158 \text { et Leblanc } 2002: 7 \text {. }\end{array}$} \\
\hline Variété & \multicolumn{2}{|c|}{$\alpha$} & $\mathrm{Fe} \delta$ \\
\hline \multirow{2}{*}{$\begin{array}{l}\mathrm{T}\left({ }^{\circ} \mathrm{C}\right) \min -\max \\
\text { Réseau }\end{array}$} & 'à 912 & $912-1394$ & $1394-1536$ \\
\hline & $\mathrm{C}$ & CFC & $\mathrm{CC}$ \\
\hline $\begin{array}{c}\text { Paramètre de la } \\
\text { maille }(\AA)\end{array}$ & 87 & 3.56 & 2.93 \\
\hline
\end{tabular}

Tableau 2 - Caractéristiques cristallographiques des allotropes du fer pur à pression ambiante.

\section{Les alliages spéciaux}

L'ajout d'éléments tels que le chrome, le molybdène ou le vanadium dans le réseau du fer permet d'obtenir des alliages aux propriétés physiques particulières : résistance à la corrosion, résistance physique, à la dureté bien supérieure. Par exemple, l'inox est un alliage qui doit ses propriétés de résistance à la corrosion à l'ajout de plus de $10 \%$ de chrome (Vander Voort 2004 : 571 ; 670).

\section{Les alliages au carbone}

L'élément qui a la plus forte incidence sur les propriétés du fer reste le carbone. L'ajout de carbone dans le réseau du fer aboutit à la formation de trois alliages principaux : le fer (ferrite), l'acier et la fonte (Tableau 1 ; (Figure 11).

Une des principales caractéristiques physiques des alliages $\mathrm{Fe}-\mathrm{C}$ est leur dureté : elle est proportionnelle à la teneur en carbone et elle varie selon les traitements thermiques appliqués ainsi que les températures de chauffe (Tableau 1, Higgins 1993 : 282).

Le point de fusion du fer pur est élevé: $1536^{\circ} \mathrm{C}$. Cependant, l'ajout de carbone dans le réseau du fer permet de réduire cette température à $1147{ }^{\circ} \mathrm{C}$ (point eutectique). Dans le cas de scories de forge, le carbone est fourni par les charbons du foyer et le monoxyde des gaz de combustion.

\section{La ferrite}

Elle dissout très peu le carbone, au maximum $0.02 \%$ (à $723^{\circ} \mathrm{C}$ ). Ses grains sont adjacents les uns avec les autres et sans relief (Figure 12 cliché 1).

La ferrite est apte à la soudure, malléable et s'utilise pour la fabrication d'objets tels que des clous, des rivets, des feuillets ou des fils de fer.

\section{Les aciers}

Entre 0.02 et $2.11 \% \mathrm{C}$, ce sont les aciers dont il existe une vaste gamme (Tableau 1).

En dessous de la composition eutectoïde (0.02-0.7\%C), il ne se forme que de la ferrite et de la perlite en quantités inversement proportionnelles. La ferrite se développe aux joints des grains, parfois avec une structure aciculaire dite de Widmanstätten. La perlite est quant à elle plutôt centrée dans le grain (clichés 2 à 7). A la composition eutectoïde (0.77 $\%$ C), l'austénite est décomposée uniquement en perlite (cliché 8). Au-dessus de la composition eutectoïde (0.8-2.11\%C), l'austénite se décompose en perlite et en cémentite qui croit aussi aux joints des grains et en bandes à l'intérieur du grain. (clichés 9 et10). 


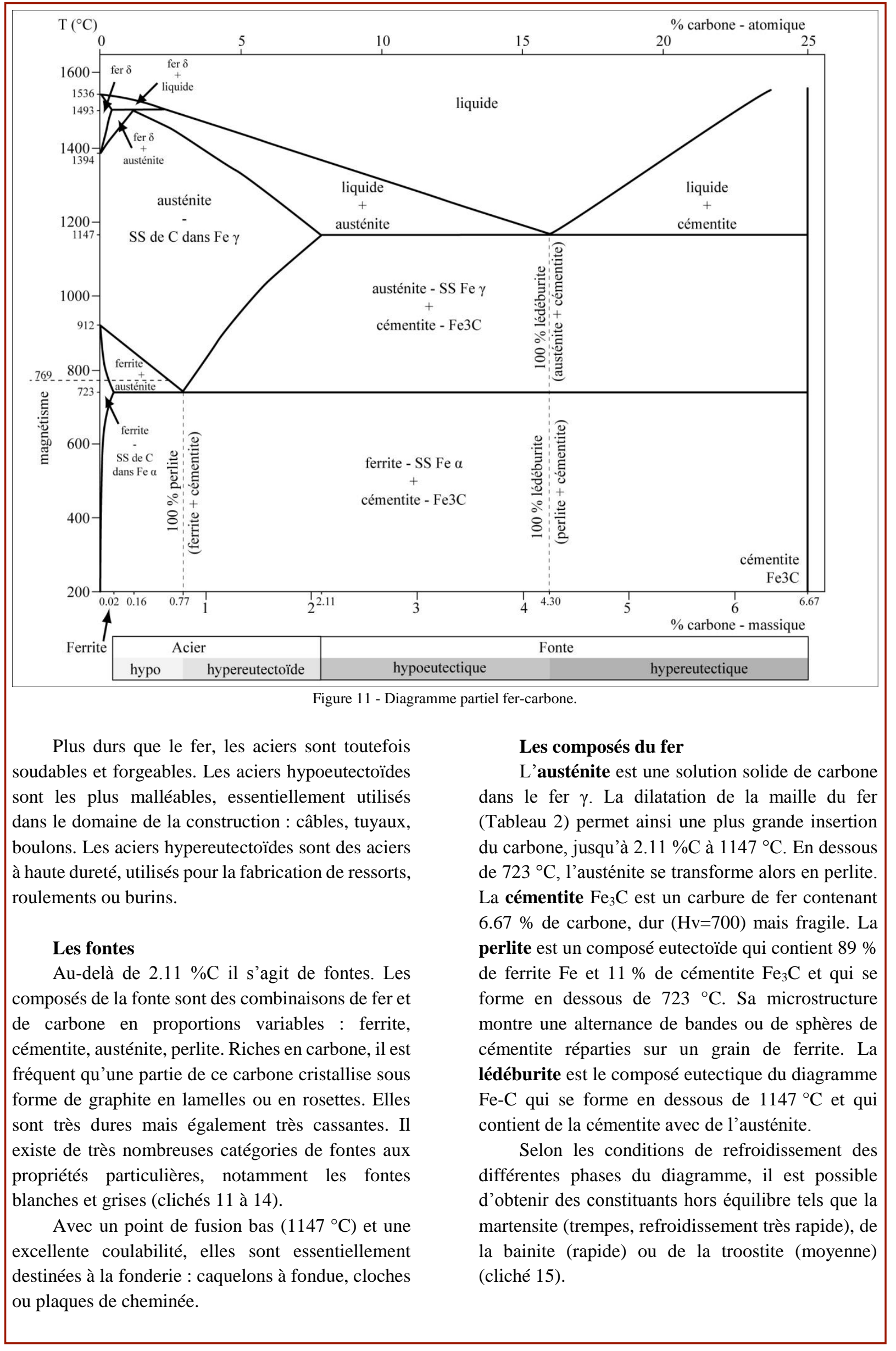




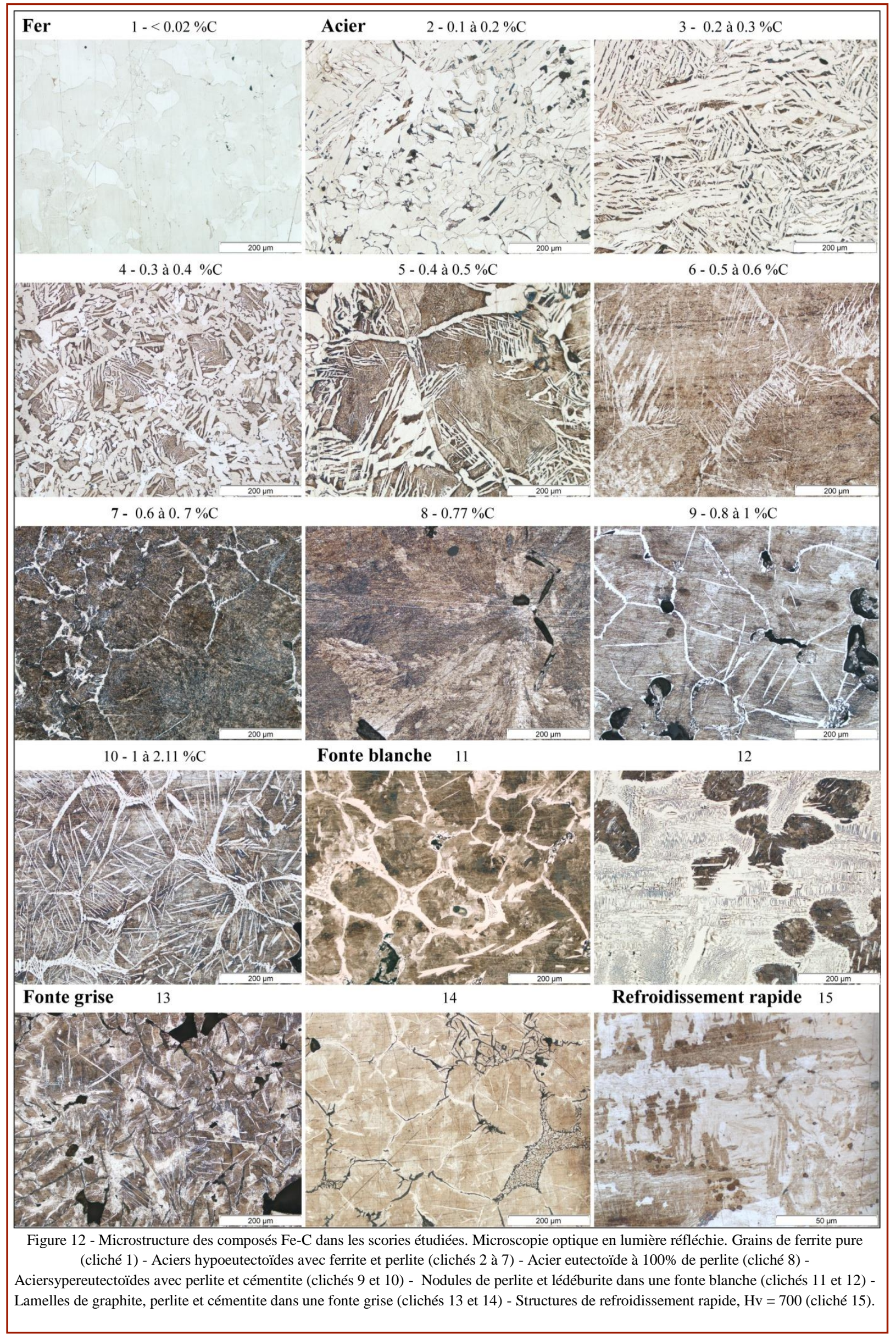




\subsection{Pays dogon et MÉtallurgie du FER}

\subsubsection{Carte d'identité}

Le Pays dogon, de par ses caractéristiques géologiques, archéologiques, ethnologiques et ses paysages exceptionnels est l'un des sites les plus imposants d'Afrique de l'Ouest. Il a été classé au patrimoine mondial de l'UNESCO en 1989. Dans cette région du monde subsistent des traditions sociales remarquables encore vivantes (masques, fêtes rituelles etc) ainsi que des paysages de falaise et plateau gréseux intégrant de très belles architectures (greniers, habitations etc).

\section{Relief et géologie}

Le Pays dogon est une région du Mali, entre le delta intérieur du Niger au nord-ouest et la frontière burkinabè au sud-est, qui comporte quatre cercles administratifs correspondant à quatre régions distinctes : Koro, Bankass, Douentza et Bandiagara (Figure 5). La zone au nord-ouest du Pays dogon est occupée par le delta intérieur du fleuve Niger et ses affluents, vaste plaine d'inondation de $20000 \mathrm{~km}^{2}$ constituée de marécages et de lacs (Figure 13).

Le plateau dogon ou plateau de Bandiagara, est formée d'une vaste dalle de grès durs entre $250 \mathrm{~m}$ et $300 \mathrm{~m}$ d'altitude dont la formation remonte au Précambrien supérieur et au Paléozoïque. Long de $250 \mathrm{~km}$ entre Douentza et Koutiala, il est incliné vers l'ouest et limité à l'est par la falaise de Bandiagara qui atteint $450 \mathrm{~m}$ de hauteur. Dans le nord, au-delà du plateau dogon, les altitudes dépassent $600 \mathrm{~m}$ et s'élèvent jusqu'à $1120 \mathrm{~m}$ dans la région de Hombori (Daveau 1959 : 13).

$\mathrm{Au}$ sud-est s'étend la plaine sableuse du Seno-Gondo, région très plate et uniforme. Au pied de la falaise se trouvent des alignements dunaires résultant d'une accumulation des sables transportés par les vents (essentiellement du Sahara) ou
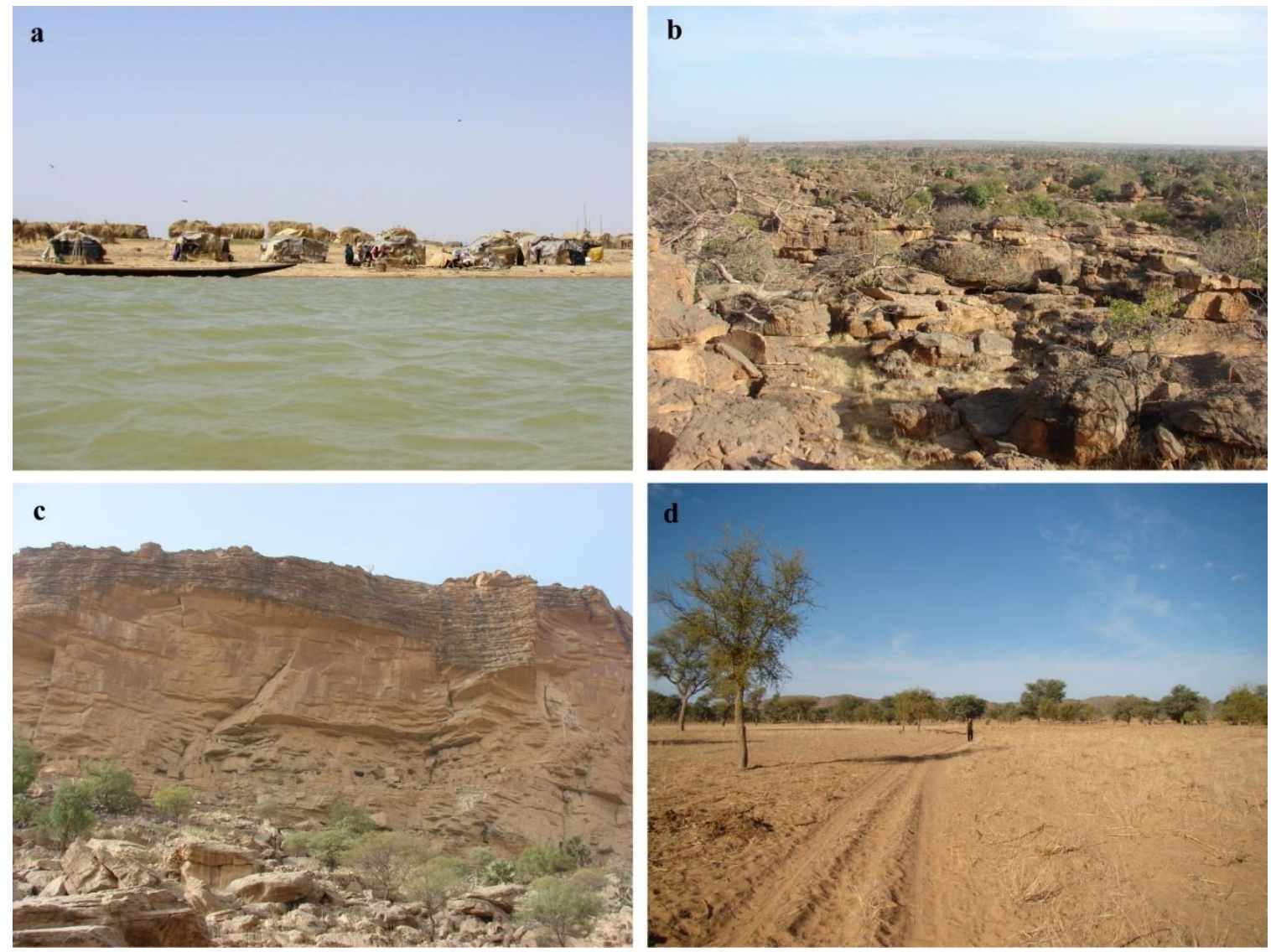

Figure 13 - a : zone humide à l'est du Pays dogon (fleuve Bani à Mopti), b : plateau rocailleux dogon (vers Dogani), $\mathrm{c}$ : falaise de Bandiagara (à Enndé), d : plaine du Séno (Doundé). 
résultant de l'érosion éolienne (Rasse et al. 2012). Sur le plateau dogon, au pied de la falaise et dans la plaine du Séno, il existe de nombreuses zones riches en minerai de fer que l'homme a exploité pour la production du fer (Daveau 1959 : carte C ; Serneels et al. $2006: 115)$.

\section{Climat et végétation}

Le Pays dogon fait partie du domaine sahélo-soudanien. Les saisons sont rythmées entre :

- une saison sèche qui s'étale de novembre à mars, caractérisée par la présence de l'harmattan qui diffuse des masses d'air sec venant du Sahara ;

- une saison humide de juin à septembre (hivernage), caractérisée par une mousson délivrant à Mopti, une quantité de pluie annuelle avoisinant $500 \mathrm{~mm}$ (Arnaud \& Collectif $2010: 82-83$ ).

La végétation sur le plateau de Bandiagara correspond à une mosaïque de savanes arborées et arbustives fortement anthropisée. Les forêts galeries sont peu présentes sur le plateau mais visibles le long de certains cours d'eau (Le Drezen \& Ballouche 2009 : 51).

\subsubsection{Métallurgie du fer}

Avant l'arrivée des dogons, le plateau de Bandiagara est occupé par des populations pré-dogon qui maitrisent déjà la métallurgie du fer. Les pré-dogon coexistent avec les forgerons Jèmè$n a$, clan de forgerons mythiques connus pour leur savoir-faire métallurgique (Robion-Brunner 2010 : 49).

La circulation d'objets de fer est déjà attestée dans les différentes régions d'Afrique, de la boucle du Niger (Mayor 2011: fig. 63; Mayor et al. 2014 : 31 ; Ozainne 2013 : 206-207), ainsi que sur le plateau de Bandiagara, la falaise et la plaine du Séno (Robion-Brunner 2010 : 118, fig. 68). La tradition métallurgique Fiko, datée du $6^{\text {ème }}$ siècle ap. J.-C., aurait ainsi été initiée par les populations pré-dogon (Figure 14).

Depuis le $6^{\text {ème }}$ siècle ap. J.-C. jusqu'à la colonisation française, le Pays dogon se trouve à l'interface de zones d'influences de plusieurs formations étatiques (Mayor 2011 : fig. 60). Le Pays dogon subit des invasions, des pillages et des enlèvements de personnes par des chasseurs d'esclaves (Gallay 1994, Mayor et al. 2005). Le plateau de Bandiagara et ses promontoires naturels servent de miradors et refuges pour des populations provenant des quatre coins de l'horizon.

Les sources orales et historiques s'accordent pour dire que les premiers Dogons émigrent du Mandé (Figure 5) et atteignent le plateau de Bandiagara entre le $13^{\text {ème }}$ et le $15^{\text {ème }}$ siècle ap. J.-C. (Huet 1994 : 39 ; Gallay et al. 1995 ; Mayor 2011 : 115). Cette arrivée a certainement été à l'origine d'une migration partielle des populations autochtones vers le Burkina-Faso (Mayor 2011 : 115). Les recherches montrent également une première phase de peuplement, par l'ouest, de forgerons venant s'installer sur le plateau de Bandiagara entre le $14^{\text {ème }}$ et le $15^{\text {ème }}$ siècle ap. J.-C, lesquels adoptent les techniques de réduction déjà mises en place (Robion-Brunner 2010 : 121, 129).

A partir du $16^{\text {ème }}$ siècle ap. J.-C. les migrations de populations, de l'ouest vers l'est du plateau de Bandiagara, s'accélèrent, provoquant la multiplication des sites de production du fer, en particulier les ateliers implantés en marge occidentale du plateau avec les traditions Ouin et Tinntam (Robion-Brunner 2010 : 123-124). Cette intensification de la production du fer conduit les métallurgistes à se regrouper en une caste, les Jèmèirin, qui regroupe des forgerons d'origine mais aussi des individus qui ont été exclus de leur catégorie sociale d'origine (agriculteurs, nobles) puis intégrés suite à des déplacements de population (guerres, besoins de main d'œuvre).

Dès le $17^{\text {ème }}$ siècle ap. J.-C., de nouveaux forgerons s'installent dans la plaine du Séno et le plateau méridional: ce sont les Jèmè-yélin. Ils émigrent du Mandé tout en maîtrisant déjà toutes les connaissances de la production et de la transformation du fer (Huysecom 2001 : 74). Ils sont à l'origine de deux nouvelles traditions métallurgiques au pied de la falaise de Bandiagara (Ama et Aridinyi). Jusqu'au $19^{\text {ème }}$ siècle ap. J.-C., certaines populations déplacées intègrent les forgerons Jèmè-irin (Robion-Brunner 2010 :129).

Dès la fin du $19^{\mathrm{e}}$ siècle, l'introduction de fer, de ferraille et d'outils en fer par les colons français a contribué à l'abandon progressif de la production locale $\mathrm{du}$ fer par le processus de réduction traditionnelle. Les sites métallurgiques de la tradition Ouin, à l'écart de la zone de contrôle des Français, se maintiennent jusque dans la première 
partie du $20^{\text {ème }}$ siècle. Enfin, les dernières réductions traditionnelles du fer ont eu lieu sur le site d'Aridinyi dans les années 1970.

Aujourd'hui la production du fer au Pays dogon est totalement stoppée, sauf reconstitutions exceptionnelles effectuées par les anciens pour les jeunes, en hommage au travail passé (Huysecom \& Agustoni 1996; Huysecom 2001). Actuellement, seules les activités de forgeage sont maintenues dans les différents villages du Pays dogon.

\subsubsection{Diversité des cultures dogon}

Le Pays dogon s'est donc peuplé selon une dynamique historique influencée par la formation des royaumes pendant plusieurs siècles, induisant la diversité actuelle des cultures dogon. Les Dogons revendiquent une identité commune et une certaine unité culturelle (traditions céramiques, traditions métallurgiques).
Actuellement, le nombre de Dogons installés au Mali est estimé à environ 500000 individus (Hochstetler et al. 2004 : 14).

Tous ne parlent pas une seule et même langue mais bien 27 langues issues d'une langue «proto-dogon» ancienne et commune (Blench \& Douyon 2006 : 184-185) et appartenant toutes, sauf une seule, à une même famille linguistique. Ces langues dogons sont réparties selon des zones géographiques bien précises qui morcellent le territoire (Figure 14).

Les Dogons cohabitent avec d'autres groupes ethniques: les Peuls (langue fulfude); les Bwa (langue bwamu) ; les Bobo (langue bobo), les Bozo (langue bozo), les Songhay (langue songhay), les Kurumba (langue kurumba), les Bambara (langue bambara), les Touareg (langue tamasheq), les Mossi (langue more) etc. Les principales langues parlées au Pays dogon restent les langues dogon, le fulfude, le bambara et le français souvent appris à l'école (Hochstetler et al. 2004 : 15, 18 ; Mayor 2011 : 8).

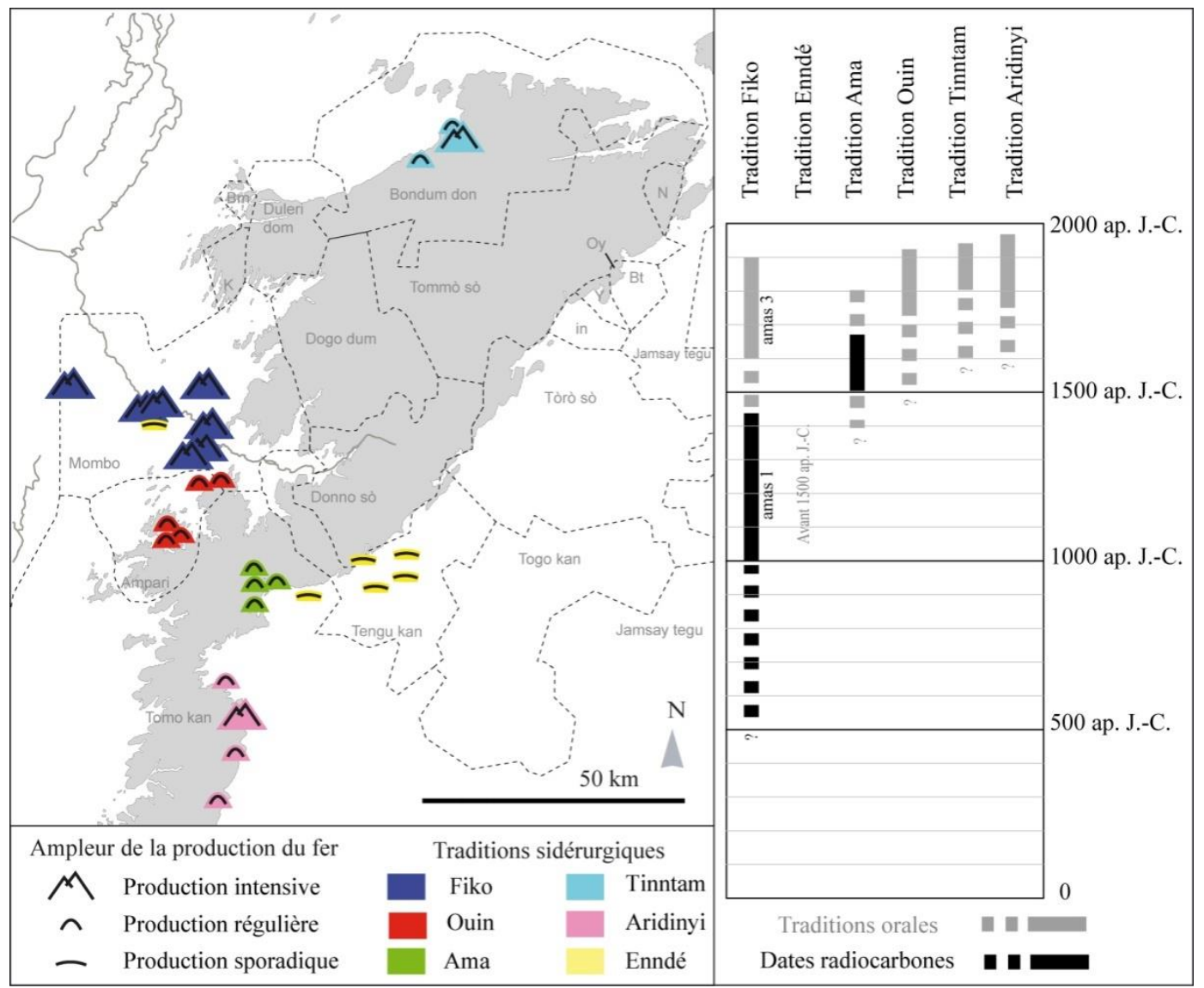

Figure 14 - Intensité et chronologie de la production du fer sur le plateau de Bandiagara. D'après Robion-Brunner 2010 (Pl.14 et Fig. 64). Morcellement du territoire selon les langues dogon. 


\subsubsection{Forgerons et société}

\section{Caste}

La société dogon est stratifiée en trois catégories sociales distinctes: les agriculteurs (les Dogons), les artisans spécialisés et les captifs. Les artisans spécialisés se répartissent en quatre castes : les forgerons, les griots/artisans du cuir, les tanneurs/teinturiers et les fabricants de plats en bois. Chez les forgerons, trois castes coexistent, réparties dans des espaces géographiques divers et possédant chacune un savoir métallurgique différent: les Jèmè-na, les Jèmè-irin et les Jèmè-yélin (RobionBrunner $2010: 9$; 14-15).

\section{Statut social}

Comme dans d'autres sociétés traditionnelles, le forgeron a un statut social particulier. La transmission de la charge de forgeron est héréditaire dans un système patrilinéaire : les fils de forgerons sont forgerons de statut. Cependant, les règles évoluent et le statut peut être très différent de l'activité professionnelle. Les femmes des forgerons, пити-тиso, pratiquent également souvent la poterie. Les forgerons forment une caste endogame; cependant on constate divers modes d'agrégation aux différents clans de forgerons (Robion-Brunner 2010).

Les forgerons s'acquittent du travail du fer mais aussi souvent de celui du bois (manches des outils). Les forgerons ont un rôle de médiateur et peuvent intervenir dans les conflits. Leur jugement est très important et il est dangereux de ne pas en tenir compte (Robion-Brunner 2010: 9). Dans certains villages, les forgerons ont le pouvoir de guérison (maladies, infertilité etc) et en particulier les blessures provoquées par le feu.

\section{Apprentissage et hiérarchie au travail}

L'apprentissage de la forge est un parcours long qui comporte plusieurs étapes (Martinelli 1995, 1996, 1998).

Les enfants sont dès le plus jeune âge confrontés au monde du fer. Ils entament d'abord un apprentissage passif de mémorisation basé sur l'ouïe et la vue : en jouant dans l'atelier, ils observent les ainés travailler puis miment eux-mêmes les gestes en jeux.

Dès l'âge d'une dizaine d'années, les jeunes apprentis sont pris en charge par un maitre. Tout d'abord ils aident à la soufflerie : le souffleur, en position assise régule la puissance de la soufflerie sur la demande du maitre de forge, afin que le foyer ait la température souhaitée. Depuis son poste, le souffleur observe et intègre toutes les étapes de la fabrication des outils.

Plus tard, le souffleur devient frappeur en battant le fer sur l'enclume avec le maître, jusqu'à participer intégralement au forgeage des outils les plus complexes. Il martèle assis ou debout et intervient en renfort lorsque beaucoup d'énergie est requise pour déformer le fer. Ensuite il s'assoit pour regarder le maître finir le travail.

A partir d'une vingtaine d'années, la formation des apprentis se termine avec des stages de formation auprès d'autres forgerons puis leur installation permanente dans un village.

Une fois devenu maître à son tour il supervisera non seulement toute la partie technique du forgeage (gestion de la température du foyer, réalisation des soudures ou traitements thermiques, finitions) mais encore il montrera l'exemple aux apprentis et prodiguera ses conseils. 


\section{PARTIE 2 . LES ATELIERS DE FORGE}


Les ateliers de forge 


\subsection{INTRODUCTION AUX ATELIERS}

La Partie 2 a pour but de décrire et confronter l'organisation et la structure de quatre ateliers de forge.

Nous rappelons que le but de cette recherche est de mettre en lien les caractéristiques observées des déchets, les scories, avec les travaux de forgeage qui les ont produites.

En allant à la rencontre de forgerons actuels qui travaillent de façon traditionnelle, nous pouvons enregistrer et décrire ces opérations de forgeage : la chaîne opératoire, les installations de forge, l'organisation hiérarchique du travail et les outils utilisés. En multipliant les visites dans des ateliers de traditions métallurgiques différentes, nous pouvons confronter les observations effectuées.

La description complète des ateliers de forge visités (identité et fonction des forgerons, foyer, soufflerie, outils) est donc obligatoire pour mettre en évidence leurs différences ainsi que l'impact de ces différences sur le déroulement du travail et les caractéristiques des scories de forge.

\subsubsection{La forge, espace de travail et de croyances}

Les activités métallurgiques présentent une grande diversité technique, culturelle mais également un symbolisme marqué sur tout le continent africain (de Heusch 1956, de Maret 2002, de Maret 1980). Certains outils sont chargés de symbolisme, notamment l'enclume qui est considérée comme la mère de tous les outils (Appia 1965 : 350). La fabrication des outils enclumes, masses ou pinces traditionnelles est aussi une activité où les mythes et croyances sont très présents (Célis \& Coulibaly 2001 : 147-155).

L'atelier de forge est le seul espace qui appartient en totalité aux forgerons. L'enclume de fondation est l'élément principal et symbolique de l'atelier. Elle appartient à une seule famille de forgerons ; une fois implantée, l'atelier peut même être construit autour (Huysecom et al. 2006 : 136).

Les ateliers de forge sont présents dans tous les villages du Pays dogon. Certaines agglomérations peuvent posséder plusieurs ateliers : dans ce cas il existe plusieurs familles de forgerons, chaque famille travaillant dans son propre atelier.

\subsubsection{Présentation des ateliers}

Le travail de terrain a été réalisé dans quatre ateliers de forge du Pays dogon : Fiko, Kakoli, Kobo, Doundé (Figure 15). Ces villages ont été choisis pour divers motifs scientifiques et logistiques :

- le travail du fer est une activité qui est toujours exercée,

- les forgerons ont la réputation de bien travailler,

- nous avons l'autorisation d'observer le travail à la forge,

- $\quad$ il est possible de s'installer pour quelques semaines dans le village,

- les contacts établis préalablement sont bons,

- le village est accessible en voiture,

- les ateliers proviennent de zones où les traditions métallurgiques sont différentes.

Les villages de Fiko et de Kakoli sont situés dans la partie la plus basse du plateau de Bandiagara. Ils font partie d'un territoire où le Mombo est la langue parlée.

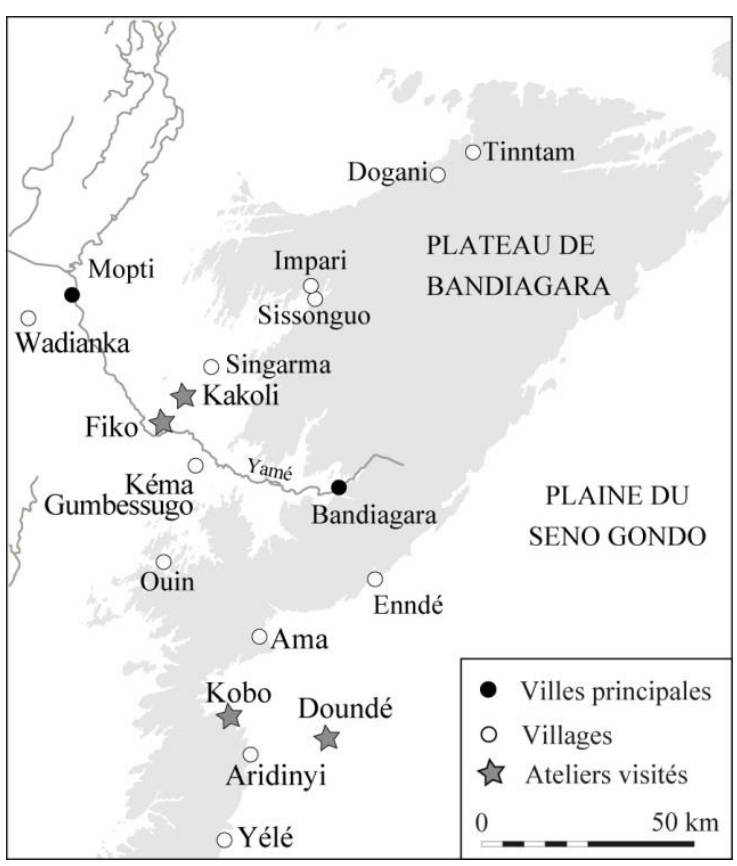

Figure 15 - Localisation de quelques villes/villages ainsi que des quatre ateliers visités au Pays dogon. 
Fiko est implanté au bord de la route goudronnée à mi-chemin entre Bandiagara et Mopti. Kakoli est un village de brousse à l'écart de la route au sommet d'un inselberg, à moins de $10 \mathrm{~km}$ au nord-est de Fiko.

Les villages de Kobo et Doundé se situent dans la plaine sableuse du Séno-Gondo, accessibles par une piste. Ces deux villages sont éclatés en plusieurs quartiers distants de quelques kilomètres les uns des autres. Dans cette zone la langue parlée est le Tomo.

Le quartier de Kobo où nous avons séjourné est implanté au pied d'un éboulis de la falaise de Bandiagara. Il comprend la concession des forgerons et celle des cordonniers. En Afrique, une concession est un ensemble d'habitations regroupé autour d'une cour, appartenant à une même famille.

Nous avons séjourné dans le quartier principal de Doundé, situé à $5 \mathrm{~km}$ à l'est de Kobo.

\subsubsection{Diversité des installations de forge}

Plusieurs modèles d'ateliers de forge, de foyers et de systèmes de soufflerie ont été observés au cours de nos visites au Pays dogon. Pour faciliter la lecture des prochaines pages, nous les présentons ici.

\section{Deux types d'ateliers}

Le hangar est un complexe spatial de travail $\left(25-45 \mathrm{~m}^{2}\right)$ qui regroupe plusieurs unités sous un même toit. Dans chaque unité de travail, les composantes (foyer, soufflerie) sont mobiles.

Les murs extérieurs du hangar sont faits de blocs de grès de formes quadrangulaires épais de 40 à $50 \mathrm{~cm}$. Pour ce bâtiment, les murs extérieurs mais aussi des piliers en blocs de grès et des poteaux de bois posés verticalement, supportent la toiture. La toiture est constituée par de longs troncs de rônier (3 m) épais (30 à $40 \mathrm{~cm}$ ). Sur cette ossature, reposent des branches plus courtes $(1.5 \mathrm{~m})$ et plus fines (10 à $20 \mathrm{~cm}$ ). Enfin, un maillage épais de feuilles de rônier recouvre le tout, ce qui confère une bonne étanchéité au hangar.

Le hangar est un ouvrage bas (1.5-1.8 m). Les frappeurs travaillant debout, ils ne peuvent pas se redresser totalement lorsqu'ils battent le fer. Enfin, des ouvertures sont présentes dans les murs dans toutes les directions.

L'abri se compose d'une seule unité spatiale de travail $\left(8 \mathrm{~m}^{2}\right)$ dont les composantes (foyer, soufflerie) sont immobiles.
Chaque unité est protégée du soleil par un abri : il s'agit de fins poteaux de bois $(10 \mathrm{~cm})$ d'une hauteur de $1.7 \mathrm{~m}$ qui soutiennent une toiture en tiges et paille de mil. Les frappeurs travaillant assis, la hauteur de l'abri importe peu. Parfois, plusieurs de ces unités sont disposées les unes à côté des autres (3 à 6) mais sans orientation préférentielle par rapport aux points cardinaux.

\section{Deux types de foyers}

Le foyer en fosse est creusé à même le sol et présente une forme elliptique. La fosse est entourée de trois blocs de grès recouverts d'une épaisse couche réfractaire de banco. Ce type de foyer ne se retrouve que dans les ateliers de type hangar. Les blocs servent à consolider les parois et à garder la chaleur à l'intérieur du foyer. Ils sont remplacés annuellement tandis qu'un rechapage régulier est nécessaire.

La tuyère, en argile et en paille, qui débouche dans le foyer est entourée de deux blocs de grès. A l'opposé de la tuyère, un dernier bloc allongé est disposé sur toute la longueur du foyer. Enfin, les deux extrémités de la structure permettent le passage du fer dans le foyer, permettant ainsi à 2 équipes de travailler en même temps.

Le foyer en coupole est formé d'une fosse circulaire hémisphérique surmontée d'une superstructure circulaire. Celle-ci est constituée de blocs de grès quadrangulaires cimentés dans du banco.

$\mathrm{Au}$ minimum trois ouvertures sont présentes : la porte du foyer qui permet le passage du fer dans le feu ; une fenêtre située en face du souffleur afin de pouvoir apprécier les couleurs des braises ; une dernière ouverture au sommet de la construction, formée par une poterie trouée et qui fait office de cheminée.

\section{Deux types de systèmes de soufflerie}

La soufflerie ventilateur est réalisée à partir d'un assemblage de matériaux de récupération : il s'agit d'une introduction moderne de la soufflerie dans l'atelier.

Le souffleur actionne le système par rotation en saisissant une poignée métallique soudée à une jante de bicyclette. Par le biais d'une courroie en toile, la jante met en mouvement une hélice qui propulse l'air à l'intérieur d'une conduite métallique tubulaire insérée dans une tuyère et débouchant dans le foyer.

Des matériaux lourds (planche, blocs de grès etc) sont posés sur la conduite d'air pour stabiliser 
l'engin pendant son fonctionnement. Avec peu d'effort pour le souffleur, cette technologie permet de fournir un débit d'air important et continu dans le foyer.

La soufflerie à double pots, se compose de deux poteries (diamètre : $20 \mathrm{~cm}$ ) disposées l'une à côté de l'autre et percées sur la panse : il s'agit d'une soufflerie traditionnelle. Deux conduites d'air tubulaires et métalliques sont insérées dans les poteries d'un côté et dans une tuyère en banco de l'autre côté.

Tout ce dispositif est solidement ancré dans une structure en banco à proximité du foyer. Une peau de chèvre est nouée au col des poteries à l'aide d'une corde. Cette peau est repliée de façon à former une poignée et une ouverture d'air que le souffleur actionne alternativement selon un rythme plus ou moins cadencé. Cette soufflerie, qui délivre un débit d'air discontinu dans le foyer, demande au souffleur une force physique importante.

\subsubsection{Outils fabriqués}

Les forgerons produisent des outils en fer pour toute la société, hommes ou femmes : agriculteurs, les chasseurs, les artisans etc (Figure 16) :

- des outils aratoires (houes, pioches),

- des outils de chasse (pièges à hyène, pointes de flèches),

- des armes (pistolets),

- des parures (bracelets et bagues torsadées),

- du matériel domestique (couteaux, passoires, louches),

- des mécanismes de fer (système de fermeture des serrures, clous),

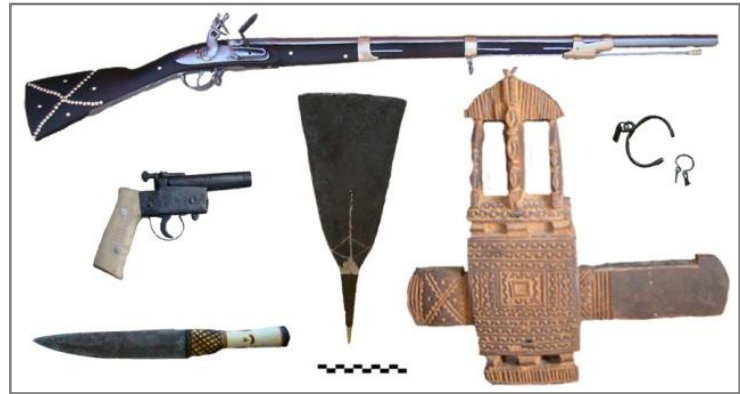

Figure 16 - Exemples d'outils fabriqués à la forge de Fiko : fusil, pistolet, houe, couteau, serrure, bijoux de fer.

- des objets de culte et cérémonie (fétiches, fusils de cérémonie),

A Fiko, les forgerons fondaient les alliages à base de cuivre à la cire perdue pour réaliser des bijoux et figurines (Huysecom et al. 2002 : 148). Le cuivre est toujours utilisé dans cet atelier pour la confection des bagues de fusils et clous de décoration des objets en bois.

D'après notre informateur Sékou Traoré de Fiko, le travail du bois peut être effectué par les dogons s'ils en ont la connaissance; cependant, ce sont les forgerons qui fabriquent et décorent les parties en bois des outils qu'ils confectionnent: manches des couteaux et des outils aratoires, crosses et fût des fusils. La fabrication des serrures est exclusivement réservée aux forgerons car, dans un cadre de partage du travail, comme « ils ne cultivent pas alors ils font les serrures » (Sékou Traoré, Fiko). A Doundé, les forgerons fabriquent des mortiers et pilons destinés au broyage des céréales.

Toutefois, c'est la maintenance du parc d'outils en fer du village qui demande le plus de travail : il faut reforger, réparer et affuter en permanence. 


\subsection{FORGE DE FIKO}

\subsubsection{Forgerons}

\section{Origine}

Les forgerons de Fiko ont pour patronyme Baguéné et ils appartiennent au clan des Jèmè-irin. La tradition orale montre qu'ils ont émigré de Wadianka dans le delta intérieur du Niger, vers Fiko à la fin du $18^{\text {ème }}$ siècle et ils appartenaient à la caste des forgerons avant leur arrivée au Pays dogon (Robion-Brunner 2010).

A cette époque, le village est alors situé sur un promontoire gréseux. Par ailleurs, les vestiges de deux anciennes forges sont encore visibles dans les ruines du village (Huysecom et al. 2003 : 54, 59). En 1961, le chef du village Allaye Koundi Traoré fonde le nouveau village de Fiko au pied du promontoire, ce qui permet un accès simplifié à l'eau et au réseau de transports tout en développant la superficie du village.

Les Baguéné sont les derniers à descendre du village perché en 1988 (Sekou Traoré, collaborateur, communication orale). La forge dans laquelle nous avons travaillé date de cette époque, même si des reconstructions saisonnières sont nécessaires.

\section{Identité et fonction}

Sekou, le fils du vieux forgeron Ali Baguéné, est un maître de forge expérimenté (Tableau 3). C'est lui qui a supervisé l'essentiel du travail. Toutefois, il a cédé à de nombreuses reprises sa place à Nou et, occasionnellement, il a aidé à frapper le fer.

\begin{tabular}{ccccc}
\hline Identité & & \multicolumn{3}{c}{ Fonction exercée } \\
Nom & Age & M & F & S \\
Sekou & 45 & 39 & 5 & 0 \\
Nou & 25 & 8 & 19 & 0 \\
Soumaïla & 15 & 0 & 29 & 0 \\
Oumar & 25 & 0 & 25 & 0 \\
Soulemane & 25 & 0 & 10 & 0 \\
Abdoulaye & 40 & 0 & 5 & 0 \\
Hamadi & 10 & 0 & 4 & 47 \\
Amadou & 15 & 0 & 2 & 0 \\
Mani & 50 & 0 & 1 & 0 \\
Nb pers./forgeage & 1 & 1 à 4 & 1 \\
\hline
\end{tabular}

Tableau 3 - Identité/fonction exercée par les forgerons à Fiko. $\mathrm{M}=$ maître-F=frappeur-S=souffleur.
De nombreux frappeurs ont participé au travail, en moyenne deux par forgeage: certains sont confirmés (Soulemane, Oumar, Nou, Abdoulaye), d'autres sont encore en apprentissage (Soumaïla, Amadou, Hamadi). Durant tout notre travail c'est Hamadi, un des fils de Sekou qui s'est chargé d'activer la soufflerie.

De plus, Mani Karambé notre chauffeur, originaire d'une famille de forgerons de Bandiagara a également souhaité battre le fer au cours d'un forgeage.

\subsubsection{Atelier}

\section{Organisation spatiale}

L'atelier est implanté au nord du village, au pied des impressionnants amas de scories de réduction (Figure 17). Il regroupe deux espaces adjacents, pour une superficie totale de $43 \mathrm{~m}^{2}$.

Le premier espace est un bâtiment de type hangar qui présente une forme carrée légèrement désaxée. La surface au sol intérieure est de $35 \mathrm{~m}^{2}$. Des ouvertures sont présentes sur trois côtés; une dernière ouverture actuellement murée, reste visible dans le mur est. Dans le hangar, trois piliers en blocs de grès, chacun formé de deux à quatre colonnes, supportent la toiture. Par ailleurs, deux poteaux en bois ont été ajoutés à la construction aux ouvertures nord et ouest.

Un second espace de $8 \mathrm{~m}^{2}$ se trouve en extérieur, en face du mur sud du hangar : il s'agit d'un espace nouveau, créé lors de la construction d'une maisonnette qui abrite un poste à souder et un générateur.

Enfin, il a été observé une zone située au nord du hangar, pour le montage des charrettes.

\section{Unités spatiales de travail}

Le hangar comprend près de neuf unités spatiales de travail. Cinq sont dédiées au travail du fer, deux au travail du bois et deux à des travaux mixtes : métaux, bois ou banco (Figure 17). 


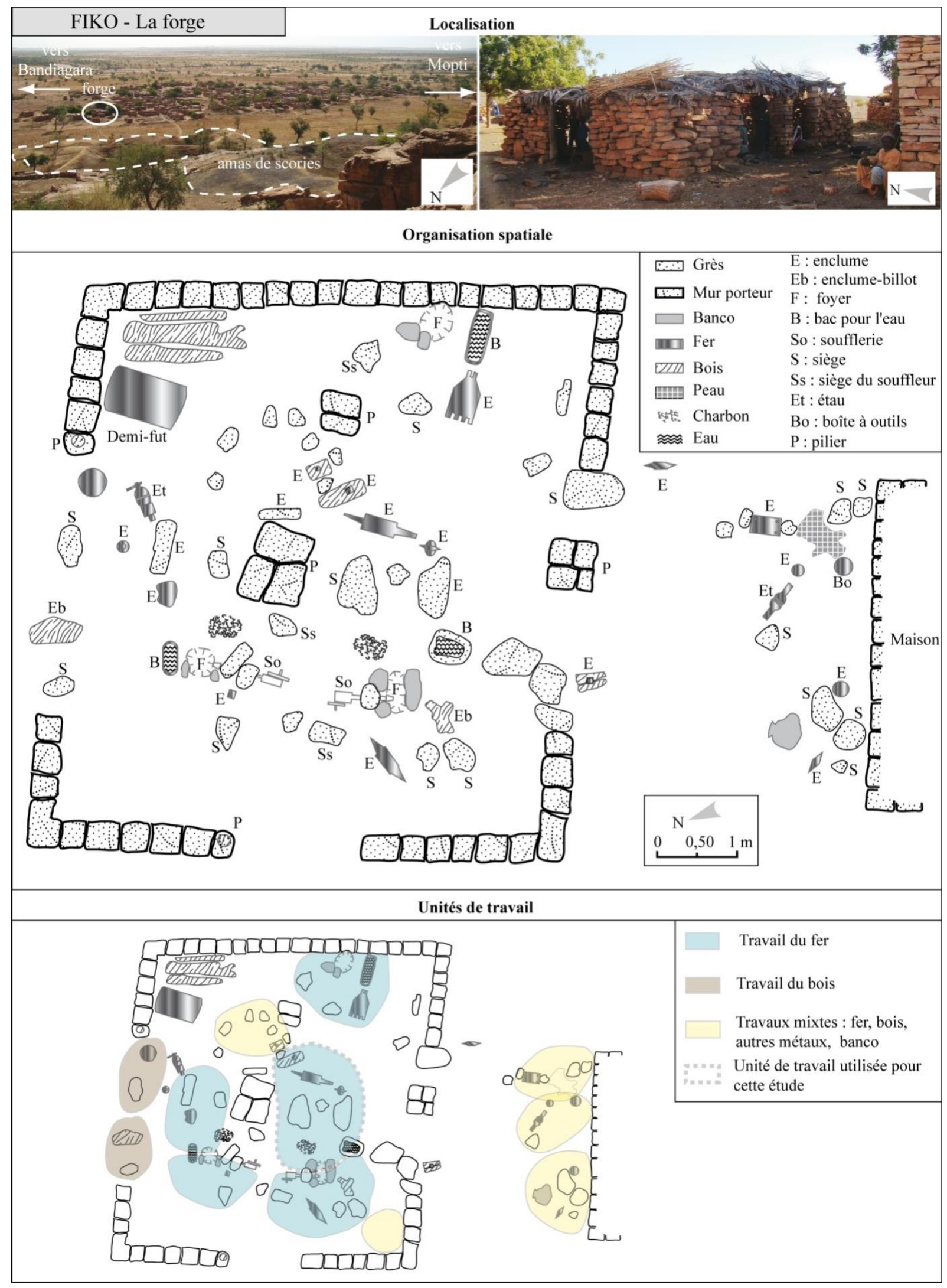

Figure 17- Présentation de l'atelier de forge de Fiko. Dao : Michel Mauvilly. 
Chaque unité de travail du fer comprend un foyer en fosse, une soufflerie ventilateur, un bac à eau, des enclumes et des pierres qui servent de siège pour le maître, le frappeur et le souffleur. Les bacs à eau sont d'anciennes meules de grès tellement usées que la dépression creusée par la mouture est assez profonde pour contenir un à deux litres d'eau. Il est aussi possible de trouver des bacs métalliques qui sont des pièces de récupération.

La soufflerie, le foyer et le bac à eau sont des éléments qui sont communs à deux unités de travail du fer et donc à deux équipes de forgerons.

Dans le cadre de notre travail, les forgeages ont tous été réalisés au sein de l'unité de travail principale de la forge (enclume de fondation), en face de l'entrée sud du hangar. A cet endroit, ce sont des outils de grande taille qui sont élaborés. Les autres unités de travail du fer sont généralement utilisées par les apprentis qui fabriquent des lames de couteaux.

Le travail $\mathrm{du}$ bois est exécuté préférentiellement du côté nord de l'atelier : ici, des apprentis confectionnent les manches des couteaux et des outils (houe par exemple). Les unités de travail mixtes sont situées dans des recoins du hangar qui ne gênent pas le passage.

L'espace extérieur est réservé aux vieux forgerons. Adossés à l'ombre, ils gardent un œil sur les travaux effectués dans l'enceinte de l'atelier et continuent à prodiguer les conseils aux plus jeunes (fils ou petits-fils). Cet espace extérieur comprend trois unités de travail dédiées à des travaux mixtes.

Enfin, il faut préciser qu'au cours des différents séjours effectués dans ce village, nous avons remarqué des légers changements quant au nombre et à l'emplacement des unités de travail. Seule l'unité de travail principale est permanente.

\section{Foyer et soufflerie}

Tous les foyers utilisés sont des foyers en fosse (Figure 18) : $30 \mathrm{~cm}$ de longueur, $12 \mathrm{~cm} \mathrm{de}$ largeur et $10 \mathrm{~cm}$ de profondeur, pouvant contenir un volume d'environ $3000 \mathrm{~cm}^{3}$. Toutefois, la zone active du foyer correspond à un volume réduit d'environ $2000 \mathrm{~cm}^{3}$.

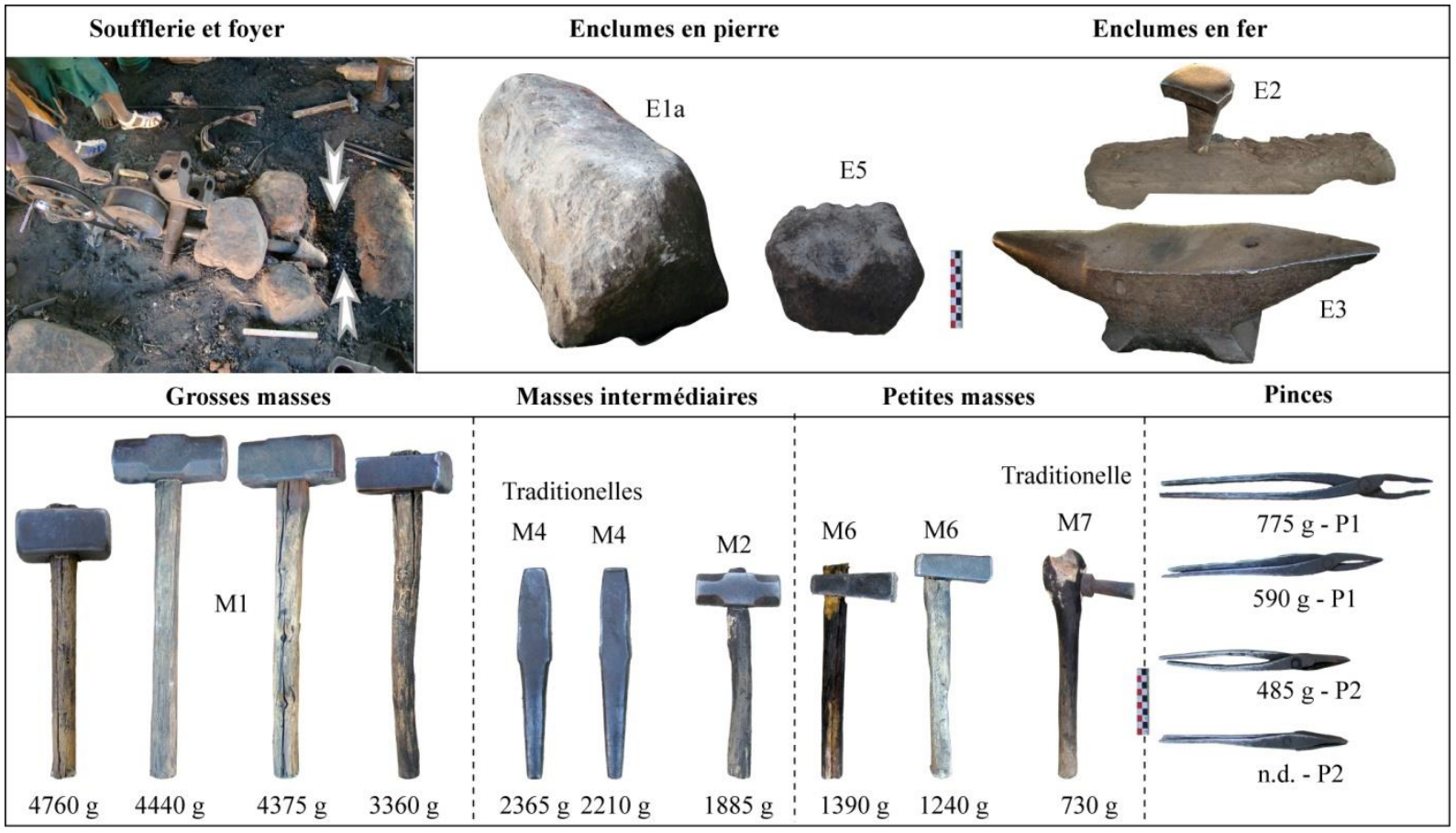

Figure 18 - Soufflerie de type ventilateur et foyer en fosse de la forge de Fiko.

E1a- grande enclume traditionnelle en grès de l'atelier (enclume de fondation) E2 - petite enclume traditionnelle en fer fichée dans son support de bois E3 : enclume européenne à bigornes E5 - enclume matrice servant à réaliser la nervure des lames de houe
M1 - grosses masses européennes à deux panes actives et emmanchement centré M2 - marteau européen à deux panes actives et emmanchement centré en bois M4 - marteaux traditionnels en fer non emmanchés de forme conique, surmontés d'un parallélépipède rectangle
M6 - petits marteaux européens à une pane active et emmanchement décentré en bois M7 - petit marteau traditionnel à emmanchement à soie en bois

$\mathrm{P} 1$ - pinces $\mathrm{P} 2$-tenailles. 
Une soufflerie ventilateur alimente en air le foyer. Ce dispositif est tout à fait mobile.

\section{Outils de la forge}

Pour les travaux demandant beaucoup de force, les frappeurs battent le fer à tour de rôle à l'aide des grosses masses européennes (M1), avec/sans le maître, sur une grande enclume en grès (E1a). Il s'agit de l'enclume de fondation de la forge. Les larges dimensions de sa table $(\mathrm{L}=80 \mathrm{~cm}, 1=25 \mathrm{~cm})$ sont idéales pour le travail de grosses masses de métal $(1 \mathrm{~kg})$.

S'il travaille seul, le maître emploie une masse traditionnelle (M4) ou une masse européenne (M2).

Au stade des finitions, il utilise des marteaux plus légers: un marteau traditionnel à emmanchement à soie (M7) ou un marteau européen (M6). Le fer est alors frappé soit sur une enclume en fer européenne (E3), soit sur une petite enclume en fer bombée de $10 \mathrm{~cm}$ de côté fichée dans un socle de bois (E2).
Une petite enclume matrice en grès (E5) est employée uniquement pour un usage spécifique : afin d'obtenir la nervure de la lame de houe, l'artisan emboutit le fer sur le profil creux de l'enclume.

Enfin, des pinces sont utilisées pour tenir le fer dans les braises (P1) et le maintenir sur l'enclume pendant le martelage (P2).

Hormis l'enclume de fondation, toutes les enclumes ainsi que les souffleries sont susceptibles d'être déplacées vers d'autres secteurs et les foyers rapidement reconstitués en d'autres places. Certains bacs à eau sont des réservoirs métalliques de récupération.

Enfin, à la fin du travail tous les outils sont laissés sur place, à même le sol, à disposition des autres forgerons qui en auraient besoin. 


\subsection{FORGE DE KAKOLI}

\subsubsection{Forgerons}

\section{Origine}

Les forgerons de Kakoli, de patronyme Yanogué, appartiennent au clan des Jèmè-irin.

$\mathrm{Au}$ cours du $15^{\text {ème }}$ siècle, les ancêtres des forgerons actuels ont quitté le Mandé d'abord pour Ségou puis pour Wadianka près du fleuve Niger. Au cours du $16^{\text {ème }}$ siècle, ils ont migré au nord-ouest du plateau de Bandiagara et ont fondé, avec la caste des cordonniers, le village d'Impari reconnu comme

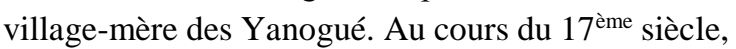
certains d'entre eux s'installèrent à Kakoli, zone riche en minerai de fer (Robion-Brunner $2010: 20$; 22-25; 47-49).

\section{Identité et fonction}

Abdou a endossé sa fonction de maître de forge une fois en totalité, six fois pour le début du travail, et il a aidé à battre le fer une seule fois (Tableau 4). Sekou a quant à lui été maître de forge une fois en entier et six fois pour terminer le travail commencé par Abdou.

Du point de vue de l'expérience du métier à la forge, Abdou est certainement plus expérimenté que Sekou. Enfin, Alimam l'apprenti forgeron a aidé à frapper le fer et il s'est aussi occupé de la soufflerie.

\begin{tabular}{ccccc}
\hline \multicolumn{2}{c}{ Identité } & \multicolumn{3}{c}{ Fonction exercée } \\
Nom & Age & $\mathrm{M}$ & $\mathrm{F}$ & $\mathrm{S}$ \\
Abdou & 40 & $1+6($ déb) & 1 & 0 \\
Sekou & 25 & $1+6($ fin $)$ & $1+6($ deb $)$ & 0 \\
Alimam & 15 & 0 & 3 & 8 \\
Nb pers./forg. & 1 & 1 à 2 & 1 \\
\hline
\end{tabular}

Tableau 4 - Identité/fonction exercée par les forgerons à Kakoli. $\mathrm{M}=$ maître-F=frappeur-S=souffleur.

\subsubsection{Atelier}

\section{Organisation spatiale}

La forge surplombe l'un des accès au sommet de l'inselberg. L'atelier de Kakoli est un hangar elliptique qui a une superficie totale au sol de $25 \mathrm{~m}^{2}$ (Figure 19). Le sol de la forge présente deux niveaux de $1.2 \mathrm{~m}$ de différence, dû au relief naturel du substrat gréseux. De plan irrégulier avec des murs courbes, le hangar correspond à l'accolement de deux espaces elliptiques.
Le premier espace mesure $10 \mathrm{~m}^{2}$ et est orienté à l'ouest sur un seul niveau haut. La hauteur sous toit est de $1.8 \mathrm{~m}$.

Le second espace possède une surface de $15 \mathrm{~m}^{2}$, il est orienté nord-sud sur deux niveaux : le niveau haut a une hauteur sous toit de $1.5 \mathrm{~m}$ et le niveau bas une hauteur de $2 \mathrm{~m}$.

Dans cette configuration, la toiture est légèrement inclinée vers le sud-est. Dans le bâtiment, trois piliers en blocs de grès et cinq poteaux en bois aident à supporter la charpente de la toiture. Le bâtiment comprend cinq ouvertures : les trois entrées au nord nécessitent de s'accroupir pour pénétrer dans la forge, ce qui n'est pas le cas pour les deux entrées du sud.

Enfin, de nombreux blocs de grès à cupules sont utilisés comme polissoir pour affuter les lames en fer des outils.

\section{Unités spatiales de travail}

Le hangar possède quatre unités spatiales de travail (Figure 19).

Le travail du fer et le forgeage des grands objets en fer est réalisé dans le niveau haut ouest de la forge. Il s'agit de l'unité de travail principale de la forge (enclume de fondation) et c'est à ce poste que nous avons travaillé.

Le niveau bas au sud de la forge est une unité de travail du bois dédiée aux apprentis et aux jeunes enfants. Ici, ils s'essaient à la taille des manches des outils fabriqués par leurs aînés.

Enfin, deux unités spatiales de travail orientent leurs activités sur le travail mixte du fer et du bois. Les petits objets de fer et des manches de bois pour les outils sont confectionnés.

Au vu du peu de place disponible au sol dans cet atelier, il semble peu probable que l'emplacement et le nombre des unités de travail varie fortement.

\section{Foyer et soufflerie}

Les foyers utilisés actuellement sont des foyers en fosse (Figure 20) : celui de l'unité de travail principale mesure $40 \mathrm{~cm}$ de longueur, $10 \mathrm{~cm}$ de largeur et $10 \mathrm{~cm}$ de profondeur, pouvant contenir 


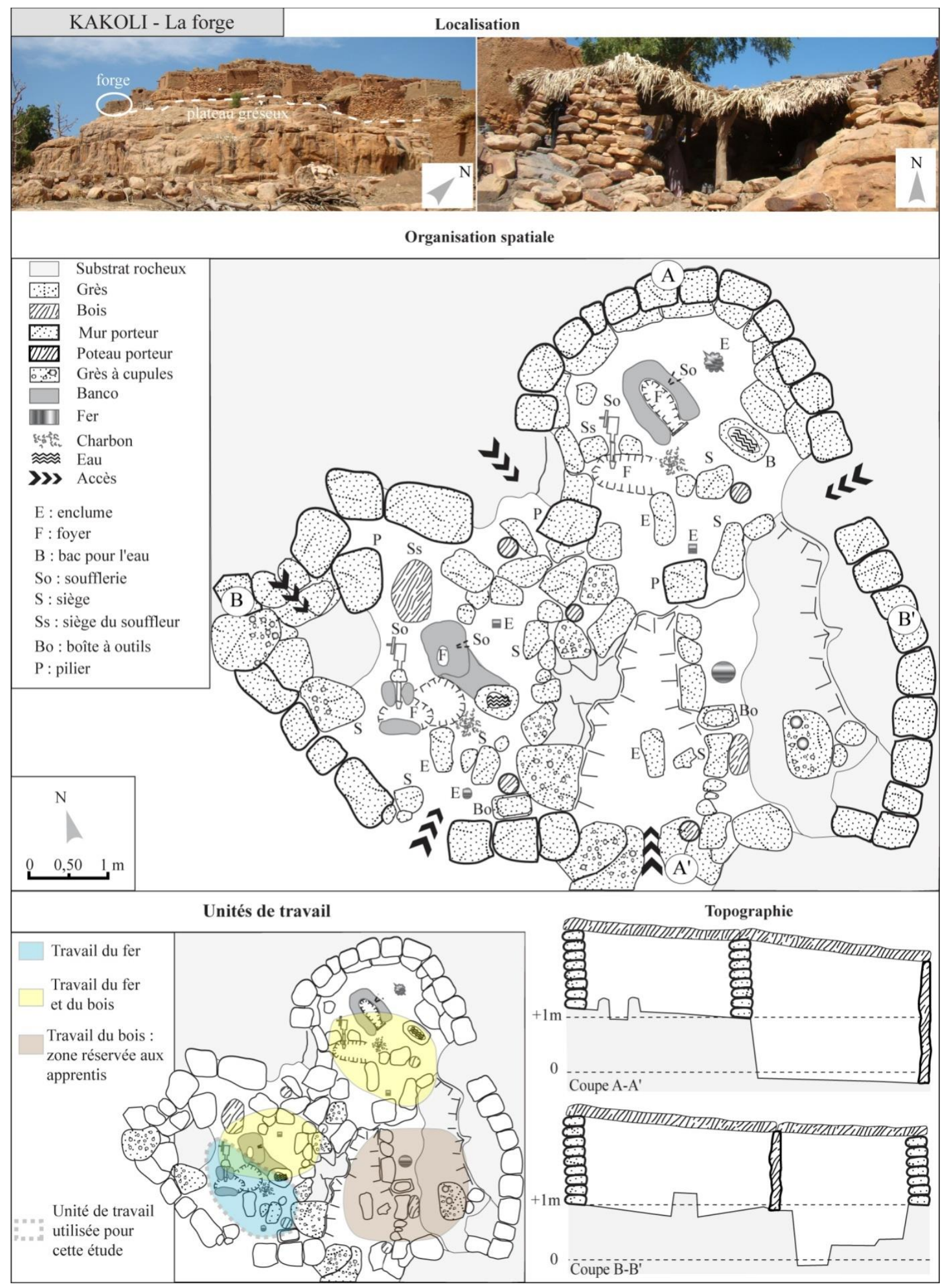

Figure 19 - Présentation de l'atelier de forge de Kakoli. Dao : Michel Mauvilly. 
un volume d'environ $4000 \mathrm{~cm}^{3}$. Toutefois, la zone active $\mathrm{du}$ foyer est plus restreinte: environ $1000 \mathrm{~cm} 3$. Les foyers étant rapprochés des murs, le fer ne peut être mis à chauffer que d'un seul côté. Ici, les blocs de grès sont probablement recouverts d'un revêtement en argile et en ciment (3.1.3). Une soufflerie ventilateur alimente en air le foyer. $\mathrm{Ce}$ dispositif est mobile.

Toutefois, il existe deux foyers anciens qui ne sont plus utilisés : il s'agit de constructions permanentes en cercle/demi-cercle comprenant des murs en banco de $40 \mathrm{~cm}$ de hauteur. La conduite d'air est introduite par un orifice cylindrique situé à la base de la structure (Figure 19). Ce type de foyer a été observé au Burkina Faso, avec un orifice situé dans le milieu de la structure (Kienon-Kaboré 2003 : 153-154). Ce foyer devait certainement être associé à un autre type de soufflerie. (Figure 26). Toutefois, nous n'avons pas fait d'enquête pour comprendre si ces foyers ont été introduits par un autre clan de forgerons ou s'il s'agit juste d'un ancien modèle moins adapté.

\section{Outils}

Pour les travaux nécessitant la déformation d'une grande quantité de métal, un frappeur bat le fer en alternance avec le maître à l'aide d'une des grosses masses (M1) sur l'enclume en grès de fondation de la forge (E1a). Sa table mesure $70 \mathrm{~cm}$ de longueur et $25 \mathrm{~cm}$ de largeur (Figure 20). Sur cette enclume, le maître utilise des marteaux traditionnels coniques en fer (M4) et un marteau européen (M3).

$\mathrm{Au}$ stade des finitions, le maître travaille sur une petite enclume bombée en fer fichée dans un support de bois enterré (E2). Il emploie alors soit un marteau traditionnel à emmanchement à soie (M7), soit un marteau européen léger (M6).

La paire de tenailles utilisée pour manipuler le fer dans le foyer est longue : $70 \mathrm{~cm}$ (P1). Trois autres pinces sont employées en alternance lors du martelage (P2). Cet atelier ne semble pas posséder d'enclume européenne à bigornes en métal. Enfin, à la fin du travail, tous les outils sont laissés au sol.

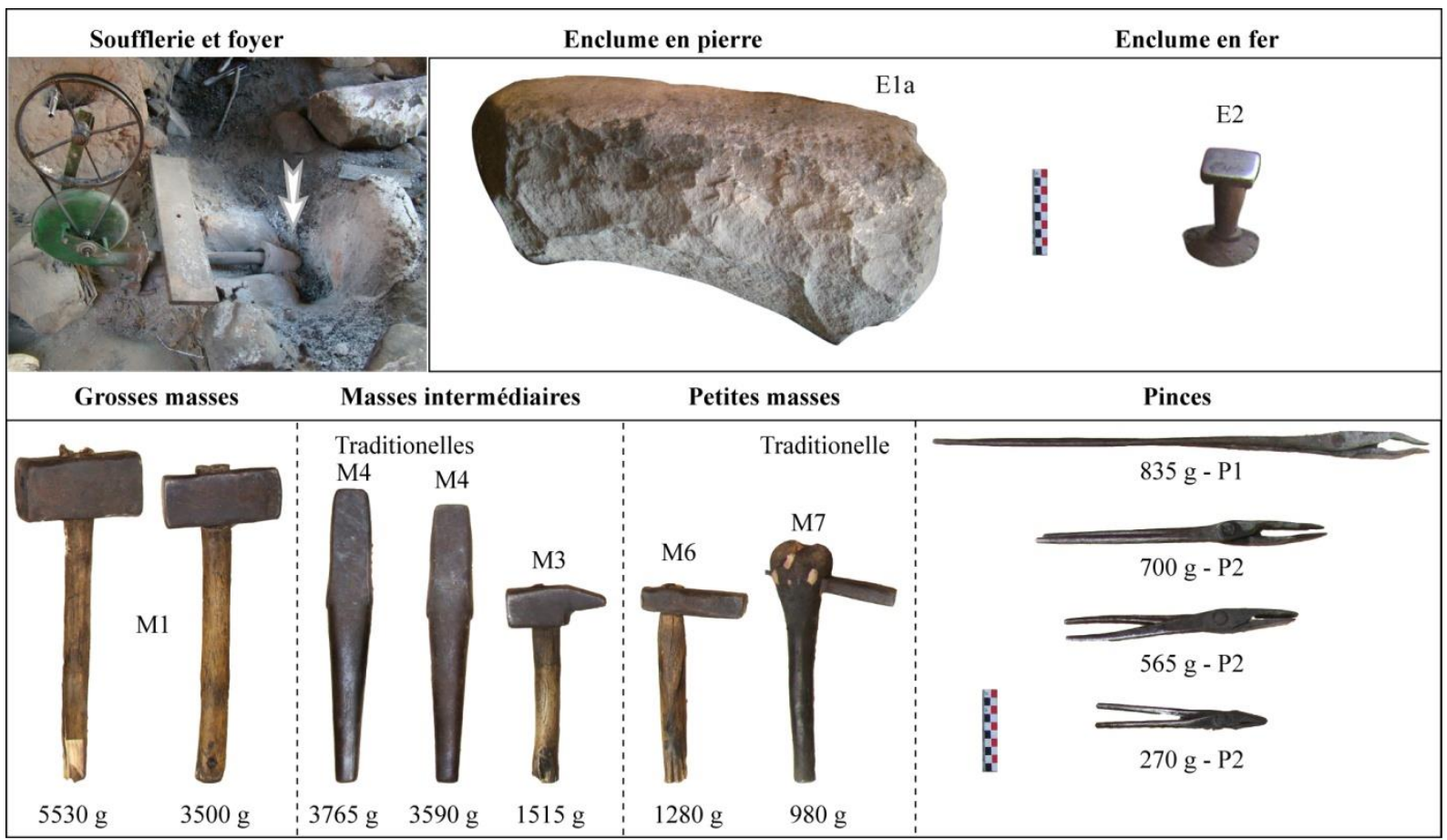

Figure 20 - Soufflerie de type ventilateur et foyer en fosse de la forge de Kakoli.

E1a- grande enclume traditionnelle en grès (enclume de fondation)

E2 - petite enclume traditionnelle en fer fichée dans son support de bois

M1 - grosses masses européennes à deux panes actives et emmanchement centré en bois
M3 - marteau européen à une pane active et emmanchement centré en bois

M4 - marteaux traditionnels en fer non emmanchés de forme conique, surmontés d'un parallélépipède rectangle

M6 - petit marteau européen à une pane active et emmanchement décentré en bois
M7 - petit marteau traditionnel à emmanchement à soie en bois P1 - pince $\mathrm{P} 2$-tenailles 


\subsection{FORGE DE KOBO}

\subsubsection{Forgerons}

\section{Origine}

Les forgerons de Kobo portent le patronyme Djo et ils appartiennent au clan des Jèmè-yélin. Ils émigrèrent du Mandé à la fin du $16^{\text {ème }}$ siècle et s'installent dans la plaine du Séno et le plateau méridional en maîtrisant déjà toutes les connaissances de la production et de la transformation du fer (Huysecom 2001 : 74).

\section{Identité et fonction}

Le travail a été réalisé en intégralité par le forgeron Tiégé qui est âgé mais très expérimenté (Tableau 5). Il est un des derniers forgerons du Pays dogon capable de maîtriser les techniques de production du fer par la méthode directe de réduction (Huysecom \& Agustoni 1996).

Les frappeurs, tous de moins de 20 ans et en formation (excepté Enné), ont été nombreux à se succéder : ses fils Malik, Kélif, Drissa et son neveu Dambo. Tous ces apprentis forgerons se sont aussi investis pour le travail à la soufflerie.

\begin{tabular}{ccccc}
\hline \multicolumn{2}{c}{ Identité } & \multicolumn{3}{c}{ Fonction exercée } \\
Nom & Age & $\mathrm{M}$ & $\mathrm{F}$ & $\mathrm{S}$ \\
Tiégé & 70 & 18 & 0 & 0 \\
Malik & 20 & 0 & 15 & 8 \\
Kélif & 15 & 0 & 13 & 3 \\
Drissa & 15 & 0 & 8 & 4 \\
Enné & 30 & 0 & 3 & 1 \\
Dambo & 10 & 0 & 2 & 2 \\
Nb pers./forgeage & 1 & 1 à 4 & 1 \\
\hline Tableau 5 - Identité/fonction exercée par les forgerons à Kobo. \\
\multicolumn{5}{c}{ M=maître-F=frappeur-S=souffleur. }
\end{tabular}

\subsubsection{Atelier}

\section{Organisation spatiale}

Dans ce quartier de Kobo, un premier atelier est implanté au sein même de la concession du maître Tiégé ; un second atelier est installé à quelques dizaines de mètres à l'extérieur de la concession, au pied de l'amoncellement des blocs effondrés de la falaise et à l'ombre d'épais feuillages d'arbres.

Dans la concession, le premier atelier correspond à un seul abri d'environ $5 \mathrm{~m}^{2}$, sans toiture au-dessus du foyer.
Le second atelier de forge de Kobo, au pied des éboulis de la falaise, a déjà fait l'objet d'une description approfondie (Huysecom et al. 2005 : 115 ; Serneels \& Huysecom A paraître).

Cet atelier se compose de trois abris distants d'une dizaine de mètres. Le premier est complètement ruiné, le second n'est pas directement en état de marche, le dernier est fonctionnel : c'est celui où le travail a été réalisé (Figure 21). La superficie d'un abri est d'environ $8 \mathrm{~m}^{2}$. Là encore, les foyers n'étaient pas couverts d'un toit de paille lors de notre passage.

De plus, les forgerons se servent d'une large enclume plate en grès située au sud-est à $4 \mathrm{~m} \mathrm{du}$ foyer. Les frappeurs et le maître doivent alors se déplacer jusqu'à cette enclume et marteler le fer avant qu'il ne refroidisse trop rapidement. Dans cette configuration, l'atelier présente une surface de près de $20 \mathrm{~m}^{2}$.

\section{Unités spatiales de travail}

Ici, chaque abri correspond à une seule unité spatiale de travail dédiée au travail du fer (Figure 21). Il comprend un foyer, un bac à eau, une soufflerie, un siège en pierre pour le souffleur, une enclume face à la porte du foyer et deux sièges en pierre pour le maître et le frappeur situés de part et d'autre de l'enclume. Ainsi, le maître attrape le fer dans le foyer avec la main gauche et frappe le fer sur l'enclume avec la main droite.

Par ailleurs, certains auteurs décrivent une zone de travail du bois à proximité des forges, ce que nous n'avons pas observé lors de notre passage en 2008 (Serneels \& Huysecom A paraître : 154).

Toutefois nous avons observé que des cages à poules en feuille de rônier sont tressées à la main par les enfants directement à l'est de l'atelier.

\section{Foyer et soufflerie}

A Kobo, ce sont les foyers en coupole qui sont utilisés. La superstructure a une hauteur de $110 \mathrm{~cm}$, un diamètre externe de $120 \mathrm{~cm}$ et interne de $75 \mathrm{~cm}$. La fosse a une profondeur de $15 \mathrm{~cm}$ et un diamètre de $20 \mathrm{~cm}$, ce qui correspond à un volume de $3100 \mathrm{~cm}^{3}$. La porte du foyer mesure $40 \mathrm{~cm}$ en longueur et en largeur. Ce foyer est alimenté en air par une soufflerie traditionnelle à double pots. 


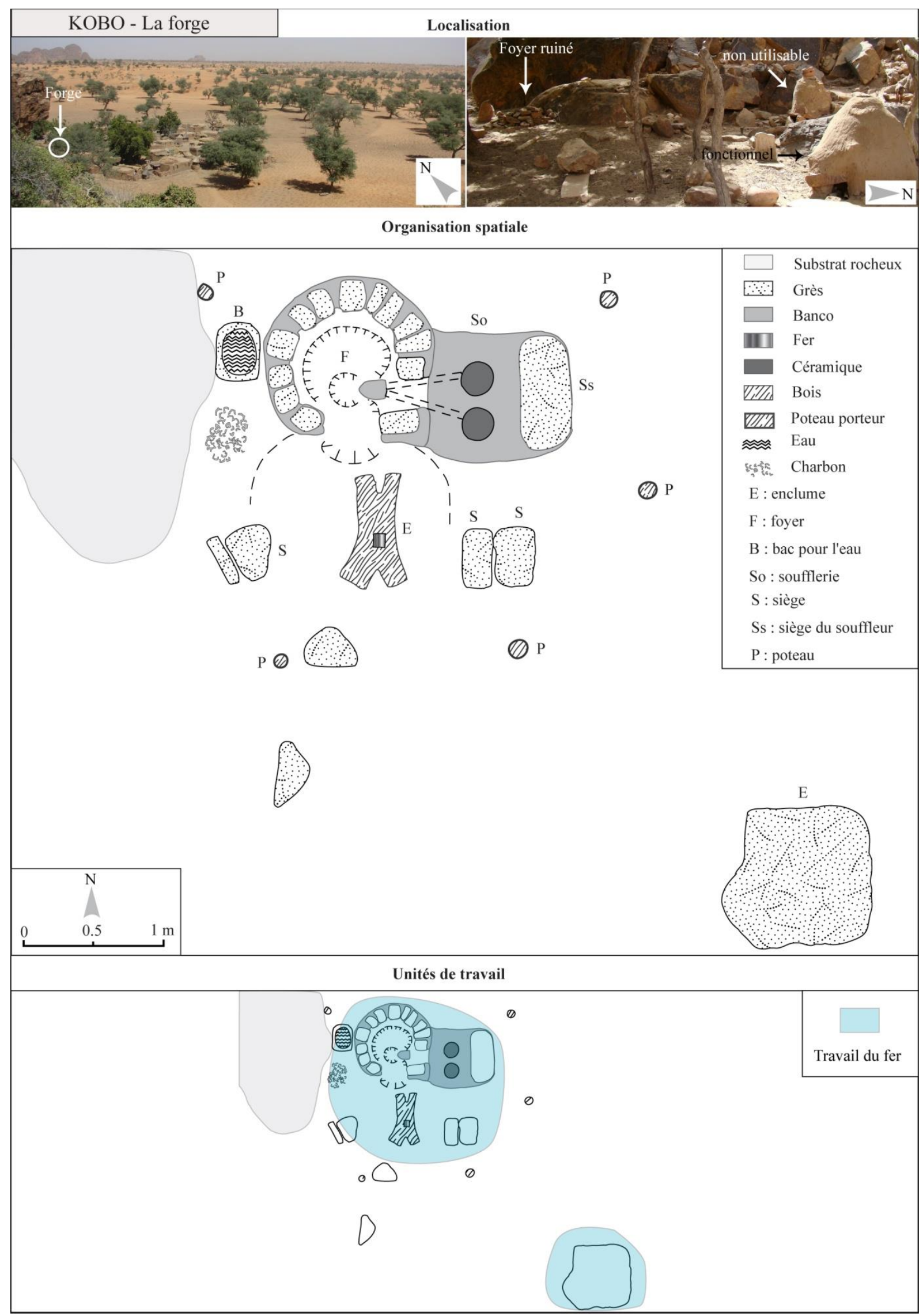

Figure 21 - Présentation de l'atelier de forge de Kobo. Un plan général des trois forges n'a pas été réalisé. D'après Huysecom et al. $2005: 115$. 


\section{Outils}

En face de la porte du foyer, une petite enclume en fer de $10 \mathrm{~cm}$ de côté est fichée dans un socle de bois (Figure 23 ; E2). Sur cette enclume, le maître n'utilise que des masses traditionnelles: l'une en forme de hachoir (M5) et une autre à emmanchement à soie uniquement pour les finitions (M7). En face de lui un frappeur se sert d'une masse européenne (M3). Une autre masse traditionnelle non emmanchée est décrite par deux auteurs (Figure 22) : il s'agit d'un bloc quadrangulaire en fer. Toutefois nous n'avons observé ni sa présence ni son utilisation car ce type de marteau ne peut pas être montré aux femmes.

Pour le travail d'une grande quantité de métal, le forgeron doit déplacer le fer chaud vers un large bloc plat en grès (E1b). Sa table de $1 \mathrm{~m}$ de longueur et $0.8 \mathrm{~cm}$ de largeur permet à plusieurs frappeurs (jusqu'à trois) de frapper le fer simultanément, debout autour de l'enclume. Pour cela ils utilisent des grosses masses européennes (M1) mais aussi le marteau européen (M3). Le maître est quant à lui accroupi et tient fortement le fer dans une pince qui sert également de tenaille (P1-P2).

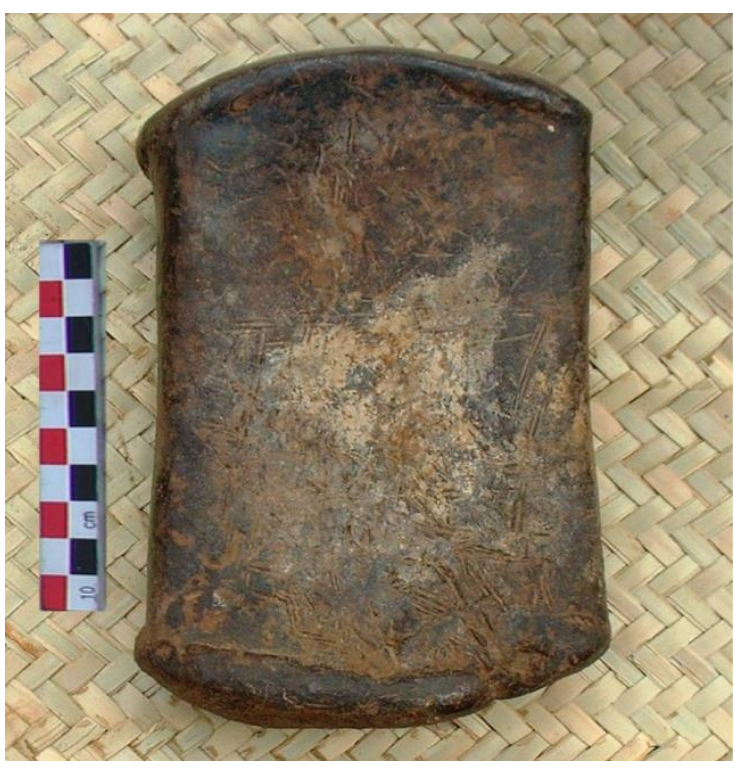

Figure 22 - Marteau traditionnel en fer 18x11x4 cm / $5 \mathrm{~kg}$. (Serneels \& Huysecom A paraître : 155)

La lame ainsi produite, le maître aiguise son tranchant et le décore à l'aide d'une lime (L). A la fin $\mathrm{du}$ forgeage, les peaux des soufflets sont soigneusement retirées et ramenées avec les marteaux dans la concession du forgeron.

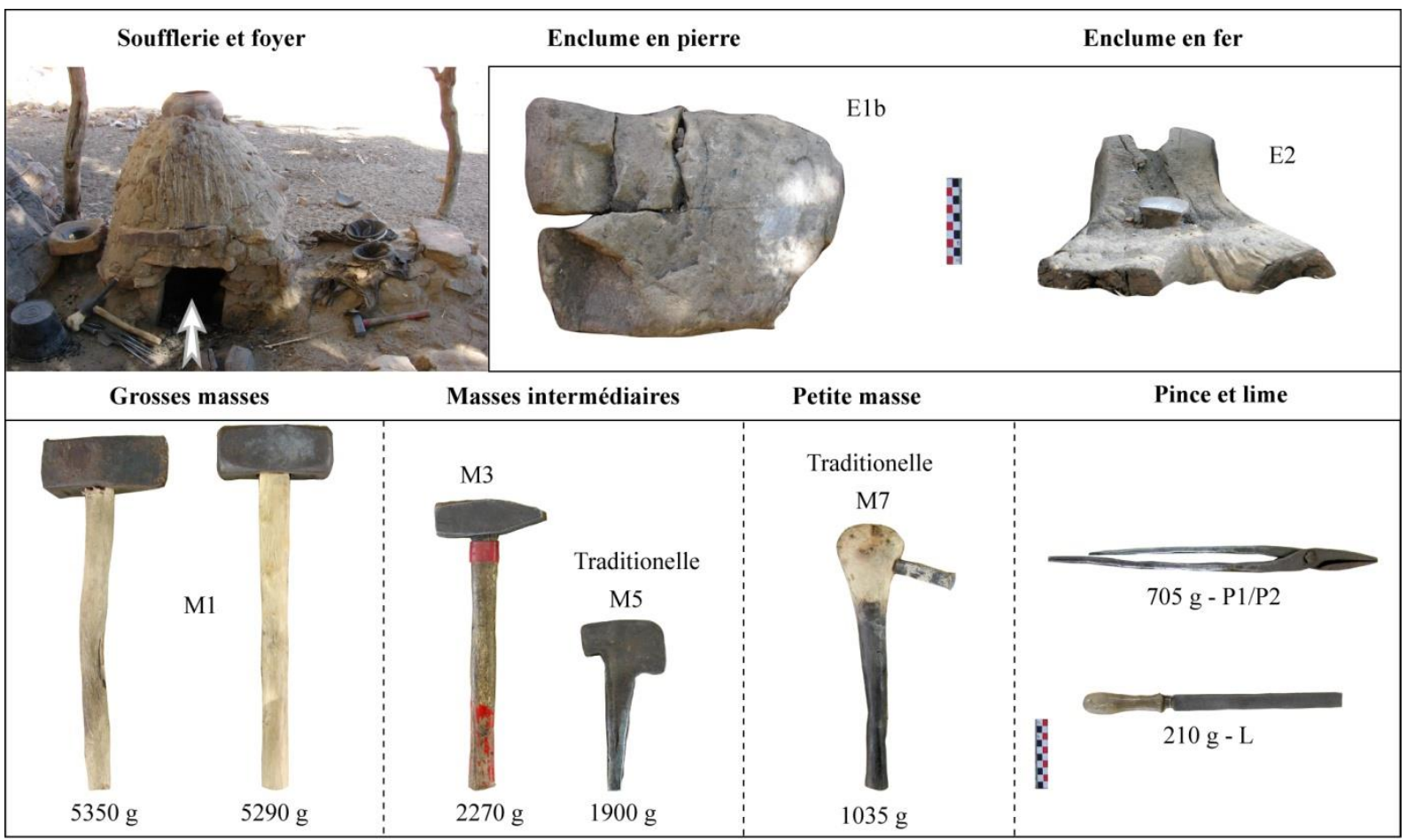

Figure 23 - Soufflerie de type double pots et foyer en coupole de la forge de Kobo.

E1b- grande enclume plate traditionnelle en grès (enclume de fondation)

E2 - petite enclume traditionnelle en fer fichée dans son support de bois
M1 - grosses masses européennes à deux panes actives et emmanchement centré en bois

M3 - marteau européen à une pane active et emmanchement centré en bois

M5 - marteau traditionnel non emmanché en forme de hachoir
M7 - petit marteau traditionnel à emmanchement à soie en bois

$\mathrm{P} 1$ - pinces

$\mathrm{P} 2$ - tenailles

L - lime. 


\subsection{FORGE DE DOUNDÉ}

\subsubsection{Forgerons}

\section{Origine}

Les forgerons de Doundé portent le patronyme Djo et ils sont parents avec les forgerons de Kobo. Ces deux familles sont issues du même ancêtre commun : Antandu Télé Jò, né vers 1820, qui eut trois fils, nés entre 1850 et 1890 , dont sont issus tous les Djo de Kobo et de Doundé (communication E. Huysecom).

Doundé n'est pas un site de réduction du fer mais plutôt un site de transformation du métal. Cependant, les forgerons allaient aider leurs voisins de Kobo à réduire le minerai de fer au pied de la falaise.

\section{Identité et fonction}

Les forgerons de Doundé forment une grande famille et le travail du fer y reste encore très présent au quotidien (Tableau 6). Les vieux forgerons sont actuellement au nombre de quatre : Carama, Sagou, Anyonron et Yaya. Seuls certains de leurs enfants sont passés maîtres de forge. Concrètement, le travail a été réalisé par deux équipes menées par Seydou et Ali B, tous deux forgerons expérimentés. L'équipe qui ne travaillait pas à la forge pouvait ainsi travailler dans les champs.

Les frappeurs, expérimentés également, furent toujours nombreux à participer au travail. A part les deux maîtres de forge cités précédemment, tout le monde a aidé à la soufflerie.

\begin{tabular}{ccccc}
\hline Identité & & \multicolumn{3}{c}{ Fonction exercée } \\
Nom & Age & M & F & S \\
Seydou & 45 & 30 & 1 & 0 \\
Ali B & 40 & 17 & 11 & 0 \\
Sekou & 40 & 4 & 14 & 4 \\
Alpha & 40 & 1 & 27 & 10 \\
Ousmane & 40 & 1 & 12 & 1 \\
Ali & 35 & 0 & 25 & 7 \\
Djibril & 35 & 0 & 24 & 6 \\
Mamadou & 35 & 0 & 22 & 1 \\
Moctar & 35 & 0 & 21 & 5 \\
Abdoulaye & 35 & 0 & 18 & 5 \\
Brama & 15 & 0 & 16 & 2 \\
Boucari & 25 & 0 & 13 & 3 \\
Yaya & 20 & 0 & 12 & 9 \\
Nb pers./forgeage & 1 & 2 à 5 & 1 \\
\hline Tableau 6 - Identité/fonction exercée par les forgerons à Doundé. &
\end{tabular}

\subsubsection{Atelier}

\section{Organisation spatiale}

La forge occupe une vaste place ombragée au cœur du village et à deux pas du marché (Figure 24).

L'atelier est composé de six abris d'environ $8 \mathrm{~m}^{2}$ chacun. Seuls quatre abris sont complets (trois ont été vus en fonctionnement), un abri n'a plus d'enclume et le dernier est complètement ruiné.

Ces six abris sont disposés en demi-cercle autour d'un arbre. Chaque abri est orienté de façon à ce que le maître de forge se trouve face à la place ombragée lorsqu'il est à sa place de travail.

\section{Unités spatiales de travail}

Chaque abri correspond à une seule unité spatiale de travail comprenant un foyer, une soufflerie, un siège pour le souffleur, une enclume en face de la porte du foyer, un siège pour le maître et un autre pour le frappeur assis face à lui. Les sièges sont des blocs quadrangulaires en bois.

Une enclume en métal longue et plate est commune à toutes les unités spatiales de travail. Il s'agit d'une pièce de récupération mobile située entre 4 et $7 \mathrm{~m}$ du foyer de chaque unité de travail. Par ailleurs, nous n'avons pas observé d'espace réservé au travail du bois.

\section{Foyer et soufflerie}

Doundé ne présente que des foyers en coupole (Figure 25). Celui où nous avons travaillé possède une superstructure circulaire d'une hauteur de $110 \mathrm{~cm}$, un diamètre externe de $115 \mathrm{~cm}$ et interne de $65 \mathrm{~cm}$. La fosse du foyer mesure $15 \mathrm{~cm}$ de profondeur et $30 \mathrm{~cm}$ de diamètre, ce qui correspond à un volume de près de $7100 \mathrm{~cm}^{3}$.

Une soufflerie à doubles pots alimente le foyer en air. 


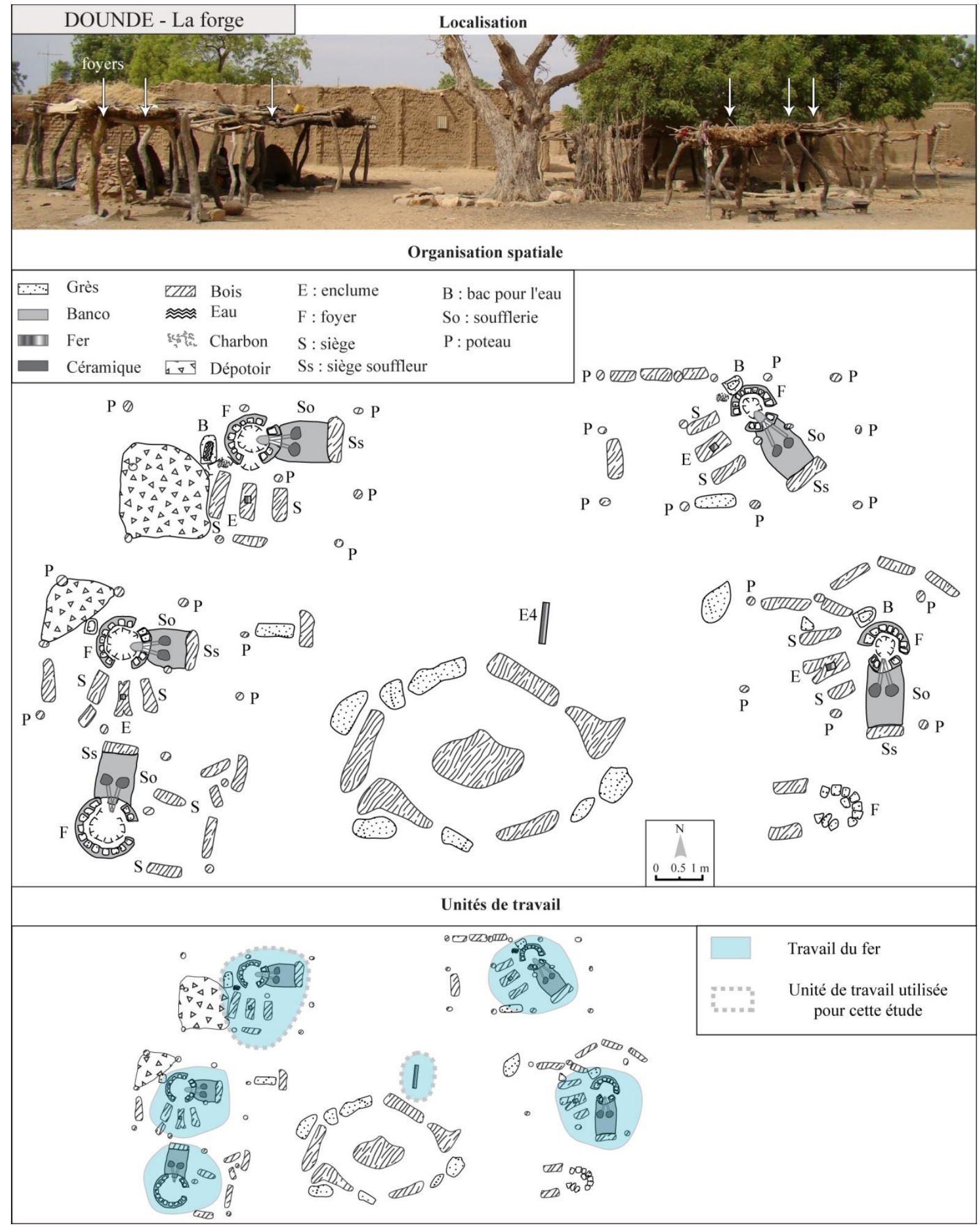

Figure 24 - Présentation de l'atelier de forge de Doundé. 


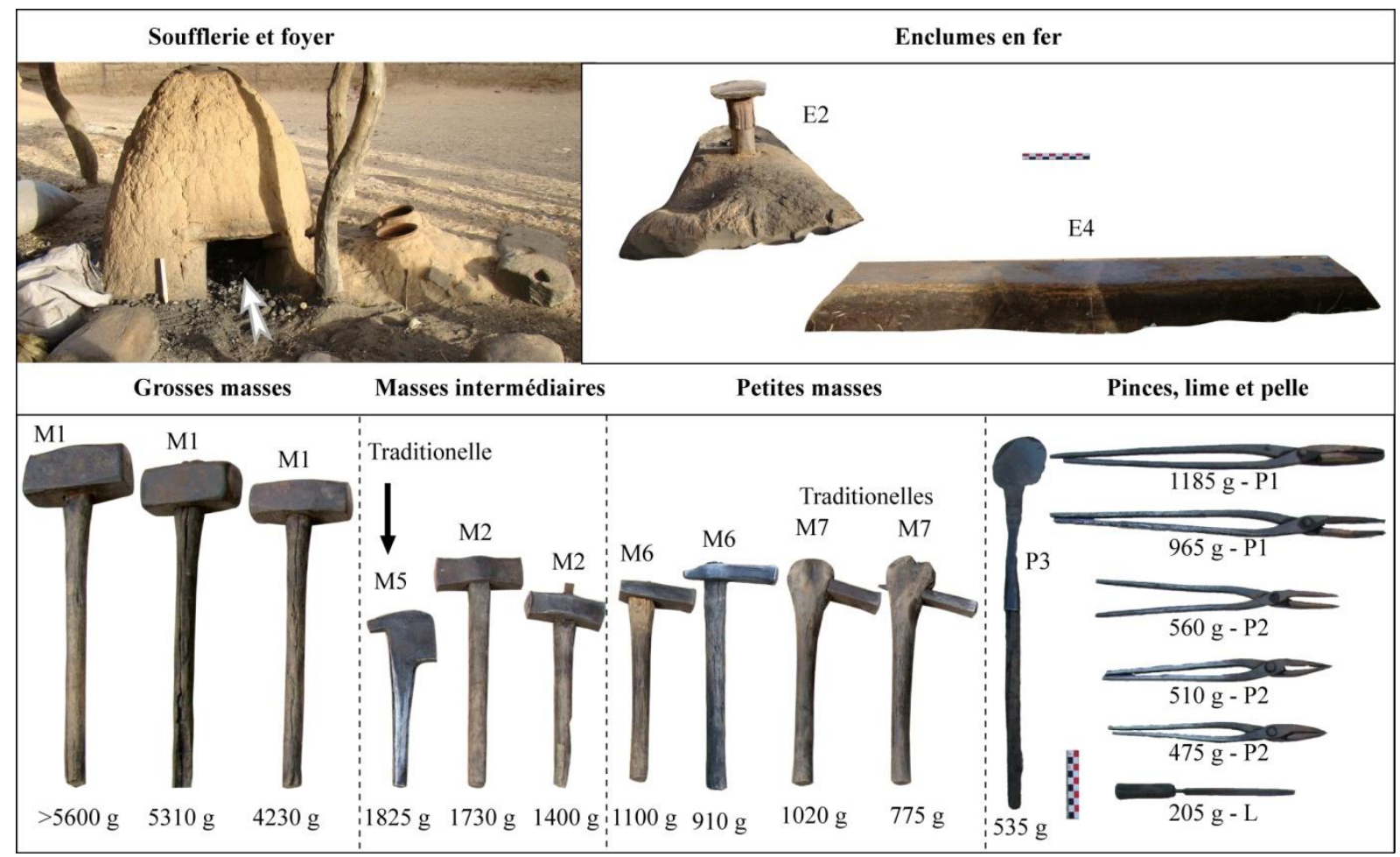

Figure 25 - Soufflerie de type double pots et foyer en coupole de la forge de Doundé.

E2 - petite enclume traditionnelle en fer fichée dans son support de bois E4 - longue enclume en fer de récupération

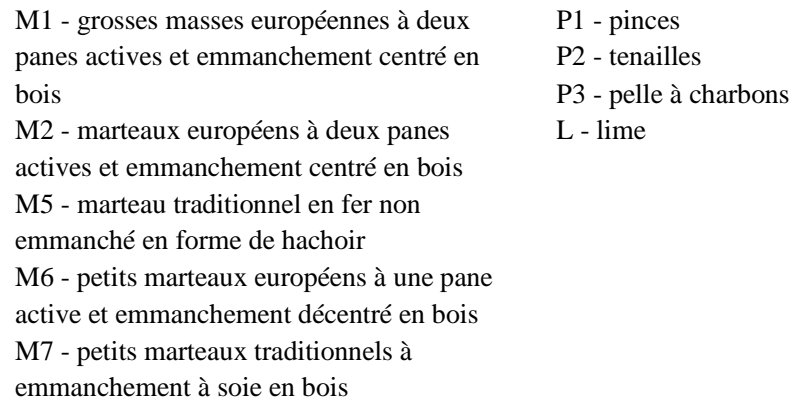
panes actives et emmanchement centré en bois

M2 - marteaux européens à deux panes actives et emmanchement centré en bois M5 - marteau traditionnel en fer non emmanché en forme de hachoir M6 - petits marteaux européens à une pane active et emmanchement décentré en bois M7 - petits marteaux traditionnels à emmanchement à soie en bois

\section{$\mathrm{P} 1$ - pinces} $\mathrm{P} 2$ - tenailles P3 - pelle à charbons L - lime

\section{Outils}

Sur la petite enclume en fer fichée dans son support en bois (E2), le maître emploie la masse traditionnelle faite d'un bloc de fer en forme de hachoir (M5) ainsi qu'un marteau européen (M2). Pour les finitions, des marteaux plus légers sont utilisés : deux de ces marteaux sont traditionnels avec un emmanchement à soie (M7), deux autres marteaux sont européens (M6).

Sur cette enclume, le frappeur utilise quant à lui un des deux marteaux européens (M2).

Pour le travail de grandes quantités de métal, le maître porte le fer chaud vers une longue et plate enclume en fer mobile (E4). Elle mesure $90 \mathrm{~cm}$ de longueur, $12 \mathrm{~cm}$ de large et sa table plane est située à $5 \mathrm{~cm}$ au-dessus du sol. Accroupi, il tient le fer à l'aide d'une pince (P2) tandis que jusqu'à quatre frappeurs battent le fer à tour de rôle en position debout à l'aide des masses (M1) et (M2).

Une pelle (P3) a servi à déplacer les charbons dans le foyer pendant la chauffe. Une fois la houe produite, le maître lime certaines arêtes de l'outil pour aiguiser le tranchant et le décorer (L).

A la fin de la journée, les marteaux sont rangés sur ou dans la toiture et les peaux sont ramenées à la concession du maître. 


\subsection{COMPARAISON ENTRE LES ATELIERS DE FORGE}

L'étude de ces quatre ateliers de forge montre des groupes de traditions différentes (Tableau 7) : ces groupes ont une origine et une culture différentes et donc des installations de forge et une manière de travailler distinctes.

A Fiko et Kakoli, l'atelier est un hangar regroupant plusieurs unités de travail dont les composantes sont globalement mobiles. A Kobo et Doundé l'atelier prend la forme d'un abri qui regroupe une seule unité de travail dont les composantes sont fixes.

L'atelier Mombo à Fiko est un hangar carré, l'atelier Mombo à Kakoli est un hangar elliptique sur 2 niveaux. La forme elliptique sur 2 niveaux a été observée dans d'autres forges de la région par V. Serneels : à Kema-Gumbessugo et à Singama par exemple. Ainsi, ce type de configuration semble être représentatif de la tradition dans la région.

Par ailleurs, parmi les deux forges retrouvées dans les ruines de l'ancien village de Fiko, la forge la plus récente (celle occupée juste avant de s'installer en bas de la colline) est déjà un hangar carré tandis que la plus ancienne présente une forme elliptique (Huysecom et al. 2003 : 54, 59). L'atelier de Fiko dans la version que l'on observe actuellement a donc été conçu en dehors de la tradition Mombo.

Dans le secteur Tomo, le village de Yélé est doté d'une forge similaire à Kobo et à Doundé (abri, foyer en coupole et soufflerie traditionnelle à double pots). Ainsi, les ateliers de Kobo/Doundé semblent traditionnels de la région Tomo.

A Fiko/Kakoli, une nouvelle forme de soufflerie est utilisée : un ventilateur moderne à fort rendement d'air. Ce type a déjà été observé au centre du Burkina-Faso (Kienon-Kaboré 2003 : 153-154). Quel système de soufflerie précédait la soufflerie ventilateur?

1. La soufflerie à doubles pots observée dans la région Tomo aurait laissé des vestiges de poterie.

2. Un système de soufflerie à soufflets à outres a été observé à Sissongo, à côté d'Impari (Figure 26). Dans cet atelier, les foyers sont similaires à ceux de Fiko mais plus profonds.
3. De plus, les foyers en banco demi-circulaires observés à Kakoli (Figure 19) fonctionneraient très bien avec des soufflets à outre.

4. Par ailleurs, Tiégé le forgeron de Kobo possède également des soufflets à outre qu'il utilise lorsqu'il se déplace.

Ainsi, nous supposons que le soufflet à outre pourrait avoir été utilisé antérieurement à la soufflerie ventilateur dans la région Mombo, et utilisé comme soufflerie traditionnelle mobile dans la région Tomo. Une enquête de terrain reste toutefois nécessaire pour compléter cette hypothèse.

On remarque que l'outillage est influencé par l'Europe puisque de nombreux marteaux (M1, M2, M3, M6) et enclumes (E3) sont de type européen. Cependant, d'autres outils traditionnels sont employés: les enclumes en grès (E1), la petite enclume en fer fichée dans un support de bois (E2) et le marteau traditionnel à emmanchement à soie (M7).

Ces deux derniers sont présents dans tous les ateliers. Par contre le marteau conique en fer (M4) n'a été observé que dans les ateliers Mombo : cette forme rappelle celle des marteaux utilisés au Burundi ou au Niger (Célis 1989 : 30 ; Célis 1994 : 403). Les marteaux en fer en forme de hachoir (M5) sont présents dans les ateliers Tomo uniquement. Le marteau traditionnel quadrangulaire en fer de Kobo (Figure 22) n'a pas été utilisé en notre présence (les femmes ne peuvent le voir). Enfin, l'enclume à bigorne européenne (E3) n'est présente que dans l'atelier de Fiko, le plus moderne.

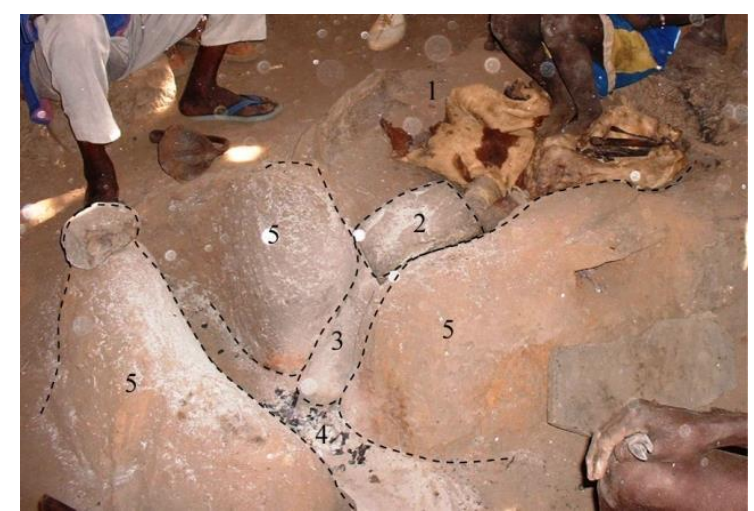

Figure 26 - Forge de Sissongo. 1 - Soufflets à outre ; 2 - Cale pour la conduite d'air ; 3 - Tuyère en banco ; 4 - Foyer en fosse à double entrée $; 5$ - Blocs de grès recouverts de banco. 


\begin{tabular}{|c|c|c|c|c|c|}
\hline & & Fiko & Kakoli & Kobo & Doundé \\
\hline \multirow{4}{*}{ 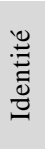 } & Localisation & Plateau & Plateau & Plaine & Plaine \\
\hline & Langue & Mombo & Mombo & Tomo & Tomo \\
\hline & Clan & Jèmè-irin & Jèmè-irin & Jèmè-yélin & Jèmè-yélin \\
\hline & Patronyme & Baguéné & Yanogué & Djo & Djo (même famille) \\
\hline \multirow{6}{*}{ 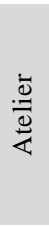 } & Type et forme & Hangar carré & Hangar elliptique & Abri sans toiture & Abri avec toiture \\
\hline & Nombre de niveaux & 1 & 2 & 1 & 1 \\
\hline & Unités spatiales de travail & 12 regroupés & 4 regroupés & 3 juxtaposés & 6 juxtaposés \\
\hline & Permanence des unités & Mobile & Mobile/Fixe & Fixe & Fixe \\
\hline & Superficie individuelle $\mathrm{m}^{2}$ & 43 & 25 & 8 & 8 \\
\hline & Superficie totale $\mathrm{m}^{2}$ & 43 & 25 & +25 & +50 \\
\hline \multirow{6}{*}{ 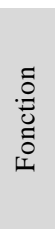 } & Maître & Adulte & Adulte & Adulte & Adulte \\
\hline & Expérience / force & +++/ +++ & $++/+++$ & $+++/+$ & $+/+++$ \\
\hline & Frappeur & Apprenti adulte & Adulte & Apprenti enfant & Adulte \\
\hline & Expérience / force & $+++/+++$ & ++ / +++ & $+1+$ & $++/+++$ \\
\hline & Souffleur & Enfant & Apprenti enfant & Apprenti enfant & Adulte \\
\hline & Expérience / force & $+++/+++$ & $+++/+++$ & $+/+$ & $+++/+++$ \\
\hline \multirow{3}{*}{ 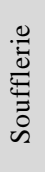 } & Type & Ventilateur & Ventilateur & Double pot & Double pot \\
\hline & Origine & Moderne & Moderne & Traditionnelle & Traditionnelle \\
\hline & Rendement* & +++ & +++ & + & +++ \\
\hline \multirow{6}{*}{$\begin{array}{l}\dot{0} \\
\underset{0}{0}\end{array}$} & Type & Fosse & Fosse & Coupole & Coupole \\
\hline & Forme de la fosse & Allongée & Allongée & Demi-circulaire & Demi-circulaire \\
\hline & $\mathrm{Nb}$ d'entrée dans le foyer & 2 & 1 & 1 & 1 \\
\hline & $\mathrm{L} \times 1 \times \mathrm{P} \mathrm{cm} / \mathrm{DxP} \mathrm{cm}$ & $30 \times 12 \times 10$ & $40 \times 10 \times 10$ & $20 x 15$ & $30 \times 15$ \\
\hline & Volume brut $\mathrm{cm} 3$ & 3000 & 4000 & 3100 & 7100 \\
\hline & Volume zone active & 2000 & 1000 & $?$ & $?$ \\
\hline \multirow{7}{*}{ 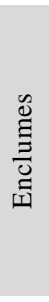 } & E1a & $\checkmark$ & $\checkmark$ & - & - \\
\hline & E1b & - & - & $\boldsymbol{v}$ & - \\
\hline & E2 & $\checkmark$ & $\checkmark$ & $\boldsymbol{v}$ & $\boldsymbol{v}$ \\
\hline & E3 & $\checkmark$ & - & - & - \\
\hline & E4 & $\sim$ & - & - & $\boldsymbol{v}$ \\
\hline & E5 & $\checkmark$ & - & - & - \\
\hline & Sol et support en bois $* *$ & $\checkmark$ & $\boldsymbol{v}$ & $\boldsymbol{v}$ & $\boldsymbol{v}$ \\
\hline \multirow{7}{*}{ 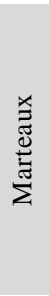 } & M1 & $\boldsymbol{V}$ frappeur & $\boldsymbol{V}$ frappeur & $\boldsymbol{V}$ frappeur & $\boldsymbol{V}$ frappeur \\
\hline & M2 & $\boldsymbol{V}$ frappeur & - & - & $\boldsymbol{V}$ maître et frappeur \\
\hline & M3 & - & $\boldsymbol{V}$ maître & $\boldsymbol{V}$ frappeur & - \\
\hline & M4 & $\boldsymbol{V}$ maître & $\boldsymbol{V}$ maître & - & - \\
\hline & M5 & - & - & $\boldsymbol{V}$ maître & $\boldsymbol{V}$ maître \\
\hline & M6 & $\boldsymbol{V}$ maître & $\boldsymbol{V}$ maître & - & $\boldsymbol{V}$ maître \\
\hline & M7 & $\boldsymbol{V}$ maître & $\boldsymbol{V}$ maître & $\checkmark$ maître & $\boldsymbol{V}$ maître \\
\hline \multirow{4}{*}{ 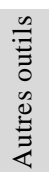 } & Pince P1 & $\checkmark$ & $\checkmark$ & $\checkmark$ & $\checkmark$ \\
\hline & Tenaille P2 & $\checkmark$ & $\checkmark$ & $\checkmark$ & $\checkmark$ \\
\hline & Lime L & - & - & $\checkmark$ & $\checkmark$ \\
\hline & Pelle P3 & - & - & - & $\boldsymbol{v}$ \\
\hline
\end{tabular}

Tableau 7 - Récapitulatif de l'identité des forgerons et des caractéristiques des ateliers de forge étudiés.

$+++=$ important, $++=$ moyen,$+=$ faible $\mathrm{L}=$ longueur, l=largeur $\mathrm{P}=$ profondeur $\mathrm{D}=$ diamètre

$\boldsymbol{V}=$ utilisé, $\sim=$ non utilisé, $-=$ absent
* : le rendement correspond à l'efficacité de la soufflerie, en prenant en compte le type de soufflerie et l'expérience/force du souffleur
** : l'observation du travail a permis de relever d'autres supports de frappe « invisibles » pour l'archéologue : le socle en bois de la petite enclume en fer (E2) et même le sol de la forge, tous 2 employés lors des finitions de l'outil. 
En résumé, l'atelier du village de Fiko est une réinterprétation moderne de la forge, qui est peu représentative de la tradition Mombo. L'atelier de Kakoli reste une forge traditionnelle Mombo, avec des éléments de modernité. Les ateliers de Kobo et
Doundé sont des forges traditionnelles Tomo (Figure 27).

Ces observations ne sont pas valables dans les autres ateliers du Pays dogon qui n'ont pas été explorés et dont nous ne possédons pas de données.

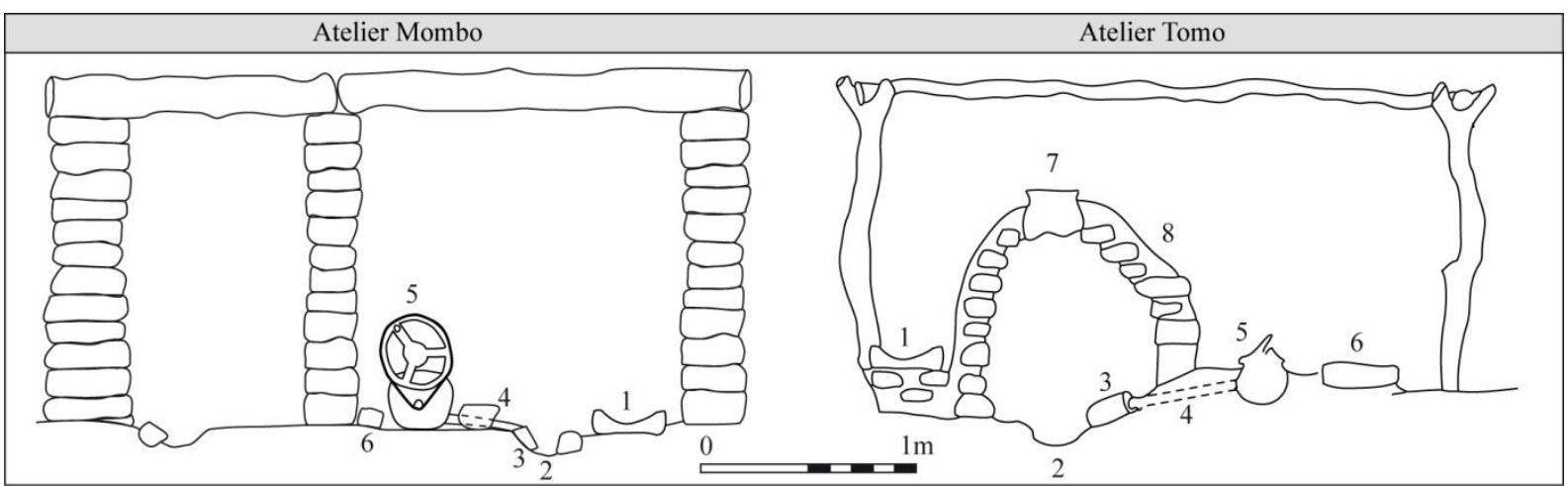

Figure 27 - Les deux configurations des ateliers de forge visités : atelier Mombo et atelier Tomo.

1 - bac à eau

2- foyer

3- tuyère
4- conduites d'air

5- soufflerie

6- siège du souffleur
7- cheminée

8- superstructure. 
Les ateliers de forge 


\section{PARTIE 3 . LES OPÉRATIONS DE FORGEAGE}


Les opérations de forgeage 


\subsection{PrÉSEnTATION DES MATÉRIAUX DE LA FORGE}

La Partie 3 de ce manuscrit est consacrée à la présentation détaillée des opérations de forgeage demandées aux forgerons dans le cadre de notre travail : variations de la chaîne opératoire et des gestes et procédés techniques, détermination des paramètres influençant la durée de travail, description du produit fabriqué, compréhension des paramètres dirigent la perte de fer au cours du forgeage.

La particularité de cette recherche est la réalisation de forgeages à partir de paramètres de départs différents, afin de comprendre l'influence de tels ou tels paramètres sur les caractéristiques observées des scories de forge.

Rappel : les paramètres de départ qui varient sont: l'atelier où se déroule le forgeage (type d'atelier, soufflerie, foyer - Partie 2), l'équipe de travail (compétence, force - Partie 2), la matière première ainsi que les gestes et procédés techniques qui sont intimement liés (Partie 3 à venir).
Le but du chapitre 3.1, est ici de définir la nature et la composition de l'ensemble des matériaux introduits au cours des travaux de forge : matière première, combustible (Annexe 2), matériaux siliceux (Annexe 3).

\subsubsection{Matière première ferreuse}

La matière première fournie aux forgerons dans chacun des ateliers pour forger les lames de houe est très diversifiée: nous en avons utilisé 23 sortes différentes (Figure 28).

Nous avons souhaité savoir quels paramètres entre leur forme des barres (trapue/allongée), leur nombre (une/plusieurs,), leur origine (moderne/ancien) et leur composition (inclusions, teneur en carbone) laissent une signature visible sur les scories de forge produites.

\begin{tabular}{|c|c|c|c|c|c|c|c|c|c|c|c|c|c|}
\hline Provenance & \multicolumn{9}{|c|}{ Industrie Moderne } & \multirow{2}{*}{\multicolumn{2}{|c|}{$\begin{array}{c}\begin{array}{c}\text { Barres anciennes } \\
\text { d'Afrique }\end{array} \\
\text { Double }\end{array}$}} & \multicolumn{2}{|c|}{ Récupération } \\
\hline Catégorie & \multicolumn{5}{|c|}{ Simple } & \multicolumn{4}{|c|}{ Double } & & & Simple & Double \\
\hline Type & M5S & M4S* & M4S & M3S & M2S & M4D & M3D & M2D & M1D & A1D & A2D & R1S & R1D \\
\hline \multicolumn{14}{|l|}{ Illustration } \\
\hline$\cdots$ & & & & & & & & & & & & & \\
\hline Section $(\mathrm{cm})$ & $5 \times 5$ & $4 \times 4$ & $4 \times 4$ & $3 \times 3$ & $2 \times 2$ & $4 \times 4$ & $3 \times 3$ & $2 \times 2$ & $1 \times 1$ & $1 \times 1$ & $\begin{array}{lr}\text { a } & 0.5 \times 1 \\
\text { b } & 4 \times 2\end{array}$ & 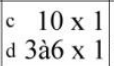 & $\begin{array}{r}10 \text { à } 16 \\
\mathrm{e} \\
\times 0.5\end{array}$ \\
\hline Longueur $(\mathrm{cm})$ & 5 & 16 & 8 & 14 & 32 & 4 & 7 & 16 & 45 & 38 à 41 & 33 à 41 & 18 à 25 & 28 à 32 \\
\hline Surface $\left(\mathrm{cm}^{2}\right)$ & 150 & 288 & 160 & 186 & 264 & 192 & 204 & 272 & 364 & $\begin{array}{c}228 \text { à } \\
332\end{array}$ & $\begin{array}{c}260 \text { à } \\
308\end{array}$ & $\begin{array}{c}375 \text { à } \\
390\end{array}$ & $\begin{array}{c}313 \text { à } \\
553\end{array}$ \\
\hline Poids total (g) & 1000 & 2000 & 1000 & 1000 & 1000 & 1000 & 1000 & 1000 & 700 & $\begin{array}{l}530 \text { à } \\
710\end{array}$ & $\begin{array}{l}675 \text { à } \\
700\end{array}$ & $\begin{array}{l}780 \text { à } \\
1620\end{array}$ & $\begin{array}{l}830 \text { à } \\
1590\end{array}$ \\
\hline $\begin{array}{c}\text { Teneur en } \\
\text { carbone }(\% \mathrm{C})\end{array}$ & $\begin{array}{c}- \\
- \\
0.45\end{array}$ & $\begin{array}{c}- \\
0.35 \\
0.45\end{array}$ & $\begin{array}{c}0.17 \\
- \\
0.45\end{array}$ & $\begin{array}{l}0.17 \\
0.35 \\
0.45\end{array}$ & $\begin{array}{c}0.17 \\
- \\
0.45\end{array}$ & $\begin{array}{c}0.17 \\
- \\
0.45\end{array}$ & $\begin{array}{l}0.17 \\
0.35 \\
0.45\end{array}$ & $\begin{array}{c}0.17 \\
- \\
0.45\end{array}$ & $\begin{array}{c}0.17 \\
0.35 \\
-\end{array}$ & & 0.8 & $\begin{array}{c}<0.1 \\
- \\
-\end{array}$ & $<0.8$ \\
\hline
\end{tabular}

Figure 28 - Caractéristiques de la matière première utilisée. 


\section{Aciers modernes}

Les barres modernes ont été achetées auprès de la société Debrunner-Acifer, fournisseur de matériaux pour le génie civil, industrie et les arts et métiers. Ces barres possèdent des sections carrées (1 à $5 \mathrm{~cm}$ ) et des longueurs différentes ( 5 à $45 \mathrm{~cm}$ ), ce qui nous a permis d'obtenir des barres individuelles trapues à très allongées de $2 \mathrm{~kg}, 1 \mathrm{~kg}, 500 \mathrm{~g}$ et $350 \mathrm{~g}$. Les barres de $2 \mathrm{~kg}$ (M4S*), ont un volume important et sont par conséquent difficilement forgeages en l'état. Les forgerons ont dû les couper en 2 morceaux (d'environ $950 \mathrm{~g}$ ) à l'aide d'un burin afin de fabriquer une lame de houe dans chacun des morceaux débités.

Parfois nous avons fourni une seule barre de $1 \mathrm{~kg}$ qui a pu être forgée telle quelle : M5S, M4S, M3S et M2S. D'autres fois, nous avons fourni deux barres de $500 \mathrm{~g}$ : M4D, M3D, M2D. Dans ce cas le forgeron est obligé de les souder ensemble avant de mettre en forme le lopin de métal résultant de la soudure. Les barres M1S de $1 \mathrm{~cm}$ de section pèsent $350 \mathrm{~g}$ chacune: nous avons fourni à chaque fois deux barres, pour un poids total de $700 \mathrm{~g}$.

Les différences de forme et de volume initial vont induire des gestes techniques de natures différentes qu'il sera intéressant de pister au sein des déchets.

\begin{tabular}{crrr}
\hline & S235 JR & \multicolumn{1}{c}{ C35 } & \multicolumn{1}{c}{ C45 } \\
$\mathrm{C} \%$ & $0.17-0.20$ & $0.32-0.39$ & $0.42-0.50$ \\
$\mathrm{Si} \%$ & - & 0.40 & 0.40 \\
$\mathrm{Mn} \%$ & 1.4 & $0.5-0.8$ & $0.5-0.8$ \\
$\mathrm{P} \%$ & 0.035 & 0.045 & 0.045 \\
$\mathrm{~S} \%$ & 0.035 & 0.045 & 0.045 \\
$\mathrm{Cu} \%$ & 0.055 & - & - \\
$\mathrm{Cr} \%$ & - & 0.40 & 0.40 \\
$\mathrm{Ni} \%$ & - & 0.40 & 0.40 \\
$\mathrm{Mo} \%$ & - & 0.10 & 0.10 \\
\hline \multicolumn{2}{c}{ Tableau 8 - Composition chimique élémentaire des aciers } \\
modernes (analyse de coulée suivant la norme EN 10277-2).
\end{tabular}

La composition chimique et la teneur en carbone de ces barres de fer est connue (Tableau 8). Trois types d'aciers ont été utilisés : un acier doux à $0.17 \% \mathrm{C}$, un acier mi-doux à $0.35 \% \mathrm{C}$ et un acier midur à $0.45 \% \mathrm{C}$ (Tableau 1) qui contiennent de très faibles teneurs en éléments autres que le fer.

La microstructure (avant forgeage) de ces différents aciers est donnée à la figure 29. La texture est homogène et montre des grains de petite taille, provenant du laminage des barres d'acier lors de leur mise en forme. Entre 0.17 et $0.45 \% \mathrm{C}$, on observe la diminution de la surface des grains de ferrite clairs au détriment de plages de perlite brune.

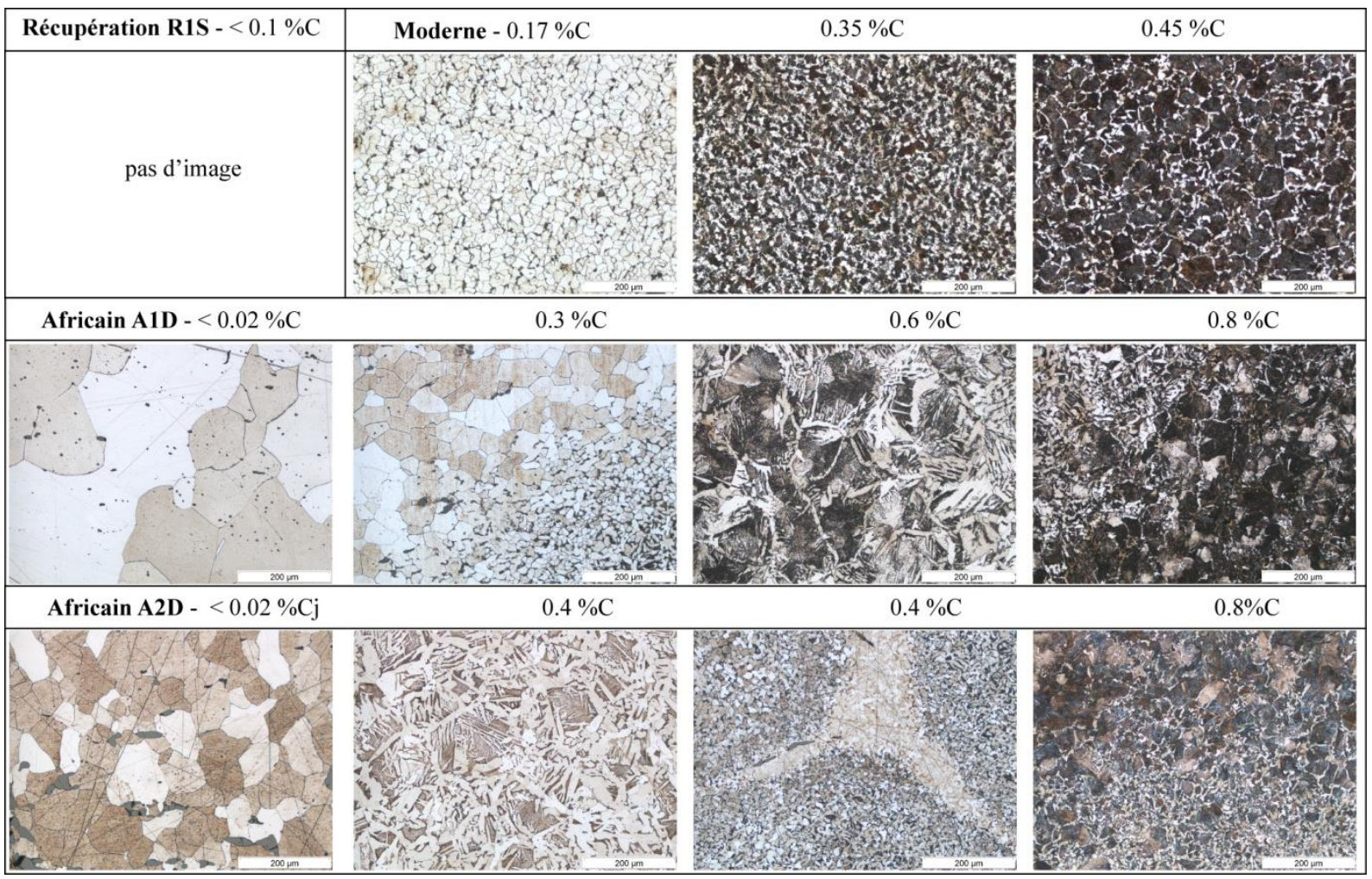

Figure 29 - Microstructure du métal des barres de fer avant forgeage $(0.17 \% \mathrm{C}, 0.35 \% \mathrm{C}, 0.45 \% \mathrm{C}, \mathrm{A} 1 \mathrm{D}$ et A2D). 


\section{Fers anciens africains}

La nature et la composition des fers contemporains peut être sensiblement différente des fers réellement forgés en contexte archéologique. Ont également été étudiées une vingtaine de barres de fers africains achetées à Bruxelles par V. Serneels dans une brocante. Deux morphologies existent : des barres droites et des barres renflées (Figure 28). Elles ne sont pas sans rappeler ces barres qui ont une fonction de monnaie, et qui se rencontrent depuis l'est du Nigeria jusqu'à l'ouest du Cameroun, entre le $16^{\mathrm{e}}$ et le $20^{\mathrm{e}}$ siècle (Johansson \& Learmonth 1967).

Les barres droites A1D se rencontrent notamment chez les yorubas, une ethnie du Nigeria. Elles sont aplaties sur leurs quatre côtés et leurs extrémités sont très légèrement épatées. Elles mesurent entre 38 et $41 \mathrm{~cm}$ de longueur pour une section d'environ $1 \times 1 \mathrm{~cm}$ et elles pèsent chacune entre $310 \mathrm{~g}$ et $390 \mathrm{~g}$. Pour toutes les barres une coupe à l'extrémité a été effectuée plutôt qu'une section au milieu de la tige. La microstructure révèle un fer plus ou moins compacté comportant de nombreuses fentes internes et inclusions d'oxydes de fer souvent aplaties par le martelage (Figure 30). Il s'agit d'un fer épuré qui a été mis sous la forme d'une barre allongée. La carburation est toujours faible (jusqu'à $0.2 \% \mathrm{C}$ ) sauf en des zones de soudure où la composition eutectoïque $(0.77 \% \mathrm{C})$ est atteinte (Figure 29).

Les barres renflées A2D sont attribuées aux groupes ethniques Fulani, Jukun et Mumuye. Elles comportent une partie centrale bi-pyramidée à quatre faces, prolongée de fines tiges aux extrémités. Ces barres mesurent entre 33 et $41 \mathrm{~cm}$ de longueur, pour une section au centre de 2 à $4 \mathrm{~cm}$

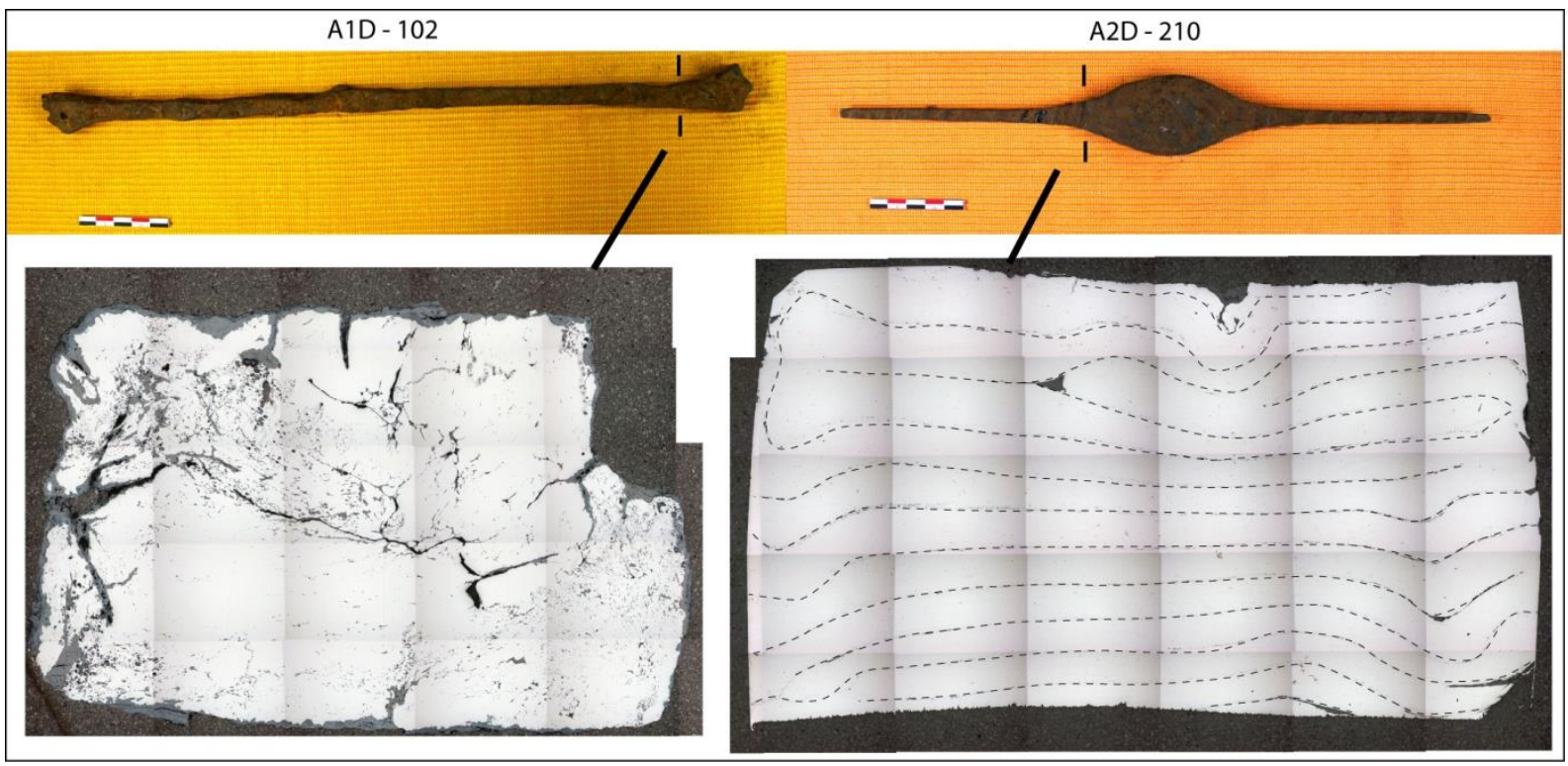

Figure 30 - Aspect mal compacté pour la barre droite A1D $\left(n^{\circ} 102\right)$ et aspect feuilleté pour la barre renflée A2D $\left(n^{\circ} 210\right)$. Même échelle.

\begin{tabular}{|c|c|c|c|c|c|c|c|c|}
\hline & Matrice/verre & $\begin{array}{l}\text { Oxydes de } \\
\text { fer }\end{array}$ & Ulvöspinelle & Perovskite & $\begin{array}{l}\text { Calcio } \\
\text { olivine }\end{array}$ & Galaxite & Jacobsite & Rutile \\
\hline $\begin{array}{l}\text { Nombre } \\
\text { analyses* }\end{array}$ & 27 & 15 & 4 & 6 & 2 & 1 & 1 & 4 \\
\hline $\mathrm{Al} \%$ & 5.6 & 1.0 & 1.6 & 0.6 & 0.5 & 31.1 & 1.8 & 2.6 \\
\hline Si $\%$ & 21.3 & 1.6 & 0.3 & 0.5 & 20.0 & n.d. & 0.1 & 4.4 \\
\hline $\mathrm{K} \%$ & 3.4 & 0.3 & 0.1 & 0.2 & 0.9 & n.d. & n.d. & 0.6 \\
\hline $\mathrm{Ca} \%$ & 11.3 & 0.8 & 0.4 & 29.9 & 31.8 & n.d. & n.d. & 1.0 \\
\hline $\mathrm{Ti} \%$ & 3.6 & 1.3 & 15.0 & 38.0 & 4.0 & n.d. & n.d. & 49.3 \\
\hline $\mathrm{V} \%$ & 0.1 & $<$ & 0.4 & n.d. & $<$ & 0.2 & n.d. & 2.5 \\
\hline $\mathrm{Cr} \%$ & 0.1 & $<$ & 0.1 & $<$ & 0.4 & 2.2 & n.d. & $<$ \\
\hline $\mathrm{Mn} \%$ & 0.4 & 0.2 & 0.8 & $<$ & n.d. & 21.8 & 53.0 & 1.1 \\
\hline $\mathrm{Fe} \%$ & 18.4 & 68.8 & 50.4 & 1.5 & 1.1 & 8.3 & 19.8 & 8.5 \\
\hline $\mathrm{Cu} \%$ & 0.1 & n.d. & n.d. & 1.3 & n.d. & n.d. & n.d. & n.d. \\
\hline $\mathrm{Zr} \%$ & 1.5 & n.d. & 2.3 & 1.0 & 2.5 & n.d. & n.d. & 0.5 \\
\hline
\end{tabular}

Tableau 9 - Composition chimique élémentaire moyenne des inclusions de scorie dans les barres africaines sur10 barres et 60 analyses. $<=$ inférieur à $0.1 \%$.. * = nombre d'analyses ponctuelles prises en compte pour la moyenne de la composition élémentaire. 
et aux extrémités de 0.5 à $1 \mathrm{~cm}$; elles pèsent de $305 \mathrm{~g}$ à $390 \mathrm{~g}$ chacune. Les barres A2D montrent soit une microstructure similaire à $\mathrm{A} 1 \mathrm{D}$, soit un feuilletage qui comporte des inclusions d'oxydes de fer entre les couches (jusqu'à 20 niveaux) (Figure 30). Ces barres semblent résulter d'un feuilletage par replis successifs lors du forgeage. La carburation est faible (jusqu'à $0.2 \% \mathrm{C}$ ) sauf quelques zones (jusqu'à 0.77 $\% \mathrm{C}$ ) de soudure. Nous avons fourni à chaque fois deux barres de chaque sorte pour que le forgeron ait à travailler une quantité de fer suffisante, soit environ $700 \mathrm{~g}$.

Du point de vue microscopique, les deux types de barres comportent des inclusions diverses de scorie de réduction. Leur étude, qui n'est pas au cœur de ce travail, a été limitée à l'analyse chimique ponctuelle d'inclusions appartenant à dix sections : cinq barres A1D et cinq barres A2D (Tableau 9). Il faudrait par la suite pouvoir réaliser une étude plus détaillée de l'ensemble des barres et de l'ensemble des inclusions pour avoir une vue plus complète de la composition de ces inclusions

Plusieurs types d'inclusions ont été observés : du verre seul, des oxydes de fer seuls (wüstite, magnétite), des oxydes de fer (avec/sans fayalite) dans une matrice vitreuse ainsi que divers autres minéraux comme des spinelles: ulvöspinelle $\mathrm{Fe}_{2} \mathrm{TiO}_{4}$ et galaxite $\mathrm{MnAl}_{2} \mathrm{O}_{4}$; une olivine : la calcioolivine $\mathrm{Ca}_{2} \mathrm{SiO}_{4}$; le rutile $\mathrm{TiO}_{2}$. Dans une moindre mesure, des minéraux proches de la perovskyte $\mathrm{CaTiO}_{3}$ et jacobsite $\mathrm{MnFe}_{2} \mathrm{O}_{4}$ (spinelle également) sont observés. La quantité des inclusions dans les barres de fer africaines reste toujours faible $(<15 \%)$.

$\mathrm{Au}$ sein de chaque groupe morphologique (droit/renflé), les inclusions ne sont pas homogènes : ainsi le fer proviendrait de plusieurs sources. De plus, les inclusions apportent des éléments traces comme $\mathrm{Ti}$ ou $\mathrm{Zr}$, dont les teneurs sont supérieures aux aciers modernes et qui devraient se retrouver dans la scorie. Au contraire, les teneurs en Mn sont inférieures à celles des aciers modernes.

\section{Fers de récupération}

Des matériaux provenant du marché de la récupération, pratique courante en forge ancienne ou contemporaine, complètent ce corpus. Il s'agit de traverses de voie ferrée et de jantes de camions prédécoupées à froid (Figure 28).
Ces matériaux ont une forme de parallélogramme de 18 à $25 \mathrm{~cm}$ de longueur sur $1 \mathrm{~cm}$ d'épaisseur. Ces barres de récupération sont systématiquement utilisées par les forgerons lorsqu'ils doivent eux-mêmes fabriquer une lame de houe. La microstructure des barres de recyclage de type R1 n'a pas été vérifiée, cependant nous supposons que leur teneur en carbone est proche de $0.1 \% \mathrm{C}$ (Figure 29).

Enfin, une partie de la matière première ayant été volée lors de son transport en Afrique, nous avons demandé aux forgerons de fabriquer une nouvelle lame à partir de deux précédentes lames comme dans le cas du recyclage des vieux outils de fer. La microstructure de la matière première n'a pas non plus été déterminée.

\subsubsection{Le combustible}

Dans les quatre ateliers, le combustible utilisé par les forgerons est un charbon local provenant majoritairement d'un arbre proche de l'acacia, le prosopis africana, déterminé par l'anthracologue Dr. B. Eicchorn pour notre étude (Figure 31, von Maydell 1990). Cet arbre est «l'arbre du forgeron $\gg$ (Huysecom 2001 : 77-78). Il pousse en abondance dans la région, il est facile à trouver, son bois est dur donc il se consume lentement, il ne produit que peu de cendres et possède une forte capacité calorifique. Seuls les arbres morts sont débités pour être transformés en charbons puis utilisés par les forgerons.

Les analyses complètes des charbons de bois prélevés à Fiko, Kakoli, Kobo et Doundé sont indiquées en annexe (Annexe 2 - Combustible).

Le calcium est le principal constituant minéral des charbons, qui représente 50 à $70 \%$ des matières minérales dans les charbons; la teneur en $\mathrm{CaO}$ dépend de nombreux facteurs, notamment l'essence et le substratum de l'arbre. Le potassium est le second constituant minéral du bois, qui représente entre 10 et $20 \%$ des matières minérales. L'apport en manganèse $(<3.5 \%)$ est significatif, celui en magnésium (2-7\%) est notable. Enfin, la teneur en sodium $(<0.5 \%)$ reste faible, celle en phosphore est variable $(0.5-3.5 \%)$. 


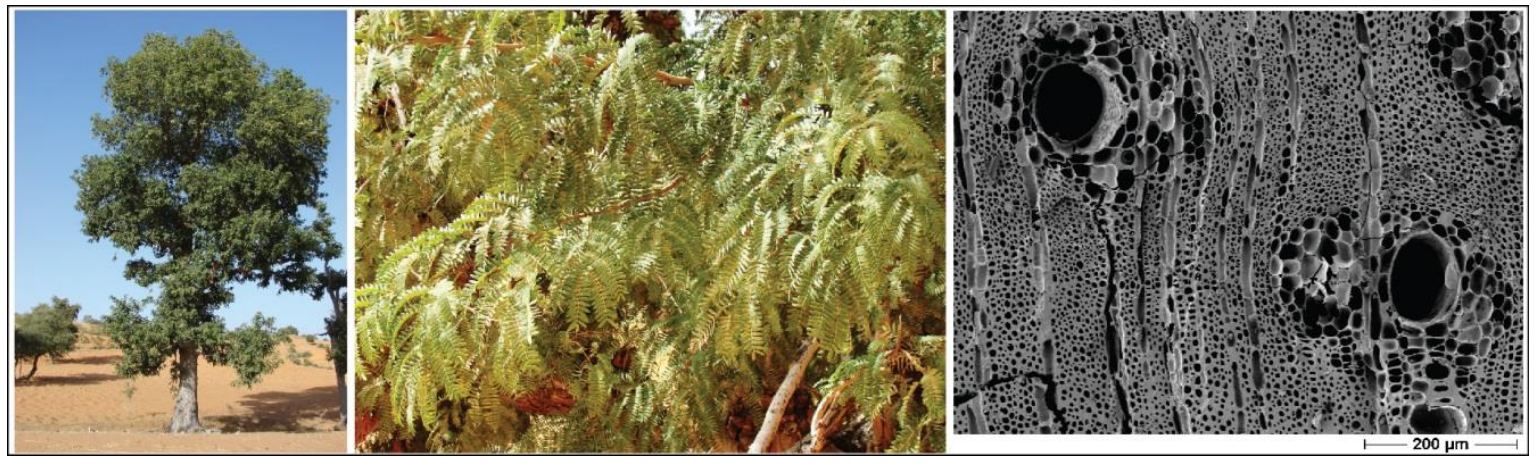

Figure 31 - Prosopis africana. Arbre et détail du feuillage ; microstructure d'un charbon de bois (dernier cliché de B. Eichhorn).

\subsubsection{Les matériaux siliceux}

\section{Ajouts volontaires}

Les forgerons utilisent divers ajouts siliceux lorsqu'ils réalisent des soudures : du grès concassé, de l'argile finement broyée, de la tuyère concassée, rarement du sable, qu'ils utilisent seuls ou mélangés entre eux. A Kobo, de la poudre de barytine $\mathrm{BaSO}_{4}$ a été de temps en temps rajoutée à notre demande pour servir de traceur géochimique (0). Le but est de former à l'interface de soudure, une gangue visqueuse (de fusion des silicates et des oxydes de fer) servant à limiter l'oxydation; celle-ci sera expulsée avec facilité lors de l'assemblage des surfaces.

De plus, dans l'atelier de Kobo, il avait déjà été observé des ajouts de sable dans le foyer lors du début du forgeage de fer de réduction directe dit « noir » (observations par E. Huysecom).

Les analyses chimiques (Annexe 3 - Matériaux Siliceux) montrent que le grès ajouté dans le foyer à
Kobo apporte presque uniquement du $\mathrm{SiO}_{2}(>90 \%)$ et peu de $\mathrm{Al}_{2} \mathrm{O}_{3}(5 \%)$. Les tuyères étant faites d'argile, les compositions de ces deux matériaux sont très similaires à Kobo et à Doundé : $\mathrm{SiO}_{2}$ est majoritaire (60\%) avec un apport important d' $\mathrm{Al}_{2} \mathrm{O}_{3}$ (25\%), puis de $\mathrm{FeO}_{\text {tot }}(6 \%)$ et de $\mathrm{K}_{2} \mathrm{O}(6 \%)$.

\section{Eléments du foyer}

Seule la paroi du foyer de Fiko a été prélevée : elle montre une composition intermédiaire entre un grès et une argile. A Kakoli la paroi n'a pas été échantillonnée ; cependant nous avons observé son rechapage avant de commencer les travaux de forge. Dans ce village, il est fort probable qu'un composant calcique comme du ciment ait été ajouté à l'argile de rechapage. De plus, la tuyère n'a été prélevée ni à Fiko et ni à Kakoli.

A Kobo et à Doundé, la tuyère utilisée au cours des forgeages n'a pas été échantillonnée. Nous avons supposé que sa composition chimique est la même que celle utilisée pour les ajouts. 


\subsection{CHAÎNE OPÉRATOIRE D'UNE LAME DE HOUE}

Le chapitre 3.2 a pour but de décrire, dans chacun des ateliers visités, la chaîne opératoire de fabrication des lames de houe et les différents gestes et procédés techniques employés, ainsi que leurs variations.

Combien d'étapes comporte la chaîne opératoire et quelles sont-elles ? Quelles techniques les forgerons utilisent-ils pour forger deux barres de fer? Comment s'organise le travail en équipe de forgerons ? Y a-t-il des échecs dans ces travaux de forge et à quoi sont-ils dus ?
L'observation du travail des forgerons a permis d'observer et d'enregistrer la mise en forme de plus d'une centaine de lames de houe (126 au total) au cours de trois missions de terrain (Tableau 10). Nos recherches montrent que la chaîne opératoire comporte toujours trois étapes : le préformage qui se déroule de manière différente en fonction de la forme de la matière première, le formage et les finitions qui sont au contraire toujours très similaires à quelques différences près (Figure 32).

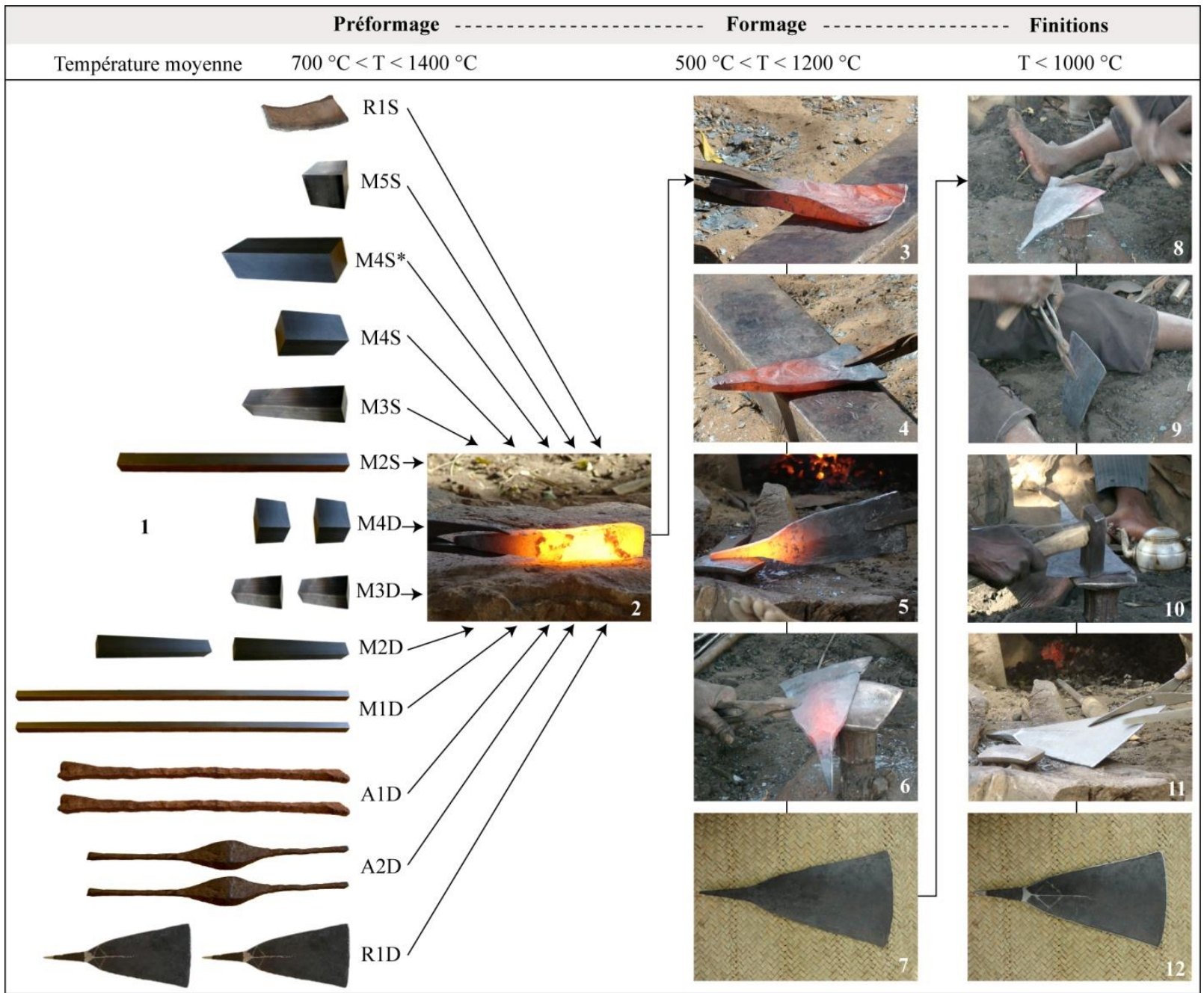

Figure 32 - Les différentes étapes de la châne opératoire.

Préformage

1 - Matière première brute

2 - Préforme
Formage

3 - Elargir la lame

4 - Former les épaulements

5 - Etirer la soie

6 - Former la nervure

7 - Outil brut
Finitions

8 - Planer la lame

9 - Egaliser les bordures

10 - Aplatir le tranchant

11 - Affuter et décorer l'outil à la lime

12 - Outil fini 


\begin{tabular}{ccccc}
\hline \multicolumn{5}{c}{ Nombre de forgeages } \\
Mission1 & Mission2 & Mission3 & Forg. / \\
village \\
Kako & Janv2008 & Nov2008 & Janv2010 & 47 \\
Kakoli & 10 & 8 & 28 & 8 \\
Kobo & - & - & 8 & 18 \\
Doundé & - & - & - & 53 \\
Forg. / \\
mission
\end{tabular}

\subsubsection{Etape 1 : le préformage}

\section{Définition}

Le préformage est l'étape de la chaîne opératoire pendant laquelle le forgeron amène la matière première (quelles que soient sa forme et sa nature), en une pyramide (tronquée ou non) à base rectangulaire à faces trapézoïdales (2) que l'on appelle «préforme». Sa longueur est d'environ $20 \mathrm{~cm}$, la grande base est un rectangle de $4 \times 5 \mathrm{~cm}$ et la petite base mesure $2 \times 1 \mathrm{~cm}$ mais elle est le plus souvent complètement effilée. Sa surface est de $200 \mathrm{~cm}^{2}$.

La préforme est une pièce qui n'est jamais retrouvée par les archéologues, cependant elle joue un rôle fondamental dans le processus de la mise en forme des outils à la forge. Chaque outil passe par une préforme qui lui est propre : la préforme d'une lame de houe sera différente de la préforme d'une lame de machette.

\section{Configuration de l'équipe}

$\mathrm{Au}$ préformage, plusieurs configurations de l'équipe de travail sont possibles : souvent, le maître martèle seul le fer pendant une courte période (quelques secondes) afin de lui donner une forme correcte pour la suite du martelage. Ensuite, soit il martèle le fer en alternance avec un frappeur, soit ce sont un ou plusieurs frappeurs qui travaillent en alternance.

Le maître emploie une masse intermédiaire mais les frappeurs utilisent toujours les grosses masses qui permettent de déformer correctement le fer.

\section{Gestes/procédés techniques}

Evidemment, le choix des gestes et des procédés techniques pour aboutir à la préforme dépend du nombre, de la matière première : forme des dimensions de la (des) barre(s) à forger.
Pour obtenir une préforme,

- les barres M5S, M4S, M3S sont allongées ;

- la barre M2S est raccourcie par refoulage ;

- les barres M4D, M3D sont allongées puis soudées entre elles ;

- les barres M2D sont raccourcies puis soudées entre elles ;

- les barres M1D, A1D, A2D et R1D sont repliées plusieurs fois, soudées à ellesmêmes puis entre elles.

Seule la barre R1S est déjà préformée : elle est prête à être élargie (formage). Enfin, pour un outil donné, la morphologie générale de la préforme varie très peu quel que soit le type de barre.

\section{Température et temps de travail}

$\mathrm{Au}$ cours d'un cycle du préformage, les températures sont élevées. Le fer est chauffé dans le foyer jusqu'à $1400{ }^{\circ} \mathrm{C}$ puis martelé sur l'enclume jusqu'à une gamme de températures entre 400 et $700{ }^{\circ} \mathrm{C}$, avant d'être remis à chauffer (Tableau 11).

Si le forgeron doit travailler une seule barre de fer, alors la température atteindra seulement $1200^{\circ} \mathrm{C}$. S'il a affaire à 2 barres de fer à souder, alors le fer est porté à blanc à $1400{ }^{\circ} \mathrm{C}$. Pour obtenir ces hautes températures, le souffleur doit produire de l'air continument et avec vigueur dans le foyer.

Au cours d'un cycle, le fer est chauffé et est martelé plus longtemps dans les ateliers Tomo que dans les ateliers Mombo. La durée de chauffe par cycle est 2.5 (ateliers Tomo) à 3.5 fois (ateliers Mombo) plus longue que le martelage.

Nous observons aussi qu'au préformage, une quantité importante de battitures est éjectée lors du martelage de la matière première sur l'enclume.

\begin{tabular}{|c|c|c|c|c|c|}
\hline & \multicolumn{3}{|c|}{ Durée moy./cycle $(\mathrm{sec})$} & \multicolumn{2}{|c|}{ Couleur $-\mathrm{T}^{\circ} \mathrm{C}$} \\
\hline & $\mathrm{C}$ & $\mathrm{M}$ & $\mathrm{R}$ & $\operatorname{Max}$ & Min \\
\hline Fiko & 174 & 48 & $3.5: 1$ & $\begin{array}{c}\text { blanc } \\
1400\end{array}$ & $\begin{array}{c}\text { rouge } \\
900\end{array}$ \\
\hline Kakoli & 194 & 55 & $3.5: 1$ & $\begin{array}{r}\text { blanc } \\
1400\end{array}$ & $\begin{array}{l}\text { brun } \\
700^{\mathrm{a}}\end{array}$ \\
\hline Kobo & 203 & 114 & $2: 1$ & $\begin{array}{r}\text { blanc } \\
1400\end{array}$ & $\begin{array}{l}\text { gris } \\
400^{a}\end{array}$ \\
\hline Doundé & 223 & 70 & $3: 1$ & $\begin{array}{r}\text { blanc } \\
1400\end{array}$ & $\begin{array}{l}\text { brun } \\
700^{\mathrm{a}}\end{array}$ \\
\hline Mombo & $\begin{array}{l}184 \\
213\end{array}$ & $\begin{array}{l}52 \\
92\end{array}$ & $\begin{array}{l}3.5: 1 \\
25 \cdot 1\end{array}$ & $\begin{array}{l}1400 \\
1400\end{array}$ & $\begin{array}{l}800^{a} \\
550^{a}\end{array}$ \\
\hline Moyenne & 200 & 72 & $3: 1$ & 1400 & $700^{\mathrm{a}}$ \\
\hline
\end{tabular}




\section{Soudures}

Au total, 123 soudures ont été exécutées au cours de 71 forgeages (sur 126). L'immense majorité de ces soudures (117) a été réalisée au cours du préformage. Ainsi, le forgeron a parfois réalisé jusqu'à quatre soudures au cours d'un seul préformage.

La soudure est certainement le procédé technique le plus complexe à réaliser :

- la température des pièces à joindre doit être supérieure $1250{ }^{\circ} \mathrm{C}$,

- il faut veiller à ne pas chauffer trop longuement afin de ne pas perdre trop de métal par oxydation,

- de plus, si le métal est mis à chauffer trop longuement, des blocs $(\mathrm{cm})$ peuvent s'en détacher,

- aucun charbon ni oxyde de fer ne doit s'insérer entre les pièces à assembler,

- le martelage visant à réunir par pression les pièces doit être énergique afin de permettre une continuité de la matière à assembler lors de la recristallisation des grains à l'interface.

Dans le cadre de nos travaux, il est à noter que nous n'avons jamais demandé la soudure de deux barres de composition différente (fer/acier).

Dans une certaine mesure, les compétences de l'artisan sont jugées par la qualité de son travail : s'il rate une soudure il perdra certainement une partie de la confiance de ses pairs et une lame de houe comportant une soudure mal faite aura certainement tendance à se briser plus facilement pendant son utilisation et sera donc jugée de mauvaise qualité.

Nous avons répertorié cinq manières différentes pour réaliser des soudures, en fonction de la dimension des barres (Figure 33).

- Soudure simple (45\%) : assemblage de deux pièces en les posant l'une sur l'autre, utilisée surtout pour les barres courtes (M4D) à moyennes (M2D).

- Soudure par repli (28\%) : pliage de la barre sur elle-même, utilisée surtout pour les barres moyennes (M2D) à longues (M1D).

- Soudure multiple ( $8 \%$ ) : superposition de fers résultant de plusieurs types de soudures, exécutée en une seule fois, utilisée pour les barres trop longues (A1D, A2D) ou trop atypiques (R1D).

- Soudure par repli avec insert (9\%) : soudure par repli à laquelle sont insérés un ou plusieurs bouts de fer qui se sont détachés de la pièce principale, utilisée pour tous types de barres.

- Soudure par consolidation $(10 \%)$ : recollage des pailles (3.4.3) qui se sont formées lors de soudures précédentes, utilisée surtout pour les barres M2D.

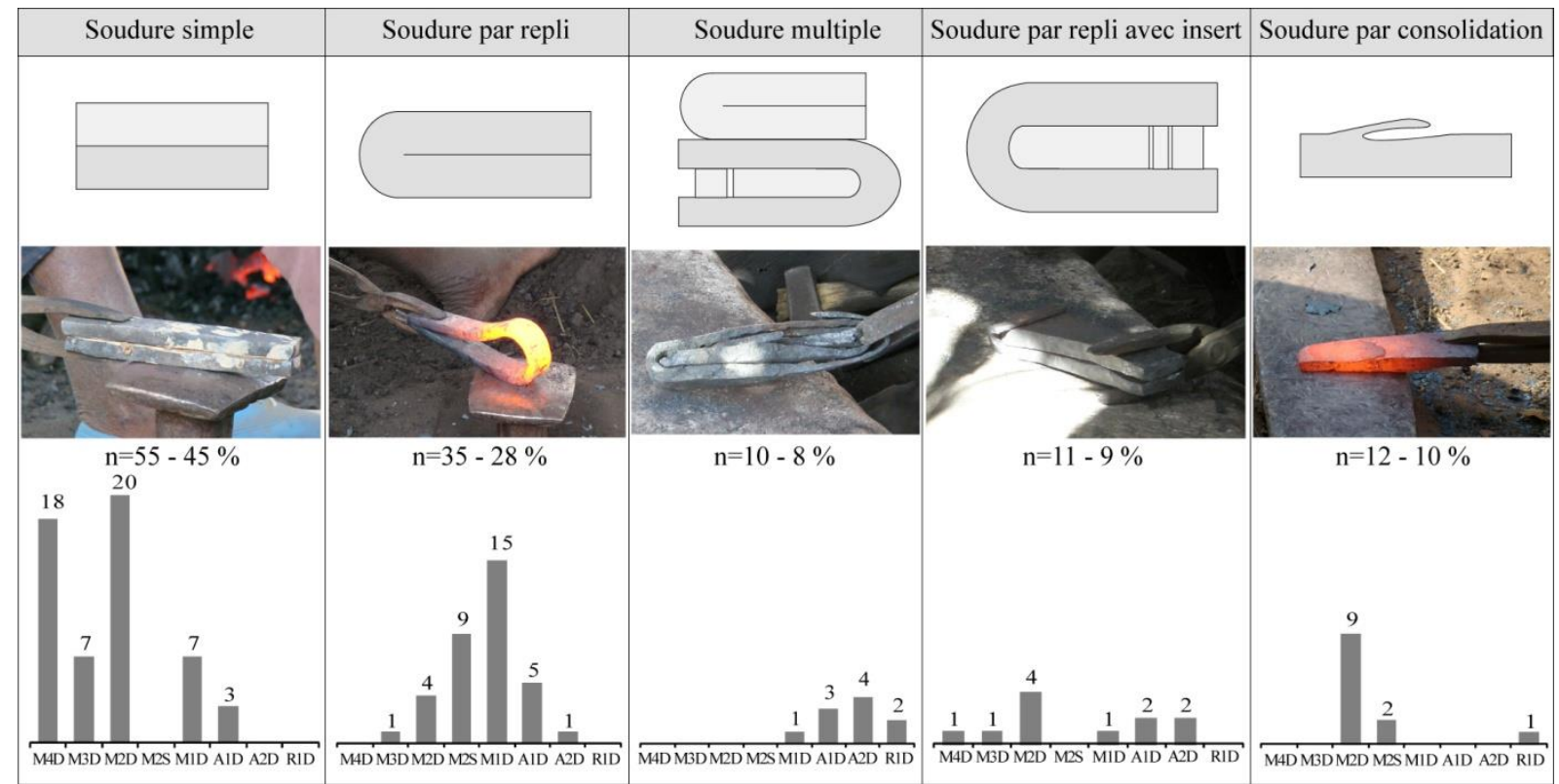

Figure 33 - Différents types de soudures et nombre d'apparition pour chaque type de barre à souder (ntot=123 soudures). 


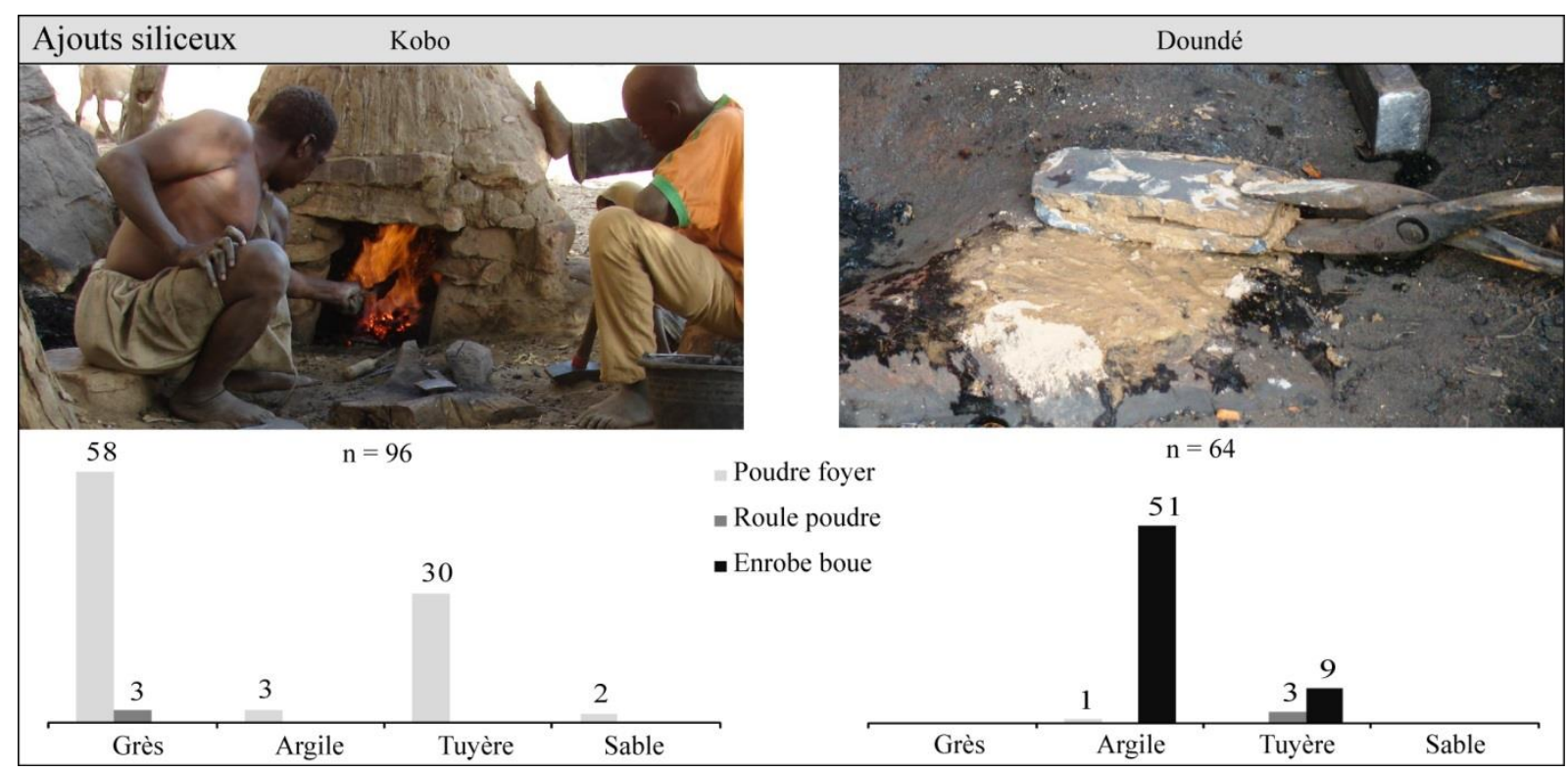

Figure 34 - Mode d'utilisation des ajouts siliceux à Kobo et Doundé.

\section{Ajouts siliceux}

La différence majeure observée entre les ateliers lors de la réalisation des soudures est l'emploi d'ajouts siliceux dans les ateliers Tomo, technique non pratiquée dans les ateliers Mombo. Nous relevons trois façons d'employer les ajouts de soudure :

- par saupoudrage dans le foyer pendant la chauffe,

- en roulant les barres de fer dans la poudre sèche,

- en réalisant une boue qui va enrober les barres à souder.

Malgré la proximité des deux villages, les forgerons n'utilisent ces ajouts ni dans les mêmes proportions ni de la même façon (Figure 34). A Doundé le grès n'est pas disponible: les forgerons ont l'habitude d'enrober les barres dans une boue d'argile ; à Kobo le forgeron a l'habitude de jeter du grès et de la tuyère concassés dans le foyer. Il a également été observé à Kobo par E. Huysecom au cours d'une mission précédente, l'utilisation d'une boue argilo-sableuse prélevée dans le fond du bac à eau (meule), pour la réalisation des soudures.

Enfin, nous avons noté que le forgeron a jeté dans le foyer trois fois de la poudre de charbon à Kakoli et trois fois également à Doundé, ce qui a eu pour effet de faire crépiter davantage le feu.

\section{« Trempes »}

Dans tous les ateliers (excepté Fiko), les forgerons ont plongé les barres de fer dans le bac à eau. Cependant le but n'était pas de durcir le métal (trempe) mais seulement de le refroidir rapidement afin de la manipuler plus facilement: il ne s'agit donc pas d'une trempe métallurgique au sens strict du terme (Encart 1 - Procédés techniques).

Au total, les barres de fer ont été refroidies de la sorte 63 fois au cours de 29 forgeages. C'est à Doundé que la majorité de ces «trempes»a été effectuée (55 fois), surtout au préformage (50 fois) afin de pouvoir appliquer directement avec les doigts la boue autour des barres à souder.

Toutefois les forgerons à Kobo (5 fois) et Kakoli (3 fois) ont aussi plongé les barres dans l'eau, surtout au préformage (sept fois) avant les phases de chauffe afin de faciliter les soudures ; au final, le fait de refroidir subitement le fer n'aura pas causé l'effet désiré de faciliter les soudures (3.2.4).

Ces refroidissements rapides, qui ne sont donc pas des trempes métallurgiques, ne remettent pas en cause le fait que les forgerons savent utiliser consciemment les trempes, notamment pour durcir l'extrémité d'outils comme des pointes de flèches (observations par E.Huysecom à Kobo avec Tiégé Djo, en 1995).

\subsubsection{Etape 2 : le formage}

\section{Définition}

C'est l'étape de la chaîne opératoire pendant laquelle le forgeron amène la préforme en une lame de houe dont la forme générale est acquise (Figure 32 - 7). D'un atelier à un autre, les procédés 
techniques exécutés sont très similaires même si leur ordre de succession peut différer.

A l'extrémité la plus épaisse, le forgeron élargit la future lame grâce à un martelage dynamique sur les deux faces de la préforme (3). Il forme les épaulements de l'outil en élargissant le centre de la barre (4). A l'autre extrémité, le forgeron étire la soie (5). Après avoir observé chaque face, il en choisit une et forme une nervure dans la lame dans la continuité de la soie (6).

Ainsi la houe se voit attribuer un « dos » (face où la nervure ressort) et un « ventre » (face où la nervure est creusée) (Figure 35).

\section{Configuration de l'équipe}

Au formage, l'élargissement de la lame est fait entre le maître et un seul frappeur qui travaillent en alternance. La formation des épaulements, de la soie et de la nervure est exécutée soit par le maître seul soit avec un frappeur en alternance, soit par le frappeur seul soit par plusieurs frappeurs.

Le maître utilise un marteau intermédiaire ou un petit marteau tandis que les frappeurs emploient des marteaux intermédiaires.

\section{Température et temps de travail}

$\mathrm{Au}$ cours d'un cycle au formage, les températures atteintes sont toujours élevées. Le fer est chauffé dans le foyer jusqu'à environ $1200^{\circ} \mathrm{C}$ puis martelé sur l'enclume jusqu'à une plage de

\begin{tabular}{|c|c|c|c|c|c|}
\hline & \multicolumn{3}{|c|}{ Durée moy./cycle (sec) } & \multicolumn{2}{|c|}{ Couleur - $\mathrm{T}^{\circ} \mathrm{C}$} \\
\hline & $\mathrm{C}$ & M & $\mathrm{R}$ & Max & Min \\
\hline Fiko & 97 & 48 & $2: 1$ & $\begin{array}{l}\text { jaune } \\
1200^{b}\end{array}$ & $\begin{array}{c}\text { brun } \\
700\end{array}$ \\
\hline Kakoli & 96 & 49 & $2: 1$ & $\begin{array}{l}\text { jaune } \\
1200^{\mathrm{b}}\end{array}$ & $\begin{array}{l}\text { brun } \\
700^{\text {a }}\end{array}$ \\
\hline Kobo & 106 & 92 & $1: 1$ & $\begin{array}{l}\text { jaune } \\
1200^{b}\end{array}$ & $\begin{array}{l}\text { gris } \\
400\end{array}$ \\
\hline Doundé & 89 & 63 & $1.5: 1$ & $\begin{array}{l}\text { jaune } \\
1200^{\mathrm{b}}\end{array}$ & $\begin{array}{l}\text { gris } \\
400^{\text {a }}\end{array}$ \\
\hline Mombo & 97 & 49 & $2: 1$ & 1200 & $700^{\mathrm{a}}$ \\
\hline Tomo & 97 & 78 & $1: 1$ & 1200 & $400^{\mathrm{a}}$ \\
\hline Moyenne & 97 & 64 & $1.5: 1$ & 1200 & $550^{\mathrm{a}}$ \\
\hline $\begin{array}{r}\text { Tableau } \\
\text { chauffe C e } \\
\text { fer et } \\
\text { supplémen }\end{array}$ & For & (12 & min - & $\begin{array}{l}\text { Irée mo } \\
\text { ort } \mathrm{R} \text { - } \\
\text { cepté tr } \\
\text { émentai }\end{array}$ & $\begin{array}{l}\text { e de } \\
\text { leurs du } \\
\text { es } \\
\text { (3.2.4). }\end{array}$ \\
\hline
\end{tabular}

températures entre 400 et $700{ }^{\circ} \mathrm{C}$, avant d'être mis de nouveau à chauffer (Tableau 12). A ce stade, le forgeron n'effectue ni soudures ni refroidissements à l'eau, sauf si des contretemps viennent perturber le déroulement du travail.

A Kobo, au cours d'un cycle de formage, le fer est chauffé et martelé plus longtemps que dans les autres ateliers. Par ailleurs, la durée de chauffe par cycle est deux fois plus longue (ateliers Mombo) ou équivalente (ateliers Tomo) à celle du martelage. De plus, au formage, la perte de battitures sur l'enclume est importante.

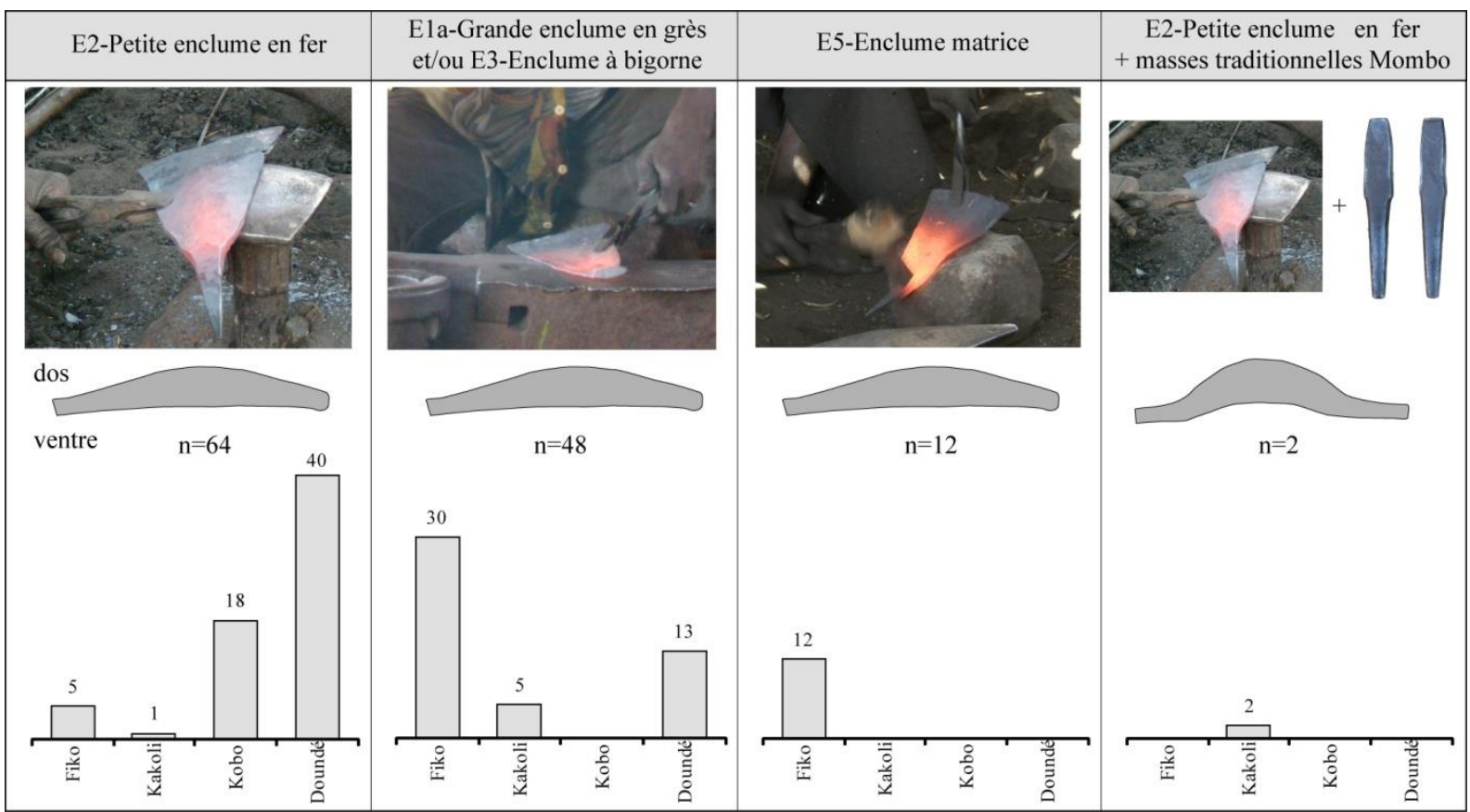

Figure 35 - Différentes manières de façonner la nervure : profil à la base de la lame, nombre d'apparition de chaque type. 


\section{Nervure centrale}

La nervure de la lame est essentielle puisqu'elle augmente sa rigidité. Avant de procéder à sa fabrication, le forgeron observe les deux faces de la lame puis choisit laquelle sera un dos ou un ventre. Diverses manières de former la nervure de la lame sont utilisées, en fonction des enclumes disponibles dans chacun des ateliers (Figure 35).

Toutes les nervures des lames de Kobo et une grande majorité de celles de Doundé sont exécutées en positionnant l'outil sur le rebord de l'enclume E2 afin de replier légèrement l'outil par martelage sur son dos. Ensuite, le forgeron retourne l'outil puis martèle sur son ventre afin de le creuser pour accentuer la nervure.

La même technique de martelage est appliquée à Fiko, Kakoli et Doundé, avec l'enclume en grès de fondation E1a et/ou l'enclume à bigorne E3.

A Kakoli, une dernière manière de former la nervure a été observée : il s'agit de replier légèrement la lame sur E2 puis de l'emboutir complètement sur les masses Mombo. De cette manière, la nervure est plus arrondie.

A Fiko, la nervure d'une partie des lames a été réalisée à l'aide de l'enclume matrice $\mathrm{E} 5$ prévue à cet effet.

\subsubsection{Etape 3 : les finitions}

\section{Définition}

Les finitions constituent l'étape de la chaîne opératoire pendant laquelle le forgeron apporte à l'outil brut quelques modifications afin d'en faire un outil fonctionnel (Figure 32 - 12). Ces interventions de finitions visent à améliorer le comportement mécanique du métal mais aussi à améliorer les qualités esthétiques de l'outil.

Pour cela, le forgeron plane les différentes parties de la lame (8), égalise les bordures et spécialement le tranchant en frappant la lame verticalement et en l'enfonçant dans le sol (9) puis il aplatit le tranchant par martelage (10). A Kobo et à Doundé, les forgerons se servent d'une lime pour affuter la lame et mais aussi décorer la nervure, la soie et les bordures de l'outil (11). Ainsi, le forgeron appose sa propre marque sur l'outil.

\section{Configuration de l'équipe}

Durant les finitions, le maître travaille toujours seul avec ses marteaux les plus petits (sauf une fois à Kobo où le frappeur a aussi participé).

\begin{tabular}{|c|c|c|c|c|c|}
\hline & \multicolumn{3}{|c|}{ Durée moy./cycle $(\mathrm{sec})$} & \multicolumn{2}{|c|}{ Couleur $-\mathrm{T}^{\circ} \mathrm{C}$} \\
\hline & $\mathrm{C}$ & M & $\mathrm{R}$ & $\operatorname{Max}$ & Min \\
\hline Fiko & 60 & 91 & $1: 1.5$ & $\begin{array}{l}\text { gris } \\
<400\end{array}$ & $\begin{array}{c}\text { orange } \\
1000\end{array}$ \\
\hline Kakoli & 55 & 122 & $1: 2$ & $\begin{array}{l}\text { gris } \\
<400\end{array}$ & $\begin{array}{c}\text { orange } \\
1000^{c}\end{array}$ \\
\hline Kobo & 52 & 166 & $1: 3$ & $\begin{array}{c}\text { gris } \\
<400\end{array}$ & $\begin{array}{c}\text { orange } \\
1000\end{array}$ \\
\hline Doundé & 37 & 97 & $1: 2.5$ & $\begin{array}{l}\text { gris } \\
<400\end{array}$ & $\begin{array}{c}\text { orange } \\
1000\end{array}$ \\
\hline Mombo & 58 & 107 & $1: 2$ & $<400$ & $1000^{c}$ \\
\hline Tomo & 45 & 132 & $1: 3$ & $<400$ & 1000 \\
\hline Moyenne & 51 & 120 & $1: 2.5$ & $<400$ & $1000^{c}$ \\
\hline
\end{tabular}

\section{Température et temps de travail}

$\mathrm{Au}$ cours d'un cycle au stade des finitions, les températures restent basses. Le fer est chauffé jusqu'à environ $1000^{\circ} \mathrm{C}$ puis il est martelé longuement sur l'enclume jusqu'au gris $\left(<400{ }^{\circ} \mathrm{C}\right)$.

Pour une lame de houe, les finitions consistent essentiellement en un martelage général à basse température (écrouissage) qui permet d'augmenter la dureté du métal et donc la solidité de l'outil (Encart 1 - Procédés techniques).

$\mathrm{Au}$ cours d'un cycle au stade des finitions, le chauffage est le plus bref à Doundé et le plus long à Fiko ; le martelage au contraire est le plus bref à Fiko et Doundé et le plus long à Kobo. De plus, davantage de temps est consacré au martelage dans les ateliers Mombo que dans les ateliers Tomo.

Enfin, nous remarquons que du fait des températures peu élevées, seulement peu, voire pas d'oxydes de fer se détachent lors du martelage.

\subsubsection{Incidents au cours du travail}

\section{Types d'incidents}

Les incidents rencontrés au cours du travail ne sont pas rares puisque ce sont près de $30 \%$ de l'ensemble des forgeages qui sont concernés (Tableau 14). C'est à Kobo (55\%) et Kakoli (50\%) que les forgeages rencontrent le plus d'incidents.

Au total, 84 incidents ont été relevés au cours des travaux à la forge (Tableau 15).

- D: défauts dans le fer (3.4.3) (38 \%) Cycles de travail supplémentaires pour colmater ces défauts par des soudures. 


\begin{tabular}{cc|cc|cc}
\hline & & \multicolumn{4}{|c}{ Forgeages } \\
& $\begin{array}{c}\text { nb } \\
\text { total }\end{array}$ & $\begin{array}{c}\text { nb } \\
\text { réussi }\end{array}$ & $\begin{array}{c}\text { nb avec } \\
\text { incident }\end{array}$ & $\begin{array}{c}\% \\
\text { réussi }\end{array}$ & $\begin{array}{c}\% \text { avec } \\
\text { incident }\end{array}$ \\
Fiko & 47 & 39 & 8 & 85 & 15 \\
Kakoli & 8 & 4 & 4 & 50 & 50 \\
Kobo & 18 & 8 & 10 & 45 & 55 \\
Doundé & 53 & 36 & 17 & 68 & 32 \\
Total & 126 & 87 & 39 & 70 & 30 \\
\hline
\end{tabular}

Tableau 14 - Récapitulatif du nombre et taux de forgeages réussis/avec incidents dans chaque atelier.

\begin{tabular}{|c|c|c|c|c|c|c|c|c|c|c|c|c|}
\hline & \multicolumn{12}{|c|}{ Nombre d'incidents } \\
\hline & \multicolumn{4}{|c|}{ Préformage } & \multicolumn{4}{|c|}{ Formage } & \multicolumn{4}{|c|}{ Finitions } \\
\hline & $\mathrm{D}$ & $\mathrm{R}$ & $\mathrm{C}$ & S & $\mathrm{D}$ & $\mathrm{R}$ & $\mathrm{C}$ & S & $\mathrm{D}$ & $\mathrm{R}$ & $\mathrm{C}$ & S \\
\hline$F$ & - & 3 & 3 & - & 1 & 2 & 1 & - & - & - & - & - \\
\hline Ka. & 1 & - & - & - & 2 & 2 & - & - & - & - & 2 & - \\
\hline Ko. & 3 & 9 & 9 & 1 & 4 & 1 & - & - & 3 & - & - & - \\
\hline $\mathrm{D}$ & 5 & 14 & 4 & - & 10 & 1 & - & - & 3 & - & - & - \\
\hline Tot & 9 & $\begin{array}{c}26 \\
52\end{array}$ & $\begin{array}{l}16 \\
\%)\end{array}$ & 1 & & $\begin{array}{l}6 \\
(36\end{array}$ & $\begin{array}{l}1 \\
\%)\end{array}$ & - & 6 & & $\begin{array}{c}2 \\
\%)\end{array}$ & - \\
\hline
\end{tabular}

Tableau 15 - Récapitulatif du nombre d'incidents rencontrés au cours de la chaîne opératoire : défauts (D), replis

supplémentaires $(\mathrm{R})$, cassures $(\mathrm{C})$, et soudure mal réalisée $(\mathrm{S})$.

- $\quad \mathrm{R}$ : replis (38 \%) - Soudures par replis supplémentaires sur les défauts euxmêmes; sur des barres M2S à Doundé qui ont été repliées au lieu d'être refoulées ou bien lorsqu'après élargissement de la lame, l'outil est trop long au lieu d'être large.

- C: cassures (23\%) - Perte d'un ou plusieurs morceaux de métal soit dans le foyer à cause d'une chauffe trop intense, soit sur l'enclume; souvent, les morceaux ont été réintégrés à la masse de fer.

- $\quad \mathrm{S}$ : soudure mal réalisée (1\%, 1 cas) - Le lopin de l'opération F020 (Kobo) a été assemblé par une soudure simple, tourné d'un quart de tour puis martelé ; l'interface de soudure s'est retrouvé au milieu de la lame, créant un travers dans sa longueur (3.4.3).

C'est essentiellement pendant le préformage (60 \%) que des incidents peuvent apparaître. Normalement le forgeron essaie par tous les moyens de les résoudre pour éviter de les propager aux étapes suivantes de la chaîne opératoire. Quand il estime que le fer est de nouveau exempt de défaut, alors il passe au formage.

Les incidents au formage sont moins fréquents $(30 \%)$ : il s'agit soit d'incidents qui étaient déjà présents au préformage, soit de nouveaux incidents.
Une fois le fer considéré propre et dépourvu de défauts, alors l'artisan passe aux finitions.

Aux finitions, les incidents sont minimes (10\%). Il s'agit de quelques défauts à supprimer dans l'outil presque achevé (provenant d'incidents antérieurs) et de cassures dues à une surchauffe locale du fer.

\section{Source des incidents}

Premièrement, tous les maitres de forge ont rencontré des incidents et le nombre d'incidents est indépendant du forgeron qui a travaillé : Sékou/Nou à Fiko (Tableau 3); Sékou/Abdou à Kakoli (Tableau 4) ; Seydou/Ali B à Doundé (Tableau 6).

Par contre à Kakoli, tous les incidents provoqués par Sékou sont des cassures aux finitions à cause d'une chauffe trop intense.

Deuxièmement, la soudure est la principale source d'apparition d'incidents. En effet, parmi les 39 forgeages avec incidents (Tableau 14), seuls 10 proviennent de barres simples mais 29 proviennent de barres doubles. De plus, la soudure n'est pas maîtrisée de la même façon dans tous les villages (Tableau 16).

- Fiko : le forgeron a l'habitude de pratiquer des soudures (ex : fabrication de houe à partir de plusieurs morceaux de houes usagées) et il réussit cette technique trois fois sur quatre.

- Kakoli : les forgerons rencontrent une fois sur deux des difficultés pendant le forgeage des outils, soudure ou non.

- Kobo : le forgeron ne pratique la soudure que rarement et il la rate très souvent; il a l'habitude de travailler à partir d'un seul morceau de fer ou de réaliser des petites réparations d'outils: c'est un sans-faute quand il s'agit de travaux sans soudure.

- Doundé : les forgerons réussissent à tous les coups les travaux sans soudure; par contre, quand ils doivent faire des soudures, ils ne la maitrisent pas totalement.

Ces différentes tendances vis-à-vis de certains travaux de forge reflètent le niveau de difficulté auquel sont confrontés les forgerons au quotidien, et donc le type de travail réalisé dans chaque forge. 


\begin{tabular}{ccc|cc}
\hline \multicolumn{3}{c}{ Barre simple } & \multicolumn{2}{c}{ Barres doubles } \\
& $\%$ & $\%$ incident & \% réussi & $\%$ incident \\
& réussi & 5 & 75 & 25 \\
Fiko & 95 & 50 & 50 & 50 \\
Kakoli & 50 & 0 & 10 & 90 \\
Kobo & 100 & 25 & 60 & 40 \\
Doundé & 75 & &
\end{tabular}

Tableau 16 - Taux de réussite des forgeages avec ou sans soudure dans chaque village.

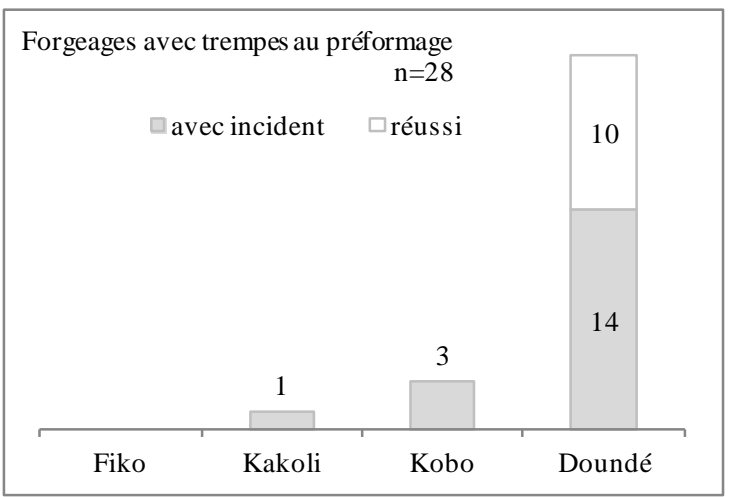

Figure 36 - Répartition par village du nombre de forgeages avec « trempes » au préformage : qui ont réussi $(n=10)$ et qui ont causé des incidents $(\mathrm{n}=18)$.

Troisièmement, les « trempes » sont la seconde source d'apparition des incidents. Au total, ce sont 29 forgeages qui ont comporté au total 63 refroidissements brusques à l'eau. Pour 28 de ces 29 forgeages, ces refroidissements ont eu lieu au préformage.

Sur ces 28 forgeages, 18 ont induit des incidents : formation de défauts dans la barre de fer et par conséquent des soudures par replis supplémentaires (Figure 36). Toutefois, 10 forgeages avec trempe (Doundé) n'ont eu aucune mauvaise conséquence pour la suite du déroulement de la chaîne opératoire.

En principe, un refroidissement brusque est sans effet sur l'acier mi-doux C17 mais il est plus important sur l'acier mi-dur C45. Toutefois, ici les 10 forgeages réussis avec «trempe » n'ont aucun lien ni avec la teneur en carbone des barres de fer ni avec un changement d'équipe (Doundé).

Ainsi, la forte proportion de forgeages avec refroidissement à l'eau provoquant des incidents montre que l'usage de ce traitement thermique n'est pas recommandé à ce stade du travail. En effet cela a pour effet un refroidissement rapide ainsi qu'un durcissement important du fer alors qu'au contraire, pendant le préformage le fer a besoin d'être le plus chaud et malléable possible.

De plus, les chauffes postérieures aux refroidissements ne permettent pas au fer de recouvrer ses propriétés initiales, créant de nombreux défauts dans le fer qu'il est impossible de résorber ni par chauffe ni par martelage additionnels.

\subsubsection{Comparaison des étapes de travail}

Dans les ateliers étudiés, l'observation de la fabrication des lames de houe a permis de mettre en évidence des variantes de la chaîne opératoire.

Dans tous les ateliers, la fabrication des lames de houe passe par la succession d'une étape de préformage très variable qui dépend des dimensions de la matière première, puis d'une étape de formage et de finitions quant à elles très peu variables.

Il existe de nombreuses combinaisons possibles pour les équipes de travail (Tableau 17). Au préformage et au formage quatre possibilités existent: le maître intervient seul ou avec un frappeur, le frappeur intervient seul ou avec un ou deux autres frappeurs. Aux finitions le maître est toujours seul (sauf une exception à Kobo).

$\mathrm{Au}$ préformage les températures atteintes sont les plus élevées $\left(700^{\circ} \mathrm{C}-1400^{\circ} \mathrm{C}\right)$ et la durée de chauffe est très supérieure à celle du martelage.

\begin{tabular}{|c|c|c|c|}
\hline & Préformage & Formage & Finitions \\
\hline Fiko & $\begin{array}{c}\mathrm{M} \\
\mathrm{M}+\mathrm{F} \\
\mathrm{F} \\
(\mathrm{F}+\mathrm{F})\end{array}$ & $\begin{array}{c}\mathrm{M} \\
\mathrm{M}+\mathrm{F} \\
(\mathrm{F}) \\
(\mathrm{F}+\mathrm{F})\end{array}$ & M \\
\hline Kakoli & $\begin{array}{c}\mathrm{M} \\
\mathrm{M}+\mathrm{F} \\
\mathrm{F} \\
\mathrm{F}+\mathrm{F}\end{array}$ & $\begin{array}{c}\mathrm{M} \\
\mathrm{M}+\mathrm{F} \\
\mathrm{F} \\
\mathrm{F}+\mathrm{F}\end{array}$ & M \\
\hline Kobo & $\begin{array}{c}\mathrm{M} \\
\mathrm{M}+\mathrm{F} \\
\mathrm{F} \\
\mathrm{F}+\mathrm{F}\end{array}$ & $\begin{array}{c}\mathrm{M} \\
\mathrm{M}+\mathrm{F} \\
\mathrm{F} \\
\mathrm{F}+\mathrm{F} \\
\mathrm{F}+\mathrm{F}+\mathrm{F}\end{array}$ & $\begin{array}{c}\mathrm{M} \\
(\mathrm{M}+\mathrm{F})\end{array}$ \\
\hline Doundé & $\begin{array}{c}\mathrm{M} \\
\mathrm{M}+\mathrm{F} \\
\mathrm{F} \\
\mathrm{F}+\mathrm{F}+\mathrm{F}\end{array}$ & $\begin{array}{c}\mathrm{M} \\
\mathrm{M}+\mathrm{F} \\
\mathrm{F} \\
\mathrm{F}+\mathrm{F} \\
\mathrm{F}+\mathrm{F}+\mathrm{F}\end{array}$ & M \\
\hline $\begin{array}{l}\text { Tablea } \\
\text { préforma }\end{array}$ & $\begin{array}{l}\text { - Combinaiso } \\
\text { ormage, finitic } \\
\text { forgeage des }\end{array}$ & $\begin{array}{l}\text { des équipes } \\
\text { dans chaqu } \\
\text { hes de houe }\end{array}$ & ier lors du \\
\hline
\end{tabular}


Au formage, les températures sont moyennes à élevées $\left(550{ }^{\circ} \mathrm{C}-1200{ }^{\circ} \mathrm{C}\right)$ et la durée de chauffe est encore supérieure à celle du martelage. Aux finitions, les températures sont moyennes à basses $\left(<1000{ }^{\circ} \mathrm{C}\right)$ et cette fois la durée de chauffe est inférieure à celle du martelage.

Il existe cinq manières de souder deux barres entre elles, en fonction de leur dimension. Une différence majeure technique reste l'emploi d'ajouts siliceux lors des soudures dans les ateliers de la plaine.

Cependant, les forgerons n'utilisent pas ces matières de la même façon : à Kobo du grès concassé et de la tuyère concassés sont jetés directement dans le foyer tandis qu'à Doundé, les barres à assembler sont enduites d'une boue d'argile.

La nervure de la houe est formée à l'aide des différentes enclumes à disposition.

Au cours de nos travaux de forge, une différence culturelle est observée lors des finitions : seuls les forgerons de la plaine utilisent une lime afin d'aiguiser le tranchant et décorer la houe. Cependant cela n'exclut pas qu'il puisse exister des houes décorées, en particulier de parades pour les danses ou premiers labours, aussi bien sur le plateau ou dans la plaine (communication E. Huysecom).

De nombreux incidents sont observés au cours de l'élaboration des lames de houe, le plus courant intervient lors $\mathrm{du}$ préformage et plus particulièrement lors de la soudure de deux barres de fer.

Il y a effectivement des questions à se poser sur le fait que les forgerons ne maitrisent pas tous de la même manière l'acte de la soudure. Les forgerons Tomo (Kobo, Doundé) maîtrisent très bien le formage et les finitions mais ils maitrisent moins bien le préformage. En effet, ils ont l'habitude de fabriquer des lames de houe à partir de fers de récupération $(\mathrm{R} 1 \mathrm{~S})$ qui sont déjà découpés à froid et préformés. Au contraire les forgerons Mombo (Fiko en particulier) maitrisent très bien les soudures car ils ont beaucoup plus l'habitude de cette pratique.

Enfin, les refroidissements brusques des barres de fer dans le bac à eau avant les soudures, favorisent considérablement l'apparition de défauts dans le métal. 


\subsection{DURÉE DU TRAVAIL}

Une large partie du travail de terrain a été consacrée à relever la durée des différentes étapes des travaux de forge à l'aide d'un chronomètre : durées de chauffe et de martelage au cours du préformage, du formage et des finitions.

Dans le chapitre 3.3 nous allons identifier, par atelier, les paramètres des forgeages qui ont une influence sur la durée du travail. Est-ce que les deux différentes souffleries ou bien la morphologie des barres de fer (etc.) induisent une différence de durée de forgeage ?

\subsubsection{Durée totale du travail}

Globalement, la durée de fabrication d'une lame de houe varie entre $1 \mathrm{~h}$ et $2 \mathrm{~h} 40$. Toutefois, cette donnée varie considérablement en fonction de l'atelier considéré, de la catégorie de barre travaillée (simple/double) et des incidents qui peuvent survenir (Figure 37).

\section{Influence de l'atelier et catégorie de barre}

Tout d'abord, Doundé et Fiko se classent toujours dans les plus rapides (1h-1h30) tandis que Kakoli et Kobo se classent toujours dans les plus lents à travailler (1h30-2h40).

Les forgeages d'une barre simple sans incident, ne sont pas en accord avec le tableau 16 : celui-ci montre que Fiko/Kobo atteignent 95-100\% de réussite donc ils devraient travailler très rapidement puisqu'ils ne rencontrent pas d'incidents ; Doundé/Kakoli atteignent $75-50 \%$ de réussite donc ils devraient travailler bien plus lentement.

Les forgeages de barres doubles sans incident, sont, par contre, en accord avec le tableau 16 : les forgerons de Fiko/Doundé atteignent $60-75 \%$ de réussite et ils sont les plus rapides à travailler ; ceux de Kakoli/Kobo atteignent 10-50 \% de réussite et ils travaillent plus lentement.

Ce classement (Doundé/Fiko versus Kakoli/Kobo) ne reflète pas non plus les différences relatives aux deux types d'ateliers (foyer et soufflerie) puisque chaque groupe comprend un atelier type Mombo et Tomo (Figure 27).

Seule une différence humaine pourrait expliquer ce classement: la qualification des souffleurs. A Fiko et Kakoli, la soufflerie ventilateur activée par des enfants ou des adultes génère un rendement important (Tableau 7). A Doundé, la soufflerie double pot activée par des souffleurs expérimentés, égale le très bon rendement de la soufflerie de type ventilateur. Au contraire, à Kobo les souffleurs (apprentis et enfants) n'ont pas encore toute l'endurance et la force nécessaires pour assurer un bon rendement d'air. Ainsi, à Kobo la chauffe est très lente, impliquant une durée totale du travail plus longue.

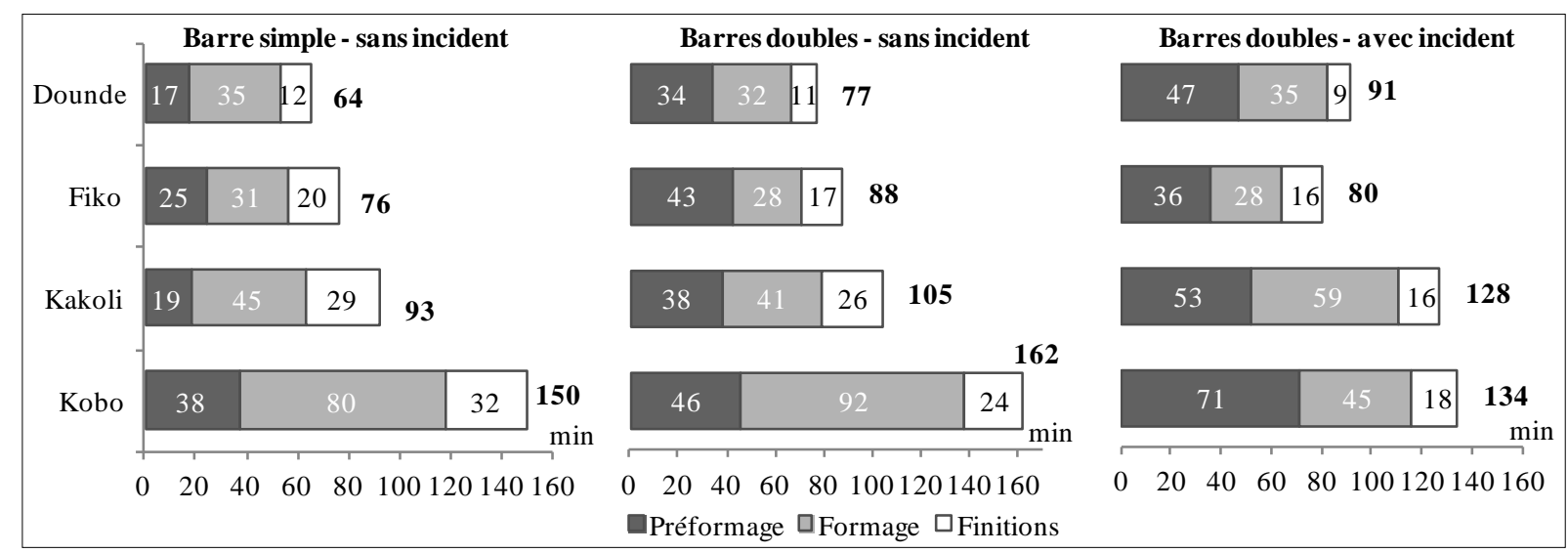

Figure 37 - Pour chaque atelier, durée totale moyenne (minutes) de fabrication d'une lame de houe pour des travaux avec une barre simple sans incident ( 52 forgeages), avec des barres doubles sans incident (35 forgeages) et avec des barres doubles avec des incidents (29 forgeages). Le temps d'utilisation de la lime à Doundé et Kobo n'est pas inclus dans la durée des finitions. 
La qualification des frappeurs peut aussi être mise en cause : les frappeurs à Kobo sont également des jeunes apprentis qui manquent de force et d'expérience. Pour finir, c'est en fait toute l'équipe de forgerons de Kobo qui travaille lentement.

Enfin, dans tous les ateliers, la durée totale du travail augmente quand il s'agit de travailler des barres doubles (sans incident). Cela est logique car il faut compter le temps de la soudure avant de préparer la préforme. Par exemple Doundé travaille en $1 \mathrm{~h} 15$ au lieu de $1 \mathrm{~h}$ et Kobo travaille en $2 \mathrm{~h} 40$ au lieu de $2 \mathrm{~h} 30$.

\section{Présence d'incidents}

En toute logique, la survenue d'incidents et leur élimination durant l'exécution de la chaîne opératoire doit allonger la durée totale de travail.

C'est le cas de Doundé (+14 min) et de Kakoli (+23 min). Cependant c'est l'opposé qui se passe à Fiko (-8 min) et à Kobo ( $-28 \mathrm{~min})$. Nous avançons une hypothèse : face à un incident, les forgerons écourtent-ils le travail en pensant que de toutes les façons cette houe ne servira probablement jamais dans un champ ?

\section{Durée de travail au préformage}

Le préformage est l'étape de la chaîne opératoire qui est la plus variable du fait que le forgeron adapte son travail à la barre (dimensions/simple/double/incidents) qu'il doit amener en une préforme.

Effectivement, dans chaque atelier la durée du préformage a varié positivement entre des forgeages d'une barre simple et de barres doubles (Figure 37). Le préformage double (x2) à Doundé, Fiko et Kakoli. Seul Kobo augmente moins rapidement (x1.2), passant de 38 min à 46 min.

Si des incidents surviennent, alors la durée du préformage augmente encore (x1.5) ou au moins reste stable (Fiko).

Il est net que la durée de préformage à Doundé, Fiko et Kakoli est toujours très similaire $: \approx 20 \mathrm{~min}$ pour des barres simples, $\approx 40$ min pour des barres doubles et $\approx 45$ min avec des incidents.

A Doundé, les nombreuses «trempes » et la réalisation de la boue pour les soudures ne semblent en tous les cas pas augmenter la durée du préformage par rapport aux autres ateliers.
Par contre, le préformage est toujours plus long à Kobo qu'ailleurs: $\approx 40 \mathrm{~min}$ pour des barres simples, $\approx 50$ min pour des barres doubles et $\approx 70 \mathrm{~min}$ avec des incidents. Ces valeurs reflètent : l'abondance des incidents au préformage (Tableau 15), le faible taux de réussite des soudures (10\%) mais aussi le manque de qualification des frappeurs et des souffleurs.

\section{Durée du travail au formage}

Le formage est une étape de la chaîne opératoire qui varie peu, quelle que soit la catégorie de barre.

La durée du formage reste globalement stable entre des travaux sur une barre simple, des barres doubles et des incidents : $\approx 35 \mathrm{~min}$ à Doundé et $\approx 30$ min à Fiko.

Par contre le temps de formage est divisé par deux à Kobo (45 min au lieu de 90 min) dans le cas de barres doubles avec incidents; le formage est augmenté à Kakoli (60 min au lieu de 40 min). Nous n'avons pas d'explication à cela.

Ensuite, la durée du formage est toujours courte à Doundé et Fiko (30-35 min), légèrement plus longue à Kakoli (50 min) et bien plus longue à Kobo (jusqu'à 90 min pour des travaux sur des barres doubles sans incident). L'allongement du temps de travail à Kakoli par rapport à Doundé et Fiko n'est pas expliqué. La faible qualification des frappeurs et souffleurs déjà évoquée à Kobo peuvent expliquer cette différence de durée du formage.

\section{Durée de travail aux finitions}

Les finitions varient peu d'un atelier à un autre, quelle que soit la catégorie de barre.

Les finitions sont toujours exécutées en $10 \mathrm{~min}$ à Doundé et 18 min à Fiko. Seuls Kobo et Kakoli réduisent à chaque fois la durée des finitions entre le forgeage de barre simple/double/incidents.

Enfin, si l'on compare atelier à atelier la durée des finitions, Doundé est toujours le plus rapide (10 min). Peut-être par habitude et répétition du travail ? Au contraire, Fiko, Kakoli et Doundé exécutent les finitions en 20 à $25 \mathrm{~min}$.

\section{Synthèse de 3.3.1}

1- Du fait des équipes de travail inégales (qualification des souffleurs et des frappeurs), Kobo 


\begin{tabular}{cccc}
\hline Classement & \multicolumn{3}{c}{ Durée globale du travail } \\
par rapidité & $\begin{array}{c}\text { Barre } \\
\text { simple }\end{array}$ & $\begin{array}{c}\text { Barre } \\
\text { double }\end{array}$ & $\begin{array}{c}\text { Barre double } \\
\text { avec incident }\end{array}$ \\
1. Doundé & $1 \mathrm{~h}$ & $1 \mathrm{~h} 15$ & $1 \mathrm{~h} 30$ \\
2. Fiko & $1 \mathrm{~h} 15$ & $1 \mathrm{~h} 30$ & $1 \mathrm{~h} 20$ \\
3. Kakoli & $1 \mathrm{~h} 30$ & $1 \mathrm{~h} 45$ & $2 \mathrm{~h} 10$ \\
4. Kobo & $2 \mathrm{~h} 30$ & $2 \mathrm{~h} 40$ & $2 \mathrm{~h} 15$ \\
\hline
\end{tabular}

Tableau 18 - Durée de fabrication d'une lame de houe dans chaque atelier pour différents types de forgeages.

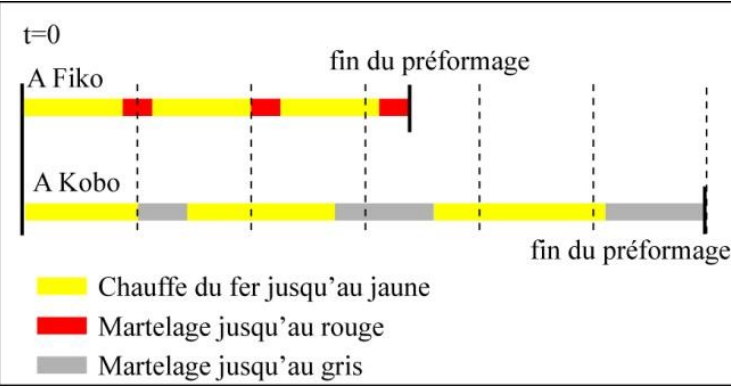

Figure 38 - A Fiko le fer est chauffé brièvement et 3 fois plus court que le martelage : cycles courts. A Kobo le fer est chauffé longtemps et 1.7 fois plus long que le martelage : cycles longs.

est toujours le plus lent à forger une lame de houe, en moyenne en $2 \mathrm{~h} 30$; dans les autres ateliers ce même travail est effectué en 1h30 (Tableau 18).

2- Dans chaque atelier, la durée globale du travail augmente (x1.2) pour le forgeage de barres doubles. Dans le cas d'incidents rencontrés au cours $\mathrm{du}$ forgeage, l'augmentation systématique de la durée totale du travail n'est pas mise en évidence.

3- Dans chaque atelier, la durée du préformage augmente lors de travaux de barres doubles (x2 sauf Kobo x1.2) et lors de travaux avec des incidents (x1 à 1.5$)$.

4- Dans chaque atelier, la durée du formage reste inchangée quelle que soit la catégorie de barres. Ceci n'est pas valable à Kobo où la durée du formage est divisée par deux lors de travaux avec incidents.

5- A Fiko et Kakoli les finitions varient peu quelle que soit la catégorie de barres. A Kakoli et Kobo la durée des finitions diminue entre des travaux sur des barres simple/double/incident. Enfin, Doundé est deux fois plus rapide que les autres ateliers.

\subsubsection{Rapport chauffe/martelage (C/M)}

Nous venons de comprendre, pour chaque village, le comportement des durées de travail à chaque stade de la chaîne opératoire et pour différentes sortes de forgeages. Pour toutes ces catégories, quelle est la part du temps dédiée au chauffage et celle dédiée au martelage (Figure 39) ?

\section{C/M au cours du préformage}

Tout d'abord, la durée de chauffe est toujours prépondérante par rapport à celle du martelage.

Dans tous les ateliers le fer est laissé à chauffer en moyenne trois fois plus qu'il n'est martelé, sauf à Kobo où la durée de chauffe n'est même pas le double (x1.7) de celle du martelage. Ainsi, à Kobo le fer est martelé en proportion plus longtemps que dans les autres ateliers.

Comme mentionné précédemment, le préformage à Kobo est toujours 2 fois plus long qu'ailleurs (Figure 37) : dans cet atelier, la durée de chauffe moyenne (33 $\mathrm{min}$ ) et de martelage (16 min) est supérieure aux autres ateliers.

Donc à Kobo :

- la proportion de martelage est plus importante que dans les autres ateliers,

- la durée de martelage est aussi plus importante.

Ainsi, le fer est trop refroidi après martelage, induisant des cycles de travail rallongés et une augmentation de la durée de fabrication des lames de houe (Figure 38).

De plus, au préformage dans chaque atelier, le fer est toujours chauffé en proportion bien plus longtemps lorsqu'il s'agit de barres doubles : à Fiko et Kakoli, le fer est chauffé dans le foyer 4 fois plus qu'il n'est martelé sur l'enclume.

\section{C/M au cours du formage}

A ce stade de la chaîne opératoire, le rapport $\mathrm{C} / \mathrm{M}$ évolue par rapport au préformage : le fer est moins chauffé et plus martelé.

Nous avions compris que dans chaque atelier, la durée du formage reste inchangée quel que soit le type de forgeage. Dans le détail, nous constatons que dans chaque atelier le rapport $\mathrm{C} / \mathrm{M}$ reste toujours le même, quel que soit le type de forgeage.

Deux groupes existent : à Fiko et Kakoli, la chauffe est en moyenne deux fois plus longue que le martelage ; à Kobo et Doundé, la chauffe est presque aussi longue que le martelage. 


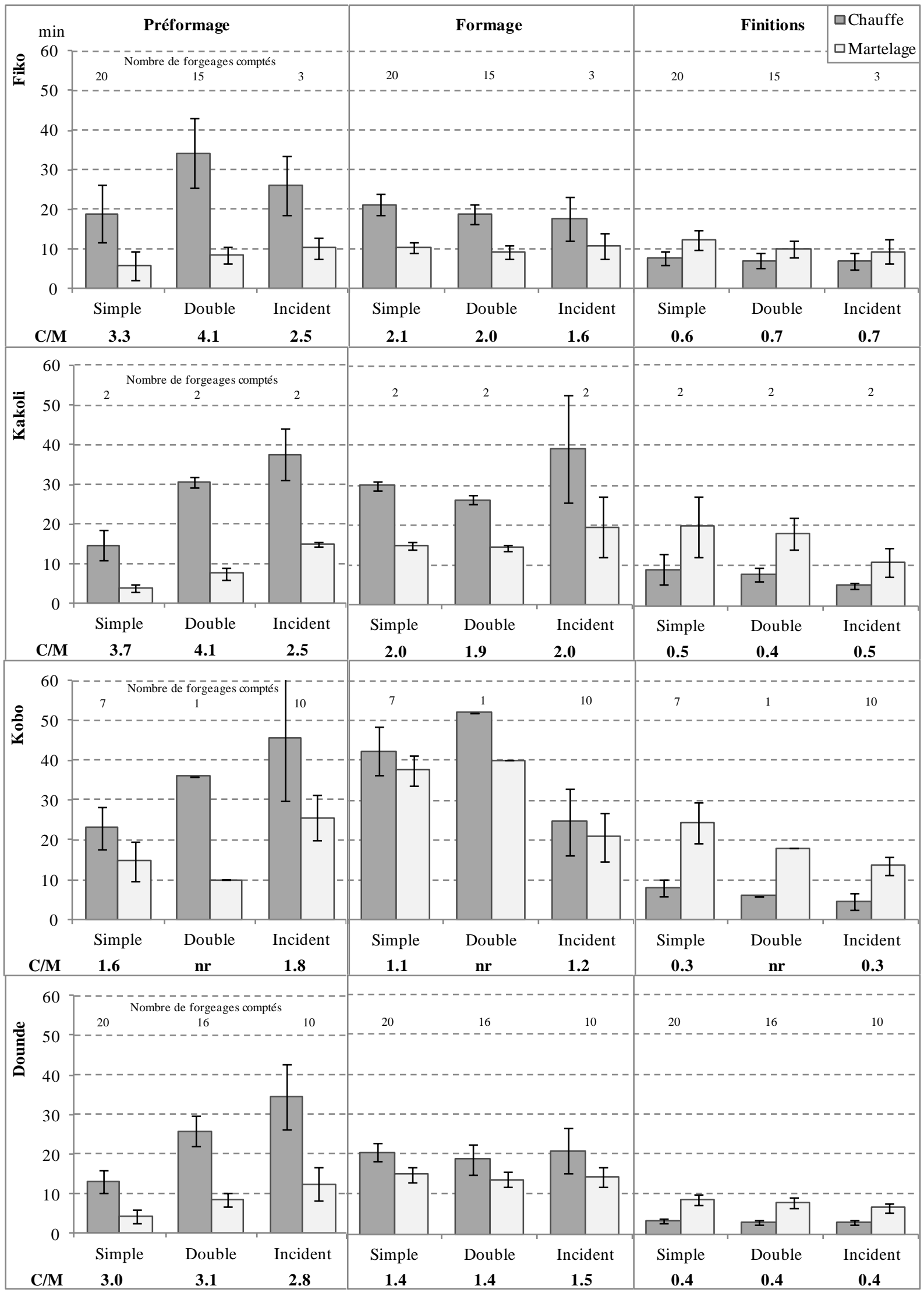

Figure 39 - Durées moyennes de chauffe (C) et de martelage (M) à chaque stade de la chaîne opératoire dans chaque atelier et pour différents forgeages : barre simple/double/double avec incident. Le rapport C/M est donné en gras sous chaque graphique. nr : non représentatif. Nombre de forgeages comptés : total 108 ; Fiko 38 ; Kakoli 6 ; Kobo 18 ; Doundé 46. 


\section{C/M au cours des finitions}

$\mathrm{Au}$ stade des finitions, le rapport $\mathrm{C} / \mathrm{M}$ est inversé car le martelage prédomine.

Comme au formage, la durée des finitions varie peu. Dans le détail, dans chaque atelier le rapport $\mathrm{C} / \mathrm{M}$ reste le même quel que soit le type de forgeage.

Les deux mêmes groupes sont mis en évidence : à Fiko et Kakoli le martelage est 1.5-2 fois plus long que la chauffe; à Kobo et Doundé le martelage est 2.5-3 fois plus long que la chauffe.

\section{Synthèse du 3.3.2}

6- Au préformage, la proportion de chauffe domine celle du martelage ; toutefois :

- plus la part de chauffe est importante $(\mathrm{C} / \mathrm{M}=3)$ et plus le martelage et donc le préformage seront brefs ;

- si le martelage est un peu plus important (C/M $<2$ ) alors le martelage et donc le préformage seront rallongés (Kobo).

7- $\mathrm{Au}$ formage, les durées de chauffe et de travail sur l'enclume s'équilibrent, chacune ayant un rôle important à ce stade : chauffer suffisamment pour pouvoir amener correctement la préforme en une lame brute. Fiko et Kakoli chauffent plus (x2) que Kobo et Doundé (x1.2)

8- Aux finitions le travail de martelage est le plus important : le forgeron durcit les différentes parties de la lame sur l'enclume, à froid, afin d'augmenter considérablement sa solidité. Kobo et Doundé martèlent plus (x3) que Fiko et Kakoli (x1.5).

\subsubsection{Type de barre}

Les forgerons ont travaillé 13 types de barres de dimensions très différentes, pour aboutir à chaque fois à une même préforme puis à un seul outil. Quelle est l'influence de la dimension des barres sur la durée de fabrication d'une houe? Seuls les forgeages sans incident ont été comparés.

\section{Taux d'aplatissement}

Pour chaque type de barre, le travail par déformation plastique consiste à amener une barre de fer en une lame de houe de $0.5 \mathrm{~cm}$ d'épaisseur

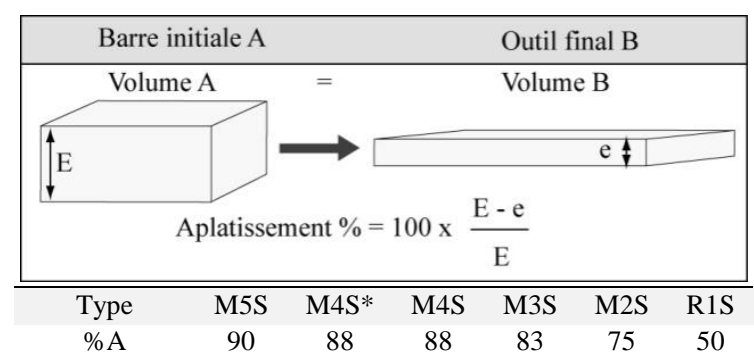

Figure 40 - Calcul du taux d'aplatissement (A) pour une barre simple. E : épaisseur initiale; e : épaisseur finale $=0.5 \mathrm{~cm}$.

moyenne. Le taux de déformation (D \%) et le principe de conservation du volume sont illustrés à la figure 40 .

Le taux de déformation peut être calculé pour chaque barre simple : le bloc M5S, de forte section, demande une forte déformation (90\%) alors qu'une barre de recyclage R1S ne nécessite qu'une faible déformation $(50 \%)$.

Au préformage, en principe la durée de travail devrait être proportionnelle au taux d'aplatissement donc en lien avec le type de barre travaillé.

\section{Influence au préformage}

Tout d'abord, le forgeage d'une barre épaisse nécessite plus de chauffe et de martelage que celui d'une barre plus fine (D élevé) (Figure 41).

La barre M5S doit être chauffée à cœur pour être correctement déformée, ce qui demande davantage de temps de chauffe. De plus, sa forme (prisme carré droit) est trop éloignée de celle de la préforme, ce qui demande également davantage de temps de martelage.

Contrairement à ce que nous pensions, le forgeage des barres M2S peut demander beaucoup de travail (excepté Doundé) : ces barres ont une forme très allongée qui nécessite d'être raccourcie. Ce travail de refoulage demande de nombreux cycles supplémentaires de chauffe et martelage.

Les taux de d'aplatissement de M3S et de M4S sont très similaires. M4S et M3S semblent demander autant de temps de préformage.

Par ailleurs il n'est constaté aucune différence de durée de travail entre les forgeages de barres de type M4S et M4S* (Figure 42). La découpe (brève) de la préforme de M4S* en 2 barres M4S compense le temps de travail (élevé) nécessaire pour déformer un volume aussi important que M4S*. 


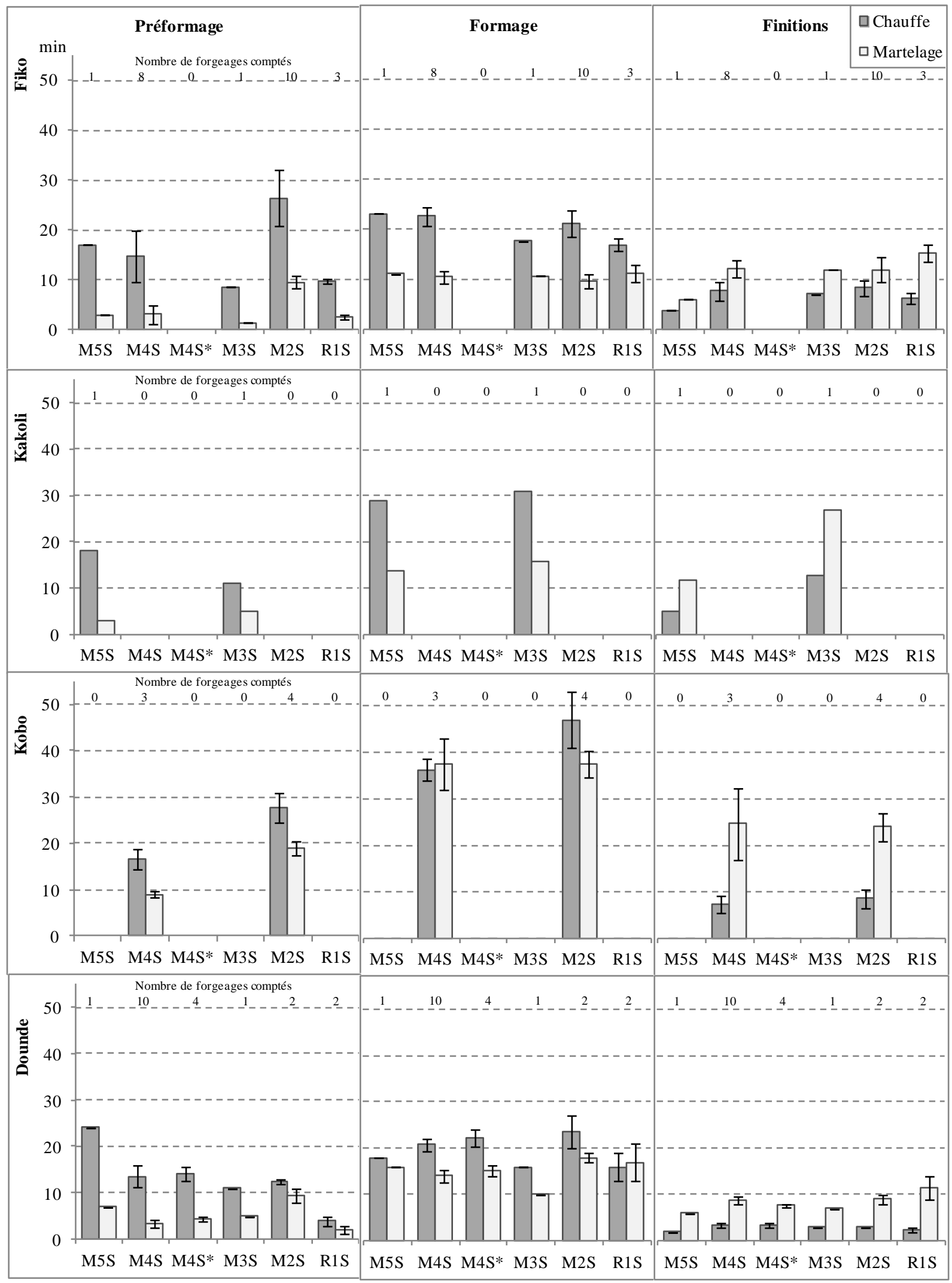

Figure 41 - Type de barre et durée du travail. Valeurs moyennes et écart-type des durées de chauffe et de martelage pour toutes les barres simples dans chacun des ateliers. Seuls les forgeages sans incidents ont été pris en compte pour une meilleure comparaison. Nombre de forgeages comptabilisés : total 52 ; Fiko 23 ; Kakoli 2 ; Kobo 7 ; Doundé 20. 


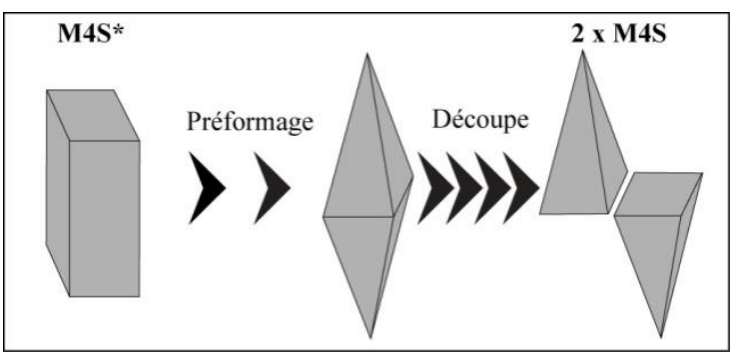

Figure 42 - Chaîne opératoire du préformage des barres de type M4S* $(2 \mathrm{~kg})$ en deux barres M4S.

La barre R1S demande le moins de travail car ses dimensions sont comparables à celle de la préforme souhaitée. D'ailleurs, D est faible.

Ainsi (excepté M2S), la durée du préformage dépend globalement de $\mathrm{D}$ et donc de la section de la barre de fer travaillée.

\section{Influence au formage et aux finitions}

Le formage débute avec une préforme standardisée. Contrairement au préformage où la durée du travail dépendait fortement des dimensions des barres, au formage et aux finitions, le type de barre n'a plus aucune influence.

La figure 41 montre que dans chaque atelier, les durées de chauffe et de martelage s'équilibrent autour d'une durée moyenne, et ce quel que soit le type de barre. Le formage à Fiko se compose d'environ 20 min de chauffe et 10 min de martelage ; les finitions comportent environ $5 \mathrm{~min}$ de chauffe et10 min de martelage.

\section{Emploi de barres doubles}

Pour les forgeages de barres doubles, la durée du travail ne dépend jamais de la section des barres travaillées. Le forgeage de barres trapues M4S ou fines M2S n'implique pas exactement les mêmes gestes techniques, cependant la durée du travail est toujours très similaire dans un atelier donné.

\section{Synthèse du 3.3.3}

9- Le choix préliminaire d'une barre de telle ou telle dimension pour la fabrication de tel ou tel objet n'est pas un acte anodin.

Pour s'affranchir d'un travail trop long de préformage, il vaut mieux choisir une barre dont les dimensions avoisinent celles de la préforme attendue. Une barre de fer trop allongée (M2S) n'est pas destinée au forgeage d'un outil large comme une lame de houe.

Classement des barres de fer dans le cas d'une lame de houe : R1S<M4S*-M4S-M3S<M2S-M5S.

10- Le type de barre employé par les forgerons n'influence pas la durée du travail au cours du formage et des finitions. Dans chaque atelier le forgeron travaille selon une durée qui lui est propre.

11- Le fait de forger et de souder des barres courtes (M4D), longues (M1D) ou atypiques (R1D) n'a aucune incidence sur la durée du travail : dans chaque atelier le forgeron travaille selon une durée qui lui est propre.

\subsubsection{Teneur en carbone}

Les forgerons reconnaissent dès les premiers coups de marteau s'ils ont à travailler un acier « mou » ou un acier «dur ». Quel est l'impact de la dureté du fer sur la durée du travail ? (Figure 43).

La catégorie de barre (simple/double) et le type de barre (moderne/africain/recyclage) influencent la durée du préformage. Pour cette raison, nous avons choisi de comparer un seul type de barre simple en $0.17 \%$ C et $0.45 \%$ C et un seul type de barres doubles en $0.17 \% \mathrm{C}$ et $0.45 \% \mathrm{C}$. Il s'agit du type qui est le plus représenté : M4S et M4S*.

\section{Influence au préformage}

L'influence de la dureté du fer varie d'un atelier à un autre.

A Fiko, les forgerons mettent toujours plus de temps à préformer un acier à $0.45 \% \mathrm{C}$ qu'un acier à $0.17 \% \mathrm{C}$, qu'il s'agisse de M4S ou M4S*. Ceci est valable à la fois pour la durée de chauffe et pour la durée du martelage.

Nous n'avons pas de données pour Kakoli et Kobo du fait du nombre limité de forgeages pour ces types de barres précis dans ces ateliers.

Contrairement à Fiko, à Doundé, la durée de préformage de M4S et de M4S* est égale, quelle que soit la teneur en carbone des barres de fer : il n'existe pas de relation entre teneur en carbone et durée du travail. 


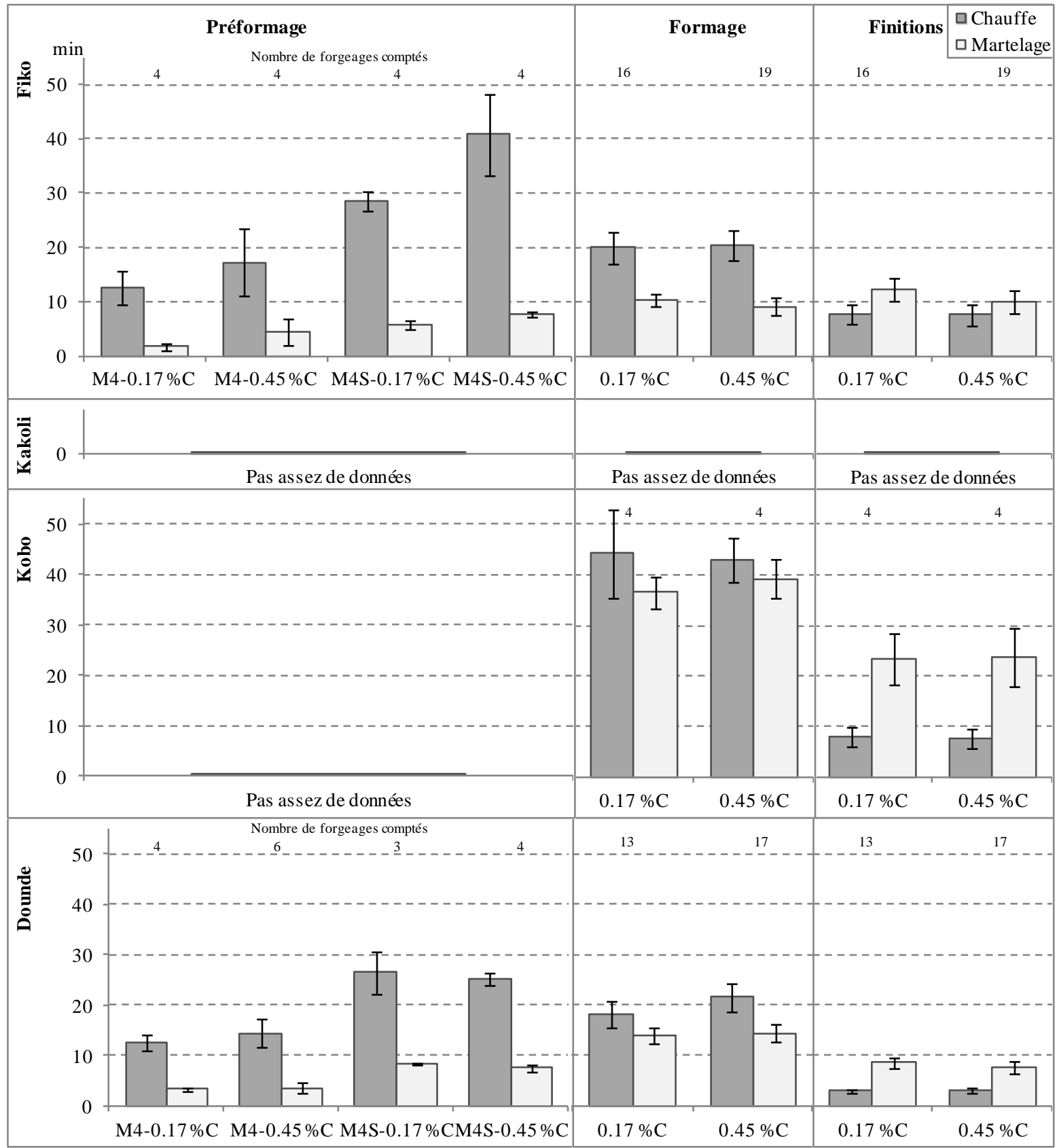

Figure 43 - Teneur en carbone du fer et durée du travail. Valeurs moyennes et écart-type des durées de chauffe et de martelage dans chacun des ateliers. Seuls les forgeages sans incidents ont été pris en compte pour une meilleure comparaison.

\section{Influence au formage et aux finitions}

$\mathrm{Au}$ formage et aux finitions, ni la catégorie ni le type de barre n'ont d'influence sur la durée du travail. Pour cette raison nous avons choisi de comparer indifféremment toutes les barres à $0.17 \% \mathrm{C}$ et toutes celles à $0.45 \% \mathrm{C}$.

A Fiko, Kobo et Doundé, il n'existe pas de différence de durée de travail entre des fers mi-doux à $0.17 \% \mathrm{C}$ et des fers mi-durs à $0.45 \% \mathrm{C}$. Le nombre restreint d'observations à Kakoli ne permet pas de se prononcer.
Il est possible de penser que le fer étant toujours très chaud pour être déformé, l'effet de la dureté du métal ne se fasse pas ressentir au cours du formage.

Cependant, l'étude du métal sur les lames de houe (3.4.4) montre que l'outil a la même teneur en carbone que le fer utilisé. Dans ce cas le forgeron doit ressentir, surtout dans le cas d'un travail à froid, une différence de dureté entre des lames à $0.17 \% \mathrm{C}$ et des lames à $0.45 \% \mathrm{C}$.

\section{Synthèse du 3.3.4}

12- Dans un des deux cas étudiés pour lequel il y a assez de données (Fiko), la composition du fer 
induit une variation de la durée du préformage : un acier mi-dur $(0.45 \% \mathrm{C})$ est travaillé plus longuement qu'un acier mi-doux $(0.17 \% \mathrm{C})$.

13- Au formage et aux finitions la durée du travail est toujours indépendante de la teneur en carbone des barres de fer utilisées.

\subsubsection{Synthèse des différents paramètres influençant la durée du travail}

Dans les quatre ateliers étudiés, la durée globale de fabrication d'une lame de houe varie entre $1 \mathrm{~h}$ et $2 \mathrm{~h} 40$. Cette durée du travail est influencée par différents paramètres (Tableau 19):

- intrinsèques à la matière première : catégorie de barre (simple/double), type de barre (forme/dimension), teneur en carbone (mou/dur) ;

humains : incidents, qualification des frappeurs et des souffleurs mais également humeur du forgeron, motivation du moment ;

- autres : période concernée par les forgeages (notamment les périodes de labour).

Tout d'abord, nous avons pu mettre en évidence qu'à Kobo, les durées de travail (chauffe/martelage) sont toujours les plus longues : la soufflerie est activée par des apprentis peu dynamiques et le martelage est assuré par ces mêmes personnes peu expérimentées.
Ensuite, le préformage est une étape clé de la chaîne opératoire, intimement liée aux paramètres intrinsèques de la matière première. Le temps de travail augmente lors :

- de forgeages de barres doubles,

- de forgeages avec incident,

- de forgeages de barres qui ont une forme générale trop éloignée de la préforme,

- de forgeage de barres de fer mi-dur à $0.45 \% \mathrm{C}$ (à Fiko seulement),

- de forgeages avec une équipe peu qualifiée (à Kobo seulement).

- de forgeages dont la durée de chauffe n'est pas au moins trois fois celle du martelage.

$\mathrm{Au}$ cours du formage, la chauffe est rattrapée par le martelage. Les ateliers du plateau ont une durée de chauffe 2 fois plus importante que celle du martelage, mais seulement 1.2 fois pour les ateliers de la plaine. Le temps de travail augmente lors :

- de forgeages avec soudure (Kobo seulement),

- de forgeages avec incident,

- de forgeages avec une équipe peu qualifiée (à Kobo seulement).

Enfin, les finitions sont une étape largement dominée par le travail de martelage, effectué trois fois plus longtemps que la chauffe dans les ateliers de la plaine, mais seulement 1.5 fois pour les ateliers du plateau. 


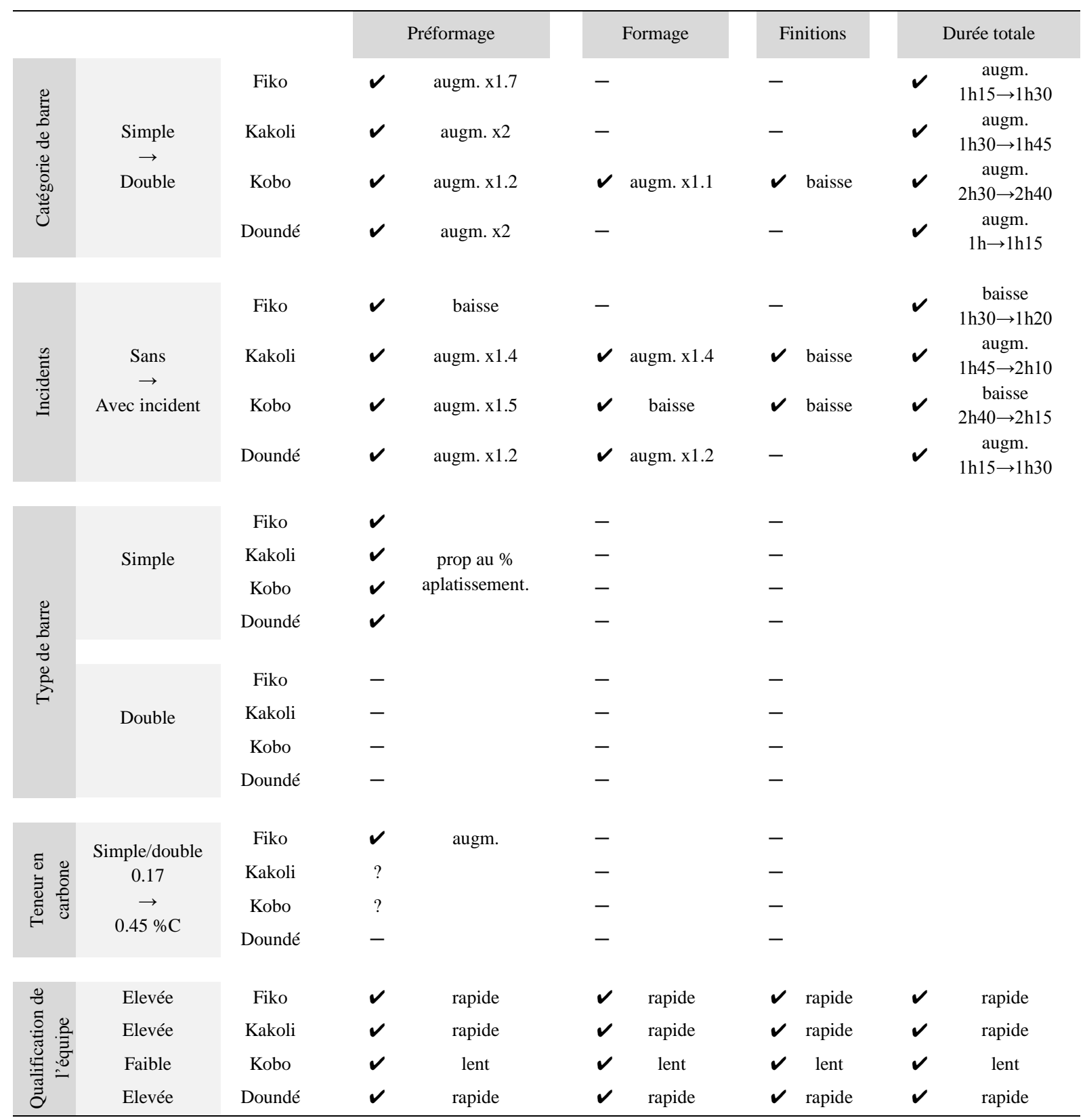

Tableau 19 - Récapitulatif des paramètres qui influencent les durées de préformage/formage/finitions dans chaque atelier de forge.

$\boldsymbol{V}=$ influence certaine $\quad ?=$ pas assez de données $\quad-=$ pas d'influence 
Le chapitre 3.4 complète la description de la chaîne opératoire, en s'intéressant au produit obtenu, le seul type de produit que nous avons demandé aux forgerons : la lame de houe.

Existe-t-il des liens entre ses caractéristiques morphométriques en relation avec le lieu de forgeage plaine/plateau? Quelle est la conséquence des incidents (3.2.4) sur les produits obtenus ? Quel est le lien entre la nature du fer de départ (teneur en carbone) et la nature du produit ?

\subsubsection{Présentation de la $d a b a$}

La houe est désignée par le terme daba en langue Manding (dabo en Malinké, inadjélé en langue Tomo). La daba est constituée d'une lame et d'un manche en bois, fixés entre eux par un emmanchement à soie ou à douille (Figure 44)

La daba est l'outil traditionnel du paysan africain, caractérisé par sa polyvalence d'emploi : elle regroupe en fait un très grand nombre de modèles, communs et très diffusés, mais aussi des modèles locaux qui conservent souvent leur propre nom (Seignobos 2000).
Au sortir de l'atelier, les lames produites sont généralement plates, elles sont vendues en l'état et ne sont pas utilisables directement. L'acheteur doit alors amener de nouveau la lame à la forge afin qu'elle soit recourbée, le dos de la lame (nervure saillante) constituant la partie concave de l'outil. Pour d'autres commandes, le forgeron peut directement recourber la lame. Les forgerons se chargent également d'emmancher la lame. Au cours de nos enquêtes, seul l'emmanchement à soie a été observé (Figure 44); cependant d'autres régions présentent un emmanchement à douille (communication E.Huysecom).

Le cultivateur, courbé, soulève la daba audessus de sa tête et ramène la terre entre ses jambes, de manière à aérer le sol en le retournant (sarclage). La daba s'use plus ou moins vite, en fonction de la nature du sol travaillé et de la fréquence d'utilisation. A mi-usure, elle peut être reprise pour d'autres menus travaux dans les champs. Une fois devenu un talon de fer (Figure 44), elle est soit donnée comme jouet aux enfants, soit assemblée de nouveau à la forge avec d'autres talons afin de former un nouvel outil (fer de recyclage).

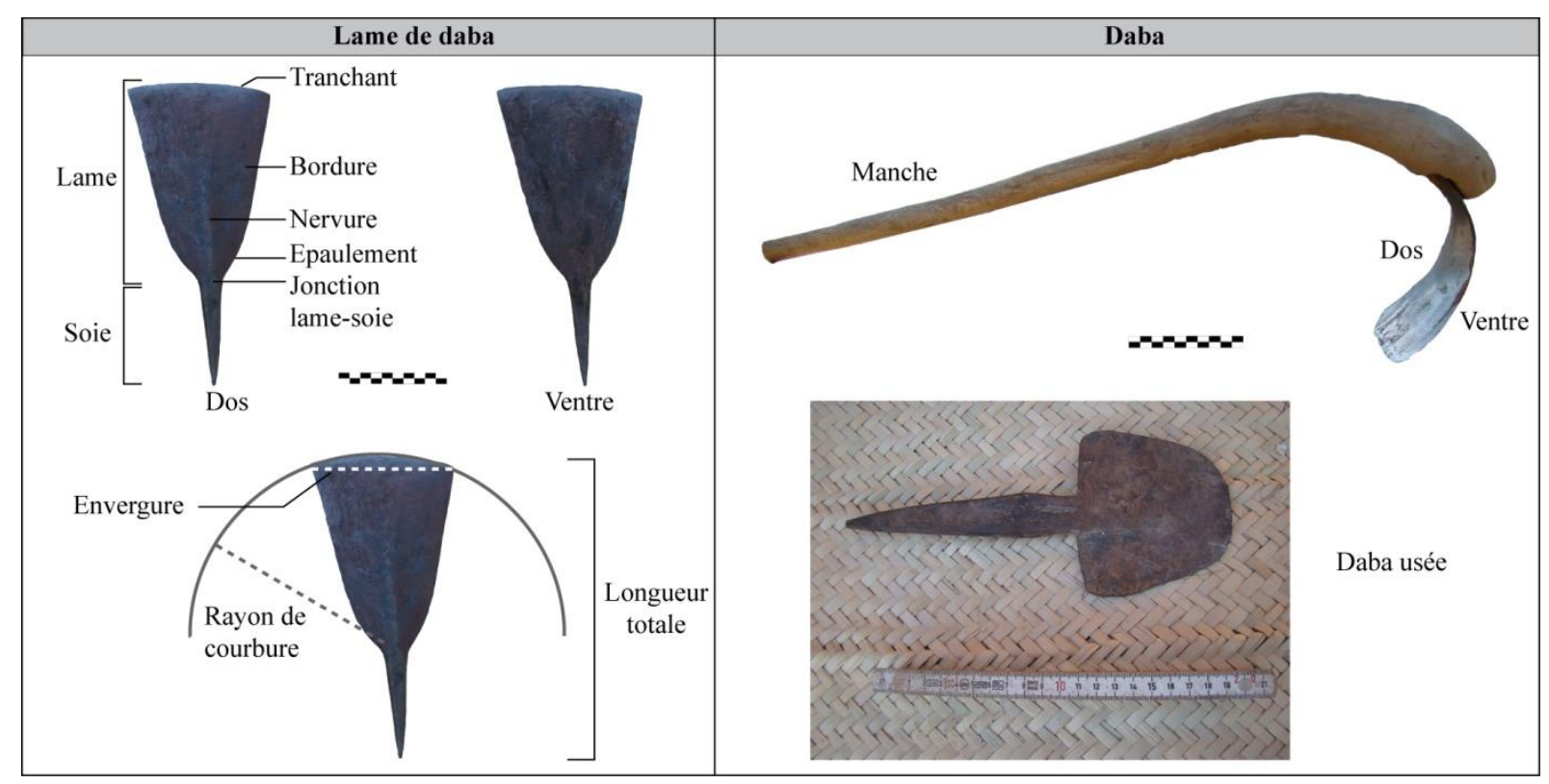

Figure 44 - Présentation de la daba. 
Les forges fonctionnent toute l'année mais la production d'outils aratoires est une activité saisonnière. De tous les ateliers visités, ceux de Fiko et Doundé sont ceux qui produisent le plus de lames de houe.

Ces outils sont très fréquemment fabriqués et ils représentent une valeur économique importante pour les forgerons. Ils sont fabriqués avant l'hivernage, à partir de morceaux de fers prédécoupés provenant de jantes de camions ou traverses de chemin de fer. Chaque morceau est acheté environ 2000 CFA (Fiko) - 1500 CFA (Doundé) et la lame de houe est revendue à 4000 CFA (Fiko) à Fiko - 3000 CFA (Doundé). Ces outils sont vendus soit directement au village, soit aux marchés alentours, soit troqués contre des céréales (mil, sorgho) ou autres biens.

Ces outils s'usent et il est nécessaire de les remplacer régulièrement. Sekou Traoré, cultivateur à Fiko, commande cinq houes et cinq pioches par année pour lui et sa famille pour cultiver ses deux champs de mil ( $\approx 4$ ha). Environ deux fois par an, il revient à la forge pour affuter et limer la lame de houe. De plus, il doit remplacer tous ses outils tous les deux ans (communication orale).

\subsubsection{Variabilités morphométriques}

Ce sont 126 daba qui ont été produites dans les quatre ateliers visités. La majorité a été redistribuée aux villageois $(n=87)$, certaines ont été échantillonnées en vue d'une investigation au laboratoire ( $\mathrm{n}=22$; seules 16 ont été étudiées), des pièces entières sont conservées dans nos réserves $(n=10)$, certaines ont servi de matière première (R1D) pour les forgeages F035 et F036 (n=4 ; D031 et D032, D033 et D034) et d'autres ont été perdues dans les transports entre l'Europe et l'Afrique ( $\mathrm{n}=3$; D024, D025 et D055).

Systématiquement les dimensions (envergurerayon de courbure-longueur de la lame), poids et les défauts observés ont été relevés. Des sections polies épaisses ont été préparées en vue d'une investigation métallographique. Toutes ces données vont être croisées afin de mieux comprendre de quels paramètres de la forge elles dépendent.

\section{Familles de daba}

Les lames peuvent être immédiatement classées en deux familles au regard de la forme des épaulements, de la couleur du fer et de l'utilisation de la lime. Les lames du plateau sont caractérisées par une couleur brune dominante (hématite) et un épaulement très marqué. Celles de la plaine sont grises (magnétite), aux épaulements droits et elles comportent des traces de lime.

\section{Poids et longueur}

Les lames de houes pèsent entre $280 \mathrm{~g}$ et $1450 \mathrm{~g}$, selon le poids de la matière première utilisée. Une lame provenant d'une barre simple est plus lourde/plus longue qu'une lame provenant de barres doubles, elle-même plus lourde/plus longue qu'une lame provenant d'un forgeage avec incident, quel que soit l'atelier considéré (Figure 45). Le poids et la longueur sont en fait conditionnés par la perte de fer qui a lieu durant le travail des forgerons (3.5).

Les lames de Fiko et Kobo sont en général plus longues que celles de Kakoli et Doundé : c'est le style de chaque atelier. A Kobo et Doundé elles sont plus lourdes que celles de Fiko et Kakoli.

\section{Rayon de courbure et envergure}

Une lame provenant d'une barre simple est plus large qu'une lame provenant de barres doubles,

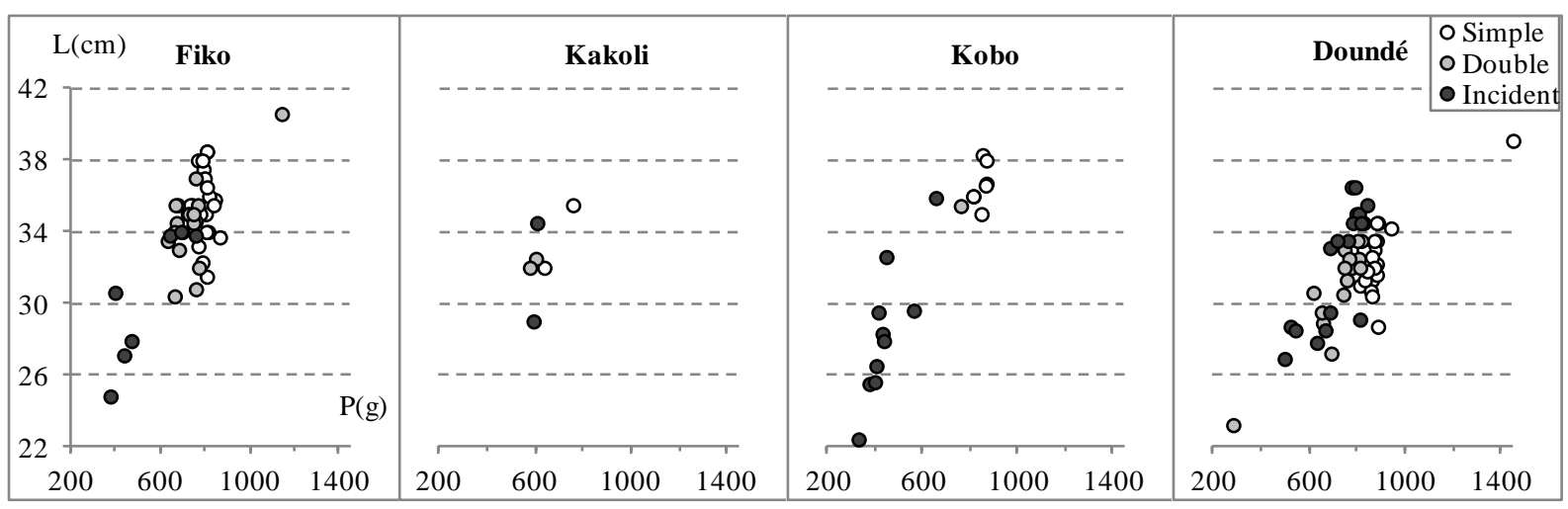

Figure 45 - Relation entre poids (P)/longueur (L) et catégorie de barre/incident. 


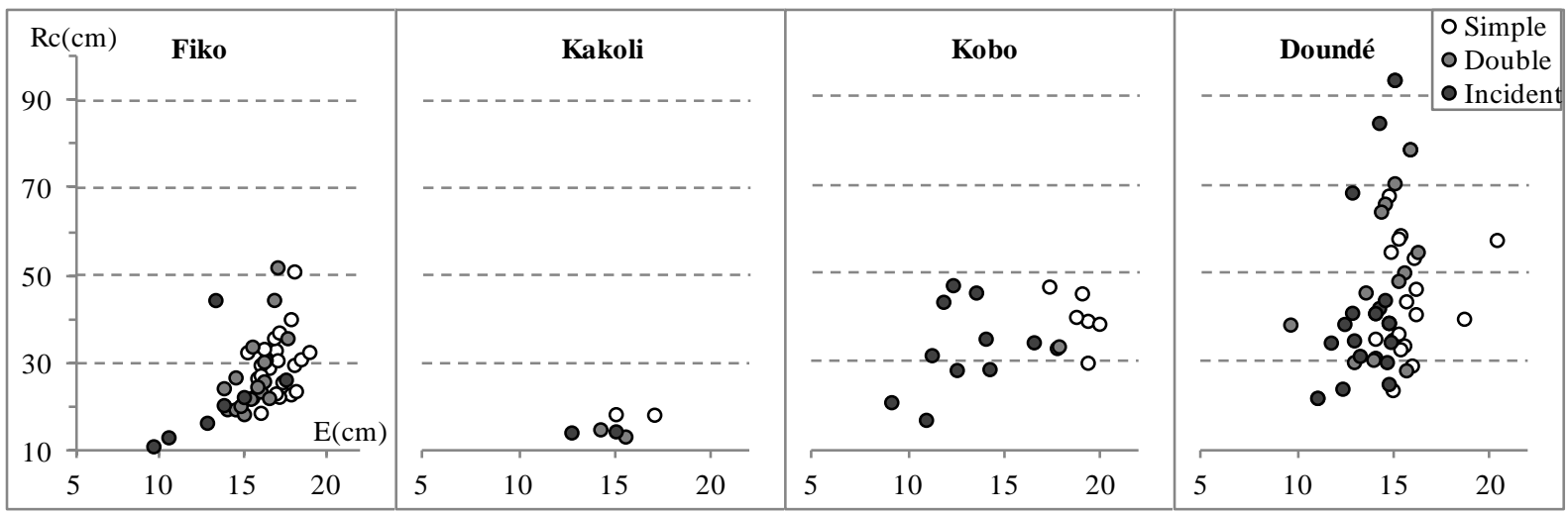

Figure 46 - Relation entre envergure (E)/rayon de courbure (Rc) et catégorie de barre/incident.

elle-même plus large qu'une lame provenant d'un forgeage avec incident, quel que soit l'atelier considéré (Figure 46). Les lames des ateliers Mombo sont plus courbées que les lames des ateliers Tomo. Kobo possède les lames les plus larges.

Il existe également un classement fonctionnel de ces lames, le degré de courbure étant lié au type d'emmanchement et à la fonction de l'outil : les houes de labour, les houes pour l'entretien des plants, pour désherber, pour les champs d'arachide des femmes etc (communication E.Huysecom).

\section{Synthèse du 3.4.2}

Les caractéristiques morphométriques des lames sont résumées dans le tableau 20. La longueur, le poids et l'envergure découlent des barres de fer employées (simple/double) et des compétences du forgeron (incident) ; le rayon de courbure dépend du style dans chaque atelier.

\begin{tabular}{|c|c|c|c|c|}
\hline & \multicolumn{2}{|c|}{ Type Mombo } & \multicolumn{2}{|c|}{ Type Tomo } \\
\hline Nombre & \multicolumn{2}{|c|}{55} & \multicolumn{2}{|c|}{71} \\
\hline Epaulements & \multirow{2}{*}{\multicolumn{2}{|c|}{$\begin{array}{c}\text { marqués } \\
\text { gris }(10-50 \%) \\
\text { brun }(90-50 \%)\end{array}$}} & \multirow{3}{*}{\multicolumn{2}{|c|}{$\begin{array}{c}\text { droits } \\
\text { gris }(90-50 \%) \\
\text { brun }(10-50 \%) \\
\text { oui }\end{array}$}} \\
\hline Couleur & & & & \\
\hline Lime & \multicolumn{2}{|c|}{ non } & & \\
\hline & Fiko & Kako & Kobo & Doundé \\
\hline Longueur & $\mathrm{XXX}$ & $\mathrm{x}$ & $\mathrm{xx}$ & $\mathrm{x}$ \\
\hline Poids & $\mathrm{x}$ & $\mathrm{x}$ & $\mathrm{xxx}$ & $\mathrm{xxx}$ \\
\hline Ray. de courbure & $\mathrm{xx}$ & $\mathrm{x}$ & $\mathrm{xx}$ & $\mathrm{xxx}$ \\
\hline Envergure & $\mathrm{X}$ & $\mathrm{x}$ & $\mathrm{xxx}$ & $\mathrm{XX}$ \\
\hline Exemple & & & & \\
\hline
\end{tabular}

Tableau 20 - Caractéristiques morphométriques des deux familles de daba. xxx : beaucoup ; $\mathrm{xx}$ : moyen ; $\mathrm{x}$ : peu

\subsubsection{Les défauts dans le métal}

Les 126 lames fabriquées montrent 199 défauts, une lame pouvant en présenter plusieurs. Les défauts dans le fer sont décrits dans les manuels de forgerons (Lagardelle 1926: 35) ou traités industriels du $19^{\text {ème }}$ siècle (Malapeyre 1843 : 4-5).

\section{Types de défauts}

Il est possible de distinguer cinq types de défauts, résultant de problèmes de soudure, de martelage ou de chauffe (Figure 47).

- Les pailles sont de fines écailles de fer qui résultent d'une soudure mal faite, parfois retirées à l'aide d'une pince.

- Les doublures sont des soudures mal faites dues à la présence de scories ou d'oxydes de fer sur la surface à souder, ou si les fers à réunir n'ont pas la même température.

- Les criques sont des fentes positionnées sur les arêtes du fer, qui résultent d'un manque de souplesse du fer pendant le martelage.

- Les travers sont des fentes placées transversalement dans le fer, de même origine que les criques.

- La lacune est un défaut de matière, résultant d'une surchauffe du métal. 


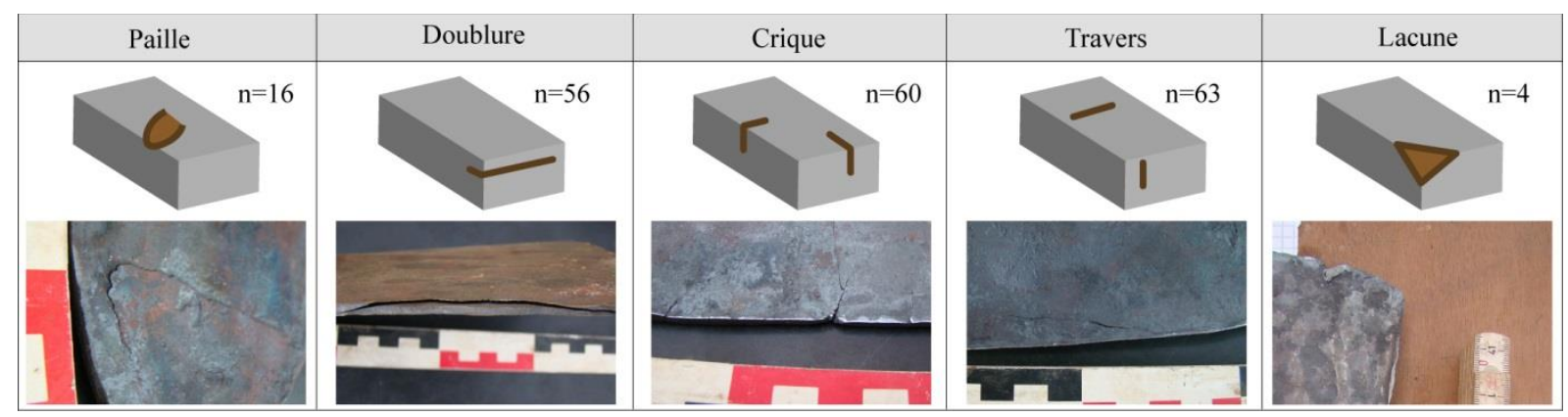

Figure 47 - Récapitulatif des cinq types de défauts répertoriés sur les lames de houe.

\section{Fréquence d'apparition}

Les défauts les plus fréquents sont les doublures, criques et travers (Figure 48). Les pailles, peu nombreuses sont tout de même rapportées sur les lames dans tous les ateliers. Des lacunes, anecdotiques mais témoins d'une chauffe intense, sont présentes à Kakoli.

On observe de grandes différences d'un atelier à un autre. Paradoxalement, ce sont dans les ateliers qui fabriquent le plus de lames de houe à l'année, que le plus de défauts sont recensés.

Les lames de l'atelier de Fiko et Doundé comportent beaucoup de criques, doublures et travers.

Au contraire, à Kakoli et à Kobo les forgerons semblent mieux éviter ces défauts.

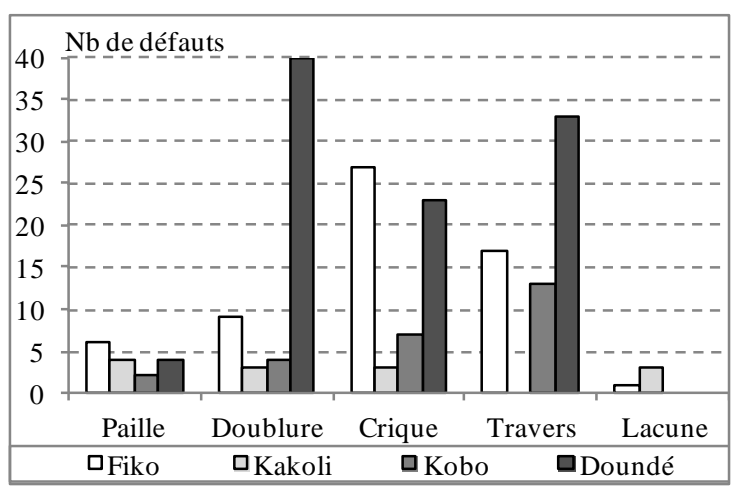

Figure 48 - Fréquence d'apparition des différents types de défauts des lames dans chaque atelier.

Ntot=199. Fiko : 60 ; Kakoli : 13 ; Kobo : 26 ; Doundé : 100.

\section{Influence des barres simples/doubles}

Les forgeages d'une barre simple donnent des lames sans ou avec peu de défauts. Au contraire, les forgeages de barres doubles engendrent souvent plusieurs défauts par lame, jusqu'à 12 à Doundé (Figure 49).

Enfin, Kobo et Doundé maîtrisent particulièrement les forgeages de barres simples (Tableau 16), puisque tous ces travaux ont permis de fabriquer des lames sans aucun défaut apparent.

\section{Influence de la teneur en carbone}

La figure 50 montre que la teneur en carbone des barres utilisées n'a aucune influence sur le nombre de défauts présents dans les lames forgées. Cependant, nous n'avons pas donné aux forgerons des barres de fer vraiment dures $(>0.5 \% \mathrm{C})$.

\section{Synthèse de 3.4.3}

- Cinq types de défauts ont été identifiés dans les dabas fabriquées.

- En général, ces défauts apparaissent lors de la fabrication de lames à partir de deux barres de fer (barres doubles).

- Le forgeron de Fiko maîtrise mieux les soudures que ceux de Doundé, car les lames de Fiko contiennent moins de défauts que celles de Doundé (jusqu'à 12 défauts).

- Enfin, il n'existe pas de relation entre le nombre de défauts dans une lame et la teneur en carbone du fer forgé : il n'y a pas plus de défauts sur des lames élaborées à partir de barres à $0.17 \% \mathrm{C}$ que sur des lames élaborées à partir de barres à $0.45 \% \mathrm{C}$. 


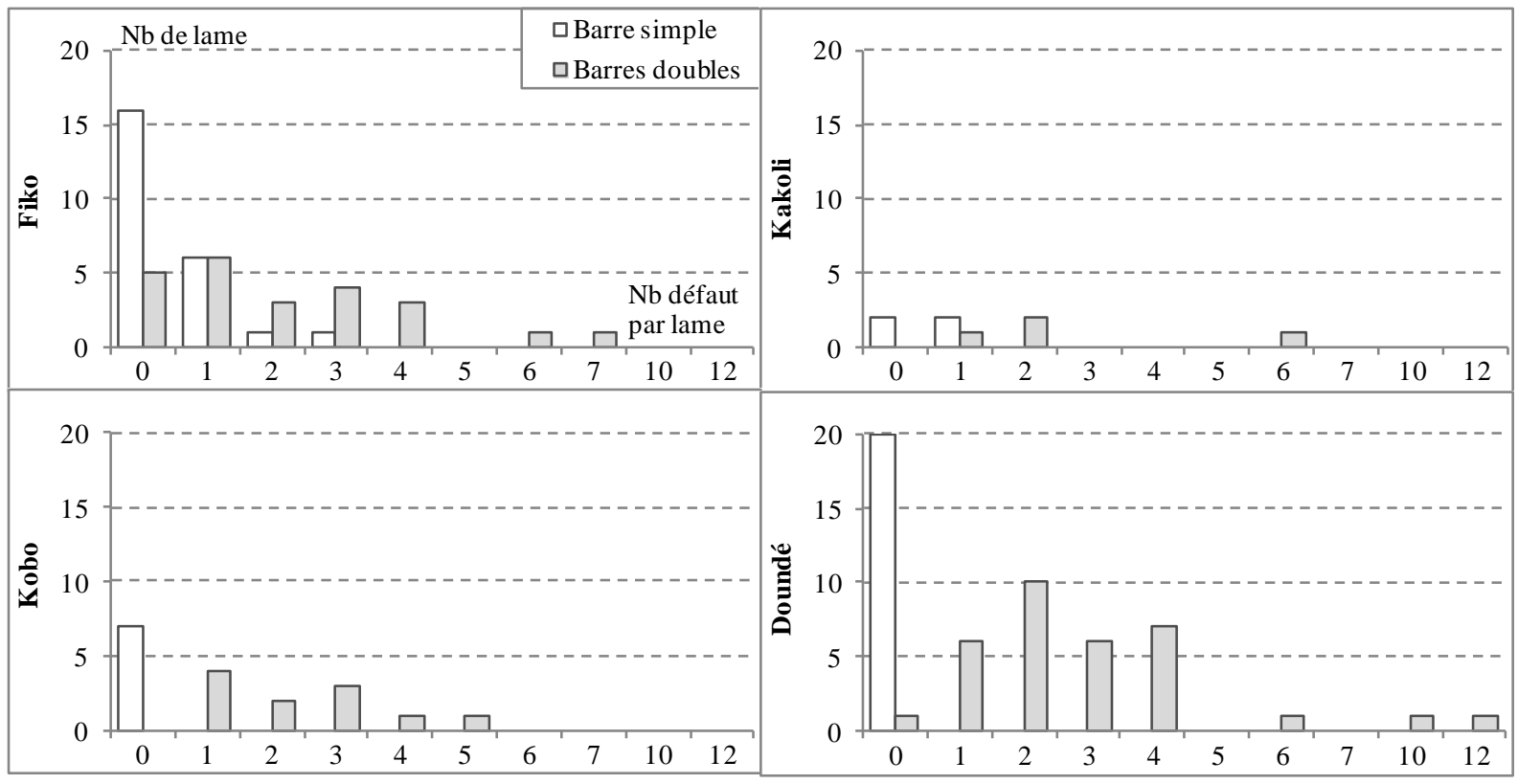

Figure 49 - Influence de la catégorie de barre sur le nombre de défauts par lame dans chaque atelier.
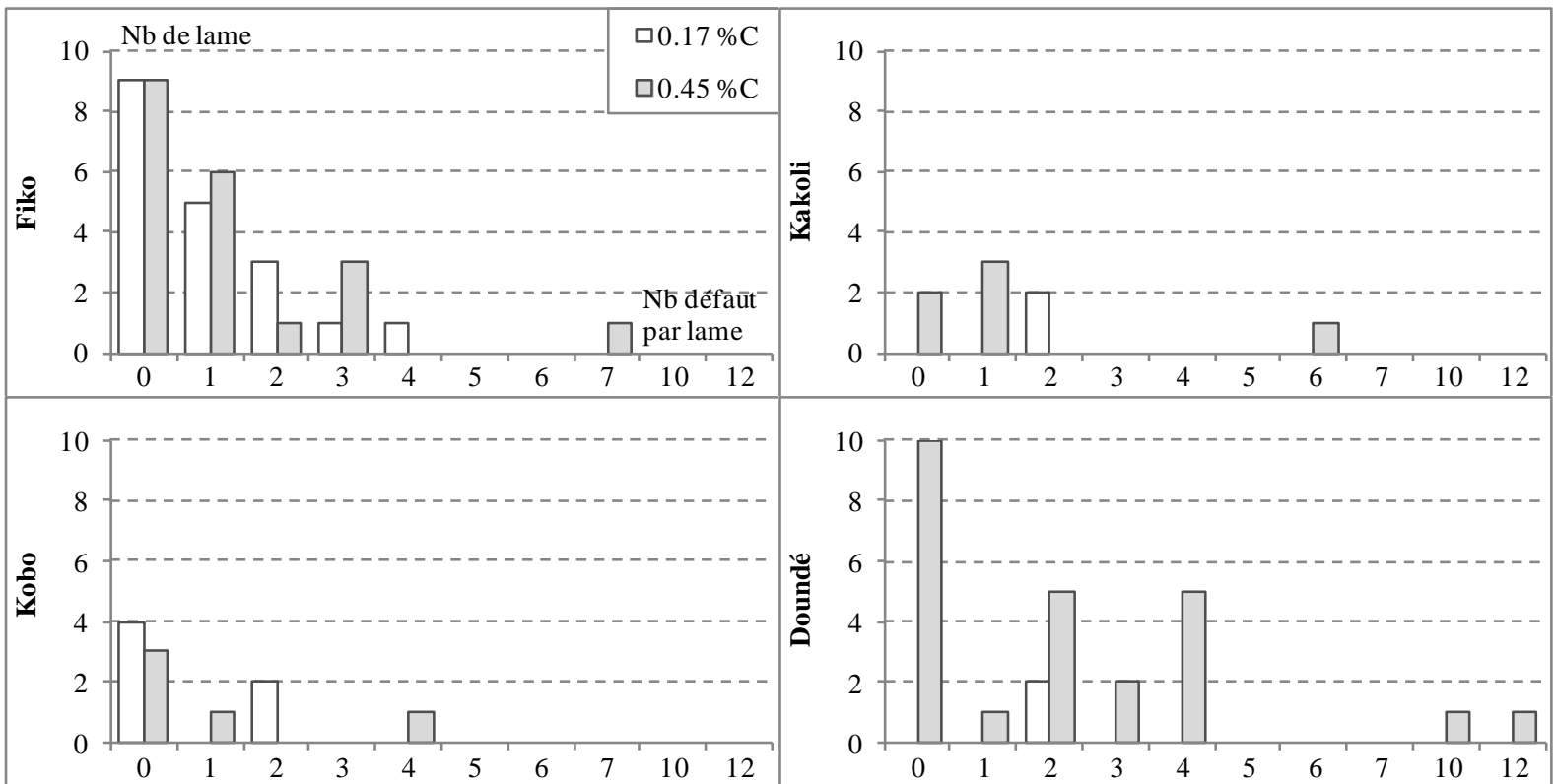

Figure 50 - Influence de la teneur en carbone des barres de fer sur le nombre de défauts par lame dans chaque atelier.

\subsubsection{Métallographie}

Dans chaque atelier, les forgerons ont fabriqué des lames de houe à partir de barres dont la teneur en carbone est connue. Ce sont 16 lames qui ont été choisies pour comprendre, dans chaque atelier, l'impact du forgeage d'une barre simple, de barres doubles ou de la teneur en carbone initiale sur la microstructure et la qualité du métal de la lame achevée (Figure 65).

\section{Lames à partir d'une barre simple}

La teneur en carbone de l'outil ne diffère pas beaucoup de la teneur en carbone du métal initial utilisé (Figure 51) : les barres à $0.17 \% \mathrm{C}$ donnent des lames à $0.1-0.2 \% \mathrm{C}$; les barres à $0.45 \% \mathrm{C}$ donnent des lames à 0.4-0.5\% ; les lames R1S sont à 0.1$0.2 \% \mathrm{C}$, donc nous supposons que leur teneur est proche de $0.1 \% \mathrm{C}$.

De plus, on observe une orientation des grains au niveau de la soie uniquement pour les lames faites 
à partir de barres à $0.17 \% \mathrm{C}$ : l'écrouissage fut la dernière action du forgeron.

Enfin, la microstructure est toujours homogène (taille des grains, carburation) sur toute la surface de la section polie. Seule la lame D012 (Doundé) montre une grande variation de la taille des grains entre le cœur et l'extrémité de la soie et une décarburation de surface sur le tranchant.

\section{Lames à partir de barres doubles}

Les lames ont une composition toujours proche de la composition du fer initial (Figure 52). Une exception : une zone du tranchant de D002 où l'on observe une interface de soudure dont la teneur en carbone est plus élevée (0.3-0.4\%C).

Toutes les micrographies révèlent la structure et la composition des interfaces de soudure. Il s'agit de zones de cristallisation des grains de fer, généralement bordées par une bande de fines inclusions vitreuses plus ou moins allongées dans le sens du martelage (D002, D035, D004). A l'interface, les grains de fer se révèlent souvent de plus grande taille que le métal alentour et il n'est pas rare de trouver des inclusions allongées d'oxydes de fer (D020, D035, D004).

Les barres R1D, du fait de nombreux replis qu'elles contiennent (Figure 30), révèlent de nombreuses interfaces de soudure.

Enfin, D020 qui avait été soudée latéralement montre une ligne de soudure verticale au niveau de la soie, de fait non visible sur le tranchant.

\section{Lames à partir de barres africaines}

Il faut noter que les barres africaines utilisées pour le forgeage des outils (10 barres A1D et 8 barres A2D) n'ont pas été décrites du point de vue de la métallographie avant leur emploi à la forge. Seules quelques-unes des barres restantes ont été étudiées postérieurement.

A priori les barres utilisées par les forgerons possèdent une microstructure et une composition similaire, c'est-à-dire jusqu'à $0.8 \% \mathrm{C}$ (Figure 29).

Les barres africaines ont dû être pliées de nombreuses fois lors de leur préformage afin d'obtenir un lopin de forme et taille adéquats. Leur microstructure montre une succession de replis dont la teneur en carbone varie entre $0.02 \% \mathrm{C}$ et $0.8 \% \mathrm{C}$, ainsi que de nombreuses inclusions de scorie, et d'oxydes de fer aplatis par le martelage (Figure 53).

Synthèse du chapitre 3.4

Cette courte étude métallographique des $d a b a$ montre plusieurs choses :

- Les lames ont la même teneur en carbone que les barres qui ont servi à les fabriquer.

- Les lames faites à partir de barres doubles montrent des interfaces de soudure horizontales pour les barres modernes ou une succession de replis pour les barres africaines.

- Quelques soient les techniques de soudure employées dans les ateliers (Figure 33, Figure 34), toutes les soudures observées sont propres, contenant très peu d'inclusions à l'interface de soudure.

- Les inclusions dans les lames sont souvent aplaties par le martelage ; elles n'ont pas été étudiées de point de vue chimique.

Il ne s'agit pas d'une étude exhaustive sur les lames de houe puisque notre intérêt est ailleurs (scories). Ce travail demande à être complété, pour les barres africaines en particulier, du point de vue de la morphologie, métallographie et chimie. Par la suite, une analyse détaillée des inclusions pourrait apporter de précieuses informations concernant la formation de particules de seconde phase, spécifiques aux opérations de forgeage. Une description de la variation de la taille des grains avant et après forgeage pourrait également être réalisée.

Toutefois, de cette étude, il ressort que les caractéristiques morphométriques des daba sont influencées par des paramètres de la matière première, mais aussi des paramètres culturels propres à chaque groupe ethnique.

Les lames de Fiko comportent le moins de défauts. Au contraire, celles de Doundé comportent plus de défauts du fait que les forgerons maitrisent moins bien les soudures.

$\mathrm{Au}$ final, quel que soit l'atelier, la microstructure des lames est toujours similaire pour un type de forgeage donné (barre simple/double/type de barre). De surcroit, les lames fabriquées avec ou sans défaut (à l'exception de la D020), sont toutes utilisables dans les champs. 


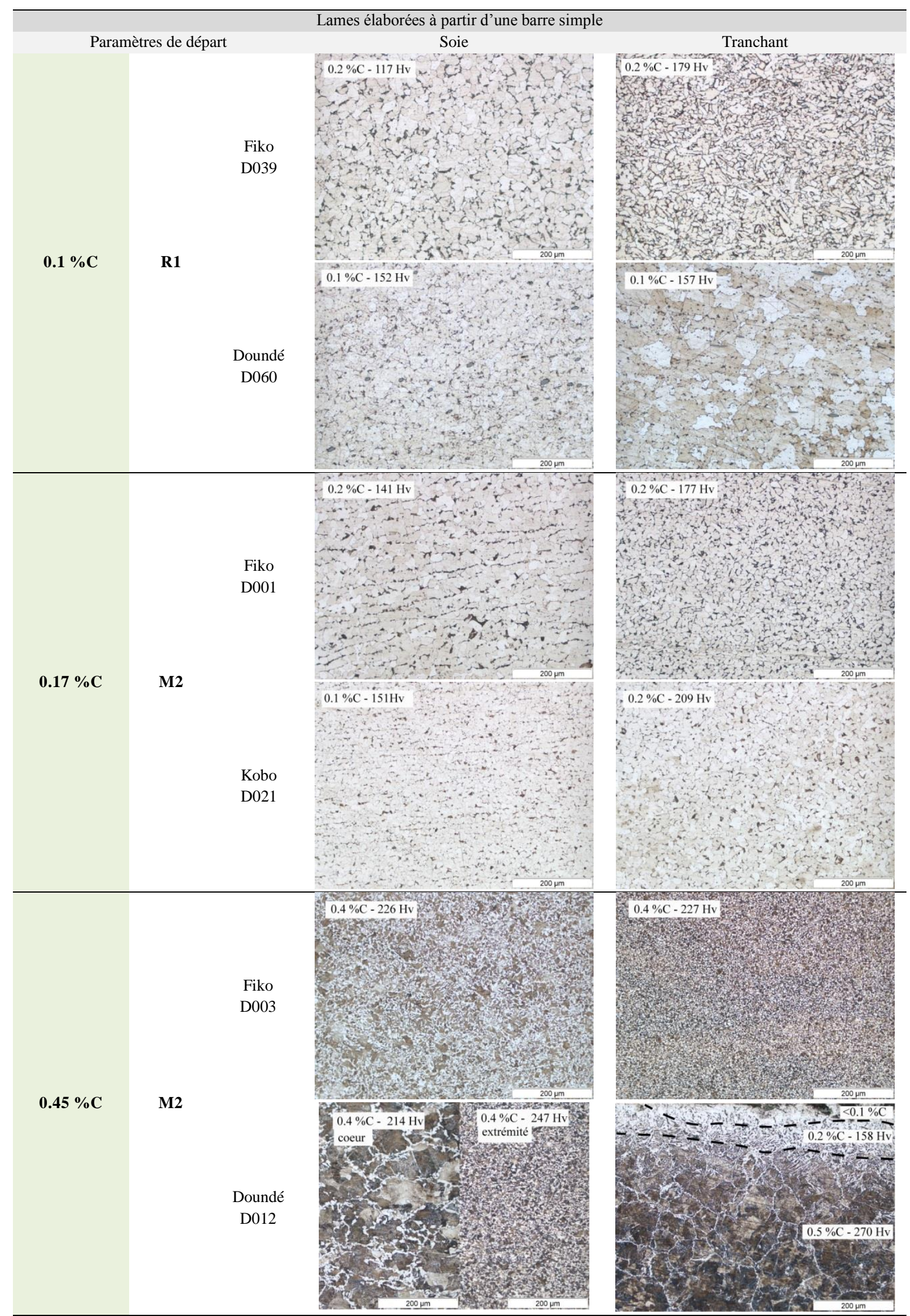

Figure 51 - Métallographie de lames élaborées à partir d'une barre simple.

Estimation de la teneur en carbone au vu de la proportion de ferrite et de perlite. Mesure de dureté (Hv). 


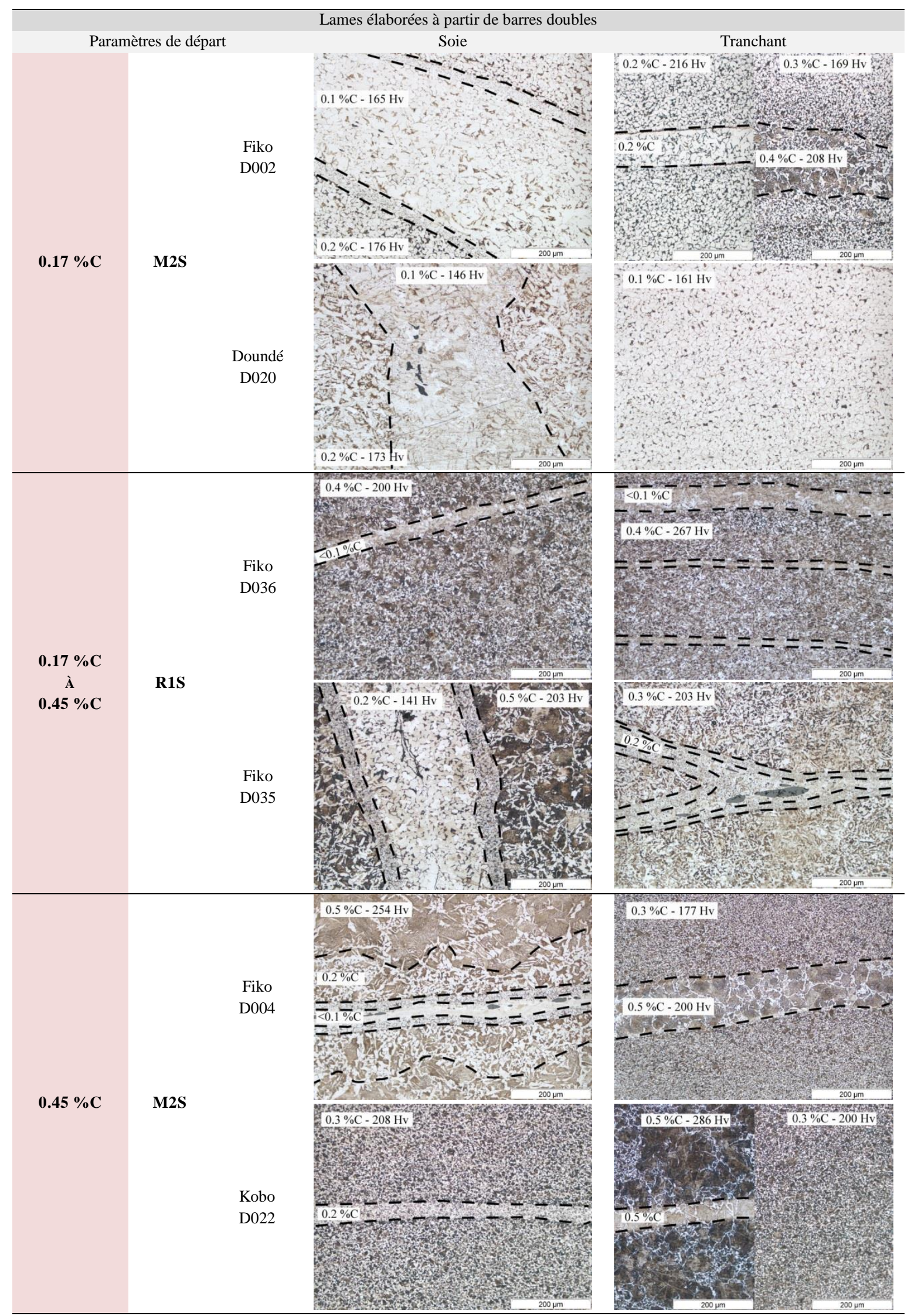

Figure 52 - Métallographie de lames élaborées à partir de barres doubles.

Estimation de la teneur en carbone au vu de la proportion de ferrite et de perlite. Mesure de dureté (Hv). 


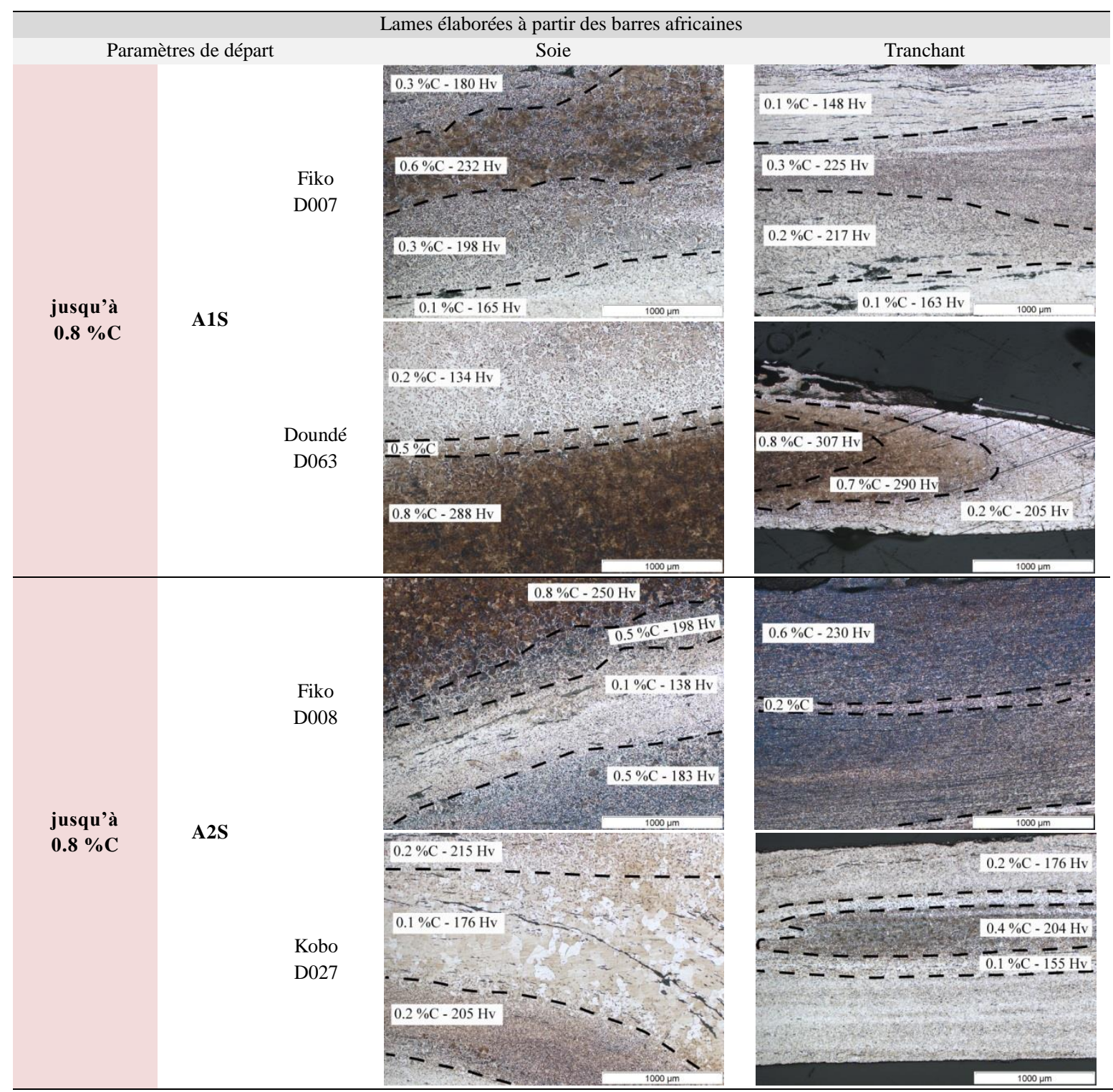

Figure 53 - Métallographie de lames élaborées à partir de barres africaines.

Estimation de la teneur en carbone au vu de la proportion de ferrite et de perlite. Mesure de dureté (Hv). 


\subsection{FORGEAGE ET PERTE EN FER}

Le chapitre 3.5 a pour but d'aider à évaluer la quantité de fer travaillée par le forgeron, à partir de l'étude des déchets retrouvés archéologiquement. Il s'agit de comprendre les procédés qui régissent la perte de matière ferreuse lors des forgeages d'une masse de métal dans un foyer et de mettre en évidence les paramètres des travaux de forge qui influent sur ces données de perte en fer.

Les données de perte en fer, liées à des observations nombreuses et répétitives de 126 travaux de fabrications de lames de houe, doivent permettre de les nuancer et de les interpréter de manière plus rigoureuse.

\subsubsection{Mesure de la perte en fer}

\section{Définition}

La perte en fer est la quantité de matière qui est perdue dans le foyer et sur l'enclume lors des travaux de forge. C'est une donnée facile à calculer : il suffit de soustraire le poids de l'outil forgé au poids de la matière première employée par le forgeron (Figure 54). Cette perte de fer se manifeste sous deux formes :

- des croûtes d'oxydes formées lors de l'oxydation du fer à chaud dans le foyer (chauffe) et des battitures sur l'enclume (martelage),

- des particules de fer à l'état de métal dans le foyer (chauffage excessif) et sur l'enclume (compactage inefficace, mauvais martelage).

L'oxydation à chaud est un phénomène physique qui est commandé par la surface exposée à l'oxydation, la température du milieu et par le temps d'oxydation (durée de chauffe) (Iordanova et al. 2000 : 160 ; Figure 76).

Plus la température est élevée et plus les oxydes de fer qui se détachent de la barre de fer sont épais, rendant la perte en fer importante (Figure 55).

Ainsi, cette oxydation est importante voire très importante au préformage et au formage lorsque le foyer atteint des températures élevées ; cependant les finitions ne génèrent que très peu d'oxydation.

\section{Perte en fer globale}

La figure 56 montre que le forgeage d'une barre simple engendre des pertes de matière, entre $8 \%$ et $37 \%$. Le forgeage d'une barre simple avec incident engendre une perte entre $16 \%$ et $34 \%$.

Le forgeage de barres doubles sans incident engendre une perte entre $18 \%$ et $47 \%$. Le forgeage de barres doubles avec présence d'incidents engendre une perte en fer entre $19 \%$ et $53 \%$.

Ainsi, les travaux de forge effectués à partir d'une seule barre de fer impliquent de faibles pertes, ceux effectués à partir de 2 barres impliquent des pertes plus importantes. Enfin, si des incidents surviennent, alors la perte de fer augmente encore davantage.

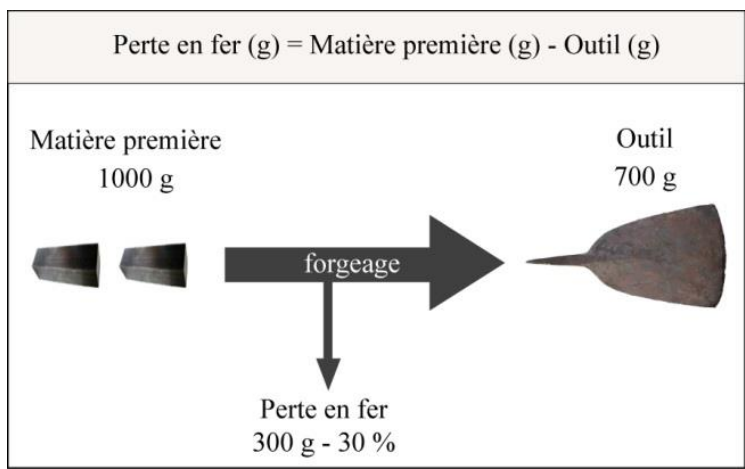

Figure 54 - Définition et schématisation de la perte en fer lors du forgeage d'un outil.

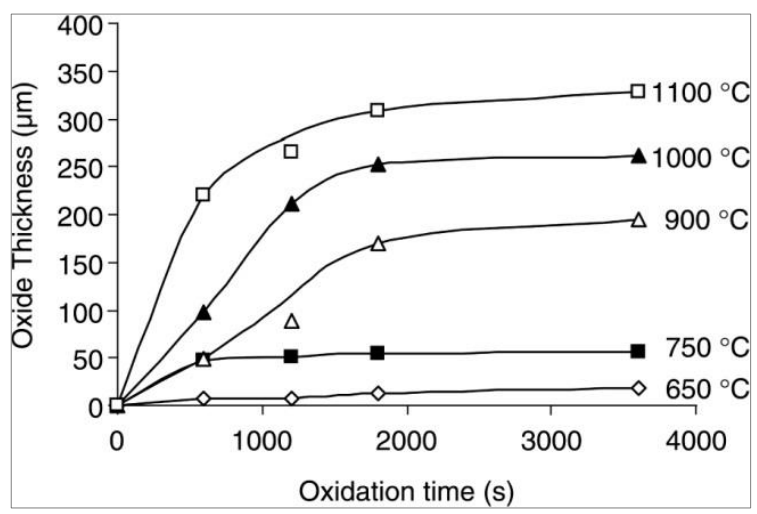

Figure 55 - Epaisseur des oxydes de fer en fonction du temps d'oxydation et de la température. D'après Birosca et al. 2004 : 236. 


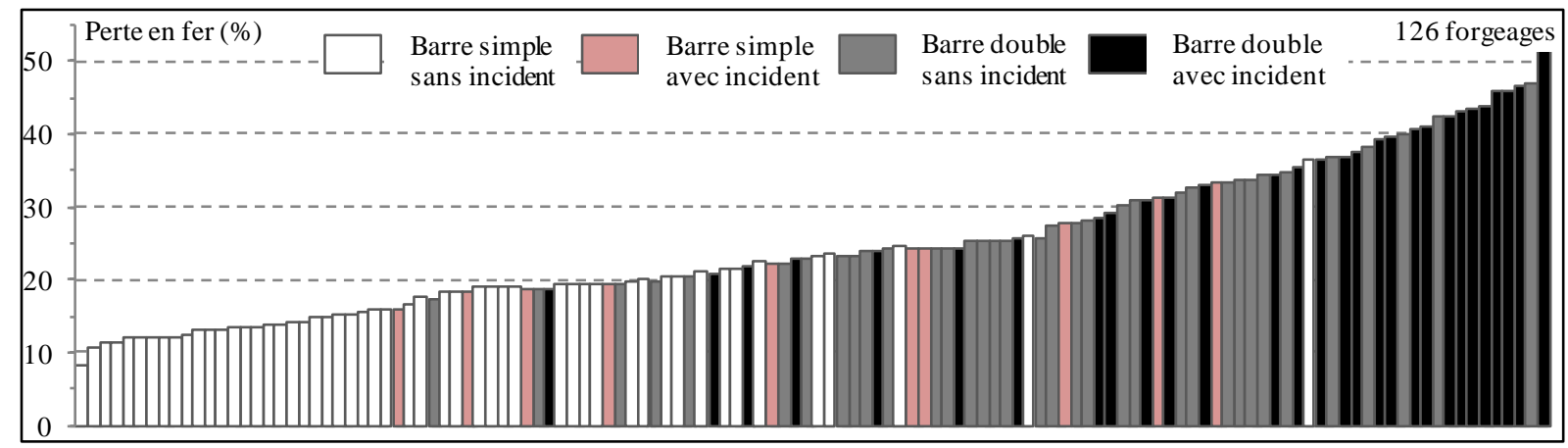

Figure 56 - Perte en fer (\%) pour les 126 forgeages. Histogramme classé selon les barres simples/doubles et avec/sans incidents Barre simple sans incident - moy : $17 \%$; Barre simple avec incident - moy : $24 \%$; Barre double sans incident - moy : $29 \%$; Barre double avec incident - moy : $35 \%$.

\subsubsection{Forgeage d'une barre de fer}

\section{Influence de la surface d'oxydation}

En théorie la perte en fer devrait être faible pour une barre de petite surface et importante pour une barre de grande surface, à température et durée de chauffe égales. La surface des barres est donnée à la figure 28. De plus, la figure 57 illustre la perte en fer des différentes barres simples. On observe trois paquets :

- R1S qui possède la surface la plus grande $\left(380 \mathrm{~cm}^{2}\right)$ génère étonnamment la plus petite perte de fer $(11 \%)$;

- M2S-M3S-M4S de surface resp. 264-186$160 \mathrm{~cm}^{2}$, ont une perte moyenne (17\%);

- M5S qui possède la surface la plus petite $\left(150 \mathrm{~cm}^{2}\right)$, implique en fait la perte la plus grande $(26 \%)$.

On obtient ici des résultats inverses à la théorie: on ne peut pas comparer directement surface de la barre et perte en fer car la température et la durée de chauffe varient au cours du forgeage, mais aussi d'une barre à l'autre, et d'un atelier à un autre.

Au moment du formage et des finitions, la durée de chauffe et la température du foyer dépendent de l'atelier dans lequel le forgeage est réalisé (Figure 41 ; Figure 59). Par contre, le taux de déformation (D) appliqué pour passer de la préforme au produit, est le même pour tous les ateliers car la surface et la section de la préforme sont toujours identiques.

Lors du forgeage d'une lame de houe à partir d'une seule barre de fer, la perte en fer produite au formage et aux finitions dépend donc seulement de l'atelier de forge.

Le préformage est quant à lui plus compliqué car les températures, durée de chauffe, déformation varient de façon importante, ce qui fait également varier la perte de fer (Figure 59).

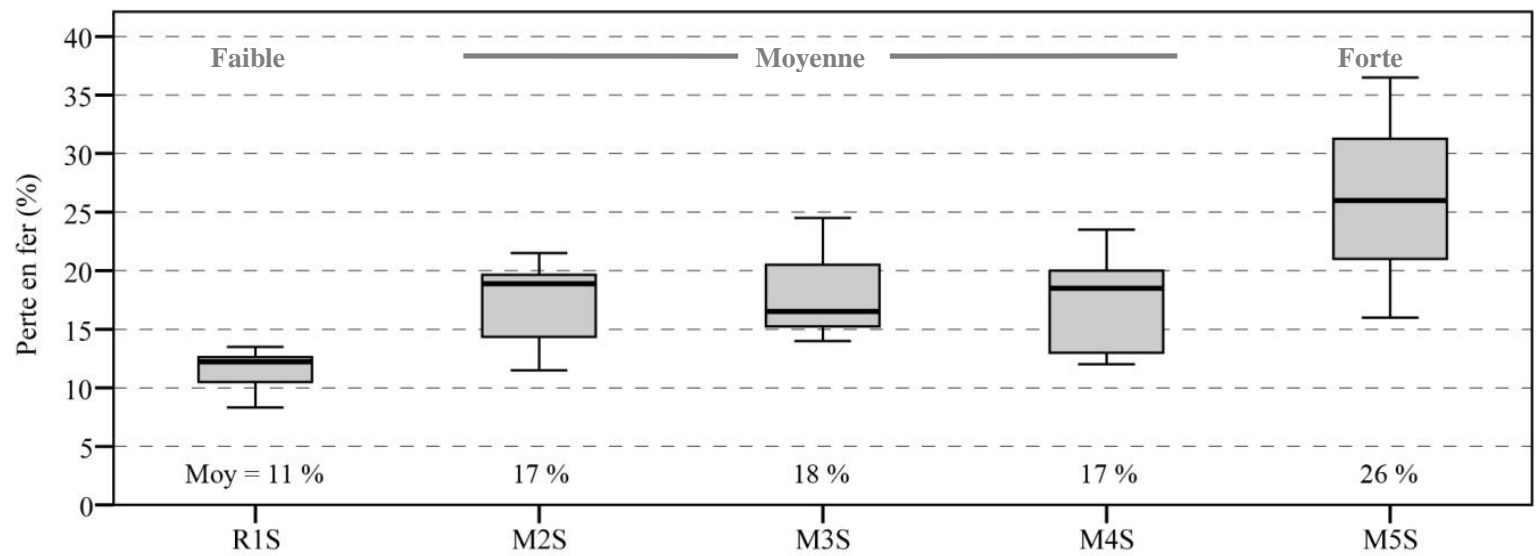

Figure 57 - Perte en fer (\%) pour les différentes sortes de barres simples (sans incident). Trait horizontale dans la boîte = médiane. $\mathrm{n}(\mathrm{R} 1 \mathrm{~S})=5$ forgeages $; \mathrm{n}(\mathrm{M} 2 \mathrm{~S})=16 ; \mathrm{n}(\mathrm{M} 3 \mathrm{~S})=3 ; \mathrm{n}(\mathrm{M} 4 \mathrm{~S})=21 ; \mathrm{n}(\mathrm{M} 5 \mathrm{~S})=3$. 


\section{Influence de la température}

La température du foyer varie d'un atelier à un autre (Tableau 21-haut). Les foyers de Kobo/Doundé sont tièdes, celui de Fiko est chaud et enfin Kakoli a le foyer le plus chaud. Ces différences entre ateliers proviennent du type/rendement de la soufflerie et du type/volume du foyer (Tableau 7).

- Doundé : le volume important du foyer associé au fort flux d'air rendent, au final, le foyer tiède.

- Kobo : le foyer est peu volumineux mais le flux d'air est très faible : le foyer est froid.

- Fiko : la zone active du foyer est restreinte et le flux d'air est très important, rendant le foyer chaud.

- Kakoli : la zone active du foyer très restreinte associée au flux d'air très important, rendent le foyer très chaud.

De plus, la perte en fer varie d'un atelier à un autre (Tableau 21-bas). Ces différences proviennent de la température du foyer : à Kobo/Doundé, le foyer froid/tiède génère une perte en fer moyenne/faible, à Fiko le foyer chaud génère une perte forte, à Kakoli le foyer très chaud génère une perte très forte.

\begin{tabular}{|c|c|c|c|c|c|}
\hline \multicolumn{5}{|c|}{ Température du foyer au préformage } & \multirow{2}{*}{$\begin{array}{c}\mathrm{T} \\
{ }^{\circ} \mathrm{C}\end{array}$} \\
\hline & Kobo & Doundé & Fiko & Kakoli & \\
\hline R1S & - & & \multirow[b]{2}{*}{ 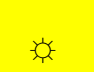 } & - & \multirow{2}{*}{$\begin{array}{l}- \\
+\end{array}$} \\
\hline $\mathrm{M} 2 \mathrm{~S}$ & & & & - & \\
\hline M3S & - & \multirow{3}{*}{ 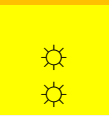 } & \multirow{3}{*}{ 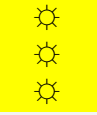 } & $-\not<-$ & \multirow{3}{*}{$\begin{array}{c}++ \\
++ \\
+++\end{array}$} \\
\hline M4S & & & & - & \\
\hline M5S & - & & & $=$ & \\
\hline \multirow[t]{3}{*}{ Foyer } & Froid & Tiède & Chaud & $\begin{array}{l}\text { Très } \\
\text { chaud }\end{array}$ & \multirow[b]{3}{*}{ Moy } \\
\hline & \multicolumn{4}{|c|}{ Perte en fer totale moyenne (\%) } & \\
\hline & Kobo & Doundé & Fiko & Kakoli & \\
\hline R1S & - & 9 & 13 & - & 11 \\
\hline $\mathrm{M} 2 \mathrm{~S}$ & 16 & 12 & 19 & - & 17 \\
\hline M3S & - & 14 & 17 & 25 & 18 \\
\hline M4S & 15 & 14 & 21 & - & 17 \\
\hline M5S & - & 16 & 26 & 36 & 26 \\
\hline Moy & 16 & 13 & 19 & 30 & \\
\hline Perte & Moyenne & Faible & Forte & Très forte & \\
\hline \multicolumn{6}{|c|}{$\begin{array}{c}\text { Tableau } 21 \text { - Lien température du foyer / perte en fer dans } \\
\text { chaque atelier et pour chaque type de barre simple } \\
\text { (sans incident). }\end{array}$} \\
\hline \multicolumn{6}{|c|}{$\begin{array}{l}\text { Couleur du fer au moment de commencer le martelage. Jaune = } \\
100{ }^{\circ} \mathrm{C} \text {, orange }=900^{\circ} \mathrm{C} \text {, l'étoile } \$ \text { - correspond à des } \\
\text { crépitements (température limite de chauffe). Température } \\
\text { moyenne par barre de fer évaluée de - à }+++.\end{array}$} \\
\hline
\end{tabular}

Perte en fer totale moyenne (\%) pour chaque barre simple dans chaque atelier (détail de Figure 57).

Enfin, dans chaque atelier le forgeron semble adapter la température du foyer à la barre qu'il est en train de forger (Tableau 21-haut) : les barres M5S sont toujours les plus chaudes $\left(1200^{\circ} \mathrm{C}\right.$ et crépitements) tandis que les barres R1S sont les moins chaudes $\left(1000{ }^{\circ} \mathrm{C}\right)$.

La température du foyer est proportionnelle à la section des barres et à la déformation de chaque barre (Figure 59). Toutefois, on remarque que M2S qui demande la plus grosse déformation n'est chauffée que faiblement : le refoulage est un acte technique qui ne s'effectue qu'à température modérée, pour ne pas « plier » la barre en deux.

Dans nos données, la perte en fer dépend de la température du foyer.

La perte en fer varie d'un atelier à un autre, chaque atelier possédant un foyer plus ou moins chaud : plus le foyer est chaud et plus la perte en fer est élevée. En fait, dans chaque atelier, la température $\mathrm{du}$ foyer résulte d'une combinaison entre le volume du foyer (zone active) et la puissance du flux d'air de la soufflerie. Ici, nous évaluons un aspect invisible et non quantitatif, mais important des travaux de forge.

$\mathrm{Au}$ préformage, la température est proportionnelle à la section des barres et à la quantité de déformation nécessaire pour fabriquer la préforme : plus la section et la déformation sont importantes et plus la température est élevée (sauf le cas de $\mathrm{M} 2 \mathrm{~S}$ ).

\section{Influence de la durée de chauffe}

La durée de chauffe varie d'un atelier à un autre. Précédemment, les analyses des forgeages ont montré que lors du préformage, la chauffe est la plus longue à Kobo (moy $23 \mathrm{~min}$ ) puis à Fiko (19 min), Kakoli (15 min) et Doundé (13 min) (Figure 39). La durée de chauffe est liée au rendement de la soufflerie: les souffleurs, enfants ou apprentis à Kobo, n'ont pas l'endurance et la force nécessaire pour produire un flux d'air permettant d'atteindre rapidement des températures de forgeage (Tableau 7).

\begin{tabular}{ccccc}
\hline & Kobo & Doundé & Fiko & Kakoli \\
Chauffe & +++ & - & ++ & + \\
Foyer & Froid & Tiède & Chaud & Très chaud \\
Perte & Moyenne & Faible & Forte & Très forte \\
\hline Tableau 22 & Lien entre durée de chauffe, température du foyer \\
et perte en fer dans chaque atelier (Tableau 21-bas).
\end{tabular}


De plus, la perte en fer est liée à la durée de chauffe des barres au cours du préformage (Tableau 22). On observe plusieurs manières de travailler :

- à Doundé, les forgerons conduisent un travail bref dans un foyer tiède, provoquant une perte de fer faible ;

- à Kobo le forgeron travaille très longuement dans un foyer froid, ce qui génère une perte de fer moyenne ;

- à Kakoli la chauffe est brève dans un foyer très chaud, générant une perte en fer très forte ;

- à Fiko enfin, la chauffe est longue dans un foyer chaud ce qui provoque une forte perte de fer.

Enfin, au moment du préformage, la durée de chauffe n'est pas identique pour toutes les barres de fer (Figure 58). Le forgeron chauffe R1S durant un temps court : 7 min en moyenne. La barre M3S est chauffée durant $10 \mathrm{~min}$, puis la barre M4S durant 15 min et la barre M5S durant $20 \mathrm{~min}$. Enfin, M2S demande la durée de chauffe la plus importante : 25 min en moyenne.

La durée de chauffe est proportionnelle à la section des barres de fer (Figure 40), sauf pour la barre M2S : ces résultats sont en accord avec les analyses des forgeages déjà effectuées (Figure 41 ; $0)$. En fait la durée de chauffe est liée à la déformation $\mathrm{D}$ que doit appliquer le forgeron pour obtenir la préforme.

Ceci explique que la barre M2S est chauffée le plus longuement : avec sa grande surface et sa petite section ( $\mathrm{D}$ élevé), le forgeron doit refouler la barre pour la raccourcir et l'épaissir. Ce travail est long en comparaison de l'allongement simple des barres M5S-M4S-M3S.

Dans nos données, la perte en fer dépend de la durée de chauffe au cours du préformage.

La perte en fer varie d'un atelier à un autre et résulte d'une combinaison entre durée de chauffe (plus ou moins longue) et température du foyer (plus ou moins chaud). En fait, dans chaque atelier la durée de chauffe est liée au rendement de la soufflerie (type de soufflerie/qualification du souffleur).

Au préformage, la durée de chauffe est liée à la déformation et à l'écart de surface entre la barre à forger et la préforme : plus la surface de la barre est proche de celle de la préforme et plus la durée de chauffe est courte.

\section{Influence de la quantité de déformation}

La quantité de déformation D ne varie pas d'un atelier à un autre puisque dans tous les ateliers le forgeron doit fabriquer une même préforme.

Cependant, la quantité de déformation à appliquer est différente pour chaque barre: le forgeron amène des barres qui ont des surfaces et sections différentes, en une préforme toujours identique (Figure 59). Pour cela il doit :

- augmenter fortement la surface en diminuant la section de M5S-M4S en une extrémité : $\mathrm{D}++$.

- augmenter légèrement la surface en resserrant M3S à une extrémité : D+.

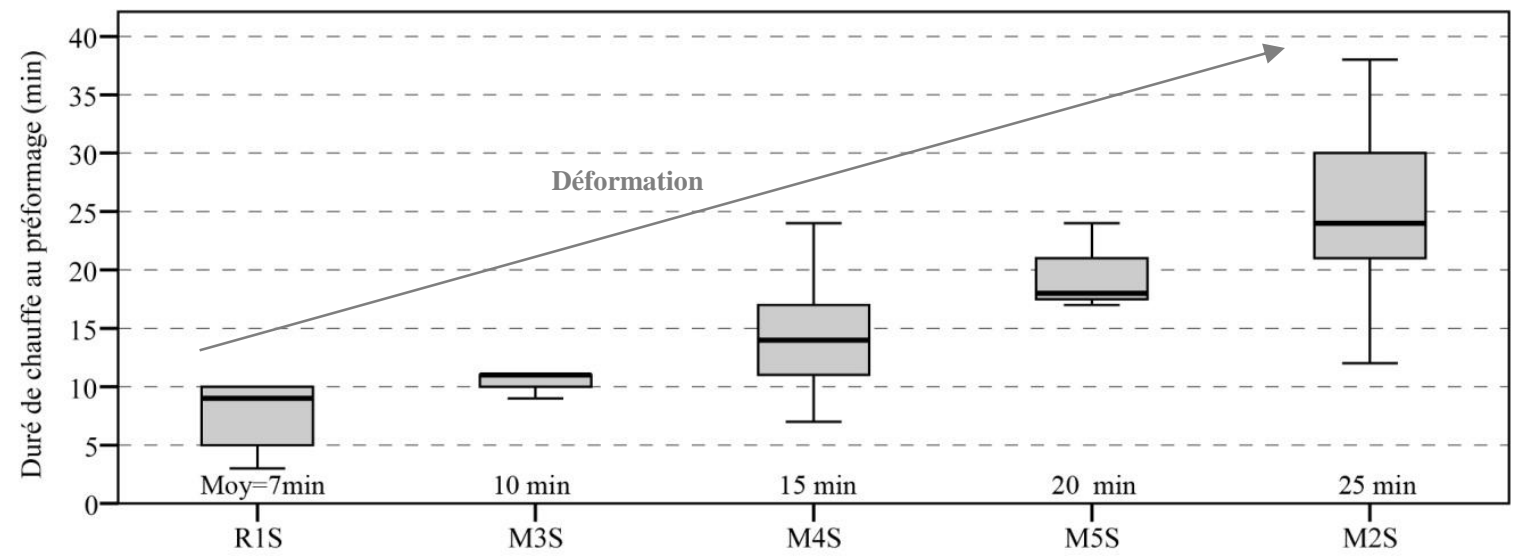

Figure 58 - Durée de chauffe des barres simples (sans incident). Trait horizontale dans la boîte $=$ médiane $\mathrm{n}(\mathrm{R} 1 \mathrm{~S})=5$ forgeages $; \mathrm{n}(\mathrm{M} 3 \mathrm{~S})=3 ; \mathrm{n}(\mathrm{M} 4 \mathrm{~S})=21 ; \mathrm{n}(\mathrm{M} 5 \mathrm{~S})=3 ; \mathrm{n}(\mathrm{M} 2 \mathrm{~S})=16$. 
- diminuer très fortement la surface en augmentant la section de M2S : D+++.

- $\quad$ (pas toujours) resserrer légèrement R1S à une extrémité et rendre la barre plus triangulaire : D -.

Comme déjà évoqué précédemment, la barre M2S a la déformation la plus importante car sa surface est trop grande et sa section trop petite par rapport à la préforme.

De plus, la figure 59 montre que certains paramètres sont dominants. Barre M2S : la température peu élevée du foyer domine le paramètre déformation : perte en fer moyenne. Barre M5S : la température très élevée du fer domine la durée de chauffe et la déformation.

\section{Influence de la teneur en carbone}

Les fers $(0.1 \% \mathrm{C})$ semblent avoir une faible perte en fer par rapport aux aciers mi-durs $(0.45 \% \mathrm{C})$ (Figure 60) : c'est uniquement parce que toutes les barres R1S sont à $0.1 \%$ C. Il n'y a en fait pas de lien entre la teneur en carbone des barres et leur perte en fer.

Dans nos données, la quantité de déformation est proportionnelle à l'écart de surface entre la barre à forger/la préforme et aussi à la durée de chauffe : plus la forme de la barre est proche de celle de la préforme (D petit) et plus la durée de chauffe est courte.

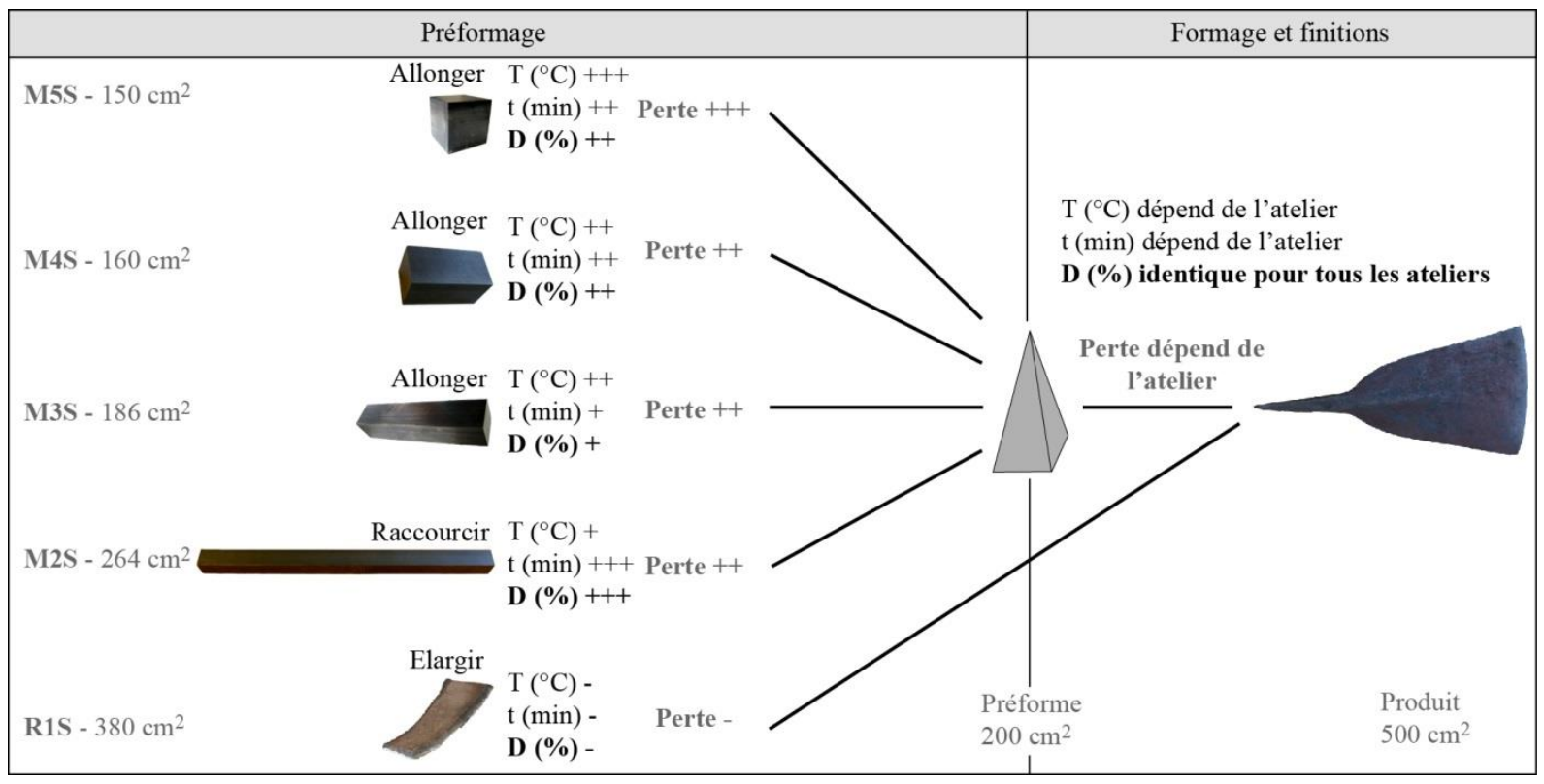

Figure 59 - Paramètres qui influencent la perte en fer. $\mathrm{t}=$ durée de chauffe $(\mathrm{min}), \mathrm{T}=$ température du foyer $\left({ }^{\circ} \mathrm{C}\right), \mathrm{D}=$ déformation $(\%)$.

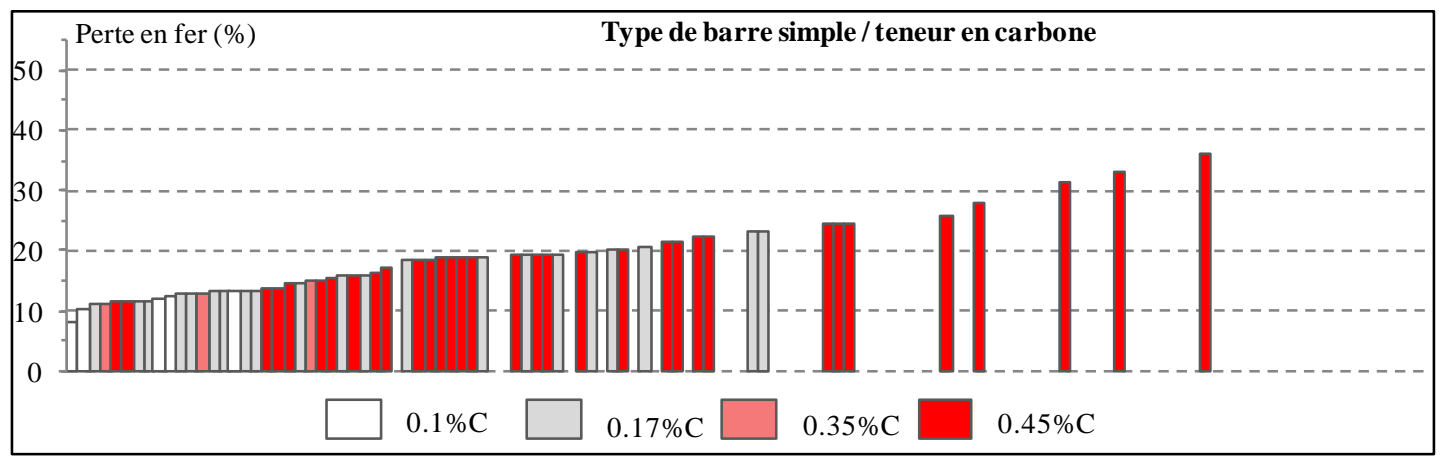

Figure 60 - Teneur en carbone des barres simples et perte en fer. 


\subsubsection{Forgeage de deux barres de fer}

\section{Influence de la température}

Contrairement au forgeage d'une seule barre de fer, le forgeage de 2 barres de fer et précisément leur soudure entre elles, implique que la température dans le foyer soit toujours très élevée.

C'est le cas puisque dans chaque atelier et pour toutes les barres de fer, le foyer est toujours porté à très haute température (Tableau 23).

\begin{tabular}{cccccc}
\hline \multicolumn{5}{c}{ Température du foyer au préformage } \\
M2D & Kobo & Doundé & Fiko & Kakoli & T ${ }^{\circ} \mathrm{C}$ \\
M3D & - & & - & - & +++ \\
M4D & - & - & - & - & +++ \\
M1D & - & - & - & - & ++ \\
A1D & - & - & - & - & ++ \\
A2D & - & - & - & - & +++ \\
R1D & - & - & - & - & +++ \\
Foyer & Très & Très & Très & Très & \\
& chaud & chaud & chaud & chaud &
\end{tabular}

Tableau 23 - Température du foyer dans chaque atelier et pour chaque type de barre double (sans incident).

\section{Influence du nombre de soudures}

$\mathrm{Au}$ cours du préformage, le nombre de soudures et la manière de réaliser ces soudures diffèrent en fonction du type de barre à forger (Figure 33).

Il s'avère que la perte en fer est globalement influencée par le nombre de soudures réalisées (Figure 62). De plus, leur réalisation demande du temps supplémentaire car elle nécessite de chauffer davantage les barres à assembler. En fait, au cours d'un forgeage, plus le forgeron doit faire de soudures et plus la durée de chauffe au préformage et la perte de fer, augmentent.

\section{Influence de la quantité de déformation}

Les barres M2S sont légèrement raccourcies avant d'être soudées entre elles. Les barres M3S et
M4S sont légèrement allongées avant d'être soudées. Toutefois, la différence de durée de chauffe entre refoulage et allongement n'est pas significative (contrairement aux barres simples) et il ne faut pas plus de temps pour souder les grosses sections (M4D) que pour souder les petites sections (M2D) (Figure 63).

La perte de fer engendrée pour ces trois types de barres s'équilibre autour de 25-30\% avec de fortes variations.

\subsubsection{Forgeage avec incidents}

Dans le cas de forgeages avec incidents, le forgeron est souvent amené à effectuer des soudures supplémentaires. Dans ce cas la durée de chauffe est augmentée (parfois très fortement - $88 \mathrm{~min}$ ) et la perte en fer produite est plus importante (Figure 62).

Pour les barres modernes, il n'y a pas de différence significative entre les forgeages sans ou avec incidents car au final leur déformation reste faible pour obtenir la préforme (Figure 63).

Par contre, les barres M1D-A1D et A2D ont des pertes bien plus élevées (moy $40 \%$ ) : leur forme est trop allongée et non adaptée pour fabriquer des dabas car le forgeron doit effectuer une succession de replis et de soudures supplémentaires. Leur durée de chauffe reste très modérée (moy $35 \mathrm{~min}$ ) car leur section très fine est rapidement réchauffée ; le travail de replis sur l'enclume est prédominant par rapport à la chauffe.

Enfin, il n'y a pas de lien entre teneur en carbone et perte en fer: les barres à $0.8 \% \mathrm{C}$ correspondent en fait toutes aux barres africaines très allongées A1D-A2D discutées précédemment (Figure 61).

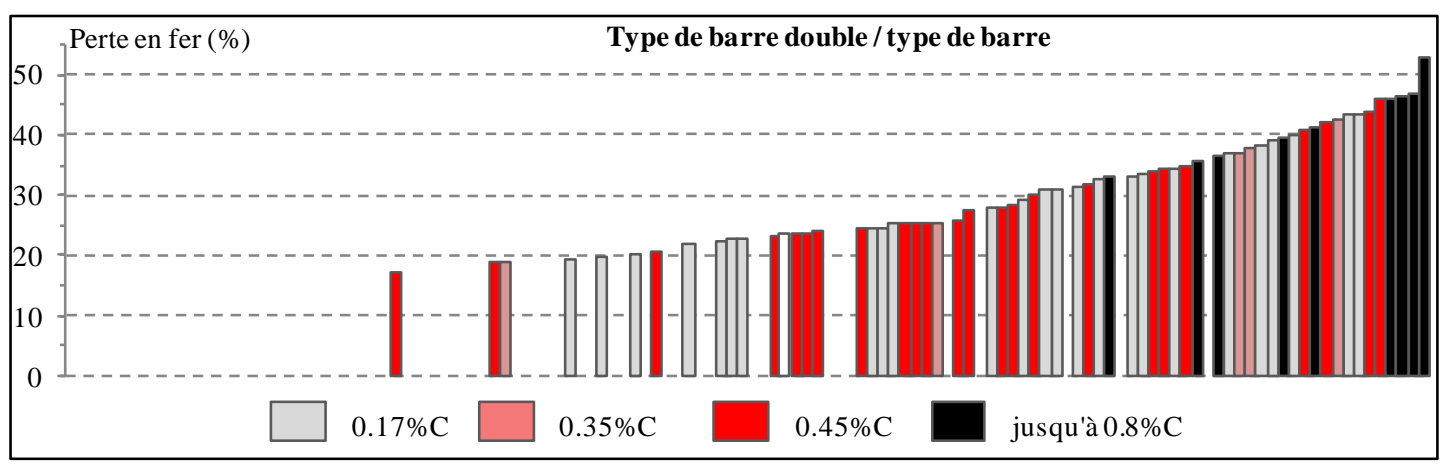

Figure 61 - Teneur en carbone des barres double et perte en fer. 

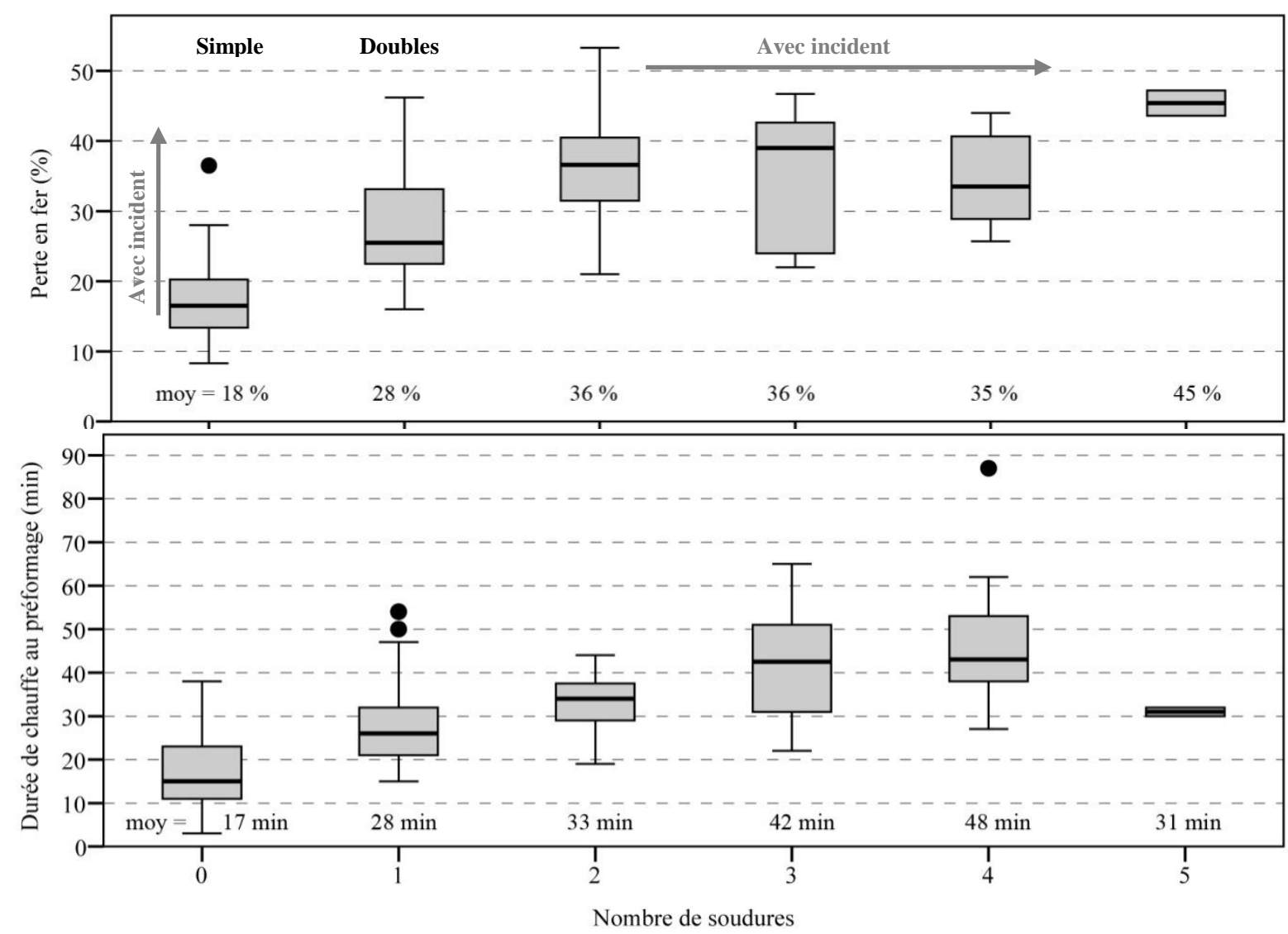

Figure 62 - Perte en fer (\%) et durée de chauffe au préformage $(\mathrm{min})$ en fonction du nombre de soudures effectuées.

Trait horizontal dans la boîte $=$ médiane. $\mathrm{n}(0$ soudure $)=55$ forgeages $; \mathrm{n}(1)=45 ; \mathrm{n}(2)=11 ; \mathrm{n}(3)=6 ; \mathrm{n}(4)=7 ; \mathrm{n}(5)=2$.
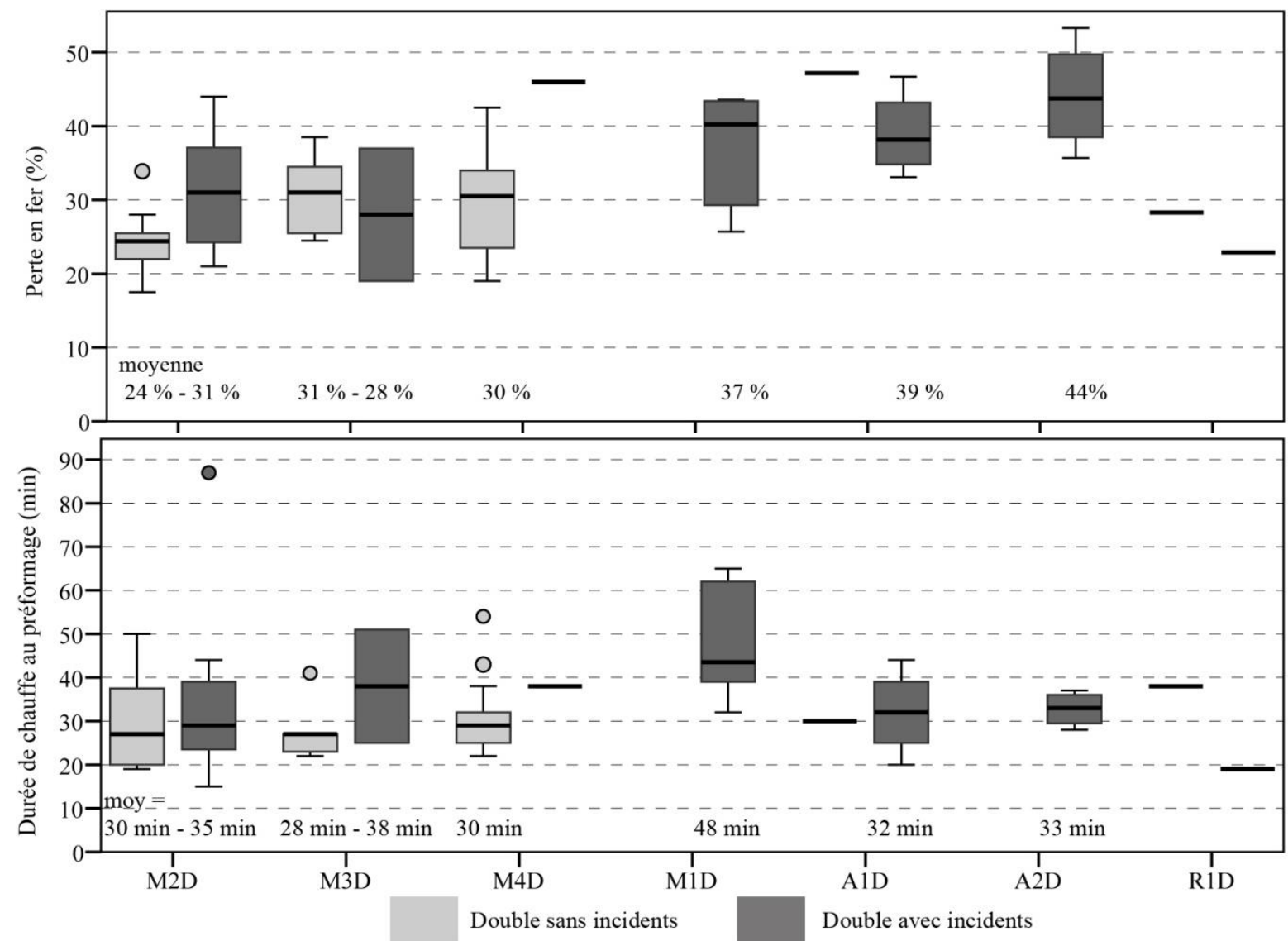

Figure 63 - Perte en fer (\%) et durée de chauffe pour les différentes sortes de barres doubles (sans-avec incidents). $\mathrm{n}(\mathrm{M} 2 \mathrm{D})=11$ (sans inc.)-11(avec inc.) forgeages $; \mathrm{n}(\mathrm{M} 3 \mathrm{D})=5-2 ; \mathrm{n}(\mathrm{M} 4 \mathrm{D})=17-1 ; \mathrm{n}(\mathrm{M} 1 \mathrm{D})=0-6 ; \mathrm{n}(\mathrm{A} 1 \mathrm{D})=1-4 ; \mathrm{n}(\mathrm{A} 2 \mathrm{D})=0-4 ; \mathrm{n}(\mathrm{R} 1 \mathrm{D})=1-1$. 


\subsubsection{Paramètres influençant la perte en fer}

La perte en fer au cours du forgeage est un processus complexe qui dépend de nombreux paramètres, avec pour conséquence une gamme de valeurs très variables, entre 8 et $53 \%$. Les paramètres sont : température du foyer (rendement soufflerie), durée de chauffe, section, forme de la barre à forger et écart entre la barre à travailler et la préforme, nombre de soudure, incidents.

La perte en fer est très variable au cours du préformage mais elle est constante au formage. Par contre, l'étape consacrée aux finitions du produit ne contribue pas du tout à la perte de fer.

Dans le cas du forgeage d'une seule barre de fer sans incident, la perte en fer prend différentes valeurs entre $8 \%$ et $37 \%$.

1- Elle varie d'un atelier à un autre et dépend à la fois de la température du foyer et de la durée de chauffe.

2- La perte en fer varie en fonction de la barre de fer que le forgeron utilise pour fabriquer sa lame : notamment sa section et sa forme.

3- La section influe à la fois sur la température dans le foyer et la durée de chauffe : plus la section est grosse et plus le foyer est chaud et la perte de fer est importante.

4- La forme de la barre de fer utilisée est également un facteur important: plus la forme de la barre de fer est éloignée de celle de la préforme et plus la quantité de déformation à appliquer sera grande et la perte en fer importante.

Dans le cas du forgeage de 2 barres de fer sans incident, la perte en fer est plus élevée : elle varie entre $18 \%$ et $47 \%$.

5- La température du foyer est toujours très élevée dans tous les ateliers et pour tous les types de barres.

6- La perte en fer est proportionnelle à la durée de chauffe au préformage et au nombre de soudures effectuées par le forgeron.

7- Dans le cas des barres modernes (dont la forme est relativement proche de celle de la préforme), la section des barres ne joue pas de rôle dans la quantité de perte en fer qui est d'ailleurs toujours très semblable.

Dans le cas de forgeages avec incidents, la perte en fer peut être très élevée, jusqu'à $53 \%$.

8- Les incidents forcent souvent le forgeron à effectuer des soudures supplémentaires et à augmenter la durée de chauffe ce qui rend la perte en fer élevée.

9- Les pertes les plus élevées ont lieu lors du forgeage de barres de fer dont la forme est vraiment trop éloignée de celle de la préforme, comme les barres africaines et la barre moderne M1D trop allongées. Le forgeron est alors contraint à un travail de replis et de soudures très important qui produit des pertes en fer très importantes.

L'oxydation des aciers à faible taux de carbone $(0.45 \% \mathrm{C})$ est normalement plus faible que celle du fer pur (Chen \& Yuen 2003 : 444). Cependant, dans nos données la teneur en carbone n'a pas d'influence sur la perte en fer, peut-être parce que les forgerons n'ont pas eu à forger un acier vraiment très dur.

Lors de forgeages avec soudure et/ou incident, l'oxydation des barres de fer n'est souvent pas majoritaire : des particules de fer à l'état de métal sont fréquemment perdues et dans de nombreux cas, en grande quantité (4.3).

Finalement la surface ne joue aucun rôle dans le processus de perte en fer, c'est l'écart entre le produit de départ et le produit d'arrivée qui est primordial : visiblement les barres africaines ne sont pas faites pour fabriquer une houe mais peut être des pointes de flèches ou des clous.

Cette réflexion permet de mieux comprendre pourquoi les forgerons que nous avons rencontrés utilisent des barres de récupération sous la forme de trapèzes plats: avec ce matériel de départ ils s'affranchissent complètement $\mathrm{du}$ travail contraignant de préformage, ce qui permet de gagner du temps et de l'énergie. Ils peuvent également produire plus d'outils et donc vendre plus dans une journée.

La barre jante de camion préformée par Sékou à Fiko est donc un pré-produit destiné à la fabrication de lames de houe, tout comme d'autres ateliers spécialisés préparent des pré-produits destinés à la fabrication de tel ou tel produit final. 


\subsection{COMPARAISON DES OPÉRATIONS DE FORGEAGE}

Le travail de terrain a fourni des données capitales pour comprendre les forgerons, leur atelier, leur travail et leurs productions.

Deux groupes ethniques existent : les ateliers de Fiko/Kakoli et ceux Kobo/Doundé. Ils ont une origine distincte, ne sont pas implantés au même endroit, ne parlent pas la même langue, leurs ateliers ne sont pas structurés de la même façon, leur foyer et soufflerie sont distincts, n'ont pas toujours les mêmes pratiques à la forge et leurs productions montrent quelques différences.

Cependant, tous ces ateliers ont en commun de travailler le fer depuis plusieurs siècles et de fabriquer des outils fonctionnels pour leur village ou leur région.

Dans tous ces ateliers, les forgerons ont fabriqué des lames de houe en suivant une chaîne opératoire précise.

Le préformage est la première étape de la chaîne opératoire, effectuée à haute/très haute température. C'est une étape qui varie énormément d'un atelier à un autre et qui compte de nombreuses différences (techniques/durées du travail).

Ces différences proviennent pour la majorité de paramètres intrinsèques à la matière première travaillée, de paramètres techniques et enfin de paramètres culturels (Tableau 24).

Par exemple, nous avons pu mettre en évidence que la présence à Kobo de jeunes apprentis comme souffleur/frappeur, augmente considérablement les durées de chauffe et de martelage dans cet atelier, par rapport aux autres.

De plus, le forgeage d'une seule barre ou de deux barres (soudure) augmente considérablement les températures dans le foyer, les durées de travail, l'apparition d'incidents ou de défauts dans les lames.

Le formage est la seconde étape de la chaîne opératoire, effectuée à moyenne/haute température. Seules quelques différences provenant de paramètres techniques et culturels influent sur les durées de travail dans chacun des ateliers : les incidents rencontrés au cours du travail, si l'équipe des forgerons n'est pas suffisamment qualifiée et la manière de façonner la nervure de la lame.

Les finitions constituent la troisième et dernière étape de la chaîne opératoire, effectuée à basse température. Il s'agit d'une étape qui varie très peu. Des différences dans la durée du travail proviennent essentiellement de paramètres techniques et culturels comme par ex. la décoration de la lame à la lime à la fin du forgeage.

Il a aussi été question de mettre en évidence les paramètres du forgeage qui influent sur la perte en fer lors de la fabrication d'une lame de houe. Globalement, le premier paramètre est la matière première. Plus la matière première présente une forme générale éloignée de la préforme et plus la perte de fer sera importante.

Ainsi, le travail d'ethno-expérimentation des forges a permis de mettre en évidence un certain nombre de paramètres qui font varier le forgeage d'une lame de houe. Nous avons pu mesurer l'influence des paramètres techniques et culturels sur le déroulement de la chaîne opératoire et le processus de perte en fer. Finalement, le rôle joué par la matière première (1 barre ou 2 barres, forme et dimensions, $\% \mathrm{C})$ semble être le plus important.

La partie 4 est dédiée à vérifier l'empreinte de tous ces paramètres sur les scories de forge issues de tous ces forgeages.

\begin{tabular}{|c|c|c|c|c|}
\hline \multicolumn{2}{|c|}{ Paramètres } & Préform. & Form. & Finit. \\
\hline \multirow{3}{*}{$\begin{array}{l}\text { Matière } \\
\text { première }\end{array}$} & Simple/double & $\checkmark$ & $\sim$ & $\sim$ \\
\hline & Forme/dimensions & $\nu$ & - & - \\
\hline & Teneur en carbone & $\sim$ & - & - \\
\hline \multirow{2}{*}{ Technique } & Incident & $\checkmark$ & $\nu$ & $\checkmark$ \\
\hline & Qualif. équipe & $\checkmark$ & $\checkmark$ & $\boldsymbol{V}$ \\
\hline \multirow{3}{*}{ Culturel } & Ajouts siliceux & $\checkmark$ & - & - \\
\hline & Type de nervure & - & $\boldsymbol{V}$ & - \\
\hline & Lime & - & - & $\boldsymbol{V}$ \\
\hline
\end{tabular}




\section{PARTIE 4 . LES DÉCHETS DE FORGEAGE}


Les déchets de forgeage 


\subsection{TECHNIQUES DE LABORATOIRE ET MÉTHODES DE CALCULS}

La Partie 4 porte sur l'approche analytique du travail, avec les investigations minéralogiques et physico-chimiques des déchets de forgeage, cœur du questionnement de la problématique de cette recherche, visant à mettre en lien les travaux de forge avec les caractéristiques des scories. Cette Partie 4 fait directement appel au catalogue en ligne.

Le chapitre 4.1 présente les méthodes d'investigations de laboratoire, ainsi que la manière dont les échantillons et les données brutes ont été traités. Les échantillons en question sont les différents matériaux rencontrés : barres de fer, parois de foyer, ajouts siliceux, scories et battitures, lesquels nécessitent une étape préliminaire indispensable au laboratoire avant d'être analysés.

\subsubsection{Préparation des échantillons}

\section{Préparation des scories de forge}

La préparation des scories (sciage, enrobage, polissage) et la perte au feu ont été effectués au laboratoire du Département des Géosciences de l'université de Fribourg par J.-P. Bourqui, P. Dietsche, C. Tamas et M. Lhemon.

Dans le cadre de cette étude, un protocole scientifique a été développé à partir d'autres protocoles déjà appliqués (Mangin et al. 2000a ; Mangin et al. 2000b ; Serneels \& Perret 2003).

Ce nouveau protocole est indiqué à la figure 64 .

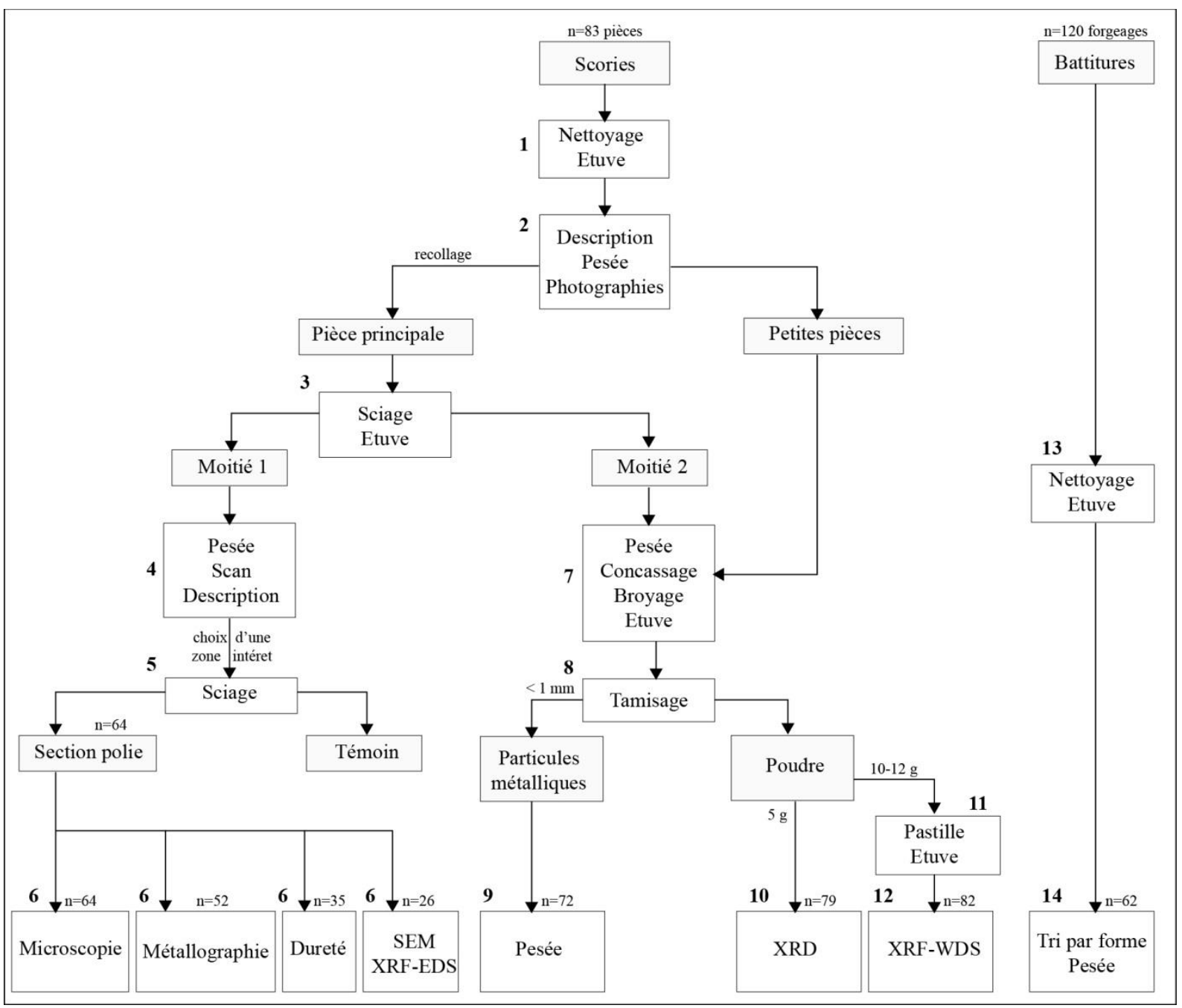

Figure 64 - Protocole pour l'étude des scories de forge et des battitures.

Les étapes (1 à 14) sont détaillées dans le texte. n correspond au nombre d'échantillons traités à chaque étape. 
1. Au laboratoire, les scories sont nettoyées sous eau avec une brosse puis séchées à l'étuve $24 \mathrm{~h}$ à $60^{\circ} \mathrm{C}$. Cette étape permet d'éliminer les matériaux qui n'appartiennent pas à la scorie et qui pourraient fausser l'interprétation des analyses chimiques (charbons, excès de terre etc.).

2. Les scories sont décrites, pesées (précision \pm 1 à $5 \mathrm{~g}$ ) et photographiées (surfaces supérieures et inférieures).

La majorité des opérations donne comme déchet une pièce principale, souvent accompagnée de fragments et petites pièces indépendantes. Les fragments sont recollés à la pièce principale

3. La pièce principale est refendue, approximativement en son milieu, à la scie diamantée. Chaque fois que cela est possible, la coupe est orientée parallèlement à l'axe de la soufflerie, lorsque les éléments de morphologie des scories permettent de le déterminer.

4. La moitié 1 (la plus lisible) est pesée. Sa coupe est scannée à très haute résolution (1200 dpi) et décrite.

L'ensemble de la coupe (pour les petites scories), ou bien une seule zone d'intérêt (pour les grandes scories) est sélectionnée. Cette zone d'intérêt représente toujours au moins $50 \%$ de la coupe : il s'agit soit d'une zone soit bien préservée, soit représentative de la coupe. Si la totalité de la coupe de la scorie n'est pas étudiée c'est surtout par manque de temps et aussi parce que la surface d'analyse du SEM+XRF-EDS est réduite à $5 \times 5 \mathrm{~cm}$. Toutefois les minéraux qui ne seraient pas présents dans la zone d'intérêt peuvent être révélés avec les autres analyses complémentaires : chimiques et minéralogiques.

5. La zone d'intérêt est débitée à la scie sur une épaisseur de $1.5 \mathrm{~cm}$ pour la réalisation d'une section polie. Les parties non utilisées de la moitié lisible sont gardées comme témoins.

Pour la réalisation de la section polie épaisse, il faut inclure la zone d'intérêt dans un moule à l'aide d'une résine (Araldite $®$ BY 158) et d'un durcisseur (Aradur® 21) pour un ratio massique de 100:28. Le séchage est réalisé à température ambiante pendant 24 h. Un pré-polissage à disque de diamant (220 puis
600) est effectué, suivi d'un polissage fin sur draps de polissage à des granulométries de 9, 6, 3 puis 1 $\mu \mathrm{m}$.

6. Les sections finement polies sont examinées à l'aide d'un microscope à lumière réfléchie : les minéraux qui forment la scorie y sont observés. Après attaque chimique au Nital, la microstructure des zones ferreuses est révélée et la dureté du métal est également testée. Seules quelques-unes des lames ont fait l'objet d'une investigation chimique supplémentaire au SEM+XRF-EDS.

7. La seconde moitié de la scorie ainsi que les petites pièces sont pesées, concassées grossièrement au marteau puis broyées en totalité dans un mortier en carbure de tungstène pendant $120 \mathrm{~s}$.

8. Pour la majorité des scories, des particules de fer à l'état métallique de plusieurs millimètres voire centimètres subsistent au milieu de la poudre de scorie après le broyage. Un tamisage est réalisé afin de les extraire.

9. Les particules de fer supérieures à $1 \mathrm{~mm}$ sont extraites puis pesées.

Le poids de ces particules de fer est important puisqu'il s'agit ensuite de les réintégrer dans les analyses chimiques finales.

10. La poudre de scorie est ponctionnée de $5 \mathrm{~g}$ pour la diffraction des rayons $\mathrm{X}$.

11. Une douzaine de grammes est nécessaire pour la préparation de pastilles pressées : selon la quantité de poudre disponible, elles ont un diamètre de 32 ou $40 \mathrm{~mm}$, pressées sous 7 tonnes pendant 90 à $120 \mathrm{~s}$. Les pastilles sont séchées à l'étuve au minimum $6 \mathrm{~h}$ à $100^{\circ} \mathrm{C}$.

12. Ces pastilles sont enfin analysées par un spectromètre en fluorescence des rayons $\mathrm{X}$ en dispersion de longueur d'onde et les intensités mesurées sont interprétées avec le programme UniQuant®.

\section{Préparation des battitures}

Dans le cadre de ce travail, il n'a pas été possible d'établir une description approfondie des battitures : uniquement des observations 
morphologiques. Il n'a pas été possible de récolter la totalité de ces matériaux car cela aurait demandé une grosse organisation de prélèvement et de traitement inadapté aux conditions de travail. Sur le terrain les battitures ont été prélevées avec des moyens très simples (pelle, balayette), de manière qualitative.

13. Au laboratoire, les battitures sont plongées dans un bac d'eau pour éliminer les poils de chèvre ou fibres végétales qui surnagent. Elles sont ensuite déposées dans un tamis de maille fine (1 mm), séparées des autres matériaux (roches $<1 \mathrm{~cm}$ par ex.) et mises à sécher à l'étuve à $80{ }^{\circ} \mathrm{C}$ pendant $24 \mathrm{~h}$.

Il est à noter que lors de leur nettoyage, environ un tiers de la masse de battitures prélevée sur le terrain, et particulièrement les particules les plus fines $(<1 \mathrm{~mm})$, a été perdu.

14. Enfin, la moitié de ces battitures a été triée par forme puis pesée. Faute de temps et de main d'œuvre, il n'a pas été réalisé d'observation au microscope ni d'analyse chimique ou minéralogique. Le but est uniquement de donner quelques ordres de grandeur et de vérifier si ce que l'on a ramassé est en accord avec les données de la bibliographie.

Finalement, pour chaque forgeage, entre la masse de fer qui a réellement pris part à la formation des battitures et la masse de fer dans les battitures ramassées par nos soins, l'écart est grand. En moyenne nous avons calculé que ce sont uniquement $15 \%$ de l'ensemble des battitures réellement produites sur l'enclume qui ont été prélevées.

\section{Préparation des autres matériaux}

\section{Matière première et produit final}

La matière première fournie aux forgerons, telle que les barres africaines (Figure 30) et les aciers modernes (Figure 29), mais aussi quelques-unes des daba élaborées ont été étudiés (Figure 51, Figure 52, Figure 53).

Tous ces matériaux ont été photographiés, décrits, prélevés à la scie diamantée suivant leur petite section. Ils ont ensuite été inclus dans une résine similaire à celle des scories puis polis jusqu'à $1 \mu \mathrm{m}$.

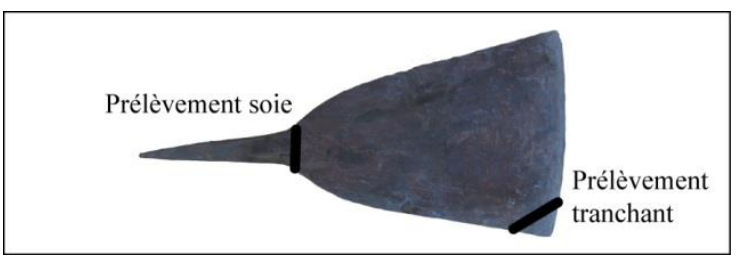

Figure 65 - Localisation des prélèvements sur les lames de houe.

La microscopie en lumière réfléchie permet d'observer la forme et la quantité des inclusions présentes dans ces matériaux (étape 6). Les observations au SEM et microanalyses à la XRF-EDS permettent de préciser leur composition chimique (étape 6). Enfin, leur microstructure, dureté et la nature des phases ferreuses ont été observées en lumière réfléchie (étape 6).

\section{Matériaux siliceux et argileux}

Quelques-uns des fondants qui ont servi à faciliter les soudures mais aussi un fragment du revêtement du foyer ont été prélevés (3.1.3).

Ces matériaux sont tout d'abord photographiés, décrits, concassés et mis à sécher à l'étuve. Ils sont ensuite broyés (étape 7) puis mis de nouveau à sécher.

De cette poudre on détermine la nature des minéraux par XRD (étape 10). Enfin, une pastille pressée est réalisée (étape 11) pour détermination de la composition chimique élémentaire à la XRFWDS.

\subsubsection{Méthodes d'analyses}

Diverses méthodes d'analyses ont été mises en œuvre pour étudier ces matériaux. Les méthodes d'analyses ponctuelles permettent de sélectionner une aire d'intérêt ou même un minéral précis puis d'en déterminer la teneur en éléments chimiques. Les méthodes globales, au contraire, s'effectuent sur une poudre homogène d'un échantillon et livrent, par conséquent, des informations relatives à la totalité du volume broyé. De plus, la seule observation ponctuelle qualitative de certains minéraux ne permet pas une généralisation à la globalité du matériau.

Dans notre étude, nous nous sommes heurtés aux particules de fer métalliques qu'il a fallu extraire avant les analyses globales, puis réintégrer dans les calculs de composition chimique. 
Les scories de forge sont souvent composées de zones chimiquement très différentes. Il est donc essentiel de choisir rigoureusement les zones à broyer : en nettoyant correctement la scorie et en broyant au moins $50 \%$ de la pièce (étapes 1 et 7), les analyses sont représentatives de l'échantillon.

\section{Microscopie optique}

L'appareil utilisé est un microscope à platine inversée Olympus GX51 utilisé en lumière réfléchie.

La surface de la section à observer doit être parfaitement plane et polie. Le grossissement total (objectif et oculaire) varie entre 50 et 1000. Le fer apparait comme une masse blanche très réfléchissante: seules les inclusions et les autres minéraux (fayalite etc) peuvent être observés.

La microscopie optique appliquée à l'étude des phases métalliques s'appelle la métallographie (Samuels 1999; Vander Voort 2004). La microstructure du fer n'apparait qu'après avoir appliqué pendant 2 à 10 secondes sur la surface de la lame une solution de Nital à $3 \%$ :

- $100 \mathrm{~mL}$ d'éthanol $\mathrm{C}_{2} \mathrm{H}_{5} \mathrm{OH}$,

- $4 \mathrm{~mL}$ d'acide nitrique $\mathrm{HNO}_{3}$ à $60 \%$.

Cette attaque chimique permet d'évaluer la teneur du carbone dans le fer $(\% \mathrm{C})$, la répartition des différentes phases carburées mais aussi la taille des grains, qui sont autant d'indices pour remonter aux températures de travail atteintes ou aux traitements thermiques appliqués.

\section{Dureté Vickers}

L'appareil utilisé est un micro-indenteur LECO LM700AT, qui fournit une valeur numérique de la dureté en Hv pour Hardness Vickers (Vander Voort 1999).

Les différentes phases ferreuses présentes dans les scories ont été soumises à test de dureté, afin de confirmer l'interprétation donnée en métallographie.

Pour simplifier, plus un grain de fer est riche en carbone et plus il sera dur. Une pointe pyramidale en diamant est fixée à un microscope optique à réflexion. Une fois la zone d'intérêt du matériau sélectionnée, cette pointe est pressée avec une force de $1 \mathrm{kgf}$ pendant $15 \mathrm{~s}$. L'empreinte résultante dans le matériau est un carré dont on mesure la longueur des diagonales. La dureté, est calculée selon la formule de la Figure 66.

$$
H v=\frac{1.8544 C}{d^{2}}
$$

Figure 66 - Dureté Vickers (Hv) avec C = la charge (kgf), d = la moyenne des diagonales ( $\mathrm{mm}$ )

A charge constante, plus le matériau est dur, plus l'empreinte et $\mathrm{d}$ sont petits et plus $\mathrm{Hv}$ sera grand. Plus le matériau est mou, plus l'empreinte sera grande et plus $\mathrm{Hv}$ sera petit. Des valeurs de dureté de fers et d'aciers sont données au tableau 1.

\section{Microscopie électronique à balayage- Spectrométrie de fluorescence des rayons $\mathrm{X}$ (XRF-EDS)}

L'appareil utilisé est un microscope électronique à balayage FEI XL 30 Sirion FEG.

$\mathrm{Du}$ fait de la faible longueur d'onde des électrons $\left(10^{-12} \mathrm{~m}\right)$ rapport aux photons de la lumière $\left(10^{-6} \mathrm{~m}\right)$, ce microscope génère des images «électroniques » dont la résolution et le grossissement sont bien plus importants qu'un microscope optique (Goldstein et al. 1992; Reed 2006).

En association avec un détecteur de rayons $\mathrm{X}$ en dispersion d'énergie et un logiciel de traitement des spectres approprié (Edax), il est également possible de déterminer des compositions chimiques ponctuelles ou de créer des cartes chimiques élémentaires de zones. Il s'agit d'une méthode d'analyse semi-quantitative. Les éléments les plus légers jusqu'à $\mathrm{N}$ ne sont pas mesurés ; les éléments légers $\mathrm{C}, \mathrm{O}$ et $\mathrm{Na}$ sont mesurés mais avec une moindre précision ; les éléments plus lourds que $\mathrm{Mg}$ sont bien mesurés.

La surface de l'échantillon est balayée par un faisceau d'électrons $(20 \mathrm{kV})$. Différentes particules ou rayonnement sont instantanément réémis par la matière et repérés par différents détecteurs ; ces rayonnements apportent des informations complémentaires :

- les électrons secondaires renseignent sur la topographie de l'échantillon ;

- les électrons rétrodiffusés fournissent des données sur l'homogénéité de la surface par contraste chimique ;

- les rayons X de fluorescence donnent des informations sur la teneur des éléments chimiques en présence : $\%$ atomique, $\%$ de 
masse de l'élément ou \% de l'oxyde correspondant.

\section{Fluorescence des rayons $\mathrm{X}$ en dispersion de longueur d'onde (XRF-WDS)}

Les mesures ont été obtenues avec un spectromètre Philips PW2400, tube PW2592/35 3kW Rhodium Anode.

La XRF en dispersion de longueur d'onde est une méthode d'analyse globale qui permet des analyses quantitatives avec l'aide de standards (Beckhoff et al. 2006; Jenkins 1999).

Couplée au logiciel UniQuant® de la société Thermoscientific (www.uniquant.com), cette méthode est utilisée pour mesurer la teneur des éléments chimiques d'un échantillon sans l'utilisation de standards: les mesures sont donc semi-quantitatives. Précision des éléments majeurs : $0.1 \%$; éléments traces : 100 ppm.

Dans le cadre de notre étude, l'échantillon se présente sous la forme d'une pastille de poudre homogène pressée de surface plane. Les éléments légers (jusqu'au fluor) sont difficilement mesurables.

La surface de l'échantillon est bombardée avec des rayons $\mathrm{X}$ primaires sous une tension variable de $60 \mathrm{kV}$ et $30 \mathrm{~mA}$ d'intensité. Ceci a pour effet de fournir assez d'énergie pour exciter les atomes de l'échantillon et éjecter les électrons des orbitales internes. L'atome se retrouve dans un état excité et retrouve son état normal uniquement quand d'autres électrons de haute énergie viennent remplir la lacune laissée sur l'orbitale interne.

C'est cette dernière transition électronique qui émet des rayons $\mathrm{X}$ de fluorescence secondaires, dont la longueur d'onde $\lambda$ est caractéristique d'un élément chimique.

Les analyses XRF sont traditionnellement exprimées en pourcentages d'oxydes. Pour les calculs, il est souvent nécessaire de convertir ces pourcentages d'oxydes en pourcentages d'éléments. Pour cela on utilise les facteurs de conversion tirés du rapport entre la masse moléculaire de l'oxyde et la masse de l'élément.

Pour les calculs sur les scories de forge, il est en particulier nécessaire de convertir les oxydes de fer $\mathrm{Fe}_{2} \mathrm{O}_{3}$ en fer élément $\mathrm{Fe}$ (Tableau 25).

\begin{tabular}{ccc}
\hline Elément/oxyde & Facteur & Elément/oxyde \\
$\mathrm{Fe}$ & x 1.2865 & $=\mathrm{FeO}$ \\
$\mathrm{Fe}$ & x 1.4298 & $=\mathrm{Fe}_{2} \mathrm{O}_{3}$ \\
$\mathrm{FeO}$ & x 1.1113 & $=\mathrm{Fe}_{2} \mathrm{O}_{3}$ \\
$\mathrm{FeO}$ & x 0.7773 & $=\mathrm{Fe}$ \\
$\mathrm{Fe}_{2} \mathrm{O}_{3}$ & x 0.6994 & $=\mathrm{Fe}$ \\
$\mathrm{Fe}_{2} \mathrm{O}_{3}$ & x 0.8998 & $=\mathrm{FeO}$ \\
\hline
\end{tabular}

Tableau 25 - Facteurs de conversion éléments/oxydes pour le fer, d'après Serneels 1993 : 193.

\section{Diffraction des rayons X $(\mathrm{XRD})$}

Les mesures ont été obtenues avec un diffractomètre Philips $\mathrm{PW} 1710$, tube $\mathrm{Cu}$ avec monochromateur en graphite.

La XRD sur poudre est une méthode d'analyse globale qui identifie les principales phases minérales cristallisées de l'échantillon (> $5 \%$ ) (Als-Nielsen \& McMorrow 2001; Warren 1990). L'échantillon se présente sous la forme d'une fine poudre homogène dont la surface d'analyse est plane.

Concrètement, un faisceau incident de rayons $\mathrm{X}$ se diffracte sur une famille de plans réticulaires d'un matériau cristallisé, uniquement à un angle d'incidence $\theta$ caractéristique du minéral. Cet angle est en relation avec la longueur d'onde des rayons $\mathrm{X}$ primaires et la distance entre les plans réticulaires qui produisent ces réflexions (Figure 67).

$$
\begin{gathered}
2 d \times \sin \theta=n \lambda \\
\mathrm{d}: \text { distance entre les plans réticulaires } \\
\theta: \text { angle des rayons } \mathrm{X} \text { incidents } \\
\mathrm{n}: \text { ordre de la réflexion } \\
\lambda: \text { longueur d'onde des rayons } \mathrm{X}\left(\mathrm{Cu}_{\mathrm{K} \alpha}: 1.54 \AA\right) \\
\text { Figure } 67 \text { - Relation de Bragg }
\end{gathered}
$$

Le faisceau de rayons $\mathrm{X}$ d'intensité $\mathrm{I}_{0}(40 \mathrm{kV}$, $40 \mathrm{~mA}$ ) se déplace avec un pas de $0.02^{\circ} / \mathrm{sec}$ entre $5^{\circ}$ et $80^{\circ}$ autour de l'échantillon. A l'angle $\theta$ caractéristique du minéral, le détecteur mesure l'intensité maximale I de la réflexion. Cette intensité dépend du volume du matériau irradié, de la taille des particules, de l'état de perfection du réseau cristallin et de la répartition des atomes dans la maille élémentaire.

Plusieurs limites existent :

- les minéraux qui ont des structures cristallines proches peuvent être confondus car leurs spectres sont très semblables; exemple : hercynite et magnétite ; 
- la détermination quantitative des proportions entre les minéraux nécessite d'appliquer la méthode d'affinage de Rietveld (Young 1996). Malheureusement par faute de temps ce traitement n'a pas pu être réalisé ;

- la matière amorphe (verre) ne donne pas de spectre interprétable et provoque une augmentation du bruit de fond ;

- $\quad$ le fer métallique induit une fluorescence qui augmente le bruit de fond et qui peut masquer certains pics.

\subsubsection{Méthode de calcul de surface}

Les coupes des scories montrent souvent différents types de matériaux. Il est possible d'évaluer leurs proportions, afin de classer les scories par pourcentage de surface des différents matériaux en présence.

La classification des scories est une étape nécessaire pour comprendre la nature et la gamme des activités de forge (Figure 3 ).

Un exemple de calcul du pourcentage de surface de chaque matériau est proposé à la Figure 68 pour la scorie S004.
1. A partir du scan de la lame polie et de son observation macroscopique mais aussi microscopique au microscope optique en lumière réfléchie, on attribue une couleur à chaque matériau présent dans la coupe.

2. Dans le logiciel GIMP® (www.gimp.org), on sélectionne une couleur donnée et on lit le nombre de pixels correspondant à cette couleur ; on en déduit le pourcentage de surface occupé par chaque matériau.

3. Par une estimation visuelle et une observation au microscope, nous estimons que les matériaux argilo-sableux et cailloux-roches contiennent en moyenne $30 \%$ de porosité ; la micro-porosité de la fayalite est négligée. On recalcule le pourcentage de surface des matériaux en tenant compte de ce facteur.

La porosité d'un matériau dépend du nombre et de la taille des pores (volume pores/volume total). Par exemple, sables: 20-50\%, grès calcaires : $<50 \%$ (Berner $2010: 52$ ), argiles : 40-60\%, verre : variable. La valeur $30 \%$ n'est pas exacte mais reste un compromis.

4. En ne tenant pas compte des charbons et de la porosité, on recalcule le pourcentage de surface des matériaux, regroupés en SAS, SGD et SFR.

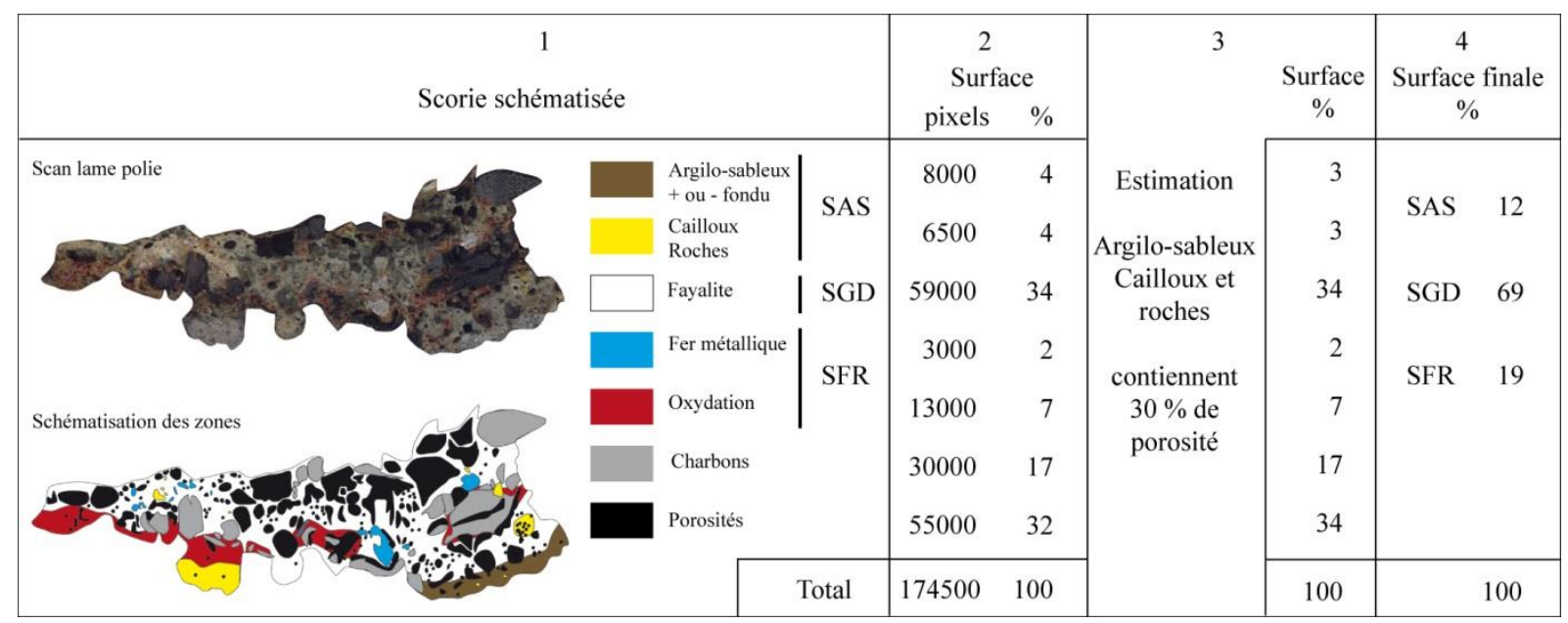

Figure 68 - Exemple de calcul de la surface des différents matériaux présents dans la coupe de la scorie S004. 


\subsubsection{Calcul du fer dans les scories et battitures}

Un des objectifs de ce travail est la compréhension de la perte en fer et de sa répartition entre les déchets : scories et battitures.

Cependant il existe des problèmes pratiques de mesure : hétérogénéité des scories, impossibilité de récolte de toutes les battitures, impossibilité de produire des poudres de scories en une seule fois à cause de la présence d'éléments métalliques de grande taille.

En prenant en compte tous ces problèmes de mesure, nous avons développé une procédure de calcul permettant de calculer la teneur en fer dans les scories ainsi que la répartition du fer entre la scorie et les battitures (Soulignac \& Serneels 2013).

\section{Calcul du fer dans les scories}

Les déchets prélevés dans le foyer de forge, généralement une pièce principale en forme de calotte et plusieurs fragments, sont lavés puis pesés. Après sciage, une moitié est conservée intacte comme témoin. Le reste, après pesage, sera broyé.
On constate alors une perte de matière variable lors du sciage (2 à $40 \%$ de perte, moyenne $10 \%$ ).

La poudre de scorie est tamisée et les particules métalliques supérieures à $1 \mathrm{~mm}$ sont extraites et pesées. La poudre seule est analysée par XRF. Cette analyse permet d'en déduire la teneur en Fe dans la poudre et de calculer la masse de Fe dans la poudre. Cette analyse chimique est représentative de l'entièreté de la scorie car la partie broyée représente au moins $50 \%$ de la pièce.

Pour déterminer la masse de Fe dans la partie analysée, il faut additionner la masse de particules de métal extraites du tamisage avec la masse de fer contenue dans la poudre. En rapportant cette masse de fer à la masse de la partie analysée, on obtient le pourcentage de fer total dans la scorie : \% $\mathrm{Fe}_{\text {tot }}$. Attention, cette valeur est non normée (vis-à-vis des autres éléments chimiques majeurs).

En tenant compte de $\% \mathrm{Fe}_{\text {tot }}$ calculé (non normé) et de la masse de la scorie initiale, on calcule la masse de fer contenue dans la scorie entière (Figure 74). Dans le Catalogue des scories, cette

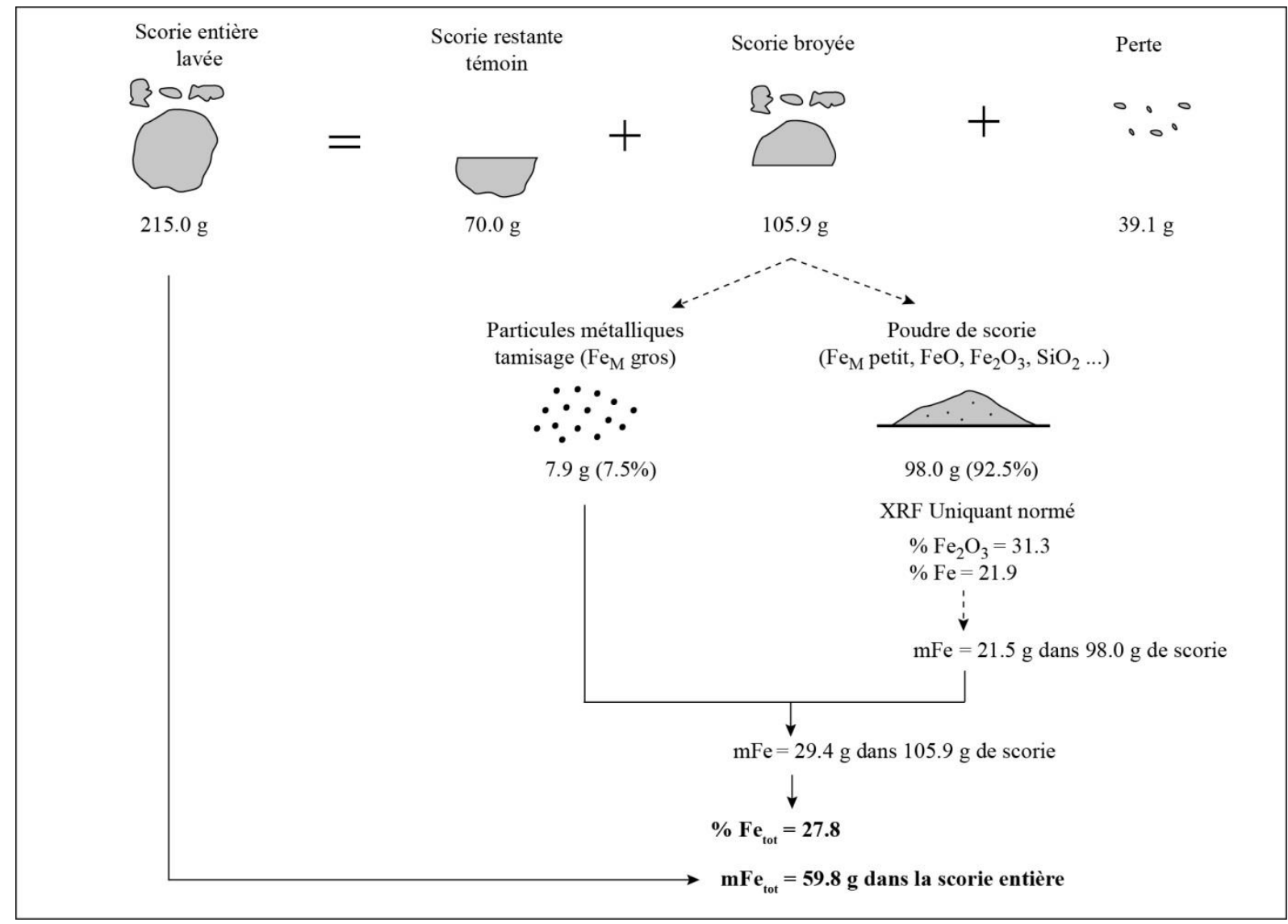

Figure 69 - Illustration du calcul de la teneur totale du fer $\mathrm{Fe}_{\text {tot }}$ dans les scories. Exemple avec les données de la scorie S004. 
valeur $\mathrm{Fe}_{\text {tot }}$ est indiquée : en la multipliant au poids de la scorie entière (pièce principale + petites pièces), on obtient la masse de fer dans la scorie entière au niveau de la ligne « Répartition scorie ».

Dans les tableaux d'analyse (Annexe 4 Scories), cette donnée a été recalculée sous forme d'oxyde $\left(\mathrm{FeO}_{\text {tot }}\right)$. Le total des majeurs variant entre $94 \%$ et $184 \%$ (à cause de la fraction métallique supplémentaire), les résultats ont été normés à $100 \%$. Du fait de cette normalisation, en

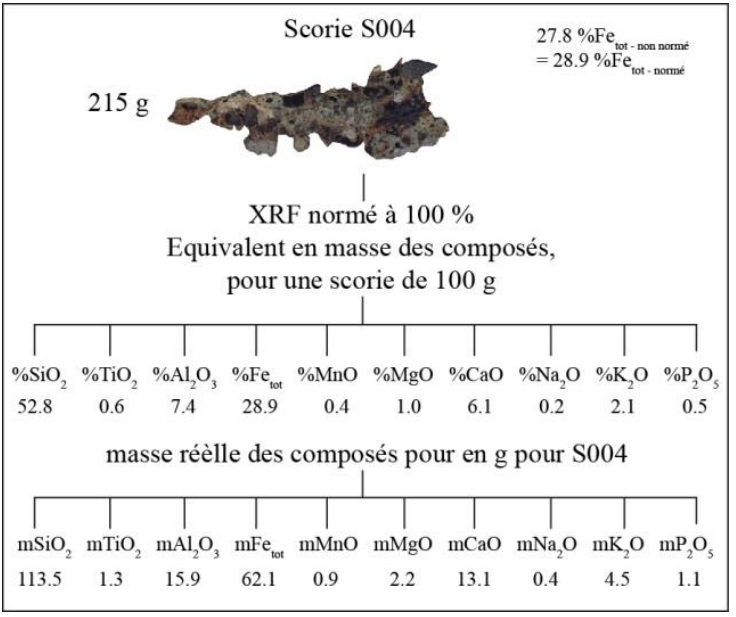

Figure 70 - Illustration du calcul de la masse des différents composés de la scorie S004.

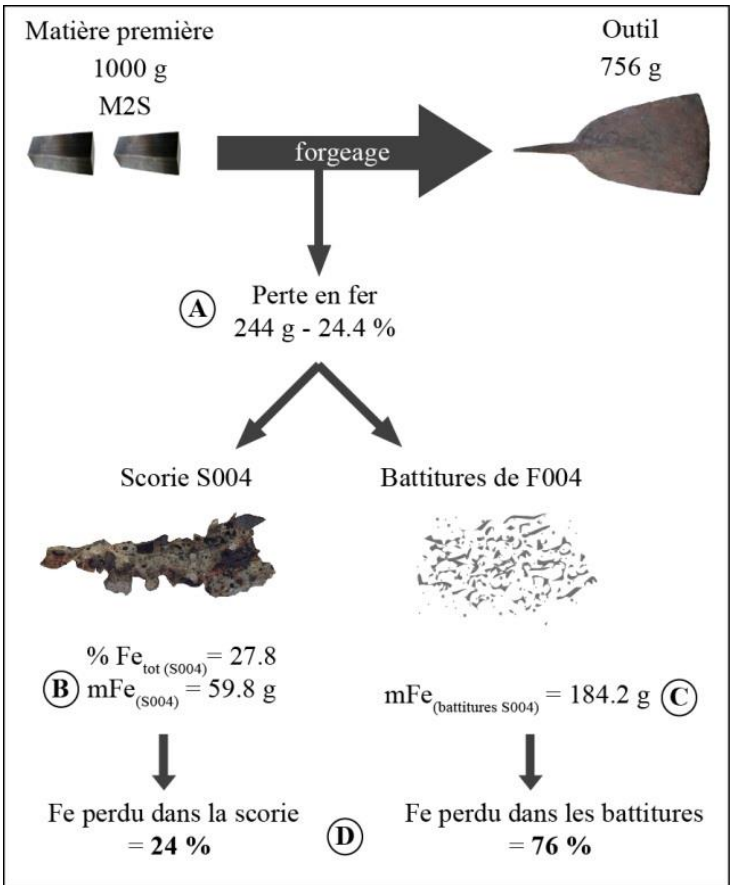

Figure 71 - Illustration du calcul de la répartition du fer perdu au cours du forgeage entre la scorie et les battitures. convertissant $\mathrm{FeO}_{\text {tot }}$ (normé) en $\mathrm{Fe}_{\text {tot }}$, on ne retrouve pas exactement la valeur $\mathrm{Fe}_{\text {tot }}$ calculée (non normée) indiquée dans le Catalogue en ligne des scories.

Calcul de la masse des différents composés des scories (Figure 70).

Le calcul de $\mathrm{Fe}_{\text {tot }}$ peut renvoyer à des totaux des composés majeurs des scories, supérieurs à 100. Il faut donc normer à 100 les teneurs des différents composés, fer y compris $\left(28.9 \% \mathrm{Fe}_{\text {tot }}\right)$. Pour une scorie de $100 \mathrm{~g}$, la masse de chaque composé (g) correspond aussi à la teneur (\%).

Pour connaitre la masse réelle des composés, il faut rapporter la masse des différents composés de la scorie de $100 \mathrm{~g}$, à la masse réelle de la scorie (215 g).

\section{Répartition du fer scorie / battitures}

La perte en fer lors du forgeage est mesurée directement lors de l'opération (Figure 54). Elle est égale à la différence entre la masse de la matière première de départ et celle de la lame de houe.

La masse de fer qui entre dans la scorie (B) est évaluée selon la procédure décrite au paragraphe précédent (Figure 69).

Le fer qui est perdu tombe soit dans le foyer lors de la chauffe (particules métalliques, croûtes d'oxydes) soit sur et autour de l'enclume lors de son martelage (particules métalliques, battitures).

La masse de fer qui est perdue sous forme de battitures (C) est considérée comme égale à la différence entre la perte totale (A) et la masse perdue dans la scorie (B).

On calcule ensuite le rapport entre (B) et (C).

Il faut cependant tenir compte du fait que même si le forgeron ramasse la scorie à notre demande après le travail, des petits fragments de fer isolés peuvent avoir été oubliés involontairement dans le foyer de forge. 


\subsection{ASPECTS DE MINÉRALOGIE ET DE CHIMIE}

La Partie 4.2 renseigne sur les aspects de minéralogie et de chimie rencontrés dans notre corpus de scories de forge.

\subsubsection{Minéralogie des silicates}

Dans ce travail, la gamme des scories de forge étudiées est limitée ; seuls sont décrits les minéraux rencontrés au cours de notre étude. Plusieurs auteurs ont décrit d'autres scories, associées à d'autres minéraux (Bachmann 1982 ; Leroy 1997 ; Serneels 1993).

\section{Groupe des olivines}

Les minéraux du groupe des olivines sont fréquents dans les scories de forge. D'une manière générale, les olivines possèdent une structure orthorhombique dans laquelle des tétraèdres de silice $\mathrm{SiO}_{4}{ }^{4-}$ sont associés à 2 ions chargés ${ }^{2+}$.

La fayalite $\mathrm{Fe}_{2} \mathrm{SiO}_{4}$ est la phase minérale typique des scories (identifiée dans $78 \%$ des pièces). Sa température de fusion est de $1205^{\circ} \mathrm{C}$, une température facilement atteinte dans un foyer de forge du fait de la soufflerie forcée (Bowen \& Schairer $1932: 200)$.

Ce minéral possède différents habitus qui varient considérablement en fonction des conditions de refroidissement, toutes les tailles de cristaux étant possibles (Figure 73 ). Un refroidissement lent conduit à des formes trapues (1 et 2). Quand les

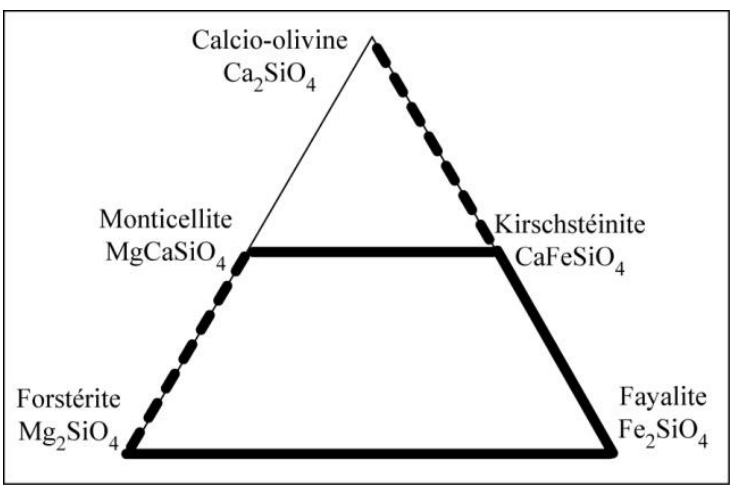

Figure 72 - Diagramme des olivines, d'après Sahama \& Hytönen 1958 : 863. En trait gras sont indiquées les solutions solides continues et en traits pointillés les solutions solides partielles. cristaux sont très développés dans un espace restreint, on observe des plages de fayalite (3). Au contraire, un refroidissement rapide conduit à la formation de cristaux allongés selon une seule direction (4).

La kirschstéinite $\mathrm{CaFeSiO}_{4}$ a été identifiée dans $10 \%$ des pièces. Sa température de fusion est de $1208{ }^{\circ} \mathrm{C}$ (Bowen et al. 1933 : 281). Deux habitus ont été observés (Figure 73) : des cristaux trapus (5) et des cristaux allongés de tailles variables (6).

Le triangle des olivines formé par la calcio-olivine $\left(\mathrm{Ca}_{2} \mathrm{SiO}_{4}\right)$, la forstérite $\left(\mathrm{Mg}_{2} \mathrm{SiO}_{4}\right)$ et la fayalite $\left(\mathrm{Fe}_{2} \mathrm{SiO}_{4}\right)$, ainsi que les olivines intermédiaires : monticellite $\left(\mathrm{MgCaSiO}_{4}\right)$ et kirschstéinite $\left(\mathrm{CaFeSiO}_{4}\right)$ ont déjà été étudiés (Figure 72).

La série forstérite-fayalite est un exemple de solution solide complète. La température du mélange augmente jusqu'à $1890{ }^{\circ} \mathrm{C}$ avec l'ajout de $\mathrm{Mg}$ (Bowen \& Schairer 1935 : 163). De plus, Mn peut être introduit comme élément trace. Dans notre cas, nous comptons deux scories dans lesquelles $\mathrm{Mg}$ remplace Fe et quatre scories dans lesquelles Mn est identifié.

Entre la fayalite et la kirschstéinite il existe également une solution solide continue (Bowen et al. 1933 : 281). Il en est de même entre la kirschstéinite et la monticellite (Schairer \& Osborn 1950 : 863).

La solution solide de la série monticelliteforstérite est partielle (Ricker \& Osborn 1954 : 136) tout comme celle de la série calcio-olivinekirschstéinite (Bowen et al. 1933 : 281).

\section{Groupe des pyroxènes}

L'hedenbergite $\mathrm{CaFeSi}_{2} \mathrm{O}_{6}$ et le diopside $\mathrm{CaMgSi}_{2} \mathrm{O}_{6}$ ont une structure monoclinique : ce sont des clinopyroxènes.

Dans notre cas, seul l'hedenbergite a été identifié de manière anecdotique ( $1 \%$ des pièces). Ces deux minéraux forment une solution solide complète connue, dans laquelle $\mathrm{Mg}$ et $\mathrm{Fe}$ se substituent l'un et l'autre dans toutes les proportions : on obtient alors une vaste gamme de 


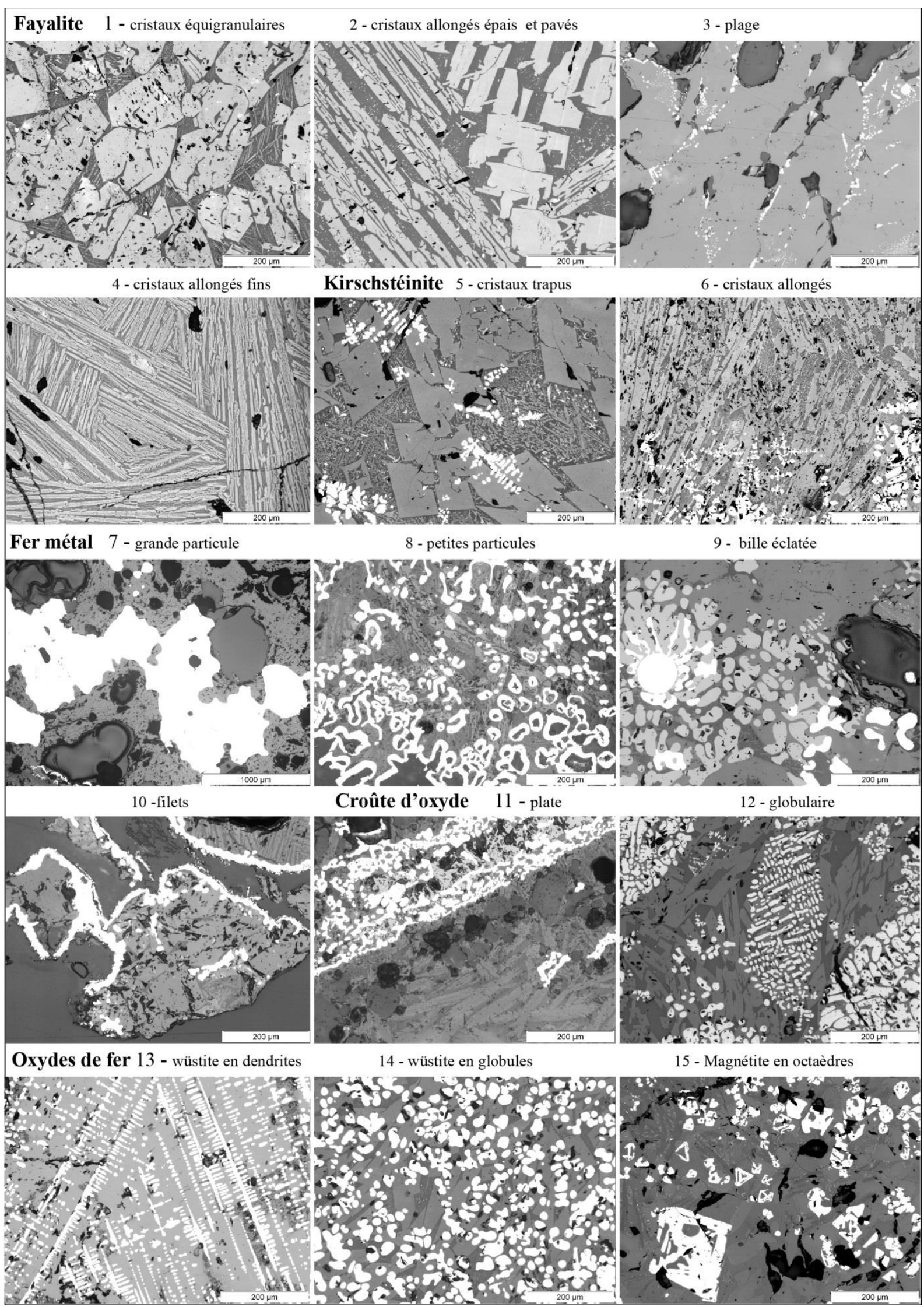

Figure 73 - Minéraux et particules ferreuses observés dans les scories de forge de notre étude. Microscopie optique en lumière réfléchie.

Différents habitus de la fayalite (clichés 1 à 4 ) et de la kirschstéinite (clichés 5 et 6) - Exemples de pertes de fer à l'état métallique dans le foyer de forge (clichés 7 à 10) - Croûtes d'oxydes de fer (clichés 11 et 12) - Structures variées des oxydes de fer (clichés 13 à 15). 
minéraux appelée augite. (Klein \& Hurlbut 1985 : 398).

L'augite est le clinopyroxène le plus commun dans la formation des roches en général mais aussi dans les scories de forge puisqu'il a été identifié dans $15 \%$ des pièces.

Les minéraux de la famille de l'augite présentent une formule chimique complexe: $(\mathbf{C a}, \mathbf{N a})(\mathbf{M g}, \mathbf{F e}, \mathbf{A l})(\mathbf{S i}, \mathbf{A l})_{2} \mathrm{O}_{6}$. En effet, $\mathrm{Ca}$ peut être substitué par $\mathrm{Na}$ tandis que $\mathrm{Mg}$ et/ou Fe et/ou Al peuvent être substitués par Al. L'augite se forme préférentiellement dans des scories où l'apport en fer est limité.

\section{Groupe des wollastonites}

$\mathrm{Si}$ les scories sont particulièrement riches en $\mathrm{Ca}$ (à partir de $15 \% \mathrm{Ca}$ ), alors pourront se former des composés comme des wollastonites $\mathrm{Ca}_{2} \mathrm{Si}_{2} \mathrm{O}_{6}$, de la bustamite $(\mathrm{Mn}, \mathrm{Ca})_{3} \mathrm{Si}_{3} \mathrm{O}_{9}$ et son équivalent ferreux la ferro-bustamite $\mathrm{Ca}(\mathrm{Fe}, \mathrm{Ca}, \mathrm{Mn}) \mathrm{Si}_{2} \mathrm{O}_{6}$.

Il a uniquement été identifié dans 2 pièces (S001 et S031, non comptabilisées) un composé proche de la ferrobustamite.

\section{Groupe des mélilites}

De formule générale $\mathrm{X}_{2} \mathrm{YZO}_{7}$, les mélilites forment une solution solide qui va d'un pôle calco-magnésien représenté par l'åkermanite $\mathrm{Ca}_{2} \mathrm{MgSi}_{2} \mathrm{O}_{7}$, à un pôle calco-alumineux, représenté par la gehlénite $\mathrm{Ca}_{2} \mathrm{Al}_{2} \mathrm{SiO}_{7}$.

Dans cette série, Ca peut être substitué partiellement par $\mathrm{Na}$ (mélilites sodiques $\left.(\mathrm{Ca}, \mathrm{Na})_{2}(\mathrm{Mg}, \mathrm{Fe}, \mathrm{Al}) \quad\left[(\mathrm{Al}, \mathrm{Si}) \mathrm{Si}_{2} \mathrm{O}_{7}\right)\right]$ et $\mathrm{Fe}$ peut remplacer $\mathrm{Mg}$ (ferro-åkermanite $\mathrm{Ca}_{2} \mathrm{FeSi}_{2} \mathrm{O}_{7}$ ). L'åkermanite et la ferro-åkermanite forment une solution solide complète (Schairer \& Osborn 1950 : 167).

Ces 2 minéraux ont été identifiés de façon récurrente puisqu'ils sont présents dans $13 \%$ des scories étudiées.

\section{Groupe des feldspathoïdes}

La leucite KAISi2 $\mathbf{O}_{6}$ est un minéral que l'on retrouve régulièrement dans les scories de forge (15\% des pièces). La leucite cristallise dans un système tétragonal bipyramidal puis cubique audessus de $625{ }^{\circ} \mathrm{C}$. Elle est le principal support du potassium qui provient des cendres du charbon de bois. Par ailleurs, elle est souvent en association symplectique avec la wüstite (Figure 74).
Il s'agit d'un équivalent pauvre en silice des feldspaths de formule (K,Na) $\mathrm{AlSi}_{3} \mathrm{O}_{8}$ : c'est-à-dire qu'elle cristallise dans des systèmes pauvres en silice. Par conséquent, à l'équilibre, la présence simultanée de leucite et de quartz est impossible : la leucite se transformerait en feldspath potassique $\mathrm{KAlSi}_{3} \mathrm{O}_{8}$ nommé orthose (Schairer \& Bowen 1938 : 293).

Dans notre assemblage de scories, les systèmes sont fréquemment non équilibrés. Les analyses XRD montrent que la leucite est toujours associée au quartz. Dans ce cas il est possible que le quartz ne provienne pas de la matrice liquide de la scorie mais de grains de quartz non fondus qui sont entrés dans le système (sol de la forge, parois, ajouts).

\section{Groupe de la silice}

Le quartz $\mathrm{SiO}_{2}$ est toujours présent dans les scories de forge (96\% des pièces), en plus ou moins grande quantité. Il y est toujours introduit sous la forme de grains millimétriques (sable) et/ou centimétriques (cailloux) non fondus et/ou partiellement fondus. Sa température de fusion est $\mathrm{T}_{\mathrm{f}}$ $=1650{ }^{\circ} \mathrm{C}$.

Le quartz est souvent associé à son polymorphe la cristobalite ( $75 \%$ des pièces). Cependant, la tridymite, autre polymorphe du quartz, n'a pas été détectée au cours de ce travail.

Lors de la chauffe, le quartz $\alpha$ se transforme en quartz $\beta$ à $573{ }^{\circ} \mathrm{C}$, en tridymite $\beta$ à $870{ }^{\circ} \mathrm{C}$ puis localement en cristobalite $\beta$ à $1470{ }^{\circ} \mathrm{C}$. En refroidissant, le quartz $\beta$ se transforme en quartz $\alpha$ à

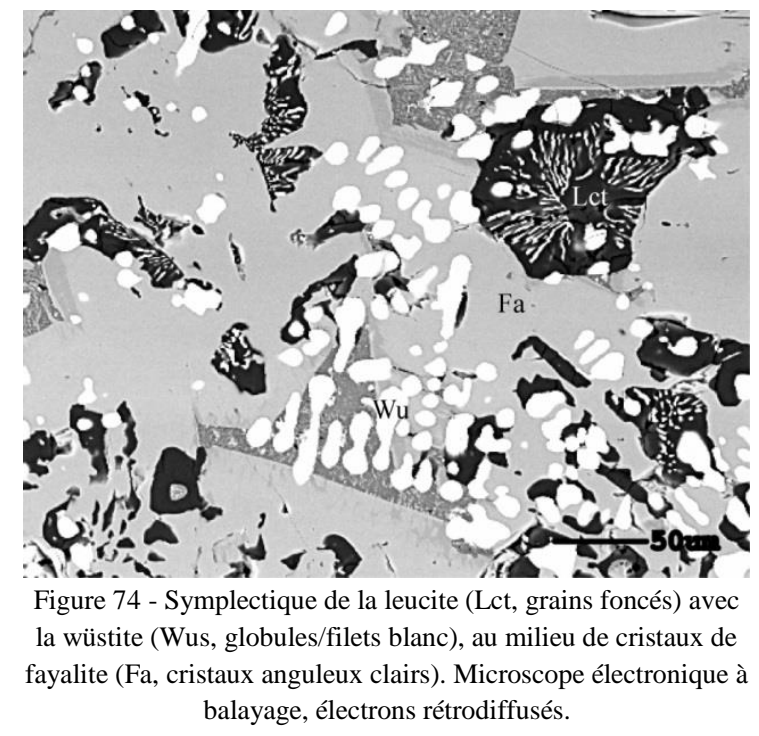


$573{ }^{\circ} \mathrm{C}$; la cristobalite $\beta$ en cristobalite $\alpha$ à $268^{\circ} \mathrm{C}$ (Klein \& Hurlbut 1985 :438). La cristobalite peut aussi être «héritée », c'est-à-dire provenir directement des parois du foyer, de la tuyère ou des roches qui tombent dans la scorie.

Cette composante vitreuse, observée au microscope, est non négligeable. Toutefois la quantification de ce verre n'a pas été réalisée. Le quartz n'ayant pas pu atteindre sa température de fusion à $1720{ }^{\circ} \mathrm{C}$, d'autres éléments comme des alcalins $(\mathrm{Na}, \mathrm{K})$ ont $\mathrm{pu}$ faire baisser cette température.

\section{Autres minéraux}

La mullite $\mathbf{3 A l}_{2} \mathrm{O}_{3} .2 \mathrm{SiO}_{2}$ est un silicate d'aluminium qui se forme à haute température et à basse pression.

Il s'agit d'un composé essentiellement trouvé dans les produits céramiques riches en silice et alumine, et dont la température de fusion se situe entre 1500 et $1800{ }^{\circ} \mathrm{C}$ selon la composition (Bowen \& Greig 1924 : 242).

Ce minéral a été identifié seulement dans $2 \%$ des pièces étudiées et pourrait provenir d'une contamination par la paroi du foyer et/ou par la tuyère utilisée.

\subsubsection{Minéralogie des oxydes}

\section{Oxydes de fer}

Dans la nature, l'oxygène est certainement l'élément chimique qui a le plus d'affinité avec les autres éléments. Les anions d'oxygène $\left(\mathrm{O}^{2-}\right)$ ont fortement tendance à s'associer avec des cations de métaux pour former des oxydes métalliques.

Le fer possède des degrés d'oxydation de 2 à +8 mais les plus courants sont +2 et +3 , ce qui conduit à la formation de 2 cations :

$$
\begin{aligned}
& \text { - l'ion ferreux } \mathrm{Fe}^{2+}:[\mathrm{Ar}] 3 \mathrm{~d}^{6} 4 \mathrm{~s}^{0}, \\
& \text { - } \quad \text { l'ion ferrique } \mathrm{Fe}^{3+}:[\mathrm{Ar}] 3 \mathrm{~d}^{5} 4 \mathrm{~s}^{0} \text {. }
\end{aligned}
$$

Selon les conditions oxydantes ou réductrices de l'atmosphère dans lesquelles ils se trouvent mais également les températures atteintes, le fer et l'oxygène forment différents oxydes de fer : 1'oxyde ferreux (wüstite $\mathbf{F e}_{1-\mathbf{x}} \mathbf{O}$ ), magnétique (magnétite $\mathrm{Fe}_{3} \mathrm{O}_{4}$ ) et ferrique (hématite $\mathrm{Fe}_{2} \mathrm{O}_{3}$ )

Dans un four de réduction du minerai du fer, on cherche à obtenir des composés à faible degré d'oxydation, en particulier du fer métallique, à partir de minerai contenant des oxydes de fer. Au contraire, dans un foyer de forge riche en oxygène, c'est le fer métallique qui est petit à petit oxydé. En dessous de $570{ }^{\circ} \mathrm{C}$, la magnétite est le produit majoritaire de la réaction (Figure 75).

Au-dessus de $570{ }^{\circ} \mathrm{C}$, à l'état solide, les oxydes de fer croissent à la surface du substrat de métal, en une triple couche de wüstite, magnétite et hématite, par des mouvements de lacunes cationiques et de défauts. Les rapports d'épaisseur de ces oxydes montrent que la wüstite est le produit principal qui est formé (Figure 76).

Si la transformation se déroule à l'état liquide alors la wüstite cristallise en globules et en dendrites (Figure 73 ; 13 et 14). La magnétite qui est un spinelle, cristallise dans le système cubique selon une structure octaédrique (15). Quant à l'hématite, elle cristallise dans le système trigonal selon une

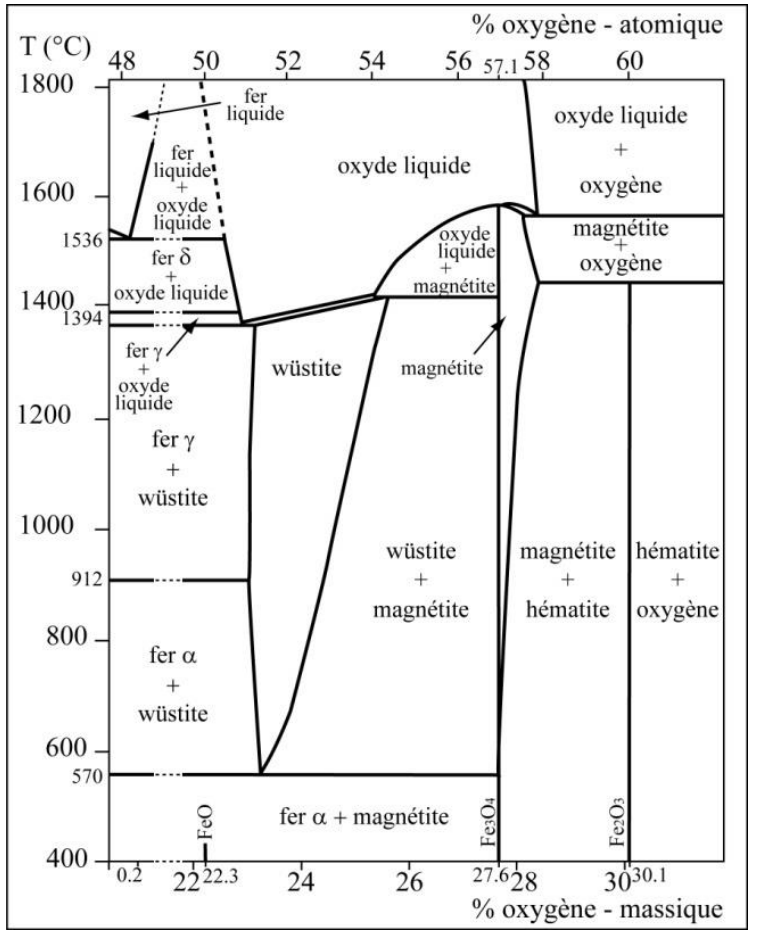

Figure 75 - Diagramme partiel fer-oxgène à 1 atm. D'après Darken \& Gurry 1946 : 799.

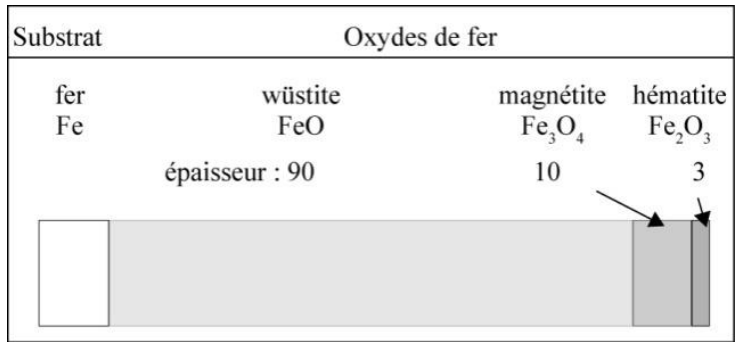

Figure 76 - Croissance des oxydes de fer à l'état solide. D'après Landolt 1993 : 350-351. 
structure tabulaire. La taille de ces formations dépend de la température et de la vitesse de refroidissement, l'orientation est fonction du sens du refroidissement.

\section{Spinelles}

Les spinelles sont une famille de minéraux qui possède une structure octaédrique $\mathrm{XY}_{2} \mathrm{O}_{4}$. Plusieurs cations peuvent entrer dans la structure et de nombreux remplacements sont possibles: spinelle $\mathrm{MgAl}_{2} \mathrm{O}_{4}$, magnétite $\mathrm{Fe}_{3} \mathrm{O}_{4}$, galaxite $\mathrm{MnAl}_{2} \mathrm{O}_{4}$, hercynite $\mathrm{FeAl}_{2} \mathrm{O}_{4}$ etc.

La hercynite $\mathrm{FeAl}_{2} \mathrm{O}_{4}$ est souvent rencontrée dans les scories de forge alumineuses. La présence de hercynite dans les scories indique un apport d'aluminium par des reliquats de scories de réduction riches en $\mathrm{Al}_{2} \mathrm{O}_{3}$, par la paroi du foyer ou par l'ajout de fondants qui contenaient de l'aluminium.

Ce minéral forme une solution solide complète avec la magnétite $\mathrm{Fe}_{3} \mathrm{O}_{4}$ uniquement au-dessus de $850{ }^{\circ} \mathrm{C}$, par remplacement mutuel de $\mathrm{Fe}$ par $\mathrm{Al}$ (Turnock \& Eugster 1962). Par ailleurs, les analyses en diffraction des rayons $\mathrm{X}$ proposent des spectres très similaires pour tous les minéraux appartenant à cette solution solide.

\section{Remarques}

Une réflexion concernant la saturation en oxydes de fer est proposée par (Serneels 1993 : 29-30). Dans le mélange liquide qui va former la scorie, le fer est l'élément le plus abondant. En refroidissant, il s'allie aux autres éléments pour former des minéraux (fayalite etc).

Si le système contient trop de fer pour former tous les minéraux, c'est la sursaturation, et l'excès de fer apparaitra sous la forme d'oxydes libres comme la wüstite et la magnétite. Si le système contient juste assez de fer pour former les minéraux, c'est l'équilibre.

Cependant, dans un système sous-saturé en fer, apparaitront des minéraux pauvres en fer comme des leucites, augites, diopsides ou hedenbergites.

\subsubsection{Chimie}

Les scories de forge peuvent inclure toutes sortes de matériaux (Figure 1) et elles peuvent donc avoir des compositions chimiques qui montrent des grandes différences.

Les composés majeurs représentent entre $99.5 \%$ et $99.9 \%$ de la scorie. Le reste correspond aux éléments dits «traces » (exprimés en ppm; $10.000 \mathrm{ppm}=1 \% \mathrm{wt}$ ) et aux éléments non comptabilisés (limite de détection de la machine, éléments légers).

Parmi les principaux composés dits « majeurs » qui font partie des scories de forge, (exprimé en pourcentage de poids d'oxyde : \%wt), on trouve le fer et la silice.

Le fer exprimé sous la forme $\mathrm{Fe}_{\text {tot }}$ ou $\mathrm{FeO}_{\text {tot }}$ provient des pertes de la barre de fer lors de la chauffe.

La silice $\mathrm{SiO}_{2}$ provient des ajouts, de la paroi $\mathrm{du}$ foyer et dans une moindre mesure de la cendre du charbon de bois dont le substratum au Pays dogon est riche en silice.

Les seconds composés majeurs des scories sont l'alumine $\mathrm{Al}_{2} \mathrm{O}_{3}$ et le calcium $\mathrm{CaO}$. L'alumine $\mathrm{Al}_{2} \mathrm{O}_{3}$ est souvent associée à la silice $\mathrm{SiO}_{2}$, toutes deux présentes dans la plupart des roches naturelles. Le rapport $\mathrm{SiO}_{2} / \mathrm{Al}_{2} \mathrm{O}_{3}$ permet de comprendre les ajouts volontaires (sable, argile etc) ou une contamination par la paroi.

Le calcium est le principal constituant minéral du bois, qui représente entre 50 et $90 \%$ de la cendre. $\mathrm{La}$ teneur en $\mathrm{CaO}$ de la cendre dépend de nombreux facteurs, notamment l'essence et le substratum. $\mathrm{CaO}$ est aussi présent dans les roches : à hauteur de moins de $1 \%$ dans les grès du plateau Dogon, 1-10 \% dans les roches magmatiques, métamorphiques, sédimentaires et à plus de $60 \%$ dans les calcaires. Le strontium $\mathrm{Sr}$ est un élément trace souvent associé au Ca dans les minéraux.

Parmi les autres composés majeurs, on trouve la soude $\mathrm{Na}_{2} \mathrm{O}$. Le sodium est apporté par le charbon de bois. Il s'agit d'un élément volatile à haute température et généralement peu présent dans les scories de forge. Le pic de $\mathrm{Na}_{2} \mathrm{O}$ est large et présente un fort bruit de fond : les teneurs de ce composé sont mesurées avec une moindre précision.

La potasse $\mathrm{K}_{2} \mathrm{O}$ est le second constituant minéral du bois, qui représente entre 10 et $50 \%$ de la cendre. Le potassium peut aussi être apporté par les roches qui tombent dans le foyer $(0.5$ à $3 \%)$. Le rubidium $\mathrm{Rb}$ est souvent lié aux minéraux contenant $\mathrm{K}_{2} \mathrm{O}$, notamment les argiles. 
Le magnésium $\mathrm{MgO}$ est présent à des teneurs variables dans les roches. Il est possible de le trouver dans la cendre de charbon de bois dans le cas où le substratum est riche en $\mathrm{MgO}$, ce qui n'est à priori pas le cas au Pays dogon.

Le phosphore $\mathrm{P}_{2} \mathrm{O}_{5}$ est présent en petite quantité dans tous les types de roches à hauteur de 0.5 à $1 \%$, et dans les cendres du charbon de bois de 1 à $5 \%$. Certains combustibles utilisant des os ou des excréments sont particulièrement riches en phosphore.

Le manganèse $\mathrm{MnO}$ et le titane $\mathrm{TiO}_{2}$ sont quant à eux présent en faibles quantités dans tous les milieux naturels, à de faibles teneurs (0.1 à $1 \%)$.

Le scandium Sc est incompatible avec le métal et il est généralement associé au grès/quartz.

Le lanthane La est quant à lui généralement lié au Sc.

Le zirconium $\mathrm{Zr}$ est un élément trace présent en faible quantité dans les roches, sous la forme de zircon $\mathrm{ZrSiO}_{4}$; il montre une affinité pour les roches siliceuses (sable, argile etc).

Le cobalt Co dans les scories est un élément trace qui peut provenir d'une pollution due au broyage des échantillons dans la meule en carbure de tungstène ; cette pollution peut apporter une dizaine de ppm de Co tout au plus.

Le cuivre $\mathrm{Cu}$ et le zinc $\mathrm{Zn}$ sont d'habitude associés ensemble.
Le chrome $\mathrm{Cr}$ est un élément que l'on trouve dans les fontes qui peut provenir des aciers modernes (Tableau 8).

Le lanthane $\mathrm{La}$ et le vanadium $\mathrm{V}$ sont quant à eux présents en faible quantité dans les roches.

Le baryum $\mathrm{Ba}$ est l'élément trace le plus abondant dans les roches (500 à 1000 ppm) ; il peut provenir de la cendre du charbon de bois et de la poudre de barytine rajoutée à notre demande aux fondants à Kobo.

Le soufre $\mathrm{S}$ est un élément volatile qui brûle au contact de l'air à faible températures (dès $300{ }^{\circ} \mathrm{C}$ ) pour former un composé gazeux $\mathrm{SO}_{2}$; il est souvent lié aux métaux $\mathrm{Fe}$ ou $\mathrm{Cu}$, au gypse $\mathrm{CaSO}_{4} 2\left(\mathrm{H}_{2} \mathrm{O}\right)$ et plus généralement au $\mathrm{Ca}$ et aux sulfates.

La composition chimique des scories de forge est représentée de façon satisfaisante par ses constituants majoritaires et projetée dans le tétraèdre $\mathrm{SiO}_{2}-\mathrm{Al}_{2} \mathrm{O}_{3}-\mathrm{CaO}-\mathrm{FeO}_{\mathrm{n}}$ (Figure 77). Les valeurs maximales sur le pôle $\mathrm{CaO}$ et $\mathrm{Al}_{2} \mathrm{O}_{3}$ ne dépassent généralement pas $15 \%$. $\mathrm{Au}$ contraire, les compositions chimiques des scories s'étendent beaucoup plus entre le pôle $\mathrm{FeO}_{\text {tot }}$ et $\mathrm{SiO}_{2}$.

Pour les éléments du foyer et les ajouts, ceuxci sont au contraire localisés principalement vers les pôles $\mathrm{SiO}_{2}$ et $\mathrm{Al}_{2} \mathrm{O}_{3}$. 


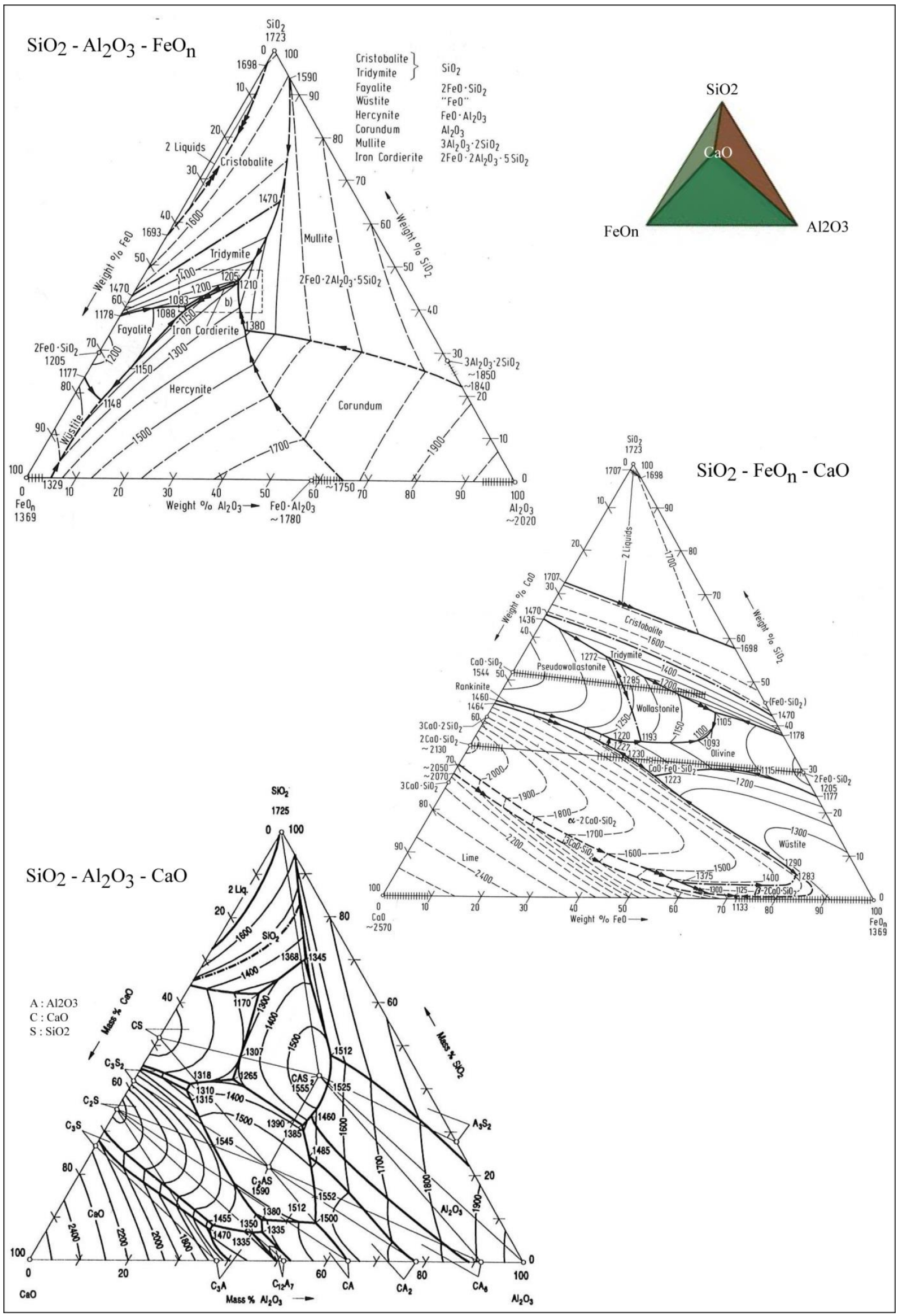

Figure 77 - Diagrammes d'équilibre du système $\mathrm{SiO}_{2}-\mathrm{Al}_{2} \mathrm{O}_{3}-\mathrm{CaO}-\mathrm{FeO}$. (VDEh 1995). 


\subsection{MATÉRIAUX ET STRUCTURE DANS LES SCORIES}

Le chapitre 4.3 a pour but de comparer les données morphologiques, minéralogiques et chimiques des scories de forge afin d'en tirer des regroupements.

\subsubsection{Description générale du corpus de scories}

\section{Rappel des conditions de forgeage}

Les opérations de forgeage réalisées au Pays dogon ont permis la réalisation de 126 lames de houes (Tableau 10) et de deux lames de machettes non pris en compte, F009 et F019 (Annexe 1 Opérations de Forgeage et Catalogue des scories, à télécharger).

Le travail à la forge a été effectué à partir de plusieurs sortes de barres de fer :

- Aciers modernes de géométrie variable contenant peu d'éléments traces $(\mathrm{Mn}, \mathrm{Cu}$, $\mathrm{Cr}$, Ni, Mo, Tableau 8) et parfois des impuretés non métalliques $(\mathrm{P}, \mathrm{S})$;

- Barres de fer africaines qui contiennent systématiquement des inclusions de scorie riche en $\mathrm{Ti}$, souvent en $\mathrm{Zr}$, parfois $\mathrm{Mn}$ et $\mathrm{V}$, rarement $\mathrm{Cr}, \mathrm{Zn}, \mathrm{P}, \mathrm{Cu}$ (Tableau 9) ;

- Fers de récupération modernes dont on ne connaît pas la nature exacte.

Les scories de forge se forment dans le fond du foyer à partir de quatre différentes contributions :

- Fer sous forme de croûte d'oxyde ou particules de métal ;
Charbon de bois) : il apporte majoritairement $\mathrm{CaO}$ et $\mathrm{K}_{2} \mathrm{O}$ mais aussi une part variable de $\mathrm{MgO}, \mathrm{P}_{2} \mathrm{O}_{5}$, $\mathrm{MnO}$ et $\mathrm{TiO}_{2}$;

Parois et tuyères du foyer :

- ) : elles apportent en particulier $\mathrm{SiO}_{2}$ et $\mathrm{Al}_{2} \mathrm{O}_{3}$ et un peu de $\mathrm{K}_{2} \mathrm{O}$; à Kakoli, elles sont riches en $\mathrm{CaO}$ (3.1.3) ;

- Ajouts volontaires de grès $\left(\mathrm{SiO}_{2}\right)$, argile $\left(\mathrm{SiO}_{2}, \mathrm{Al}_{2} \mathrm{O}_{3}, \mathrm{~K}_{2} \mathrm{O}\right)$ et barytine $(\mathrm{Ba})$.

Les 126 opérations de forgeage ont livré 80 scories de forge qui ont été étudiées : 56 sont simples (une scorie $=$ un forgeage), 6 sont doubles (une scorie $=$ deux forgeages) et 18 sont triples (une scorie $=$ trois forgeages) (Tableau 26).

L'état de conservation des scories est en effet un critère important à prendre en compte. L'étude morphologique des scories de forge est préférable sur des pièces complètes, afin de prendre en compte la globalité des paramètres quantitatifs et qualitatifs utilisés pour décrire ces matériaux. Toutefois, même si la scorie est cassée, il est possible de décrire chaque fragment et de conclure avec prudence sur l'entièreté de la pièce.

Lorsque des scories sont mises au jour sur des sites d'anciennes forges, seules les pièces les plus solides sont conservées intactes. Pour les autres, il est important de recoller ensemble les différentes parties si c'est possible.

\begin{tabular}{|c|c|c|c|c|c|}
\hline Type de scorie & $\begin{array}{c}\text { Nombre de } \\
\text { scories }\end{array}$ & $\begin{array}{l}\text { Equivalent nombre } \\
\text { de forgeages }\end{array}$ & Scorie en calotte & Scorie réduite & Scorie "informe" \\
\hline Simple & 56 & 56 & 39 & 6 & 11 \\
\hline Double & 6 & 12 & 5 & - & 1 \\
\hline Triple & 18 & 54 & 16 & - & 2 \\
\hline Nombre étudiés & 80 & 122 & 60 & 6 & 14 \\
\hline Non pris en compte & 6 & $\begin{array}{l}\text { F001 foyer sale } \\
\text { F009 machette } \\
\text { F019 machette } \\
\text { F031 foyer sale } \\
\text { F102 pas de scorie } \\
\text { F104 scorie cassée }\end{array}$ & $\begin{array}{l}\text { S001 } \\
\text { S009 } \\
\text { S019 } \\
\text { S031 }\end{array}$ & - & $\begin{array}{l}\text { S102 n'existe pas } \\
\text { S104 cassée }\end{array}$ \\
\hline Nombre total & 86 & 128 & 64 & 6 & 16 \\
\hline
\end{tabular}

Tableau 26 - Récapitulatif du nombre de scories et opérations de forgeage prises en compte. 


\section{0 scories en calotte}

Sur les 80 scories, 60 sont en forme de calotte, comportant plus ou moins de petites pièces, ainsi que 6 scories en calotte réduite. Pour les 60 scories en calotte, il a été facile de récupérer la globalité du matériel car le foyer était vidangé avant chaque forgeage ou groupement de forgeage. Les scories cassées ont systématiquement été remontées. Elles sont toutes conservées quasiment en intégralité : 90 à $100 \%$.

\section{6 scories réduites}

Les 6 scories en calotte réduite correspondent en fait à des pièces qui étaient entières à leur sortie du foyer, mais qui ont été considérablement réduites et fractionnées lors de leur transport (casse) et de leur lavage (friabilité) : perte entre 50 et $70 \%$ de leur poids initial. Elles ne ressemblent donc plus à des scories en calotte conventionnelles.

\section{4 scories « informes »}

A ces scories en calotte s'ajoutent 14 scories « informes » qui sont des petites pièces indépendantes de formes irrégulières. Il n'est pas exclu que certaines pièces n'aient pas été ramassées et soient restées dans le lit de charbons du foyer (scorie et charbon ont une taille, couleur et forme similaires).

Dans notre corpus de scories, le groupe de scories « informes » rassemble :

- des fragments de scorie pour lesquels il est impossible de déterminer la forme de départ; l'état de surface montre des cassures ;

essentiellement des pièces individuelles avec des surfaces naturelles de refroidissement, lisses ou déchiquetées. Alors que les scories en calotte se forment par accumulation de matériaux au fond du foyer, les pièces individuelles pourraient s'assembler dans un endroit quelconque du foyer, dans le lit de charbons, sous des conditions de formation diverses: hétérogénéité de la température par exemple.

\section{Scories non comptabilisées}

On notera que 6 scories (correspondant à 6 forgeages) ne sont ni comptabilisées ni étudiées par la suite :
- S001 et S031 pour lesquelles le foyer n'a pas été nettoyé,

- S009 et S019 qui résultent du travail d'une lame de machette,

- S102 n'existe pas car le forgeage F102 (Doundé) n'a pas donné de scorie,

- S104 qui, trop fragmentée, et n'a pas pu être remontée.

\section{Quatre groupes de scories}

Ce travail permet de distinguer quatre groupes de scories. Les critères de morphométrie, de nature des matériaux et de minéralogie, les conditions des forgeages et de stratification seront présentés un à un pour chacun des quatre groupes de scories.

1. Le groupe des scories argilo-sableuses SAS. Ce groupe comprend deux sous-groupes : SAS avec des scories qui ont la particularité de ne contenir que très peu $(<10 \%)$ voire pas du tout de scorie grise dense à base de fayalite (SGD), et SAS-M avec des scories qui contiennent jusqu'à $20 \%$ de matériel SGD.

2. Le groupe des scories grises denses SGD réparties en trois sous-groupes : SGD-B avec des scories comportant un bourrelet argilo-sableux; SGD-SFR avec des scories à base de fayalite et de fer ; enfin SGD-K rassemble des scories grises et denses, à base d'olivine calcique (kirschstéinite).

3. Le groupe des scories ferreuses rouillées SFR. Il s'agit de scories montrant une prédominance de fer à l'état de métal et plus ou moins d'oxydes de fer

4. Le groupe des scories «informes» SI. Seules les plus grandes pièces ont pu être coupées et étudiées. Concernant les scories qui n'ont pas pu être coupées, nous avons des données minéralogiques et chimiques mais aucune donnée de texture.

Un aperçu de l'ensemble de ces pièces est donné dans le Catalogue des scories, à télécharger sur internet. 


\section{Encart 3 - Code-couleur et nomenclature}

Pour chaque atelier, les données sont indiquées par des couleurs différentes :

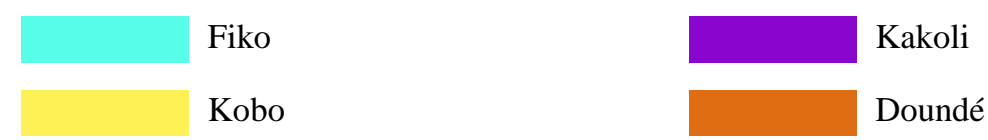

Les matériaux contenus dans les scories de forge sont différenciés par le code de couleurs suivant :

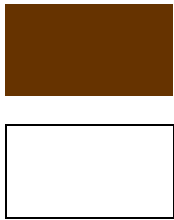

Argilo-sableux : matériau

siliceux plus ou moins fondu

Fayalitique : matériau grisé ou jaunâtre plus ou moins poreux

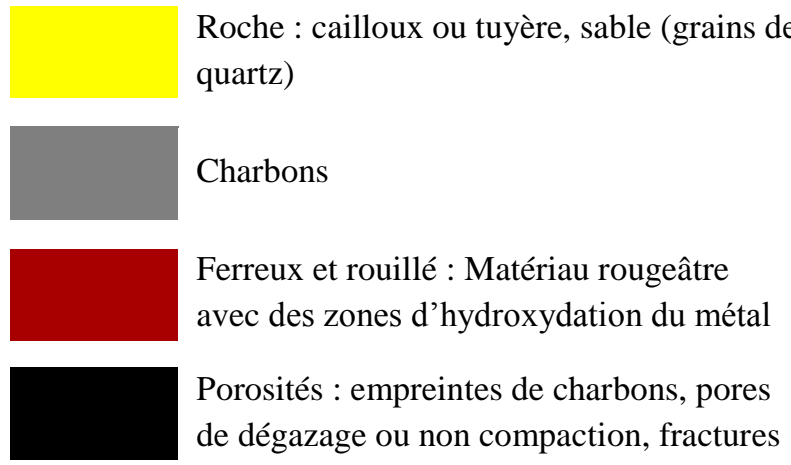

Autres matériaux

de dégazage ou non compaction, fractures

Ce code couleur est appliqué dans la méthode de calcul de surface (Figure 68). Cette méthode permet, grâce à une observation de la coupe des scories de forge, macroscopique en premier lieu puis microscopique en lumière réfléchie, de calculer le pourcentage de surface de chaque matériau. Les pourcentages de surface obtenus sont à l'origine de la classification des scories de forge (Figure 3).

Nomenclature : $\mathrm{S}+\mathrm{n}^{\circ} \mathrm{du}$ (des) forgeage (s)

$\mathrm{S} x \mathrm{xx}$ : scorie simple (récoltée après un forgeage)

$\mathrm{S}$ xxx-Xxx : scorie multiple (après deux forgeages)

$\mathrm{S} \mathrm{xxx}-\mathrm{Xxx}-\mathrm{Xxx}$ : scorie multiple (trois forgeages)

\subsubsection{Morphométrie}

Sur les 80 scories, $20 \%$ sont composées d'une seule et unique pièce, tandis que $80 \%$ sont accompagnées de plusieurs petites pièces. En moyenne ce sont cinq pièces de scorie qui sont récoltées : c'est-à-dire une pièce principale et quatre petites pièces. Archéologiquement, ces petites pièces indépendantes sont retrouvées mais il est alors impossible de les relier à une pièce principale de scorie.

\section{Scories SAS}

Le groupe des scories SAS comporte :

- 7 scories SAS

- 7 scories SAS-M (Figure 78).

Les scories se partagent entre des formes en calotte $(n=8)$ et pièces réduites $(n=6)$, ces dernières provenant toutes de l'atelier de Kobo.
Pour l'ensemble des SAS, le poids varie entre $20 \mathrm{~g}$ (S026) et $300 \mathrm{~g}$ (S003), avec une moyenne assez faible de $115 \mathrm{~g}$ pour les SAS et $130 \mathrm{~g}$ pour les SAS-M (Figure 82). Le diamètre maximal varie entre $7 \mathrm{~cm}$ et $16 \mathrm{~cm}$, avec une moyenne de $11 \mathrm{~cm}$ pour les SAS et $10 \mathrm{~cm}$ pour les SAS-M. Par ailleurs, les trois scories multiples (SAS-M) correspondent aux pièces les plus lourdes, mais elles ne sont pas les plus volumineuses.

Près de $50 \%$ des pièces sont concaves, environ $30 \%$ sont irrégulières (scories réduites de Kobo), le reste $(20 \%)$ correspondant à des scories planes et plano-convexes.

\section{Scories SGD}

Le groupe des scories SGD comporte 33 scories en calotte dont :

- 15 scories SGD-B,

- 10 scories SGD-SFR,

- 8 scories SGD-K (Figure 79).

Pour l'ensemble des scories, le poids varie entre $135 \mathrm{~g}$ (S067) et $860 \mathrm{~g}$ (S099), avec une 
moyenne de $360 \mathrm{~g}$ pour les SGD-B, de $230 \mathrm{~g}$ pour les SGD-SFR et de $300 \mathrm{~g}$ pour les SGD-K (Figure 82). Leur diamètre maximal varie entre $7 \mathrm{~cm}$ (S117) et $17 \mathrm{~cm}$ (S074) avec une moyenne relativement similaire de $13 \mathrm{~cm}$ pour les SGD-B, de $11 \mathrm{~cm}$ pour les SGD-SFR et de $12 \mathrm{~cm}$ pour les SGD-K. Par ailleurs, ces dernières ont toutes des dimensions très standardisées puisqu'elles varient uniquement dans la plage 12.0-13.5 cm. Enfin, les scories multiples ne sont ni les plus lourdes et ni les plus volumineuses.

Les SGD-B sont toutes des scories à bourrelet uniquement $(100 \%)$ tandis que les SGD-SFR montrent différents types de profils: biconvexe (40\%), plano-convexe $(30 \%)$, plan $(20 \%)$ et concave $(10 \%)$. Les scories SGD-K sont quant à elles majoritairement concaves (62\%), plano-convexes $(25 \%)$ mais aussi planes $(13 \%)$.

\section{Scories SFR}

Le groupe des scories SFR compte 15 scories en calotte (Figure 80). A la fois le poids et la longueur des scories varient fortement (Figure 82).
Le poids est situé entre $90 \mathrm{~g}$ (S056) et $860 \mathrm{~g}$ (S045), avec une moyenne de $215 \mathrm{~g}$. Le diamètre maximal est compris entre $6.5 \mathrm{~cm}$ (S120) et $12.8 \mathrm{~cm}$ (S045), avec une moyenne située à $9 \mathrm{~cm}$. Par ailleurs, les scories multiples qui sont les plus lourdes ne sont pas forcément les plus volumineuses.

Les scories SFR ont des profils très divers : les pièces biconvexes $(40 \%)$ et concaves $(33 \%)$ sont les plus représentées, mais on trouve aussi des pièces planes $(20 \%)$ puis plano-convexes $(7 \%)$.

\section{Scories SI}

Le groupe des scories «informes» SI rassemble 18 scories (Figure 81). Pour l'ensemble des pièces, le poids varie entre $3 \mathrm{~g}$ (S040) et $60 \mathrm{~g}$ (S037), avec une moyenne très faible de $25 \mathrm{~g}$ (Figure 82 ). Le diamètre maximal varie quant à lui entre $1.5 \mathrm{~cm}$ (S038, S040) et $7.5 \mathrm{~cm}$ (S037), avec une moyenne peu élevée de $4 \mathrm{~cm}$. Il s'agit de scories au profil irrégulier (billes, rognons, nodules) composées de plusieurs petites pièces, jusqu'à 14 éléments. Seule S032 est véritablement plane (6\%).

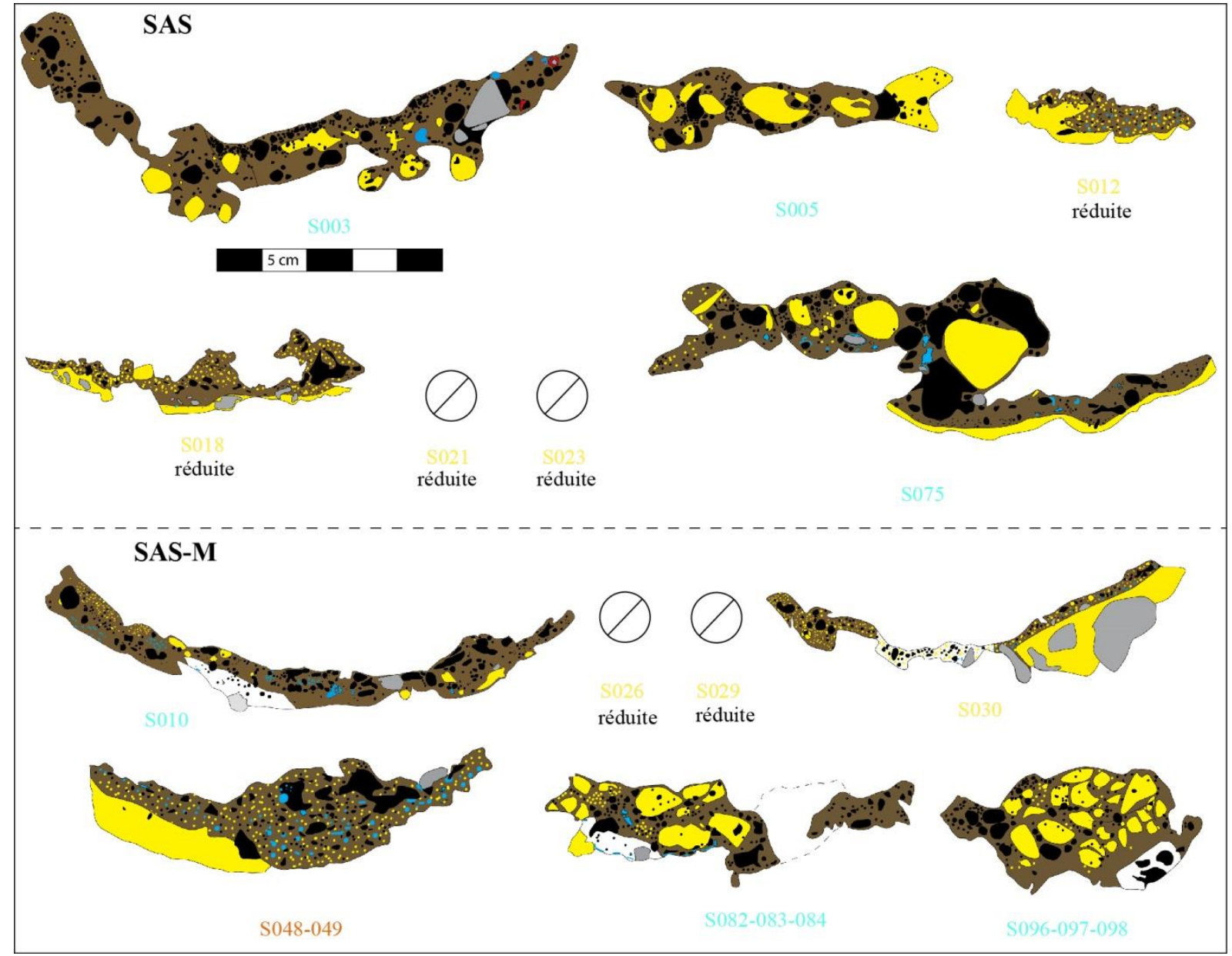

Figure 78 - Les scories du groupe SAS (Encart 3 - Code-couleur).

Cercles barrés : scories trop friables pour lesquelles une coupe et une section polie n'ont pas pu être réalisées. 


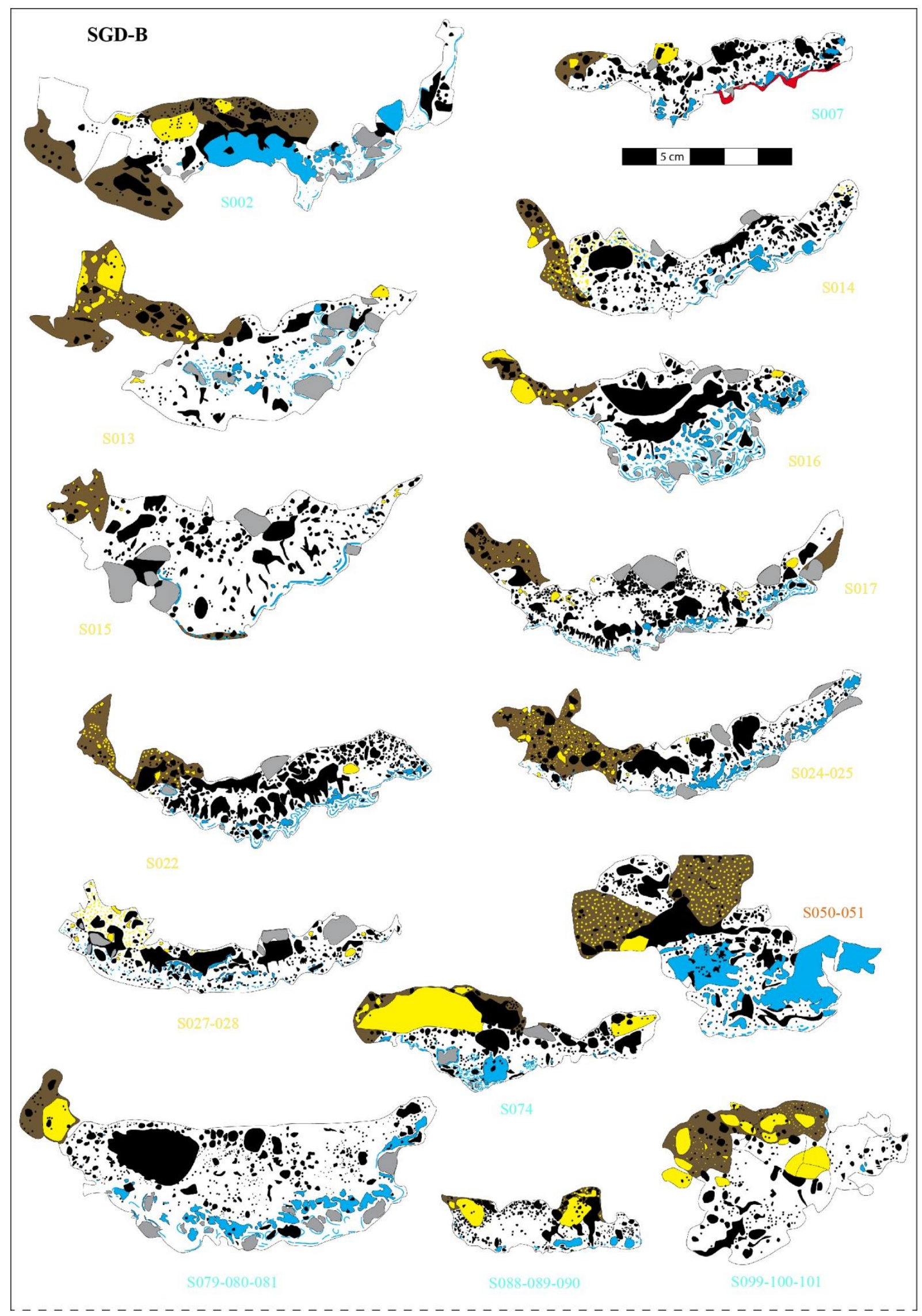

Figure 79 - Première partie des scories du groupe SGD (Encart 3 - Code-couleur ). Ici les SGB à bourrelet. 


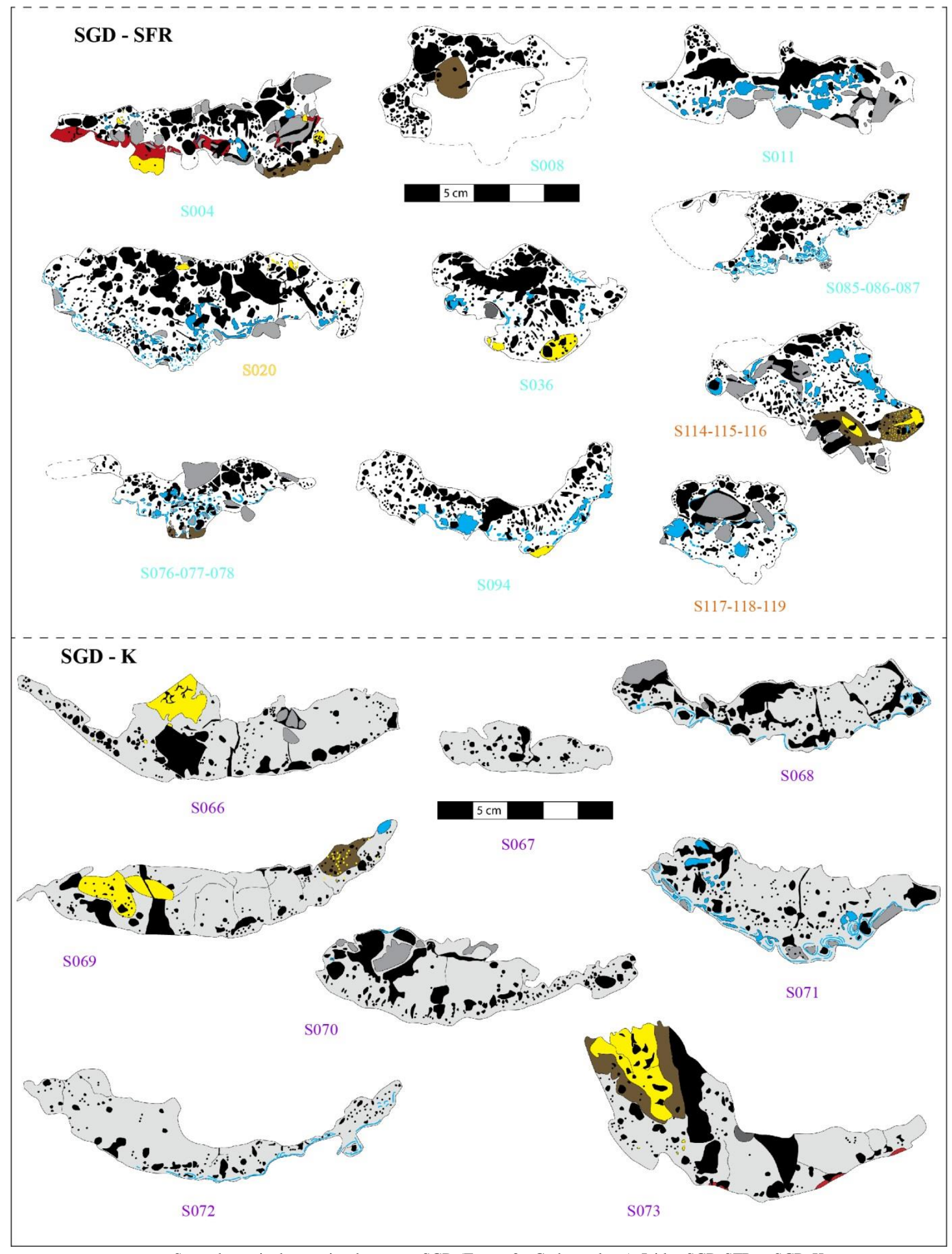

Seconde partie des scories du groupe SGD (Encart 3 - Code-couleur ). Ici les SGD-SFR et SGD-K. 


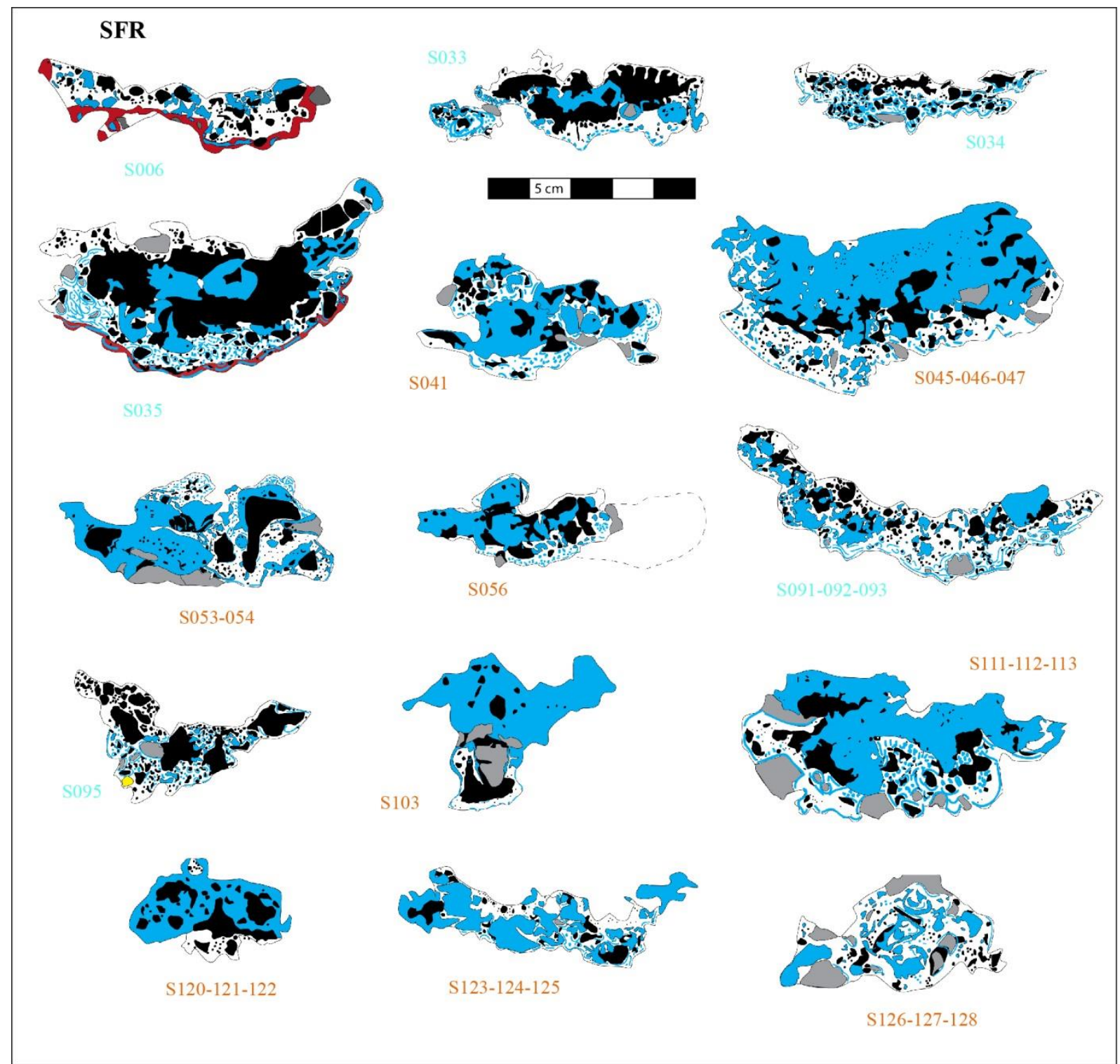

Figure 80 - Les scories du groupe SFR (Encart 3 - Code-couleur ).

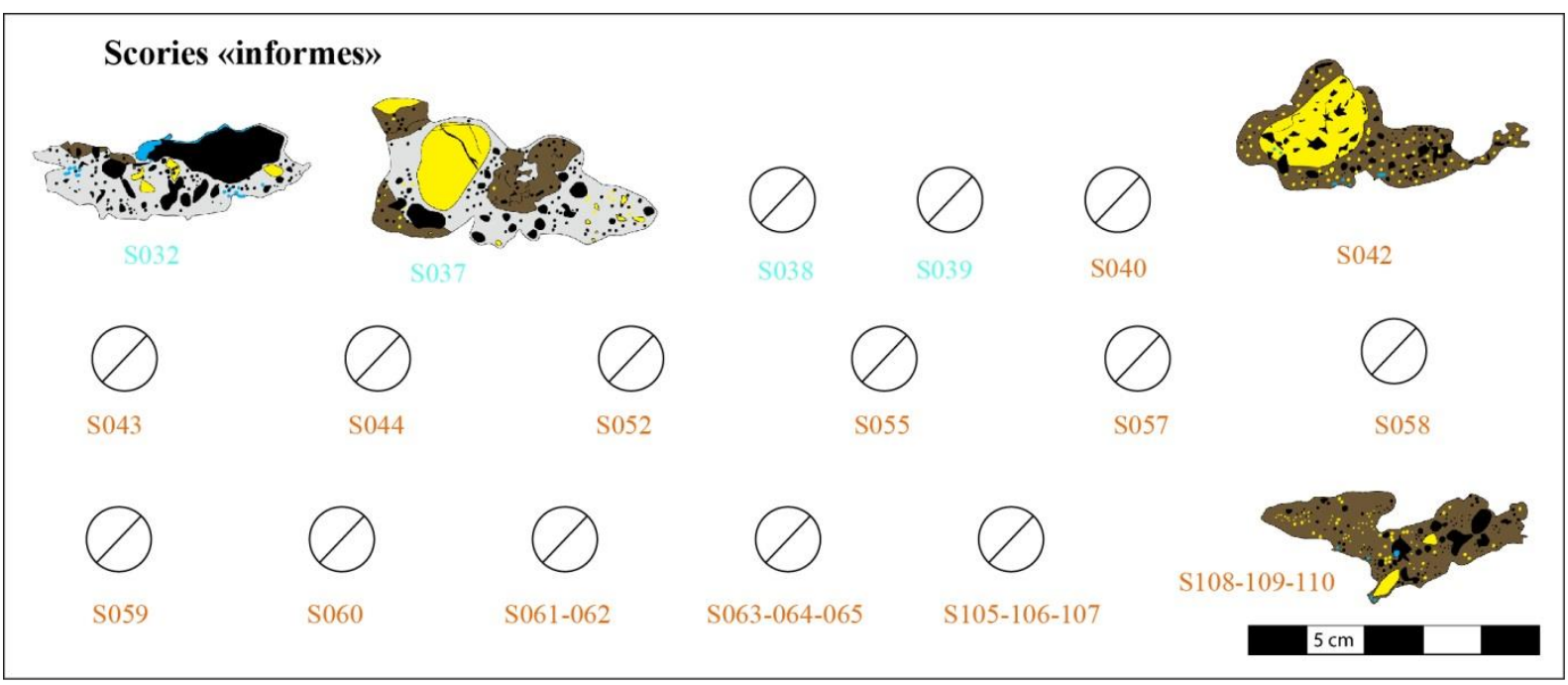

Figure 81 - Les scories du groupe SI (Encart 3 - Code-couleur ).

Cercles barrés : scories de dimensions trop petites, pas de coupe, voir le catalogue des scories de forge pour une vue complète de la pièce. 


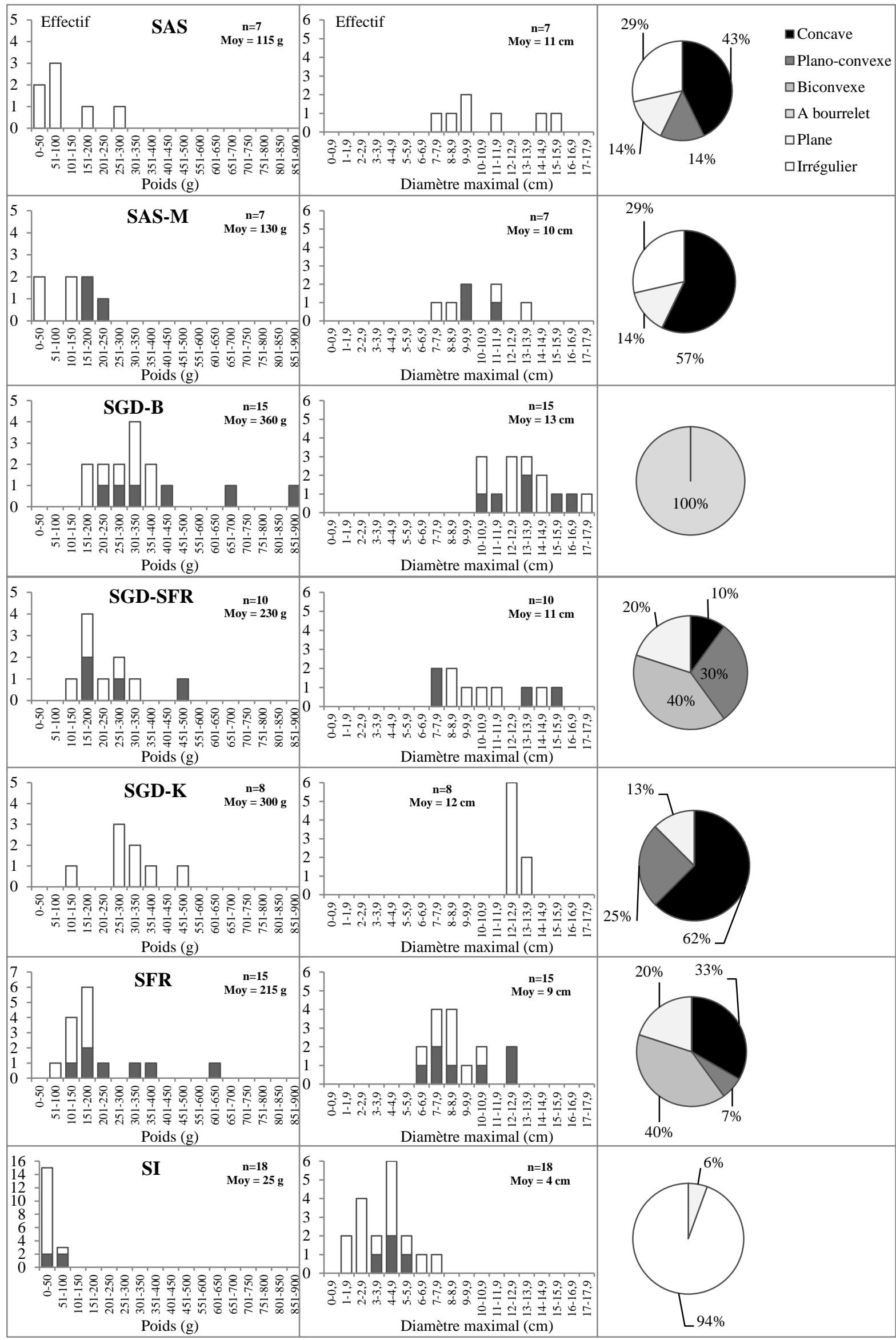

Figure 82 - Poids, diamètre maximal et profil des scories. Les barres sombres des histogrammes correspondent à des scories multiples. 


\section{Taille et poids}

La taille et poids sont influencés par plusieurs paramètres contrôlés ou non contrôlés du forgeage (Figure 83).

Pour les SAS et les SI, le poids est proportionnel à la longueur (A) des scories (sauf pour certaines pièces plus lourdes qui correspondent à des scories issues de plusieurs forgeages) ; SI restent plus petites et les moins lourdes ; les SAS ont la plus faible densité ; pour les SGD et les SFR le poids reste proportionnel à la longueur dans une certaine gamme et leur densité est plus élevée. Certaines pièces SGD et SFR sont quant à elles bien plus lourdes et plus denses que les autres.

Pour comprendre l'influence de l'apport des fondants siliceux sur le poids des scories, seules les

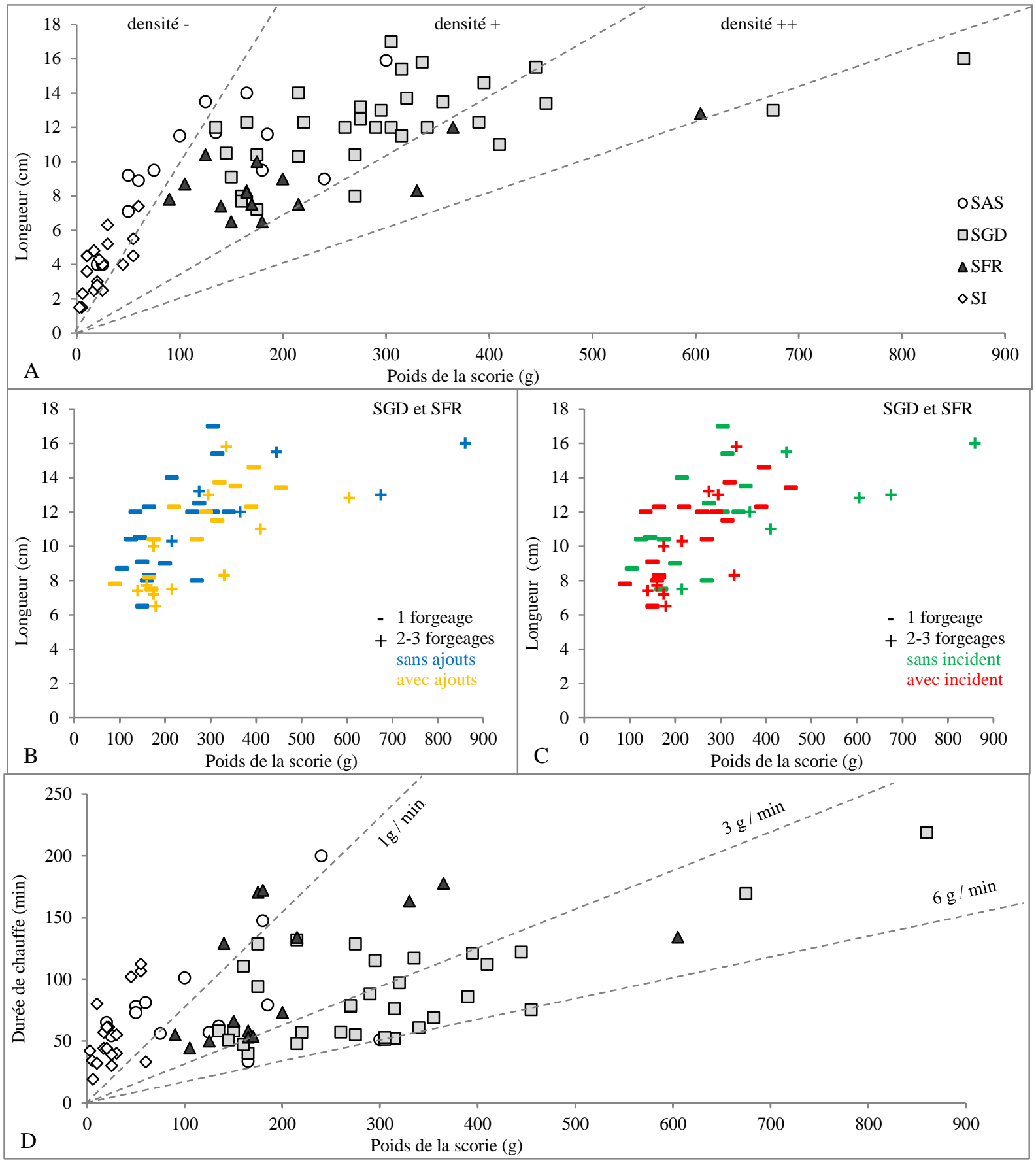

Figure 83 - Relations poids/longueur des scories par type de scorie (A), nombre de forgeages et ajouts (B) et incidents (C). Relation poids/durée de chauffe par type de scorie (D). 
SGD et SFR sont comparées (B) : les scories qui ont reçu un apport volontaire de matières siliceuses ont tendance à être plus lourdes que les autres scories ; de même, les scories issues d'une journée de travail (2-3 forgeages) ont aussi tendance à être plus lourdes que celles issues d'un seul forgeage. Toutefois, ces tendances ne sont pas très marquées.

Les incidents qui surviennent au cours des forgeages n'ont pas d'influence sur le poids ou la taille des scories (C).

Les divers types de scories présentent des taux de formation dans le foyer très différents les uns des autres (D). Pour les SAS et SI, moins de $1 \mathrm{~g}$ de scorie est formé par minute de chauffe ; les SFR et des SGD se forment entre 1 à $6 \mathrm{~g} / \mathrm{min}$ de chauffe. Ces taux de formation reflètent les conditions de forgeage : pour des forgeages qui nécessitent de souder des barres de fer, la température élevée du foyer implique une augmentation de l'apport en fer (croûtes d'oxydes, particules de métal) et en silice (ajouts, paroi) et donc aussi une augmentation du taux de formation des scories SGD et SFR par rapport aux scories SAS et SI.

\section{Ellipticité et aplatissement}

Après avoir déterminé ou supposé l'orientation des scories dans le foyer, il est possible de calculer leur ellipticité et aplatissement qui définit leur forme générale (Figure 84). Cette forme reflète en réalité celle de la zone la plus chaude à l'intérieur du foyer.
Tout d'abord, la forme des scories (ronde, plate etc) est indépendante de leur nature (SAS etc) (A).

Ensuite, les scories de Kobo et de Kakoli montrent une certaine standardisation puisqu'ils fournissent des scories rondes et leur aplatissement est : $A=2.5 \pm 0.5$ pour Kobo et $A=3.0 \pm 0.5$ pour Kakoli (A).

Si plusieurs forgeages successifs sont réalisés dans la journée, la scorie résultante devrait être plus haute qu'une scorie résultant d'un seul et unique forgeage (accumulation successive de plusieurs scories). Cependant cette hypothèse ne semble pas être vérifiée $(B)$.

Une soudure entraîne une perte de matière supplémentaire par rapport à un forgeage sans soudure : les scories provenant de forgeages avec soudures devraient donc être plus hautes que celles qui proviennent de forgeages sans soudure. Effectivement, l'aplatissement est fort pour un forgeage sans soudure (scorie basse) et il est faible pour un forgeage avec soudure (scorie haute) (B).

L'étape du préformage avec la soudure est de nouveau mise en évidence comme un paramètre important du forgeage qui influe sur la morphologie des scories qui se forment dans le foyer.

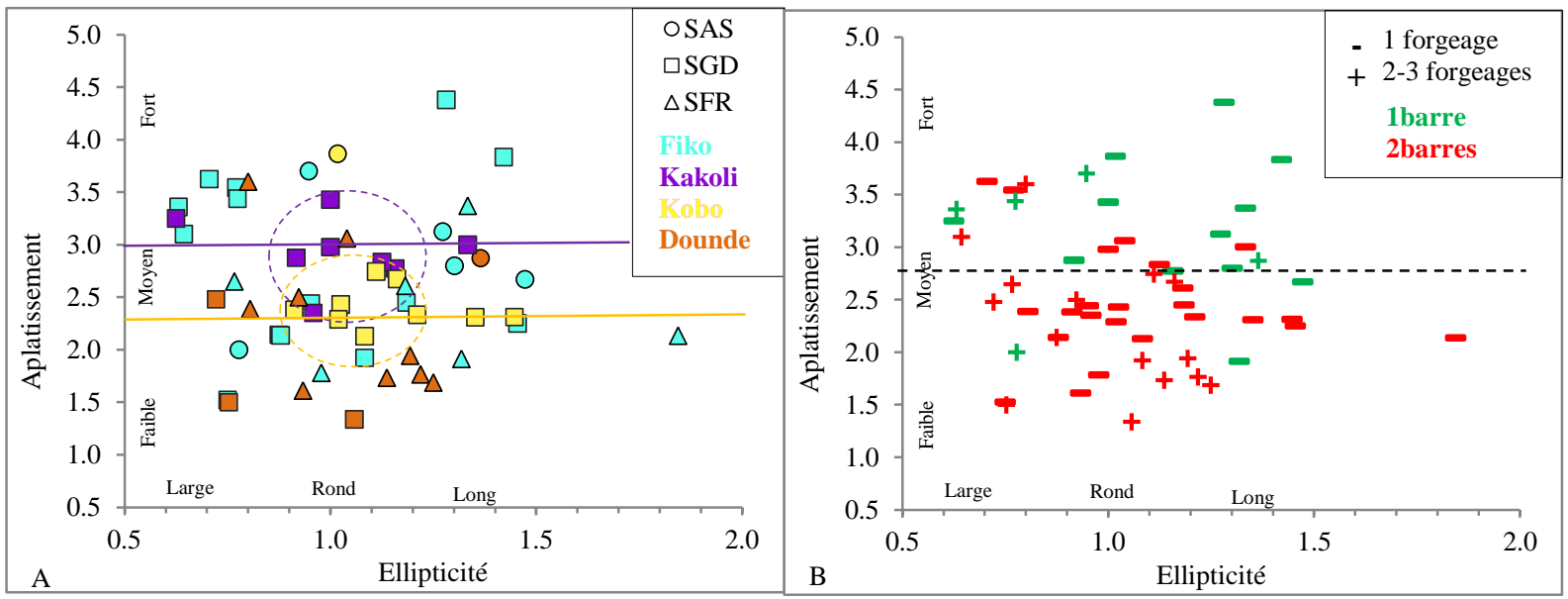

Figure 84 - Ellipticité et aplatissement des scories SAS, SGD et SFR produites dans chaque atelier.

$\mathrm{N}=56$. Scories réduites et classe SI non comptées. 


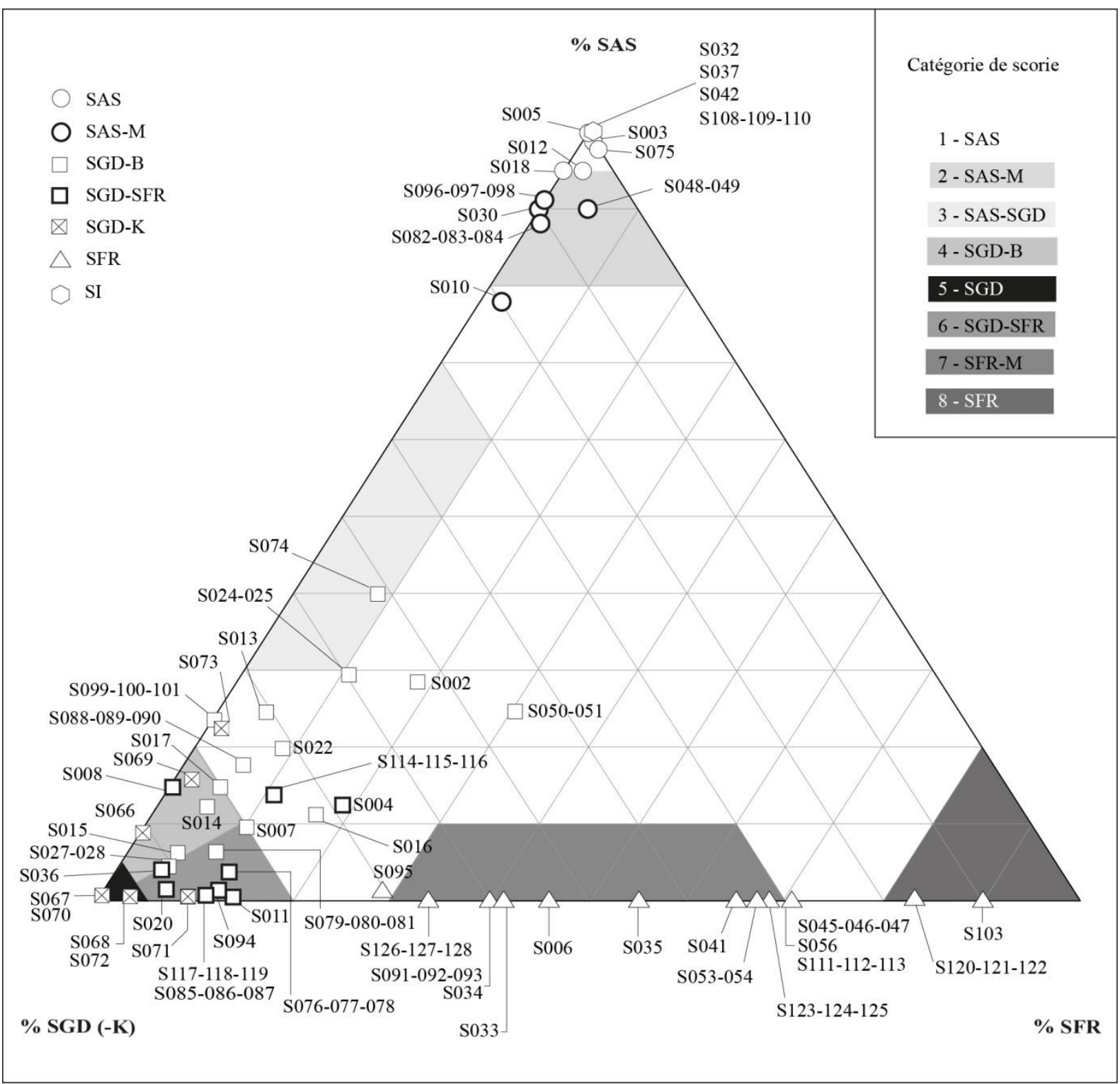

Figure 85 - Classification des scories de forge selon le type de matériaux ( $n=78$ ), d'après Serneels \& Perret $2003: 475$ et Figure 3. Les scories réduites et «informes » (sauf les quatre plus grandes pièces) ne sont pas représentées.

S008 n'est pas à sa juste place car elle comportait en réalité un noyau métallique qui a été expulsé lors du sciage de la pièce.

\subsubsection{Matériaux et minéralogie}

Le détail de l'étude et de la classification minéralogique en groupe est indiqué dans l'Annexe 4 - Scories.

\section{Scories SAS}

Les scories SAS et SAS-M sont situées au pôle argilo-sableux dans le triangle de classification selon les matériaux (Figure 85).

Les SAS sont composées quasi exclusivement de grains de quartz plus ou moins fondus, cailloux et roches centimétriques non fondus dans un verre plus ou moins riche en fer (Figure 86). L'étude en XRD montre que les SAS appartiennent au groupe minéralogique $\mathrm{Q}$ pour lequel la silice (quartz et cristobalite pris ensemble) est le constituant principal à hauteur de 75-100\%. Dans trois scories, du fer métallique et de l'augite sont identifiés. Dans les quatre autres scories, seule de la fayalite est identifiée de manière accessoire $(<5 \%)$.

Les SAS-M sont composées de grains de quartz plus ou moins fondus, et de roches, cimentés dans un verre contenant des cristaux de fayalite naissants et des petites particules de métal (Figure 87). En totalité, cinq scories ont leur surface inférieure comportant une couche compacte de sable aggloméré. Les SAS-M appartiennent quant à elles au groupe minéralogique $\mathrm{QF}$ dans lequel la silice reste le constituant principal (45-80\%) mais cette fois-ci avec une faible part de fayalite (15-40\%). 
Plusieurs paragénèses existent: avec ou sans fer métallique et/ou augite.

Pour l'ensemble des SAS la porosité est abondante, de $25 \%$ à plus de $50 \%$ dans la coupe. Cette porosité provient des matériaux argilo-sableux eux-mêmes poreux et des bulles de gaz qui sont restées piégées dans la scorie lors de son refroidissement (Figure 86). Les pores sont de forme arrondie : les plus gros mesurent jusqu'à $1 \mathrm{~cm}$ et les plus fins sont inférieurs à $1 \mathrm{~mm}$. Ils sont répartis aléatoirement dans les scories.

En coupe, il est observé en moyenne $2 \%$ de particules ferreuses (min 0\%-max $5 \%$ ), le reste du métal perdu ayant été assimilé par le système puis cristallisé sous la forme de minéraux comme la fayalite $\mathrm{Fe}_{2} \mathrm{SiO}_{4}$. Les particules observées en coupe correspondent à des fins filaments de métal, quelques billes de métal, des petites particules jusqu'à $5 \mathrm{~mm}$ mais surtout systématiquement des croûtes d'oxydes plates.

L'aimantation des scories est faible localement, voire nulle. Ces particules sont constituées uniquement de ferrite dans le cas des

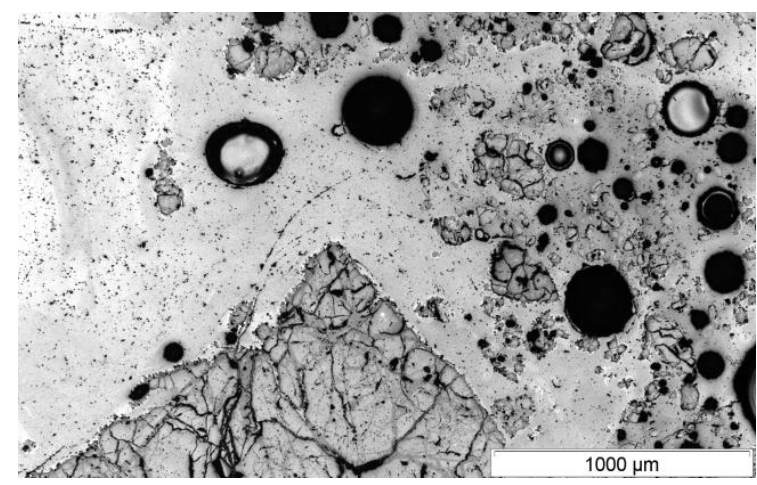

Figure 86 - SAS : grains de quartz et porosités circulaires dans une matrice vitreuse plus ou moins ferreuse (MM_S003_1). Microscope optique en lumière réfléchie.

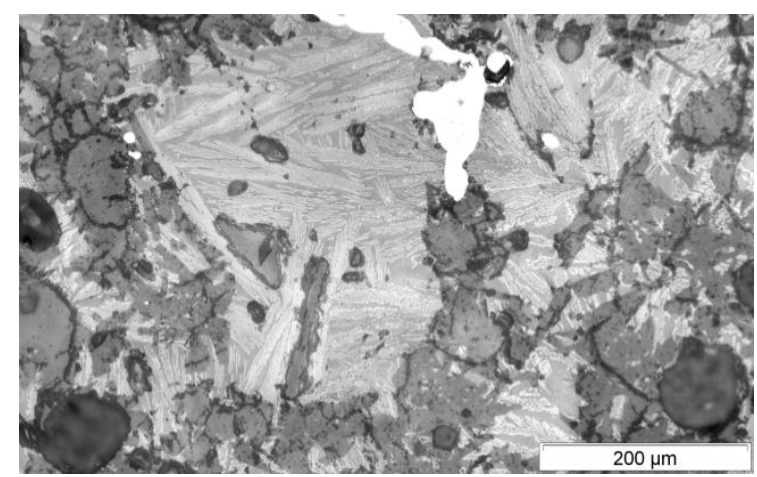

Figure 87 - SAS-M : cristaux naissants de fayalite (gris clair) au milieu de grains de quartz (gris foncé). Peu de verre interstitiel. Croûte d'oxyde plate brisée (MM_S048-049_1a). Microscopie optique en lumière réfléchie.
SAS. Pour les SAS-M, $50 \%$ des particules sont constituées de ferrite, le reste des particules est composé d'acier qui peut parfois atteindre plus que $0.8 \% \mathrm{C}$

\section{Scories SGD}

Les scories SGD occupent le pôle SGD du triangle de classification d'une manière large (Figure $85)$.

Les SGD-B sont surmontées d'un bourrelet argilo-sableux plus ou moins proéminent : bourrelet massif (S050) à la couche vitreuse superficielle (S074). Les SGD-SFR contiennent quant à elles une part limitée de matériel argilo-sableux et de métal ; elles occupent le pôle SGD de manière plus restreinte.

A l'œil nu, deux faciès existent et peuvent coexister : des scories à dominante brune et sombre et des scories à dominante jaune et claire. Au microscope, les scories sombres possèdent des cristaux de fayalite bien formés sous divers habitus (Figure 88); les scories claires ont des cristaux uniquement en baguettes / pavés moins bien formés

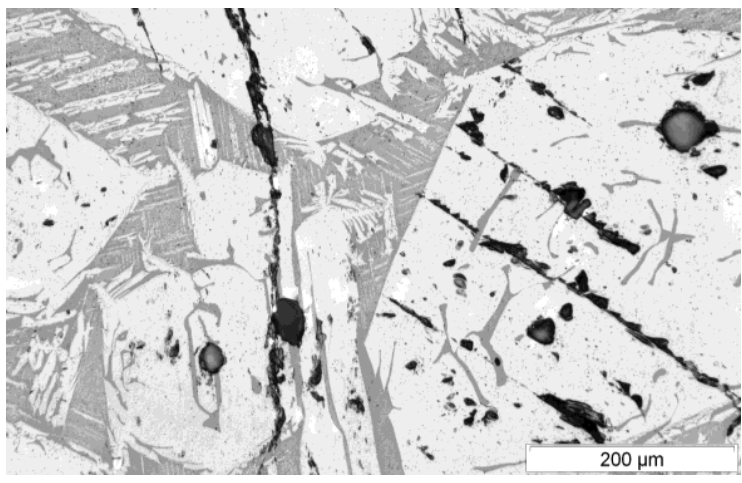

Figure 88 - SGD-B et SGD-SFR, scories sombres : cristaux de fayalite bien formés, sous différents habitus (MM_S015_1a). Microscope optique en lumière réfléchie.

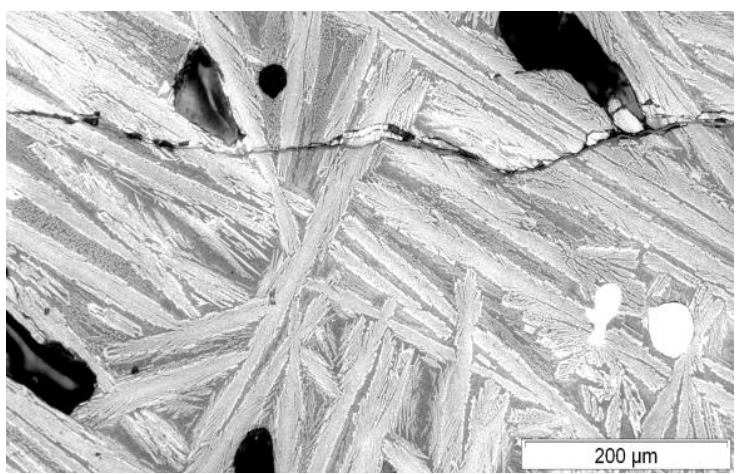

Figure 89 - SGD-B et SGD-SFR, scories claires : cristaux de fayalite en baguettes desserrées (MM_S050-051_5a). Microscope optique en lumière réfléchie. 
(Figure 89). De plus, S013, S027, S099 montrent un empilement de zones de cristaux de fayalite possédants divers habitus; S004 et S094 sont formées de cristaux trapus en partie basse et de cristaux en baguettes et en pavés en partie haute. Dans quatre cas, du matériel argilo-sableux est disposé dans la base des scories et provient de l'arrachement $\mathrm{du}$ fond $\mathrm{du}$ foyer (agglomération thermique) et/ou des parois du foyer.

Concernant l'étude minéralogique en XRD, les scories SGD-B comportent une part variable de quartz et cristobalite inversement proportionnelle à la part de fayalite: QF à dominante de silice (45$80 \%)$, FQ à dominante de fayalite (60-80\%), F qui comporte quasi-uniquement de la fayalite (75$100 \%$ ), ne comprend qu'une seule scorie. De l'augite est détectée en XRD en faible quantité $(<5 \%)$ dans trois pièces (S002, S007, S099) et des minéraux de leucite sont identifiés dans S016. Ces minéraux n'ont cependant pas été observés au microscope.

Les scories SGD-SFR appartiennent également aux trois groupes minéralogiques précédents mais cette fois la fayalite y est plus présente. De l'augite est identifiée dans S004 et de la leucite est détectée dans S036. Ce dernier minéral est observé en association symplectique avec la wüstite (Figure 74).

La porosité de ces deux groupes de scories n'est pas négligeable puisqu'elle est comprise entre 20 et $40 \%$ (moy $30 \%$ ). Les plus gros pores sont disposés horizontalement et affleurent la surface. Sinon, ils sont dans le milieu ou la base de la scorie, en fines bulles verticales. Les pores les plus fins sont répartis aléatoirement.

Les SGD-B et SGD-SFR comptent en moyenne $10 \%$ de particules ferreuses en coupe (min $0 \%$-max $31 \%$ pour S050). Toutefois les SGD-B ont une aimantation faible à moyenne alors que les SGDSFR ont une aimantation moyenne à forte.

Parmi les particules ferreuses observées, on compte surtout des particules $<5 \mathrm{~mm}$ dont des filaments, des croûtes d'oxydes et des billes éclatées disposées dans le fond ou le milieu des pièces (Figure 73, cliché 12). La présence d'oxydes de fer provient de l'oxydation à chaud du métal lors de sa chauffe (11 et 12) mais aussi de la formation d'oxydes sur le métal contenu par la scorie du fait de l'atmosphère oxydante du foyer de forge. Le faciès métallographique de ces particules de métal est très variable : chaque scorie peut comporter à la fois des particules de ferrite et des particules d'acier.
Pour information: S002 et S050-051 contiennent uniquement des blocs de fer $(>10 \mathrm{~mm})$; S008 ne montre pas de fer en coupe, cependant elle renfermait un noyau de fer qui a été expulsé lors du sciage de la pièce.

Les scories SGD-K sont composées d'une matrice d'olivine calcique (silicate de fer et de calcium), la kirschstéinite $\mathrm{CaFeSiO}_{4}(0)$, comportant des fragments de roche (S066, S069) ou de tuyère (S073). La kirschstéinite est représentée à hauteur de 75 à $100 \%$ dans la coupe (Figure 85 ).

$\mathrm{Au}$ microscope, les cristaux de kirschstéinite sont en présence de très nombreux oxydes de fer tels wüstite et magnétite (Figure 90, Figure 91). Ce minéral, déjà identifié dans des cas de scorie de réduction (Leroy 1997), est toutefois rarement cité dans le cas de scories de forge (Oudbashi et al. 2009 : 409-Figure2). Dans certaines scories, il s'agit soit d'un minéral situé entre une fayalite et une kirschstéinite (Joly et al. 2011: 28), soit d'un minéral «comme de la fayalite » riche en calcium $(18-50 \% \mathrm{CaO})$ et également associé à des oxydes de fer (Renzi \& Rovira 2009 : Table1-Figure2a).

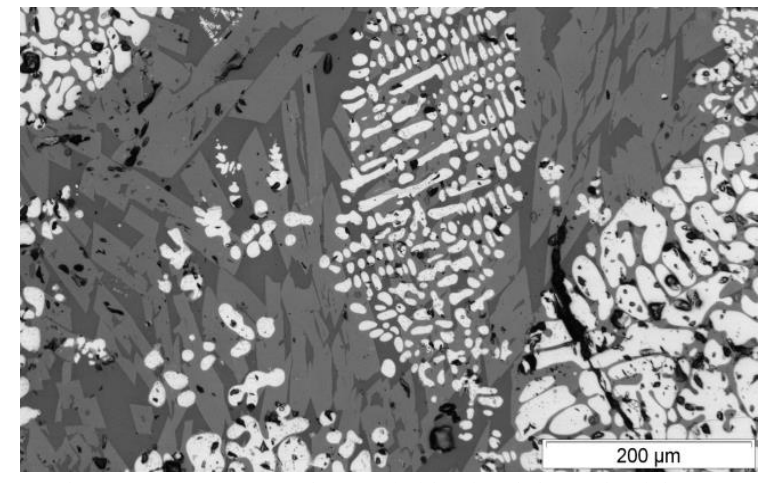

Figure 90 - SGD-K : cristaux de kirschstéinite (gris clair) et croûtes d'oxydes (wüstite) dans un verre sombre (MM_S070_1a). Microscope optique en lumière réfléchie.

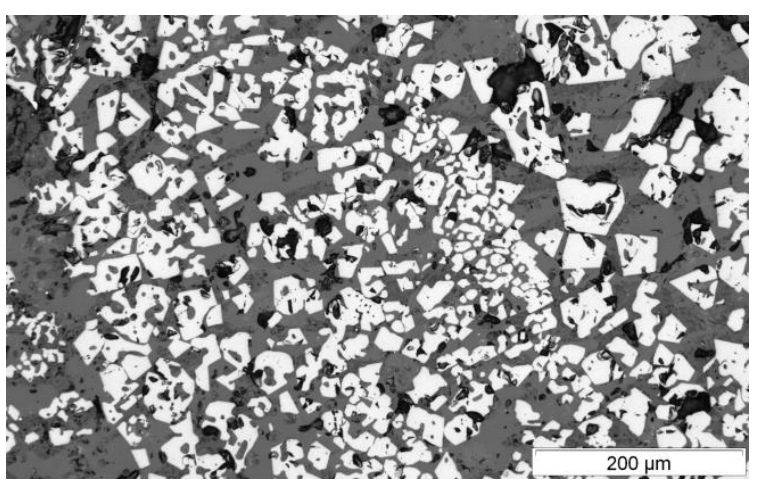

Figure 91 - SGD-K : cristaux de kirschstéinite (gris clair) et cristaux de magnétite (blanc) (MM_S072_1a). Microscope optique en lumière réfléchie. 
En XRD, les scories SGD-K montrent une minéralogie complexe avec de la kirschstéinite, des oxydes de fer et d'autres minéraux tels la leucite et l'akermanite. Dans ce groupe, quasiment chaque pièce montre un spectre de diffraction singulier et donc une paragénèse particulière. Les SGD-K appartiennent au groupe minéralogique $\mathrm{K}$ pour lequel la kirchstéinite est présente à hauteur de 15 à $60 \%$.

Ces scories sont compactes : la porosité y est faible, seulement de 10 à $30 \%$ (moy $20 \%$ ). Cette porosité provient des bulles de gaz qui sont restées piégées dans la scorie lors de son refroidissement. Les pores les plus gros sont situés le long des surfaces supérieures ou inférieures des scories. Ils ont souvent une forme arrondie mais peuvent parfois se trouver allongés verticalement (S070) ou horizontalement (S068). Cette faible porosité laisse penser qu'elles ont subi une forte fusion.

Les scories SGD-K sont un peu différentes des autres SGD. Elles ont une aimantation très variable, faible à forte et comportent très peu de particules ferreuses, en moyenne uniquement $2 \%$ en coupe. La quasi-totalité de ces particules observées sont en réalité des croûtes d'oxydes de fer plates, disposées aléatoirement (Figure 90). Toutes sont constituées de ferrite pure $(<0.02 \% \mathrm{C})$.

\section{Scories SFR}

Les scories SFR occupent la base du triangle des matériaux, entre les pôles SGD et SFR et avec seulement 0 à $2 \%$ SAS (Figure 85 ).

Les scories sont composées de cristaux de fayalite sombre et de fer à l'état de métal (petites ou grosses particules) en proportion importante, jusqu'à $90 \%$ en coupe pour S103 (Figure 92). La présence de fer sous forme métallique s'explique de différentes manières. Il peut se produire un détachement mécanique d'un fragment non compact du métal (Figure 73 ; cliché 7), une perte de petites particules de fer (8), une perte de billes de fer à l'état liquide (9) et une perte de chapelets de métal à l'état liquide (10), ou bien la réduction d'une partie des oxydes de fer par du monoxyde de carbone (CO) présent dans les porosités et par le carbone contenu par les charbons (10). Ceci a lieu le long des pores et sur le contour des charbons.

On observe différentes combinaisons d'empilement («stratification ») entre les pores des scories, les plages de fayalite et les zones ferreuses : soit ces trois composantes sont totalement mélangées, soit c'est le métal qui surmonte la fayalite. Pour la moitié des pièces (huit scories), de grandes plages d'oxydes de fer telles la wüstite ou de magnétite s'ajoutent aux matériaux déjà cités (Figure 93).

Concernant l'étude en XRF, les scories SFR appartiennent en majorité au groupe minéralogique MO pour lequel le métal est les oxydes sont représentés ensembles à hauteur de 20 à $45 \%$. Les oxydes en question sont la wüstite en grande partie puis la magnétite. De nombreuses paragénèses existent du fait des différents rapports entre les minéraux. Les autres scories appartiennent aux groupes minéralogiques $\mathrm{F}$ et $\mathrm{FQ}$, mettant en évidence la présence de fayalite. Une seule pièce (S120) montre une minéralogie différente des autres pièces du groupe $(\mathrm{QF})$ : le métal ne pouvant être broyé c'est la composante siliceuse des petites pièces de scorie associées qui a été analysée. De plus, des cristaux d'augite et de leucite ont été détectés (XRF) dans quatre scories.

La porosité est faible à forte puisqu'elle varie entre $13 \%$ (S103) et $45 \%$ (S095, S035). Les grosses porosités sont de forme irrégulière et correspondent aux interstices non comblés entre les particules ou blocs de fer après leur dépôt dans la scorie. Les

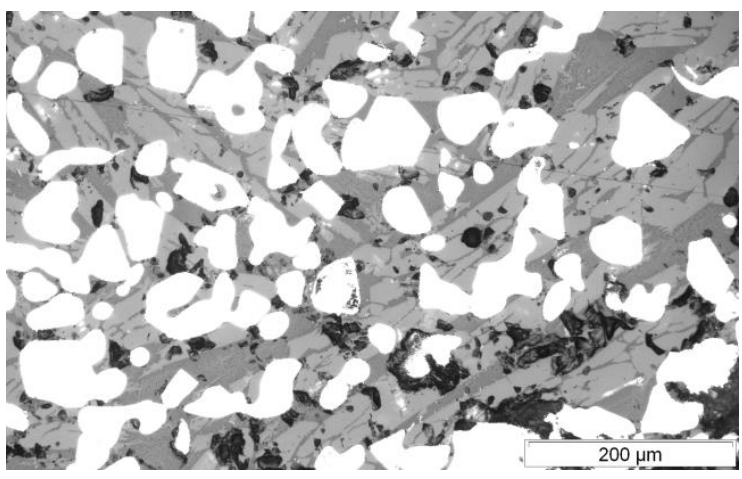

Figure 92 - SFR : petites particules métalliques dispersées dans la scorie (MM_S035_3a). Microscopie optique en lumière réfléchie.

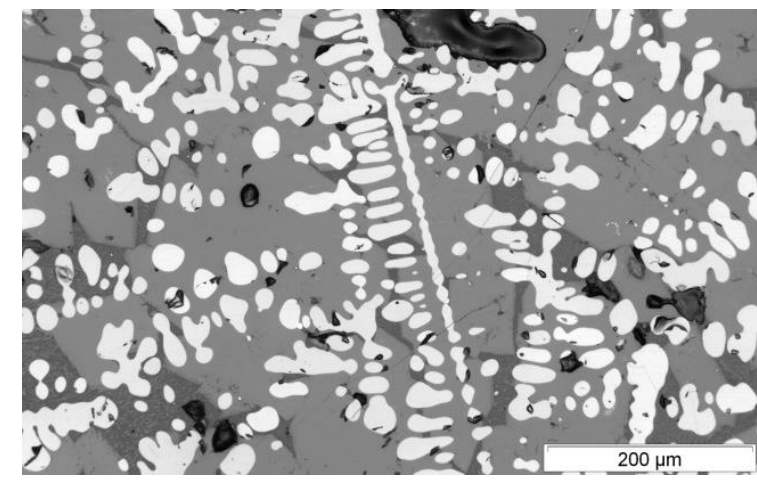

Figure 93 - SFR : dendrites et globules de wüstite dans des plages de fayalite (gris moyen) (MM_S006_4a). Microscopie optique en lumière réfléchie. 
fines porosités sont dans la fayalite.

L'aimantation des scories est toujours forte (sauf S095, moyenne et locale). En coupe, il est observé de 28 à $90 \%$ de particules ferreuses. Ces particules se répartissent sous la forme de fins filaments, de particules de différentes tailles et de billes mais surtout (sauf S006), de larges blocs de métal > $10 \mathrm{~mm}$.

L'étude métallographique révèle de grandes variations de la teneur en carbone des particules ferreuses : bien souvent, chaque scorie comprend à la fois un acier doux $(<0.2 \% \mathrm{C})$ un acier hypoeutectoïde $(0.2$ à $0.8 \% \mathrm{C})$ et hypereutectoïde $(>0.8 \% \mathrm{C})$. Enfin, 2 pièces sont composées de fontes blanches (S103) et grises à graphite (S103, S120) (Figure 12).

\section{Scories SI}

Les scories SI sont situées au pôle argilosableux dans le triangle de classification selon les matériaux (Figure 85).

Ces scories ont une matrice qui n'est ni à base de fayalite ni à base de kirchstéinite : ici il s'agit d'une combinaison de minéraux pauvres en fer comme l'augite, la leucite et d'hedenbergite dans un verre (Figure 94, Figure 95). Pour les quatre scories étudiées, la porosité est moyenne à forte, dans la gamme $27-44 \%$. Les autres scories SI sont composites car elles comprennent des petites pièces de SAS et/ou de SGD et/ou de SFR.

L'analyse des matériaux montrait déjà une certaine diversité du fait que ces matériaux sont composites.

L'assemblage des scories SI reflète également une certaine diversité de leur profil minéralogique. Les groupes minéralogiques Q, QF et F, standards pour des scories de forge et déjà rencontrés au cours de ce travail, rassemblent la moitié des scories SI. L'autre moitié des scories appartient au groupe MO et A : pour ce dernier groupe, il s'agit de scories riches en silice et en minéraux (10-60\%) que l'on trouve dans des systèmes pauvres en fer. Ces minéraux sont la leucite, l'augite, l'hedenbergite et l'akermanite (4.2.1). De la mullite est identifiée dans deux scories (S039, S061), à hauteur de 5 à $15 \%$.

D'autre part, deux scories (S040, S043) du groupe minéralogique A contiennent d'autres minéraux mal identifiés, mais qui semblent toutefois être très riches en $\mathrm{Ca}, \mathrm{Na}, \mathrm{Mg}$ et $\mathrm{P}$.

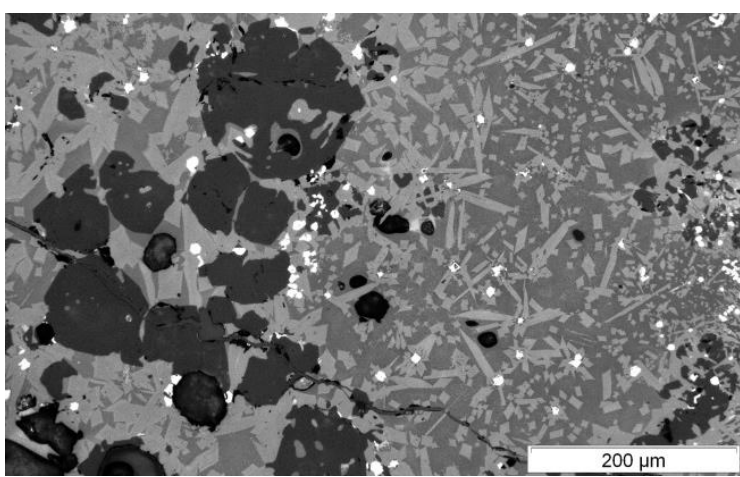

Figure 94 - SI : grains de leucite (sombre) dans un verre (gris foncé), baguettes d'augite (gris clair) et magnétites (blanc) (MM_S037_4a). Microscopie optique en lumière réfléchie.

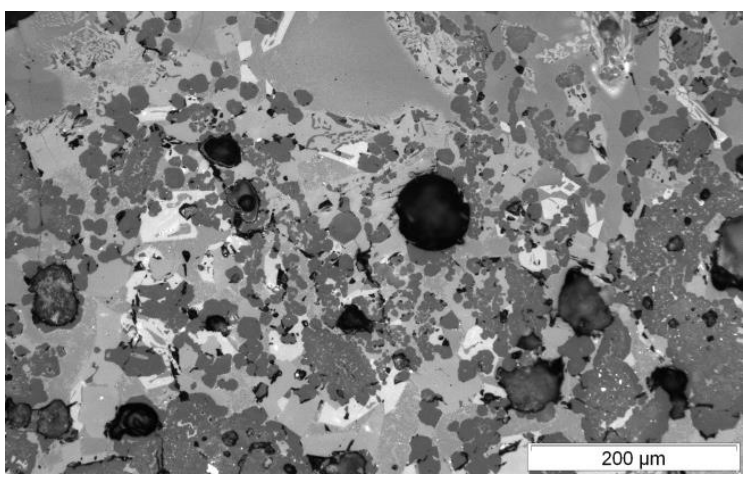

Figure 95 - SI : cristaux de leucite (sombre), de fayalite (gris très clair, présence rare) et d'hedenbergite (gris moyen) dans un verre (gris foncé) (MM_S032_1a). Microscopie optique en lumière réfléchie.

Ces différences marquées de la minéralogie reflètent sans doute des conditions de formation instables pour ces pièces à peine centimétriques.

Seules S032 et S037 ont une aimantation faible localement, et contiennent en moyenne $1 \%$ de particules ferreuses en coupe ( $\min 0 \%$-max $5 \%$ ). Il s'agit essentiellement de petites particules (billes, croûtes d'oxydes et particules $<1 \mathrm{~mm}$ ) de ferrite $(50 \%)$ et d'acier proche de l'eutectoïde (50\%).

\subsubsection{Conditions de forgeage}

\section{Scories SAS}

Les scories du groupe SAS proviennent des ateliers de Fiko et de Kobo, en plus d'une seule pièce provenant de Doundé (S048) (annexe 4 - Scories). Elles ont toutes été produites au cours de forgeages d'une seule barre de fer (M2S, M3S, M4S), sans soudure, sans ajouts et sans incidents. Les pièces ont été collectées après un seul forgeage $(80 \%)$, deux forgeages $(5 \%)$ et trois forgeages $(15 \%)$. 
Les SAS résultent donc de travaux de forge sans soudure globalement rapides. Il s'agit de mettre en forme une seule barre de fer mais ce travail demande tout de même une phase de préformage courte indispensable: les barres M2S et M3S doivent être refoulées car elles ont une forme allongée qui nécessite d'être raccourcie et la barre M4S est allongée.

Ainsi, les scories SAS sont des scories «de formage » qui peuvent compter une petite composante « de préformage ».

\section{Scories SGD}

Des SGD-B et des SGD-SFR ont été produites à Fiko, Kobo et Doundé. Elles ont été produites majoritairement au cours de travaux impliquant la mise en forme de 2 barres de fer, indifféremment de la provenance (modernes, africaines, récupération) et de la forme (barres trapues, longues etc). Ainsi, au minimum une soudure est nécessaire au cours du préformage et plus de la moitié des forgeages compte des incidents. A Kobo et Doundé, les forgerons ont systématiquement employé des ajouts siliceux (Figure 34). A Fiko et Kakoli aucun ajout n'a été effectué. Les pièces ont été collectées après un seul forgeage $(60 \%)$, deux forgeages $(10 \%)$ et trois forgeages $(30 \%)$.

A Kobo, ces scories apparaissent lorsque le forgeron travaille des barres plus compliquées : M1D, A1D et A2D. L'utilisation de ces barres a ainsi systématiquement généré des incidents. Il s'agit donc de travaux relativement complexes.

Les scories SGD-K proviennent quant à elles toutes de l'atelier de Kakoli. Elles ont été produites au cours de travaux dont les conditions de départ étaient très différentes : forgeage d'une barre de fer $(50 \%)$, de deux barres de fer $(50 \%)$, sans incident $(50 \%)$, avec incident $(50 \%)$. Aucun ajout n'a été employé. Par contre, toutes les scories ont été récoltées après un seul forgeage (scorie simple). Les scories SGD résultent donc de travaux impliquant le plus souvent des soudures ( $85 \%$ des forgeages) au cours du préformage suivi d'un formage et de finitions habituels

Ainsi, les scories SGD sont des scories « de préformage ». Les SGD-B comportent un bourrelet argilo-sableux de type SAS, correspondant à la composante «de formage » décrite précédemment. Les SGD-SFR et SGD-K ne comportent pas de bourrelet : celui-ci aurait pu être englouti dans le corps pâteux ou liquide de la scorie au cours du formage.

\section{Scories SGD}

Les scories SFR proviennent des ateliers de Fiko et Doundé. Elles ont été produites au cours de forgeages impliquant deux barres de fer et jusqu'à sept soudures ont été réalisées (S123) avec ou sans ajout selon les ateliers. Près de la moitié de ces travaux compte un ou plusieurs incidents ainsi que de possibles trempes. Enfin, $50 \%$ de ces scories a été collectée après un seul forgeage, et $50 \%$ après une journée de travail, soit plusieurs forgeages. Les scories SFR résultent donc de travaux impliquant des soudures (90\% des forgeages) au préformage.

Ainsi, les scories SFR sont des scories «de préformage ».

\section{Scories SI}

Les scories SI proviennent de l'atelier de Doundé $(80 \%)$ et de Fiko $(20 \%)$. Elles ont été produites essentiellement au cours du forgeage d'une seule barre de fer (M4S et R1S), donc sans soudure et sans ajout. Seulement peu d'incidents se sont produits au cours de ces forgeages $(20 \%)$. Environ $75 \%$ des pièces sont des scories simples, le reste étant des scories multiples récoltées après une journée de travail.

Les scories SI résultent donc de travaux de forge globalement rapides. Il s'agit également de scories « de formage ».

\subsubsection{Stratification}

La formation d'une stratification dans les scories peut s'expliquer de différentes manières :

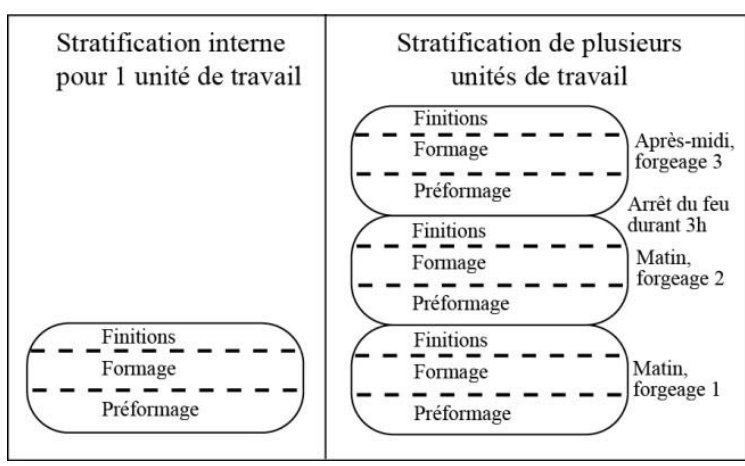

Figure 96 - Modèle théorique pour la stratification des scories lors de leur formation dans le foyer. 
- la stratification à l'intérieur d'une unité de travail avec ou sans extinction du feu : stratification interne d'une scorie simple où la scorie est collectée après un forgeage ;

- la stratification de plusieurs unités de travail : scorie multiple où la scorie est collectée après plusieurs forgeages, avec ou sans extinction du feu.

Dans le cas d'un seul forgeage, on peut s'attendre à ce que les différentes phases (préformage, formage et finitions) soient responsables de la formation de strates superposées de natures différentes (Figure 96, gauche). Lorsque des forgeages successifs sont entrepris sans vidange $\mathrm{du}$ foyer, on peut s'attendre à une stratification de plusieurs scories; on peut aussi penser que l'extinction ou le refroidissement $\mathrm{du}$ foyer soit marqué (Figure 96, droite).

\section{Stratification d'une seule unité de travail}

Après sciage en deux parties et observation au microscope de la section polie des 56 scories récoltées après un seul forgeage, on observe une stratification interne pour 26 scories. Toutes ces scories ont été produites sans extinction du feu, sauf S013. Cette stratification est exprimée dans les scories par un ou plusieurs indices à la fois :

- Un bourrelet argilo-sableux proéminent à l'extrémité proximale de la scorie ou d'une fine couche superficielle argilo-sableuse.

- Du métal réparti en couche horizontale soit dans le fond, soit dans la partie médiane soit dans la partie supérieure de la scorie; on observe des situations préférentielles : les filaments et les particules $<10 \mathrm{~mm}$ sont plutôt situés dans la partie médiane et/ou basse de la scorie, les blocs >10 mm sont plutôt concentrés dans la partie médiane et/ou supérieure des pièces.

- Une porosité répartie dans la partie inférieure ou médiane ou supérieure de la scorie. Les larges pores affleurent généralement la surface supérieure des scories tandis que les pores moyens et fins peuvent se retrouver partout.

Conclusions des observations de la figure 97 :
1. Les scories qui ne sont pas stratifiées sont les scories SAS et SI. Les scories SAS arrachent souvent un peu du fond du foyer dans lequel elles se sont formées, ce qui n'est toutefois pas de la stratification. Ces scories résultent de forgeages dont le préformage est très limité : pour simplifier, nous les appellerons scories « de formage ».

2. Les scories qui montrent une stratification sont essentiellement les SGD. Ces scories sont le résultat de forgeages nécessitant un travail de préformage relativement important: nous les nommerons scories « de préformage ».

Les scories SGD-B sont composées une scorie SGD de préformage contenant des pores larges dans la partie supérieure ainsi que des particules de métal dans le fond des pièces, puis surmonté d'un bourrelet argilo-sableux de type SAS de formage.

Les scories SGD-SFR sont uniquement composées d'un corps fayalitique contenant des pores larges dans la partie supérieure et du métal dans le fond des pièces. Les scories SGD-K possèdent un corps de préformage contenant des petites porosités et du métal tous deux dans le fond des pièces. Dans ces deux derniers cas, la composante de formage n'est pas visible car visiblement englouti dans le reste de la scorie.

3. Quelques scories SFR montrent également une stratification (Fiko) : on observe des grosses particules de métal dans de larges pores au milieu de la scorie alors que des petites particules de métal et le corps fayalitique sont répartis dans le fond des pièces.

4. Le métal est souvent réparti dans le fond des pièces : la masse volumique du fer $\left(7.88 \mathrm{~g} . \mathrm{cm}^{-3}\right)$ étant supérieure à celle de la fayalite $\left(4.39 \mathrm{~g} . \mathrm{cm}^{-3}\right)$, les particules de fer s'y déposent par gravité au cours d'un forgeage à haute température et donc lorsque la fayalite est peu visqueuse (préformage et formage).

La porosité est issue des produits de dégazage des réactions chimiques entre le fer métallique, l'oxygène apporté par la soufflerie et le carbone du charbon. Les pores les plus larges (cm) affleurent généralement la surface. Pendant le préformage, les gaz remontent jusqu'à la surface (du fait de leur faible densité) dans la fayalite peu visqueuse puis semblent se faire piégés lors du formage : à cette étape du forgeage la baisse de température pourrait rendre la fayalite trop pâteuse pour évacuer les pores. Seules les scories SGD-K, denses, ont une porosité peu abondante : produites dans un foyer plus chaud 
avec un point de fusion plus bas, la scorie reste fluide même le temps du formage, permettant aux gaz de s'évacuer facilement.

5. Le bourrelet argilo-sableux se constitue pendant le formage et non pas lors des finitions car le forgeron n'ajoute jamais de matières siliceuses au cours des finitions. De plus, la température est bien trop faible $\left(<500{ }^{\circ} \mathrm{C}\right)$ pour faire fondre complètement voire partiellement des grains de quartz qui proviendraient soient de la tuyère, de la paroi ou des potentiels ajouts siliceux.

6. Dans aucun cas il n'a été possible de mettre en évidence la composante «de finitions»des scories : ces travaux, réalisés à basses températures essentiellement sur l'enclume, ne semblent pas contribuer à la formation de la scorie dans le foyer.

7. Une seule scorie (S013) a été produite sur deux journées avec une extinction du feu d'au moins $12 \mathrm{~h}$. Le préformage a été réalisé l'après-midi du $1^{\mathrm{er}}$ jour et le formage a été effectué le matin du $2^{\text {nd }}$ jour : on observe alors une scorie de préformage contenant du métal en ligne horizontale dans le milieu de la pièce (jour 1), surmonté d'un bourrelet argilo-sableux de formage (jour 2) (Figure 97). Il semble toutefois étonnant que le métal n'ait pas eu le temps de se déposer dans le fond de la scorie.

\section{Stratification de plusieurs unités de travail}

Les 18 scories triples ont toutes été produites avec extinction du feu dans le foyer pendant la pause de midi : deux forgeages successifs le matin et un forgeage l'après-midi. Pour les six scories doubles, trois ont été produites sans arrêt du feu (S048, S053 et $\mathrm{S} 061)$ et trois ont été produites avec arrêt du feu (S024 et S027: $1^{\text {er }}$ forgeage le matin et $2^{\text {nd }}$ forgeage l'après-midi ; S050: $1^{\text {er }}$ forgeage l'après-midi et $2^{\text {nd }}$ forgeage le lendemain matin).

8. Les scories SAS et SI qui ont été produites par des opérations doubles et triples ne sont pas stratifiées (Figure 97).

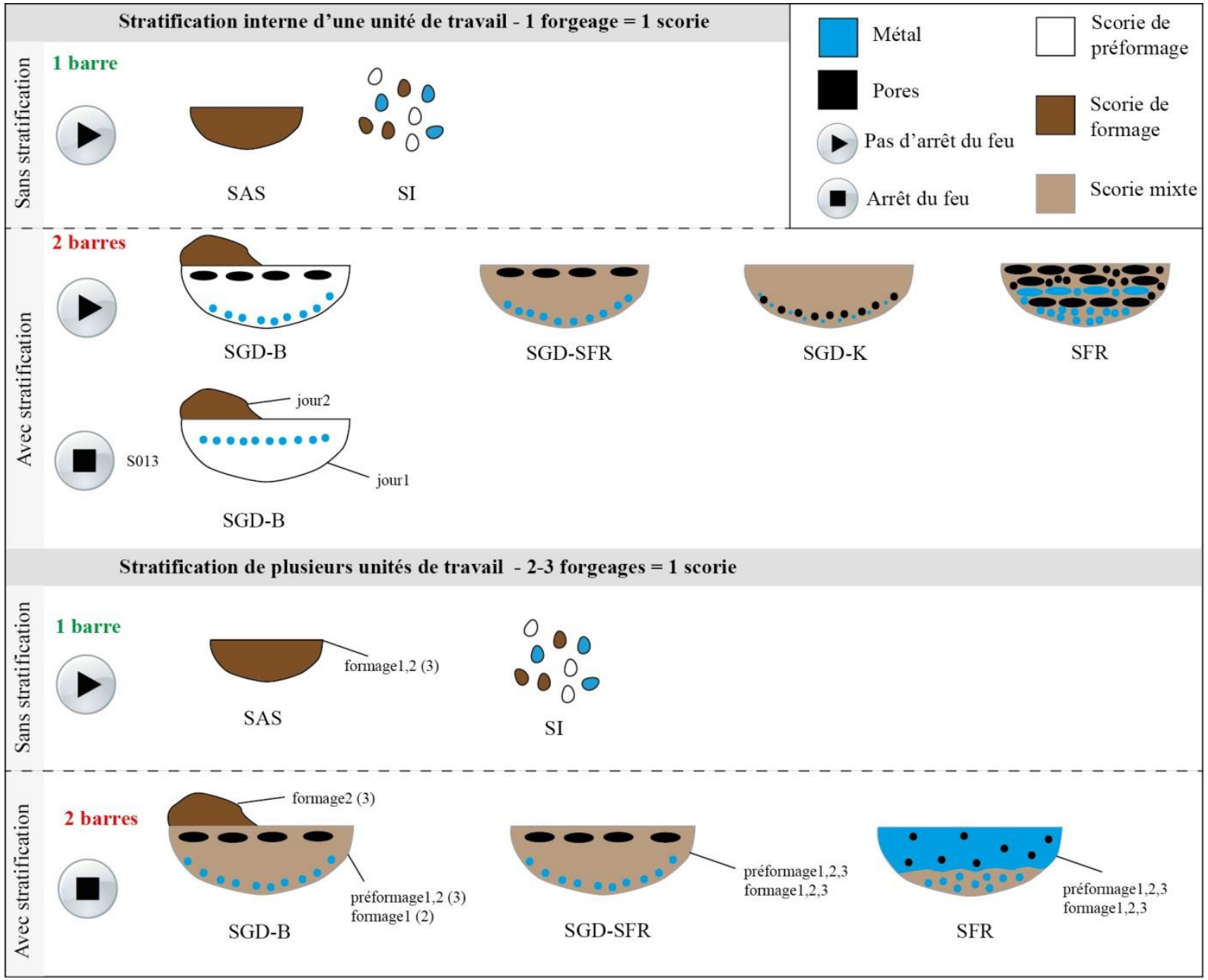

Figure 97 - Situations observées concernant la stratification dans les scories de forge. 
9. Les scories SGD issues de forgeages doubles et triples montrent une stratification : le métal tombe dans le fond de la scorie, les pores les plus larges affleurent la surface et un bourrelet argilo-sableux (ou pas) correspondant à la dernière phase de travail se rajoute sur le corps de la scorie.

10. Pour trois scories SFR (S045, S111, S122) la stratification est particulière puisque le métal est concentré dans la partie supérieure et la fayalite se trouve en partie inférieure de la scorie : le métal, accumulé par petites particules, s'agglomère petit à petit ; tant que les particules ne sont pas jointives, la fayalite fluide s'écoule par percolation.

11. Il semble que lorsqu'un nouveau forgeage est entrepris (forgeage de l'après-midi), la précédente scorie se ramollisse de nouveau et incorpore la nouvelle scorie, re-mélangeant tout le système. Par exemple pour une scorie SGD-B : le corps de la scorie est constitué de matériel accumulé au cours du préformage et du formage de plusieurs forgeages; le bourrelet sableux correspondrait au formage du dernier forgeage.

Dans aucun cas on a observé une stratification pouvant correspondre à deux ou trois unités de travail superposées avec une surface de refroidissement entre les scories, alors que dans plusieurs cas le refroidissement a été effectif (Figure 96, droite).

Ces scories stratifiées sont pourtant souvent observées et décrites sur les sites de forge anciens (Bauvais 2007a; Bauvais \& Fluzin 2005; Dunikowski et al. 1996; Eschenlohr et al. 1991; Mauvilly et al. 1998; Serneels 1993; Serneels \& Perret 2007). Elles se forment dans des conditions différentes des conditions existantes au Pays dogon.

Nous supposons quelques hypothèses :

- dans le cas d'un foyer qui n'est pas chaud, la première scorie peut ne pas se ramollir $\left(\mathrm{n}^{\circ} 11\right)$, ce qui permet à la nouvelle scorie de se former par-dessus ;

- dans le cas où le sol du foyer de forge est meuble, la première scorie s'enfonce dans le sol et sert de support à la nouvelle scorie.

\subsubsection{Comparaison des groupes de scories}

Un résultat inattendu est que les scories SAS et SI ne semblent se former que lors des étapes de formage rapide, sans ajout ni soudure, où le préformage est quasi-inexistant. Ces scories se forment donc majoritairement pendant la phase de formage de la lame de houe. Scories « de formage », légères et peu denses, elles ne montrent pas de stratification.

$\mathrm{Au}$ contraire, les opérations de forgeage plus longues avec des soudures utilisant des ajouts mais surtout générant des pertes de fer importantes, vont être à l'origine de la formation des scories à dominante fayalitique. Quand ces pertes de fer sont encore plus importantes (incidents au cours du préformage), on assistera à la formation de scories ferreuses. Ces scories «de préformage» sont plus volumineuses et plus denses que les précédentes. De plus, la succession des étapes de la chaîne opératoire et la succession des forgeages, éventuellement avec refroidissement $\mathrm{du}$ foyer, n'entraîne pas forcément une stratification: si les scories sont suffisamment chaudes, les matériaux pâteux ou liquides vont s'ordonner verticalement en fonction de leur densité, et non en fonction des opérations successives.

On attribue donc les types de scorie aux étapes du travail : SI et SAS liées au formage ne générant que peu de pertes de métal et SGD et SFR liées à l'étape de préformage générant beaucoup de pertes de métal. En revanche, la complexité et la variabilité des processus de formation ne permettent pas d'atteindre une interprétation plus fine des gestes techniques.

\section{Scories SAS}

Les scories SAS sont composées de 80 à $100 \%$ de matériaux siliceux, roches et verre, et de peu ou pas de cristaux fayalite et particules de métal $(<20 \%)$; il s'agit de croûtes d'oxydes et des particules métalliques $<1 \mathrm{~mm}$ décarburées. La présence de verre peut s'expliquer par la présence d'éléments alcalins (moy $\mathrm{Na}_{2} \mathrm{O}+\mathrm{K}_{2} \mathrm{O}$ wt $\%: 1 \%$ ) qui font baisser la température de fusion de la silice $\left(\mathrm{T}_{\mathrm{f}}=1650{ }^{\circ} \mathrm{C}\right)$, ou bien par la fusion partielle de silice à l'endroit le plus chaud du foyer, sous la tuyère. La cristobalite en présence est soit héritée de l'argile du foyer, soit provient des transformations de phase du quartz lors de son refroidissement.

Ces scories se forment dans un système soussaturé en fer. Ce dernier provient de la faible perte 
du métal au cours des travaux de forge ; le matériel argilo-sableux provient à la fois du revêtement du foyer, de la tuyère, du fond du foyer et du sable ; la fayalite est une combinaison entre ces deux composés.

La phase de préformage, courte, ne nécessite pas des températures extrêmement élevées $\left(<1200{ }^{\circ} \mathrm{C}\right)$ pour le type de barre de fer travaillée : il n'est pas rare que l'ensemble des gains de quartz ne soit que partiellement fondu ou bien non fondu (Figure 86). En addition, si ces scories sont produites dans un foyer «froid» comme c'est le cas de l'atelier de Kobo, alors, du fait d'un manque de cohésion des matériaux siliceux, elles seront très friables au point de n'en avoir plus de trace en contexte archéologique : ce sont les scories réduites. Dans un foyer «chaud», comme celui de Fiko, peuvent se former des scories plus compactes.

\section{Scories SI}

Les SI quant à elles sont des petites pièces composites de différentes natures (SAS, SGD et SFR). Les scories contiennent en moyenne uniquement $1 \%$ de particules en coupe : elles se présentent sous la forme de filaments, de petites particules $<1 \mathrm{~mm}$, de croûtes d'oxydes mais surtout de petites billes. Dans cet environnement sous-saturé en fer, on observe la formation des minéraux d'augite, de leucite, d'akermanite et hedenbergite. La mullite pourrait provenir d'un fragment de céramique de la voute du foyer ou de la tuyère. Les autres minéraux non identifiés de S040 et S043 pourraient provenir d'une contamination du foyer par des excréments d'animaux.

Les scories SI montrent à la fois des compositions minéralogiques variées et une morphologie inhabituelle pour des scories de forge : ceci reflète certainement des conditions de formation difficiles dans le foyer de l'atelier de Doundé. La seule différence évidente du foyer de l'atelier de Doundé par rapport à celui de Kobo (et des autres ateliers par ailleurs) est le volume de son foyer qui est d'une contenance bien supérieure : $7100 \mathrm{~cm}^{3}$ à Doundé et $3100 \mathrm{~cm}^{3}$ à Kobo (Tableau 7).

Les scories se forment ainsi de manière désordonnée au milieu des charbons et non pas dans le fond du foyer. De plus, malgré de bonnes conditions de soufflerie, le foyer de l'atelier de Doundé est «tiède », ce qui limite d'autant plus l'agrégation des matériaux dans le foyer. Par ailleurs, le forgeage F102 qui n'a pas produit de scorie provient également de cet atelier.

\section{Scories SGD}

Les SGD contiennent entre 50 et $100 \%$ de fayalite et $10 \%$ de particules ferreuses en coupe (Kakoli : kirschstéinite et $<2 \%$ de fer en coupe). C'est au cours du préformage, réalisé dans des foyers « très chauds » que se forme le corps de la scorie : les croûtes d'oxydes qui intègrent la scorie, ainsi que la silice des ajouts et du foyer forment une matrice de $\mathrm{Fe}_{2} \mathrm{SiO}_{4}$ ou de $\mathrm{CaFeSiO}_{4}$. A Kakoli, ces oxydes de fer (wüstite et de magnétite) sont très nombreux (Figure 90, Figure 91) et l'excès de calcium favorise la formation d'akermanite $\mathrm{Ca}_{2} \mathrm{MgSi}_{2} \mathrm{O}_{7}$, minéral détecté quasi-systématiquement (XRD). Certaines rares pièces comportent des augites et des leucites.

Des particules métalliques se déposent dans le fond des scories par gravité ; toutes les carburations sont observées : le lien avec la microstructure n'est pas évident mais la quantité et la taille des particules de métal peuvent jouer un rôle. A Kakoli, il est étonnant de constater que les scories ne comportent pas ou peu de fer à l'état de métal : malgré une chauffe intense, le feu à Kakoli semble bien mieux maitrisé qu'à Fiko et Kobo.

Les nombreuses porosités observées en surface des pièces témoignent d'un système qui a été pâteux et qui n'a pas permis l'évacuation totale des gaz (température plus faible qu'au préformage). Cet état pâteux facilite le dépôt des matières argilosableuses, au cours du formage, sous la forme d'un bourrelet (SGD-B). A Kakoli, les scories comportent une faible porosité (moy $20 \%$ ) ; sous le coup d'une forte soufflerie dans un petit foyer, et avec un point de fusion plus bas à cause du calcium, la scorie est restée très liquide facilitant l'évacuation des gaz.

\section{Scories SFR}

Dans le cadre de notre étude, les scories SFR proviennent de forgeages ratés ou avec des trempes et sont formées dans des conditions où les pertes de métal sont particulièrement extrêmes. Elles contiennent entre 30 et $90 \%$ de matériel métallique, des petites particules et gros blocs $(\mathrm{cm})$ en plus des particules métalliques, ainsi que de 20 à $45 \%$ d'oxydes de fer pour la moitié des pièces.

Les forgerons de Doundé ont pratiqué de nombreuses trempes au cours du préformage afin d'appliquer sur les barres à réunir, avec les doigts, 
une boue d'argile et de tuyère. Cependant, l'usage de ce traitement thermique a un effet négatif sur le métal : après un refroidissement brusque de $1200{ }^{\circ} \mathrm{C}$ à la température ambiante dans un bain d'eau, le fait de reprendre les cycles de chauffe et de martelage contribue de manière significative à fragiliser le métal et conduit à des pertes de fer volumineuses dans le foyer.

Plusieurs carburations sont observées, entre ferrite et acier hypereutectoïde. Par ailleurs S103 et S120 sont constituées de fonte blanche et grise. Ces scories de Doundé font partie des pièces les plus petites (resp. $7.5 \mathrm{~cm}$ et $6.5 \mathrm{~cm}$ ) et ce sont celles qui comportent le plus de fer en coupe (resp. $90 \%$ et $83 \%$ ). Elles ont pu s'enrichir fortement en carbone au contact des charbons dans le foyer.

Concernant leur empilement, on a deux configurations: un mélange entre fayalite, petites particules de fer et pores, soit des grosses particules ou blocs qui surmontent un bloc de fayalite. 


\subsection{DIFFÉRENTES CONTRIBUTIONS À LA FORMATION DES SCORIES}

La scorie se forme dans le foyer de forge à partir de différentes contributions : la matière première (le fer), le combustible (charbon), les éléments du foyer en argile (paroi, tuyère) et les ajouts volontaires de matières siliceuses (Figure 98). Les matériaux prélevés ont été analysés en XRF ; toutefois il faut rappeler que nous n'avons pas de données de composition chimique concernant la paroi du foyer de Kakoli ainsi que la tuyère de Fiko et Kakoli.

Dans les deux ateliers du plateau (Fiko, Kakoli) les forgerons n'ont jamais utilisé d'ajouts siliceux pour aider à la réalisation des soudures. A Kobo, le forgeron emploie surtout du grès, un peu de tuyère concassée et d'argile et parfois de la barytine qui a été mise à disposition. A Doundé le forgeron n'ajoute jamais de grès, surtout de l'argile fine et un peu de tuyère concassée. Dans ces ateliers la morphologie des foyers de forge implique qu'il n'y a pas de contact entre la scorie et une paroi en terre cuite à proprement dite, comme c'est le cas dans les foyers en fosse (Fiko, Kakoli).
Le chapitre 4.4 a pour but de mesurer l'influence qualitative ou quantitative de ces différents apports, qui contribuent à la formation des scories. Cela permettra de mieux comprendre et de mieux prendre en compte la nature et la quantité de tous ces différents matériaux, dans le cas des scories de forge archéologiques.

\subsubsection{Matière première métallique}

La perte de fer au cours du forgeage varie de 8 à $53 \%$ et dépend de nombreux paramètres $(3.5)$ : le nombre de barres de fer, le nombre d'incidents, le nombre de soudures, la température du foyer et la durée de chauffe, la section et la forme de la barre à forger, tous ces paramètres étant étroitement liés à l'étape du préformage. Ce fer est perdu sous la forme de croûtes d'oxydes et de particules métalliques dans le foyer de forge d'une part, et sous la forme de battitures sur l'enclume d'autre part.

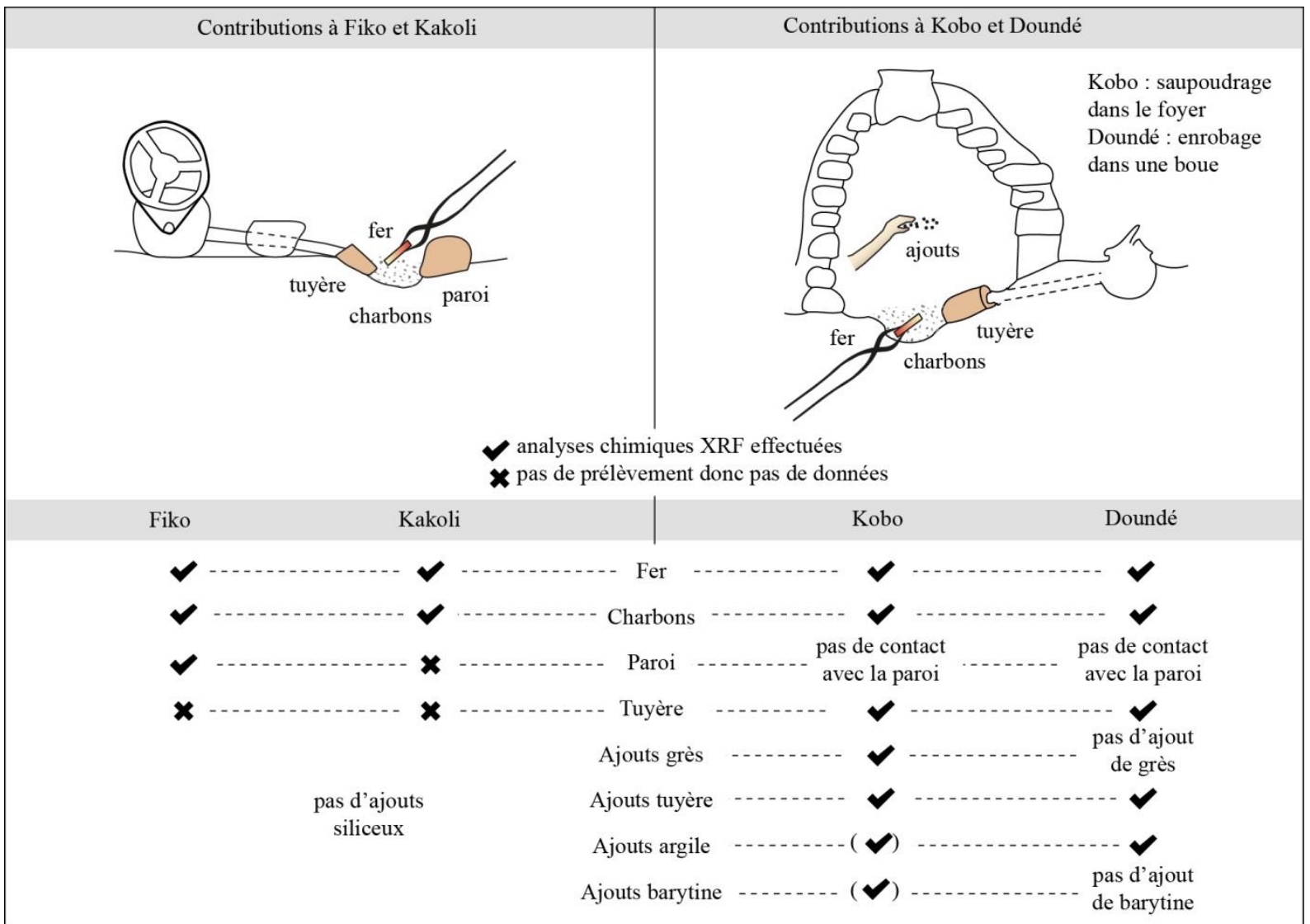

Figure 98 - Récapitulatif des différents matériaux (probables contributions aux scories) que l'on peut rencontrer dans chaque atelier de forge. 


\section{Répartition du fer scorie / battitures}

Ce paragraphe se base sur le calcul de répartition établi à la Figure 71.

Dans le cas du forgeage d'une seule barre de fer sans soudure, la température dans le foyer diffère d'un atelier à un autre (Tableau 21). La température, combinaison entre le volume du foyer et la puissance du flux d'air, conditionne la perte en fer.

Dans les ateliers de la plaine où la perte en fer est faible à moyenne (8-37 \%), au final très peu de métal perdu intègre la scorie (Figure 99) :

- à Kobo le foyer est froid, le travail est très long, la perte en fer est moyenne (moy $16 \%$ ) et seulement en moyenne $4 \%$ du fer perdu intègre la scorie ;

- à Doundé le foyer est tiède, le travail est bref, la perte est faible (moy $13 \%$ ) et seulement $7 \%$ du fer perdu va dans le foyer.

Dans les ateliers du plateau où la perte est forte à très forte, une part plus importante de métal intègre la scorie :
- à Fiko le foyer est chaud, le travail est long, la perte est forte (moy $19 \%$ ) et en moyenne $10 \%$ du fer perdu est intégré à la scorie ;

- à Kakoli le foyer est très chaud, le travail est court, la perte est très forte (moy $30 \%$ ) et en moyenne $30 \%$ du fer perdu se retrouve dans le foyer de forge.

Dans le cas du forgeage de plusieurs barres de fer avec des incidents, la durée de chauffe, la température du foyer, la perte en fer (jusqu'à $53 \%$ ) et la proportion de ce fer perdu qui intègre le foyer et la scorie augmentent également (Figure 100).

Les groupes de scories mis en évidence reflètent également les différents forgeages qui ont été réalisés. Les scories SI et SAS proviennent de forgeages simples : respectivement $5 \%$ et $8 \%$ du fer perdu contribue à la formation de la scorie (Figure 101). Les scories SGD et SFR résultent de forgeages avec soudure : en moyenne $29 \%$ et $26 \%$ du fer qui intègre la scorie.

C'est ici la première fois que des données sur la répartition de la perte en fer entre scories et battitures sont clairement établies.

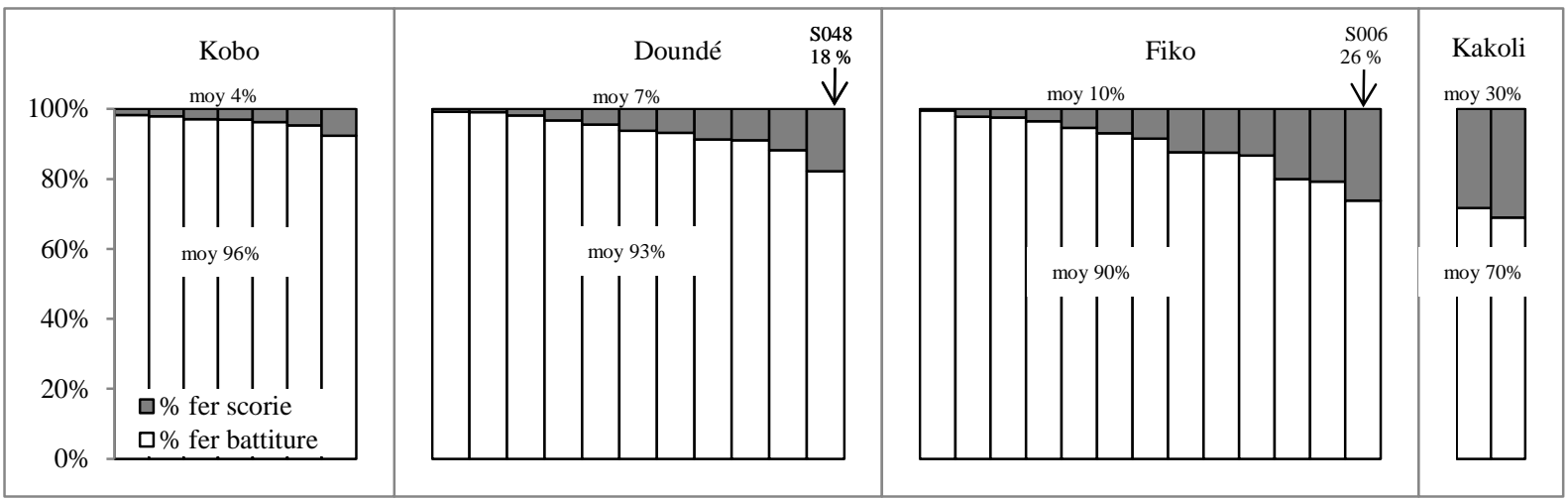

Figure 99 - Répartition du fer perdu entre scorie et battitures dans chaque atelier pour des forgeages sans soudure et sans incident.

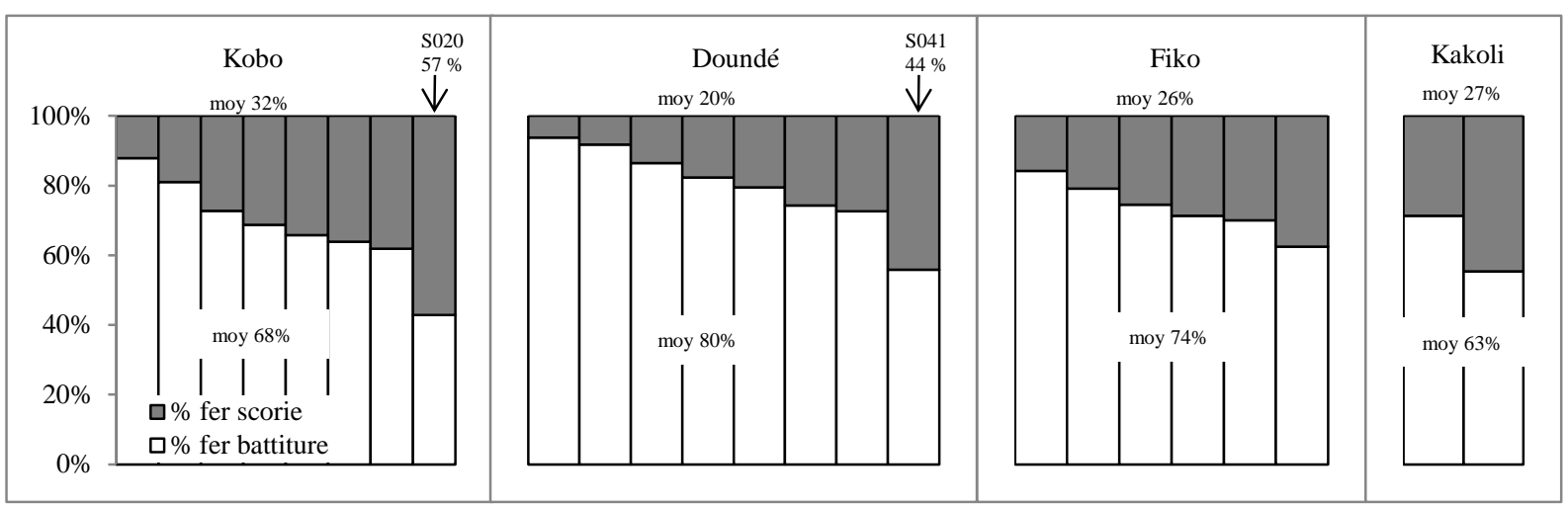

Figure 100 - Répartition du fer perdu entre scorie et battitures dans chaque atelier pour des forgeages avec des soudures et des incidents. 


\section{Quantité de fer métallique extraite}

Une fois la demi-scorie broyée, il est obtenu une poudre fine dans laquelle subsistent des particules métalliques impossibles à broyer. La poudre est tamisée et le métal est facilement extrait. La poudre de scorie contient des petites particules métalliques $(<1 \mathrm{~mm})$ et tous les autres matériaux : oxydes de fer (FeO etc), silicates ( $\mathrm{SiO} 2, \mathrm{Fe}_{2} \mathrm{SiO}_{4}$ etc) etc. Le fer extrait correspond à des grosses particules de métal supérieures à1 mm jusqu'à des blocs de plusieurs centimètres. Cette perte sous la forme de grosses particules se répartit différemment en fonction des conditions de travail.

A Kobo et Kakoli, les scories issues d'un forgeage sans soudure sont globalement pauvres en fer et ne contiennent pas de grosses particules de fer métallique (Figure 102). A Fiko la tendance est la même si ce n'est qu'il y a souvent une certaine proportion de grosses particules métalliques. A Doundé la quantité de grandes particules est beaucoup plus importante : il s'agit en fait des SI qui sont des matériaux composites comportant souvent des pièces métalliques.

Pour des forgeages avec soudures et incidents, dans tous les ateliers excepté Kakoli, la teneur globale de fer augmente ainsi que la proportion de fer sous la forme de grosses particules (Figure 103).
A Fiko, S008 est la scorie qui comportait un noyau ferreux extrait lors du sciage de la pièce ; Doundé regroupe en fait une majorité des scories SFR très riches en fer métallique.

La trempe, systématiquement pratiquée à Doundé, sert à refroidir les pièces métalliques avant la soudure afin de les enduire d'argile. Cette pratique provoque manifestement des pertes importantes (Figure 104). Les scories formées lors de forgeages avec des trempes ont tendance à contenir plus de particules métalliques que les scories résultant de forgeages sans trempes.

Les quatre groupes de scories comportent une quantité totale de fer et une quantité de fer métallique qui varient fortement à cause des différentes conditions de forgeages (Figure 105).

- Les scories SI ont une composition très variable du fait des différentes petites pièces aux natures diverses qui les composent; pour $100 \mathrm{~g}$ de scorie, elles peuvent comporter jusqu'à $90 \mathrm{~g}$ de fer.

- Les scories SAS sont issues de forgeages sans soudures et elles restent les moins riches en fer : en moyenne $11 \mathrm{~g}$ de poudre et $0.5 \mathrm{~g}$ de fer métallique extrait ; seules quelques rares pièces contiennent jusqu'à $12 \mathrm{~g}$ de fer métallique extrait.

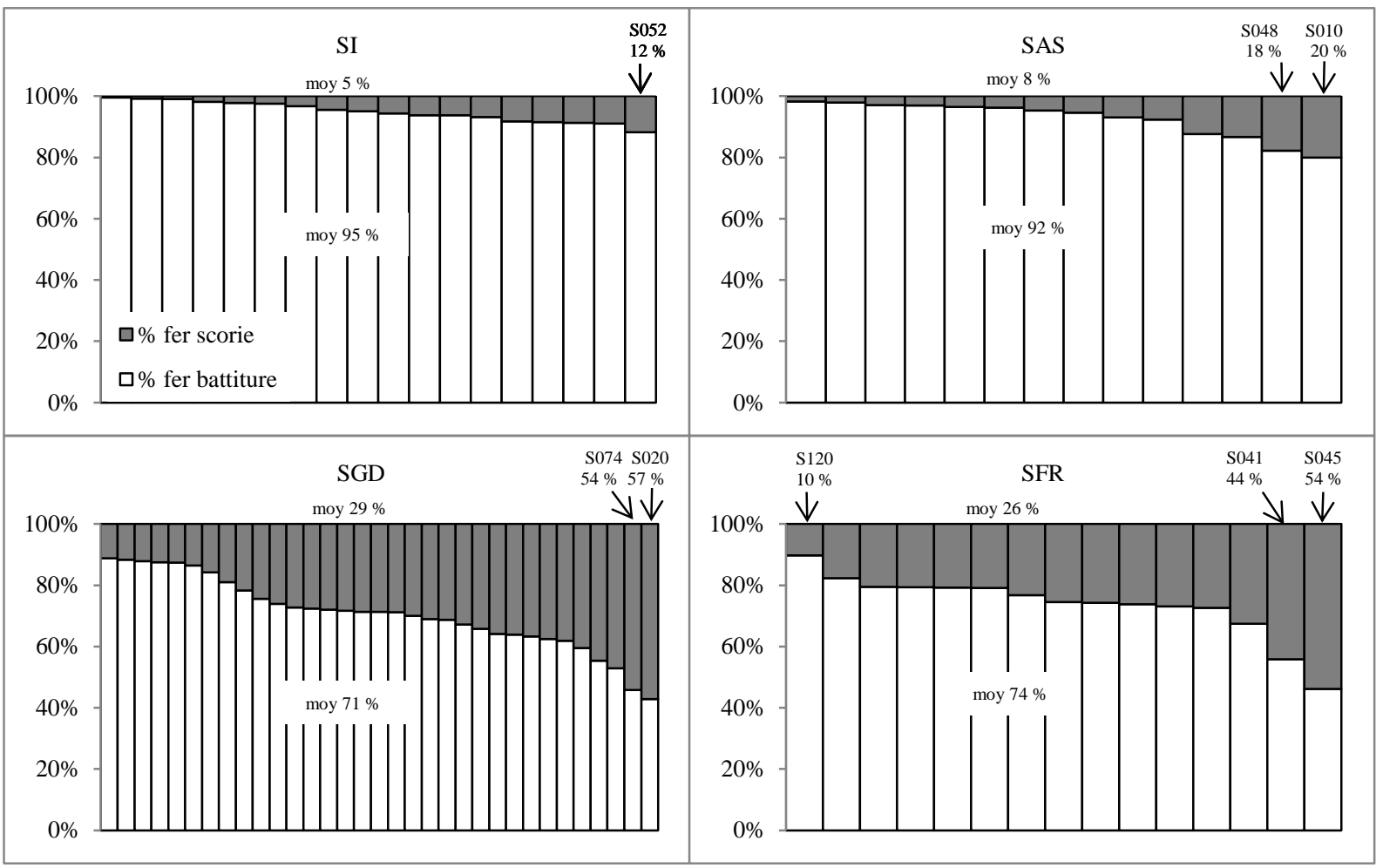

Figure 101 - Répartition du fer perdu entre la scorie (foyer) et les battitures (enclume), pour chaque groupe de scorie. 


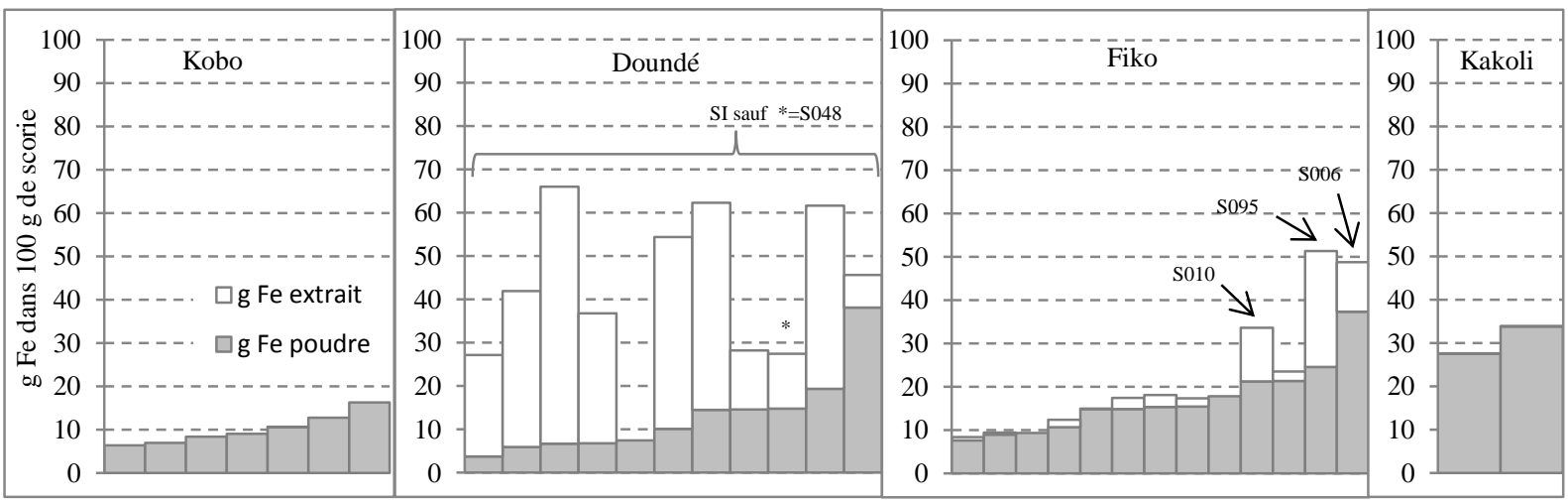

Figure 102 - Poids du fer extrait par tamisage et du fer présent dans la poudre de scorie (XRF) pour une scorie de $100 \mathrm{~g}$. Scories issues de forgeages sans soudure et sans incident.

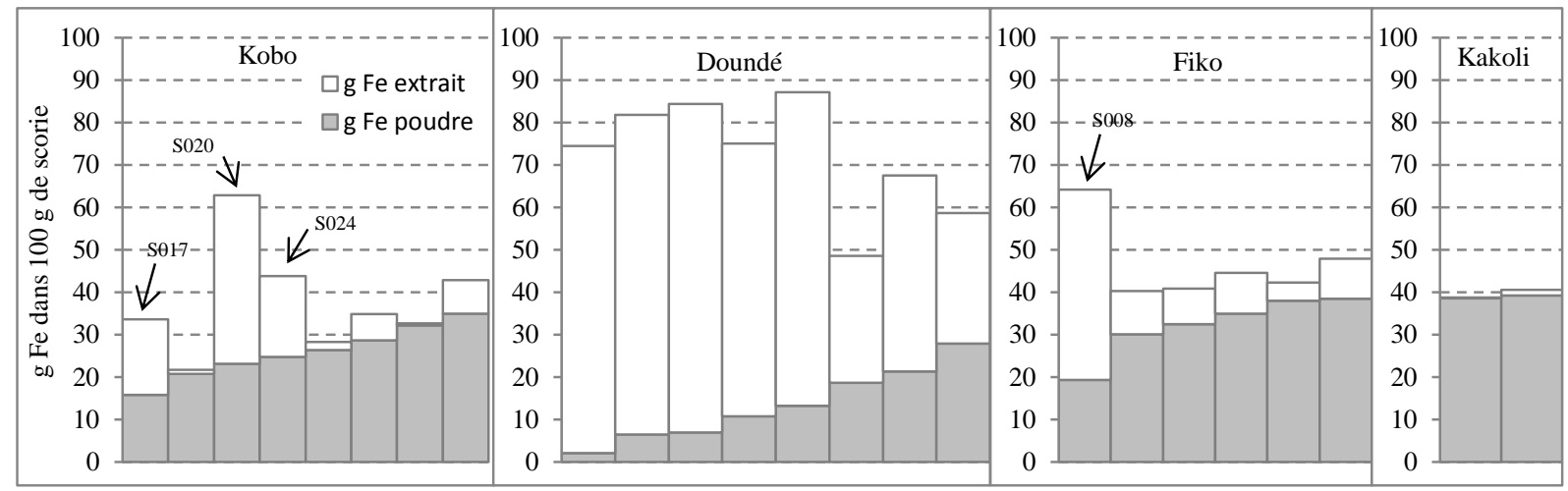

Figure 103 - Scories issues de forgeages avec soudure et avec incidents.

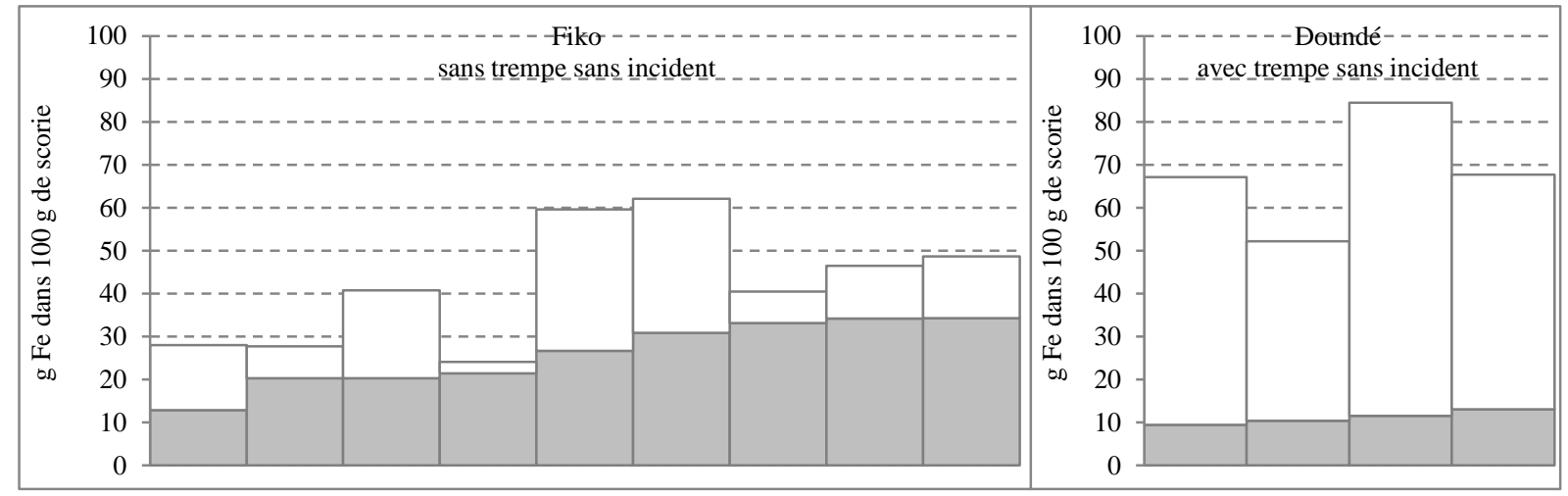

Figure 104 - Comparaison de la quantité de fer des scories issues de forgeages sans trempes (Fiko) et avec trempes (Doundé). 


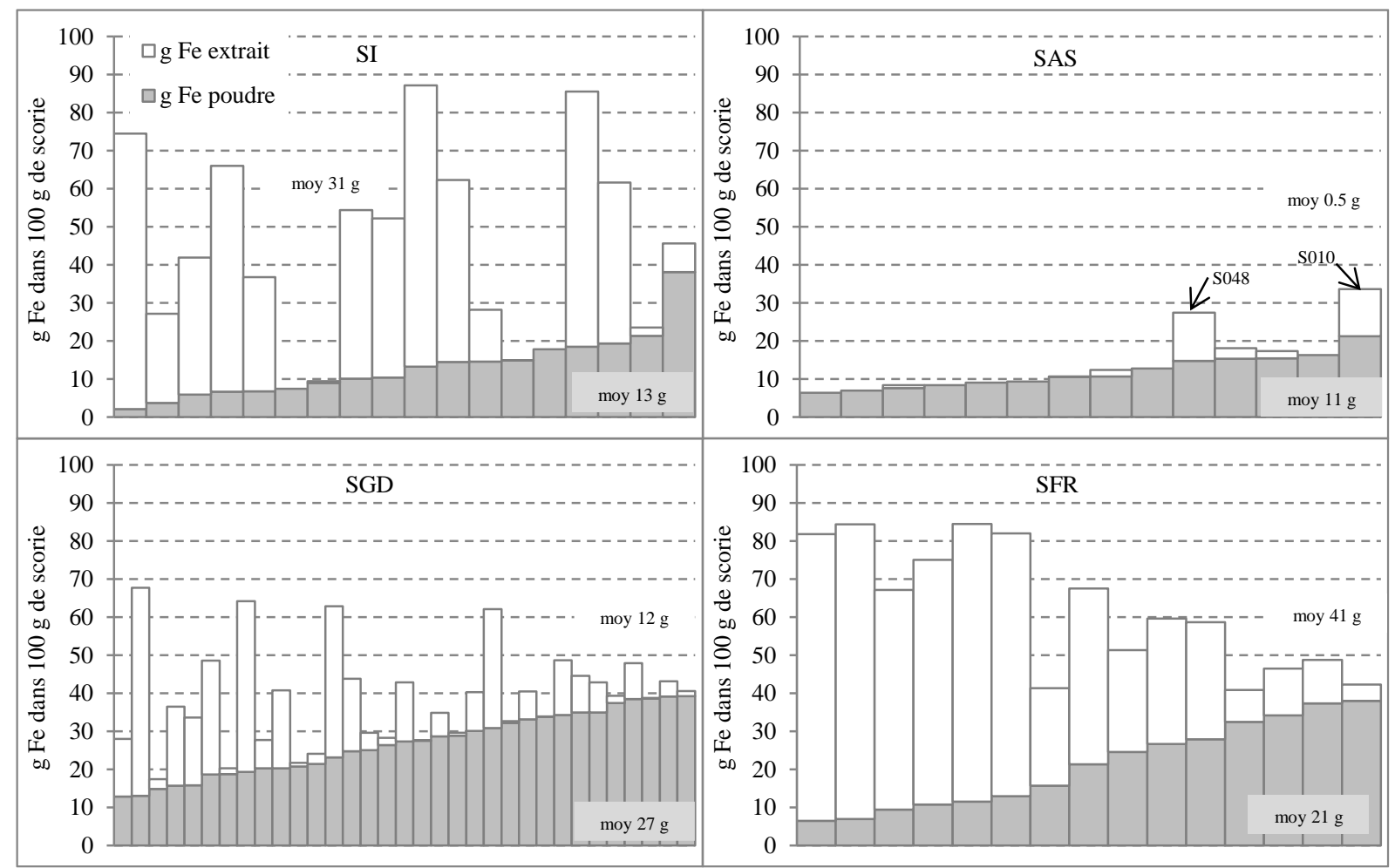

Figure 105 - Poids du fer extrait par tamisage et du fer présent dans la poudre de scorie (XRF) dans la représentation d'une scorie de $100 \mathrm{~g}$, pour les quatre groupes de scorie.

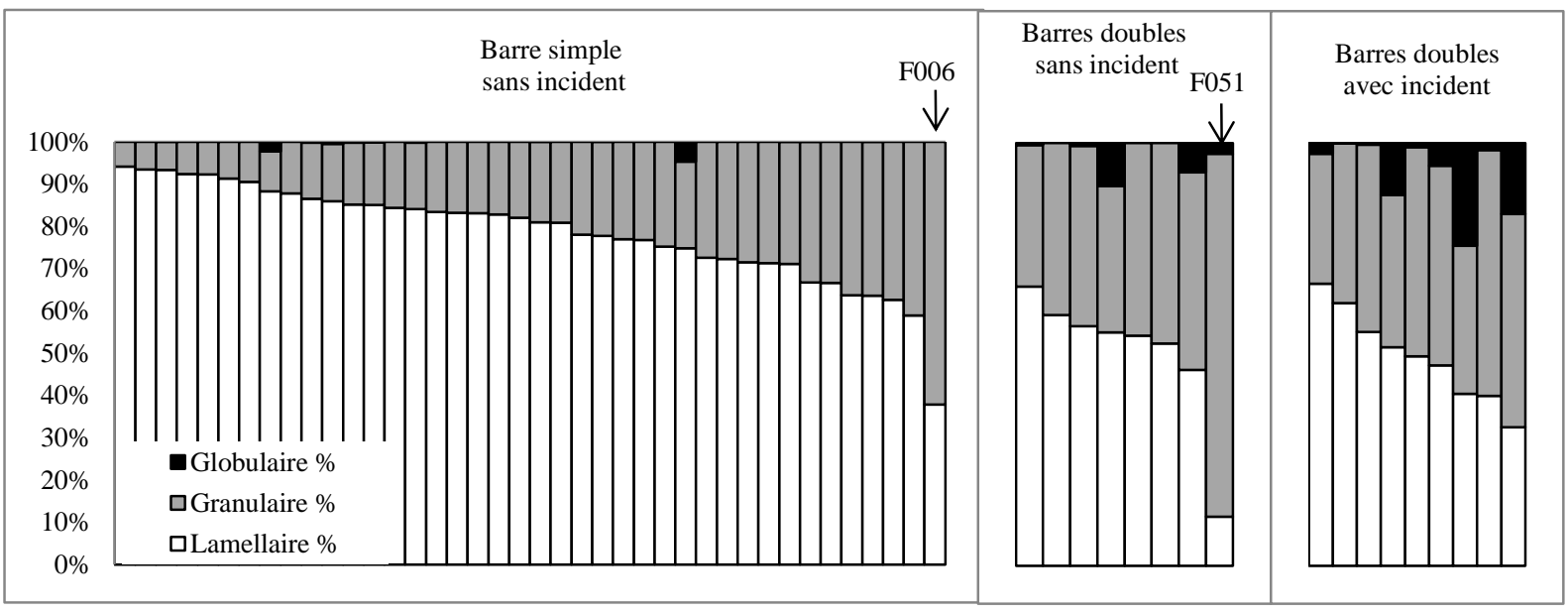

Figure 106 - Répartition des différents types de battitures dans le cas de forgeages d'une barre, deux barres, sans / avec incident. $\mathrm{N}=62$ forgeages comptabilisés.

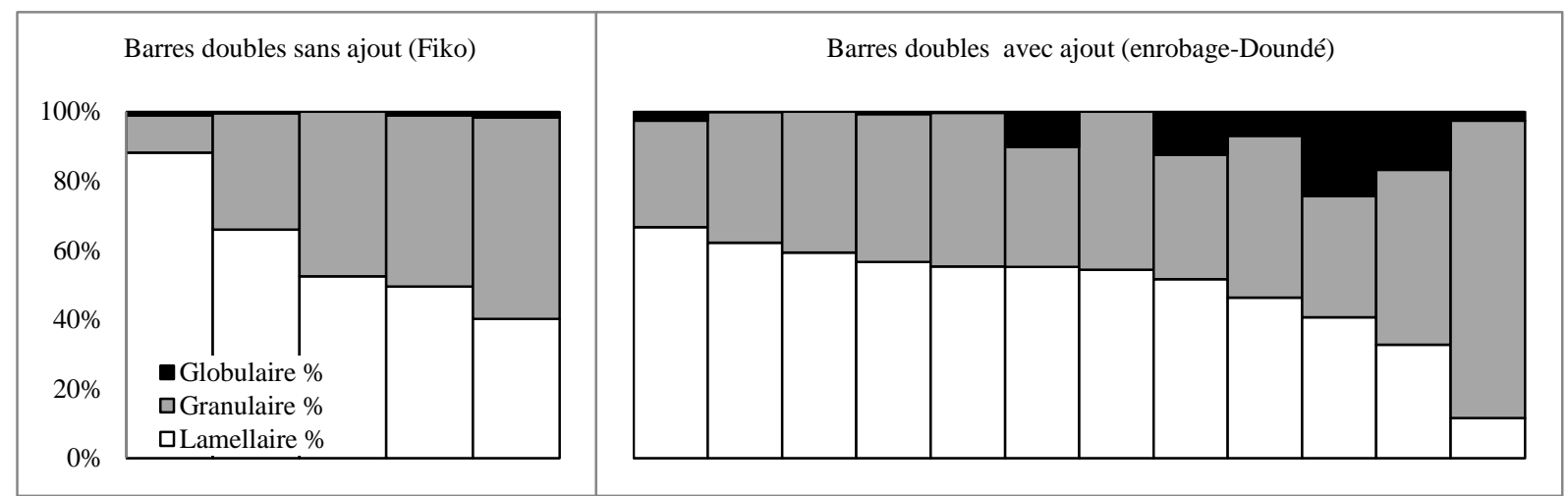

Figure 107 - Répartition des différents types de battitures dans le cas d'ajouts siliceux au cours des soudures. N=17 forgeages comptabilisés. 
- Les scories SGD comportent le plus de fer dans la poudre (moy: $27 \mathrm{~g}$ ) avec une part variable de fer métallique (moy : $12 \mathrm{~g}$ ).

- Les scories SFR, issues de forgeages avec des trempes, comportent quant à elles moins de fer dans la poudre $(21 \mathrm{~g})$ mais bien plus de fer métallique extrait (moy : $41 \mathrm{~g}$ ).

Finalement, les forgeages comportant des soudures et des trempes provoquent une augmentation de la quantité de fer dans la scorie ainsi qu'une augmentation importante de la part de grosses particules de fer métalliques. En fait, plus les temps de chauffe sont longs et nombreux, plus on retrouve du fer dans la scorie, sous la forme de particules et d'oxydes.

\section{Quantité de battitures produites}

Les battitures ont été prélevées pour 120 forgeages, cependant seulement la moitié de ces matériaux a été trié par forme et pesé. Ces données restent superficielles car les échantillonnages ne sont pas quantitatifs et les analyses chimiques et minéralogiques n'ont pas été menées à bien, faute de temps. Les trois morphologies de battitures (lamellaires, granulaires et globulaires) sont observées (Figure 10).

Pour des forgeages sans soudure, la morphologie lamellaire est clairement la plus abondante : il s'agit de martelage à chaud (Figure 106). Cependant la morphologie granulaire est aussi toujours représentée en faible proportion (5-40\%). Seule exception : le forgeage F006 (S006, SFR) qui a produit $60 \%$ de battitures granulaires; la scorie S006 fait d'ailleurs elle-même partie de la classe SFR : pour ce forgeage, il semble visiblement que le foyer a été porté involontairement à très haute température.

Pour des forgeages de deux barres de fer sans incident, ce sont des battitures lamellaires et granulaires qui sont produites, presque à parts égales. Une exception : le forgeage F051 a produit $85 \%$ de battitures granulaires. Rarement des battitures globulaires sont observées; elles représentent au maximum $10 \%$ des particules.

Pour des forgeages de deux barres de fer avec des incidents, les battitures globulaires sont quasiment toujours produites en plus des deux autres morphologies. Par ailleurs, dans le cas d'une surchauffe du métal comme cela est arrivé au cours de nos expérimentations, les battitures produites sont intégralement globulaires (Dungworth \& Wilkes 2007 : 15-16).

L'emploi d'ajouts siliceux a pour effet l'apparition d'un fluide de silicate à l'interface des pièces de fer à assembler. Lors du martelage, cette pellicule fluide est expulsée et coalesce rapidement en une bille après l'impact du marteau (Dungworth \& Wilkes 2007 : 18). Ces battitures montrent en général une matrice de silicate contenant des cristaux de wüstite (Leblanc 2002).

L'emploi d'ajouts sous la forme d'un enrobage (boue) favorise la création de battitures globulaires (Doundé), en comparaison avec des forgeages sans ajouts (Fiko-Kakoli) (Figure 107). Cependant, il n'y a pas de données concernant la répartition des types de battitures à Kobo, où le forgeron jette directement les ajouts dans le foyer.

\section{Nature du fer contenu dans les scories}

Le métal dans les scories est présent sous différentes formes : de fins filaments de quelques $\mu \mathrm{m}$ (Figure 73, cliché 10) jusqu'à des particules de quelques $\mathrm{cm}$ (Figure 73, cliché 7), et en quantité variable, de 0 à $90 \%$ dans la coupe de la scorie.

Durant l'opération de forgeage, les composés comme le carbone (provenant du charbon de bois) peuvent diffuser dans le métal, ce qui augmente sa teneur en carbone; d'un autre côté, l'oxygène apporté par l'air de la soufflerie emporte le carbone du métal, ce qui diminue sa teneur en carbone. Au final, la nature du métal dans les scories est très variée, du fer pur $(<0.02 \% \mathrm{C})$ à de l'acier hypereutectoïde $(>0.8 \% \mathrm{C})$ avec de rares cas de fonte (> $2.1 \%$ C) (Figure 12).

Nous cherchons à déterminer quels paramètres du forgeage influencent la quantité et la nature du métal rencontré dans les scories de forge. Pour une meilleure comparaison des données, il sera uniquement question des scories issues d'un seul forgeage (scorie simple) et de trois forgeages consécutifs (scorie multiple) pour des barres de fer à $0.17 \% \mathrm{C}$ et à $0.45 \% \mathrm{C}$ (Figure 108).

Résultats issus de la figure 108 :

1. Si le métal est contenu en petite quantité sous la forme de petites particules $(<5 \%$ de métal en coupe et jusqu'à $1 \mathrm{~mm}$ de longueur), on observe à chaque fois de la ferrite (uniquement), quelle que soit la nature du fer de départ. Si le métal est présent en grande quantité sous la forme de grosses 
particules, alors la tendance est clairement à une augmentation de la teneur en carbone.

2. Pour des scories formées lors de forgeages sans soudure (SAS et SI), à part les exceptions S006 et $\mathrm{S} 095$, la perte en fer est faible et le fer est souvent décarburé (cf. point 1). Pour des scories issues de forgeages avec soudure (SGD, SFR), la perte en fer est plus importante : la teneur en carbone est variable (ferrite-acier hypereutectoïde) mais souvent dans le sens d'une carburation du métal (cf. point 1).

3. Les scories produites dans les ateliers de Fiko, Kobo et Doundé présentent des faciès métallographiques très différents. Les scories de Kakoli sont les seules, qui, indépendamment des conditions de forgeage, contiennent peu de métal ; il s'agit de fins filaments (Figure 79) constitués en intégralité de ferrite pure (cf. point 1). A Kakoli, le flux d'air important généré par une soufflerie efficace et la température élevée du foyer provoquent manifestement la décarburation totale des particules de métal qui tombent dans la scorie.

4. La durée totale du forgeage ou la durée de chauffe pourraient certainement influencer la teneur en carbone du métal contenu dans les scories, comme le montrent les variations importantes de la carburation du métal dans les scories avec soudures. Cependant, les données ne sont pas assez cohérentes pour évaluer ce paramètre plus en détail.

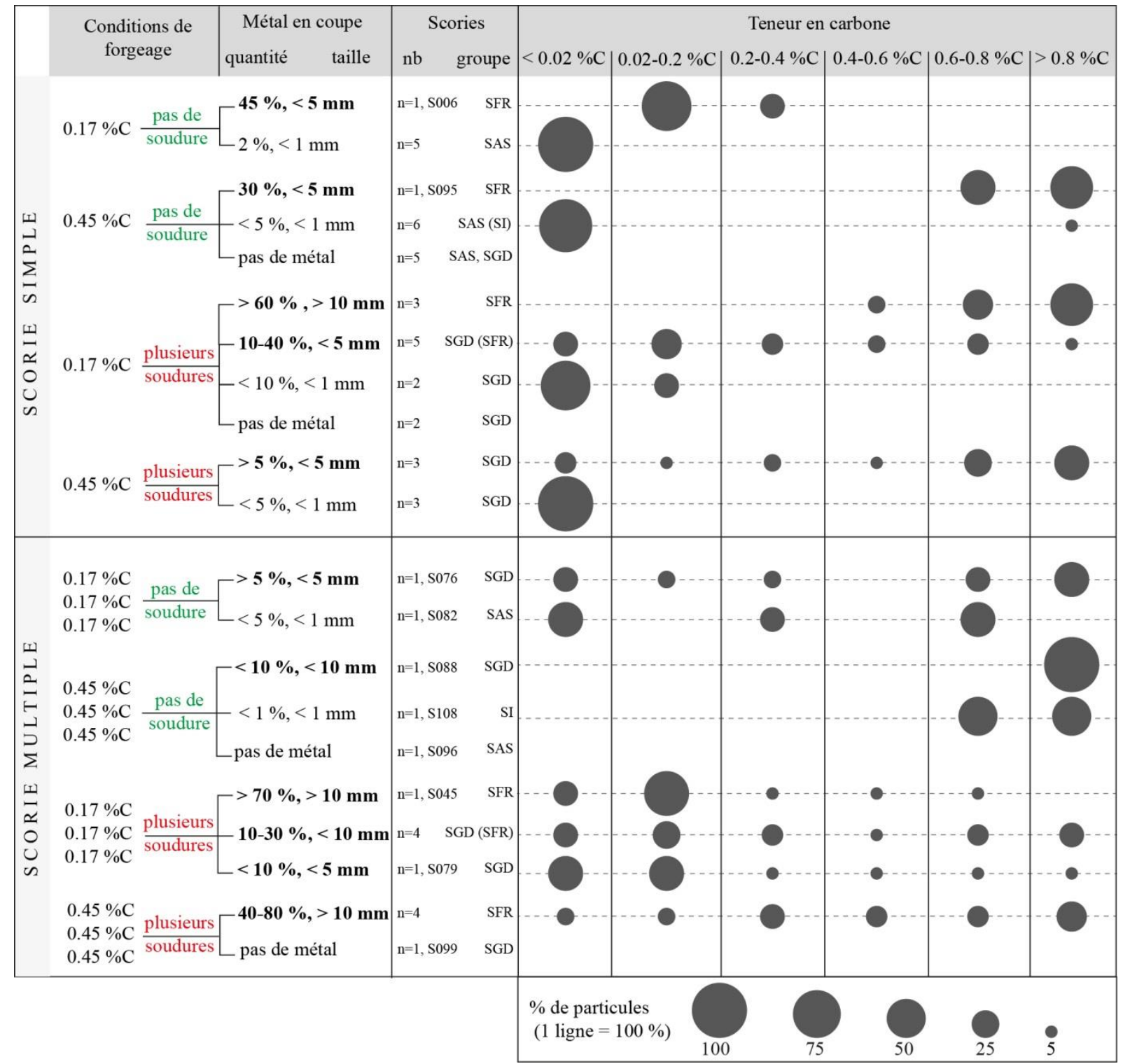

Figure 108 - Quantité et nature du métal contenu dans les scories simples et les scories multiples au cours de forgeages de barres de fer à $0.17 \% \mathrm{C}$ et $0.45 \% \mathrm{C}$, sans/avec soudure. Gras : particules nombreuses et grosses ; ( ) : type de scorie peu représenté. 
5. Dans le cas des scories multiples, le métal montre toujours des faciès très divers. Pour chaque protocole de forgeage, la microstructure est variable dans la gamme ferrite-acier hypereutectoïde, avec une tendance à la carburation du métal. Il n'est toutefois pas possible de différencier des forgeages avec/sans soudure uniquement en étudiant la microstructure du métal.

6. Quatre cas de fonte ont été recensés dans les scories : soit quelques particules de fonte blanche (S094) ou grise (S117), soit toute la pièce principale qui est en réalité une fonte grise (S120) ou bien blanche et grise (S103). Points communs entre ces scories: trois pièces sur quatre proviennent de Doundé, relativement de petites dimensions (diamètre min-max : $5-8 \mathrm{~cm}$ ), poids variable (170$270 \mathrm{~g}$ ), au moins une soudure. Ces scories témoignent d'une carburation importante.

7. Seule la scorie S076 contient une particule montrant des structures de refroidissement rapide. Cependant aucune trempe n'a été effectuée durant les forgeages correspondants. De plus, le métal contenu dans les scories provenant de forgeages avec trempe, ne montre pas des faciès de refroidissement.

Dans la littérature sur les scories de forge archéologiques, on admet généralement que la teneur en carbone maximum observée reflète celle travaillée. Nos données expérimentales montrent un résultat important: il semble à priori difficile d'interpréter la teneur en carbone du métal forgé à partir de celle des fragments de métal emprisonnés dans les scories. On savait que des phénomènes de décarburation limitaient ces interprétations mais il semble également que, en fonction des conditions thermodynamiques locales, des phénomènes de carburation significatifs soient également générés.

Il reste donc très hasardeux d'utiliser les déchets pour identifier la qualité de la matière première ou du produit mais il est peut-être nécessaire d'étudier les scories de manière plus complète (observation sur toute la section par exemple), ce qui n'a pas pu être fait pour des raisons techniques.

\section{Evaluation de la masse de fer travaillée}

En prenant en compte les données calculées de la perte en fer lors des forgeages (Figure 54) et de la répartition de ce fer (Figure 101), il est possible d'évaluer la masse de fer initialement travaillée (Figure 109). Il en ressort que l'estimation de la quantité de métal initialement forgé est influencée par la nature de la scorie (argilo-sableuse ou fayalitique)

Pour des scories SAS et SI qui contiennent $10 \mathrm{~g}$ de fer, cela correspond à une perte en fer de 100 à $200 \mathrm{~g}$ et donc à une masse travaillée de $500 \mathrm{~g}$ à 2000 g, soit moins de $2 \%$ de la masse du fer travaillée.

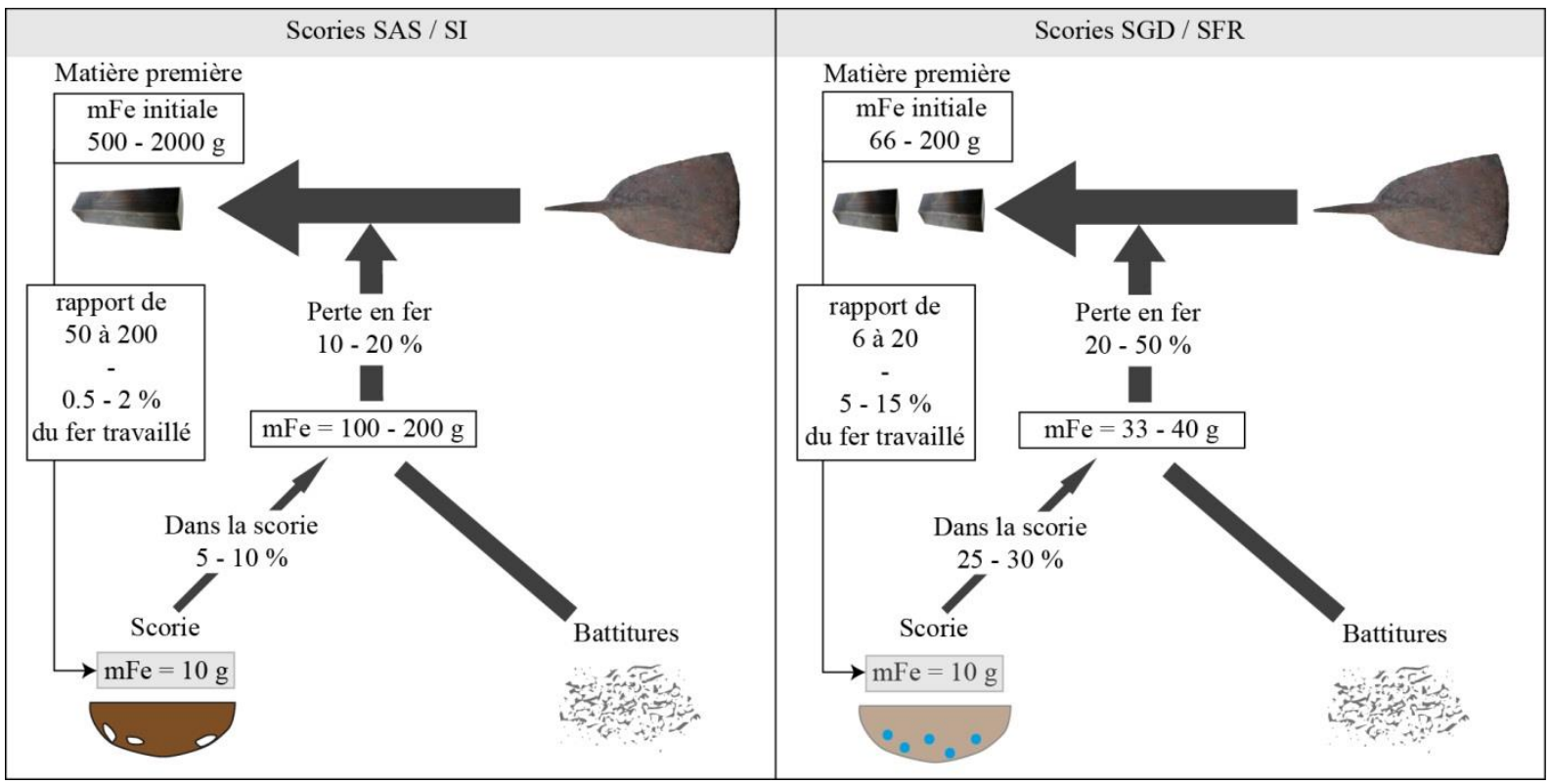

Figure 109 - Calcul de la masse de fer initiale à partir d'une masse de fer de $10 \mathrm{~g}$ dans des scories $\mathrm{SAS} / \mathrm{SI}$ et $\mathrm{SGD} / \mathrm{SFR}$. 
Pour des scories SGD et SFR qui contiennent $10 \mathrm{~g}$ de fer, cela correspond à une perte en fer de 33 à $40 \mathrm{~g}$ et à une masse de fer travaillée de 66 à $200 \mathrm{~g}$, soit 5 à $15 \%$ de la masse du fer initiale.

Les données de perte en fer pour les petits objets sont souvent estimées à $10 \%$, celles de gros objets à $20 \%$, la perte de métal dans la scorie entre 5 et $25 \%$ (Perret 2002, 2009; Serneels 2006; Serneels \& Perret 2007). Ces estimations s'approchent des valeurs que nous avons calculées.

\subsubsection{Combustible}

\section{Consommation de charbon}

Tout le charbon qui a été utilisé a été pesé. La consommation de charbon de bois en gramme par minute de chauffe peut permettre de mettre en évidence les forgeages dont la consommation est soit la plus faible soit la plus forte. Prendre en compte la durée de chauffe permet de rester plus précis car c'est durant la chauffe que l'air provenant de la soufflerie permet leur combustion; durant le martelage, la soufflerie est inactive et la combustion est finalement assez limitée.

Cette consommation de charbon de bois varie entre $40 \mathrm{~g} / \mathrm{min}$ et $120 \mathrm{~g} / \mathrm{min}$. Il est tentant d'imaginer que les forgeages avec soudure voient leur consommation de charbons augmenter du fait de travail rallongé et d'un besoin évident de plus de combustible (consommation autour du trend à $120 \mathrm{~g} / \mathrm{min}$ ). Cependant l'écart de consommation entre des forgeages d'une barre de fer, de deux barres de fer, sans ou avec des incidents n'est pas mis en évidence (Figure 110, A).

Par contre, la consommation du charbon de bois varie en fonction de l'atelier (B). Elle est la plus importante à Doundé et à Kakoli (60-120 g/min) et la plus faible à Kobo et Fiko ( $60 \mathrm{~g} / \mathrm{min})$. Quelques forgeages de Fiko montrent une consommation plus faible ( $40 \mathrm{~g} / \mathrm{min})$. A Doundé, le volume important du foyer peut expliquer une consommation importante ; cependant ce volume est restreint à Kakoli et ne permet pas de comprendre la forte consommation de charbon dans cet atelier.

Enfin, la différence de consommation entre les différents types de scories n'est pas mise en évidence (C).

L'incorporation des cendres au cours du forgeage est aléatoire dans les scories de forge, leur proportion est inconnue et non mesurable.

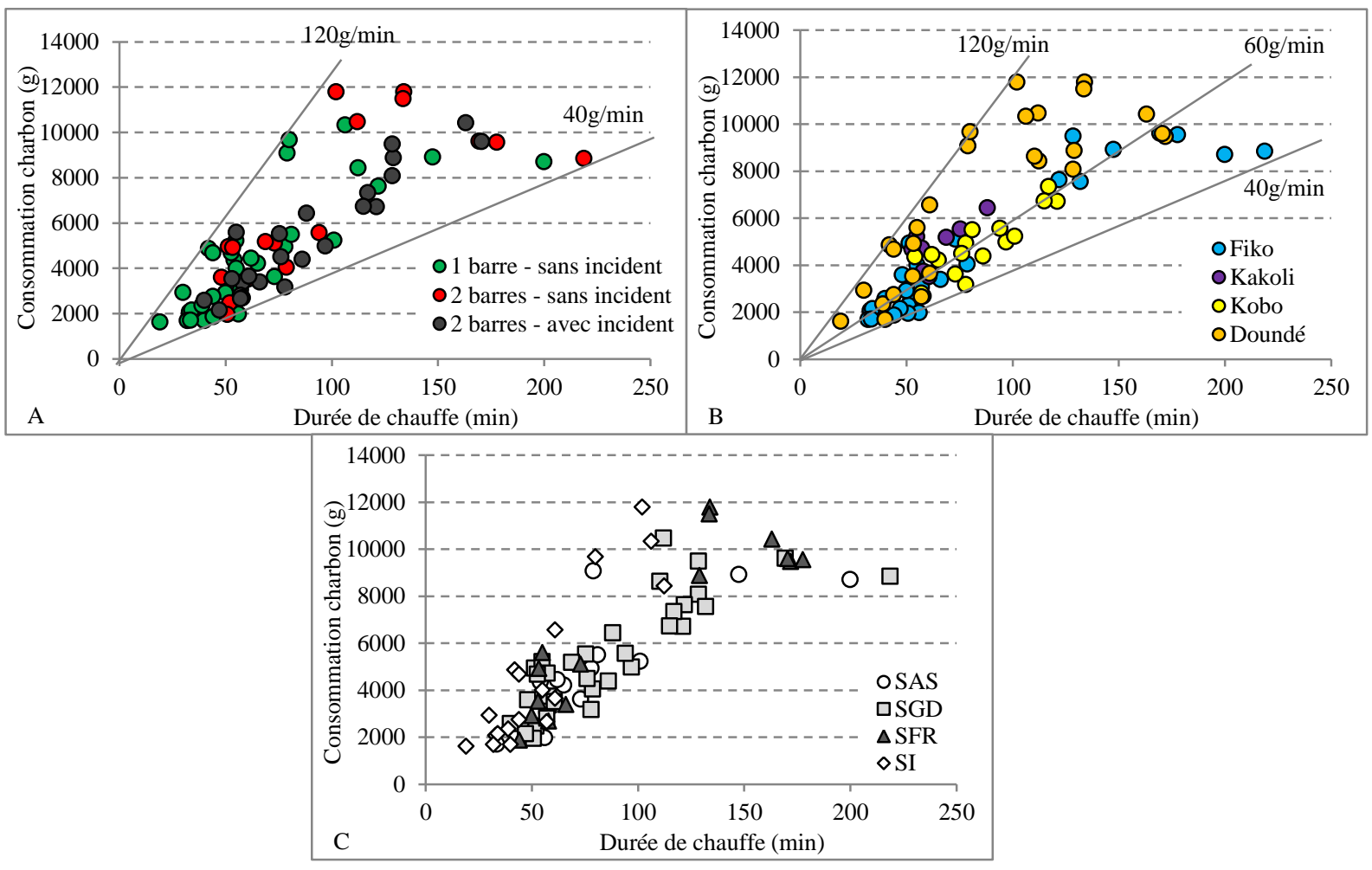

Figure 110 - Consommation de charbon (g) par minute de chauffe.

$\mathrm{A}$ : par nombre de barres à forger et incidents ; B : par atelier ; C : par type de scorie. 
En effet, si $3 \mathrm{~kg}$ de charbons ont été utilisés, alors peut-être uniquement $2 \mathrm{~kg}$ ont été consumés et seulement une faible fraction a pris part à la scorie.

Pour cela, il est impossible de restituer la quantité de charbons réellement consommée et incorporée aux scories de forge.

\section{Apport chimique du combustible}

Le calcium $\mathrm{CaO}$ est en parfaite corrélation avec le strontium $\mathrm{Sr}$ dans les scories de tous les ateliers (Figure 111-A). Le rapport Sr:Ca est élevé et est le même à Kobo et Doundé (plaine), ce qui montre que dans cette région le substratum pour le charbon de bois est le même; le rapport Sr:Ca est plus faible à Fiko et Kakoli (plateau), avec un apport particulièrement important en $\mathrm{CaO}$ à cause de l'emploi de ciment pour le rechapage du foyer.
Le charbon de bois qui apporte $\mathrm{Ca}$ et $\mathrm{Sr}$ apporte également du phosphore $\mathrm{P}$, même si quelques valeurs de Doundé sont polluées (B).

Sr est corrélé à $\mathrm{MgO}$ pour tous les ateliers de forge (C). $\mathrm{MgO}$ et $\mathrm{Rb}$ sont également corrélés, avec une tendance pour les ateliers du plateau et une autre pour ceux de la plaine (D); ces deux tendances montrent que $\mathrm{Rb}$ est aussi apporté par une autre source comme l'argile.

La corrélation entre $\mathrm{CaO}$ et $\mathrm{K}_{2} \mathrm{O}$ est bonne (E) ; les rapports $\mathrm{K}_{2} \mathrm{O}: \mathrm{CaO}$ diffèrent pour les mêmes raisons ; de plus, $\mathrm{K}_{2} \mathrm{O}$ est aussi normalement apporté par les argiles.

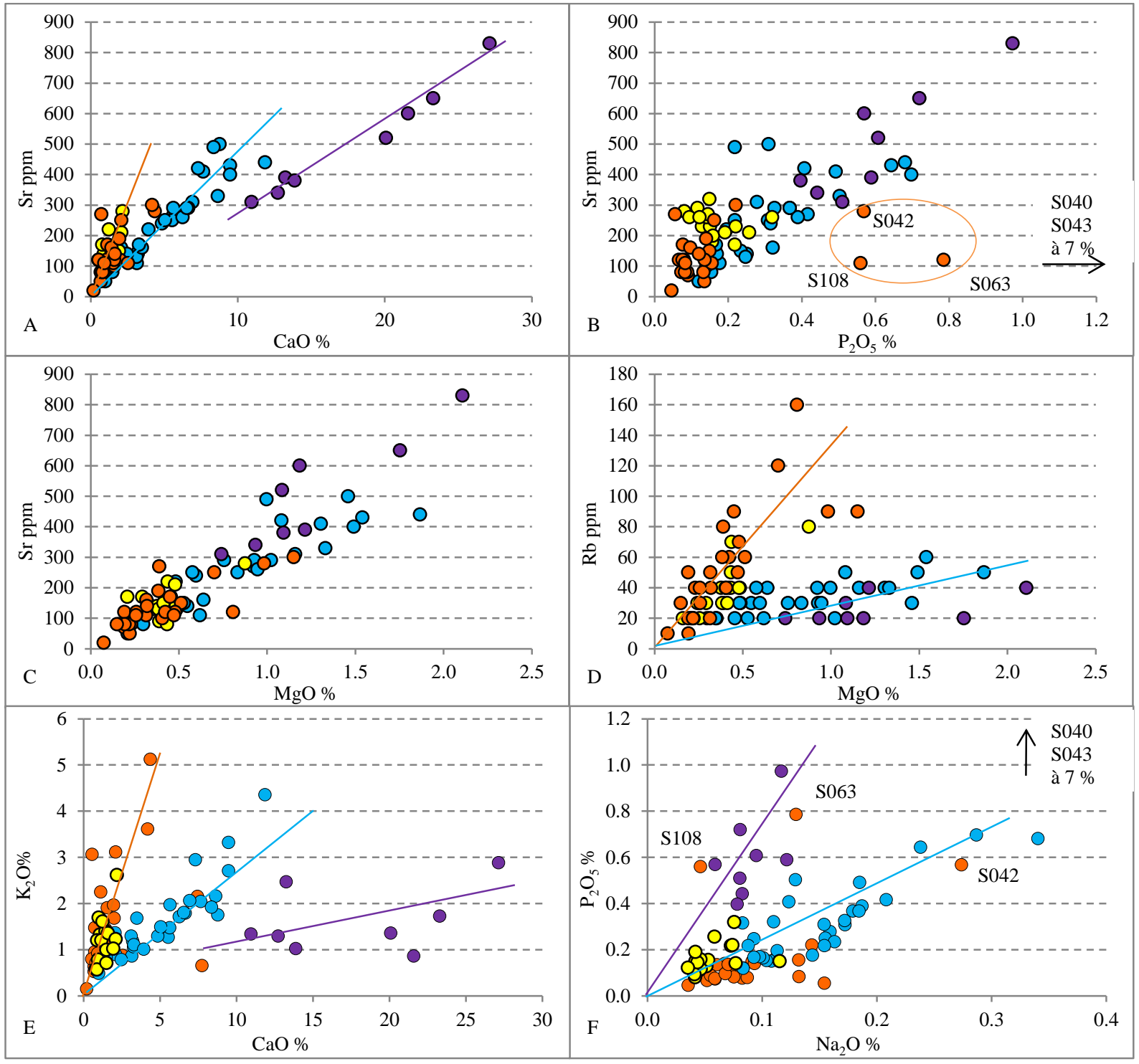

Figure 111 - Apport chimique du combustible dans les scories de forge, dans les quatre ateliers (Encart 3 - Code-couleur). 
Enfin, $\mathrm{P}_{2} \mathrm{O}_{5}$ est corrélé à $\mathrm{Na}_{2} \mathrm{O}(\mathrm{F})$ pour les ateliers du plateau ; cela est moins évident pour ceux de la plaine.

En conclusion, les charbons de bois apportent plusieurs éléments chimiques dans les scories de forge : $\mathrm{Ca}, \mathrm{Mg}, \mathrm{P}, \mathrm{K}, \mathrm{Na}, \mathrm{Sr}$ (et Rb)

\subsubsection{Inclusions des barres de fer}

$\mathrm{Au}$ cours des travaux de forgeage, les inclusions contenues dans les fers sont susceptibles de tomber dans le foyer et donc prendre part à la scorie. Les éléments chimiques concernés sont donnés au tableau 27.

Les fers modernes utilisés ont été choisis en fonction de leur faible teneur en éléments d'alliage (Tableau 8). Ils contiennent un peu de manganèse (Mn 0.5-1.4\%), de chrome ( $\mathrm{Cr}<0.4 \%)$ et de nickel (Ni $<0.4 \%$ ). Dans les scories de forge on pourrait s'attendre à une influence négligeable.

Les fers africains utilisés contiennent une proportion significative d'inclusions contenant des minéraux de titane (ulvöspinelle, perovskite, rutile) et de manganèse (galaxite, jacobsite). Il faut aussi noter des valeurs élevées en zirconium dans certaines phases (Tableau 9).

Pour comprendre l'apport de ces éléments chimiques sur la composition chimique des scories, nous avons comparé le corpus des scories issues du forgeage de deux barres africaines (Af), avec un corpus de scories issues du forgeage de deux barres d'acier moderne (Mod). Les scories formées à partir des barres africaines (A1D, A2D) sont au nombre de 6 : Fiko (S007, S008) et Kobo (S014, S015, S024 et S027). Les barres modernes sont des M1D, M2D et M4D, en version simple ou triple, dans ces mêmes ateliers.

Tout d'abord, le nickel Ni est absent dans plus de la moitié des scories. Dans l'autre moitié, les teneurs sont très faibles (jusqu'à $70 \mathrm{ppm}$ ) excepté 2 pièces à 110 ppm (S103) et 160 ppm (S069); de plus $\mathrm{Ni}$ est un élément qui s'oxyde moins vite que le fer et qui a donc tendance à rester dans le métal plutôt que dans la scorie. Le molybdène Mo présente quant à lui de faibles variations et des teneurs négligeables (50 à 100 ppm). Les teneurs en zinc Zn ne sont pas significatives $(<100 \mathrm{ppm})$.

Le titane $\mathrm{TiO}_{2}$ (absent des aciers modernes) et la silice $\mathrm{SiO}_{2}$ sont tous deux associés ensemble (Figure 112 - A). Le titane varie peu, de 0.1 à $0.5 \%$. Le rapport $\mathrm{TiO}_{2}: \mathrm{SiO}_{2}$ est plus important pour les scories de Fiko par rapport à celles de Kobo : les scories de Fiko contiennent plus de titane : il s'agit vraisemblablement de la composition du sol qui est plus riche en titane à Fiko. Dans ces ateliers, le rapport $\mathrm{TiO}_{2}: \mathrm{SiO}_{2}$ est plus important pour les scories formées avec des barres de fer africaines : le titane est donc un élément apporté par les inclusions de scorie de réduction.

Le même effet est constaté entre le zirconium $\mathrm{Zr}$ et la silice $\mathrm{SiO}_{2}$ (B). Cet élément est apporté par les inclusions de scorie et augmente la teneur en $\mathrm{Zr}$ des scories issues de forgeages de barres africaines.

C'est encore la même chose entre le vanadium $\mathrm{V}$ (absent des aciers modernes) et la silice $\mathrm{SiO}_{2}(\mathrm{C})$. Le vanadium est compris entre 20 et 50 ppm pour les scories de Kobo et entre 30 et 100 ppm pour les scories de Fiko. De plus, les scories provenant de fers africains sont encore plus riches en $\mathrm{V}$, que ce soit à Fiko ou à Kobo.

Les teneurs en cuivre $\mathrm{Cu}$ dans les scories d'aciers modernes (100-1090 ppm) sont supérieures aux teneurs en $\mathrm{Cu}$ dans les scories de fers africains (0-140 ppm) (D) : les aciers modernes apportent l'élément $\mathrm{Cu}$; de plus, pour les scories provenant de Kobo, les teneurs en $\mathrm{Cu}$ (0-340 ppm) restent toujours plus faibles que pour les scories de Fiko (01090 ppm). Concernant le soufre S, les teneurs dans les aciers modernes ou fers africains à Kobo sont similaires $(<300 \mathrm{ppm})$ et très basses, donc $\mathrm{S}$ provient d'une autre source; à Fiko la fourchette de $\mathrm{S}$ est grande (100-1350 ppm), sans distinction entre les 2 types de fer donc ici aussi $\mathrm{S}$ vient aussi d'ailleurs.

\begin{tabular}{|c|c|c|c|c|c|c|c|c|c|c|c|}
\hline & $\mathrm{Si}$ & $\mathrm{Ti}$ & $\mathrm{Mn}$ & $\mathrm{P}$ & $\mathrm{Cu}$ & $\mathrm{Cr}$ & $\mathrm{Zr}$ & $\mathrm{V}$ & $\mathrm{S}$ & $\mathrm{Ni}$ & Mo \\
\hline Fers africains & $\boldsymbol{V}$ & 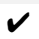 & $\checkmark$ & $\mathcal{V}$ & $\checkmark$ & $\boldsymbol{V}$ & $\boldsymbol{V}$ & $\checkmark$ & ? & & \\
\hline Aciers modernes & $\boldsymbol{V}$ & & $\nu$ & $\checkmark$ & $\nu$ & $\checkmark$ & & & $\boldsymbol{V}$ & $\boldsymbol{v}$ & 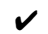 \\
\hline
\end{tabular}

Tableau 27 - Liste des éléments chimiques présents dans les fers africains et les aciers modernes, d'après l'analyse élémentaire des inclusions dans les fers africains (Tableau 9) et les données du fabricant pour les aciers modernes (Tableau 8). 


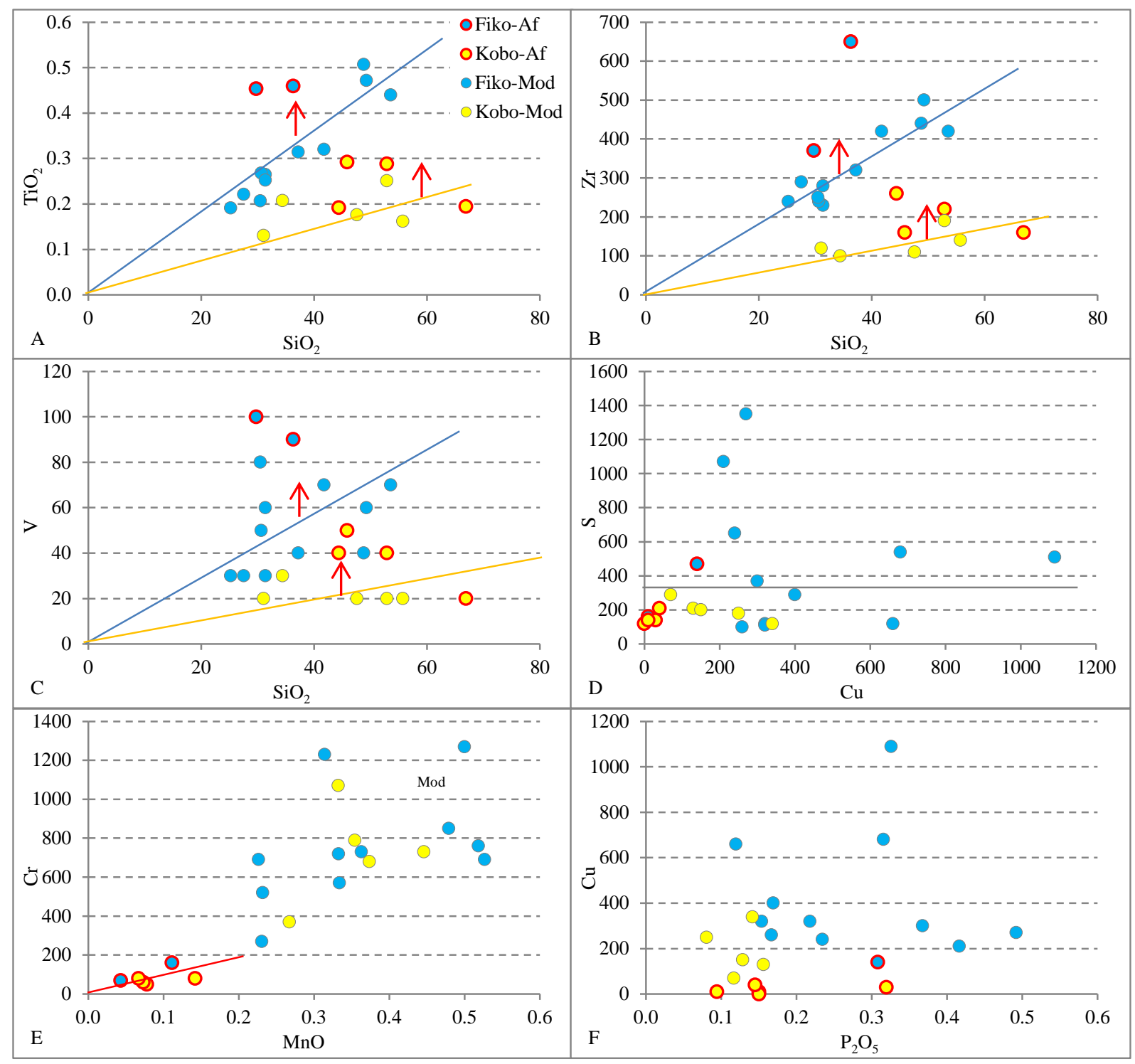

Figure 112 - Apport chimique des aciers modernes et fers africains dans les scories de forge. Fers africains (bordure rouge) - Aciers modernes (bordure noire) - Ateliers de Fiko (bleu) et de Kobo (jaune).

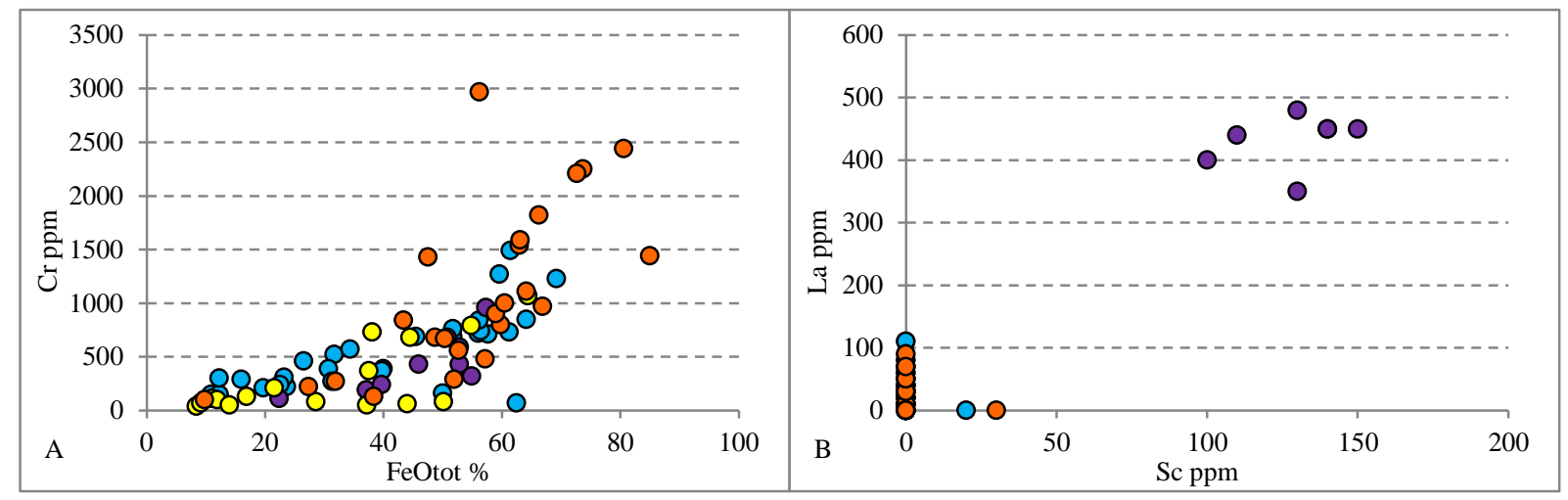

Figure 113 - Apport chimique du fer dans les scories de forge, dans les quatre ateliers (Encart 3 - Code-couleur ) 
Les teneurs en chrome $\mathrm{Cr}$ et manganèse $\mathrm{MnO}$ sont différentes dans les deux types de fers employés (E) : dans les fers africains $\mathrm{Cr}$ varie entre 50 et 160 ppm et $\mathrm{MnO}$ reste inférieur à $0.2 \%$; dans les aciers modernes $\mathrm{Cr}$ varie entre 270 et $1270 \mathrm{ppm}$ et $\mathrm{MnO}$ est compris entre 0.2 et $0.5 \%$. Ces 2 éléments sont associés ensemble pour les scories produites à partir des fers africains ce qui montre qu'ils sont apportés ensemble uniquement par les inclusions de scorie ; la corrélation pour les aciers modernes est moins évidente, cependant $\mathrm{Cr}$ et $\mathrm{MnO}$ semblent tout de même apportés par les impuretés d'aciers modernes. Enfin, le rapport $\mathrm{MnO}: \mathrm{Cr}$ est plus faible pour les aciers modernes : on a certainement un $2^{\text {nd }}$ apport en $\mathrm{Cr}$.

Le phosphore $\mathrm{P}_{2} \mathrm{O}_{5}$ ne semble corrélé à aucun élément en particulier et aucune relation en lien avec les scories venant de fers africains ou d'aciers modernes $(\mathrm{F})$. Les différences de teneurs entre les ateliers (0.1-0.2\% à Kobo et $0.2-0.5 \%$ à Fiko) semblent seulement refléter la qualité du sol : le sol de Fiko est certainement plus riche en phosphore que celui de Kobo.

Enfin, le chrome $\mathrm{Cr}$ (tout comme le manganèse $\mathrm{Mn}$ ) est définitivement lié au métal Fe dans tous les ateliers de forge (Figure 113 - A). Le lanthane La et le scandium Sc se retrouvent uniquement dans les scories de Kakoli (B) ; alors que pour l'ensemble des scories les teneurs sont $<100 \mathrm{ppm}$ pour $\mathrm{La}$ et $<30$ ppm pour Sc, celles de Kakoli ont des teneurs situées entre 350 et 500 ppm pour La et entre 100 et 150 ppm pour Sc. Ces éléments sont généralement associés aux roches siliceuses ; étant donné qu'elles ne peuvent pas provenir du fer ou du charbon, que nous n'avons pas de données concernant l'argile de Kakoli, nous supposons que La et Sc sont présents dans l'argile industrielle qui a servi à fabriquer le ciment qui a servi lors du rechapage du foyer de forge à Kakoli.

Finalement, les analyses chimiques montrent que les fers africains et aciers modernes apportent différents éléments chimiques dans les scories de forge, en plus de Fe.

- Les barres de fer africaines apportent des inclusions de scorie de réduction qui induisent des variations faibles mais évidentes de certains éléments comme $\mathrm{Ti}, \mathrm{V}, \mathrm{Zr}, \mathrm{MnO}$ et $\mathrm{Cr}$.

- Les aciers modernes apportent quant à eux des éléments tels que $\mathrm{Cu}, \mathrm{Cr}$ et $\mathrm{Mn}$.

\subsubsection{Ajouts siliceux}

\section{Argiles}

Dans les scories, la corrélation entre le rubidium $\mathrm{Rb}$ et $\mathrm{Al}_{2} \mathrm{O}_{3}$ ou $\mathrm{K}_{2} \mathrm{O}$ sont bonnes ; on observe toutefois une différence plaine/plateau liée à la nature du sol (Figure 114-A). Le baryum Ba est également lié à $\mathrm{K}_{2} \mathrm{O}$ et à $\mathrm{Al}_{2} \mathrm{O}_{3}$ dans les argiles et dans les ateliers de Fiko, Kakoli et Doundé ; à Kobo certaines scories sont contaminées en $\mathrm{Ba}(\mathrm{B})$.

Dans les scories, par l'apport des argiles, $\mathrm{Al}_{2} \mathrm{O}_{3}$ et $\mathrm{K}_{2} \mathrm{O}$ apportent $\mathrm{Rb}$ et $\mathrm{Ba}$.

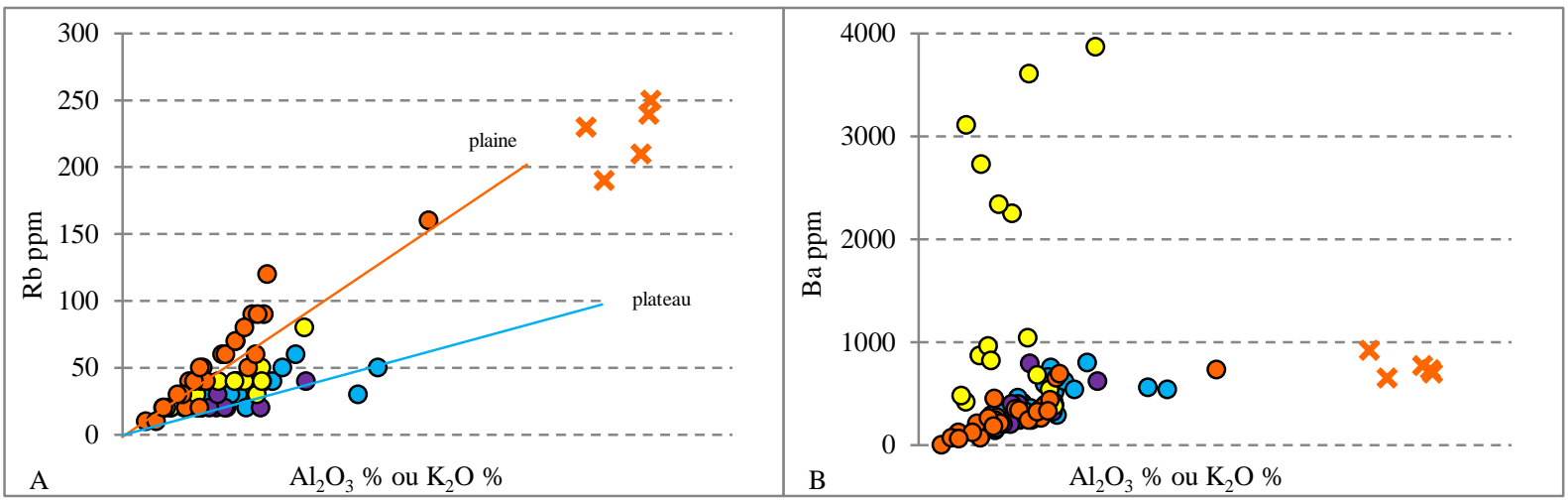

Figure 114 - Apport chimique en $\mathrm{Rb}$ et $\mathrm{Ba}$ dans les scories. $\mathrm{X}=$ tuyère-argile 


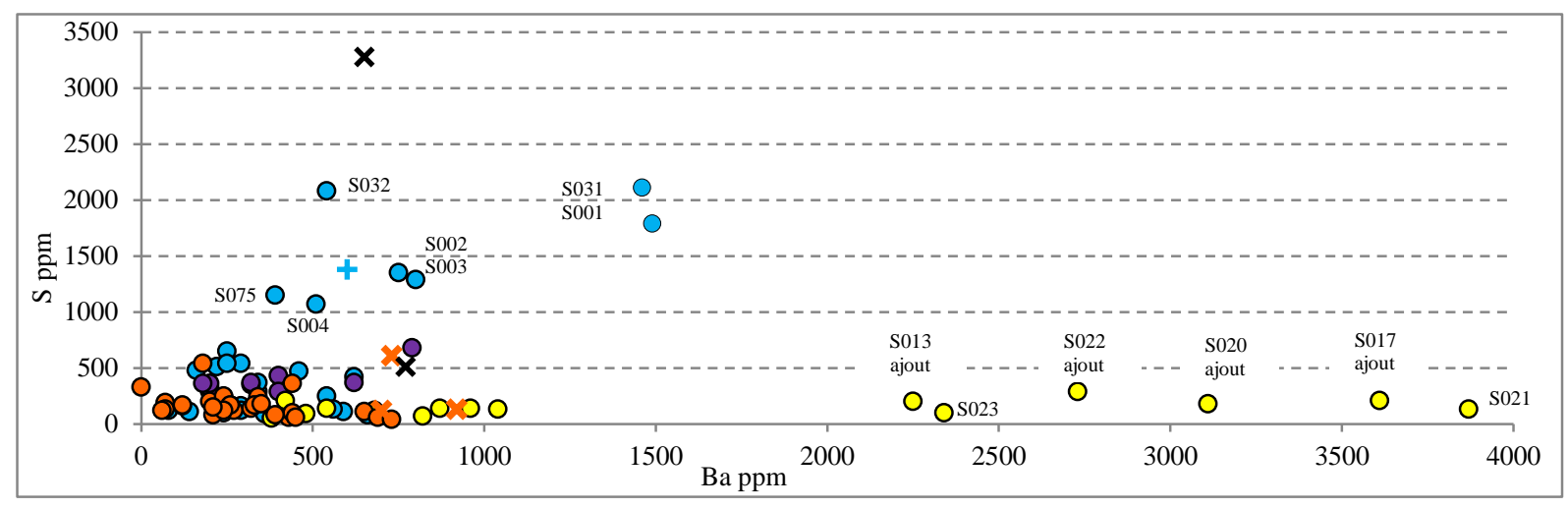

Figure 115 - Teneurs en baryum Ba et en soufre $S$ dans les scories de forge. $X$ : tuyère-argile,+ : paroi

\section{Barytine}

Les scories contiennent normalement jusqu'à 1000 ppm de $\mathrm{Ba}$ (Figure 115). De la barytine $\mathrm{BaSO}_{4}$ a été ajoutée à notre demande dans le mélange de fondants utilisé par Tiégé (Kobo). En présence d'un ajout siliceux contenant du baryum, les scories de Kobo montrent un enrichissement entre 2000 et 4000 ppm. Sur les six scories très riches en $\mathrm{Ba}$, quatre proviennent effectivement de forgeages où la barytine a été employée et deux scories montrent clairement une contamination de cette barytine. Un renouvellement complet des charbons et un bon nettoyage du foyer peut expliquer que les teneurs en Ba des autres scories de Kobo restent $<1000$ ppm.

Un futur travail est l'examen des coupes de scorie pour voir où se trouve $\mathrm{Ba}$ introduit comme ajout.

Il n'y a pas de corrélation entre le baryum Ba et le soufre $\mathrm{S}$ qui est apporté avec $\mathrm{BaSO}_{4}$, vraisemblablement brûlé au cours des forgeages. Le soufre est présent dans les scories jusqu'à 600 ppm ; cependant plusieurs scories montrent des teneurs variant de 1000 à 2000 ppm. Parmi ces pièces (S002, S003, S004 et S032), les teneurs en soufre semblent provenir d'une contamination par les forgeages F001 et F031. Pour ces scories correspondantes, le fond du foyer n'a pas été nettoyé et un apport extérieur de soufre au cours des forgeages précédents est indéniable.

Enfin, certains éléments du foyer (paroi, argile, tuyère) contiennent des teneurs variables en soufre : $120 \mathrm{ppm}<\mathrm{S}<3280 \mathrm{ppm}$. La teneur en soufre pour S075 (S : 1150 ppm) reste inexpliquée.

\subsubsection{Apport chimique par atelier}

Dans cette partie seront distingués les éléments chimiques apportés par l'argile $\left(\mathrm{Al}_{2} \mathrm{O}_{3}\right.$, paroi, tuyère, ajouts), par le sol $\left(\mathrm{SiO}_{2}\right.$, sable, grès) et par le combustible $(\mathrm{CaO}$, charbons) d'atelier à atelier.

\section{Fiko}

Les scories de Fiko montrent clairement un apport concernant la paroi du foyer de forge et le sol (Figure 116-A, B et E).

Le diagramme $\mathrm{SiO}_{2}: \mathrm{Al}_{2} \mathrm{O}_{3}$ (A) montre un rapport constant mais légèrement décalé par rapport à la paroi du foyer : on observe en effet un apport en $\mathrm{SiO}_{2}$ provenant du sable du sol du foyer. Le diagramme $\mathrm{B}$ montre quant à lui une bonne corrélation entre $\mathrm{Al}_{2} \mathrm{O}_{3}$ et $\mathrm{TiO}_{2}$ : ces éléments sont apportés par la paroi. La corrélation en $\mathrm{E}$ montre qu'au moins une partie de $\mathrm{MgO}$ est apporté, avec $\mathrm{SiO}_{2}$, par la paroi.

On observe un enrichissement en $\mathrm{TiO}_{2}$ pour les scories S007 et S008 qui proviennent de forgeages de barres de fer africaines (Figure 112-A). Enfin, on note que les scories S037, S038 et S039, faites à partir de fers de recyclage (jante de camion), ont une teneur plus importante en $\mathrm{Al}_{2} \mathrm{O}_{3}$ que les autres scories.

Les scories de Fiko reçoivent également un apport chimique mixte de la paroi du foyer et du charbon de bois (C et D).

Les corrélations $\mathrm{Al}_{2} \mathrm{O}_{3}: \mathrm{K}_{2} \mathrm{O}$ et $\mathrm{Al}_{2} \mathrm{O}_{3}: \mathrm{CaO}$ montrent une forte contribution de la paroi et une faible contribution du charbon de bois.

L'apport chimique concernant le charbon de bois est visible dans les diagrammes $\mathrm{F}, \mathrm{G}$ et $\mathrm{H}$.

La corrélation $\mathrm{K}_{2} \mathrm{O}: \mathrm{CaO}(\mathrm{F})$ est parfaite, ce qui montre que ces éléments sont apportés par les charbons. Enfin, $\mathrm{CaO}, \mathrm{MgO}$ et $\mathrm{P}_{2} \mathrm{O}_{5}$ sont également apportés par les charbons mais on note toutefois un enrichissement en magnésium $(\mathrm{G})$ et en phosphore pouvant provenir d'une autre source. 


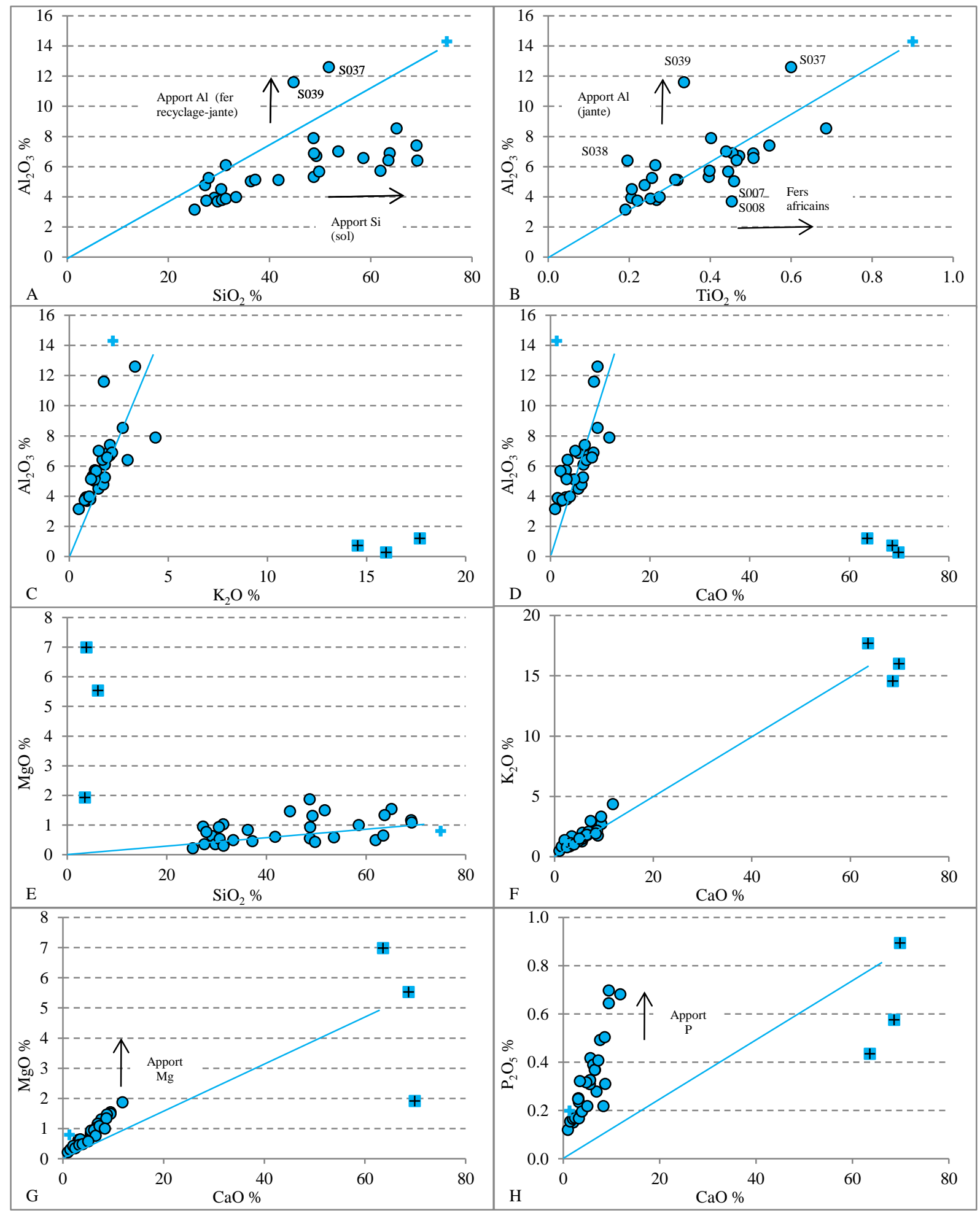

Figure 116 - Apport des éléments chimiques dans les scories de Fiko.

$\begin{array}{lll}+ \text { paroi } & \mathbf{x} \text { argile - tuyère } & \boxplus \text { charbons } \\ * \text { grès } & \bigcirc \text { scorie sans ajout siliceux } & \bigcirc \text { scorie avec ajout siliceux }\end{array}$




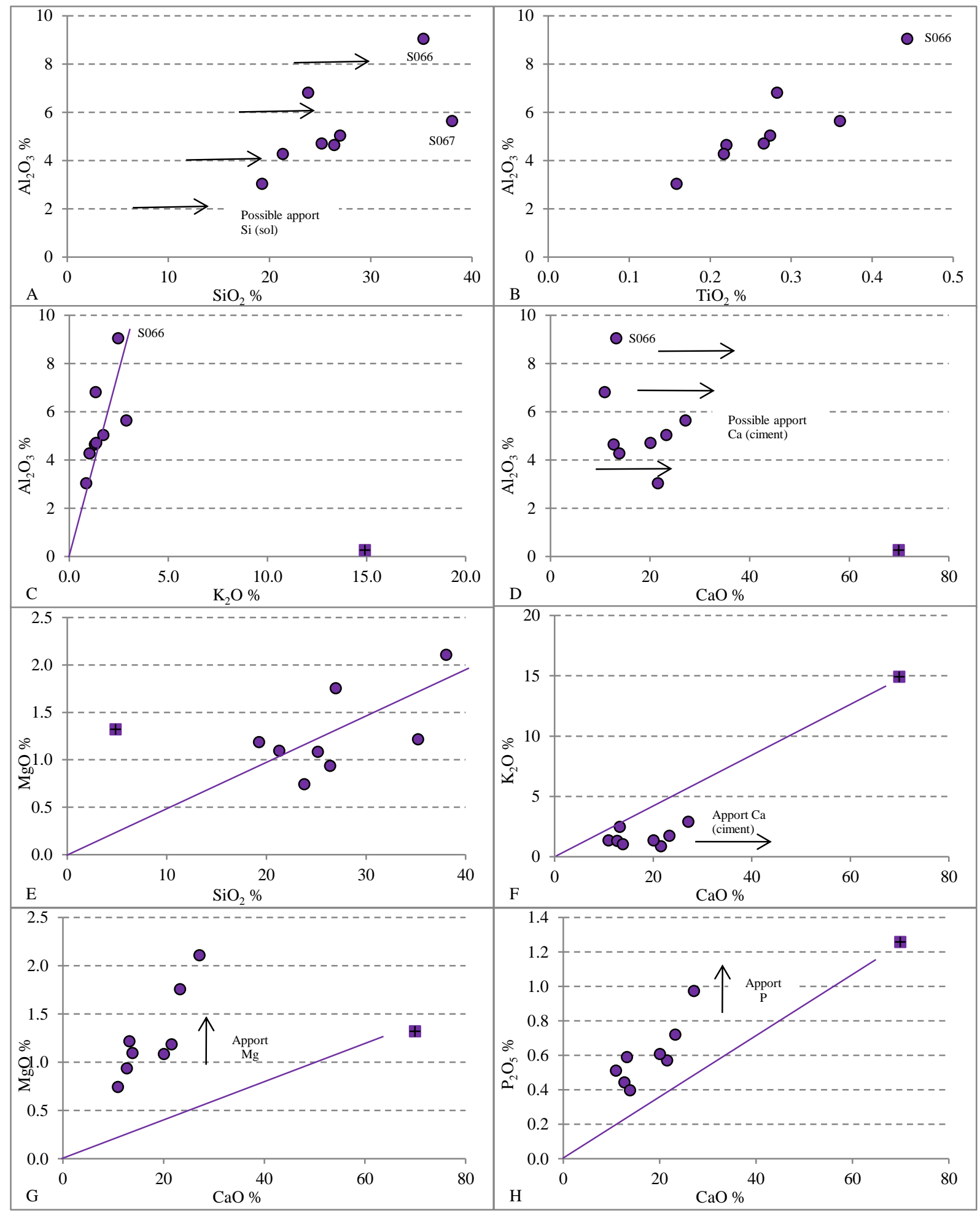

Figure 117 - Apport des éléments chimiques dans les scories de Kakoli.

$\begin{array}{lll}+ \text { paroi } & \mathbf{x} \text { argile - tuyère } & \text { 由 charbons } \\ * \text { grès } & \bigcirc \text { scorie sans ajout siliceux } & \bigcirc \text { scorie avec ajout siliceux }\end{array}$




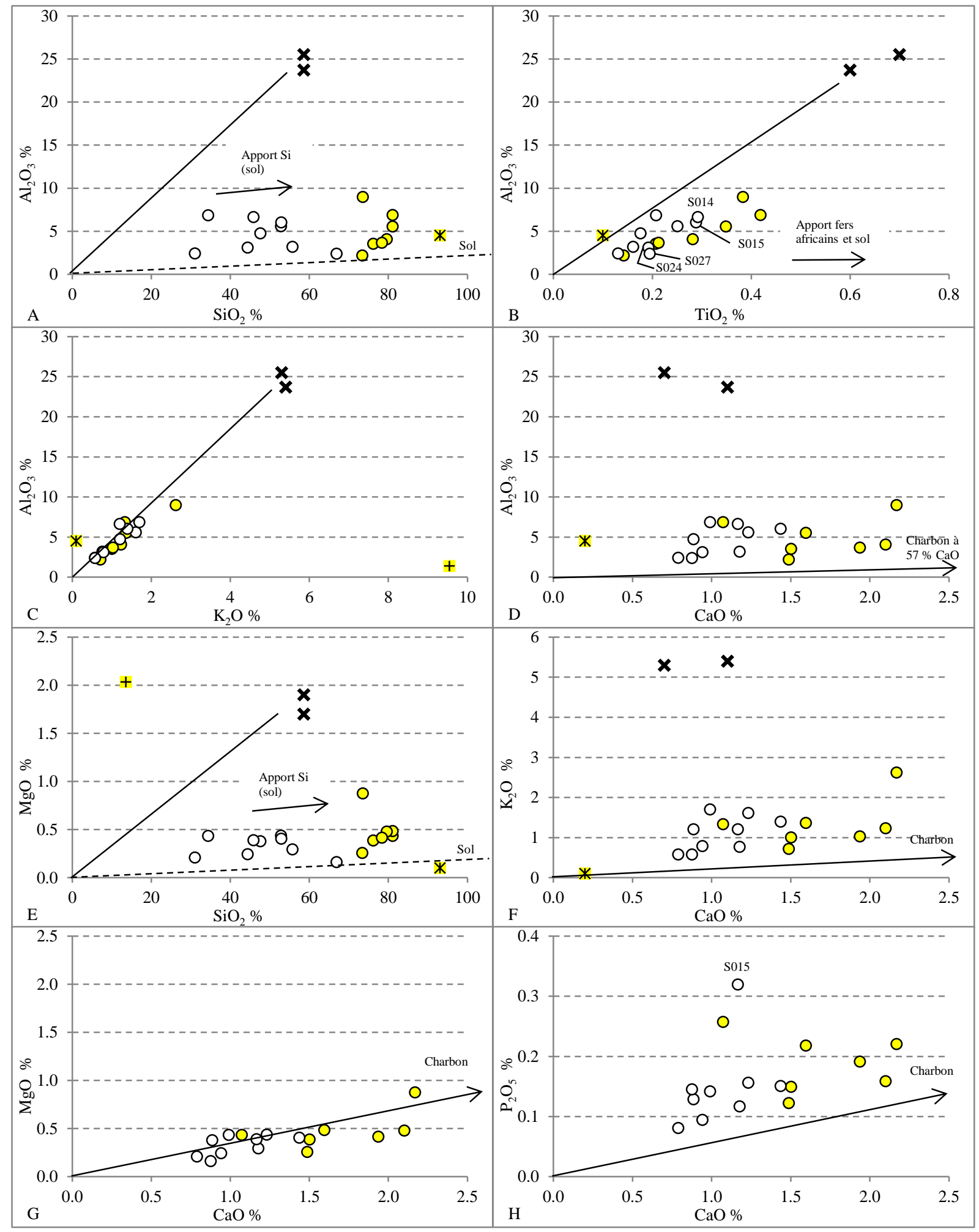

Figure 118 - Apport des éléments chimiques dans les scories de Kobo. 


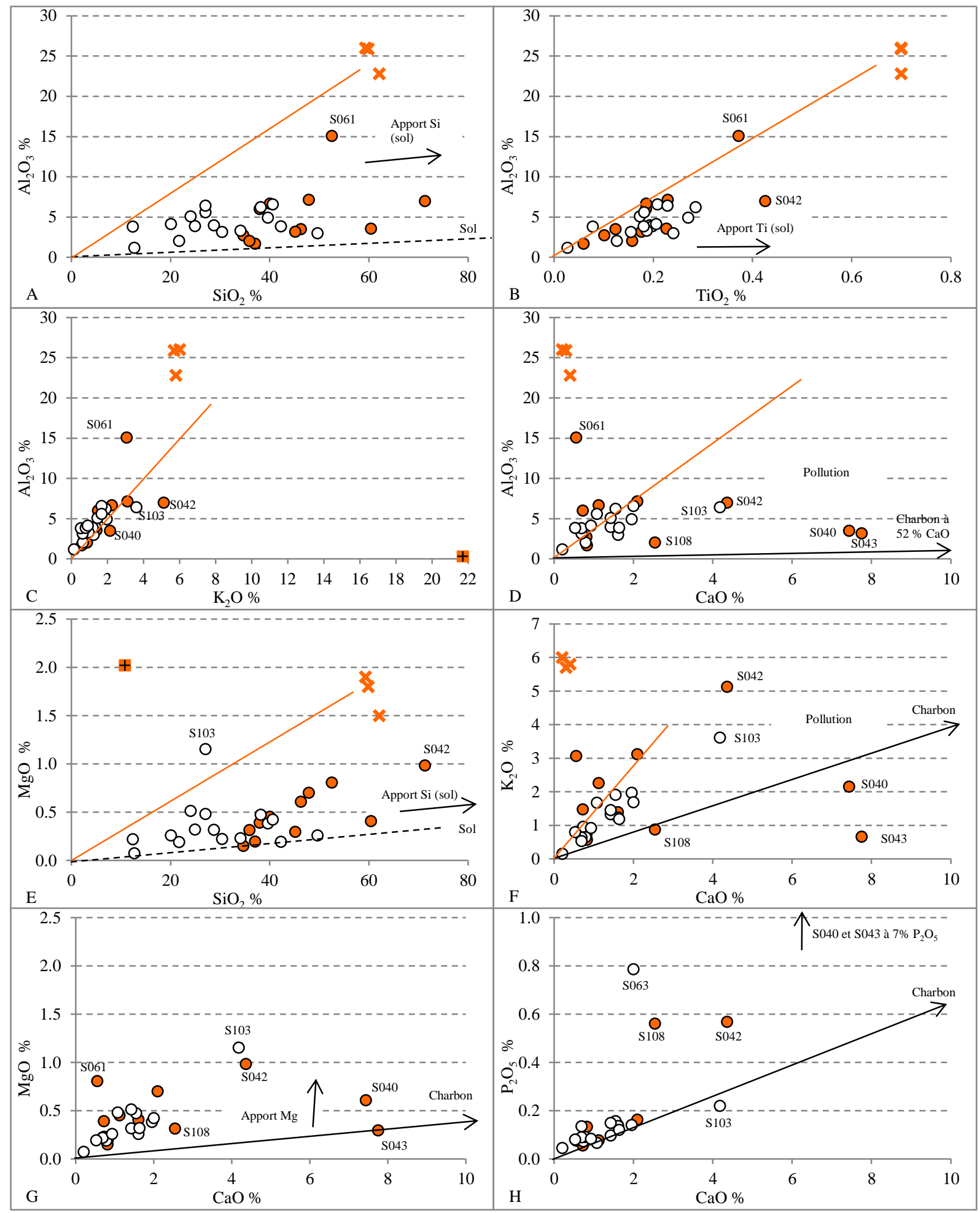

Figure 119 - Apport des éléments chimiques dans les scories de Doundé.

$\begin{array}{lll}+ \text { paroi } & \mathbf{x} \text { argile - tuyère } & \text { 由 charbons } \\ * \text { grès } & \bigcirc \text { scorie sans ajout siliceux } & \bigcirc \text { scorie avec ajout siliceux }\end{array}$




\section{Kakoli}

Pour cet atelier nous ne disposons pas des données chimiques de la paroi et de la tuyère, uniquement des charbons (Figure 117).

Les diagrammes $\mathrm{Al}_{2} \mathrm{O}_{3}: \mathrm{SiO}_{2}(\mathrm{~A}), \mathrm{Al}_{2} \mathrm{O}_{3}: \mathrm{TiO}_{2}$ (B) et $\mathrm{MgO}: \mathrm{SiO}_{2}$ (E) montrent des rapports constants; cependant nous ne pouvons pas nous prononcer davantage sur l'origine (paroi ?) et sur une possible contamination en silice dans les scories (sol). On note toutefois que S066 qui comporte les teneurs les plus élevées en $\mathrm{Al}_{2} \mathrm{O}_{3}, \mathrm{SiO}_{2}$ et $\mathrm{TiO}_{2}$, comporte un fragment de tuyère (Figure 79).

On note une bonne corrélation sur le diagramme $\mathrm{C}$ : $\mathrm{Al} 2 \mathrm{O} 3$ et $\mathrm{K} 2 \mathrm{O}$ qui ne proviennent pas des charbons mais qui pourraient provenir de la paroi du foyer.

La corrélation pour $\mathrm{K}_{2} \mathrm{O}: \mathrm{CaO}(\mathrm{F})$ n'est pas tout à fait dans l'alignement des charbons de bois à cause d'un enrichissement en calcium. Nous supposons que ce calcium provient de la paroi du foyer de forge et que cet élément a été ajouté à l'argile de rechapage avec du ciment. Pour cela, on peut penser qu'il y a également un enrichissement en calcium dans le diagramme $\mathrm{D}$.

Les éléments $\mathrm{MgO}, \mathrm{CaO}, \mathrm{P}_{2} \mathrm{O}_{5}$ sont apportés par les charbons de bois (avec $\mathrm{K}_{2} \mathrm{O}, \mathrm{F}$ ) mais $\mathrm{G}$ montre un apport supplémentaire en manganèse et $\mathrm{H}$ un apport en phosphore. La source de cet apport n'est pas identifiée.

\section{Kobo}

Des ajouts siliceux (grès surtout, argile un peu) sont utilisés dans les deux ateliers Tomo (Kobo et Doundé) pour réaliser les soudures de deux barres de fer (Figure 34). Dans ces ateliers, les scories formées sans ajouts sont de type SAS à Kobo et SI à Doundé ; elles sont riches en silice car l'apport en fer est limité. Pour comprendre l'influence chimique de l'apport des ajouts siliceux, il faut regarder uniquement les SGD et non pas les SAS/SI.

De plus, dans ces ateliers, comme il s'agit de foyers en fosse, il n'y a pas de paroi en argile cuite comme dans les deux ateliers du plateau.

Les scories de forge produites à Kobo montrent un clair apport mixte entre la tuyère/ajouts de grès et d'argile, et le sol du foyer (Figure 118-A, B et E).

Le diagramme $\mathrm{SiO}_{2}: \mathrm{Al}_{2} \mathrm{O}_{3}$ (A) montre un rapport très décalé par rapport à la paroi du foyer. Pour les SGD on a un apport important de $\mathrm{SiO}_{2}$ dû aux ajouts siliceux et au sol du foyer, pour les SAS cet apport ne provient que du fond du foyer de forge ; d'ailleurs on notera que la plupart des SAS comportent une couche sableuse (Figure 78). Cet influence du sol est très marquée (voir teneurs du grès) et la contribution de l'argile (tuyère) est minime.

Les diagrammes $B$ et $E$ montrent une corrélation avec la tuyère/argile et toujours un apport très important du sol du foyer. On observe que les scories provenant de forgeages avec des barres africaines (S014, S015, S024, S025) montrent un apport supplémentaire en titane (B).

La corrélation est très bonne dans le cas du rapport $\mathrm{Al}_{2} \mathrm{O}_{3}: \mathrm{K}_{2} \mathrm{O}(\mathrm{C})$ : dans ce cas ces éléments sont apportés tous les deux par la tuyère du foyer via l'argile.

Le diagramme D montre que l'apport de $\mathrm{Al}_{2} \mathrm{O}_{3}$ et de $\mathrm{CaO}$ provient de deux sources : une faible contribution de l'argile mais une forte contribution du charbon de bois.

Enfin, les corrélations observées sur les diagrammes F, $\mathrm{G}$ et $\mathrm{H}$ montrent que $\mathrm{K}_{2} \mathrm{O}, \mathrm{CaO}, \mathrm{MgO}$ et $\mathrm{P}_{2} \mathrm{O}_{5}$ sont apportés uniquement par le charbon de bois. On notera que la scorie $\mathrm{S} 015$ a une teneur plus élevée en phosphore que les autres pièces.

\section{Doundé}

Les scories de forge de Doundé montrent également un apport mixte entre la tuyère/ajouts d'argile et le sol du foyer (Figure 119-A, B et E).

La contribution du sol est très importante. $\mathrm{Al}_{2} \mathrm{O}_{3}, \mathrm{SiO}_{2}, \mathrm{TiO}_{2}$ et $\mathrm{MgO}$ sont apportés par la tuyère et ajouts d'argile (pour les SGD) mais on observe toujours un fort apport provenant du foyer de forge $\left(\mathrm{SiO}_{2}\right.$ et $\left.\mathrm{TiO}_{2}\right)$. De plus, on notera que S061 (scorie $\mathrm{SI})$ est particulièrement riche en aluminium (15\% $\mathrm{Al}_{2} \mathrm{O}_{3}$ ).

Les diagrammes $\mathrm{C}$ et $\mathrm{D}$ montrent une contribution mixte de l'argile et des charbons. Les composés $\mathrm{Al}_{2} \mathrm{O}_{3}, \mathrm{~K}_{2} \mathrm{O}$ et $\mathrm{CaO}$ sont apportés dans les scories de forge par ces deux sources.

On observe également une pollution qui concerne cinq scories: S040, S042, S043, S103 et S108. Celles-ci ont des teneurs importantes et anormales en $\mathrm{CaO}, \mathrm{K}_{2} \mathrm{O}$ et $\mathrm{P}_{2} \mathrm{O}_{5}$ à Doundé. Dans cet atelier de forge, il se peut qu'elles aient été contaminées par des excréments d'animaux (apport 
d'apatite) avant les travaux de forge, les animaux pouvant aller et venir aisément sur le site de forge.

Enfin, les corrélations vis-à-vis du charbon de bois sont bonnes, excepté les scories polluées spécifiées ci-dessus $(\mathrm{F}, \mathrm{G}$ et $\mathrm{H})$. Les composés $\mathrm{K}_{2} \mathrm{O}$, $\mathrm{CaO}, \mathrm{MgO}$ et $\mathrm{P}_{2} \mathrm{O}_{5}$ sont apportés par le combustible. On notera toutefois un léger apport en magnésium dans le cas de la corrélation $\mathrm{MgO}: \mathrm{CaO}(\mathrm{G})$.

En conclusion, l'argile est toujours liée aux composés $\mathrm{Al}_{2} \mathrm{O}_{3}, \mathrm{SiO}_{2}, \mathrm{TiO}_{2}$ et $\mathrm{MgO}$, parfois $\mathrm{K}_{2} \mathrm{O}$ et $\mathrm{CaO}$ à Fiko et Doundé. Dans les 2 ateliers de la plaine on observe un apport très important de silice provenant des ajouts siliceux dans le cas des scories SGD et SFR. L'apport de $\mathrm{CaO}$ par le ciment à Kakoli est également mesuré.

Le sol est lié à $\mathrm{SiO}_{2}, \mathrm{TiO}_{2}, \mathrm{Al}_{2} \mathrm{O}_{3}$ et parfois $\mathrm{MgO}$ à Kobo et Doundé. Même à Fiko où les ajouts siliceux sont exclus, on observe tout de même un léger enrichissement en silice qui provient du sol du foyer. Dans les 2 ateliers de la plaine on note un apport très important de silice pour les scories SAS.

Le charbon est lié quant à lui à $\mathrm{K}_{2} \mathrm{O}, \mathrm{CaO}, \mathrm{MgO}$ et $\mathrm{P}_{2} \mathrm{O}_{5}$. A Fiko et Doundé, une source indéterminée apporte également une part de $\mathrm{MgO}$ et $\mathrm{P}_{2} \mathrm{O}_{5}$.

Enfin, d'autres apports ont pu être mesurés : celui de $\mathrm{TiO}_{2}$ dans les fers africains, celui de $\mathrm{Al}_{2} \mathrm{O}_{3}$ dans les fers de recyclage.

\subsubsection{Récapitulatif des apports dans les scories}

Les scories se forment à partir des éléments chimiques fournis par différents matériaux en présence lors de leur formation. Les proportions de ces différentes contributions varient selon les ateliers et les paramètres des travaux de forge. Cette étude nous a permis de mesurer quantitativement (masse ou pourcentage chimique) l'influence de ces différents apports de matériaux.

L'apport le plus important concerne le fer Fe, la matière première travaillée par l'artisan.

Pour un forgeage sans soudure ni incident (SAS/SI), ce fer est perdu au cours du travail, essentiellement sur l'enclume sous la forme de battitures lamellaires et granulaires; seuls 0.5 à $2 \%$ de la masse initiale du fer prennent part à la scorie. Les scories ne contiennent donc pas beaucoup de fer, mais en plus elles ne comptent que très peu de particules sous leur état métallique : dans ce cas il s'agit de petites particules décarburées (scorie simple) ou de teneur variable en carbone (scorie multiple).

Pour un forgeage avec soudure et incident (SGD/SFR), le fer est perdu sur l'enclume sous la forme de battitures lamellaires, granulaires mais aussi globulaires; la proportion de battitures globulaires augmente avec l'emploi d'ajouts siliceux.

\begin{tabular}{|c|c|c|c|c|}
\hline & Fiko & Kakoli & Kobo & Doundé \\
\hline Fer moderne & $\mathrm{FeO}, \mathrm{Cu}, \mathrm{Cr}, \mathrm{Mn}$ & $\mathrm{FeO}, \mathrm{Cu}, \mathrm{Cr}, \mathrm{Mn}$ & $\mathrm{FeO}, \mathrm{Cu}, \mathrm{Cr}, \mathrm{Mn}$ & $\mathrm{FeO}, \mathrm{Cu}, \mathrm{Cr}, \mathrm{Mn}$ \\
\hline Fer africain & $\begin{array}{l}\mathrm{FeO}, \mathrm{Ti}, \mathrm{V}, \\
\mathrm{Zr}, \mathrm{MnO}, \mathrm{Cr}\end{array}$ & - & $\begin{array}{l}\mathrm{FeO}, \mathrm{Ti}, \mathrm{V} \\
\mathrm{Zr}, \mathrm{MnO}, \mathrm{Cr}\end{array}$ & $?$ \\
\hline Fer de recyclage & $\mathrm{Al}_{2} \mathrm{O}_{3}$ & - & - & $?$ \\
\hline $\begin{array}{l}\text { Argile - Paroi } \\
\text { Ajouts }\end{array}$ & $\begin{array}{c}\mathrm{SiO}_{2}, \mathrm{Al}_{2} \mathrm{O}_{3}, \mathrm{TiO}_{2}, \mathrm{MgO}, \\
\left(\mathrm{K}_{2} \mathrm{O}\right),(\mathrm{CaO}) \\
\mathrm{Rb}, \mathrm{Ba}\end{array}$ & $\begin{array}{c}\mathrm{SiO}_{2}, \mathrm{Al}_{2} \mathrm{O}_{3}, \mathrm{TiO}_{2}, \mathrm{MgO} \\
\mathrm{Rb}, \mathrm{Ba} \\
\mathrm{Sc}, \mathrm{La}\end{array}$ & $\begin{array}{l}\mathrm{SiO}_{2}, \mathrm{Al}_{2} \mathrm{O}_{3}, \mathrm{TiO}_{2}, \\
\mathrm{MgO}, \mathrm{Rb}, \mathrm{Ba}\end{array}$ & $\begin{array}{c}\mathrm{SiO}_{2}, \mathrm{Al}_{2} \mathrm{O}_{3}, \mathrm{TiO}_{2}, \mathrm{MgO}, \\
\left(\mathrm{K}_{2} \mathrm{O}\right),(\mathrm{CaO}) \\
\mathrm{Rb}, \mathrm{Ba}\end{array}$ \\
\hline Sol - Ajouts & $\mathrm{SiO}_{2}, \mathrm{TiO}_{2}, \mathrm{Al}_{2} \mathrm{O}_{3}$ & $?$ & $\begin{array}{c}\mathrm{SiO}_{2}, \mathrm{TiO}_{2}, \mathrm{Al}_{2} \mathrm{O}_{3}, \\
\mathrm{MgO}\end{array}$ & $\mathrm{SiO}_{2}, \mathrm{TiO}_{2}, \mathrm{Al}_{2} \mathrm{O}_{3}, \mathrm{MgO}$ \\
\hline Charbons & $\begin{array}{c}\mathrm{CaO}, \mathrm{K}_{2} \mathrm{O}, \underset{\mathrm{MgO}, \mathrm{P}_{2} \mathrm{O}_{5}, \mathrm{Sr}}{\mathrm{Rb}} \\
.\end{array}$ & $\mathrm{CaO}, \mathrm{K}_{2} \mathrm{O}, \mathrm{MgO}, \mathrm{P}_{2} \mathrm{O}_{5}, \mathrm{Sr}, \mathrm{Rb}$ & $\begin{array}{c}\mathrm{CaO}, \mathrm{K}_{2} \mathrm{O}, \mathrm{MgO}, \\
\mathrm{P}_{2} \mathrm{O}_{5}, \mathrm{Sr}, \mathrm{Rb}\end{array}$ & 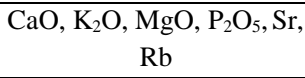 \\
\hline Autres apports & $\mathrm{MgO}, \mathrm{P}_{2} \mathrm{O}_{5}$ & $\mathrm{MgO}, \mathrm{P}_{2} \mathrm{O}_{5}$ & - & $\mathrm{MgO}$ \\
\hline Remarques & Apport faible de $\mathrm{SiO}_{2}$ & $\begin{array}{c}\text { Possible apport en } \mathrm{SiO}_{2} \\
\text { Apport de } \mathrm{Ca}, \mathrm{Sc} \text { et } \mathrm{La} \text { avec le } \\
\text { ciment du foyer }\end{array}$ & $\begin{array}{l}\text { Apport très important } \\
\text { de } \mathrm{SiO}_{2} \\
\text { Apport de } \mathrm{Ba} \text { avec la } \\
\text { barytine }\end{array}$ & $\begin{array}{l}\text { Apport très important de } \\
\qquad \mathrm{SiO}_{2} \\
\text { Pollution de cinq scories } \\
\text { en apatite }\end{array}$ \\
\hline
\end{tabular}

Tableau 28 - Récapitulatif des apports chimiques dans les scories de forge (par atelier). 
Environ 5 à $15 \%$ de la masse du fer travaillée prend part à la scorie dans le foyer de forge. Les scories contiennent beaucoup plus de fer mais en plus, elles comptent bien plus de particules sous leur état métallique. Leur nombre augmente lorsque le forgeron réalise des trempes (qui fragilisent le métal); la taille et la teneur en carbone de ces particules varient sensiblement d'une pièce à une autre.

$\mathrm{Du}$ point de vue de la chimie, les aciers modernes apportent d'autres éléments comme $\mathrm{Cu}$, $\mathrm{Mn}$ et $\mathrm{Cr}$, les fers africains apportent des inclusions de scorie de réduction composées d'éléments comme $\mathrm{TiO}_{2}, \mathrm{~V}, \mathrm{Zr}, \mathrm{MnO}, \mathrm{Cr}$, les fers de recyclage apportent $\mathrm{Al}_{2} \mathrm{O}_{3}$ (Tableau 28).

Le second apport le plus important concerne la silice $\mathrm{SiO}_{2}$, qui est apportée par plusieurs sources : l'argile (tuyère, ajouts), le foyer (paroi, fond du foyer). Les analyses chimiques montrent que les scories sont toujours un peu contaminées par le sol du foyer de forge, même dans les ateliers où les ajouts siliceux ne sont pas utilisés; l'emploi des ajouts siliceux occasionne la formation de scories
SGD/SFR encore plus riches en silice notamment. Dans les ateliers de la plaine, l'apport de l'argile $\left(\mathrm{Al}_{2} \mathrm{O}_{3}\right)$ est très faible en comparaison de celui du sol et des ajouts $\left(\mathrm{SiO}_{2}\right)$.

L'argile (tuyère, ajouts) et le foyer (paroi, sol) apportent $\mathrm{SiO}_{2}, \mathrm{Al}_{2} \mathrm{O}_{3}, \mathrm{TiO}_{2}, \mathrm{MgO}$, éventuellement $\mathrm{K}_{2} \mathrm{O}$ et $\mathrm{CaO}$, ainsi que $\mathrm{Rb}, \mathrm{Ba}, \mathrm{Zr}$ comme traces ; à Kakoli l'argile du ciment apporte La et Sc.

Enfin, la scorie incorpore une certaine quantité de cendres de charbon de bois. Ce charbon apporte $\mathrm{CaO}, \mathrm{K}_{2} \mathrm{O}, \mathrm{MgO}, \mathrm{P}_{2} \mathrm{O}_{5}$ ainsi que $\mathrm{Sr}$ et $\mathrm{Rb}$ en traces. Un apport très important en calcium dans les scories de Kakoli (10-30 \% CaO) suggère que le revêtement réfractaire du foyer de forge est un mélange entre de l'argile et un ciment (riche en $\mathrm{CaO}$ ).

En résumé, il faut parler de plusieurs origines pour comprendre les différentes contributions des matériaux dans les scories de forge. Il s'agit d'un apport complexe (un élément chimique est apporté par plusieurs sources) et chaque scorie a une histoire de formation particulière (conditions de forgeages, atelier). 


\subsection{RELATIONS ENTRE FORGEAGES ET SCORIES}

L'ensemble des travaux de forge a produit un assemblage de scories de forge de natures très diverses, comparable aux scories que l'on peut trouver sur des sites d'anciens ateliers de forge. Les critères morphologiques, minéralogiques et chimiques des scories, en lien avec la nature des travaux de forge, ont été comparés (Tableau 29). Il en ressort que l'étape du préformage est une étape cruciale qui conditionne complètement la nature des déchets qui sont produits.

Au cours du forgeage d'une seule barre de fer, le forgeron effectue un travail rapide sans incident. Le préformage y est bref ou quasi inexistant : la perte de fer pendant le travail est faible $(15 \%$ de la masse de fer initiale), très peu de métal se retrouve dans la scorie (0.5 à $2 \%$ ) : il s'agit essentiellement de petites particules de ferrite des croûtes d'oxydes. La contribution du fer est faible et celle du sol et du foyer est forte. Ce travail sur une seule barre de fer génère peu ou pas de scorie.

Les scories SAS issues de ces forgeages rapides sont dépourvues de fayalite $(<5 \%)$ et elles résultent de l'agglomération du fond et de la paroi du foyer de forge. Les scories SAS-M contiennent quant à elles jusqu'à $15 \%$ de fayalite. Ces scories sont à la fois légères $(<150 \mathrm{~g})$ et poreuses $(>40 \%)$. Si le foyer est trop froid (Kobo), les matériaux siliceux ne s'agrègent pas assez et la scorie devient très friable : c'est le cas des scories réduites. Si le foyer est plus chaud les matériaux sont bien plus cohérents (Fiko).

Un autre type de scorie est observé pour le même type de travaux de forge mais dans d'autres conditions de travail. Si le foyer est volumineux et la température inhomogène, la simple accumulation des matériaux dans le foyer est perturbée : c'est le cas des scories SI. Ces petites pièces sont souvent nombreuses et composites car elles peuvent comporter à la fois une composante sableuse, ferreuse ou fayalitique ; elles ont une morphologie très inhabituelle pour des scories de forge et certaines pièces montrent une minéralogie et une chimie complexes. Une contribution importante pour les SI est le charbon de bois auprès duquel elles s'enrichissent très souvent: teneurs en carbone augmentées dans certaines pièces.

Le préformage étant rapide et exécuté à des températures peu élevées, les apports de matériaux à ce stade sont limités. Le formage quant à lui nécessite des températures plus importantes, pour un travail standardisé par outil et par atelier de forge : c'est à ce stade que l'essentiel de la scorie se forme. Les finitions, effectuées à basse température sur l'enclume, ne participent pas à la formation de la scorie dans le foyer. Les scories SAS et SI sont toutes des scories « de formage ».

Avec deux barres de fer, le temps de préformage est rallongé, le foyer est chauffé davantage et cette étape occupe à présent la place principale dans la chaîne opératoire. Le forgeron doit en effet mettre en forme puis assembler les deux morceaux de métal avant de mettre en forme le lopin réalisé (préforme). A ce stade, la perte de fer générale est conséquente puisque ce sont en moyenne $30 \%$ de la masse du fer initiale qui est perdue au cours du forgeage et jusqu'à $15 \%$ du fer initial qui atterrit directement dans la scorie de forge. La contribution du fer plus importante et le foyer plus chaud permettent la formation d'une scorie plus massive, à la fois plus lourde (jusqu'à $400 \mathrm{~g}$ ) et moins poreuse (20 à $40 \%$ ).

La soudure, si elle est bien maitrisée, induit des pertes de petites particules ferreuses $(<10 \mathrm{~mm})$, comme c'est le cas des scories SGD. Si elle est mal maitrisée alors des blocs entiers (> $10 \mathrm{~mm}$ ) tombent dans le foyer, comme pour les scories SFR. Lorsque des trempes sont effectuées comme à Doundé, dans le but d'enduire les barres de fer d'une boue d'argile avant la soudure, le fer a tendance à se fragiliser et des gros blocs de métal tombent dans la scorie de forge : tant que les particules ne sont pas jointives, la fayalite plus fluide s'écoule par percolation; pour la scorie qui résulte de ce genre de travaux, la fayalite est située en partie basse de la scorie et le métal en partie haute de la pièce. 


\begin{tabular}{|c|c|c|c|c|c|c|c|c|}
\hline & & \multicolumn{2}{|c|}{ SAS } & \multicolumn{3}{|c|}{ SGD } & \multirow{2}{*}{ SFR } & \multirow{2}{*}{ SI } \\
\hline & & SAS & SAS-M & SGD-B & SGD-SFR & SGD-K & & \\
\hline \multirow{6}{*}{$\begin{array}{l}\text { Condition } \\
\mathrm{du} \\
\text { forgeage }\end{array}$} & \multirow{4}{*}{ Ateliers } & Fiko & Fiko & Fiko & Fiko & - & Fiko & Fiko \\
\hline & & - & - & - & - & Kakoli & - & - \\
\hline & & Kobo & Kobo & Kobo & (Kobo) & - & - & - \\
\hline & & - & (Doundé) & (Doundé)- & Doundé & - & Doundé & Doundé \\
\hline & Barres & 1 & 1 & 2 & 2 & 1 ou 2 & 2 & 1 \\
\hline & Incidents & - & - & $(\boldsymbol{V})$ & $(\boldsymbol{V})$ & $(\boldsymbol{V})$ & $(\boldsymbol{V})$ & - \\
\hline \multirow{3}{*}{$\begin{array}{c}\text { Nombre } \\
\text { Poids } \\
\text { Taille }\end{array}$} & $\mathrm{nb}$ & 7 & 7 & 15 & 10 & 8 & 15 & 18 \\
\hline & moyen & $115 \mathrm{~g}$ & $130 \mathrm{~g}$ & $360 \mathrm{~g}$ & $230 \mathrm{~g}$ & $300 \mathrm{~g}$ & $215 \mathrm{~g}$ & $25 \mathrm{~g}$ \\
\hline & moyenne & $11 \mathrm{~cm}$ & $10 \mathrm{~cm}$ & $13 \mathrm{~cm}$ & $11 \mathrm{~cm}$ & $12 \mathrm{~cm}$ & $9 \mathrm{~cm}$ & $4 \mathrm{~cm}$ \\
\hline Scorie & à base de & silice & silice & fayalite & fayalite & kirchstéi. & fayalite & variable \\
\hline \multirow{3}{*}{$\begin{array}{c}\text { Matériau } \\
\%\end{array}$} & SAS & $95-100$ & $80-95$ & $5-40$ & $0-15$ & $0-20$ & 0 & $0-100$ \\
\hline & SGD & $0-5$ & $5-20$ & $40-90$ & $70-95$ & $75-100$ & $10-70$ & $0-100$ \\
\hline & SFR & $0-5$ & $5-20$ & $0-30$ & $0-20$ & $0-10$ & $30-90$ & $0-100$ \\
\hline Porosité & $\%$ & +40 & 40 & 30 & 30 & 20 & 30 & variable \\
\hline \multirow{7}{*}{$\begin{array}{l}\text { Minéralo- } \\
\text { gie }\end{array}$} & $\mathrm{Qz}$ & $v$ & - & - & - & - & - & $\checkmark$ \\
\hline & Qz-Fa & - & $\checkmark$ & $\checkmark$ & $v$ & - & $(\boldsymbol{\sim})$ & $\checkmark$ \\
\hline & Fa-Qz & - & - & $v$ & $v$ & - & $v$ & $\checkmark$ \\
\hline & $\mathrm{Fa}$ & - & - & $(\boldsymbol{\sim})$ & $v$ & - & $\checkmark$ & - \\
\hline & Kir & - & - & - & - & $\checkmark$ & - & - \\
\hline & $\begin{array}{c}\text { MO } \\
\text { métal+ox }\end{array}$ & - & - & - & - & - & $\checkmark$ & $\checkmark$ \\
\hline & Autres & - & - & - & - & - & - & $\checkmark$ \\
\hline \multirow{2}{*}{$\begin{array}{l}\text { Perte en } \\
\text { fer }\end{array}$} & gamme & $13-20 \%$ & $13-20 \%$ & $23-53 \%$ & $18-46 \%$ & $24-42 \%$ & $22-44 \%$ & $8-34 \%$ \\
\hline & moyenne & $17 \%$ & $17 \%$ & $36 \%$ & $27 \%$ & $35 \%$ & $30 \%$ & $17 \%$ \\
\hline \multirow{2}{*}{$\begin{array}{l}\text { Réparti- } \\
\text { tion }\end{array}$} & scorie & $6 \%$ & $9 \%$ & $30 \%$ & $27 \%$ & $30 \%$ & $26 \%$ & $5 \%$ \\
\hline & battiture & $94 \%$ & $91 \%$ & $70 \%$ & $73 \%$ & $70 \%$ & $74 \%$ & $95 \%$ \\
\hline Métal & coupe & $1 \%$ & $2 \%$ & $10 \%$ & $10 \%$ & $2 \%$ & $<90 \%$ & variable \\
\hline \multirow{5}{*}{$\begin{array}{l}\text { Nature } \\
\text { parti- } \\
\text { cules } \\
\text { ferreuses }\end{array}$} & filaments & $(\boldsymbol{\sim})$ & $(\boldsymbol{\sim})$ & $(\boldsymbol{\sim})$ & $(\boldsymbol{V})$ & $(\boldsymbol{V})$ & $(\boldsymbol{V})$ & $(\boldsymbol{V})$ \\
\hline & croûtes ox & $v$ & $\checkmark$ & $(\boldsymbol{V})$ & $(\boldsymbol{\sim})$ & $v$ & - & $(\boldsymbol{V})$ \\
\hline & billes & $(\boldsymbol{\sim})$ & $(\boldsymbol{v})$ & $(\boldsymbol{V})$ & $(\boldsymbol{V})$ & $(\boldsymbol{V})$ & $(\boldsymbol{\sim})$ & $(\boldsymbol{v})$ \\
\hline & dimension & $<5 \mathrm{~mm}$ & $<5 \mathrm{~mm}$ & $<10 \mathrm{~mm}$ & $<10 \mathrm{~mm}$ & $<1 \mathrm{~mm}$ & $>10 \mathrm{~mm}$ & varie \\
\hline & $\% \mathrm{C}$ & $<0.02$ & $<0.02$ & $<0.02 \grave{a}>0.8$ & $<0.02 \mathrm{a}>0.8$ & $<0.02$ & $<0.02 \mathrm{a}>0.8$ & $<0.02$ \\
\hline \multirow{5}{*}{$\begin{array}{l}\text { Contri- } \\
\text { butions } \\
\text { approxi. }\end{array}$} & Fer & 10 & 15 & 30 & 50 & 60 & 50 & 40 \\
\hline & Sol & 60 & 65 & 20 & 10 & $<$ & 10 & 10 \\
\hline & Paroi/tuy. & 20 & 10 & 30 & 10 & 30 & 10 & 30 \\
\hline & Ajouts & - & - & 20 & 10 & - & 20 & - \\
\hline & Charbon & 10 & 10 & $<$ & 10 & 10 & 10 & 20 \\
\hline \multicolumn{2}{|c|}{$\begin{array}{l}\text { Conditions de } \\
\text { préformage }\end{array}$} & $\begin{array}{l}\text { rapide } \\
\text { foyer } \\
\text { chaud }\end{array}$ & $\begin{array}{l}\text { rapide } \\
\text { foyer } \\
\text { chaud }\end{array}$ & $\begin{array}{c}\text { maitrisé } \\
\text { foyer chaud }\end{array}$ & $\begin{array}{c}\text { maitrisé } \\
\text { foyer chaud }\end{array}$ & $\begin{array}{l}\text { maitrisé } \\
\text { foyer chaud } \\
+ \text { paroi } \\
\text { calcique }\end{array}$ & $\begin{array}{c}\text { non maitrisé } \\
\text { foyer chaud } \\
\text { (Fiko) + } \\
\text { trempes } \\
\text { (Dounde) }\end{array}$ & $\begin{array}{l}\text { sans } \\
\text { préf. (F) } \\
\text { ou préf. } \\
\text { foyer } \\
\text { tiède } \\
\text { (D) }\end{array}$ \\
\hline \multicolumn{2}{|c|}{ Conditions de formage } & $\begin{array}{l}\text { foyer } \\
\text { froid }\end{array}$ & $\begin{array}{l}\text { foyer } \\
\text { plus } \\
\text { chaud }\end{array}$ & foyer tiède & foyer chaud & foyer chaud & & $\begin{array}{l}\text { foyer } \\
\text { tiède }\end{array}$ \\
\hline \multicolumn{2}{|c|}{ Scorie de } & formage & formage & préf+form & préf+form & préf+form & préf+form & formage \\
\hline \multicolumn{2}{|c|}{ Schéma } & & & & & & Fiko & $\therefore 0$ \\
\hline
\end{tabular}

Tableau 29 - Récapitulatif des conditions de formation et des caractéristiques des différents groupes de scories de forge. 
Dans le cas de forgeages avec soudures, simples ou multiples, la teneur en carbone dans le fer contenu dans les scories peut varier considérablement dans le sens d'une carburation ou bien même d'une décarburation.

La paroi du foyer, les ajouts, le sol contribuent de manière aussi importante à la scorie que le métal. Dans les ateliers où des ajouts siliceux sont employés pour la réalisation des soudures, un apport siliceux supplémentaire est mesuré. La contribution des charbons de bois reste faible $(<10 \%)$ sauf à Kakoli : le foyer semble avoir été rechapé avec un ciment qui apporte une proportion importante de calcium; les scories SGD-K comportent une matrice de kirschstéinite, équivalent calcique de la fayalite, ainsi que des minéraux riches en calcium. Cet apport en calcium dans les scories de Kakoli favorise la baisse du point de fusion de la scorie qui devient liquide et permet un dégazage important : ces scories sont très compactes.

L'étape du préformage, à haute température et réalisée principalement à la chauffe du fer dans le foyer, forme l'essentiel de la scorie de forge. Si la température du foyer diminue entre le préformage et le formage, la scorie de formage SAS vient se déposer sur la scorie de préformage devenue très pâteuse : le bourrelet sableux vient de se former (SGD-B). Si le foyer reste toujours très chaud entre ces deux étapes, la scorie de formage est engloutie dans la scorie de préformage et aucun bourrelet n'est visible (SGD-SFR, SGD-K et SFR).

Là encore, si le formage contribue de manière peu importante à la formation de la scorie, les finitions, effectuées à basse température essentiellement sur l'enclume, ne contribuent pas du tout à la formation de la scorie dans le foyer de forge.

En résumé, les forgeages rapides d'une barre de fer simple à travailler génèrent peu ou pas de déchets, de nature argilo-sableuse ; si ces pièces sont fragiles et petites, elles seront détruites avant que les archéologues ne puissent les retrouver; les scories petites pièces de SI sont, elles aussi, souvent retournées sur les sites de forge.

Les autres travaux de forge impliquant des soudures, des ratés, des ajouts, des trempes génèrent quant à eux des scories plus volumineuses, de nature fayalitique et/ou ferreuse. Ces scories sont effectivement le plus fréquemment retrouvées par les archéologues car moins fragiles. 
PARTIE 5 . CONCLUSION 
Conclusion 
Ce travail concerne l'étude de scories de forge du Pays dogon (Mali). Les scories de forge sont fréquemment retrouvées sur les sites archéologiques. Leur étude, depuis de nombreuses années, montre une grande variabilité de ces matériaux sur plusieurs aspects. Ces variabilités sont le reflet de la grande diversité des travaux de forge, lesquels sont souvent difficiles à restituer.

Cette recherche originale a comporté l'élaboration d'un protocole d'observation d'ateliers et de travaux de forge ainsi qu'une étude des scories en laboratoire. Le but est d'identifier et de comprendre les facteurs des travaux de forge qui influencent la formation des scories, afin de fournir des clés de réponses aux archéologues pour mieux interpréter ces matériaux.

En observant seulement une gamme limitée de travaux de forge, car nous avons observé la fabrication d'un seul type d'outil, nous avons pu identifier de nombreux paramètres du travail qui ont une influence certaine sur les scories de forge :

- Nombre de barres à forger,

- Forme de la barre de fer,

- Volume du foyer et la puissance de la soufflerie,

- La température du foyer,

- L'équipe de travail,

- Le savoir-faire des forgerons.

\section{Les ateliers de forge}

$\mathrm{Au}$ cours de la phase d'observation ethnoarchéologique, le déroulement des activités de forge a été enregistré dans quatre ateliers de travail $\mathrm{du}$ fer appartenant à des traditions techniques distinctes : Fiko et Kakoli dans la région Mombo, Kobo et Doundé dans la région Tomo.

Dans la région Mombo, l'atelier de forge est un hangar regroupant plusieurs unités de travail dont les composantes sont globalement mobiles. Cet atelier est un hangar carré à Fiko conçu en dehors de la tradition Mombo. Il s'agit d'un hangar elliptique sur deux niveaux à Kakoli ; cette configuration semble être représentative de la tradition dans la région. Dans ces ateliers, les artisans travaillent dans des foyers en fosse elliptiques avec une nouvelle forme de soufflerie : un ventilateur moderne à fort rendement d'air.

Nous avons montré que dans ces ateliers, la morphologie des foyers en fosse associés à la forte soufflerie ventilateur, génèrent la formation de scories compactes (argilo-sableux et fayalitique).

A Kobo et Doundé, l'atelier prend la forme d'un abri qui regroupe une seule unité de travail dont les composantes sont fixes ; il est représentatif de la région. Les forgerons travaillent quant à eux dans des foyers en coupole, avec une soufflerie à double pots traditionnelle de la région.

Ici nous avons montré qu'un foyer profond (Doundé) rend difficile l'agrégation des matériaux composant les scories, ce qui forme des scories « informes », une morphologie souvent retrouvée sur les sites archéologiques et pour laquelle nous pouvons à présent proposer un mode de formation.

L'outillage est aujourd'hui influencé par l'Europe, cependant d'autres outils traditionnels sont employés comme des enclumes en grès et des marteaux faits d'un bloc d'acier. Les outils de forge, traditionnels ou modernes n'ont ici à priori aucune sorte d'influence sur les scories de forge produites.

Ces résultats confirment l'importance de réunir un maximum d'informations sur les structures de forge qui ont pu être employées par les artisans.

\section{La chaîne opératoire}

Dans chaque atelier visité, il a été fourni au forgeron une seule sorte de matière première parmi 23 différentes et il a été demandé de fabriquer à chaque fois une lame de houe. Le foyer est nettoyé avant de procéder aux forgeages. L'intégralité du déroulement de la chaîne opératoire est retranscrite sur une fiche d'enregistrement. Après chaque phase de martelage, les battitures sont récupérées sur et à proximité immédiate de l'enclume.

La chaîne opératoire du forgeage de 126 lames de houe a été comparée. Notre recherche met en évidence que la fabrication des lames passe par la succession de trois étapes de travail complémentaires. Le travail commence par une étape de préformage très variable, qui est fortement influencée par la nature de la matière première (dimensions, nombre de barres).

Puis vient une étape de formage et de finitions quant à elles toutes deux très peu variables d'un atelier à un autre. La durée globale de fabrication d'une lame de houe varie entre $1 \mathrm{~h}$ et $2 \mathrm{~h} 40$. Notre recherche montre que cette durée du travail est influencée par plusieurs paramètres intrinsèques à la 
matière première (une/deux barres, forme/dimension, fer mou/dur) ou humains (incidents, qualification des frappeurs et des souffleurs).

$\mathrm{Au}$ préformage et au formage plusieurs possibilités de travail existent : le maître intervient seul ou avec un frappeur, le frappeur intervient seul ou avec un ou deux autres frappeurs. Aux finitions le maître est toujours seul. Nous avons montré qu'à Kobo, les durées travail (chauffe/martelage) sont toujours les plus longues : la soufflerie est activée par des apprentis peu dynamiques et le martelage est assuré par ces mêmes personnes peu expérimentées.

$\mathrm{Au}$ préformage les températures atteintes très élevées $\left(700^{\circ} \mathrm{C}-1400^{\circ} \mathrm{C}\right)$ et la durée de chauffe est très supérieure à celle du martelage. Il existe plusieurs manières de souder deux barres entre elles ; nos recherches ne permettent pas d'évaluer si les différentes manières de souder influent sur les scories. Une différence majeure technique reste l'emploi d'ajouts siliceux lors des soudures dans les ateliers de la plaine. A Kobo du grès concassé et de la tuyère concassés sont jetés directement dans le foyer tandis qu'à Doundé, les barres à assembler sont enduites d'une boue d'argile. Notre travail montre que lors de travaux avec ajouts siliceux, les scories formées sont principalement fayalitiques, ce qui remet en question un certain nombre d'écrits qui stipulent que l'emploi d'ajouts siliceux forme des scories sableuses.

Les refroidissements rapides dans l'eau des barres de fer, avant soudures (Doundé), favorisent l'apparition de défauts dans le métal ainsi que la production de scories ferreuses. La fréquence de ces «trempes » nous fait nous interroger sur la représentativité des forgeages dans cet atelier, les forgerons ayant un peu perdu la pratique des soudures. Les «trempes» sont ainsi un mode de formation des scories ferreuses.

De plus, le temps de travail augmente lors de travaux sur deux barres de fer, lors de travaux avec incidents, lors de travaux sur des barres de fer qui ont une forme trop éloignée de l'outil à forger, travaux d'aciers mi-dur (Fiko) et lors de travaux avec une équipe peu efficace (Kobo).

Au formage, les températures sont moyennes à élevées $\left(550{ }^{\circ} \mathrm{C}-1200{ }^{\circ} \mathrm{C}\right)$ et la durée de chauffe est encore supérieure à celle du martelage. Lors de cette étape, réalisée à plus basse température, la contribution du fer est moins importante et la paroi/tuyère du foyer peuvent contribuer, sous certaines conditions de température, à former l'appendice sableux des scories fayalitiques à bourrelet. Cette hypothèse de formation du bourrelet devra être appuyée dans le futur par une étude des sections des scories qui ont reçu le traceur de barytine, afin d'observer le lieu de leur dépôt.

Aux finitions, les températures sont moyennes à basses $\left(<1000{ }^{\circ} \mathrm{C}\right)$ et cette fois la durée de chauffe est inférieure à celle du martelage. Les observations de terrain montrent que les finitions ne génèrent quasiment aucune scorie dans le foyer ni déchet sur l'enclume. Une différence culturelle est observée lors des finitions : dans le cadre de nos forgeages contrôlés, seuls les forgerons de la plaine utilisent une lime afin d'aiguiser le tranchant et décorer la houe.

Les forgerons Tomo (Kobo, Doundé) maîtrisent très bien le formage et les finitions mais ils maitrisent beaucoup moins bien le préformage et ratent souvent les travaux demandés : en effet, ils ont l'habitude de fabriquer des lames de houe à partir de fers de récupération (R1S) qui sont déjà découpés à froid et préformés.

Nous avons ainsi pu mettre en évidence que, suite à des incidents, le forgeron peut rattraper son erreur mais cela engendre des pertes de fer importantes et la formation de scories ferreuses. Le savoir-faire de l'artisan est ainsi un paramètre important invisible à prendre en compte pour l'analyse des scories et le décryptage de la chaîne opératoire.

Dans notre étude nous avons observé la fabrication d'un seul type d'outil alors que le panel d'outils fabriqués par les forgerons est très large. La mise en forme de lames de houe demande en réalité une certaine masse de métal et un certain temps de travail, ce qui donne un temps suffisant pour former des scories. Le travail d'autres outils comme des clous n'aurait pas permis la production de déchets.

\section{La perte en fer au cours du forgeage}

La perte en fer est la quantité de matière première métallique perdue dans le foyer et sur l'enclume lors des travaux de forge. Ce fer perdu correspond à des croûtes d'oxydes qui tombent dans le foyer (chauffe), des battitures sur l'enclume (martelage), des particules de fer métallique dans le foyer (chauffage excessif) et sur l'enclume 
(compactage inefficace, mauvais martelage). Cette notion de perte en fer permet d'aider à évaluer la quantité de fer produit à partir de l'examen des déchets retrouvés archéologiquement.

Nous avons pu montrer qu'il s'agit d'un processus complexe qui résulte de la synergie de plusieurs paramètres, principalement au préformage : température du foyer, durée de chauffe, section/forme de la barre, écart entre la barre travaillée et la préforme, nombre de soudure et d'incidents.

La perte en fer, mesurée systématiquement au cours de 126 forgeages permet d'obtenir de nombreuses données de qualité, dans un domaine où cette information n'est souvent pas accessible. Cette perte en fer est variable : 8 à $37 \%$ pour un forgeage sans soudure, 18 à $47 \%$ pour un forgeage avec soudure sans incident, jusqu'à $53 \%$ pour un forgeage avec des incidents.

Ces résultats mettent l'accent sur l'importance du choix de la matière première par le forgeron, ce choix étant essentiel pour la suite des opérations car il essaie en général de perdre le moins de matériel possible.

\section{Les lames de houe}

Systématiquement la lame de houe produite a été pesée, ses dimensions et les défauts qu'elle comporte ont été relevés. La longueur, le poids et l'envergure sont liés aux barres de fer employées (une/deux barres) et aux compétences du forgeron (incident); le rayon de courbure dépend du style dans chaque atelier.

Cinq types de défauts, apparaissant lors des incidents au cours du travail, ont été identifiés dans les lames de houe: ces défauts apparaissent généralement lors de l'assemblage de deux barres de fer; les lames de Fiko contiennent moins de défauts que celles de Doundé, les forgeages à Fiko sont également mieux réussis que ceux de Doundé.

Nous avons montré que les lames ont la même teneur en carbone que les barres qui ont servi à les fabriquer, ce qui confirme les résultats attendus. Les lames réalisées à partir de deux barres de fer montrent des interfaces de soudure: toutes les soudures observées sont propres, contenant très peu d'inclusions aux interfaces; les inclusions dans les lames sont souvent aplaties par le martelage.

\section{Les scories de forge}

La scorie a été récoltée dans le foyer à la fin d'une opération de forgeage ou bien à la fin d'une journée de travail, soit deux à trois forgeages. $\mathrm{Au}$ laboratoire, un protocole d'analyse a été mis en œuvre : celui-ci implique une description détaillée de chacune des pièces par une étude morphométrique approfondie, ainsi que l'utilisation de plusieurs méthodes de caractérisation physicochimiques, en particulier les analyses chimiques par fluorescence des rayons $\mathrm{X}$, la diffraction des rayons $\mathrm{X}$, la microscopie optique et la microscopie électronique à balayage.

La scorie se forme dans le foyer à partir de plusieurs sources : la matière première (fer), le combustible (charbon), les éléments du foyer en argile (paroi, tuyère) ou en grès (sol/fond de foyer) et les ajouts volontaires de matières siliceuses.

Les analyses montrent un apport faible mais significatif des éléments chimiques contenus dans les barres de fer utilisées (inclusions et impuretés) : les aciers modernes apportent $\mathrm{Cu}, \mathrm{Mn}$ et $\mathrm{Cr}$; les fers africains apportent $\mathrm{TiO}_{2}, \mathrm{~V}, \mathrm{Zr}, \mathrm{MnO}, \mathrm{Cr}$ avec les inclusions de scorie de réduction, les fers de recyclage apportent $\mathrm{Al}_{2} \mathrm{O}_{3}$.

Les scories sont toujours un peu contaminées par le sol du foyer de forge, même dans les ateliers où les ajouts siliceux ne sont pas utilisés ; l'emploi des ajouts siliceux forme des scories encore plus riches en silice. Dans les ateliers de la plaine, l'apport de l'argile $\left(\mathrm{Al}_{2} \mathrm{O}_{3}\right)$ est très faible en comparaison de celui du sol et des ajouts $\left(\mathrm{SiO}_{2}\right)$. L'argile et le foyer apportent $\mathrm{SiO}_{2}, \mathrm{Al}_{2} \mathrm{O}_{3}, \mathrm{TiO}_{2}$, $\mathrm{MgO}$, éventuellement $\mathrm{K}_{2} \mathrm{O}$ et $\mathrm{CaO}$, ainsi que $\mathrm{Rb}, \mathrm{Ba}$, $\mathrm{Zr}$ comme traces; à Kakoli l'argile du ciment apporte $\mathrm{La}$ et $\mathrm{Sc}$.

Le charbon apporte $\mathrm{CaO}, \mathrm{K}_{2} \mathrm{O}, \mathrm{MgO}, \mathrm{P}_{2} \mathrm{O}_{5}$ ainsi que $\mathrm{Sr}$ et $\mathrm{Rb}$ en traces. Un apport très important en calcium dans les scories de Kakoli (10-30\% $\mathrm{CaO}$ ) suggère que le revêtement réfractaire du foyer de forge est un mélange entre de l'argile et un ciment (riche en $\mathrm{CaO}$ ).

Les résultats confirment qu'il faut réunir le plus d'éléments possibles du système (paroi, charbon, métal) afin d'en déterminer les contributions. Ce travail met en effet clairement en évidence les apports qualitatifs des différentes sources de contribution mais il souligne également la difficulté d'interpréter ces apports de manière quantitative. 
Pour un forgeage où le préformage est bref, la scorie se forme principalement pendant le formage ; il s'agit donc de scories «de formage » : les scories argilo-sableuses SAS et les scories informes SI. Pour ces forgeages, le fer est perdu au cours du travail essentiellement sur l'enclume sous la forme de battitures lamellaires et granulaires, morphologies attendues pour ces travaux sans soudure.

Nous avons pu remonter au résultat suivant : seuls 0.5 à $2 \%$ de la masse initiale du fer prennent part à la scorie SAS. Finalement, en déterminant la masse de fer dans une scorie archéologique SAS, nous pouvons donner un ordre de grandeur de la masse de fer travaillée par l'artisan.

Les SAS contiennent de 80 à $100 \%$ de matériaux siliceux, roches et verre, et de peu ou pas de fayalite $(<20 \%)$. Ces scories se forment dans un système sous-saturé en fer. La phase de préformage est courte et les températures sont peu élevées : il n'est pas rare que l'ensemble des gains de quartz ne soit que partiellement fondu ou bien non fondu. Si ces scories sont produites dans un foyer «froid», alors du fait d'un manque de cohésion des matériaux siliceux, elles seront très friables et fragiles : ce sont les scories réduites de Kobo. Dans un foyer « chaud », comme celui de Fiko, les scories sont plus compactes.

Les scories SI sont quasiment toutes produites à Doundé ; il s'agit de petites pièces composites de différentes natures. Elles contiennent beaucoup de minéraux d'augite, de leucite, d'akermanite et hedenbergite, parfois mullite. Elles montrent des compositions minéralogiques variées et une morphologie inhabituelles, qui reflètent des conditions de formation difficiles dans le foyer Doundé (volume important et foyer « tiède »).

Pour un forgeage avec soudure, la scorie se forme pendant l'étape du préformage qui est conséquente; il s'agit donc de scories «de préformage »: les scories grises denses SGD et les scories ferreuses rouillées SFR. Pour ces forgeages le fer est perdu sur l'enclume aussi sous la forme de battitures globulaires, dont la proportion augmente avec l'emploi d'ajouts siliceux; autre ordre de grandeur déterminé : environ 5 à $15 \%$ de la masse du fer travaillée prend part à ces scories dans le foyer de forge. Les scories SGD et SFR contiennent plus de fer $(20-70 \% \mathrm{FeO})$ et possèdent $10 \%$ de particules métalliques en coupe; leur nombre augmente davantage lorsque le forgeron réalise des trempes; la taille et la teneur en carbone des particules varie d'une pièce à une autre.

Notre recherche met également en évidence que dans le cas de forgeages où les matériaux restent liquides ou pâteux dans le foyer, même lors de l'interruption $\mathrm{du}$ feu, on observe une stratification verticale due à la gravité des différents composants. Nous pouvons donc nous interroger sur les conditions de formation des scories stratifiées archéologiques, qui doivent se former dans d'autres conditions de travail que les nôtres.

Les SGD contiennent entre 50 et $100 \%$ de fayalite et $10 \%$ de particules ferreuses en coupe (Kakoli : kirschstéinite et $<2 \%$ de fer en coupe). A Kakoli, les oxydes de fer (wüstite et de magnétite) sont très nombreux et l'excès de calcium favorise la formation d'akermanite. Certaines rares pièces comportent des augites et des leucites.

Les observations des particules ferreuses permettant un nouvel éclairage sur l'extrême variation du degré de carburation du fer dans les déchets, quelque soit la nature du fer de départ, ce qui remet en cause un certain nombre d'écrits. Ici, la quantité et la taille des particules de métal peuvent jouer un rôle.

Les nombreuses porosités observées en surface des pièces témoignent d'un système qui a été pâteux et qui n'a pas permis l'évacuation totale des gaz. Cet état pâteux facilite le dépôt des matières argilosableuses, au cours du formage, sous la forme d'un bourrelet (SGD-B). A Kakoli, les scories sont très compactes (moy $20 \%$ ) ; sous le coup d'une forte soufflerie dans un petit foyer, et avec un point de fusion plus bas à cause de l'apport en calcium, la scorie est restée très liquide facilitant l'évacuation des gaz.

Dans notre étude, les scories SFR proviennent souvent de forgeages ratés ou avec des refroidissements des barres de fer dans de l'eau. Nous avons mis en évidence un mode de formation de scories SFR, il en existe bien d'autres, au regard des très nombreuses scories ferreuses retrouvées sur les sites métallurgiques car il est impossible qu'il s'agisse uniquement d'opérations ratées.

Les scories SFR contiennent jusqu'à $90 \%$ de matériel métallique, souvent en gros blocs $(\mathrm{cm})$ et 20 à $45 \%$ d'oxydes de fer pour la moitié des pièces. Les forgerons de Doundé ont pratiqué de nombreuses « trempes » au cours du préformage ; cependant, ce traitement thermique conduit à des pertes de fer importantes. Les carburations sont variables, entre ferrite et acier hypereutectoïde avec cependant 
quelques cas de fontes non expliqués sont observés sur des petites pièces à Doundé.

Ce travail est une étape importante dans les tentatives de compréhension des gestes techniques et des activités de forgeage à travers les vestiges de déchets de travail retrouvés sur les sites archéologiques. Le corpus de scories de forge constitué, même s'il est loin de représenter l'ensemble des configurations possibles du travail du forgeage du fer, est unique et il faudra encore plusieurs études de ce type pour balayer l'ensemble des questionnements. 
Conclusion 
Annexes 


\section{Annexe 1 - Opérations de forgeage}

Chaque forgeage a fait l'objet une fiche détaillée (soit 126 fiches et pages au total), comprenant de nombreuses informations (voir ci-dessous, aussi Figure 6) : durées, températures, outils utilisés etc.

L'Annexe 1 fournit un résumé de chaque fiche, sur une seule ligne, en se contentant de fournir les principales informations : paramètres de départ, durées, soudures, ajouts et particularité du forgeage.

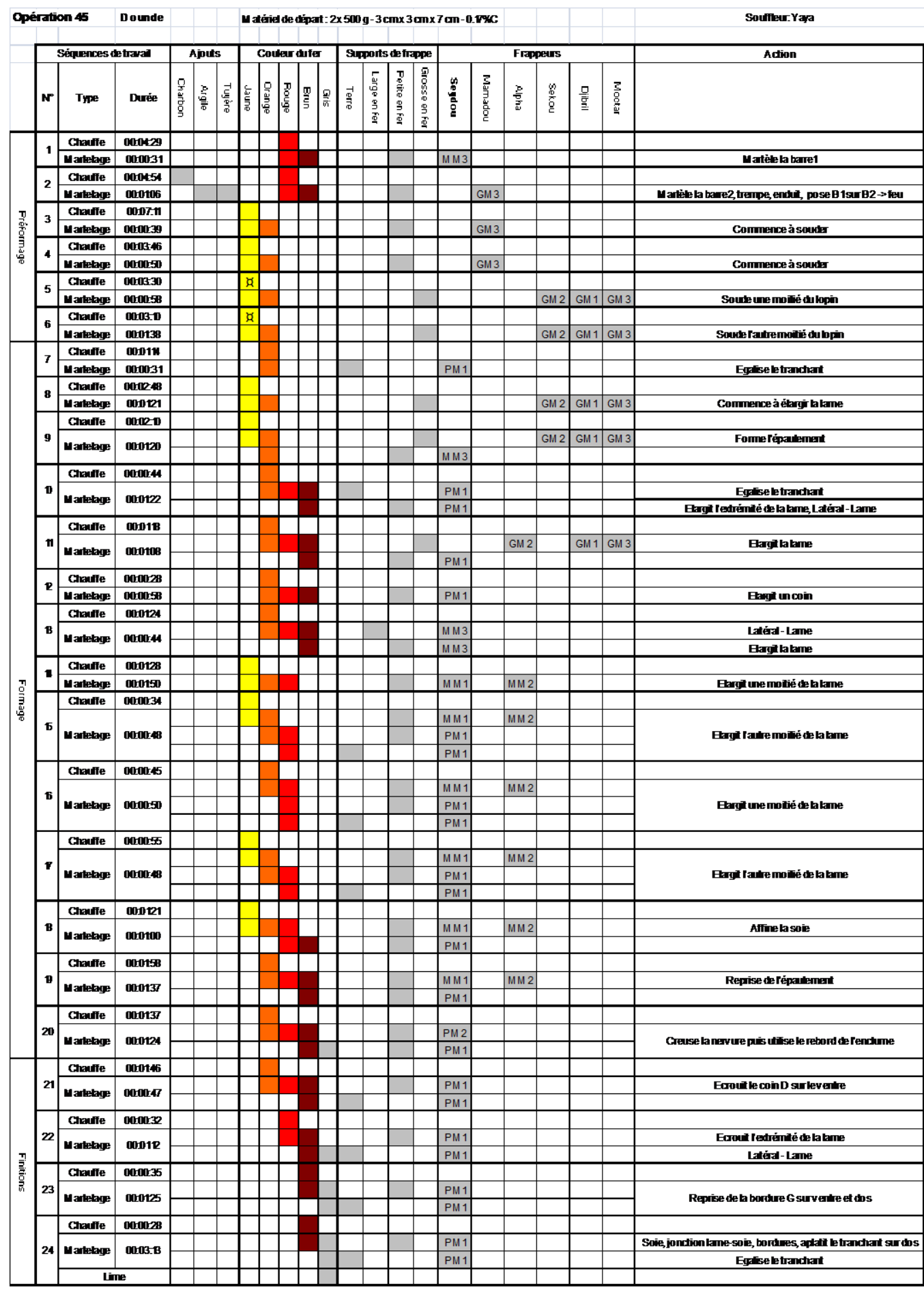




\begin{tabular}{|c|c|c|c|c|c|c|c|c|c|c|c|}
\hline \multirow{2}{*}{ Forgeage } & \multirow{2}{*}{ Barre } & \multirow{2}{*}{ Poids } & \multirow{2}{*}{$\% \mathrm{C}$} & \multirow{2}{*}{ Arrêt du feu } & \multicolumn{2}{|c|}{ Préformage } & \multicolumn{2}{|c|}{ Formage } & \multicolumn{2}{|c|}{ Finitions } & \multirow{2}{*}{$\begin{array}{l}\text { Durée } \\
\text { totale }\end{array}$} \\
\hline & & & & & $\mathrm{C}$ & M & $\mathrm{C}$ & $\mathrm{M}$ & $\mathrm{C}$ & M & \\
\hline F001 & M2S & 1000 & 0.17 & - & 20 & 10 & 17 & 10 & 12 & 19 & 88 \\
\hline F002 & M2D & $500+500$ & 0.17 & - & 36 & 18 & 10 & 8 & 6 & 12 & 90 \\
\hline F003 & M2S & 1000 & 0.45 & - & 26 & 11 & 16 & 9 & 9 & 12 & 83 \\
\hline F004 & M2D & $500+500$ & 0.45 & - & 20 & 7 & 16 & 10 & 12 & 14 & 79 \\
\hline F005 & M2S & 1000 & 0.45 & - & 22 & 9 & 23 & 12 & 11 & 14 & 91 \\
\hline F006 & M4S & 1000 & 0.17 & - & 18 & 3 & 21 & 9 & 11 & 15 & 77 \\
\hline F007 & A1D & $310+390$ & $<0.8$ & - & 20 & 8 & 13 & 9 & 7 & 12 & 69 \\
\hline F008 & $\mathrm{A} 2 \mathrm{D}$ & $345+380$ & $<0.8$ & - & 28 & 9 & 21 & 10 & 9 & 8 & 85 \\
\hline F009 & M2D & $500+500$ & $0.17+0.45$ & - & - & - & - & - & - & - & - \\
\hline F010 & M2S & 1000 & 0.17 & - & 23 & 7 & 25 & 13 & 9 & 15 & 92 \\
\hline F011 & M2D & 1000 & 0.17 & - & 25 & 6 & 19 & 10 & 7 & 11 & 78 \\
\hline F012 & M2S & 1000 & 0.45 & - & 29 & 20 & 40 & 32 & 9 & 24 & 154 \\
\hline F013 & M2D & $500+500$ & 0.45 & $\begin{array}{l}\text { Form.+fini. } \\
\text { lendemain }\end{array}$ & 87 & 38 & 23 & 13 & 11 & 10 & 182 \\
\hline F014 & A1D & $365+335$ & $<0.8$ & - & 44 & 25 & 10 & 12 & 3 & 13 & 107 \\
\hline F015 & $\mathrm{A} 2 \mathrm{D}$ & $380+320$ & $<0.8$ & - & 37 & 22 & 46 & 29 & 3 & 16 & 153 \\
\hline F016 & M1D & $350+350$ & 0.17 & - & 62 & 30 & 13 & 12 & 3 & 9 & 129 \\
\hline F017 & M1D & $350+350$ & 0.35 & - & 65 & 35 & 26 & 18 & 6 & 11 & 161 \\
\hline F018 & $\mathrm{M} 2 \mathrm{~S}$ & 1000 & 0.17 & - & 33 & 21 & 56 & 38 & 12 & 28 & 188 \\
\hline F019 & M2D & $500+500$ & $0.17+0.45$ & $\begin{array}{l}\text { Form.+fini. } \\
\text { lendemain }\end{array}$ & - & - & - & - & - & - & - \\
\hline F020 & M2D & $500+500$ & 0.17 & - & 29 & 11 & 44 & 31 & 3 & 13 & 131 \\
\hline F021 & M2S & 1000 & 0.17 & - & 23 & 19 & 50 & 41 & 8 & 26 & 167 \\
\hline F022 & M2D & $500+500$ & 0.45 & - & 36 & 10 & 52 & 40 & 6 & 18 & 162 \\
\hline F023 & $\mathrm{M} 2 \mathrm{~S}$ & 1000 & 0.45 & - & 26 & 16 & 42 & 39 & 5 & 18 & 146 \\
\hline F024 & A1D & $345+365$ & $<0.8$ & - & 34 & 28 & 20 & 25 & 6 & 19 & 132 \\
\hline F025 & A1D & $380+320$ & $<0.8$ & - & 30 & 26 & 25 & 26 & 2 & 14 & 123 \\
\hline F026 & M4S & 1000 & 0.17 & - & 20 & 9 & 38 & 33 & 7 & 26 & 133 \\
\hline F027 & A2D & $325+375$ & $<0.8$ & - & 31 & 23 & 23 & 24 & 6 & 17 & 124 \\
\hline F028 & A2D & $345+330$ & $<0.8$ & - & 35 & 18 & 16 & 18 & 4 & 14 & 105 \\
\hline F029 & M4S & 1000 & 0.17 & - & 16 & 10 & 33 & 34 & 5 & 13 & 111 \\
\hline F030 & M4S & 1000 & 0.45 & - & 14 & 8 & 38 & 46 & 10 & 35 & 151 \\
\hline F031 & M4S & 1000 & 0.45 & - & 24 & 5 & 19 & 8 & 9 & 13 & 78 \\
\hline F032 & M4S & 1000 & 0.45 & - & 23 & 9 & 22 & 12 & 10 & 14 & 90 \\
\hline F033 & M1D & $350+350$ & 0.35 & - & 44 & 19 & 9 & 7 & 5 & 8 & 92 \\
\hline F034 & M1D & $350+350$ & 0.35 & - & 32 & 11 & 32 & 22 & 2 & 2 & 101 \\
\hline F035 & R1D & $805+785$ & $?$ & - & 38 & 13 & 30 & 14 & 5 & 14 & 114 \\
\hline F036 & R1D & $435+395$ & $?$ & - & 19 & 10 & 16 & 7 & 12 & 16 & 80 \\
\hline F037 & R1S & 860 & $<0.1$ & - & 10 & 3 & 15 & 10 & 8 & 18 & 64 \\
\hline F038 & R1S & 835 & $<0.1$ & - & 10 & 2 & 19 & 10 & 5 & 13 & 59 \\
\hline F039 & R1S & 780 & $<0.1$ & - & 9 & 3 & 17 & 14 & 6 & 15 & 64 \\
\hline F040 & M2S & 1000 & 0.17 & - & 12 & 8 & 27 & 19 & 3 & 8 & 77 \\
\hline F041 & M2D & $500+500$ & 0.17 & - & 28 & 9 & 22 & 16 & 3 & 7 & 85 \\
\hline F042 & M4S & 1000 & 0.45 & - & 14 & 3 & 21 & 15 & 5 & 9 & 67 \\
\hline F043 & M4S & 1000 & 0.45 & - & 20 & 6 & 20 & 17 & 4 & 9 & 76 \\
\hline F044 & M4S & 1000 & 0.45 & - & 17 & 4 & 19 & 13 & 3 & 7 & 63 \\
\hline F045 & M3D & $500+500$ & 0.17 & - & 27 & 6 & 19 & 16 & 3 & 7 & 78 \\
\hline F046 & M3D & $500+500$ & 0.17 & - & 22 & 7 & 16 & 12 & 2 & 8 & 67 \\
\hline F047 & M3D & $500+500$ & 0.17 & - & 27 & 5 & 16 & 14 & 2 & 6 & 70 \\
\hline F048 & M4S* & 985 & 0.35 & - & 13 & 4 & 22 & 14 & 4 & 8 & 65 \\
\hline F049 & M4S* & 1015 & 0.35 & - & 17 & 4 & 20 & 14 & 3 & 8 & 66 \\
\hline F050 & M3D & $500+500$ & 0.45 & - & 41 & 12 & 24 & 12 & 3 & 5 & 97 \\
\hline F051 & M3D & $500+500$ & 0.45 & - & 23 & 7 & 18 & 15 & 3 & 9 & 75 \\
\hline F052 & M3S & 1000 & 0.45 & - & 11 & 5 & 16 & 10 & 3 & 7 & 52 \\
\hline F053 & M3D & $500+500$ & 0.35 & - & 51 & 19 & 17 & 13 & 3 & 5 & 108 \\
\hline F054 & M3D & $500+500$ & 0.35 & - & 25 & 5 & 29 & 15 & 4 & 9 & 87 \\
\hline F055 & M5S & 1000 & 0.45 & - & 24 & 7 & 18 & 16 & 2 & 6 & 73 \\
\hline
\end{tabular}

Poids en grammes - Durée de chauffe (C), de martelage (M) et durée totale du forgeage en minutes - Vert : 1 barre - Rouge : 2 barres 


\begin{tabular}{|c|c|c|}
\hline Soudures : Type (Nombre) & Ajouts (Utilisation) & Particularités du forgeage \\
\hline- & - & Fond du foyer non nettoyé, scorie non comptabilisée \\
\hline Repli avec insert(1) & - & - \\
\hline- & - & - \\
\hline Simple(1) & - & - \\
\hline- & - & - \\
\hline- & - & - \\
\hline Multiple(1) & - & Un fragment de fer perdu est réintégré par soudure \\
\hline Multiple(1) & - & Un fragment de fer perdu est réintégré par soudure \\
\hline & - & Forgeage non pris en compte, lame de machette \\
\hline - & - & - \\
\hline Simple(1) & - & - \\
\hline- & - & - \\
\hline Simple(2),repli(2) & Argile,grès,barytine(F,R) & Présence de défauts dans le fer, fragment de fer réintégré \\
\hline Repli(1),insert(2) & Grès,tuyère(F) & Cassure des extrémités des barres lors de leur repli \\
\hline Multiple(1),insert(1) & Sable,grès,tuyère(F) & Cassure des extrémités des barres lors de leur repli \\
\hline Simple(2),multiple(1),insert(1) & Grès,tuyère $(F)$ & Chaque barre est coupée en 2 ,fragment de fer réintégré \\
\hline Simple(1),repli(2) & Grès,tuyère,barytine(F) & Trempe \\
\hline- & - & - \\
\hline & Argile,grès,barytine(F,R) & Forgeage non pris en compte, lame de machette \\
\hline Simple(1) & Grès,tuyère,barytine $(\mathrm{F})$ & Mauvaise soudure, la lame s'ouvre en son milieu \\
\hline- & - & - \\
\hline Simple(1) & Grès,barytine $(\mathrm{F})$ & - \\
\hline- & - & - \\
\hline Repli(1),multiple(1) & Grès(F) & Plie chaque barre en 3 avant soudure \\
\hline Repli(1),multiple(1) & Grès(F) & Plie chaque barre en 3 avant soudure \\
\hline & - & - \\
\hline Repli(1),multiple(1) & Grès $(F)$ & Plie chaque barre en 3 avant soudure, 1 trempe, replie le lopin \\
\hline Multiple(1),insert(1) & Grès(F) & Plie chaque barre en 3,1 trempe,cassure d'une tige réintégrée et repli \\
\hline- & - & - \\
\hline- & - & - \\
\hline- & - & - \\
\hline- & - & - \\
\hline Simple(1),repli(3) & - & Le lopin formé trop long est replié sur $1 / 3$ de sa longueur \\
\hline Simple(1),repli(4) & - & Chaque barre pliée en 3, côté de la lame et coin déformés sont repliés \\
\hline Multiple(1) & - & Chaque daba : bordures, épaulements et lame repliés avant soudure \\
\hline Multiple(1),consolidation(1) & - & Idem F035, un fragment de la soie détaché est réintégré par soudure \\
\hline- & - & - \\
\hline- & - & - \\
\hline- & - & - \\
\hline 4. - - & -4 & - \\
\hline Simple(1),consolidation(1) & $\operatorname{Argile}(\mathrm{F}, \mathrm{B})$, charbon $(\mathrm{F})$ & Fragment de fer non réintégré, 2 trempes réalisées \\
\hline- & - & - \\
\hline- & - & - \\
\hline- & - & - \\
\hline Simple(1) & Charbon(F),argile,tuyère(B) & Trempe \\
\hline Simple(1) & Charbon(F),argile,tuyère(B) & Trempe \\
\hline Simple(1) & Charbon(F),argile,tuyère(B) & Trempe \\
\hline- & - & Une barre de $2 \mathrm{~kg}$ coupée en deux à l'aide d'un burin \\
\hline- & - & - \\
\hline Simple(1) & Charbon $(\mathrm{F}), \operatorname{argile}(\mathrm{B})$ & Trempe \\
\hline Simple(1) & Charbon $(\mathrm{F}), \operatorname{argile}(\mathrm{B})$ & Trempe \\
\hline- & - & - \\
\hline Simple(1),repli(1),insert(1) & Argile(B) & Trempe, fragments perdus réintégrés, repli d'une extrémité du lopin \\
\hline Simple(1) & Argile(B) & Trempe \\
\hline- & - & - \\
\hline
\end{tabular}




\begin{tabular}{|c|c|c|c|c|c|c|c|c|c|c|c|}
\hline \multirow{2}{*}{ Forgeage } & \multirow{2}{*}{ Barre } & \multirow{2}{*}{ Poids } & \multirow{2}{*}{$\% \mathrm{C}$} & \multirow{2}{*}{ Arrêt du feu } & \multicolumn{2}{|c|}{ Préformage } & \multicolumn{2}{|c|}{ Formage } & \multicolumn{2}{|c|}{ Finitions } & \multirow{2}{*}{$\begin{array}{l}\text { Durée } \\
\text { totale }\end{array}$} \\
\hline & & & & & $\mathrm{C}$ & M & $\mathrm{C}$ & $\mathrm{M}$ & $\mathrm{C}$ & M & \\
\hline F056 & M1D & $350+350$ & 0.17 & - & 39 & 12 & 14 & 11 & 2 & 6 & 84 \\
\hline F057 & M1D & $350+350$ & 0.35 & - & 43 & 19 & 11 & 9 & 3 & 6 & 91 \\
\hline F058 & M2S & 1000 & 0.45 & - & 27 & 12 & 32 & 22 & 2 & 5 & 100 \\
\hline F059 & M2D & $500+500$ & 0.45 & - & 41 & 17 & 17 & 15 & 3 & 7 & 100 \\
\hline F060 & R1S & 1025 & 0.1 & - & 3 & 1 & 13 & 13 & 3 & 14 & 47 \\
\hline F061 & M4S* & 1020 & 0.45 & - & 13 & 5 & 21 & 17 & 3 & 7 & 66 \\
\hline F062 & M4S* & 980 & 0.45 & - & 13 & 5 & 26 & 16 & 4 & 8 & 72 \\
\hline F063 & A1D & 530 & $<0.8$ & - & 30 & 15 & 9 & 7 & 1 & 5 & 67 \\
\hline F064 & $\mathrm{M} 2 \mathrm{~S}$ & 1000 & 0.35 & - & 13 & 11 & 20 & 17 & 3 & 10 & 74 \\
\hline F065 & R1S & 1620 & 0.1 & - & 5 & 3 & 19 & 21 & 2 & 9 & 59 \\
\hline F066 & M3S & 1000 & 0.45 & - & 11 & 5 & 31 & 16 & 13 & 27 & 103 \\
\hline F067 & $\mathrm{M} 2 \mathrm{~S}$ & 1000 & 0.45 & - & 26 & 13 & 25 & 16 & 7 & 18 & 105 \\
\hline F068 & M2D & $500+500$ & 0.45 & - & 31 & 14 & 53 & 27 & 4 & 7 & 137 \\
\hline F069 & M2D & $500+500$ & 0.17 & - & 44 & 16 & 26 & 12 & 6 & 14 & 117 \\
\hline F070 & M4S & 1000 & 0.45 & - & 11 & 2 & 39 & 18 & 7 & 13 & 90 \\
\hline F071 & M4D & $500+500$ & 0.17 & - & 29 & 6 & 25 & 13 & 6 & 14 & 94 \\
\hline F072 & M4D & $500+500$ & 0.45 & - & 32 & 9 & 28 & 15 & 9 & 22 & 115 \\
\hline F073 & M5S & 1000 & 0.45 & - & 18 & 3 & 29 & 14 & 5 & 12 & 82 \\
\hline F074 & M4D & $500+500$ & 0.17 & - & 29 & 5 & 18 & 10 & 4 & 9 & 76 \\
\hline F075 & M3S & 1000 & 0.45 & - & 9 & 1 & 18 & 11 & 7 & 12 & 58 \\
\hline F076 & M4S & 1000 & 0.17 & - & 10 & 1 & 26 & 11 & 7 & 12 & 67 \\
\hline F077 & M4S & 1000 & 0.17 & - & 9 & 2 & 22 & 12 & 6 & 14 & 65 \\
\hline F078 & M4S & 1000 & 0.17 & - & 13 & 1 & 24 & 11 & 5 & 11 & 66 \\
\hline F079 & M4D & $500+500$ & 0.17 & - & 25 & 5 & 19 & 13 & 8 & 11 & 80 \\
\hline F080 & M4D & $500+500$ & 0.17 & - & 31 & 7 & 22 & 11 & 7 & 10 & 89 \\
\hline F081 & M4D & $500+500$ & 0.17 & - & 29 & 6 & 20 & 9 & 8 & 12 & 84 \\
\hline F082 & $\mathrm{M} 2 \mathrm{~S}$ & 1000 & 0.17 & - & 25 & 11 & 18 & 11 & 7 & 12 & 84 \\
\hline F083 & M2S & 1000 & 0.17 & - & 17 & 7 & 21 & 10 & 7 & 10 & 72 \\
\hline F084 & M2S & 1000 & 0.17 & - & 23 & 10 & 22 & 10 & 7 & 10 & 82 \\
\hline F085 & M2D & $500+500$ & 0.17 & - & 15 & 8 & 13 & 10 & 6 & 8 & 61 \\
\hline F086 & M2D & $500+500$ & 0.17 & - & 20 & 8 & 16 & 8 & 7 & 9 & 69 \\
\hline F087 & M2D & $500+500$ & 0.17 & - & 21 & 9 & 19 & 9 & 11 & 14 & 83 \\
\hline F088 & M4S & 1000 & 0.45 & - & 16 & 3 & 24 & 12 & 7 & 8 & 70 \\
\hline F089 & M4S & 1000 & 0.45 & - & 7 & 1 & 26 & 12 & 6 & 10 & 63 \\
\hline F090 & M4S & 1000 & 0.45 & - & 15 & 2 & 22 & 9 & 8 & 9 & 66 \\
\hline F091 & M4D & $500+500$ & 0.45 & - & 29 & 8 & 19 & 11 & 5 & 8 & 79 \\
\hline F092 & M4D & $500+500$ & 0.45 & - & 38 & 7 & 17 & 9 & 4 & 5 & 80 \\
\hline F093 & M4D & $500+500$ & 0.45 & - & 43 & 9 & 17 & 8 & 7 & 10 & 94 \\
\hline F094 & M4D & $500+500$ & 0.45 & - & 54 & 7 & 18 & 8 & 6 & 9 & 103 \\
\hline F095 & M5S & 1000 & 0.45 & - & 17 & 3 & 23 & 11 & 4 & 6 & 64 \\
\hline F096 & $\mathrm{M} 2 \mathrm{~S}$ & 1000 & 0.45 & - & 38 & 12 & 24 & 8 & 5 & 7 & 93 \\
\hline F097 & $\mathrm{M} 2 \mathrm{~S}$ & 1000 & 0.45 & - & 31 & 9 & 21 & 7 & 9 & 10 & 86 \\
\hline F098 & M2S & 1000 & 0.45 & - & 38 & 10 & 26 & 7 & 9 & 10 & 101 \\
\hline F099 & M2D & $500+500$ & 0.45 & - & 50 & 10 & 21 & 6 & 6 & 8 & 101 \\
\hline F100 & M2D & $500+500$ & 0.45 & - & 39 & 9 & 22 & 7 & 9 & 9 & 93 \\
\hline F101 & M2D & $500+500$ & 0.45 & - & 47 & 7 & 18 & 6 & 9 & 8 & 95 \\
\hline F102 & M4S & 1000 & 0.17 & - & 14 & 4 & 20 & 14 & 3 & 9 & 64 \\
\hline F103 & M4D & $500+500$ & 0.17 & - & 25 & 11 & 25 & 15 & 4 & 9 & 88 \\
\hline F104 & M4D & $500+500$ & 0.45 & - & 25 & 8 & 21 & 16 & 2 & 10 & 82 \\
\hline F105 & M4S & 1000 & 0.17 & - & 14 & 3 & 22 & 15 & 4 & 9 & 67 \\
\hline F106 & M4S & 1000 & 0.17 & - & 12 & 3 & 21 & 15 & 3 & 11 & 65 \\
\hline F107 & M4S & 1000 & 0.17 & - & 10 & 3 & 18 & 15 & 3 & 10 & 58 \\
\hline F108 & M4S & 1000 & 0.45 & - & 11 & 2 & 22 & 11 & 3 & 7 & 57 \\
\hline F109 & M4S & 1000 & 0.45 & - & 11 & 3 & 20 & 12 & 3 & 9 & 58 \\
\hline F110 & M4S & 1000 & 0.45 & - & 12 & 3 & 25 & 14 & 5 & 8 & 66 \\
\hline F111 & M4D & $500+500$ & 0.45 & - & 38 & 13 & 16 & 13 & 3 & 6 & 88 \\
\hline F112 & M4D & $500+500$ & 0.45 & - & 23 & 6 & 25 & 14 & 3 & 7 & 78 \\
\hline F113 & M4D & $500+500$ & 0.45 & - & 26 & 8 & 28 & 18 & 2 & 5 & 87 \\
\hline F114 & $\mathrm{M} 2 \mathrm{~S}$ & 1000 & 0.17 & - & 15 & 6 & 20 & 15 & 3 & 9 & 67 \\
\hline F115 & $\mathrm{M} 2 \mathrm{~S}$ & 1000 & 0.17 & - & 15 & 6 & 15 & 14 & 3 & 12 & 65 \\
\hline F116 & $\mathrm{M} 2 \mathrm{~S}$ & 1000 & 0.17 & - & 18 & 5 & 20 & 13 & 3 & 7 & 66 \\
\hline
\end{tabular}




\begin{tabular}{|c|c|c|}
\hline Soudures : Type (Nombre) & Ajouts (Utilisation) & Particularités du forgeage \\
\hline Simple(1),repli(3) & Argile(B) & Trempes \\
\hline Simple(1),repli(3) & Argile(B) & Trempes, présence de nombreux défauts dans le fer \\
\hline Repli(2),consolidation(2) & Argile(B) & Barre repliée au lieu d'être refoulée, présence de défauts dans le fer, trempes \\
\hline Simple(1), consolidation(2) & Argile(B) & Trempe, présence de nombreux défauts dans le fer \\
\hline- & - & - \\
\hline- & - & Découpe d'une barre de de $2 \mathrm{~kg}$ au burin \\
\hline- & - & - \\
\hline Simple(3),repli(2) & Argile(B) & Trempe \\
\hline 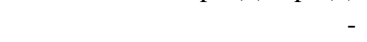 & - & - \\
\hline- & - & - \\
\hline- & - & - \\
\hline- & - & Un coin casse lors des finitions \\
\hline Repli(2),repli insert(1) & Charbon(F) & Apparition de défauts, replis de la lame et de la soie sur le reste de la lame \\
\hline Repli insert(1),consolidation(1) & Charbon $(\mathrm{F})$ & La soudure se décolle, trempe, apparition d'une paille sur la lame \\
\hline- & - & - \\
\hline Simple(1) & - & - \\
\hline Simple(1) & Charbon $(\mathrm{F})$ & - \\
\hline- & - & - \\
\hline Simple(1) & - & - \\
\hline- & - & - \\
\hline- & - & - \\
\hline- & - & - \\
\hline- & - & - \\
\hline Simple(1) & - & - \\
\hline Simple(1) & - & - \\
\hline Simple(1) & - & - \\
\hline- & - & - \\
\hline- & - & - \\
\hline- & - & - \\
\hline Simple(1) & - & Un coin de la lame se détache lors du formage \\
\hline Simple(1) & - & - \\
\hline Simple(1) & - & - \\
\hline- & - & Présence de défauts sur le fer lors du formage \\
\hline- & - & - \\
\hline- & - & - \\
\hline Simple(1) & - & - \\
\hline Simple(1) & - & - \\
\hline Simple(1) & - & - \\
\hline Simple(1) & - & - \\
\hline- & - & - \\
\hline- & - & - \\
\hline- & - & - \\
\hline- & - & - \\
\hline Simple(1) & - & - \\
\hline Simple(1) & - & - \\
\hline Simple(1) & - & - \\
\hline- & - & - \\
\hline Simple(1) & Tuyère(B) & Trempe \\
\hline Simple(1) & Tuyère(B) & Trempe \\
\hline- & - & - \\
\hline - & - & - \\
\hline - & - & - \\
\hline - & - & - \\
\hline - & - & - \\
\hline - & - & - \\
\hline Simple(1), insert(1) & Argile,tuyère(B) & Trempe, 2 fragments se détachent, une paille est retirée lors du formage \\
\hline Simple $(1)$ & Argile(B) & Trempe \\
\hline Simple(1) & Argile(B) & Trempe \\
\hline Repli(1) & Argile(B) & La barre est repliée au lieu d'être refoulée, trempe \\
\hline Repli(1) & Argile(B) & La barre est repliée au lieu d'être refoulée, trempe \\
\hline Repli(1) & Argile(B) & La barre est repliée au lieu d'être refoulée, trempe \\
\hline
\end{tabular}


Annexes

\begin{tabular}{|c|c|c|c|c|c|c|c|c|c|c|c|}
\hline \multirow{2}{*}{ Forgeage } & \multirow{2}{*}{ Barre } & \multirow{2}{*}{ Poids } & \multirow{2}{*}{$\% \mathrm{C}$} & \multirow{2}{*}{ Arrêt du feu } & \multicolumn{2}{|c|}{ Préformage } & \multicolumn{2}{|c|}{ Formage } & \multicolumn{2}{|c|}{ Finitions } & \multirow{2}{*}{$\begin{array}{l}\text { Durée } \\
\text { totale }\end{array}$} \\
\hline & & & & & $\mathrm{C}$ & M & $\mathrm{C}$ & M & $\mathrm{C}$ & M & \\
\hline F117 & M2D & $500+500$ & 0.17 & - & 22 & 8 & 23 & 13 & 4 & 8 & 78 \\
\hline F118 & M2D & $500+500$ & 0.17 & - & 20 & 9 & 17 & 13 & 4 & 10 & 72 \\
\hline F119 & M2D & $500+500$ & 0.17 & - & 19 & 7 & 17 & 15 & 3 & 9 & 70 \\
\hline $\mathrm{F} 120$ & $\mathrm{M} 2 \mathrm{~S}$ & 1000 & 0.45 & - & 17 & 5 & 33 & 17 & 3 & 8 & 83 \\
\hline F121 & $\mathrm{M} 2 \mathrm{~S}$ & 1000 & 0.45 & - & 44 & 20 & 18 & 12 & 3 & 7 & 105 \\
\hline F122 & $\mathrm{M} 2 \mathrm{~S}$ & 1000 & 0.45 & - & 17 & 6 & 33 & 20 & 3 & 7 & 86 \\
\hline $\mathrm{F} 123$ & M2D & $500+500$ & 0.45 & - & 37 & 16 & 25 & 17 & 2 & 4 & 101 \\
\hline F124 & M2D & $500+500$ & 0.45 & - & 22 & 7 & 34 & 20 & 3 & 7 & 93 \\
\hline $\mathrm{F} 125$ & M2D & $500+500$ & 0.45 & - & 27 & 9 & 18 & 14 & 3 & 9 & 79 \\
\hline F126 & M4D & $500+500$ & 0.17 & - & 33 & 9 & 17 & 10 & 4 & 10 & 82 \\
\hline F127 & M4D & $500+500$ & 0.17 & - & 25 & 8 & 15 & 13 & 3 & 7 & 72 \\
\hline F128 & M4D & $500+500$ & 0.17 & - & 22 & 8 & 13 & 12 & 3 & 8 & 66 \\
\hline
\end{tabular}


Annexes

\begin{tabular}{|c|c|c|}
\hline Soudures : Type (Nombre) & $\begin{array}{c}\text { Ajouts } \\
\text { (Utilisation) }\end{array}$ & Particularités du forgeage \\
\hline Simple(1),consolidation(2) & Argile(B) & Nombreuses trempes car les soudures ne tiennent pas \\
\hline Simple(1) & Argile(B) & - \\
\hline Simple(1) & Argile(B) & - \\
\hline Replis(1) & Argile(B) & Replie la barre en deux au lieu de refouler \\
\hline Replis(2) & Argile(B) & Replie la barre en deux au lieu de refouler, le lopin trop long est replié \\
\hline Simple(1) & Argile(B) & Replie la barre en deux au lieu de refouler \\
\hline Simple(1),insert(1),consolidation(2) & Argile,tuyère(B) & $\begin{array}{l}\text { Plusieurs fragments de fer se détachent puis réintégrés par soudure, } \\
\text { trempe }\end{array}$ \\
\hline Simple(1),consolidation(1) & Argile,tuyère(B) & Trempe, présence de défauts dans le fer \\
\hline Simple(1) & Tuyère(B) & Trempe \\
\hline Simple(1) & Tuyère(R) & - \\
\hline Simple(1) & Tuyère(R) & - \\
\hline Simple(1) & Tuyère(R) & - \\
\hline
\end{tabular}


Annexes 
Annexes

Annexe 2 - Combustible (XRF-WDS)

\begin{tabular}{crrrrrr}
\hline & CH-Fiko-1 & CH-Fiko-2 & CH-Fiko-3 & CH-Kakoli-3 & CH-Kobo-1 & CH-Dounde-2 \\
$\mathrm{SiO}_{2}$ & 3.9 & 6.2 & 3.6 & 4.9 & 13.5 & 10.8 \\
$\mathrm{TiO}_{2}$ & 1.2 & 1.0 & 1.3 & 1.8 & 3.1 & 2.3 \\
$\mathrm{Al}_{2} \mathrm{O}_{3}$ & 1.2 & 0.7 & 0.3 & 0.3 & 1.4 & 0.3 \\
$\mathrm{FeO}_{\text {tot }}$ & 3.6 & 2.3 & 3.9 & 2.6 & 5.7 & 3.5 \\
$\mathrm{MnO}$ & 0.9 & 0.3 & 2.0 & 2.9 & 3.4 & 3.5 \\
$\mathrm{MgO}$ & 7.0 & 5.5 & 1.9 & 1.3 & 2.0 & 2.0 \\
$\mathrm{CaO}$ & 63.6 & 68.7 & 69.9 & 70.0 & 57.4 & 52.3 \\
$\mathrm{Na} \mathrm{O}_{2}$ & 0.5 & 0.2 & 0.2 & 0.2 & 0.4 & 0.2 \\
$\mathrm{~K}_{2} \mathrm{O}$ & 17.7 & 14.6 & 16.0 & 14.9 & 9.5 & 21.7 \\
$\mathrm{P}_{2} \mathrm{O}_{5}$ & 0.4 & 0.6 & 0.9 & 1.3 & 3.4 & 3.3 \\
$\mathrm{Total} \mathrm{majeurs}$ & 100 & 100 & 100 & 100 & 100 & 100 \\
\hline $\mathrm{S}$ & 10540 & 22540 & 11540 & 17990 & 17510 & 23650 \\
$\mathrm{Sc}$ & 4380 & 3370 & 5670 & 7900 & 13910 & 10490 \\
$\mathrm{Cu}$ & 1830 & 1130 & 4070 & 6490 & 10030 & 7140 \\
$\mathrm{Zn}$ & 1180 & 1030 & 3390 & 5170 & 8820 & 6700 \\
$\mathrm{Sr}$ & 5290 & 7980 & 8990 & 10320 & 8490 & 3850 \\
$\mathrm{La}$ & 17240 & 12540 & 20500 & 30710 & 47320 & 36970 \\
$\mathrm{~Pb}$ & 4200 & 3600 & 8230 & 14320 & 31950 & 26310 \\
$\mathrm{Total}$ traces & 44660 & 52190 & 62390 & 92900 & 138030 & 115510 \\
\hline
\end{tabular}

Analyses semi-quantitatives XRF Uniquant à $\pm 1 \%$, normé à 100 - Traces en ppm - 1,2,3 : mission 1,2,3 
Annexes 


\section{Annexe 3 - Matériaux siliceux (XRF-WDS)}

\begin{tabular}{|c|c|c|c|c|c|c|c|}
\hline & $\begin{array}{c}\text { P-Fiko-1 } \\
- \\
\text { paroi }\end{array}$ & $\begin{array}{l}\text { G-Kobo-1 } \\
\text { ajout vol. } \\
-\end{array}$ & $\begin{array}{l}\text { T-Kobo-1 } \\
\text { ajout vol. } \\
\text { tuyère }\end{array}$ & $\begin{array}{c}\text { A-Kobo-1 } \\
\text { ajout vol. } \\
-\end{array}$ & $\begin{array}{l}\text { T-Dounde-2 } \\
\text { ajout vol. } \\
\text { tuyère }\end{array}$ & $\begin{array}{l}\text { T-Dounde-3 } \\
\text { ajout vol. } \\
\text { tuyère }\end{array}$ & $\begin{array}{c}\text { A-Dounde-3 } \\
\text { ajout vol. } \\
-\end{array}$ \\
\hline $\mathrm{SiO} 2$ & 75.0 & 93.1 & 58.6 & 58.6 & 62.1 & 59.9 & 59.3 \\
\hline $\mathrm{TiO}_{2}$ & 0.9 & 0.1 & 0.6 & 0.7 & 0.7 & 0.7 & 0.7 \\
\hline $\mathrm{Al}_{2} \mathrm{O}_{3}$ & 14.3 & 4.5 & 23.7 & 25.5 & 22.8 & 25.9 & 26.0 \\
\hline FeOtot & 4.9 & 1.6 & 8.3 & 7.2 & 6.3 & 5.5 & 5.7 \\
\hline $\mathrm{MnO}$ & 0.1 & 0.0 & 0.1 & 0.0 & 0.1 & 0.0 & 0.0 \\
\hline $\mathrm{MgO}$ & 0.8 & 0.1 & 1.9 & 1.7 & 1.5 & 1.8 & 1.9 \\
\hline $\mathrm{CaO}$ & 1.3 & 0.2 & 1.1 & 0.7 & 0.4 & 0.3 & 0.2 \\
\hline $\mathrm{Na}_{2} \mathrm{O}$ & 0.3 & 0.0 & 0.1 & 0.0 & 0.1 & 0.1 & 0.1 \\
\hline $\mathrm{K}_{2} \mathrm{O}$ & 2.2 & 0.1 & 5.4 & 5.3 & 5.8 & 5.7 & 6.0 \\
\hline $\mathrm{P}_{2} \mathrm{O}_{5}$ & 0.2 & 0.3 & 0.3 & 0.2 & 0.1 & 0.1 & 0.1 \\
\hline Total majeurs & 100 & 100 & 100 & 100 & 100 & 100 & 100 \\
\hline $\mathrm{Rb}$ & 60 & $<$ & 190 & 210 & 230 & 240 & 250 \\
\hline $\mathrm{Sr}$ & 100 & 190 & 130 & 130 & 140 & 110 & 120 \\
\hline Co & 110 & 220 & 100 & 90 & 150 & 80 & 60 \\
\hline $\mathrm{Cu}$ & 70 & $<$ & 40 & 40 & 50 & 40 & 30 \\
\hline $\mathrm{Zn}$ & 180 & 10 & 70 & 50 & 110 & 80 & 60 \\
\hline $\mathrm{Ba}$ & 600 & 220 & 650 & 670 & 920 & 730 & 700 \\
\hline Sc & $<$ & $<$ & $<$ & $<$ & $<$ & $<$ & $<$ \\
\hline $\mathrm{Cr}$ & 60 & $<$ & 80 & 90 & 90 & 90 & 90 \\
\hline $\mathrm{La}$ & 20 & 70 & 20 & 30 & 30 & 40 & $<$ \\
\hline $\mathrm{Zr}$ & 840 & 70 & 220 & 200 & 230 & 180 & 140 \\
\hline V & 50 & $<$ & 70 & 80 & 80 & 100 & 80 \\
\hline S & 1380 & 220 & 3280 & 510 & 130 & 610 & 120 \\
\hline Total traces & 3470 & 1000 & 4850 & 2100 & 2160 & 2300 & 1650 \\
\hline
\end{tabular}

Analyses semi-quantitatives XRF Uniquant à $\pm 1 \%$, normé à 100 - Traces en ppm - 1,2,3 : mission 1,2,3 $P$ : paroi - $G$ : grès - $T$ : tuyère - $A$ : argile 
Annexes 


\section{Annexe 4 - Scories (XRF-WDS et XRD)}

\begin{tabular}{|c|c|c|c|c|c|c|c|c|c|c|c|c|c|c|}
\hline & \multicolumn{7}{|c|}{ SAS } & \multicolumn{7}{|c|}{ SAS-M } \\
\hline & S003 & S005 & S012 & S018 & S021 & S023 & S075 & S010 & S026 & S029 & S030 & $\begin{array}{c}\text { S048 } \\
049\end{array}$ & $\begin{array}{c}\text { S082 } \\
083 \\
084\end{array}$ & $\begin{array}{c}\text { S096 } \\
097 \\
098\end{array}$ \\
\hline Barre & M2S & M2S & M2S & M2S & M2S & M2S & M3S & M2S & M4S & M4S & M4S & $\begin{array}{l}\text { M4S* } \\
\text { M4S* }\end{array}$ & $\begin{array}{l}\text { M2S } \\
\text { M2S } \\
\text { M2S }\end{array}$ & $\begin{array}{l}\text { M2S } \\
\text { M2S } \\
\text { M2S }\end{array}$ \\
\hline$\% \mathrm{C}$ & 0.45 & 0.45 & 0.45 & 0.17 & 0.17 & 0.45 & 0.45 & 0.17 & 0.17 & 0.17 & 0.45 & $\begin{array}{l}0.35 \\
0.35\end{array}$ & $\begin{array}{l}0.17 \\
0.17 \\
0.17\end{array}$ & $\begin{array}{l}0.45 \\
0.45 \\
0.45\end{array}$ \\
\hline Fond. & - & - & - & - & - & - & - & - & - & - & - & - & - & - \\
\hline Incid. & - & - & - & - & - & - & - & - & - & - & - & - & - & - \\
\hline PEF & 19 & 20 & 19 & 15 & 13 & 16 & 17 & 16 & 13 & 14 & 19 & 14 & 20 & 20 \\
\hline Poids & 300 & 75 & 50 & 100 & 60 & 50 & 165 & 125 & 20 & 25 & 135 & 185 & 180 & 240 \\
\hline $\mathrm{rt}=1 \mathrm{bar}$ & Pono- & 0 & teneur & arbone de & arres de & Fond. / & $\mathrm{id} .=\mathrm{y} a$ & 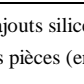 & / des ir & & & ; - = no & & \\
\hline $\mathrm{SiO}_{2}$ & 65.1 & 69.1 & 81.1 & 81.1 & 73.5 & 79.6 & 63.7 & 48.7 & 76.2 & 73.4 & 78.4 & 60.4 & 63.5 & 58.6 \\
\hline $\mathrm{TiO}_{2}$ & 0.7 & 0.5 & 0.4 & 0.3 & 0.4 & 0.3 & 0.5 & 0.4 & 0.2 & 0.1 & 0.2 & 0.2 & 0.5 & 0.5 \\
\hline $\mathrm{Al}_{2} \mathrm{O}_{3}$ & 8.5 & 7.4 & 6.9 & 5.5 & 9.0 & 4.1 & 6.9 & 5.3 & 3.5 & 2.2 & 3.7 & 3.5 & 6.4 & 6.6 \\
\hline $\mathrm{FeO}_{\text {tot }}$ & 10.9 & 12.2 & 8.4 & 9.2 & 11.0 & 11.9 & 15.9 & 40.0 & 16.9 & 21.5 & 14.0 & 31.9 & 23.2 & 22.5 \\
\hline $\mathrm{MnO}$ & 0.2 & 0.1 & 0.1 & 0.1 & 0.1 & 0.1 & 0.2 & 0.2 & 0.1 & 0.1 & 0.1 & 0.3 & 0.2 & 0.2 \\
\hline $\mathrm{MgO}$ & 1.5 & 1.2 & 0.4 & 0.5 & 0.9 & 0.5 & 1.3 & 0.5 & 0.4 & 0.3 & 0.4 & 0.4 & 0.6 & 1.0 \\
\hline $\mathrm{CaO}$ & 9.5 & 6.9 & 1.1 & 1.6 & 2.2 & 2.1 & 8.7 & 3.2 & 1.5 & 1.5 & 1.9 & 1.6 & 3.5 & 8.4 \\
\hline $\mathrm{Na}_{2} \mathrm{O}$ & 0.2 & 0.2 & 0.1 & 0.1 & 0.1 & 0.0 & 0.1 & 0.2 & 0.0 & 0.0 & 0.0 & 0.1 & 0.1 & 0.2 \\
\hline $\mathrm{K}_{2} \mathrm{O}$ & 2.7 & 2.1 & 1.3 & 1.4 & 2.6 & 1.2 & 2.2 & 1.2 & 1.0 & 0.7 & 1.0 & 1.4 & 1.7 & 1.9 \\
\hline $\mathrm{P}_{2} \mathrm{O}_{5}$ & 0.6 & 0.3 & 0.3 & 0.2 & 0.2 & 0.2 & 0.5 & 0.3 & 0.1 & 0.1 & 0.2 & 0.1 & 0.3 & 0.2 \\
\hline $\mathrm{Rb}$ & 60 & 40 & 40 & 40 & 80 & 40 & 40 & 30 & 30 & 20 & 30 & 40 & 40 & 40 \\
\hline $\mathrm{Sr}$ & 430 & 310 & 110 & 140 & 280 & 210 & 330 & 140 & 130 & 110 & 150 & 100 & 160 & 490 \\
\hline Co & 150 & 240 & 210 & 170 & 230 & 200 & 140 & 260 & 320 & 260 & 210 & 200 & 160 & 150 \\
\hline $\mathrm{Cu}$ & 280 & 110 & 20 & 50 & 60 & 30 & 250 & 770 & 80 & 90 & 30 & 50 & 230 & 380 \\
\hline $\mathrm{Zn}$ & 140 & 40 & $<$ & $<$ & $<$ & $<$ & 240 & 270 & $<$ & $<$ & $<$ & $<$ & 180 & 50 \\
\hline $\mathrm{Ba}$ & 800 & 620 & 380 & 1040 & 3870 & 2340 & 390 & 340 & 960 & 480 & 820 & 260 & 350 & 660 \\
\hline $\mathrm{Sc}$ & $<$ & $<$ & $<$ & $<$ & $<$ & $<$ & $<$ & $<$ & $<$ & $<$ & $<$ & $<$ & $<$ & $<$ \\
\hline $\mathrm{Cr}$ & 150 & 150 & 40 & 70 & 110 & 100 & 290 & 390 & 130 & 210 & 50 & 270 & 310 & 240 \\
\hline $\mathrm{La}$ & $<$ & $<$ & 20 & 10 & 10 & $<$ & $<$ & 30 & 0 & 30 & 30 & 60 & 20 & 10 \\
\hline $\mathrm{Zr}$ & 620 & 520 & 360 & 320 & 240 & 260 & 490 & 370 & 190 & 130 & 210 & 190 & 490 & 580 \\
\hline $\mathrm{V}$ & 50 & 70 & 40 & 20 & 30 & 10 & 80 & 60 & 20 & 10 & 10 & 20 & 50 & 60 \\
\hline $\mathrm{S}$ & 1290 & 420 & 50 & 130 & 130 & 100 & 1150 & 290 & 140 & 90 & 70 & 170 & 140 & 80 \\
\hline
\end{tabular}

\begin{tabular}{|c|c|c|c|c|c|c|c|c|c|c|c|c|c|c|}
\hline $\mathrm{Fe}$ & (x) & (x) & - & - & - & - & (x) & (x) & - & - & - & - & - & (x) \\
\hline Wus & - & - & - & - & - & - & - & - & - & - & - & - & - & - \\
\hline Mag & - & - & - & - & - & - & - & - & - & - & - & - & - & - \\
\hline Hem & - & - & - & - & - & - & - & - & - & - & - & - & - & - \\
\hline $\mathrm{Fa}$ & - & - & (x) & (x) & (x) & (x) & - & $\mathrm{x}$ & $\mathrm{x}$ & $\mathrm{x}$ & $\mathrm{x}$ & $\mathrm{x}$ & $\mathrm{x}$ & $\mathrm{x}$ \\
\hline Kir & - & - & - & - & - & - & - & - & - & - & - & - & - & - \\
\hline Aug & (x) & (x) & - & - & - & - & (x) & - & - & - & - & - & (x) & (x) \\
\hline $\mathrm{Hd}$ & - & - & - & - & - & - & - & - & - & - & - & - & - & - \\
\hline $\mathrm{Ak}$ & - & - & - & - & - & - & - & - & - & - & - & - & - & - \\
\hline $\mathrm{Qz}$ & $\operatorname{xxxx}$ & xxxx & xxxx & $\mathrm{xxxx}$ & $\operatorname{xxxx}$ & $\mathrm{xxxx}$ & $\mathrm{xxxx}$ & $\mathrm{xxx}$ & $\mathrm{xxx}$ & $\mathrm{xxx}$ & $\mathrm{xxx}$ & $\mathrm{xxx}$ & $\mathrm{xxx}$ & $\mathrm{xxx}$ \\
\hline Crs & $\mathrm{x}$ & $\mathrm{x}$ & $\mathrm{x}$ & $\mathrm{x}$ & $\mathrm{x}$ & $\mathrm{x}$ & $\mathrm{x}$ & $\mathrm{x}$ & $\mathrm{x}$ & $\mathrm{x}$ & $\mathrm{x}$ & $\mathrm{x}$ & $\mathrm{x}$ & $\mathrm{x}$ \\
\hline Lct & - & - & - & - & - & - & - & - & - & - & - & - & - & - \\
\hline Mul & - & - & - & - & - & - & - & - & - & - & - & - & - & - \\
\hline n.i. & - & - & - & - & - & - & - & - & - & - & - & - & - & - \\
\hline Groupe & Q & Q & Q & Q & Q & $\mathrm{Q}$ & Q & $\mathrm{QF}$ & $\mathrm{QF}$ & $\mathrm{QF}$ & $\mathrm{QF}$ & QF & $\mathrm{QF}$ & QF \\
\hline
\end{tabular}




\begin{tabular}{|c|c|c|c|c|c|c|c|c|c|c|c|c|c|c|c|}
\hline & & & & & & & & SGD-B & & & & & & & \\
\hline & S002 & S007 & S013 & S014 & S015 & S016 & S017 & S022 & $\begin{array}{c}\text { S024 } \\
025\end{array}$ & $\begin{array}{c}\mathrm{S} 027 \\
028\end{array}$ & $\begin{array}{c}\text { S050 } \\
051\end{array}$ & S074 & $\begin{array}{c}\text { S079 } \\
080 \\
081\end{array}$ & $\begin{array}{c}\text { S088 } \\
089 \\
090\end{array}$ & $\begin{array}{c}\text { S099 } \\
100 \\
101\end{array}$ \\
\hline Barre & M2D & A1D & M2D & A1D & A2D & M1D & M1D & M2D & $\begin{array}{l}\text { A1D } \\
\text { A1D }\end{array}$ & $\begin{array}{l}\text { A2D } \\
\text { A2D }\end{array}$ & $\begin{array}{l}\text { M3D } \\
\text { M3D }\end{array}$ & M4D & $\begin{array}{l}\text { M4D } \\
\text { M4D } \\
\text { M4D }\end{array}$ & $\begin{array}{l}\text { M4S } \\
\text { M4S } \\
\text { M4S }\end{array}$ & $\begin{array}{l}\text { M2D } \\
\text { M2D } \\
\text { M2D }\end{array}$ \\
\hline$\% \mathrm{C}$ & 0.17 & varie & 0.45 & varie & varie & 0.17 & 0.35 & 0.45 & $\begin{array}{l}\text { varie } \\
\text { varie }\end{array}$ & $\begin{array}{l}\text { varie } \\
\text { varie }\end{array}$ & $\begin{array}{l}0.45 \\
0.45\end{array}$ & 0.17 & $\begin{array}{l}0.17 \\
0.17 \\
0.17\end{array}$ & $\begin{array}{l}0.45 \\
0.45 \\
0.45\end{array}$ & $\begin{array}{l}0.45 \\
0.45 \\
0.45\end{array}$ \\
\hline Fond. & - & - & $v$ & $v$ & $\checkmark$ & $\checkmark$ & $\checkmark$ & $v$ & $\checkmark$ & $\checkmark$ & $\checkmark$ & - & - & - & - \\
\hline Incid. & - & $v$ & $v$ & $\checkmark$ & $\checkmark$ & $\checkmark$ & $\checkmark$ & - & 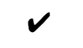 & $\checkmark$ & - & - & - & $\checkmark$ & - \\
\hline PEF & 34 & 33 & 44 & 47 & 53 & 43 & 43 & 24 & 38 & 39 & 30 & 23 & 35 & 23 & 25 \\
\hline Poids & 315 & 165 & 395 & 220 & 390 & 270 & 320 & 175 & 335 & 295 & 410 & 305 & 675 & 215 & 860 \\
\hline $\mathrm{SiO}_{2}$ & 49.3 & 36.3 & 47.6 & 52.9 & 45.8 & 34.4 & 52.9 & 55.7 & 44.4 & 66.9 & 34.1 & 41.8 & 25.2 & 49.8 & 53.6 \\
\hline $\mathrm{TiO}_{2}$ & 0.5 & 0.5 & 0.2 & 0.3 & 0.3 & 0.2 & 0.3 & 0.2 & 0.2 & 0.2 & 0.2 & 0.3 & 0.2 & 0.4 & 0.4 \\
\hline $\mathrm{Al}_{2} \mathrm{O}_{3}$ & 6.7 & 5.0 & 4.7 & 6.0 & 6.6 & 6.8 & 5.6 & 3.2 & 3.1 & 2.4 & 3.3 & 5.1 & 3.1 & 5.7 & 7.0 \\
\hline $\mathrm{FeO}_{\text {tot }}$ & 31.6 & 50.0 & 44.5 & 37.2 & 44.0 & 54.8 & 37.5 & 38.1 & 50.2 & 28.6 & 59.8 & 45.4 & 69.2 & 39.7 & 31.3 \\
\hline $\mathrm{MnO}$ & 0.2 & 0.1 & 0.4 & 0.1 & 0.1 & 0.4 & 0.3 & 0.4 & 0.1 & 0.1 & 0.5 & 0.2 & 0.3 & 0.3 & 0.2 \\
\hline $\mathrm{MgO}$ & 1.3 & 0.8 & 0.4 & 0.4 & 0.4 & 0.4 & 0.4 & 0.3 & 0.2 & 0.2 & 0.2 & 0.6 & 0.2 & 0.4 & 0.6 \\
\hline $\mathrm{CaO}$ & 7.7 & 5.5 & 0.9 & 1.4 & 1.2 & 1.0 & 1.2 & 1.2 & 0.9 & 0.9 & 0.7 & 4.8 & 1.0 & 2.1 & 5.1 \\
\hline $\mathrm{Na}_{2} \mathrm{O}$ & 0.2 & 0.2 & 0.1 & 0.1 & 0.1 & 0.1 & 0.1 & 0.0 & 0.0 & 0.0 & 0.1 & 0.1 & 0.1 & 0.1 & 0.1 \\
\hline $\mathrm{K}_{2} \mathrm{O}$ & 2.0 & 1.3 & 1.2 & 1.4 & 1.2 & 1.7 & 1.6 & 0.8 & 0.8 & 0.6 & 1.0 & 1.3 & 0.5 & 1.4 & 1.5 \\
\hline $\mathrm{P}_{2} \mathrm{O}_{5}$ & 0.5 & 0.3 & 0.1 & 0.2 & 0.3 & 0.1 & 0.2 & 0.1 & 0.1 & 0.1 & 0.1 & 0.3 & 0.1 & 0.2 & 0.2 \\
\hline $\mathrm{Rb}$ & 40 & 30 & 40 & 40 & 30 & 50 & 70 & 30 & 30 & 20 & 40 & 30 & 20 & 40 & 40 \\
\hline $\mathrm{Sr}$ & 410 & 250 & 140 & 100 & 90 & 80 & 220 & 170 & 110 & 80 & 80 & 240 & 50 & 160 & 250 \\
\hline Co & 190 & 310 & 230 & 230 & 260 & 260 & 180 & 290 & 260 & 270 & 210 & 190 & 220 & 140 & 200 \\
\hline $\mathrm{Cu}$ & 270 & 140 & 150 & $<$ & 30 & 340 & 130 & 70 & 10 & 40 & 80 & 680 & 660 & 190 & 320 \\
\hline $\mathrm{Zn}$ & 140 & 40 & $<$ & $<$ & 10 & $<$ & $<$ & 20 & $<$ & $<$ & $<$ & 100 & $<$ & 230 & 40 \\
\hline $\mathrm{Ba}$ & 750 & 460 & 2250 & 680 & 540 & 420 & 3610 & 2730 & 870 & 420 & 200 & 250 & 80 & 360 & 290 \\
\hline $\mathrm{Sc}$ & $<$ & $<$ & $<$ & $<$ & $<$ & $<$ & $<$ & $<$ & $<$ & $<$ & $<$ & $<$ & $<$ & $<$ & $<$ \\
\hline $\mathrm{Cr}$ & 520 & 160 & 680 & 50 & 60 & 790 & 370 & 730 & 80 & 80 & 800 & 690 & 1230 & 370 & 270 \\
\hline $\mathrm{La}$ & $<$ & 50 & 70 & 50 & 40 & 40 & 30 & 40 & 10 & 30 & 90 & 30 & 110 & 20 & 40 \\
\hline $\mathrm{Zr}$ & 500 & 650 & 110 & 220 & 160 & 100 & 190 & 140 & 260 & 160 & 190 & 420 & 240 & 550 & 420 \\
\hline V & 60 & 90 & 20 & 40 & 50 & 30 & 20 & 20 & 40 & 20 & 20 & 70 & 30 & 70 & 70 \\
\hline $\mathrm{S}$ & 1350 & 470 & 200 & 120 & 140 & 120 & 210 & 290 & 140 & 210 & 200 & 540 & 120 & 90 & 120 \\
\hline $\mathrm{Fe}$ & (x) & - & (x) & (x) & (x) & (x) & (x) & (x) & (x) & (x) & (x) & (x) & (x) & - & - \\
\hline Wus & - & - & - & - & - & - & - & - & - & - & (x) & - & - & - & - \\
\hline Mag & - & - & - & - & - & - & - & - & - & - & - & - & - & (x) & (x) \\
\hline Hem & - & - & - & - & - & - & - & - & - & - & - & - & - & - & (x) \\
\hline $\mathrm{Fa}$ & $\mathrm{xx}$ & $\mathrm{xxxx}$ & $\mathrm{xx}$ & $\mathrm{xx}$ & $\mathrm{xxxx}$ & XXXXX & $\mathrm{x}$ & $\mathrm{xx}$ & $\mathrm{xxxx}$ & $\mathrm{xx}$ & $\mathrm{x}$ & Xxxx & $\mathrm{xxxx}$ & $\mathrm{x}$ & $\mathrm{x}$ \\
\hline Kir & - & - & - & - & - & - & - & - & - & - & - & - & - & - & - \\
\hline Aug & (x) & (x) & - & - & - & - & - & - & - & - & - & - & - & - & (x) \\
\hline $\mathrm{Hd}$ & - & - & - & - & - & - & - & - & - & - & - & - & - & - & - \\
\hline $\mathrm{Ak}$ & - & - & - & - & - & - & - & - & - & - & - & - & - & - & - \\
\hline Qz & $\mathrm{xxx}$ & $\mathrm{x}$ & $\mathrm{xx}$ & $\mathrm{xx}$ & $\mathrm{x}$ & (x) & $\mathrm{xxxx}$ & $\mathrm{xx}$ & $\mathrm{x}$ & $\mathrm{xx}$ & $\mathrm{xxx}$ & $\mathrm{x}$ & $\mathrm{x}$ & $\mathrm{xxx}$ & $\mathrm{xxx}$ \\
\hline Crs & (x) & (x) & $\mathrm{x}$ & $\mathrm{x}$ & (x) & - & (x) & $\mathrm{x}$ & (x) & $\mathrm{x}$ & $\mathrm{x}$ & (x) & (x) & $\mathrm{x}$ & $\mathrm{x}$ \\
\hline Lct & - & - & - & - & - & (x) & - & - & - & - & - & - & - & - & - \\
\hline Mul & - & - & - & - & - & - & - & - & - & - & - & - & - & - & - \\
\hline n.i. & - & - & - & - & - & - & - & - & - & - & - & - & - & - & - \\
\hline Groupe & $\mathrm{QF}$ & FQ & $\mathrm{QF}$ & $\mathrm{QF}$ & FQ & $\mathrm{F}$ & $\mathrm{QF}$ & $\mathrm{QF}$ & FQ & $\mathrm{QF}$ & $\mathrm{QF}$ & FQ & FQ & $\mathrm{QF}$ & $\mathrm{QF}$ \\
\hline
\end{tabular}




\begin{tabular}{|c|c|c|c|c|c|c|c|c|c|c|}
\hline & \multicolumn{10}{|c|}{ SGD-SFR } \\
\hline & S004 & S008 & S011 & S020 & S036 & $\begin{array}{c}\text { S076 } \\
077 \\
078\end{array}$ & $\begin{array}{c}\text { S085 } \\
086 \\
087\end{array}$ & S094 & $\begin{array}{l}\text { S114 } \\
115 \\
116\end{array}$ & $\begin{array}{l}\text { S117 } \\
118 \\
119\end{array}$ \\
\hline \multirow{3}{*}{ Barre } & \multirow{3}{*}{ M2D } & \multirow{3}{*}{$\mathrm{A} 2 \mathrm{D}$} & \multirow{3}{*}{ M2D } & \multirow{3}{*}{ M2D } & \multirow{3}{*}{ R1D } & $\mathrm{M} 4 \mathrm{~S}$ & M2D & & $\mathrm{M} 2 \mathrm{~S}$ & M2D \\
\hline & & & & & & M4S & M2D & M4D & $\mathrm{M} 2 \mathrm{~S}$ & M2D \\
\hline & & & & & & M4S & M2D & & $\mathrm{M} 2 \mathrm{~S}$ & M2D \\
\hline \multirow{3}{*}{$\% \mathrm{C}$} & \multirow{3}{*}{0.45} & \multirow{3}{*}{ varie } & \multirow{3}{*}{0.17} & \multirow{3}{*}{0.17} & \multirow{3}{*}{$\begin{array}{l}0.35 \\
0.17\end{array}$} & 0.17 & 0.17 & \multirow{3}{*}{ M4D } & 0.17 & 0.17 \\
\hline & & & & & & 0.17 & 0.17 & & 0.17 & 0.17 \\
\hline & & & & & & 0.17 & 0.17 & & 0.17 & 0.17 \\
\hline Fond. & - & - & - & $\checkmark$ & - & - & - & - & $v$ & $v$ \\
\hline Incid. & - & $\checkmark$ & - & $\checkmark$ & $\checkmark$ & - & $\checkmark$ & - & $\checkmark$ & $\checkmark$ \\
\hline PEF & 24 & 46 & 25 & 35 & 23 & 21 & 28 & 31 & 18 & 21 \\
\hline Poids & 215 & 150 & 145 & 315 & 160 & 445 & 275 & 270 & 160 & 175 \\
\hline $\mathrm{SiO}_{2}$ & 48.8 & 29.8 & 30.6 & 31.0 & 28.0 & 61.9 & 31.4 & 37.2 & 38.2 & 39.6 \\
\hline $\mathrm{TiO}_{2}$ & 0.5 & 0.5 & 0.3 & 0.1 & 0.3 & 0.4 & 0.3 & 0.3 & 0.3 & 0.3 \\
\hline $\mathrm{Al}_{2} \mathrm{O}_{3}$ & 6.9 & 3.7 & 3.8 & 2.4 & 5.2 & 5.7 & 3.9 & 5.1 & 6.2 & 4.9 \\
\hline $\mathrm{FeO}_{\text {tot }}$ & 34.3 & 62.5 & 59.6 & 64.4 & 56.4 & 26.5 & 61.3 & 51.7 & 50.8 & 50.3 \\
\hline $\mathrm{MnO}$ & 0.3 & 0.0 & 0.5 & 0.3 & 0.5 & 0.2 & 0.4 & 0.5 & 0.3 & 0.3 \\
\hline $\mathrm{MgO}$ & 0.9 & 0.4 & 0.5 & 0.2 & 0.8 & 0.5 & 0.3 & 0.5 & 0.5 & 0.4 \\
\hline $\mathrm{CaO}$ & 5.7 & 2.1 & 3.3 & 0.8 & 6.5 & 3.1 & 1.5 & 3.3 & 1.5 & 2.0 \\
\hline $\mathrm{Na}_{2} \mathrm{O}$ & 0.2 & 0.1 & 0.2 & 0.0 & 0.2 & 0.1 & 0.1 & 0.1 & 0.1 & 0.1 \\
\hline $\mathrm{K}_{2} \mathrm{O}$ & 2.0 & 0.9 & 1.1 & 0.6 & 1.8 & 1.3 & 0.8 & 1.1 & 1.9 & 2.0 \\
\hline $\mathrm{P}_{2} \mathrm{O}_{5}$ & 0.4 & 0.2 & 0.2 & 0.1 & 0.4 & 0.2 & 0.2 & 0.2 & 0.2 & 0.1 \\
\hline $\mathrm{Rb}$ & 40 & 20 & 20 & 20 & 30 & 30 & 20 & 20 & 50 & 60 \\
\hline $\mathrm{Sr}$ & 270 & 130 & 150 & 170 & 290 & 130 & 80 & 170 & 110 & 190 \\
\hline Co & 190 & 270 & 260 & 280 & 280 & 180 & 280 & 240 & 240 & 210 \\
\hline $\mathrm{Cu}$ & 210 & 10 & 240 & 250 & 320 & 320 & 320 & 260 & 140 & 120 \\
\hline $\mathrm{Zn}$ & 60 & 0 & 20 & 0 & 0 & 50 & 0 & 20 & 20 & 10 \\
\hline $\mathrm{Ba}$ & 510 & 290 & 250 & 3110 & 410 & 240 & 140 & 240 & 260 & 350 \\
\hline $\mathrm{Sc}$ & 0 & 0 & 0 & 0 & 0 & 0 & 0 & 0 & 0 & 0 \\
\hline $\mathrm{Cr}$ & 570 & 70 & 1270 & 1070 & 740 & 460 & 730 & 760 & 680 & 670 \\
\hline $\mathrm{La}$ & 10 & 30 & 30 & 30 & 20 & 50 & 80 & 110 & 60 & 90 \\
\hline $\mathrm{Zr}$ & 440 & 370 & 240 & 120 & 200 & 430 & 280 & 320 & 160 & 210 \\
\hline $\mathrm{V}$ & 40 & 100 & 50 & 20 & 50 & 40 & 30 & 40 & 20 & 20 \\
\hline$S$ & 1070 & 160 & 650 & 180 & 290 & 240 & 110 & 100 & 170 & 180 \\
\hline $\mathrm{Fe}$ & (x) & (x) & (x) & (x) & (x) & (x) & (x) & (x) & (x) & (x) \\
\hline Wus & - & - & - & - & (x) & - & - & - & (x) & (x) \\
\hline Mag & - & - & - & - & (x) & - & - & - & - & - \\
\hline Hem & - & - & - & - & - & - & - & - & - & - \\
\hline $\mathrm{Fa}$ & $x x$ & $\operatorname{xxxxx}$ & $\operatorname{xxxxx}$ & xxxx & $\operatorname{xxxx}$ & $\mathrm{x}$ & $\operatorname{xxxxx}$ & $\operatorname{xxxxx}$ & xxxx & $x x$ \\
\hline Kir & - & - & - & - & - & - & - & - & - & - \\
\hline Aug & (x) & - & - & - & - & - & - & - & - & - \\
\hline $\mathrm{Hd}$ & - & - & - & - & - & - & - & - & - & - \\
\hline $\mathrm{Ak}$ & - & - & - & - & - & - & - & - & - & - \\
\hline $\mathrm{Qz}$ & $\mathrm{xxx}$ & (x) & (x) & $\mathrm{x}$ & (x) & $\mathrm{xxx}$ & (x) & (x) & $\mathrm{x}$ & $\mathrm{xxx}$ \\
\hline $\mathrm{Crs}$ & (x) & (x) & - & (x) & - & $\mathrm{x}$ & (x) & (x) & (x) & (x) \\
\hline Lct & - & - & - & - & (x) & - & - & - & - & - \\
\hline Mul & - & - & - & - & - & - & - & - & - & - \\
\hline n.i. & - & - & - & - & - & - & - & - & - & - \\
\hline Groupe & $\mathrm{QF}$ & $\mathrm{F}$ & $\mathrm{F}$ & $\mathrm{FQ}$ & $\mathrm{F}$ & $\mathrm{QF}$ & $\mathrm{F}$ & $\mathrm{F}$ & FQ & $\mathrm{QF}$ \\
\hline
\end{tabular}




\begin{tabular}{|c|c|c|c|c|c|c|c|c|}
\hline & \multicolumn{8}{|c|}{ SGD-K } \\
\hline & S066 & S067 & S068 & S069 & S070 & S071 & S072 & S073 \\
\hline Barre & M3S & M2S & M2D & M2D & M4S & M4D & M4D & M5S \\
\hline$\% \mathrm{C}$ & 0.45 & 0.45 & 0.45 & 0.17 & 0.45 & 0.17 & 0.45 & 0.45 \\
\hline Fond. & - & - & - & - & - & - & - & - \\
\hline Incid. & - & $\checkmark$ & $\checkmark$ & $\checkmark$ & $\checkmark$ & - & - & - \\
\hline PEF & 25 & 25 & 41 & 40 & 28 & 40 & 43 & 37 \\
\hline Poids & 275 & 135 & 290 & 455 & 260 & 340 & 355 & 305 \\
\hline $\mathrm{SiO}_{2}$ & 35.2 & 38.0 & 23.8 & 26.4 & 27.0 & 21.3 & 19.3 & 25.2 \\
\hline $\mathrm{TiO}_{2}$ & 0.4 & 0.4 & 0.3 & 0.2 & 0.3 & 0.2 & 0.2 & 0.3 \\
\hline $\mathrm{Al}_{2} \mathrm{O}_{3}$ & 9.0 & 5.6 & 6.8 & 4.6 & 5.0 & 4.3 & 3.0 & 4.7 \\
\hline $\mathrm{FeO}_{\text {tot }}$ & 37.1 & 22.4 & 54.9 & 52.9 & 39.7 & 57.3 & 52.8 & 45.9 \\
\hline $\mathrm{MnO}$ & 0.6 & 0.3 & 0.6 & 0.4 & 0.5 & 0.4 & 0.4 & 0.7 \\
\hline $\mathrm{MgO}$ & 1.2 & 2.1 & 0.7 & 0.9 & 1.8 & 1.1 & 1.2 & 1.1 \\
\hline $\mathrm{CaO}$ & 13.2 & 27.1 & 10.9 & 12.7 & 23.3 & 13.9 & 21.6 & 20.1 \\
\hline $\mathrm{Na}_{2} \mathrm{O}$ & 0.1 & 0.1 & 0.1 & 0.1 & 0.1 & 0.1 & 0.1 & 0.1 \\
\hline $\mathrm{K}_{2} \mathrm{O}$ & 2.5 & 2.9 & 1.3 & 1.3 & 1.7 & 1.0 & 0.9 & 1.4 \\
\hline $\mathrm{P}_{2} \mathrm{O}_{5}$ & 0.6 & 1.0 & 0.5 & 0.4 & 0.7 & 0.4 & 0.6 & 0.6 \\
\hline $\mathrm{Rb}$ & 40 & 40 & 20 & 20 & 20 & 20 & 20 & 30 \\
\hline $\mathrm{Sr}$ & 390 & 830 & 310 & 340 & 650 & 380 & 600 & 520 \\
\hline Co & 240 & 150 & 260 & 280 & 250 & 300 & 300 & 290 \\
\hline $\mathrm{Cu}$ & 170 & 120 & 250 & 600 & 180 & 540 & 550 & 460 \\
\hline $\mathrm{Zn}$ & 30 & 40 & 10 & 40 & 10 & $<$ & 10 & 20 \\
\hline $\mathrm{Ba}$ & 620 & 790 & 320 & 200 & 400 & 200 & 180 & 400 \\
\hline $\mathrm{Sc}$ & 100 & 130 & 0 & 110 & 140 & 130 & 150 & 140 \\
\hline $\mathrm{Cr}$ & 190 & 110 & 320 & 430 & 240 & 960 & 590 & 430 \\
\hline $\mathrm{La}$ & 400 & 350 & 10 & 440 & 450 & 480 & 450 & 450 \\
\hline $\mathrm{Zr}$ & 250 & 270 & 180 & 150 & 170 & 120 & 90 & 130 \\
\hline $\mathrm{V}$ & 90 & 50 & 90 & 20 & 60 & 30 & 30 & 20 \\
\hline $\mathrm{S}$ & 370 & 680 & 370 & 320 & 430 & 360 & 360 & 290 \\
\hline $\mathrm{Fe}$ & - & (x) & (x) & (x) & (x) & (x) & (x) & - \\
\hline Wus & $\mathrm{x}$ & (x) & $\mathrm{xx}$ & $\mathrm{xx}$ & $\mathrm{x}$ & $\mathrm{xx}$ & $\mathrm{xx}$ & $\mathrm{x}$ \\
\hline Mag & $\mathrm{x}$ & (x) & (x) & $\mathrm{x}$ & (x) & (x) & $\mathrm{x}$ & $\mathrm{x}$ \\
\hline Hem & - & - & - & - & - & - & - & - \\
\hline $\mathrm{Fa}$ & - & - & - & - & - & - & - & - \\
\hline Kir & $\mathrm{x}$ & $\mathrm{x}$ & $\mathrm{xx}$ & $\mathrm{x}$ & $\mathrm{xxx}$ & $\mathrm{xxx}$ & $\mathrm{x}$ & $\mathrm{xx}$ \\
\hline Aug & - & - & - & - & - & - & - & - \\
\hline $\mathrm{Hd}$ & - & - & - & - & - & - & - & - \\
\hline $\mathrm{Ak}$ & $\mathrm{x}$ & $\mathrm{xx}$ & - & (x) & $\mathrm{x}$ & (x) & $\mathrm{x}$ & $\mathrm{x}$ \\
\hline $\mathrm{Qz}$ & (x) & (x) & $\mathrm{x}$ & $\mathrm{x}$ & - & - & (x) & (x) \\
\hline Crs & - & (x) & - & - & - & - & - & - \\
\hline Lct & $\mathrm{x}$ & $\mathrm{x}$ & - & (x) & - & - & - & - \\
\hline Mul & - & - & - & - & - & - & - & - \\
\hline n.i. & - & - & - & - & - & - & - & - \\
\hline Groupe & $\mathrm{K}$ & K & K & $\mathrm{K}$ & $\mathrm{K}$ & K & $\mathrm{K}$ & $\mathrm{K}$ \\
\hline
\end{tabular}




\begin{tabular}{|c|c|c|c|c|c|c|c|c|c|c|c|c|c|c|c|}
\hline & & & & & & & & SFR & & & & & & & \\
\hline & S006 & S033 & S034 & S035 & S041 & $\begin{array}{c}\text { S045 } \\
046 \\
047\end{array}$ & $\begin{array}{c}\text { S053 } \\
054\end{array}$ & S056 & $\begin{array}{c}\text { S091 } \\
092 \\
093\end{array}$ & S095 & S103 & $\begin{array}{c}\text { S111 } \\
112 \\
113\end{array}$ & $\begin{array}{c}\mathrm{S} 120 \\
121 \\
122\end{array}$ & $\begin{array}{c}\text { S123 } \\
124 \\
125\end{array}$ & $\begin{array}{c}\text { S126 } \\
127 \\
128\end{array}$ \\
\hline Barre & M4S & M1D & M1D & R1D & M2D & $\begin{array}{l}\text { M3D } \\
\text { M3D } \\
\text { M3D }\end{array}$ & $\begin{array}{l}\text { M3D } \\
\text { M3D }\end{array}$ & M1D & $\begin{array}{l}\text { M4D } \\
\text { M4D } \\
\text { M4D }\end{array}$ & M5S & M4D & $\begin{array}{l}\text { M4D } \\
\text { M4D } \\
\text { M4D }\end{array}$ & $\begin{array}{l}\text { M2S } \\
\text { M2S } \\
\text { M2S }\end{array}$ & $\begin{array}{l}\text { M2D } \\
\text { M2D } \\
\text { M2D }\end{array}$ & $\begin{array}{l}\text { M4D } \\
\text { M4D } \\
\text { M4D }\end{array}$ \\
\hline$\% \mathrm{C}$ & 0.17 & 0.35 & 0.17 & $\begin{array}{l}0.45 \\
0.45\end{array}$ & 0.17 & $\begin{array}{l}0.17 \\
0.17 \\
0.17\end{array}$ & $\begin{array}{l}0.35 \\
0.35\end{array}$ & 0.17 & $\begin{array}{l}0.45 \\
0.45 \\
0.45\end{array}$ & 0.45 & 0.17 & $\begin{array}{l}0.45 \\
0.45 \\
0.45\end{array}$ & $\begin{array}{l}0.45 \\
0.45 \\
0.45\end{array}$ & $\begin{array}{l}0.45 \\
0.45 \\
0.45\end{array}$ & $\begin{array}{l}0.17 \\
0.17 \\
0.17\end{array}$ \\
\hline Fond. & - & - & - & - & $v$ & $\boldsymbol{v}$ & $\boldsymbol{V}$ & $v$ & - & - & $v$ & $v$ & $\boldsymbol{v}$ & $v$ & $\boldsymbol{v}$ \\
\hline Incid. & - & $\checkmark$ & $\boldsymbol{v}$ & - & $\boldsymbol{V}$ & - & $\checkmark$ & 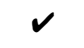 & - & - & - & $\checkmark$ & $\checkmark$ & $\checkmark$ & - \\
\hline PEF & 23 & 38 & 44 & 28 & 32 & 32 & 28 & 29 & 31 & 26 & 35 & 30 & 24 & 22 & 22 \\
\hline Poids & 125 & 165 & 150 & 200 & 165 & 605 & 140 & 90 & 365 & 105 & 170 & 330 & 180 & 175 & 215 \\
\hline $\mathrm{SiO}_{2}$ & 29.1 & 31.3 & 30.5 & 27.3 & 24.1 & 21.7 & 27.0 & 30.4 & 27.5 & 33.4 & 27.1 & 28.7 & 49.7 & 25.0 & 20.1 \\
\hline $\mathrm{TiO}_{2}$ & 0.2 & 0.3 & 0.2 & 0.2 & 0.2 & 0.1 & 0.2 & 0.2 & 0.2 & 0.3 & 0.2 & 0.2 & 0.2 & 0.2 & 0.2 \\
\hline $\mathrm{Al}_{2} \mathrm{O}_{3}$ & 3.9 & 6.1 & 4.5 & 4.8 & 5.1 & 2.0 & 5.6 & 3.1 & 3.7 & 4.0 & 6.4 & 4.0 & 3.0 & 3.9 & 4.1 \\
\hline $\mathrm{FeO}_{\text {tot }}$ & 61.4 & 51.8 & 56.0 & 57.6 & 66.3 & 73.7 & 63.0 & 64.1 & 64.1 & 56.1 & 56.2 & 63.1 & 43.4 & 66.8 & 72.7 \\
\hline $\mathrm{MnO}$ & 0.4 & 0.5 & 0.3 & 0.6 & 0.8 & 0.6 & 0.9 & 0.4 & 0.5 & 0.5 & 0.8 & 0.8 & 0.4 & 0.8 & 0.6 \\
\hline $\mathrm{MgO}$ & 0.6 & 1.0 & 0.9 & 0.9 & 0.5 & 0.2 & 0.5 & 0.2 & 0.3 & 0.5 & 1.2 & 0.3 & 0.3 & 0.3 & 0.3 \\
\hline $\mathrm{CaO}$ & 3.1 & 6.6 & 5.6 & 6.3 & 1.4 & 0.8 & 1.1 & 0.7 & 2.5 & 3.9 & 4.2 & 1.4 & 1.6 & 1.6 & 0.9 \\
\hline $\mathrm{Na}_{2} \mathrm{O}$ & 0.1 & 0.2 & 0.2 & 0.2 & 0.1 & 0.1 & 0.1 & 0.1 & 0.1 & 0.1 & 0.1 & 0.1 & 0.1 & 0.1 & 0.1 \\
\hline $\mathrm{K}_{2} \mathrm{O}$ & 0.9 & 1.8 & 1.5 & 1.7 & 1.5 & 0.6 & 1.7 & 0.6 & 0.8 & 1.0 & 3.6 & 1.3 & 1.2 & 1.2 & 0.9 \\
\hline $\mathrm{P}_{2} \mathrm{O}_{5}$ & 0.2 & 0.4 & 0.3 & 0.4 & 0.1 & 0.1 & 0.1 & 0.1 & 0.2 & 0.2 & 0.2 & 0.1 & 0.1 & 0.1 & 0.1 \\
\hline $\mathrm{Rb}$ & 20 & 20 & 30 & 30 & 60 & 20 & 70 & 20 & 20 & 30 & 90 & 50 & 30 & 40 & 40 \\
\hline $\mathrm{Sr}$ & 110 & 290 & 290 & 260 & 150 & 70 & 120 & 50 & 140 & 220 & 300 & 160 & 120 & 140 & 110 \\
\hline Co & 280 & 250 & 290 & 250 & 370 & 370 & 330 & 310 & 240 & 240 & 280 & 260 & 190 & 280 & 280 \\
\hline $\mathrm{Cu}$ & 200 & 300 & 1090 & 150 & 130 & 180 & 90 & 200 & 400 & 110 & 260 & 140 & 170 & 120 & 190 \\
\hline $\mathrm{Zn}$ & $<$ & $<$ & 120 & $<$ & $<$ & $<$ & $<$ & $<$ & 10 & 60 & 20 & 30 & $<$ & 50 & 50 \\
\hline $\mathrm{Ba}$ & 160 & 340 & 220 & 290 & 340 & 120 & 240 & 70 & 200 & 320 & 390 & 270 & 210 & 240 & 210 \\
\hline $\mathrm{Sc}$ & $<$ & $<$ & $<$ & $<$ & $<$ & $<$ & $<$ & $<$ & $<$ & $<$ & $<$ & $<$ & $<$ & $<$ & $<$ \\
\hline $\mathrm{Cr}$ & 1490 & 690 & 720 & 710 & 1820 & 2250 & 1540 & 1110 & 850 & 840 & 2970 & 1590 & 840 & 970 & 2210 \\
\hline $\mathrm{La}$ & 30 & 20 & 10 & 10 & $<$ & 80 & 10 & 40 & 70 & 70 & $<$ & 60 & 50 & 50 & 70 \\
\hline $\mathrm{Zr}$ & 160 & 230 & 250 & 190 & 130 & 90 & 120 & 120 & 290 & 360 & 140 & 200 & 230 & 150 & 150 \\
\hline $\mathrm{V}$ & 30 & 60 & 80 & 40 & 40 & 30 & 50 & 30 & 30 & 40 & 50 & 30 & 40 & 30 & 40 \\
\hline $\mathrm{S}$ & 480 & 370 & 510 & 540 & 240 & 160 & 250 & 190 & 290 & 350 & 80 & 120 & 80 & 120 & 150 \\
\hline $\mathrm{Fe}$ & (x) & (x) & (x) & (x) & (x) & (x) & $\mathrm{x}$ & (x) & (x) & (x) & $\mathrm{x}$ & (x) & (x) & (x) & (x) \\
\hline Wus & $\mathrm{xx}$ & $\mathrm{x}$ & (x) & (x) & - & $\mathrm{x}$ & $\mathrm{x}$ & - & - & - & $\mathrm{x}$ & - & - & - & $\mathrm{x}$ \\
\hline Mag & - & - & (x) & (x) & - & - & - & - & - & - & - & - & - & - & - \\
\hline Hem & - & - & - & - & - & - & - & - & - & - & - & - & - & - & - \\
\hline $\mathrm{Fa}$ & $\mathrm{xx}$ & $\mathrm{xxx}$ & $\mathrm{xxx}$ & $\mathrm{xxx}$ & $\mathrm{xxxx}$ & $\mathrm{xxx}$ & $\mathrm{xx}$ & $\operatorname{xxxx}$ & $\operatorname{xxxxx}$ & $\operatorname{xxxx}$ & $\mathrm{x}$ & $\operatorname{xxxx}$ & $\mathrm{x}$ & $\begin{array}{c}\mathrm{xxxx} \\
\mathrm{x}\end{array}$ & $\mathrm{xxx}$ \\
\hline Kir & - & - & - & - & - & - & - & - & - & - & - & - & - & - & - \\
\hline Aug & - & (x) & - & - & - & - & - & - & - & - & - & - & - & - & - \\
\hline $\mathrm{Hd}$ & - & - & - & - & - & - & - & - & - & - & - & - & - & - & - \\
\hline $\mathrm{Ak}$ & - & - & - & - & - & - & - & - & - & - & - & - & - & - & - \\
\hline $\mathrm{Qz}$ & $\mathrm{x}$ & $\mathrm{x}$ & $\mathrm{x}$ & $\mathrm{x}$ & $\mathrm{x}$ & $\mathrm{x}$ & $\mathrm{x}$ & $\mathrm{x}$ & (x) & $\mathrm{x}$ & $x x$ & $\mathrm{x}$ & $\mathrm{xxx}$ & (x) & $\mathrm{x}$ \\
\hline Crs & - & - & - & - & - & (x) & (x) & (x) & - & (x) & (x) & (x) & $\mathrm{x}$ & - & - \\
\hline Lct & - & (x) & - & (x) & - & (x) & - & - & - & - & (x) & - & - & - & - \\
\hline Mul & - & - & - & - & - & - & - & - & - & - & - & - & - & - & - \\
\hline n.i. & - & - & - & - & - & - & - & - & - & - & - & - & - & - & - \\
\hline Gpe & MO & MO & MO & MO & FQ & MO & MO & FQ & F & FQ & MO & FQ & $\mathrm{QF}$ & $\mathrm{F}$ & MO \\
\hline
\end{tabular}




\section{SI}

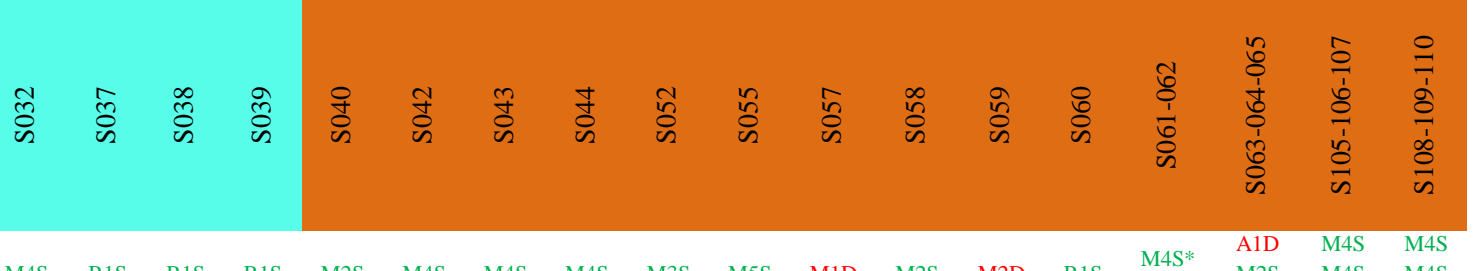

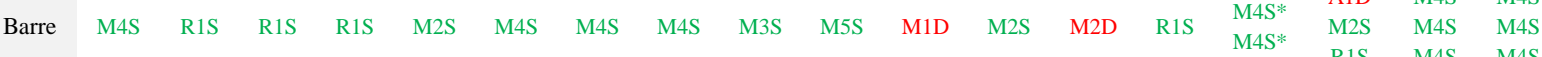

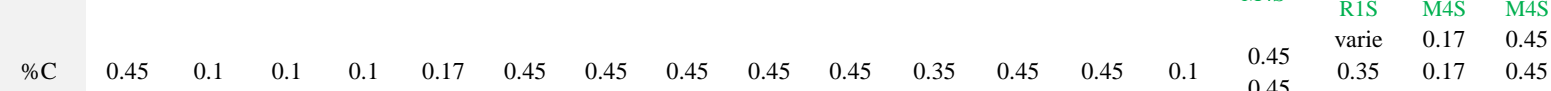

Incid.

$\begin{array}{lllllllllllllllllll}\text { PEF } & 22 & 12 & 13 & 14 & 12 & 12 & 14 & 12 & 14 & 16 & 26 & 34 & 24 & 8 & 15 & 23 & 13 & 18\end{array}$

$\begin{array}{lllllllllllllllllll}\text { Poids } & 30 & 60 & 5 & 10 & 3 & 30 & 17 & 25 & 25 & 20 & 17 & 22 & 20 & 6 & 10 & 45 & 55 & 55\end{array}$

\begin{tabular}{|c|c|c|c|c|c|c|c|c|c|c|c|c|c|c|c|c|c|c|}
\hline $\mathrm{SiO}_{2}$ & 48.7 & 51.7 & 69.2 & 44.8 & 46.3 & 71.3 & 45.2 & 47.9 & 38.0 & 40.1 & 12.4 & 12.7 & 42.3 & 37.1 & 52.5 & 40.6 & 34.7 & 35.9 \\
\hline $\mathrm{TiO}_{2}$ & 0.4 & 0.6 & 0.2 & 0.3 & 0.1 & 0.4 & 0.2 & 0.2 & 0.2 & 0.2 & 0.1 & 0.0 & 0.2 & 0.1 & 0.4 & 0.2 & 0.1 & 0.2 \\
\hline $\mathrm{Al}_{2} \mathrm{O}_{3}$ & 7.9 & 12.6 & 6.4 & 11.6 & 3.5 & 7.0 & 3.2 & 7.1 & 6.0 & 6.7 & 3.8 & 1.2 & 3.8 & 1.7 & 15.1 & 6.6 & 2.7 & 2.0 \\
\hline $\mathrm{FeO}_{\text {tot }}$ & 23.6 & 19.7 & 12.3 & 30.7 & 32.5 & 9.8 & 35.5 & 38.4 & 52.7 & 48.7 & 80.5 & 85.0 & 51.9 & 58.9 & 27.4 & 47.5 & 60.4 & 57.2 \\
\hline $\mathrm{MnO}$ & 0.3 & 0.2 & 0.1 & 0.2 & 0.2 & 0.2 & 0.2 & 0.1 & 0.3 & 0.4 & 1.6 & 0.6 & 0.1 & 0.5 & 0.1 & 0.1 & 0.2 & 0.4 \\
\hline $\mathrm{MgO}$ & 1.9 & 1.5 & 1.1 & 1.5 & 0.6 & 1.0 & 0.3 & 0.7 & 0.4 & 0.5 & 0.2 & 0.1 & 0.2 & 0.2 & 0.8 & 0.4 & 0.1 & 0.3 \\
\hline $\mathrm{CaO}$ & 11.9 & 9.5 & 7.3 & 8.8 & 7.4 & 4.4 & 7.8 & 2.1 & 0.7 & 1.1 & 0.7 & 0.2 & 0.5 & 0.8 & 0.6 & 2.0 & 0.8 & 2.5 \\
\hline $\mathrm{Na}_{2} \mathrm{O}$ & 0.3 & 0.3 & 0.1 & 0.2 & 0.3 & 0.3 & 0.1 & 0.1 & 0.2 & 0.1 & 0.1 & 0.0 & 0.1 & 0.1 & 0.1 & 0.1 & 0.1 & 0.0 \\
\hline $\mathrm{K}_{2} \mathrm{O}$ & 4.4 & 3.3 & 3.0 & 1.8 & 2.2 & 5.1 & 0.7 & 3.1 & 1.5 & 2.3 & 0.5 & 0.2 & 0.8 & 0.6 & 3.1 & 1.7 & 0.7 & 0.9 \\
\hline $\mathrm{P}_{2} \mathrm{O}_{5}$ & 0.7 & 0.7 & 0.4 & 0.3 & 6.9 & 0.6 & 7.0 & 0.2 & 0.1 & 0.1 & 0.1 & 0.0 & 0.1 & 0.1 & 0.1 & 0.8 & 0.1 & 0.6 \\
\hline $\mathrm{Rb}$ & 50 & 50 & 50 & 30 & 40 & 90 & 30 & 120 & 80 & 90 & 20 & 10 & 50 & 10 & 160 & 60 & 30 & 20 \\
\hline $\mathrm{Sr}$ & 440 & 400 & 420 & 500 & 160 & 280 & 150 & 250 & 270 & 170 & 80 & 20 & 120 & 80 & 120 & 120 & 80 & 110 \\
\hline Co & 280 & 210 & 90 & 230 & 140 & 260 & 110 & 110 & 130 & 190 & 330 & 100 & 90 & 340 & 60 & 130 & 200 & 310 \\
\hline $\mathrm{Cu}$ & 430 & 170 & 40 & 60 & 60 & 90 & 110 & 60 & 70 & 40 & 120 & 240 & 130 & 250 & 10 & 30 & 360 & 180 \\
\hline $\mathrm{Zn}$ & 120 & 70 & 60 & 10 & 10 & 20 & $<$ & 40 & 10 & 10 & 10 & $<$ & 20 & 40 & 30 & 10 & $<$ & 30 \\
\hline $\mathrm{Ba}$ & 540 & 540 & 590 & 560 & 440 & 650 & 430 & 690 & 320 & 440 & 180 & $<$ & 450 & 70 & 730 & 330 & 120 & 60 \\
\hline $\mathrm{Sc}$ & $<$ & $<$ & $<$ & 20 & $<$ & $<$ & 30 & $<$ & $<$ & $<$ & $<$ & $<$ & $<$ & 30 & $<$ & $<$ & $<$ & $<$ \\
\hline $\mathrm{Cr}$ & 220 & 210 & 300 & 390 & 570 & 100 & 480 & 130 & 560 & 680 & 2440 & 1440 & 290 & 900 & 220 & 1430 & 1000 & 480 \\
\hline $\mathrm{La}$ & $<$ & $<$ & $<$ & $<$ & $<$ & $<$ & $<$ & $<$ & 20 & $<$ & $<$ & $<$ & $<$ & $<$ & $<$ & $<$ & $<$ & 30 \\
\hline $\mathrm{Zr}$ & 340 & 430 & 370 & 460 & 160 & 300 & 400 & 370 & 260 & 260 & 70 & 60 & 500 & 90 & 340 & 350 & 200 & 130 \\
\hline V & 70 & 200 & 310 & 370 & 30 & 80 & 60 & 60 & 70 & 80 & 90 & 40 & 50 & 40 & 100 & 100 & 70 & 30 \\
\hline S & 2080 & 250 & 110 & 130 & 360 & 110 & 60 & 60 & 140 & 100 & 540 & 330 & 60 & 130 & 40 & 170 & 170 & 120 \\
\hline
\end{tabular}

\begin{tabular}{|c|c|c|c|c|c|c|c|c|c|c|c|c|c|c|c|c|c|c|}
\hline $\mathrm{Fe}$ & (x) & - & - & (x) & - & - & - & (x) & (x) & (x) & $\mathrm{x}$ & (x) & - & (x) & - & (x) & (x) & (x) \\
\hline Wus & (x) & - & - & - & (x) & - & (x) & - & - & - & $x x$ & $x x$ & - & $x x$ & - & (x) & - & (x) \\
\hline Mag & (x) & (x) & - & $x x$ & - & - & - & - & - & - & - & (x) & - & - & - & (x) & - & (x) \\
\hline Hem & (x) & (x) & - & $\mathrm{x}$ & - & - & - & - & - & - & - & - & - & - & - & (x) & - & (x) \\
\hline $\mathrm{Fa}$ & - & - & - & - & - & $\mathrm{x}$ & - & $\mathrm{x}$ & $\mathrm{x}$ & $\mathrm{x}$ & $x x$ & $x x$ & (x) & $\mathrm{x}$ & - & $\mathrm{x}$ & XXXX & $\mathrm{x}$ \\
\hline Kir & - & - & - & - & - & - & - & - & - & - & - & - & - & - & - & - & - & - \\
\hline Aug & - & $x x$ & - & - & - & (x) & - & - & - & - & - & - & - & - & - & - & - & - \\
\hline $\mathrm{Hd}$ & $\mathrm{x}$ & - & - & - & - & - & - & - & - & - & - & - & - & - & - & - & - & - \\
\hline $\mathrm{Ak}$ & - & - & - & - & (x) & - & (x) & - & - & - & - & - & - & - & (x) & - & - & - \\
\hline Qz & $\mathrm{x}$ & $\mathrm{x}$ & $\mathrm{xxx}$ & $x x$ & $\mathrm{xxxx}$ & xxxx & $x x$ & $\mathrm{xxx}$ & $x x$ & $x x x$ & - & $\mathrm{x}$ & $\mathrm{XXXX}$ & $x x$ & $\mathrm{xxx}$ & $x x x$ & $\mathrm{x}$ & $x x$ \\
\hline Crs & (x) & (x) & $x x$ & (x) & (x) & $(\mathrm{x})$ & $x x$ & $x$ & $x x$ & $\mathrm{x}$ & - & (x) & $\mathrm{x}$ & (x) & $\mathrm{x}$ & $\mathrm{x}$ & (x) & $\mathrm{x}$ \\
\hline Lct & $x x$ & $\mathrm{x}$ & - & - & - & - & - & - & - & - & - & - & - & - & - & - & - & - \\
\hline Mul & - & - & - & (x) & - & - & - & - & - & - & - & - & - & - & $x$ & - & - & - \\
\hline n.i. & - & - & - & - & (x) & - & (x) & - & - & - & - & - & - & - & - & - & - & - \\
\hline Gpe & A & A & Q & MO & A & $\mathrm{QF}$ & A & $\mathrm{QF}$ & $\mathrm{QF}$ & $\mathrm{QF}$ & MO & MO & Q & $\mathrm{MO}$ & A & $\mathrm{QF}$ & FQ & $\mathrm{QF}$ \\
\hline
\end{tabular}

$\mathrm{Q}=$ quartz+cristobalite $75-100 \%$ et fayalite $<5 \%$

$\mathrm{QF}=$ quartz+cristobalite $45-80 \%$ et fayalite $15-40 \%$

$\mathrm{FQ}=$ fayalite $60-80 \%$ et quartz+cristobalite $20-25 \%$

$\mathrm{F}=$ fayalite $75-100 \%$ et quartz+cristobalite $<10 \%$

$\mathrm{MO}=$ fer métallique +oxydes $20-45 \%$

$\mathrm{A}=$ autres minéraux 10-60\% 


\section{Annexe 5 - Diagrammes chimiques}

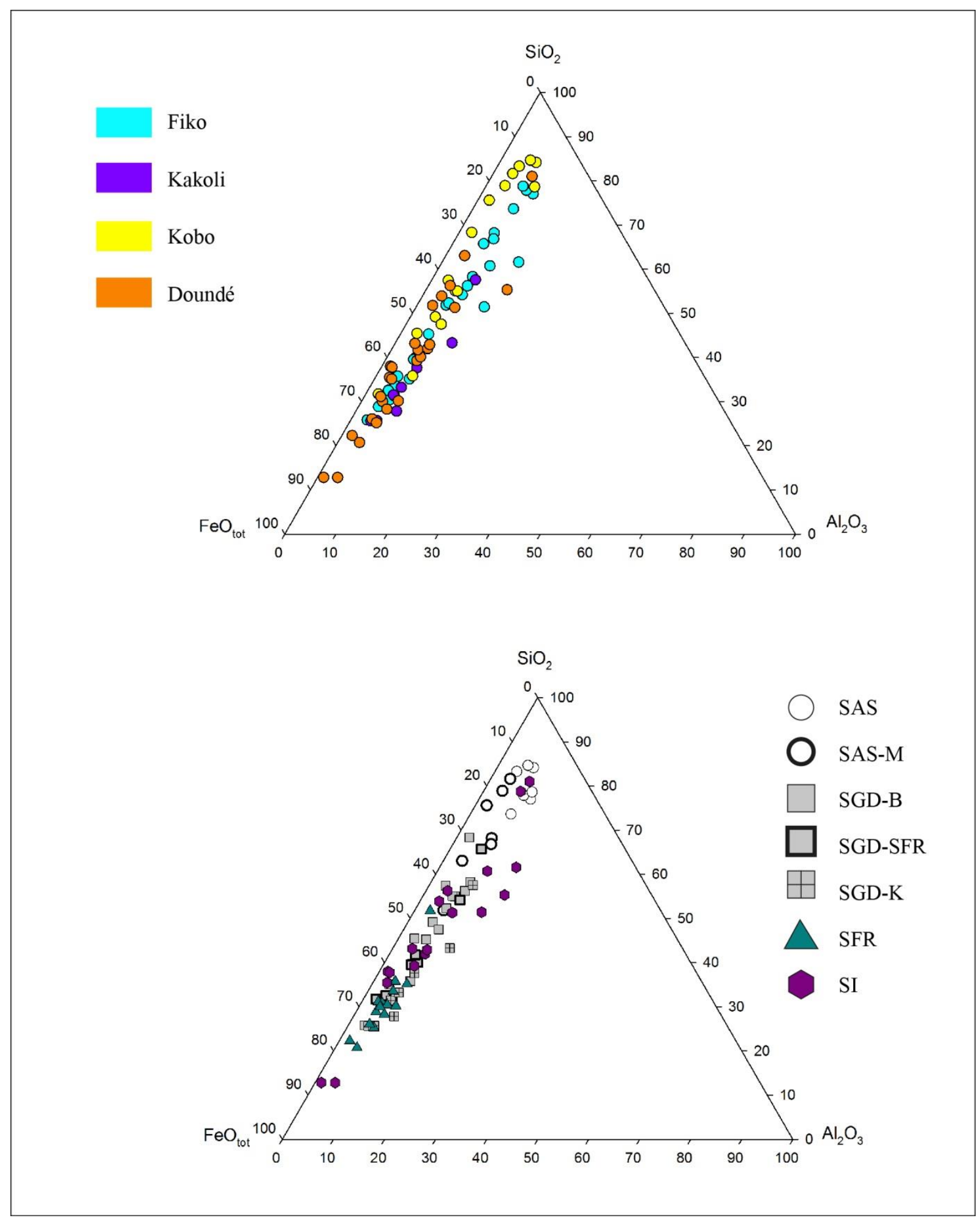

Diagrammes ternaires $\mathrm{Al}_{2} \mathrm{O}_{3}-\mathrm{SiO}_{2}-\mathrm{FeO}_{\text {tot }}$ par atelier et par groupe de scorie 


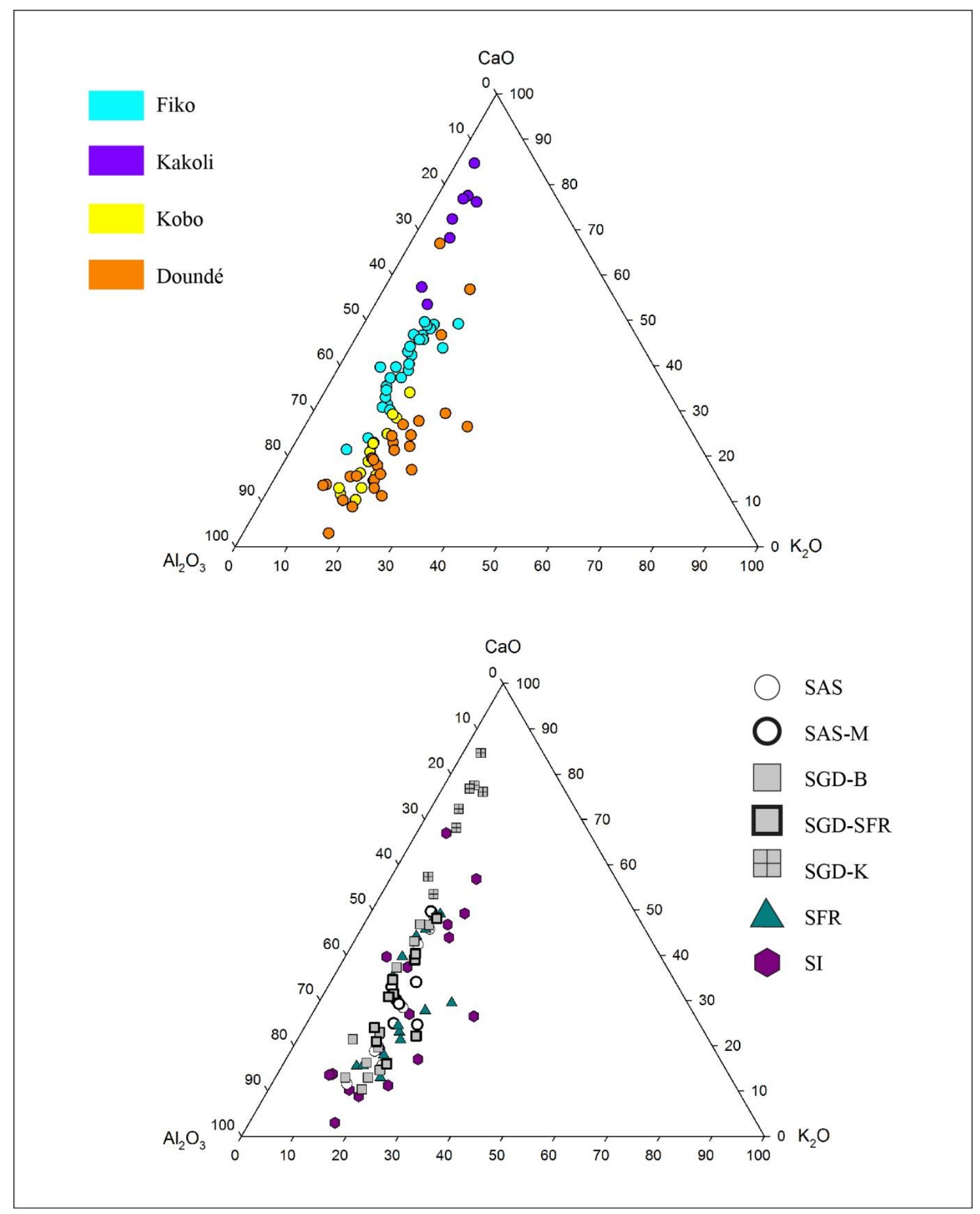

Diagrammes ternaires $\mathrm{K}_{2} \mathrm{O}-\mathrm{CaO}-\mathrm{Al}_{2} \mathrm{O}_{3}$ par atelier et par groupe de scorie 


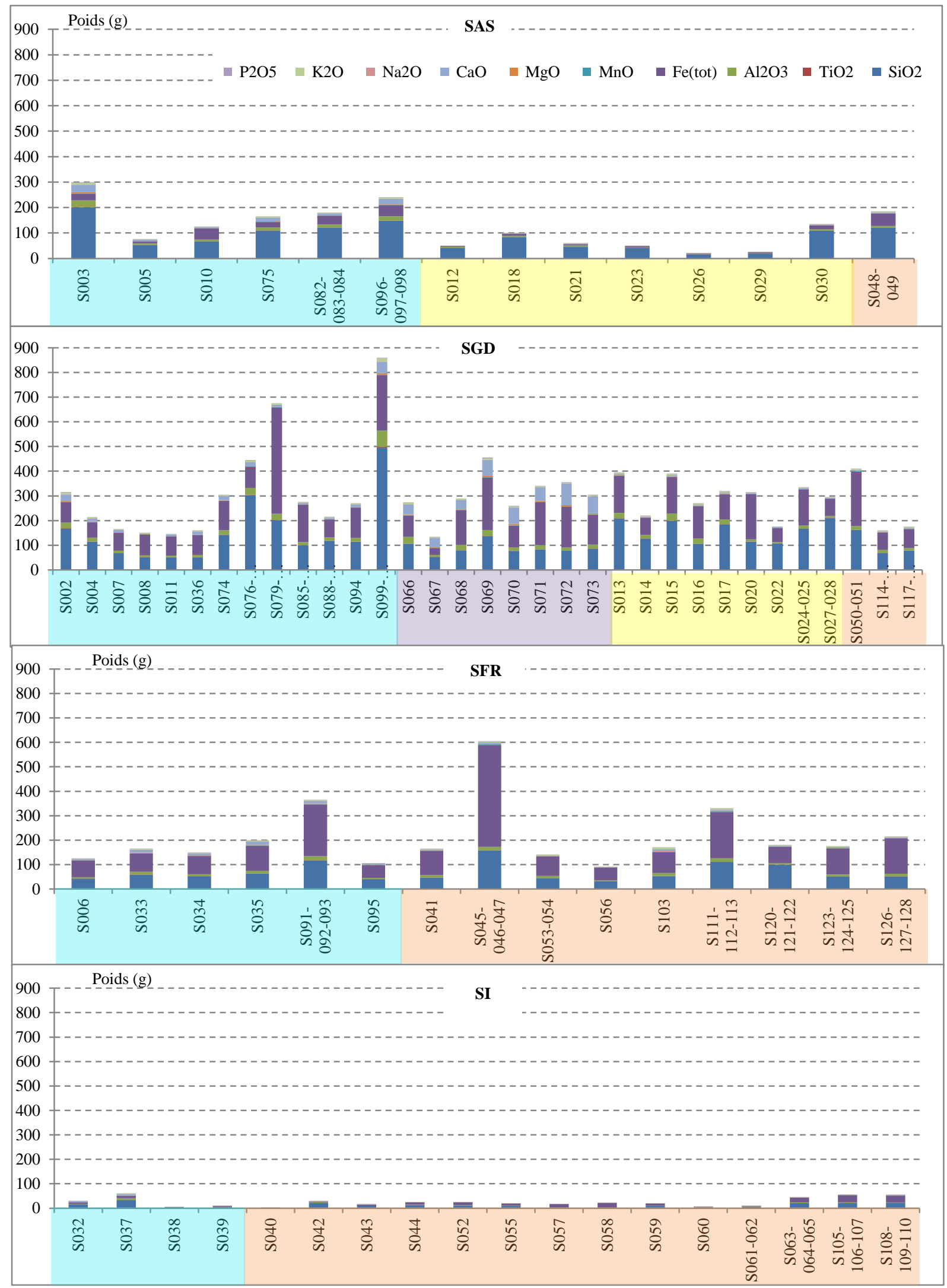

Poids (g) des différents composés dans les scories de forge 


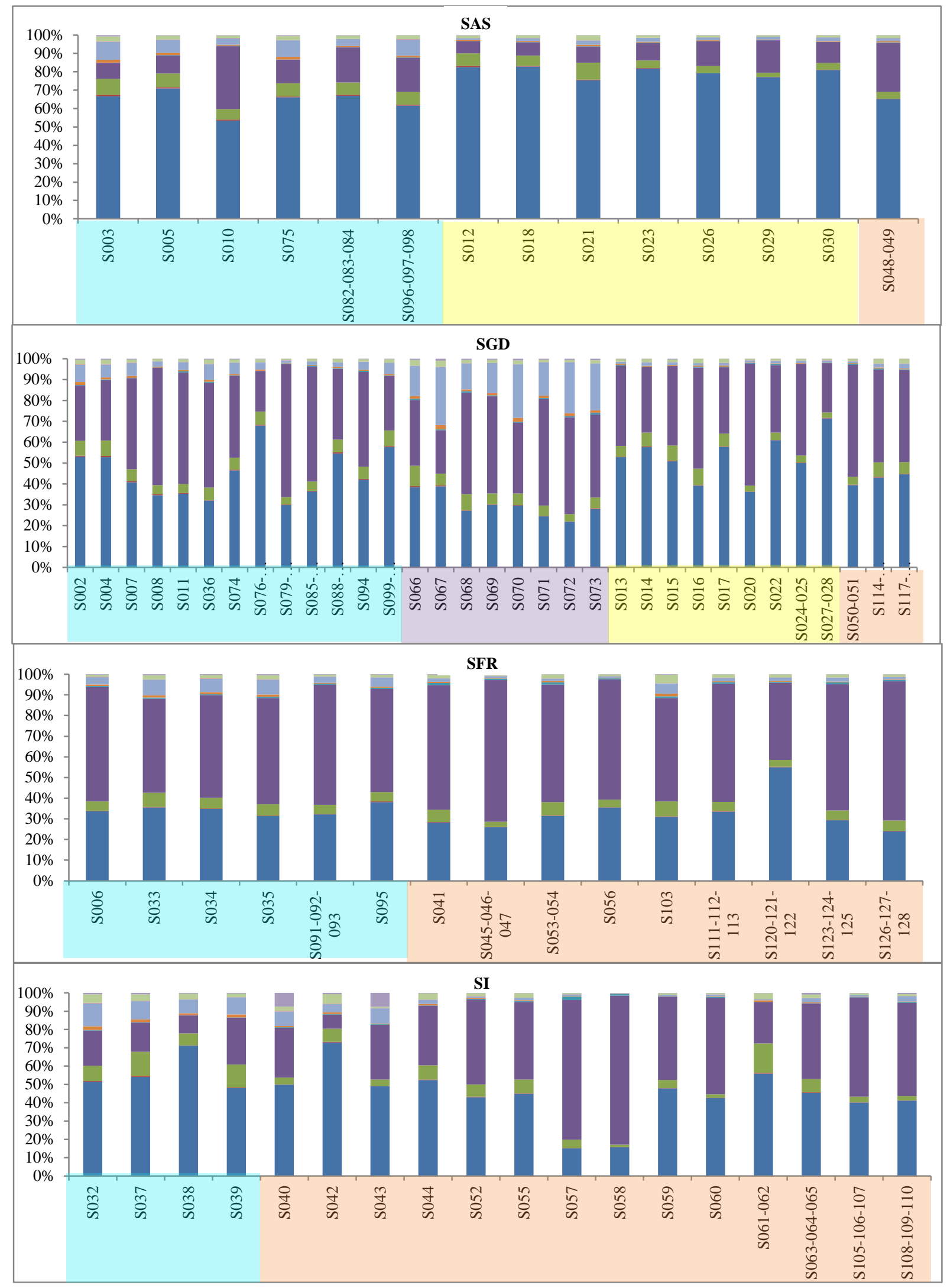

Poids normalisé (\%) des différents composés dans les scories de forge 


\section{PARTIE 7 . BIBLIOGRAPHIE}


Bibliographie 
Allen, J.R.L. 1986. Interpretation of some RomanoBritish smithing slag from Awre in Gloucestershire. Historical metallurgy 20, 97-104.

Als-Nielsen, J. \& McMorrow, D. 2001. Elements of modern X-ray Physics. John Wiley \& Sons, Inc., Chichester.

Anderson, T.J., Agustoni, C., Duvauchelle, A., Serneels, V. \& Castella, D. 2003. Des artisans à la campagne. Carrière de meules, forge et voie gallo-romaines à Châbles (FR). Archéologie fribourgeoise 19. Academic Press Fribourg, Fribourg.

Anderson, T.J. \& Castella, D. 2007. Une ferme gauloise à Courgevaux (Fribourg, Suisse). Archéologie fribourgeoise 21. Academic Press Fribourg, Fribourg.

Anderson, T.J., Duvauchelle, A., Serneels, V. \& Agustoni, C. 2000. Stone and metal working on the Roman site of Châbles-Les Saux (Canton Fribourg, Switzerland). In: Iron, Blacksmiths and Tools. Ancient European Crafts, Acts of the Instrumentum Conference, April 1999, Podsreda Editions Monique Mergoil, Montagnac, pp. 103108.

Appia, B. 1965. Les forgerons du Fouta-Djallon. Journal de la Société des Africanistes 35(2), 317-352.

Arnaud, J.-C. \& Collectif 2010. Atlas de l'Afrique, Mali. Les Editions Jeune Afrique, Paris.

Bachmann, H.-G. 1982. The identification of slags from archaeological sites. Occasional Publication 6. The Institute of Archaeology, London.

Bauvais, S. 2007a. Evolution de l'organisation des activités de forge dans le nord du Bassin parisien au second Age du fer. Etudes pluridisciplinaires de la chaîne opératoire en métallugie $d u$ fer. Vol 2.Thèse de doctorat, Université de Technologie de Belfort-Montbeliard, Belfort.

Bauvais, S. 2007b. Evolution de l'organisation des activités de forge dans le nord du Bassin parisien au second Age du fer. Etudes pluridisciplinaires de la chaîne opératoire en métallugie $d u$ fer. Vol 1.Thèse de doctorat, Université de Technologie de Belfort-Montbeliard, Belfort.
Bauvais, S. \& Fluzin, P. 2005. Analyses métallographiques des déchets de forge du site de Bazoches-sur-Vesle "Les Chantraines" (Aisne). Confrontation et perspective régionale. Revue archéologique de Picardie 22, 115-130.

Bauvais, S., Gaudefroy, S., Gransar, F., Malrain, F. \& Fluzin, P. 2007. Premières réflexions sur l'organisation des activités de forge en contexte rural à La Tène finale en Picardie. In: L'économie du fer protohistorique : de la production à la consommation du métal, Actes du XXVIII colloque de l'AFEAF, 2023 mai 2004, Toulouse. Fédération Aquitania, Bordeaux, pp. 239-261.

Beckhoff, B., Kanngiesser, B., Langhoff, N., Wedell, R. \& Wolff, H. 2006. Handbook of Practical X-Ray Fluorescence Analysis. Springer, Berlin Heidelberg.

Berner, P. 2010. Archaeometry of historical lime mortars. Thèse de doctorat, Département des Geosciences de l'Université de Fribourg, Fribourg.

Berranger, M. \& Fluzin, P. 2007. Organisation de la chaîne opératoire en métallurgie du fer aux II $^{\mathrm{e}}-\mathrm{I}^{\mathrm{er}}$ siècle av. J.-C.,sur l'oppidum d'Entremont (Aix-en-Provence, Bouchesdu-Rhône) : la circulation du métal. Archeosciences 31, 7-22.

Berti Rossi, S. \& May Castella, C. 2005. La fouille de Vidy "Chavannes 11" 1989-1990. Archéologie, architecture et urbanisme. Cahier d'Archéologie Romande 102. Lausanne.

Birosca, S., Dingley, D. \& Higinson, R.L. 2004. Microstructural and microtextural characterization of oxide scale on steel using electron backscatted diffraction. Journal of Microscopy 213, 235-240.

Blench, R. \& Douyon, D. 2006. L'apport de la linguistique pour décrypter la préhistoire du Plateau dogon. Etudes Maliennes 65, 183189.

Bowen, N.L. \& Greig, J.W. 1924. The System : $\mathrm{Al}_{2} \mathrm{O}_{3}-\mathrm{SiO}_{2}$. Journal of the American Ceramic Society 7(4), 238-254.

Bowen, N.L. \& Schairer, J.F. 1932. The system FeO$\mathrm{SiO}_{2}$. American Journal of Science 24(141), 177-213. 
Bowen, N.L. \& Schairer, J.F. 1935. The system $\mathrm{MgO}-\mathrm{FeO}-\mathrm{SiO}_{2}$. American Journal of Science 29(170), 151-217.

Bowen, N.L., Schairer, J.F. \& Posnjak, E. 1933. The system $\mathrm{Ca}_{2} \mathrm{SiO}_{4}-\mathrm{Fe}_{2} \mathrm{SiO}_{4}$. American Journal of Science 25(148), 273-297.

Célis, G.R. 1989. La métallurgie traditionnelle au Burundi, au Rwanda et au Buha. Essai de synthèse. Anthropos 84, 25-46.

Célis, G.R. 1994. La métallurgie traditionnelle chez les Songhaï (Niger). Techniques et croyances : la reconstitution de Yelwani. Anthropos 89, 401-420.

Célis, G.R. \& Coulibaly, Y.T. 2001. Métallurgies traditionnelles du fer sénoufo, malinké et somono. Côte d'Ivoire, Burkina Faso et Mali. Archives d'anthropologie 32. Musée royal de l'Afrique centrale, Tervuren.

Chardron-Picault, P. \& Pernot, M. 1999. Un quartier antique d'artisanat métallurgique à Autun (Saône et Loire). Le site du Lycée militaire. Documents d'archéologie française. Editions de la Maison des Sciences de l'Homme, Paris.

Chen, R.Y. \& Yuen, W.Y. 2003. Review of the hightemperature oxidation of iron and carbon steels in air or oxygen. Oxidation of Metals 59, 433-468.

Crew, P. 1991. The Experimental Production of Prehistoric Bar Iron. Historical metallurgy 25(1), 21-36

Darken, L.S. \& Gurry, R.W. 1946. The System IronOxygen II. Equilibrium and Thermodynamics of Liquid Oxide and Other Phases. Journal of the American Chemistry Society 68(5), 798-816.

Daveau, S. 1959. Recherches morphologiques sur la région de Bandiagara. Mémoires de l'Institut Français d'Afrique Noire 56. IFAN, Dakar.

de Heusch, L. 1956. Le Symbolisme du Forgeron en Afrique. Reflets du Monde 10, 57-70.

de Maret, P. 1980. Ceux qui jouent avec le feu : la place du forgeron en Afrique Centrale. Africa 50(3), 263-279.

de Maret, P. 2002. L' Afrique centrale : le "savoirfer". In: Bocoum, H. (ed.), Aux origines de la métallurgie du fer en Afrique. Une ancienneté méconnue. Editions Unesco, Paris, pp. 123-131.

Dillmann, P. \& Fluzin, P. 2003. Analyse des matériaux et histoire de la sidérurgie. Apport de la métallographie et de l'analyse à l'étude de l'élaboration et de l'utilisation des fers anciens. Technè 18, 20-26.

Doswald, C., Duvauchelle, A., Eschenlohr, L., Fasnacht, W., Schaltenbrand-Obrecht, V., Senn-Luder, M. \& Serneels, V. 1997. Technique des fouilles. Minerai, scories, fer. Cours d'initiation à l'étude de la métallurgie ancienne et à l'identification des déchets de cette industrie. GSAF/ASTFA, Basel.

Dungworth, D. \& Wilkes, R. 2007. An investigation of hammerscale, technology report. Research Department Report 26. English heritage, London.

Dunikowski, C., Leroy, M., Merluzzo, P. \& Ploquin, A. 1996. L'atelier de forge gallo-romain de Nailly Les Bordes (Yonne), contribution à la caractérisation des déchets de production. Revue archéologique de l'Est et du Centre-Est 47, 97-121.

Duvauchelle, A. \& Agustoni, C. 2002. Couper du fer : outils et gestes des forgerons d' hier et d' aujourd'hui. Annuaire de la Société Suisse de Préhistoire et d'Archéologie 85, 261266.

Eschenlohr, L., Friedli, V., Robert-Charrue Linder, C., Senn, M. \& Fellner, R. 2007. DevelierCourtételle, un habitat rural mérovingien. Métallurgie du fer et mobilier métallique. Cahier d'archéologie jurassienne 14. Office de la culture et Société jurassienne d'Emulation, Porrentruy.

Eschenlohr, L., Serneels, V. \& Hiltpold, B. 1991. Les bas fourneaux mérovingiens de Boécourt, Les Boulies (Jura, Suisse) Cahier d'Archéologie Jurassienne 3. Office du patrimoine historique et Société jurassienne d'émulation, Porrentruy.

Faivre, A., Mangin, M. \& Raïssouni, B. 1998. Forges et forgerons dans les villes et les campagnes de l'Est des Gaules romaines : l' exemple du Haut-Auxois (Côte-d' Or) et du Finage dolois (Jura). In: Les métaux antiques : travail et restauration, Actes du colloque de Poitiers, 28-30 sept. 1995, Poitiers. Editions Monique Mergoil, Montagnac, pp. 13-18. 
Fluzin, P. 2000. Etudes métallographiques préliminaires concernant les vestiges sidérurgiques du site de Montagney. Minaria Helvetica 20b, 91-104.

Fluzin, P. 2002. La chaîne opératoire en sidérurgie : matériaux archéologiques et procédés. Apports des études métallographiques. In: Bocoum, H. (ed.), Aux origines de la métallurgie $d u$ fer en Afrique. Une ancienneté méconnue. Editions Unesco, Paris, pp. 59-91.

Fluzin, P., Ploquin, A. \& Dabosi, F. 2004. Approches métallurgiques et archéométriques. In: Mangin, M. (ed.), Le fer. Editions Errance, Paris, pp. 113-173.

Fluzin, P., Ploquin, A. \& Serneels, V. 2000. Archéométrie des déchets de production sidérurgique. Moyens et méthodes d'identification des différents éléments de la chaîne opératoire directe. Gallia 57, 101121.

Forbes, R.J. 1963. Ancient geology, Ancient mining and quarrying, Ancient mining techniques. Studies in Ancient Technology 7. Brill Academic Pub, Leiden.

Gallay, A. 1994. Sociétés englobées et traditions céramiques. Les cas du Pays dogon (Mali) depuis le XIIIe siècle. In: Terre cuite et Société. La céramique, document technique, économique et culturel, Actes des XIV rencontres internationales d'Archéologie et d'Histoire d'Antibes, 2123 Octobre 1993, Antibes. Editions APDCA, Antibes, pp. 435-457.

Gallay, A., Huysecom, E. \& Mayor, A. 1995. Archéologie, histoire et traditions orales: trois clés pour découvrir le passé Dogon. In: Homberger, L. (ed.), Die Kunst der Dogon. Museum Rietberg, Zürich, pp. 19-43.

Goldstein, J., Newbury, D.E., Echlin, P., Joy, D.C., Roming, A.D., Lyman, C.E., Fiori, C. \& Lifshin, E. 1992. Scanning Electron Microscopy and X-Ray Microanalysis. A text for Biologists, Material Scientists, and Geologists. Plenum Press, New-York.

Gruat, P., Abraham, P., Mahé-Le Carlier, C. \& Ploquin, A. 2007. L'artisanat du fer en milieu caussenard : l'exemple de l'enceinte du Puech de Mus à Sainte-Eulalie-deCernon (Aveyron) aux $\mathrm{V}^{\mathrm{e}}$ et $\mathrm{IV}^{\mathrm{e}}$ s. a.C. In: L'économie du fer protohistorique : de la production à la consommation du métal, Actes du XXVIII colloque de l'AFEAF, 2023 mai 2004, Toulouse. Fédération Aquitania, Bordeaux, pp. 177-212.

Guillaumet, J.-P. 1998. Les pinces dites "de forgeron". In: Les métaux antiques : travail et restauration, Actes $d u$ colloque de Poitiers, 28-30 sept. 1995, Poitiers. Editions Monique Mergoil, Montagnac, pp. 1-6.

Higgins, R.A. 1993. Engineering Metallurgy. Applied Physical Metallurgy (6th edition). Arnold, London.

Hochstetler, J.L., Durieux, J.A. \& Durieux-Boon, E.I.K. 2004. Sociolinguistic Survey of the Dogon Language Area. SIL Electronic Survey Reports 4. SIL International, Dallas, e-publication http://www.sil.org/silesr/abstract.asp?ref= 2004-004.

Hofmann Rognon, P. 2005. Le Landeron-Les Carougets. Vestiges protohistoriques, villa romaine, tombe et habitat médiévaux. Archéologie neuchâteloise 32. Service et musée cantonal d'archéologie de Neuchâtel, Neuchâtel.

Huet, J.-C. 1994. Villages perchés des Dogon du Mali. Habitat, espace et société. L'Harmattan, Paris.

Huysecom, E. 2001. Technique et croyance des forgerons africains : éléments pour une approche ethnoarchéologique. In: The Origins of Iron Metallurgy, Proceedings of the First International Colloquium on The Archaeology of Africa and the Mediterranean Basin, 4-7 June 1999, Geneva. Mediterranean Archaeology, Sydney, pp. 73-82.

Huysecom, E. 2002. Paleoenvironnement and human population in West Africa : an international research project in Mali. Antiquity 76, 335-336.

Huysecom, E. \& Agustoni, B. 1996. Inagina, l'ultime maison du fer. Genève, 52 min.

Huysecom, E., Ballouche, A., Boëda, E., Cappa, L., Cissé, L., Dembélé, A., Gallay, A., Doulaye, K., Mayor, A., Ozainne, S., Raeli, F., Rasse, M., Robert, A., Robion, C., Sanogo, K., Soriano, S., Sow, O. \& Stokes, S. 2002. Cinquième Campagne de Recherches à Ounjougou (Mali). Résultats 
préliminaires. In: SLSA Jahresbericht 2001. Fondation Suisse-Liechtenstein pour les recherches Archéologiques à l'étranger, Zurich-Vaduz, pp. 103-161.

Huysecom, E., Ballouche, A., Cissé, L., Gallay, A., Doulaye, K., Mayor, A., Neumann, K., Ozainne, S., Perret, S., Rasse, M., Robert, A., Robion, C., Sanogo, K., Serneels, V., Soriano, S. \& Stokes, S. 2003. Paléoenvironnement et peuplement humain en Afrique de l'Ouest : rapport de la sixième campagne de recherches à Ounjougou (Mali). In: SLSA Jahresbericht 2002. Fondation Suisse-Liechtenstein pour les recherches Archéologiques à l'étranger, Zurich-Vaduz, pp. 27-68.

Huysecom, E., Ballouche, A., Gallay, A., Guindo, N., Kéita, D., Kouti, S., Le Drezen, Y., Mayor, A., Neumann, K., Ozainne, S., Perret, S., Rasse, M., Robion-Brunner, C., Schaer, K., Serneels, V., Soriano, S., Stokes, S. \& Tribolo, C. 2005. La septième campagne de terrain à Ounjougou (Mali) et ses apports au programme interdisciplinaire Paléoenvironnement et peuplement humain en Afrique de l'Ouest. In: SLSA Jahresbericht 2004. Fondation SuisseLiechtenstein pour les recherches Archéologiques à l'étranger, Zurich-Vaduz, pp. 57-142.

Huysecom, E., Mayor, A., Ozainne, S., Rasse, M., Schaer, K. \& Soriano, S. 2004. Ounjougou : plus de 100000 ans d'histoire en pays dogon (Mali). Archéologie Suisse 27(3), 213.

Huysecom, E., Ozainne, S., Schaer, K., Ballouche, A., Blench, R., Douyon, D., Guindo, N., Kéita, D., Le Drezen, Y., Neumann, K., Perret, S., Rasse, M., Robion-Brunner, C., Serneels, V., Soriano, S. \& Tribolo, C. 2006. Peuplement humain et évolution paléoclimatique en Afrique de l'Ouest : apports de la huitième année de recherches interdisiplinaires. In: SLSA Jahresbericht 2005. Fondation Suisse-Liechtenstein pour les recherches Archéologiques à l'étranger, Zurich-Vaduz, pp. 79-160.

Iordanova, I., Surtchey, M., Forcey, K.S. \& Krastev, V. 2000. High-temperature surface oxidation of low-carbon rimming steel. Surface and Interface Analysis 30, 158160.
Jenkins, R. 1999. X-Ray Fluorescence Spectrometry. Analytical Chemistry and its Application 152. John Wiley \& Sons, New-York.

Johansson, S.-O. \& Learmonth, J. 1967. Nigerian currencies: manillas, cowries, and others. Alfa-Tryck, Norrköping.

Joly, S., Mercey, F., Filipinni, A., Abenzoar, V., Liard, M. \& Poupon, F. 2011. Un nouvel habitat du Bronze final IIIb dans le Val d'Orléans et ses traces de métallurgie du fer : Bonnée, les Terres à l'Est du Bourg (Centre, Loiret). Revue archéologique $d u$ Centre de la France 50, 1-43.

Kienon-Kaboré, T.H. 2003. La métallurgie ancienne du fer au Burkina Faso. Approche ethnologique, historique, archéologique et métallographique. L'Harmattan, Paris.

Klein, C. \& Hurlbut, C.S.J. 1985. Manual of Mineralogy - 20th edition. John Wiley and Sons, New York.

König, K. 2011. Finsterhennen, Uf der Höchi. Eine hochmittelalterliche Wüstung im Berner Seeland. Verlag Rub Media, Berne.

Lagardelle, G. 1926. Manuel $d u$ forgeron (reédition). Editions Emotion Primitive, Fontaine.

Landolt, D. 1993. Corrosion et chimie de surfaces des métaux. Traité des Matériaux 12. Presses polytechniques et universitaires romandes, Lausanne.

Le Carlier, C., Leroy, M. \& Merluzzo, P. 2007. L'apport de l'analyse morphologique, microscopique et chimique des scories en forme de culot à la restitution des activités de forge. Archeosciences 31, 23-35.

Le Drezen, Y. \& Ballouche, A. 2009. Dynamiques récentes des paysages végétaux en domaine soudano-sahélien. L'exemple de la moyenne vallée du Yamé (Ounjougou, Pays Dogon, Mali). Bulletin de l'Association des Géographes Français 1, 46-66.

Leblanc, J.-C. 2002. Sur l'identification de la chaîne opératoire de forgeage du fer antique, associée à l'archéométrie des battitures. Thèse de doctorat, Université Paul Sabatier, Toulouse

Leroy, M. 1997. La Sidérurgie en Lorraine avant le haut fourneau. L'utilisation du minerai de 
fer oolithique en réduction directe. Monographie du CRA 18. CNRS-Editions, Paris.

Leroy, M. \& Merluzzo, P. 2004. La réduction : du minerai au métal. In: Mangin, M. (ed.), Le fer. Editions Errance, Paris, pp. 49-80.

Leroy, M. \& Merluzzo, P. 2007. Déchets d'activités sidérurgiques de Saint-Martin-desChamps. In: Milcent, P.-Y. (ed.), BourgesAvaricum : un centre proto-urbain celtique du $V^{e}$ s. av. J.-C. Les fouilles de SaintMartin-des-Champs et les découvertes des établissements militaires. Editions de la ville de Bourges, Bourges, pp. 168-188.

Malapeyre, F. 1843. Le Technologiste. Archives des progrès de l'industrie française et étrangère 4. Université Royale de France, Paris.

Mangin, M. 2004. Introduction. In: Mangin, M. (ed.), Le fer. Editions Errance, Paris, pp. 523.

Mangin, M., Courtadon, J.-L., Fluzin, P. \& de Laclos, E. 2000a. Village, forges et parcellaire aux Sources de la Seine. L'agglomération antique de BlesseySalmaise (Côte-d'Or). Annales littéraires de l'Université de Franche-Comté 699, Série "Environnement, Sociétés, Archéologie". Presses universitaires franccomtoises, Marmagne.

Mangin, M., Dabosi, F., Domergue, C., Fluzin, P., Leroy, M., Merluzzo, P., Ploquin, A. \& Serneels, V. 2004. Le fer. Collection "Archéologiques". Editions Errance, Paris.

Mangin, M., Fluzin, P., Courtadon, J.-L. \& Fontaine, M.-J. 2000b. Forgerons et paysans des campagnes d'Alésia (Haut-Auxois, Côte$\left.d^{\prime} O r\right), I^{e r}$ siècle avant-VIII ${ }^{e}$ siècle après J.C. Monographie du CRA 22. CNRS Editions Paris.

Martinelli, B. 1995. Transmission de savoir et évolution des techniques métallurgiques dans la boucle du Niger. In: $L a$ transmission des connaissances techniques, Actes des tables rondes d'Aixen-Provence, Avril 1993-Mai 1994, Aix-enProvence. Publications de l'Université de Provence, Aix-en-Provence, pp. 163-188.

Martinelli, B. 1996. Sous le regard de l'apprenti. Paliers de savoir et d'insertion chez les forgerons Moose du Yatenga (Burkina Faso). Techniques et cultures 58, 9-47.
Martinelli, B. 1998. La mémoire en travail. A propos de la forge au Burkina Faso. Les Territoires du Travail 1, 65-76.

Mauvilly, M., Antenen, I., Garcia Cristobal, E., Ruffieux, M. \& Serneels, V. 1998. Sévaz "Tudingues" : chronique d'un atelier de métallurgistes du début de La Tène dans la Broye. Archéologie Suisse 21(4), 144-154.

Mayor, A. 2011. Traditions céramiques dans la boucle du Niger. Ethnoarchéologie et histoire du peuplement au temps des empires précoloniaux. Journal of African Archaeology Monograph Series 7, Peuplement humain et paléoenvironnement en Afrique de l'Ouest 2. Africa Magna Verlag, Frankfurt am Main.

Mayor, A., Huysecom, E., Gallay, A., Rasse, M. \& Ballouche, A. 2005. Population dynamics and paleoclimate over the padt 3000 years in the Dogon Country, Mali. Journal of Anthropological Archaeology 24, 25-61.

Mayor, A., Huysecom, E., Ozainne, S. \& Magnavita, S. 2014. Early social complexity in the Dogon country (Mali) as evidenced by a new chronology of funerary practices. Journal of Anthropological Archaeology 34, 17-41.

McDonnell, J.G. 1984. The Study of Early Iron Smithing Residues. In: The Crafts of the Blacksmith, Symposium of the Comite pour la sidérurgie ancienne de l'UISPP, 16-21 September 1984, Belfast. UISPP Belfast, pp. 47-52.

McDonnell, J.G. 1986. The classification of Early Ironworking Slags. Thèse de doctorat, University of Aston, Birmingham.

McDonnell, J.G. 1991. A model for the formation of smithing slags. In: Materialy Archeologiczne XXVI, Symposium of the Comité pour la sidérurgie ancienne de l'UISPP, 18-22 September 1989, KielceAmeliówka. Muzeum Archeologiczne w Krakowie, Kraków, pp. 23-26.

Menez, Y., Vivet, J.-B., Chanson, K. \& Dupré, M. 2007. La forge de Paule (Côtes-d' Armor). In: L'économie du fer protohistorique : de la production à la consommation du métal, Actes du XXVIII colloque de l'AFEAF, 2023 mai 2004, Toulouse. Fédération Aquitania, Bordeaux, pp. 213-237. 
Milcent, P.-Y. 2007. Bourges-Avaricum : un centre proto-urbain celtique du $V^{e} s$. av. J.-C. Les fouilles de Saint-Martin-des-Champs et les découvertes des établissements militaires. Bituriga, Archéologie de la Cité 1. Editions de la ville de Bourges, Bourges.

Mills, A. \& McDonnell, J.-G. 1992. The identification and analysis of the hammerscale from Burton Dassett, Warwickshire. Ancient Monuments Laboratory 47. English Heritage, London.

Nuoffer, P. \& Menna, F. 2001. Le vallon de Pomy et Cuarny (VD) de l'âge du Bronze au haut Moyen Age. Cahiers d'Archéologie Romande 82. Lausanne.

Orengo, L., Frénée, E. \& Fluzin, P. 2000. Un atelier de forge de l' âge du Fer au "Bois du Jarrier 3", commune de La-Celle-sur-Loire (Nièvre, France). Archéologie et archéométrie. In: Iron, Blacksmiths and Tools. Ancient European Crafts, Acts of the Instrumentum Conference, April 1999, Podsreda (Slovenia). Editions Monique Mergoil, Montagnac, pp. 45-66.

Oudbashi, O., Mohammadamin Emani, S. \& Bakhshandehfard, H. 2009. Preliminary archaeometallurgical studies on mineralogical structure and chemical slag from Haft Tepe, Khuzestan, South-West Ian (middle Elamite period). In: Proceedings of the $36^{\text {th }}$ International Symposium on Archaeometry, 3-6 May 2006, Quebec City. CELAT/Université de Laval, Quebec, pp. 407-412.

Ozainne, S. 2013. Un Néolithique Ouest-Africain. Cadre chrono-culturel, économique et environnemental de l'Holocène récent en Pays dogon (Mali). Journal of African Archaeology Monograph Series 8, Peuplement humain et paléoenvironnement en Afrique de l'Ouest 3. Africa Magna Verlag, Frankfurt am Main.

Pagès, G., Schneider, L. \& Fluzin, P. 2005. Le travail du fer dans l'établissement perché tardoantique du Roc de Pampelune (Argelliers, Hérault) : l'apport des analyses métallographiques. Archeosciences 29, 107-116.

Perret, S. 2002. Etude quantitative et technologique des vestiges sidérurgiques d'une forge d'époque romaine à Etagnières (Vaud). Travail de diplôme non publié, Université de Genève, Genève.
Perret, S. 2009. Les vestiges sidérurgiques. In: Reymond, S. (ed.), La villa romaine du Buy et sa forge. Dernières découvertes à Cheseaux, Morrens et Etagnières (canton de Vaud, Suisse). Cahiers d'Archéologie Romande, Lausanne, pp. 83-98.

Perret, S. \& Serneels, V. 2009. Technological characterisation and quantification of a large-scale iron smelting site in Fiko (Dogon Plateau, Mali). In: Proceedings of the $36^{\text {th }}$ International Symposium on Archaeometry, 3-6 May 2006, Quebec City. CELAT/Université de Laval, Quebec, pp. 453-463.

Philibert, J., Vignes, A., Bréchet, Y. \& Combrade, P. 1997. Métallurgie, du minerai au matériau. Masson, Paris.

Ploquin, A., Orzechowski, S. \& Briand, B. 1999. Paléométallurgie à Mleiha : une première approche. In: Mleiha 1. Environnement, Stratégies de subsistance et artisanats. Maison de l'Orient et de la Méditerranée, Lyon, pp. 171-190.

Rasse, M., Tribolo, C., Soriano, S. \& Huysecom, E. 2012. Premières données chronostratigraphiques sur les formations du Pléistocène supérieur de la "falaise" de Bandiagara (Mali, Afrique de l'ouest). Quaternaire 23(1), 3-21.

Reed, S.J.B. 2006. Electron Microprobe Analysis and Scanning Electron Microscopy in Geology. Cambridge University Press, Cambridge.

Renzi, M. \& Rovira, S. 2009. Slags of early iron production in the iberian peninsula. In: Proceedings of the $36^{\text {th }}$ International Symposium on Archaeometry, 3-6 May 2006, Quebec City. CELAT/Université de Laval, Quebec, pp. 399-406.

Reymond, S., Eschbach, F. \& Perret, S. 2009. La villa romaine du Buy et sa forge. Dernières découvertes à Cheseaux, Morrens et Etagnières (canton de Vaud, Suisse). Cahiers d'Archéologie Romande 115. Lausanne.

Ricker, R.W. \& Osborn, E.F. 1954. Additional Phase Equilibrium Data for the System CaO$\mathrm{MgO}-\mathrm{SiO}_{2}$. Journal of the American Ceramic Society 37(3), 133-139. 
Robion-Brunner, C. 2010. Forgerons et sidérurgie en pays dogon. Vers une histoire de la production du fer sur le plateau de Bandiagara (Mali) durant les empires précoloniaux. Journal of African Archaeology Monograph Series 3, Peuplement humain et paléoenvironnement en Afrique de l'Ouest 1. Africa Magna Verlag, Frankfurt am Main.

Sahama, T.G. \& Hytönen, K. 1958. Calcium-bearing magnesium-iron olivines. The American Mineralogist 43(sept-oct), 862-871.

Samuels, L.E. 1999. Light Microscopy of Carbon Steels. ASM International, Ohio.

Schairer, J.F. \& Bowen, N.L. 1938. The System, Leucite-Diopside-Silica. American Journal of Science 35A, 189-309.

Schairer, J.F. \& Osborn, E.F. 1950. Additionel Phase Equilibrium Data for the System CaO$\mathrm{MgO}-\mathrm{FeO}-\mathrm{SiO}_{2}$. Preliminary Data on the Join $\mathrm{CaSiO}_{3}-\mathrm{MgO}-\mathrm{FeO}$. Journal of the American Ceramic Society 33(5), 160-167.

Schucany, C. 2006. Die römische Villa von BiberistSpitalhof (SO) (Grabungen 1982, 1983, 1986-1989). Ausgrabunden und Forschungen 4, Band 2. Verlag Bernhard Albert Greiner, Remshalden.

Seignobos, C. 2000. Nomenclature commentée des instruments aratoires du Cameroun. In: Seignobos, C., Marzouk, Y. \& Signaut, F. (eds.), Outils aratoires en Afrique. Innovation, normes et traces. Karthala/IRD, Paris, pp. 297-337.

Serneels, V. 1993. Archéométrie des scories de fer. Recherches sur la sidérurgie ancienne en Suisse occidentale. Cahiers d'Archéologie Romande 61. Lausanne.

Serneels, V. 2001. Les déchets métallurgiques des sites de Pomy-Cuarny. In: Nuoffer, P. \& Menna, F. (eds.), Le vallon de Pomy et Cuarny (VD) de l'âge du Bronze au haut Moyen Age. Cahiers d'Archéologie Romande 82, Lausanne, pp. 245-249.

Serneels, V. 2004. Le fer et ses minerais. In: Mangin, M. (ed.), Le fer. Editions Errance, Paris, pp. 25-48.

Serneels, V. 2006. Les déchets métallurgiques. In: Schucany, C. (ed.), Die römische Villa von Biberist-Spitalhof (SO) (Grabungen 1982,
1983, 1986-1989). Verlag Bernhard Albert Greiner, Remshalden, pp. 520-533.

Serneels, V. 2011. Scories. In: König, K. (ed.), Finsterhennen, Uf der Höchi. Eine hochmittelalterliche Wüstung im Berner Seeland. Verlag Rub Media, Berne, pp. 124-135.

Serneels, V., Chardron-Picault, P. \& Ploquin, A. 1999. Le travail du fer et les objets en fer In: Chardron-Picault, P. \& Pernot, M. (eds.), Un quartier antique d'artisanat métallurgique à Autun (Saône et Loire). Le site $d u$ Lycée militaire. Editions de la Maison des Sciences de l'Homme, Paris, pp. 201-213.

Serneels, V. \& Doswald, C. 2005. Etude des déchets métallurgiques. In: Hofmann Rognon, P. (ed.), Le Landeron-Les Carougets. Vestiges protohistoriques, villa romaine, tombe et habitat médiévaux. Service et musée cantonal d'archéologie de Neuchâtel, Neuchâtel, pp. 117-125.

Serneels, V. \& Huysecom, E. A paraître. Tiégé Jo et le savoir-faire des forgerons dogon (Afrique occidentale, Mali) : étude d'une forge. In: Actes du congrès international "Orfèvres et forgerons", 17 octobre 2001, Université Toulouse 2 - Le Mirail. Editions Errance, Paris.

Serneels, V., Huysecom, E. \& Fluzin, P. 1997. Inagina Iron, slags and eutectoïd steel. In: Early Ironworking in Europe, archaeology and experiment, Abstracts of the International Conference, 19-25 September 1997, Plas Tan y Bwlch. Maentwrog, pp. 104-110.

Serneels, V., May Castella, C. \& Berti Rossi, S. 2005. Les traces de métallurgie dans le vicus de Lousonna. In: Berti Rossi, S. \& May Castella, C. (eds.), La fouille de Vidy "Chavannes 11" 1989-1990. Archéologie, architecture et urbanisme. Cahier d'Archéologie Romande 102, Lausanne, pp. 287-302.

Serneels, V., Merluzzo, P. \& Leroy, M. 2004. Les activités de forge : le travail du métal. In: Mangin, M. (ed.), Le fer. Editions Errance, Paris, pp. 81-112.

Serneels, V. \& Perret, S. 2003. Quantification of smithing activities based on the investigation of slag and other material remains. In: Archaeometallurgy in Europe, 
Proceedings of the International Conference, 24-25-26 september 2003, Milan. Associazione italiana di metallurgia, Milan, pp. 469-478.

Serneels, V. \& Perret, S. 2007. Les scories de forge de La Tène finale. In: Anderson, T. \& Castella, D. (eds.), Une ferme gauloise à Courgevaux (Fribourg, Suisse). Academic Press Fribourg, Fribourg, pp. 135-152.

Serneels, V., Robion-Brunner, C. \& Perret, S. 2006. La sidérurgie en pays dogon : problématiques, premiers résultats et perspectives. Etudes Maliennes 65, 113126.

Sim, D. 1998. Beyond the Bloom. Bloom refining and iron artifact production in the Roman world. BAR International Series 725. Archeopress, Oxford.

Soulignac, R. \& Serneels, V. 2013. Forging with Dogon smiths (Mali). In: Dungworth, D. \& Doonan, R.C.P. (eds.), Accidental and Experimental Archaeometallurgy. The Historical Metallurgy Society, 119-126.

Starley, D. 1995. Hammerscale. HMS Archaeometallurgy Datasheet 10, epublication : $\quad$ http://histmet.org/datasheets.html.

Tauber, J. 1998. Die Eisenwerker im Röserntal. In: Ewald, J. \& Tauber, J. (eds.), Tatort Vergangenheit: Ergebnisse aus der
Archäologie heute. Wiese Verlag Basel, Basel, pp. 241-266.

Turnock, A.C. \& Eugster, H.P. 1962. Fe-Al Oxides: Phase Relationships below $1000^{\circ} \mathrm{C}$. Journal of Petrology 3(3), 533-565.

Unglik, H. 1991. Observations on the structures and formation of microscopic smithing residues from Bixby Blacksmith Shop at Barre Four Corners, Massachussetts, 1824-55. Historical metallurgy 25(2), 92-98.

Vander Voort, G.F. 1999. Metallography. Principles and Practice. ASM International, Ohio.

Vander Voort, G.F. 2004. Metallography and Microstructures. ASM Handbook 9. ASM International, Ohio.

VDEh 1995. Slag Atlas, 2nd Edition. Verlag Stahleisen GmbH, Düsseldorf.

von Maydell, H.-J. 1990. Arbres et arbustes du Sahel: leurs caractéristiques et leurs utilisations. Verlag Josef Margraf, Weikersheim.

Warren, B.E. 1990. X-Ray Diffraction. Dover publications, New-York.

Young, R.A. 1996. The Rietveld Method. International Union of Crystallography Monographs on Crystal 5. Oxford University Press, Oxford. 
This publication is also available open-access on

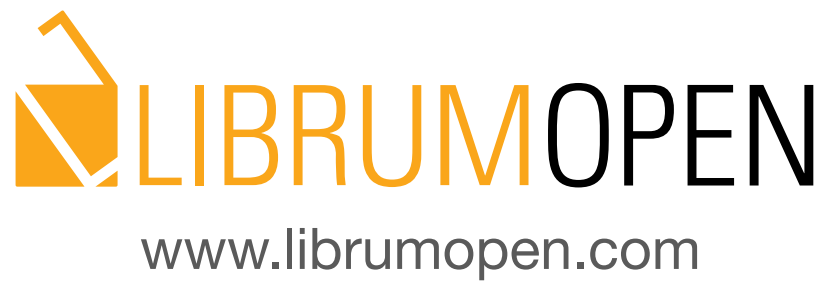

or as printed version at

\section{SLIBRUMSTORE www.librumstore.com}

